\begin{tabular}{|l|l|}
\hline \begin{tabular}{l} 
2. To: $\begin{array}{c}\text { (Receiving Organization) } \\
\text { Distribution }\end{array}$ \\
\hline $\begin{array}{l}\text { 5. Proj./Prog./Dept./Div.: } \\
\text { Tank 241-T-204/Waste } \\
\text { Management/PPC/AS }\end{array}$
\end{tabular} & $\begin{array}{l}\text { 6. Design Authority/ Design Agent/Cog. } \\
\text { Engr.: } \\
\text { Jennifer L. Nuzum } \\
\text { and Contronting Organization) }\end{array}$ \\
\hline $\begin{array}{l}\text { 8. Originator Remarks: } \\
\text { This document is being released into the supporting document } \\
\text { systme for retrievability purposes. }\end{array}$
\end{tabular}

11. Receiver Remarks: 11A. Design Baseline Document? [] Yes [X] No For release.

4. Related EDT No.:
N/A

9. Equip./Component No.: N/A

10. System/Bldg./Facil ity: 241-T-204

12. Major Assm. Dwg. No.: N/A

13. Permit/Permit Application No.: N/A

14. Required Response Date: $07 / 28 / 97$

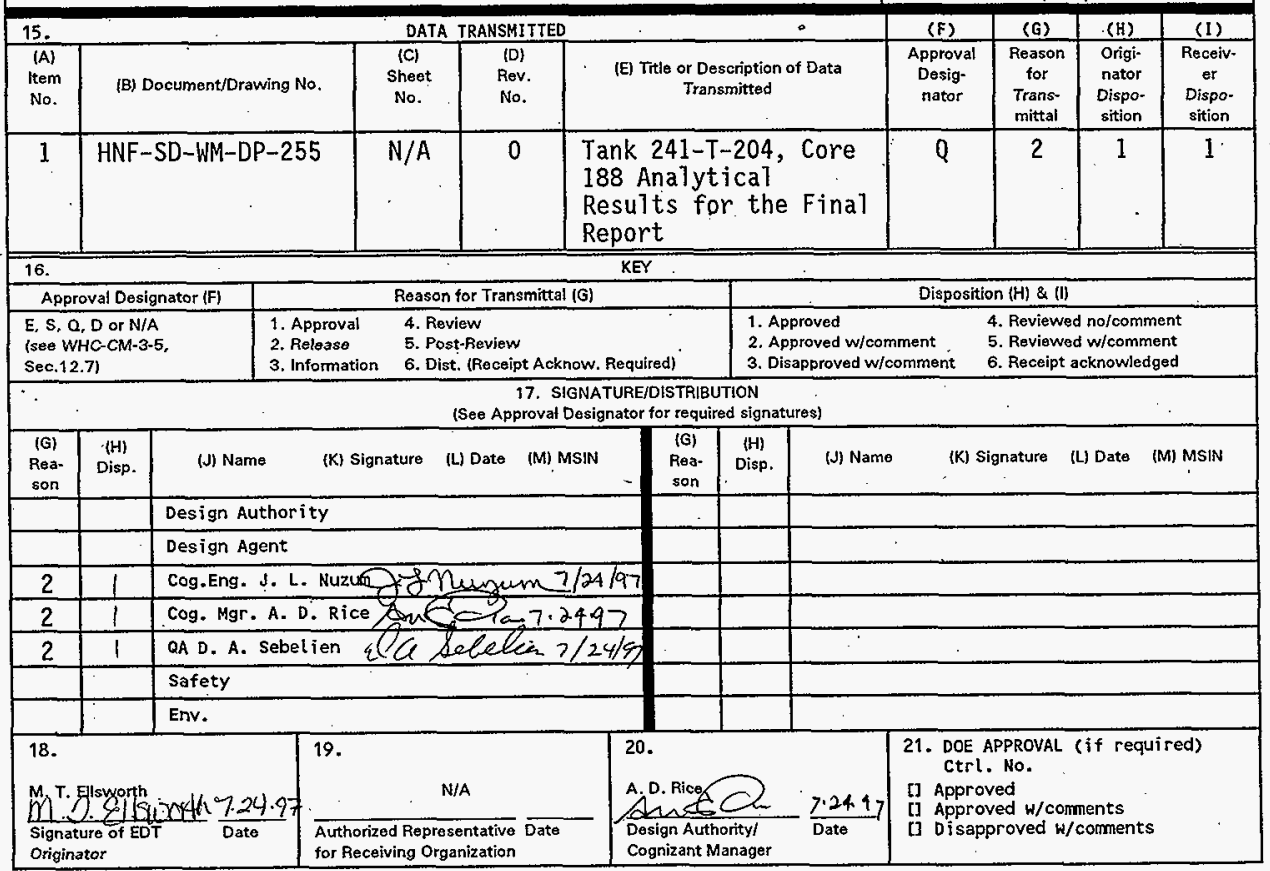




\title{
Tank 241-T-204, Core 188 Analytical Results for the Final Report
}

\author{
Jennifer L. Nuzum \\ Waste Management of Hanford, Inc., Richland, WA 99352 \\ U.S. Department of Energy Contract DE-AC06-96RL13200
}

EDT/ECN: EDT-620412 UC: 2070

Org Code: $7576 \mathrm{~B}$

Charge Code: MD378

B\&R Code: EW 3120074 Total Pages: for

Key Words: Tank 241-T-204, 241-T-204, T-204, Core 188, Analytical Results, Final Report

Abstract: $N / A$

TRADEMARK DISCLAIMER. Reference herein to any specific comercial product, process, or service by trade name, trademark, manufacturer, or otherwise, does not necessarily constitute or imply its endorsement, recommendation, or favoring by the United States Government or any agency thereof or its contractors or subcontractors.

Printed in the United States of America. To obtain copies of this document, contact: Document Control Services, P.0. Box 950, Mailstop H6-08, Richland WA 99352, Phone (509) 372-2420; Fax (509) 376-4989.
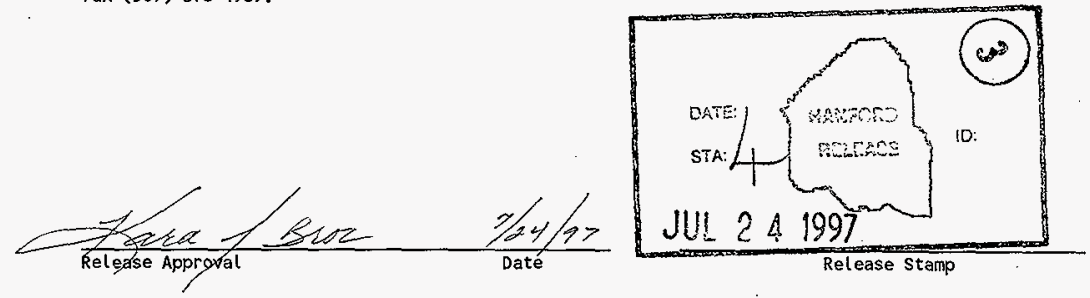
HNF-SD-WM-DP-255, REV, 0

ANALYTICAL SERVICES

TANK 241-T-204, CORE 188, ANALYTICAL RESULTS FOR THE FINAL REPORT

Project Coordinator: JENNIFER L. NUZUM

Prepared for the U.S. Department of Energy

office of Environmental Restoration and Waste Management

by

222-S Laboratory

Waste Management of Hanford, Inc.

P.0. Box 700

Richland, Washington 
HNF-SD-WM-DP-255, REV. 0

THIS PAGE INTENTIONALLY LEFT BLANK 
HNF-SD-HM-DP-255, REV. 0

TABLE OF CONTENTS

Narrative ...................... 1

T-204 Sample Breakdown (Attachment 1) . . . . . . . . I3

Sample Data Summary . . . . . . . . . . . . . . . . 27

Chain of Custody Forms .................... 43

Photographs .................... . 56

Composite Worksheets ................... 69

Sample Handling . . . . . . . . . . . . . . . . 77

Extrusion Workl ist \# $17438(451)$. . . . . . . . . . . . 79

Extrusion Worklist \# $17566(534)$. . . . . . . . . . . . . 80

Extrusion Worklist \# $17567(535) \ldots \ldots$. . . . . . . . 81

Extrusion Worklist \# $17568(536)$. . . . . . . . . . . . .82

Extrusion Workl ist \# $17569(565) \ldots . . . . . . . . .83$

Extrusion Workl ist \# $17570(564)$. . . . . . . . . . . 84

Extrusion Workl ist \# $17571(563)$. . . . . . . . . . . 85

Extrusion Workl ist \# $17602(566)$. . . . . . . . . . . . .86

Extrusion Workl ist \# $17603(568)$. . . . . . . . . . . .87

Extrusion Work1 ist \# $17604(567) \quad \ldots . . . . . . . . . .88$

Extrusion Worklist \# $17605(569)$............ . 89

Sample Preparations ................. . . 90

Fusion Digest Worklist \# $17711(587,588,589) \ldots \ldots . . . . . . .92$

Fusion Digest Worklist \# $17712(590,621,622) \ldots \ldots . . . . . .93$

Fusion Digest Worklist \# $17713(623,624,625,626) \ldots \ldots . . . . .94$

Fusion Digest Work1 ist \# 18345 (1198) ........... 96

H20 Digest Work1 ist \#18346 (1200) . . . . . . . . . . . . 97

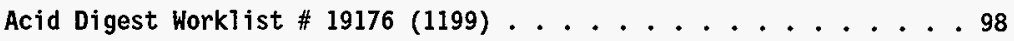

Bulk Density Worksheets . . . . . . . . . . . . . 99

Inorganic Analysis ................. . . 112

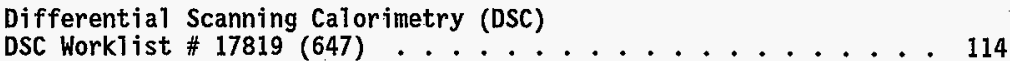

DSC Work1 ist \# $17819(647), 49 . \cdots \ldots \ldots$

DSC Work7 ist \# $17829(574,575) \ldots \ldots \ldots \ldots \ldots$

DSC Workl ist \# $17830(576,580) \ldots \ldots \ldots \ldots . . \ldots . . \ldots 131$

DSC Work1 ist \# $17831(603,604) \ldots \ldots . . . . \ldots . . . . .138$

DSC Work1 ist \#17832 $(605,606) \ldots \ldots . \ldots . \ldots 145$ 
HNF-SD-WM-DP-255, REV. 0

TABLE OF CONTENTS (Continued)

DSC Workl ist \# $17833(607,608) \ldots \ldots \ldots \ldots$

DSC Worklist \# $17834(633,634) \ldots \ldots \ldots \ldots . . \ldots \ldots$

DSC Work1 ist \# $17835(635,636) \ldots \ldots \ldots \ldots . . . . . . . . .166$

DSC Work1 ist \# $17836(637,638) \ldots \ldots . . . . . . . . .173$

DSC Workl ist \# $18349(498,499,574,575,576,580,603,604,605) 180$

DSC Work1 ist \# $18350(606,607,608,633,634,635,636,637,638) 182$

DSC Workl.ist \# 18351 (647) . . . . . . . . . . . . . 184

DSC Worklist \# 18689 (1197) . . . . . . . . . . . . . 185

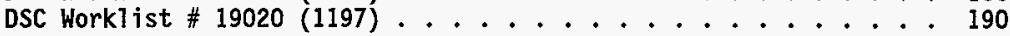

Thermogravimetric Analysis (TGA)

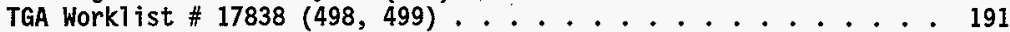

TGA Work1 ist \# $17839(574,575) . . \ldots . . . . . . . . .197$

TGA Work1 ist \# $17842(605,606) \ldots . . . . . . . . .204$

TGA Workl ist \# $17843(607,608) \ldots . . . . . . . . . .211$

TGA Work1 ist \# $17844(633,634) \ldots . . . . . . . . . . .218$

TGA Workl ist \# $17845(635,636) \ldots \ldots . . . . . . . .225$

TGA Work1 ist \# $17846(637,638) \ldots . . . . . . . . . . .232$

TGA Workl ist \# 17847 (647) .................. . 239

TGA Workl ist \# $18239(576,580,603) \ldots . . . . . . . . .243$

TGA Workl ist \# $18240(604,637,638) \ldots . . . . . . . .252$

TGA Workl ist \# 18685 (1197) . . . . . . . . . . . 260

Specific Gravity AnaTysis (SpG)

SpG Workl ist \# $17820(647) \ldots \ldots . . \ldots 265$

Ion Chromatography (IC)

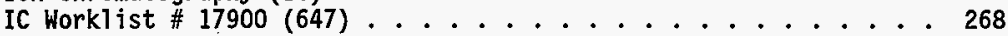

IC Workl ist \# $19440(1200) \ldots \ldots . \ldots . . \ldots 273.1$

Inductively Coupled Plasma Spectrophotometer (ICP)

ICP Work1 ist \# $17851(647)$. . . . . . . . . . . . . . . 282

ICP Worklist \# $19295(1199) \ldots . . . . . . . . . .288$

Total Inorganic / Total Organic (TICTOC)

TICTOC Worklist \# 18752 (1197) . . . . . . . . . . . 307

Radiochemical Anatysis ................ 326

Total Alpha (AT)

AT Workl ist \# 17815 (647) . . . . . . . . . . . . . . . 328

AT Work 1 ist \# $17817(590,621,622) \ldots \ldots . . . . . . . . .334$

AT Work 1 ist \# $17901(623,624,625,626) \ldots . . . . . . .345$

AT Workl ist \# $17950(587,588,589) \ldots . . . . . . . .357$ 
HNF-SD-WM-DP-255, REV. 0

TABLE OF CONTENTS (Continued)

Gamma Energy Analysis (GEA)

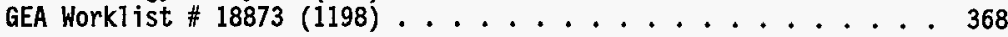

Strontium 89/90 Analysis (Sr90)

Sr90 Worklist \# $18756(1198)$. . . . . . . . . . . . . 387

This Document consists of pages 1 through 392. Pages $i i, 2,14,28,44,57$, $70,78,91,100,113$ and 327 were intentionally left blank.

TRADEMARKS:

Perkin-Elmer is a Registered Trademark of Research and Manufacturing Company, Inc. Mettler is a Registered Trademark of Mettler Electronics. 
HNF-SD-WM-DP-255, REV. 0

NARRATIVE 


\title{
HNF-SD-WM-DP-255, REV. 0
}

\section{2-S ANALYTICAL SERVICES}

\author{
TANK 241-T-204, CORE 188, \\ ANALYTICAL RESULTS FOR THE FINAL REPORT
}

\section{Summary}

This document is the final laboratory report for Tank 241-T-204. Push mode core segments were removed from Riser 3 between March 27, 1997, and April 11, 1997. Segments were received and extruded at 222-S Laboratory. Analyses were performed in accordance with Tank 241-T-204 Push Mode Core Sampling and Analysis Plan (TSAP) (Winkleman, 1997), Letter of Instruction for Core Sample Analysis of Tanks 241-T-201, 241-T-202, 241-T-203, and 241-T-204 (LOI) (Bell, 1997), and Safety Screening Data Quality Objective (DQO) (Dukelow, et al., 1995).

None of the subsamples submitted for total alpha activity (AT) or differential scanning calorimetry (DSC) analyses exceeded the notification limits stated in DQO. The statistical results of the $95 \%$ confidence interval on the mean calculations are provided by the Tank Waste Remediation Systems Technical Basis Group and are not considered in this report.

\section{Appearance and Sample Handling}

One core of eleven segments was expected from Tank 241-T-204. One core (Core 188) of ten segments was obtained.

Attachment 1 illustrates subsamples generated in the laboratory for analysis and identifies their sources. This reference also relates tank farm identification numbers to their corresponding 222-S Laboratory sample numbers.

\section{Core 188}

Segments were removed from Tank 241-T-204 Riser 3 between March 27, 1997, and April 11, 1997. All segments were received by 222-S Laboratory between April 1, 1997, and April 14, 1997. Table 1 summarizes extrusion information.

\section{Field Blank}

A field blank was provided to 222-S Laboratory with Core 188 . This sample was treated as a drainable liquid in accordance with TSAP. 


\section{HNF-SD-WM-DP-255, REV. 0}

\section{Hydrostatic Head Fluid Blank}

There was no indication of the use of hydrostatic head fluid (HHF) in sampling, nor a HHF blank provided to 222-S Laboratory.

\section{Core Composite}

One solid core composite was made from Core 188 in accordance with LOI. The core composite worksheets are included in this document. 
HNF-SD-WM-DP-255, REV. 0

Table 1. Sample Receipt and Extrusion Information for T-204, Core 188.

\begin{tabular}{|c|c|c|c|c|c|c|c|c|}
\hline 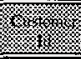 & 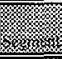 & 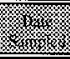 & 1. & 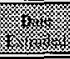 & 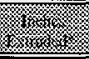 & 19x & 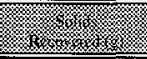 & 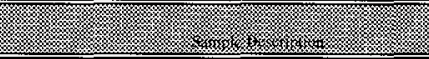 \\
\hline $\begin{array}{l}\text { Field } \\
\text { Blank }\end{array}$ & $\begin{array}{l}\text { Field } \\
\text { Blank }\end{array}$ & $4 / 11 / 97$ & $4 / 15 / 97$ & $4 / 21 / 97$ & 0.0 & 272.8 & 0.0 & Drainable liquid was clear and colorless with no organic layer. \\
\hline $97-60$ & 1 & $3 / 27 / 97$ & $4 / 1 / 97$ & 4/9/97 & 18.0 & 0.0 & $\begin{array}{l}\text { 150.3--Lower Half } \\
\text { 161.2--Upper Half }\end{array}$ & Solids were black and resembled a wet sludge slumy. \\
\hline $97-61$ & 2 & $4 / 11 / 97$ & $4 / 11 / 97$ & $4 / 16 / 97$ & 4.0 & 0.0 & 94.2--Lower Half & Solids were black and resembled a wet sludge. \\
\hline $97-62$ & 3 & $4 / 11 / 97$ & $4 / 11 / 97$ & $4 / 16 / 97$ & 13.0 & 0.0 & 216.6--Lower Half & Solids were black and resembled a wet sludge. \\
\hline 97.63 & 4 & $4 / 11 / 97$ & $4 / 11 / 97$ & $4 / 16 / 97$ & 19.0 & 0.0 & $\begin{array}{l}\text { 166.5--Lower Half } \\
\text { 189.4--Upper Half }\end{array}$ & Solids were black and resembled a dry sludge. \\
\hline $97-64$ & 5 & $4 / 11 / 97$ & $4 / 15 / 97$ & $4 / 18 / 97$ & 19.0 & 0.0 & $\begin{array}{l}\text { 178.1---Lower Half } \\
\text { 177.3--Upper Half }\end{array}$ & Solids were black and resembled a dry sludge. \\
\hline 97.065 & 6 & $4 / 11 / 97$ & $4 / 15 / 97$ & $4 / 18 / 97$ & 19.0 & 0.0 & $\begin{array}{l}\text { 166.6--Lower Half } \\
\text { 194.8--Upper Half }\end{array}$ & Solids were black and resembled a dry sludge. \\
\hline $97-066$ & 7 & $4 / 11 / 97$ & $4 / 15 / 97$ & $4 / 18 / 97$ & 19.0 & 0.0 & $\begin{array}{l}\text { 183.2--Lower Half } \\
\text { 172.7--Upper Half }\end{array}$ & Solids were black and resembled a dry sludge. \\
\hline $97-67$ & 8 & $4 / 11 / 97$ & $4 / 15 / 97$ & $4 / 18 / 97$ & 19.0 & 0.0 & $\begin{array}{l}\text { 193.1--Lower Half } \\
\text { 159.5--Upper Half }\end{array}$ & Solids were black and resembled a dry sludge. \\
\hline $97-68$ & 9 & $4 / 11 / 97$ & $4 / 15 / 97$ & $4 / 21 / 97$ & 17.0 & 0.0 & $\begin{array}{l}\text { 153.7--Lower Half } \\
\text { 173.9--Upper Half }\end{array}$ & Solids were black and resembled a dry sludge. \\
\hline $97-69$ & 10 & $4 / 11 / 97$ & $4 / 15 / 97$ & $4 / 21 / 97$ & 19.0 & 0.0 & $\begin{array}{l}\text { 151.9--Lower Half } \\
\text { 195.3--Upper Half }\end{array}$ & Solids were black and resembled a dry sludge. \\
\hline
\end{tabular}




\section{HNF-SD-WM-DP-255, REV. 0}

\section{Analytical Results Summary}

The Data Summary Table (Table 2) included in this report compiles analytical results associated with each subsample submitted in accordance with TSAP, LOI, and DQO.

Liquid subsamples prepared for analysis by an acid adjustment of the direct subsample are indicated by a " $\mathrm{D}$ " in the A\# column in Table 2 . Solid subsamples prepared for analysis by performing a fusion digest are indicated by a " $F$ " in the A\# column in Table 2. Solid subsamples prepared for analysis by performing a water digest are indicated by a " $\mathrm{W}$ " in the A\# column of Table 2.

\section{Inorganic Analyses}

\section{Differential Scanning Calorimetry (DSC) Analysis}

DSC analysis was performed on all direct subsamples. The exothermic energy based on dry weight of subsample was calculated for all subsamples. The average of the thermogravimetric analysis (TGA) results for each subsample was used in the dry weight correction for that subsample.

DSC analysis results for all subsamples submitted were 0.00 Joules $/ g$. There were no exceptions to the quality control (QC) parameters stated in TSAP for these subsamples.

\section{Thermogravimetric Analysis (TGA)}

TGA was performed on all direct subsamples. TGA results were typically determined by summing weight loss steps below $200^{\circ} \mathrm{C}$. Weight loss steps above this were not used to determine the result. More information may be obtained by examining the raw data.

Upper Half Segment 4 of Core 188 (S97T000580) was analyzed in triplicate because an instrument fluctuation occurred while analyzing the duplicate. The duplicate value was considered invalid, and the triplicate result is shown as the duplicate in Table 2. More information may be obtained by observing the raw data. Upper Half Segment 10 of Core 188 (S97T000638) had a relative percent difference (RPD) of 30.8 between sample and duplicate. This RPD was reproducible due to inhomogeneity between sample and duplicate. Further analysis was not requested. 


\section{HNF-SD-WM-DP-255, REV. 0}

\section{Density}

Bulk density was performed on solid lower half segments and the core composite. Results from bulk density tests ranged from $1.15 \mathrm{~g} / \mathrm{mL}$ to $1.21 \mathrm{~g} / \mathrm{mL}$. There were no QC parameters stated in TSAP for bulk density analysis.

\section{Specific Gravity (SpG)}

Specific gravity analyses were performed on direct liquid subsamples. There were no exceptions to the QC parameters stated in TSAP for these subsamples.

\section{Ion Chromatography (IC)}

IC analysis was performed on direct liquid subsamples and the core composite. For direct liquid subsamples, only the $\mathrm{QC}$ for $\mathrm{Br}$ was reviewed for this report in accordance with TSAP. There were no exceptions to the QC parameters stated in TSAP for these subsamples.

For the core composite (S97T001199), only the QC for requested analytes $\left(\mathrm{F}, \mathrm{Cl}, \mathrm{NO}_{2}, \mathrm{NO}_{3}\right.$, $\mathrm{SO}_{4}, \mathrm{PO}_{4}$, and Oxalate) were reviewed for this report in accordance with LOI. Other opportunistic analyte results are included in Table 2. These analytes do not have customer defined QC parameters and are not discussed. The preparation blank showed $\mathrm{NO}_{2}$ and $\mathrm{NO}_{3}$ results above the detection level. The levels of these analytes in the preparation blank are inconsequential when compared to results for the sample, and do not impact sample data quality. A matrix spike was not performed on S97T001199.. At this time, the IC instrument is out of service. If further analysis of $\mathrm{S} 97 \mathrm{~T} 001199$ is requested, the results will be presented in a revision to this document.

\section{Inductively Coupled Plasma Spectrophotometry (ICP)}

ICP was performed on direct liquid subsamples and the core composite. For direct liquid subsamples, only the QC for Li was reviewed for this report in accordance with TSAP. There were no exceptions to the QC parameters stated in TSAP for these subsamples.

For the core composite (S97T001199), only the QC for requested analytes ( $\mathrm{Al}, \mathrm{Ba}, \mathrm{Bi}, \mathrm{Ca}, \mathrm{Cr}$, $\mathrm{Fe}, \mathrm{La}, \mathrm{Mn}, \mathrm{Ni}, \mathrm{P}, \mathrm{K}, \mathrm{Si}, \mathrm{S}, \mathrm{Na}, \mathrm{Sr}$, and U) were reviewed for this report in accordance with LOI. Other opportunistic analyte results are included in Table 2. These analytes do not have customer defined QC parameters and are not discussed. 


\section{HINF-SD-WM-DP-255, REV. 0}

Sample S97T001199 spike recoveries reported in Table 2 for $\mathrm{Bi}, \mathrm{Fe}, \mathrm{K}, \mathrm{La}, \mathrm{Mn}$, and $\mathrm{Na}$ are invalid because the spike concentration $(1 \mathrm{ppm})$ is too low in comparison to the sample concentration (less than $25 \%$ ). Second spikes with an analyte level of $10 \mathrm{ppm}$ were performed, and the resulting spike recoveries were $104.4 \%, 101.2 \%, 94.7 \%, 102.9 \%, 101.9 \%$, and $99.5 \%$, respectively. These calculations can be found in the raw data. The preparation blank showed $\mathrm{Al}$, $\mathrm{Ca}, \mathrm{Na}$, and $\mathrm{Si}$ results above the detection level. The levels of these analytes in the preparation blank are inconsequential when compared to the results for the sample, and do not impact sample data quality.

\section{Total Organic Carbon (TOC)}

TOC analysis was performed on the core composite (S97T001199). RPD between sample and duplicate was 21.8. Elevated RPD is due to inhomogeneity between sample and duplicate. Further analysis was not requested.

\section{Total Inorganic Carbon (TIC)}

TIC analysis was performed on the core composite (S97T001199). There were no exception to the QC parameters stated in LOI for this sample.

For all TIC analyses, a "Total Inorganic Carbon Analysis Report" worksheet is included as raw data. Due to programming limitations with the TIC instrument software, the sample size listed on this worksheet is incorrect. This value is not used in the final calculations (also included) and has no bearing on the results in Table 2 .

\section{Radiochemistry Analyses}

\section{Total Alpha (AT) Analysis}

$\mathrm{AT}$ analyses were requested for direct liquid subsamples and solid lower half segments. Liquid AT results were below the total alpha activity notification limit of $61.5 \mu \mathrm{Ci} / \mathrm{mL}$. All solid AT results were below the total alpha activity notification limit of $41 \mu \mathrm{Ci} / \mathrm{g}$.

RPD between sample and duplicate was 26.0 for Lower Half Segment 5 of Core 188 (S97T000621). Replicates indicated the elevated RPD was due to inhomogeneity between sample and duplicate. Rerun analysis was not requested.

One of the preparation blanks showed AT results above the detection level. The level in the preparation blank is inconsequential when considering the elevated counting error, and does not impact sample data quality. 


\section{HNF-SD-WM-DP-255, REV. 0}

\section{Gamma Energy Analysis (GEA)}

GEA was performed on the core composite. There were no exceptions to the QC parameters stated in LOI for this subsample.

\section{Strontium $90\left({ }^{90} \mathrm{Sr}\right)$}

${ }^{90} \mathrm{Sr}$ analysis was performed on the core composite (S97T001198). A tracer was used instead of a spike for QC purposes. RPD between sample and duplicate was 49.6. Count rates for both sample and duplicate were very low, with high counting uncertainties, resulting in an elevated RPD. The preparation blank showed ${ }^{90} \mathrm{Sr}$ results above the detection level. The level in the preparation blank is inconsequential when considering the high counting error, and does not impact sample data quality. Rerun analysis was not requested. 


\section{HNF-SD-WM-DP-255, REV. 0}

\section{Procedures}

Table 3 lists the analytical procedures used for performing sample analyses. Abbreviations for analyses are defined in the table notes.

Table 3. Analytical Procedures

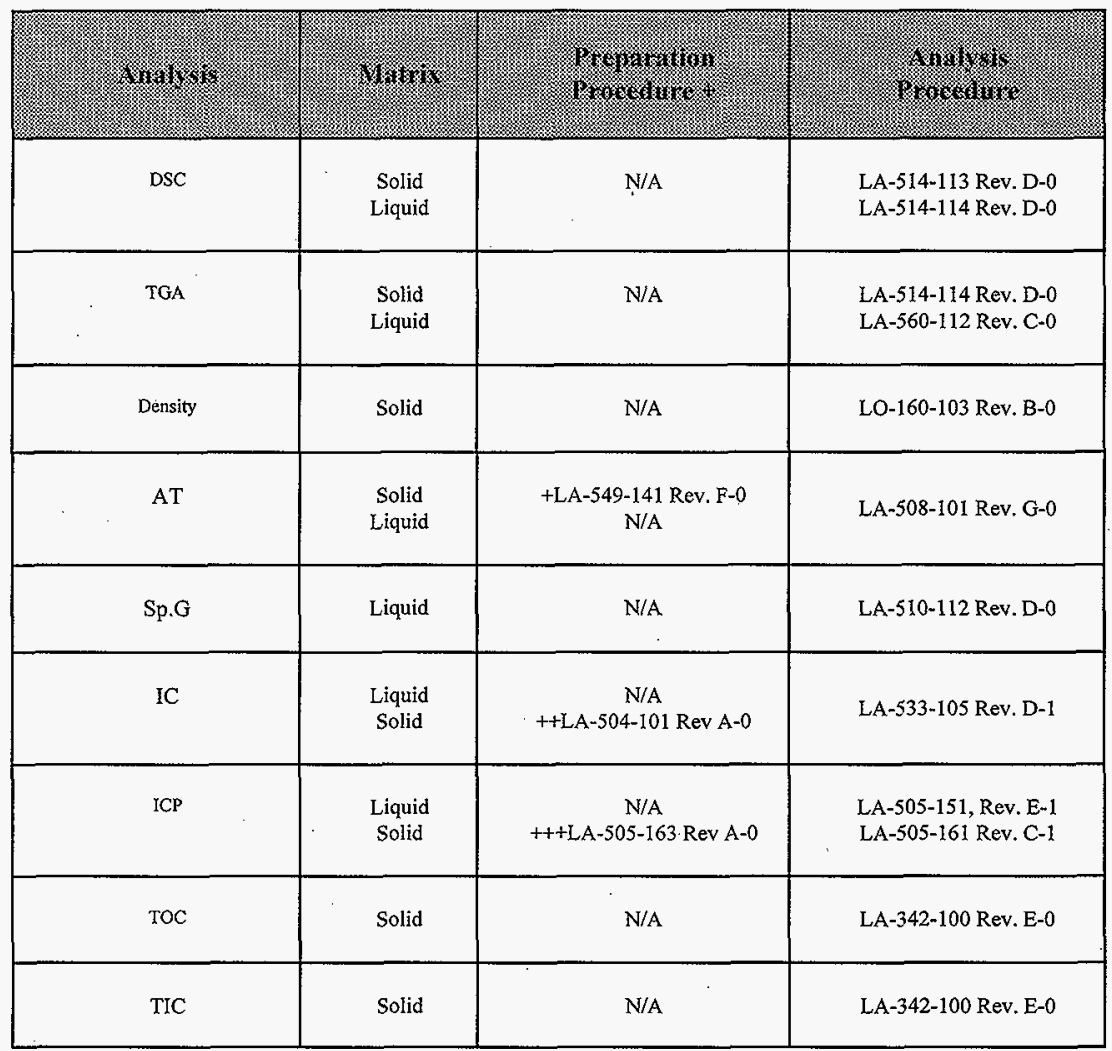


HNF-SD-WM-DP-255, REV. 0

\begin{tabular}{|c|c|c|c|}
\hline GEA & Solid & +LA-549-141 Rev. F-0 & LA-548-121 Rev. B-0 \\
\hline $90 \mathrm{Sr}$ & Solid & +LA-549-141 Rev. F-0 & LA-220-101 Rev. E-2 \\
\hline
\end{tabular}

Notes:

$\begin{array}{ll}\text { N/A } & \text { not applicable (these are direct samples) } \\ \text { DSC } & \text { differential scanning calorimetry } \\ \text { TGA } & \text { thermogravimetric analysis } \\ \text { Density } & \text { bulk density } \\ \text { AT } & \text { total alpha } \\ \text { Sp.G } & \text { specific gravity } \\ \text { IC } & \text { ion chromatography } \\ \text { ICP } & \text { inductively coupled plasma } \\ \text { TOC } & \text { total organic carbon } \\ \text { TIC } & \text { total inorganic carbon } \\ \text { GEA } & \text { gamma energy analysis } \\ 90 \mathrm{Sr} & \text { strontium } 90 \\ & \\ + & \text { fusion digest } \\ ++ & \text { water digest } \\ +++ & \text { acid digest }\end{array}$




\section{HNF-SD-WM-DP-255, REV. 0}

\section{References}

Dukelow, G. T., J. W. Hunt, H. Babad, and J. E. Meacham, 1995, Tank Safety Screening Data Quality Objective, WHC-SD-WM-SP-004, Rev. 2, Westinghouse Hanford Company, Richland, WA 99352.

Winkleman, W.D., 1997, Tank 24I-T-204 Push Mode Core Sampling and Analysis Plan, HNF-SD-WM-TSAP-119, Rev. 0, Lockheed Martin Hanford Corporation, Richland, WA 99352.

Bell, K.E., 1997, Letter of Instruction for Core Sample Analysis of Tanks 241-T-201, 241-T-202, 241-T-203, and 241-T-204, Memo 74620-97-195, Lockheed Martin Hanford Corporation, Richland, WA 99352.

Meznarich, H. K., 1995, Quality Assurance Plan for the 222-S Laboratories, WHC-SD-CPQAPP-016, Rev. 0, Westinghouse Hanford Company, Richland, WA 99352. 
HNF-SD-WM-DP-255, REV. 0

T-204 SAMPLE BREAKDOWN (ATTACHMENT 1) 
HNF-SD-WM-DP-255, REV. 0

THIS PAGE INTENTIONALLY LEFT BLANK 
T-204

Core:188

Seg: Field Blank

S97Tg00568

Field

Blank

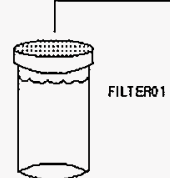

S97T000645

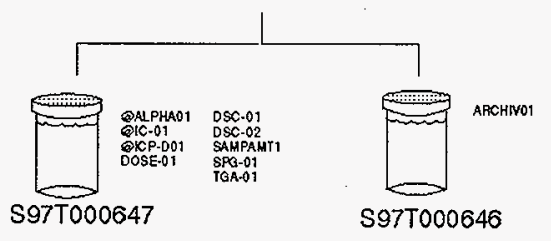

㭊 
Core:188

Seg: 1

S97T000451
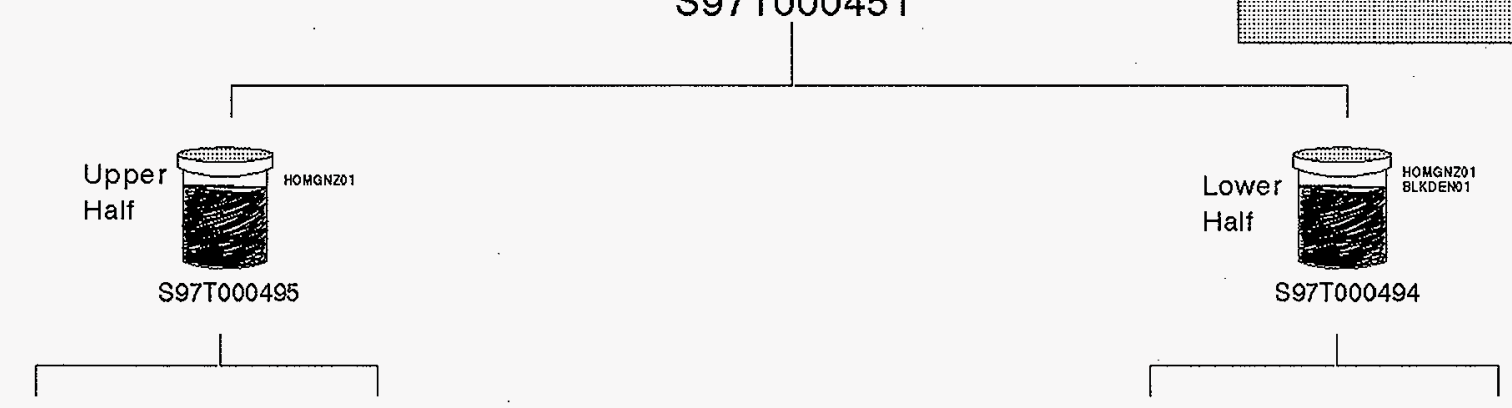

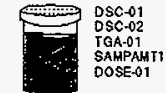

S97T000489

$\theta$

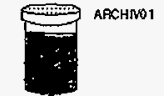

S97T000497

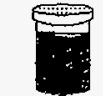

S97T000498
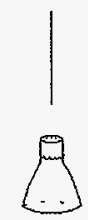

FUSAONO 1

DSSE-OZ

s97T000587 
Core: 188

Seg: 2

S97T000534 
Core: 188

Seg: 3

S97T000535

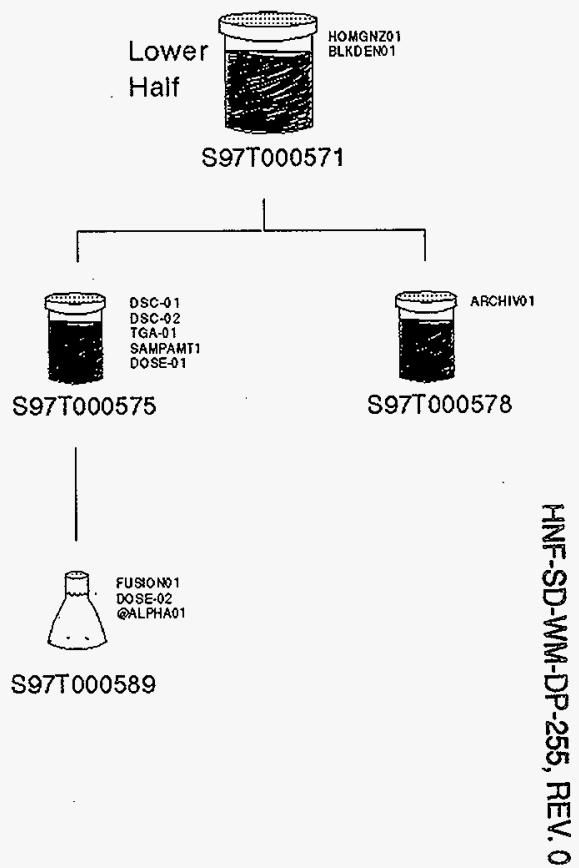


T-204

Core:188

Seg: 4

S97T000536
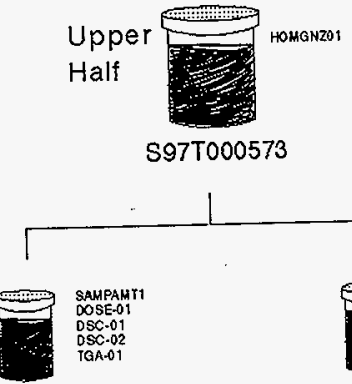

S97T000580

S97TO00581
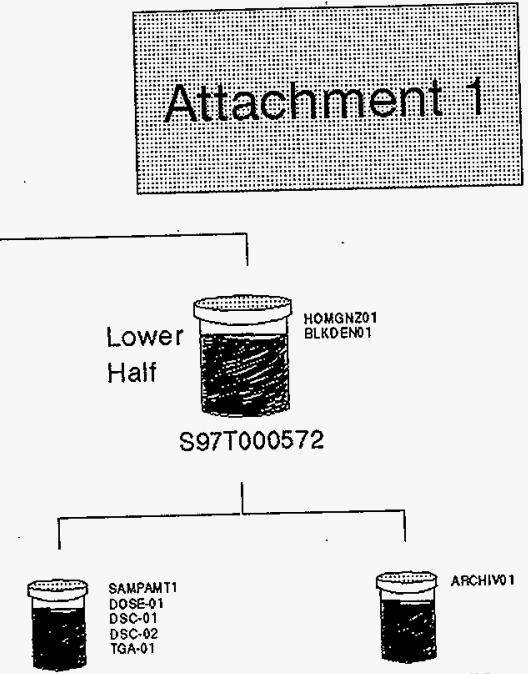

SAMPAMI1

DOSE-O

DSC-01

TQA.01

S97T000576

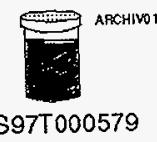

S97T000579 

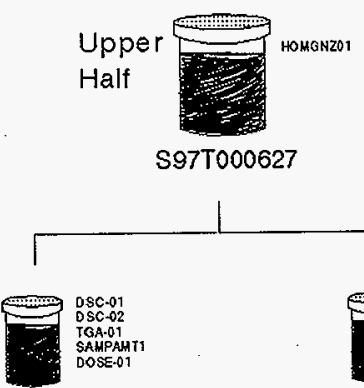

S97T000633

$\infty$

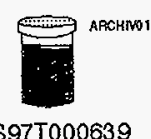

S97T000639
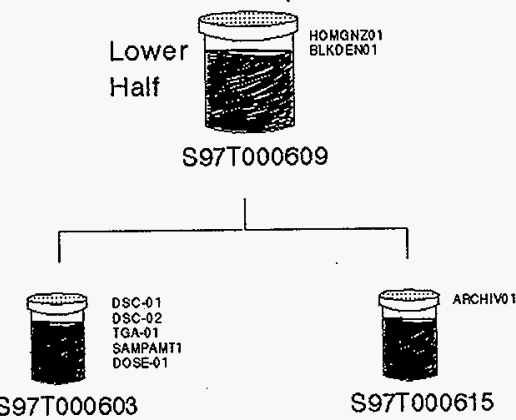

S97T000603

\$97T000615

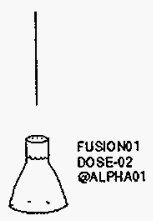

S97T000621 
Core:188

Seg: 6

S97T000564

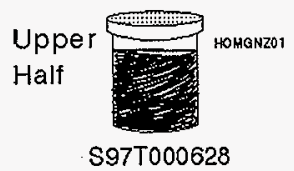

S97T000628
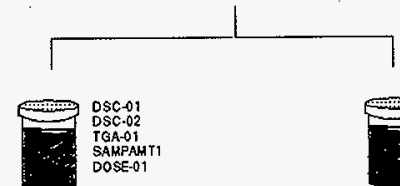

S97T000634

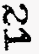

Attachment 1
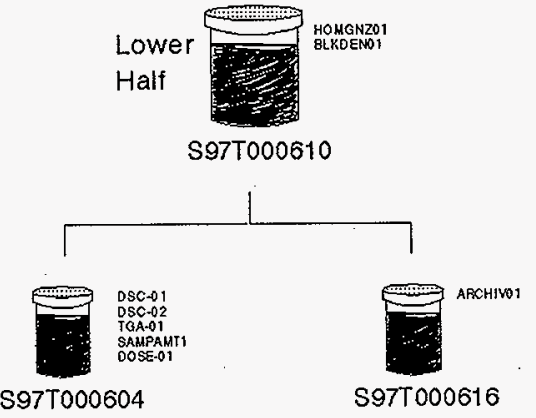
Core:188

Seg: 7

S97T000563
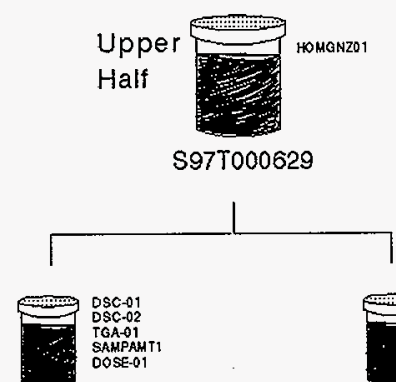

S97T000635

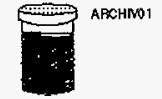

S97T000641

N
Attachment 1
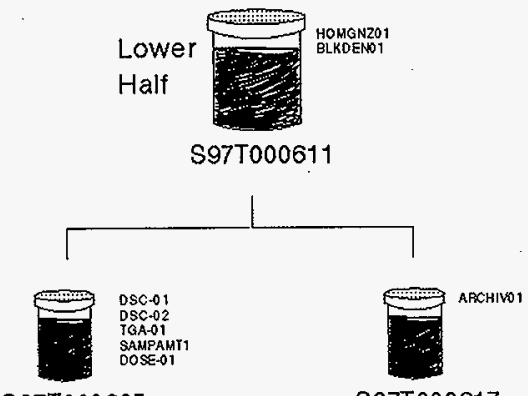

S97T000605

S97T000617

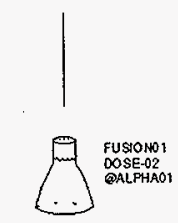

S97T000623 
Core: 188

Seg: 8

S97T.000566

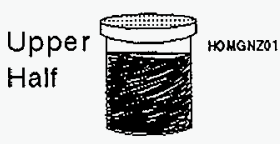

S97T000630
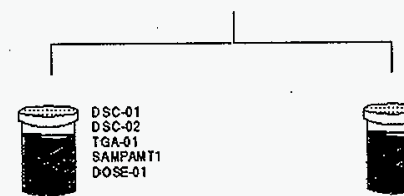

S97T000636

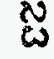
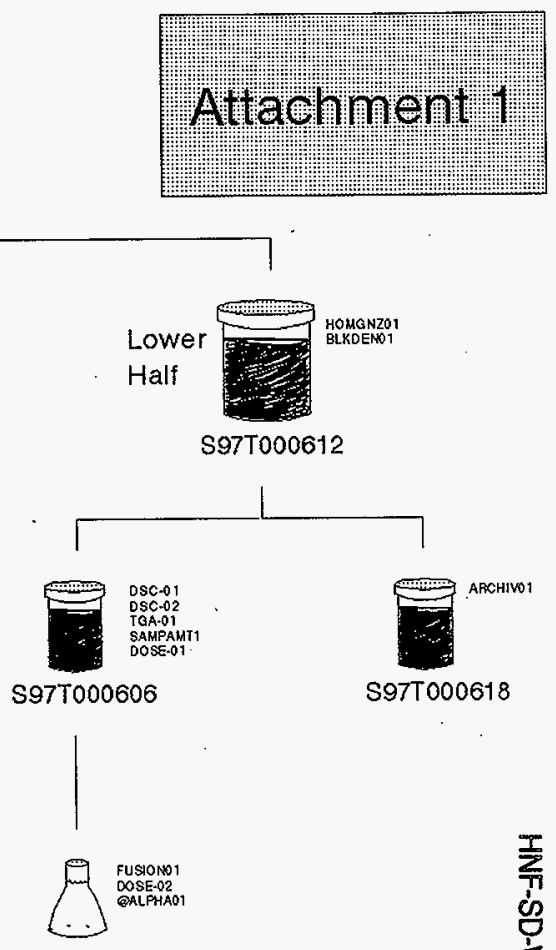

S97T000624 
Core:188

Seg: 9

S97T000567

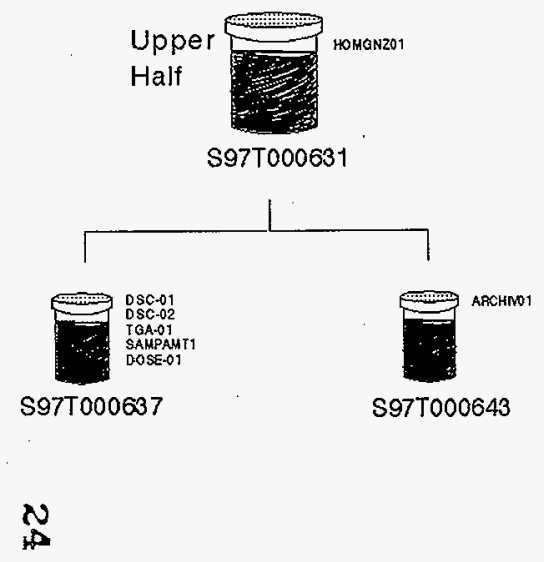

\$97T000631
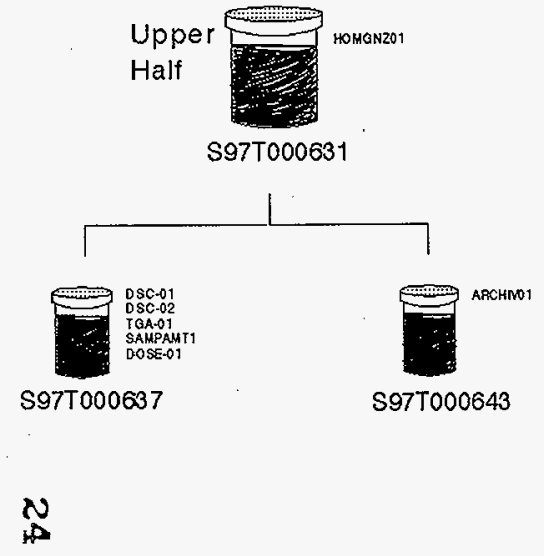

S97T000637

$N$

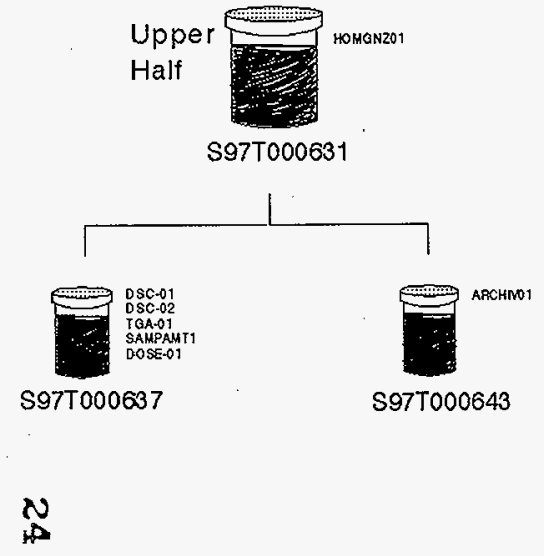

S97T000643

is
Attachiment 1

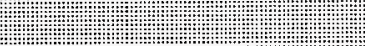

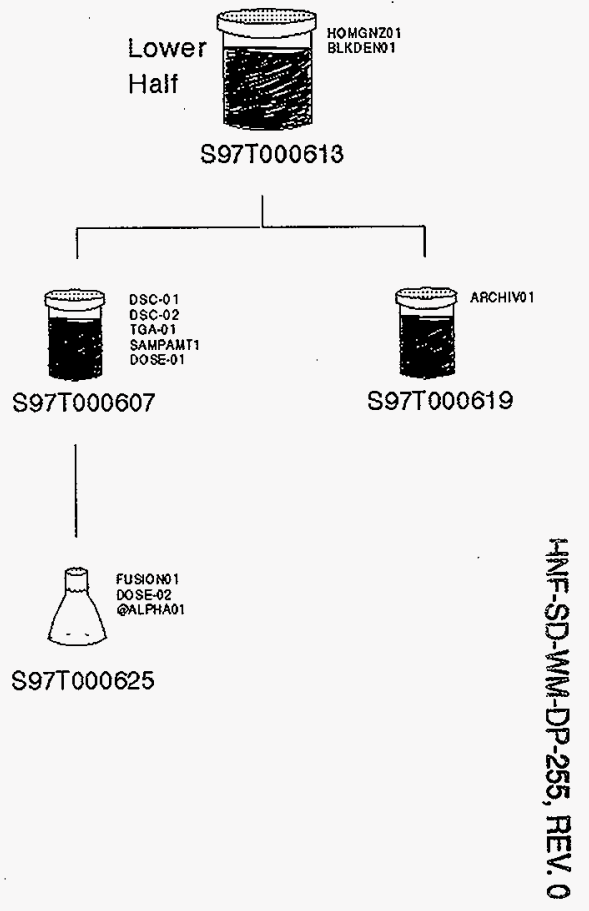




\section{T-204}

Core:188

Seg: 10

Attachment 1 S97T000569

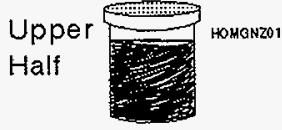

S97T000632

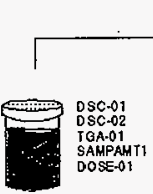

S97T000238

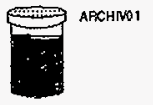

S97T000644

U

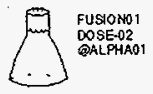

S97T000626 


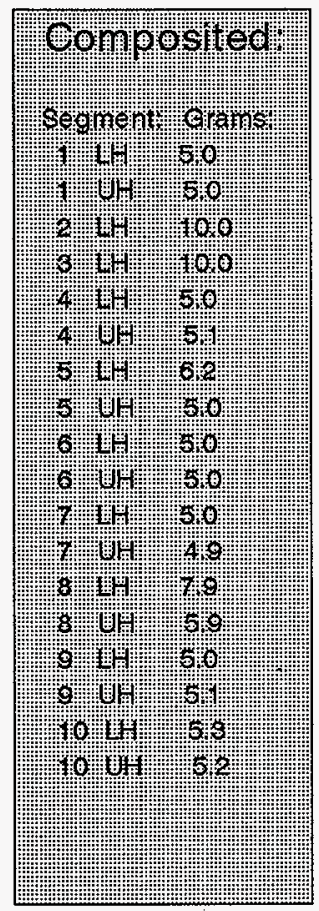

T-204

Attachment 1

Core:188

Composite:Solids

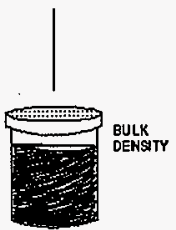

S97T001191

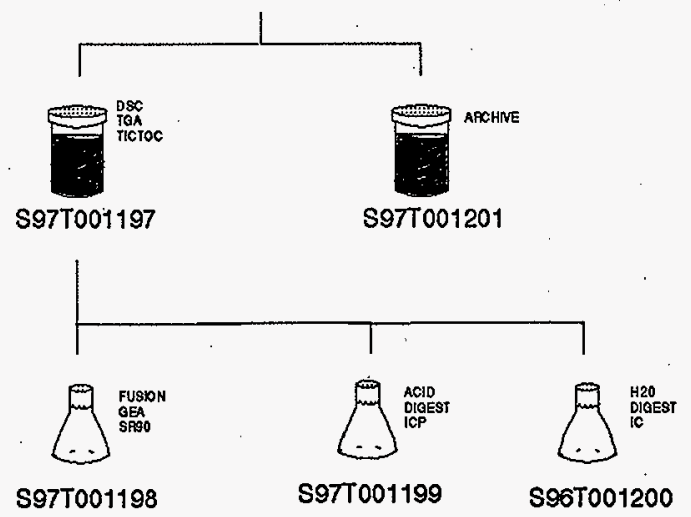

0 
HNF-SD-WM-DP-255, REV. 0

SAMPLE DATA SUMMARY 
HNF-SD-WM-DP-255, REV. 0

THIS PAGE INTENTIONALLY LEFT BLANK 
CORE NUMBER: 188

SEGMENT \#: Core Composite

SEGMENT PORTION: Core Composite

\begin{tabular}{|c|c|c|c|c|c|c|c|c|c|c|c|c|}
\hline Sample\# & $\mathbf{R}$ A\# & Analyte & Unit & Standard \% & Blank & Result & Duplicate & Average & RPO \% & Spk Rec \% & Det Limit & Count Err\% \\
\hline S97T001191 & & Bulk Density of Sample & $g / m L$ & $n / a$ & $\mathrm{n} / \mathrm{a}$ & 1.210 & $n / a$ & $\mathrm{n} / \mathrm{a}$ & $\mathrm{n} / \mathrm{a}$ & $n / a$ & $5.00 e-01$ & $\mathrm{n} / \mathrm{a}$ \\
\hline S97T001197 & & DSC Exotherm Dry Calculated & Joules/g Dry & n/a & $\mathrm{n} / \mathrm{a}$ & $0.00 \mathrm{e}+00$ & $0.00 \mathrm{e}+00$ & $0.00 \mathrm{e}+00$ & 0.00 & $n / a$ & $n / a$ & $n / a$ \\
\hline \$97T001197 & & DSC Exotherm on Perkin Elmer & Joules/g & 96.94 & $\mathrm{n} / \mathrm{a}$ & $0.00 \mathrm{e}+00$ & $0.00 e+00$ & $0.00 \mathrm{e}+00$ & 0.00 & $n / a$ & $\mathrm{n} / \mathrm{a}$ & $n / a$ \\
\hline \$97T001197 & & $\%$ Water by JGA on Perkin Etmer & $\%$ & 98.59 & $n / a$ & 75.87 & 74.32 & 75.09 & 2.06 & $\mathrm{n} / \mathrm{a}$ & $\mathrm{n} / \mathrm{a}$ & $\mathrm{n} / \mathrm{a}$ \\
\hline S97T001197 & & TIC by Acid/Coul ometry & ug $/ 9$ & 97.34 & 3.100 & $1.41 \mathrm{e}+03$ & $1.38 \mathrm{e}+03$ & $1.40 \mathrm{e}+03$ & 2.15 & 86.30 & 5.000 & $\mathrm{n} / \mathrm{a}$ \\
\hline S97T001197 & & Toc by Persulfate/Coulometry & $49 / 9$ & 101.0 & 1.800 & $3.46 \mathrm{e}+02$ & 278.0 & 312.0 & 21.8 & 83.30 & 40.00 & $n / a$ \\
\hline 5971001198 & $F$ & Strontium- $89 / 90$ High Level & uci/g & 103.0 & $3.00 \mathrm{e}-03$ & $3.46 \mathrm{e}-03$ & $5.74 e^{-03}$ & $4.60 e-03$ & 49.6 & $n / a$ & $1.00 \mathrm{e}-03$ & $3.23 E+01$ \\
\hline 5977001198 & $F$ & Cobalt -60 by GEA & $\mathrm{uCi} / \mathrm{g}$ & 100.0 & $<2.93 \mathrm{e}-06$ & $<1.71 \mathrm{e}-03$ & $\leq 1.48 \mathrm{e}-3$ & $n / a$ & $\mathrm{n} / \mathrm{a}$ & $n / a$ & $2.00 \mathrm{e}-03$ & $\mathrm{n} / \mathrm{a}$ \\
\hline S97T001198 & F & Cesium -137 by GEA & $\mathrm{uCi} / \mathrm{g}$ & 100.9 & $<4.20 \mathrm{e}-06$ & $7.75 e^{-03}$ & $7.77 \mathrm{e}-03$ & $7.76 \mathrm{e}-03$ & 0.26 & $\mathrm{n} / \mathrm{a}$ & $\mathrm{n} / \mathrm{a}$ & 28.1 \\
\hline$\$ 971001198$ & $F$ & Europium -154 by GEA & $\mathrm{uCi} / \mathrm{g}$ & $\mathrm{n} / \mathrm{a}$ & $<8.98 \mathrm{e}-06$ & $<4.39 e-03$ & $<4.03 \mathrm{e}-3$ & $n / a$ & $n / a$ & $n / a$ & $4.00 \mathrm{e}-03$ & $\mathrm{n} / \mathrm{a}$ \\
\hline S97T001198 & $F$ & Europium- 155 by GEA & $\mathrm{uCi} / \mathrm{g}$ & $\mathrm{n} / \mathrm{a}$ & $<7.34 \mathrm{e}-06$ & $<3.53 e^{-03}$ & $<3.29 e-3$ & $\mathrm{n} / \mathrm{a}$ & $\mathrm{n} / \mathrm{a}$ & $\mathrm{n} / \mathrm{a}$ & $4.00 e-03$ & $n / a$ \\
\hline S97TC & $\mathbf{F}$ & Americium-241 by GEA & $\mathrm{uCi} / \mathrm{g}$ & $\mathrm{n} / \mathrm{a}$ & $<1.92 e-05$ & $2.26 e-02$ & $2.62 e-02$ & $2.44 \mathrm{e}-02$ & 14.8 & $\mathrm{n} / \mathrm{a}$ & $\mathrm{n} / \mathrm{a}$ & 39.5 \\
\hline S97TC & $\mathrm{A}$ & Silver -ICP-ACid Digest & $\mathrm{ug} / \mathrm{g}$ & 120.0 & $<1.00 \mathrm{e}-02$ & $<2.860$ & $<2.81 \mathrm{e} 0$ & n/a & $n / a$ & 127.0 & 2.860 & $n / a$ \\
\hline S971001199 & $\bar{A}$ & Aluminium -ICP-Acid Digest & ug/g & 102.8 & $1.69 \mathrm{e}-01$ & 52.90 & 54.30 & 53.60 & 2.61 & 103.0 & 14.30 & $\mathrm{n} / \mathrm{a}$ \\
\hline S971001199 & $A$ & Arsenic -ICP-Acid Digest & ug/g & 105.0 & $<1.00 \mathrm{e}-01$ & $2.07 \mathrm{e}+02$ & 209.0 & 208.0 & 0.96 & 107.8 & 28.60 & $\mathrm{n} / \mathrm{a}$ \\
\hline S971 & A & Boron - ICP-Acid Digest & $\mathrm{ug} / \mathrm{g}$ & 99.20 & $1.19 \mathrm{e}-01$ & 67.20 & 123.0 & 95.10 & 58.7 & 106.4 & 14.30 & $\mathrm{n} / \mathrm{a}$ \\
\hline S971001199 & A & Barium -ICP-Acid Digest & $\mathrm{ug} / \mathrm{g}$ & 101.2 & $<5.00 \mathrm{e}-02$ & 14.30 & $<1.40 \mathrm{e} 1$ & $n / a$ & n/a & 101.2 & 14.30 & $\mathrm{n} / \mathrm{a}$ \\
\hline 5971001199 & A & Beryllium -ICP-Acid Digest & ug/g & 107.0 & $<5.00 \mathrm{e}-03$ & 1.430 & $<1.40 \mathrm{e} 0$ & $\mathrm{n} / \mathrm{a}$ & $n / a$ & 103.4 & 1.430 & $\mathrm{n} / \mathrm{a}$ \\
\hline \$97T & $\mathrm{A}$ & Bismuth - ICP-Acid Digest & ug/g & 99.20 & $<1.00 e-01$ & $5.11 e+04$ & $5.19 \mathrm{e}+04$ & $5.15 e+04$ & 1.55 & 181.2 & 28.60 & $\mathrm{n} / \mathrm{a}$ \\
\hline S97I & $A$ & Calciun -ICP-Acid Digest & ug/g & 99.60 & $2.08 \mathrm{e}-01$ & $2.04 \mathrm{e}+02$ & 208.0 & 206.0 & 1.94 & 98.60 & 28.60 & $\mathrm{n} / \mathrm{a}$ \\
\hline 1199 & A & Cadmium-ICP-Acid Digest & ug/g & 99.20 & $<5.00 e^{-03}$ & 7.430 & $<1.40 \mathrm{e} 0$ & $\mathrm{n} / \mathrm{a}$ & $n / a$ & 101.6 & 1.430 & $\mathrm{n} / \mathrm{a}$ \\
\hline 1199 & A & Cerium -ICP-Acid Digest & ug/g & 104.6 & $<1.00 \mathrm{e}-01$ & 61.60 & 64.30 & 62.95 & 4.29 & 107.0 & 28.60 & $n / a$ \\
\hline $597 \mathrm{~T}$ & A & Cobalt - ICP-ACid Digest & $u g / g$ & 101.6 & $<2.00 e^{-02}$ & 6.120 & 6.920 & 6.520 & 12.3 & 104.4 & 5.720 & $n / a$ \\
\hline S97T & A & Chromium -ICP-ACid Digest & $u g / g$ & 98.20 & $<1.00 \mathrm{e}-02$ & $4.51 \mathrm{e}+03$ & $4.47 e+03$ & $4.49 e+03$ & 0.89 & 119.4 & 2.860 & $n / a$ \\
\hline S97T & A & Copper - ICP-Acid Digest & ug/g & 95.40 & $1.70 \mathrm{e}-02$ & 7.250 & 6.420 & 6.835 & 12.1 & 95.20 & 2.860 & n/a \\
\hline S97T & A. & Iron-ICP-Acid Digest & $\mathrm{ug} / \mathrm{g}$ & 98.00 & $<5.00 e^{-02}$ & $3.86 \mathrm{e}+03$ & $4.21 \mathrm{e}+03$ & $4.04 \mathrm{e}+03$ & 8.67 & $1.34 e+03$ & 14.30 & $n / a$ \\
\hline 99 & A & Potassium -ICP-Acid Digest & $\mathrm{ug} / \mathrm{g}$ & 92.40 & $<5.00 \mathrm{e}-01$ & $6.09 e+03$ & $6.15 e+03$ & $6.12 \mathrm{e}+03$ & 0.98 & 138.8 & 143.0 & $n / a$ \\
\hline S97T & A & Lanthanum -ICP-Acid Digest & ug/g & 102.2 & $<5.00 \mathrm{e}-02$ & $1.13 e+04$ & $1.17 \mathrm{e}+04$ & $1.15 e+04$ & 3.48 & 157.6 & 14.30 & $n / a$ \\
\hline$\$ 97 \mathrm{~T}$ & A & Lithium -ICP-Acid Digest & ug/g & 99.20 & $<1.00 \mathrm{e}-02$ & 2.860 & $<2.81 \mathrm{e} 0$ & $n / a$ & $\mathrm{n} / \mathrm{a}$ & 97.00 & 2.860 & $\mathrm{n} / \mathrm{a}$ \\
\hline \$97T & A & Magnes ium -ICP-Acid Digest & $\mathrm{ug} / \mathrm{g}$ & 101.0 & $\leq 1.00 \mathrm{e}-01$ & 35.10 & 34.10 & 34.60 & 2.89 & 103.6 & 28.60 & $n / a$ \\
\hline 99 & A & Manganese -ICP-Acid Digest & $49 / 9$ & 99.40 & $<1.00 \mathrm{e}-02$ & $1.41 \mathrm{e}+04$ & $1.40 \mathrm{e}+04$ & $1.40 \mathrm{e}+04$ & 0.71 & 155.8 & 2.860 & $n / a$ \\
\hline 199 & A & Molybdenum -ICP-ACid Digest & $\mathrm{ug} / \mathrm{g}$ & 99.60 & $<5.00 \mathrm{e}-02$ & 14.30 & $<1.40 \mathrm{e} 1$ & $n / a$ & $\mathrm{n} / \mathrm{a}$ & 101.4 & 14.30 & $\mathrm{n} / \mathrm{a}$ \\
\hline S97TC & $\bar{A}$ & Sodium - ICP-ACId Digest & ug/g & 102.8 & $3.36 e-01$ & $3.17 e+04$ & $3.19 \mathrm{e}+04$ & $3.18 \mathrm{e}+04$ & 0.63 & 208.0 & 28.60 & $\mathrm{n} / \mathrm{a}$ \\
\hline S97T & A & Neodymium -ICP-Acid Digest & $\mathrm{ug} / \mathrm{g}$ & 101.6 & $<1.00 e-01$ & 28.60 & $<2.81 \mathrm{e} 1$ & $n / a$ & $n / a$ & 101.0 & 28.60 & $n / a$ \\
\hline 199 & A & Nickel -ICP-Acid Digest & $u g / g$ & 102.0 & $<2.00 \mathrm{e}-02$ & $2.42 \mathrm{e}+02$ & 241.0 & 241.5 & 0.41 & 105.4 & 5.720 & $\mathrm{n} / \mathrm{a}$ \\
\hline 199 & A & Phosphorus - ICP-Acid Digest & ug $/ g$ & 100.6 & $<2.00 \mathrm{e}-01$ & $2.63 \mathrm{e}+03$ & $2.66 \mathrm{e}+03$ & $2.64 \mathrm{e}+03$ & 7.13 & 114.2 & 57.20 & $n / a$ \\
\hline \$97T & A & Lead - ICP-Acid Digest & $\mathrm{ug} / \mathrm{g}$ & 96.00 & $<1.00 \mathrm{e}-01$ & $3.08 \mathrm{e}+02$ & 312.0 & 310.0 & 1.29 & 100.4 & 28.60 & $n / a$ \\
\hline 199 & A & Sulfur -ICP-ACid Digest & $49 / 9$ & 117.6 & 1.300 & 28.60 & $<2.81 \mathrm{e} 1$ & n/a & $\mathrm{n} / \mathrm{a}$ & 97.20 & 28.60 & $\mathrm{n} / \mathrm{a}$ \\
\hline 199 & A & Antimony -ICP-Acid Digest & ug/g & 96.40 & $<6.00 \mathrm{e}-02$ & 30.50 & 36.80 & 33.65 & 18.7 & 97.00 & 17.20 & n/a \\
\hline 199 & A & Silicon-ICP-Acid Digest & $\mathrm{ug} / \mathrm{g}$ & 160.4 & 2.450 & $1.52 \mathrm{e}+03$ & $1.47 e+03$ & $50 \mathrm{e}+03$ & 3.34 & 108.0 & 14.30 & $n / a$ \\
\hline 1199 & A & Samar ium -ICP-Acid Digest & $\mathrm{ug} / \mathrm{g}$ & 102.6 & $<1.00 e-01$ & 28.60 & $<2.81 \mathrm{e} 1$ & $\mathrm{n} / \mathrm{a}$ & $n / a$ & 98.60 & 28.60 & $n / a$ \\
\hline 1199 & $\bar{A}$ & Strontium - ICP-Acid Digest & ug/g & 101.6 & $<1.00 e-02$ & $4.95 e+02$ & 502.0 & 498.5 & 1.40 & 99.80 & 2.860 & $\mathrm{n} / \mathrm{a}$ \\
\hline 199 & $\bar{A}$ & Titanium-ICP-ACid Digest & $\mathrm{ug} / \mathrm{g}$ & 89.40 & $<7.00 \mathrm{e}-02$ & 3.470 & 3.460 & 3.465 & 0.29 & 90.60 & 2.860 & $n / a$ \\
\hline 199 & & Thallium -ICP-Acid Digest & ug/g & 97.80 & $<2.00 e-01$ & $2.90 \mathrm{e}+02$ & 308.0 & 299.0 & 6.02 & 105.6 & 57.20 & $n / a$ \\
\hline
\end{tabular}

S97T001199 A Thallium-ICP-Acid Digest 


\begin{tabular}{|c|c|c|c|c|c|c|c|c|c|c|c|c|}
\hline Sample\# & $\mathbf{R} \mid \mathbf{A} A$ & \# Analyte & Unit & standard \% & Blank: & Result & Duplicate & Average & RPD \% & Spk Rec \% & Det Limit & Count Err\% \\
\hline \$977001199 & A & Uranium-ICP-ACid Digest & $\mathrm{ug} / \mathrm{g}$ & 96.90 & $<5.00 \mathrm{e}-01$ & $<1.43 \mathrm{e}+02$ & $<1.40 \mathrm{e} 2$ & $\mathrm{n} / \mathrm{a}$ & $n / a$ & 77.90 & 143.0 & $\mathrm{n} / \mathrm{a}$ \\
\hline S97T001199 & A & Vanadium -ICP-Acid Digest & ug/g & 98.00 & $<5.00 \mathrm{e}-02$ & $\leq \quad 14.30$ & $<1.40 \mathrm{e} 1$ & $n / a$ & $n / a$ & 100.8 & 14.30 & $\mathrm{n} / \mathrm{a}$ \\
\hline \$97T001.199 & A & Zinc-ICP-Acid Digest & ug/g & 97.40 & $3.70 \mathrm{e}-02$ & 54.20 & 71.20 & 62.70 & 27.1 & 100.2 & 2.860 & $\mathrm{n} / \mathrm{a}$ \\
\hline S97T001199 & $A$ & Zirconium -ICP-Acid Digest & $\mathrm{ug} / \mathrm{g}$ & 98.60 & $<1.00 e-02$ & 2.860 & $<2.81 \mathrm{e} 0$ & $n / a$ & $n / a$ & 100.0 & 2.860 & n/a \\
\hline \$97T001200 & $W$ & Fluoride-IC-Dionex $4000 / 4500$ & ug/g & 94.92 & $<1.20 \mathrm{e}-02$ & $6.06 \mathrm{e}+03$ & $5.82 e+03$ & $5.94 \mathrm{e}+03$ & 4.04 & $n / a$ & 27.21 & $n / a$ \\
\hline S97T001200 & $W$ & Chloride-IC-Dionex $4000 / 4500$ & ug/g & 99.11 & $<1.70 \mathrm{e}-02$ & $6.94 \mathrm{e}+02$ & 653.0 & 673.5 & 6.09 & $n / a$ & 38.54 & $n / a$ \\
\hline $597 T 001200$ & $W$ & Nitrite-IC - Dionex $4000 / 4500$ & $4 \mathrm{~g} / \mathrm{g}$ & 101.5 & $2.32 \mathrm{e}-01$ & $3.00 \mathrm{e}+02$ & 267,0 & 283.6 & 11.6 & $n / a$ & 244.9 & $\mathrm{n} / \mathrm{a}$ \\
\hline 597.001200 & W & Bromide by Ion Chromatograph & ug/g & 100.7 & $<1.25 \mathrm{e}-01$ & $<2.83 \mathrm{e}+02$ & $<2.82 \mathrm{e} 2$ & $n / a$ & $n / a$ & $n / a$ & 283.5 & $n / a$ \\
\hline 5971001200 & W & Nitrate by IC-Dionex $4000 / 4500$ & $\mathrm{ug} / \mathrm{g}$ & 101.5 & $1.39 \mathrm{e}-01$ & $5.74 e+04$ & $5.29 e+04$ & $5.52 \mathrm{e}+04$ & 8.16 & $\mathrm{n} / \mathrm{a}$ & 315.1 & $\mathrm{n} / \mathrm{a}$ \\
\hline 5971001200 & W & Phosphate-IC-Dionex $4000 / 4500$ & ug/g & 94.68 & $<1.20 \mathrm{e}-01$ & $2.55 e+03$ & $2.36 e+03$ & $2.46 \mathrm{e}+03$ & 7.74 & $n / a$ & 272.1 & $n / a$ \\
\hline$\$ 971001200$ & W & Sulfate by IC-Dionex $4000 / 4500$ & ug/g & 100.0 & $<1.38 \mathrm{e}-01$ & $4.14 e+02$ & $<3.12 \mathrm{e} 2$ & $n / a$ & $n / a$ & $\mathrm{n} / \mathrm{a}$ & 312.9 & $n / a$ \\
\hline S97T001200 & W & Oxalate-IC-Dionex $4000 / 4500$ & ug/g & 103.0 & $<1.05 \mathrm{e}-01$ & $1.35 e+03$ & $1.31 \mathrm{e}+03$ & $1.33 e+03$ & 3.01 & $\mathrm{n} / \mathrm{a}$ & 238.1 & $n / a$ \\
\hline
\end{tabular}


Table 2. Data Summary Table

T-204

CORE NUMBER: 188

SEGMENT \#: Field Blank

SEGMENT PORTION: Field Blank

\begin{tabular}{|c|c|c|c|c|c|c|c|c|c|c|c|c|}
\hline Sample\# & $\mathrm{R}$ A\# & Analyte & Unit & Standard \% & Blank & Result & Dupl icate & Average & RPD \% & Spk Rec $\%$ & Det Limit & Count Err\% \\
\hline S97T000647 & & DSC Exotherm using Mettler & Joules/g & 95.96 & $n / a$ & $0.00 \mathrm{e}+00$ & $0.00 \mathrm{e}+00$ & $0.00 e+00$ & 0.00 & $\mathrm{n} / \mathrm{a}$ & $\mathrm{n} / \mathrm{a}$ & $\mathrm{n} / \mathrm{a}$ \\
\hline$\$ 971000647$ & & DSC Exotherm Dry Calculated & Joules/g Dry & $n / a$ & $\mathrm{n} / \mathrm{a}$ & $0.00 \mathrm{e}+00$ & $0.00 \mathrm{e}+00$ & $0.00 \mathrm{e}+00$ & 0.00 & $\mathrm{n} / \mathrm{a}$ & $\mathrm{n} / \mathrm{a}$ & $n / a$ \\
\hline S97T000647 & & Specific Gravity & Sp.G. & 100.5 & $n / a$ & 1.006 & 1.203 & 1.105 & 17.8 & n/a & $1.00 \mathrm{e}-02$ & $n / a$ \\
\hline S97T000647 & & $\%$ Water by TGA using Mettler & $\%$ & 99.58 & $n / a$ & $1.01 \mathrm{e}+02$ & 100.3 & 100.5 & 0.35 & $n / a$ & $n / a$ & n/a \\
\hline 5977000647 & D & Silver-ICP-Acid Dil. & $\mathrm{ug} / \mathrm{mL}$ & 98.60 & $<1.00 \mathrm{e}-02$ & $<2.00 e^{-02}$ & $<2.00 \mathrm{e}-2$ & $\mathrm{n} / \mathrm{a}$ & $\mathrm{n} / \mathrm{a}$ & $n / a$ & $2.00 e-02$ & $n / a$ \\
\hline 5971000647 & $\frac{D}{D}$ & Aluminium-ICP-Acid Dil. & $\mathrm{ug} / \mathrm{mL}$ & 98.40 & $<5.00 e-02$ & $<1.00 \mathrm{e}-01$ & $<1.00 \mathrm{e}-1$ & $\mathrm{n} / \mathrm{a}$ & n/a & $n / a$ & $1.00 \mathrm{e}-01$ & $\mathrm{n} / \mathrm{a}$ \\
\hline S97T000647 & D & Arsenic-ICP-Acid Dil. & $\mathrm{ug} / \mathrm{mL}$ & 104.4 & $<1.00 \mathrm{e}-01$ & $<2.00 \mathrm{e}-01$ & $<2.00 \mathrm{e}-1$ & $\mathrm{n} / \mathrm{a}$ & $\mathrm{n} / \mathrm{a}$ & $n / a$ & $2.00 e^{-01}$ & $\mathrm{n} / \mathrm{a}$ \\
\hline 5971000647 & D & Boron-ICP-Acid Dil. & $\mathrm{ug} / \mathrm{mL}$ & 101.8 & $<5.00 e^{-02}$ & 1.680 & 1.710 & 1.695 & 1.77 & $n / a$ & $1.00 \mathrm{e}-01$ & $\mathrm{n} / \mathrm{a}$ \\
\hline \$97T000647 & D & Barium-ICP-Acid Dil. & $\mathrm{ug} / \mathrm{mL}$ & 100.6 & $<5.00 e-02$ & $<1.00 \mathrm{e}-01$ & $<1.00 \mathrm{e}-1$ & $\mathrm{n} / \mathrm{a}$ & $\mathrm{n} / \mathrm{a}$ & $n / a$ & $1.00 e-01$ & $\mathrm{n} / \mathrm{a}$ \\
\hline \$97T000647 & D & Beryllium-ICP-Acid Dil. & $\mathrm{ug} / \mathrm{mL}$ & 102.2 & $<5.00 \mathrm{e}-03$ & $<1.00 \mathrm{e}-02$ & $<1.00 \mathrm{e}-2$ & $\mathrm{n} / \mathrm{a}$ & $\mathrm{n} / \mathrm{a}$ & $n / a$ & $1.00 \mathrm{e}-02$ & $n / a$ \\
\hline \$97T000647 & $\bar{D}$ & Bismuth-ICP-Acid Dil. & $\mathrm{ug} / \mathrm{mL}$ & 100.8 & $<1.00 \mathrm{e}-01$ & $<2.00 \mathrm{e}-01$ & $<2.00 e^{-1}$ & $\mathrm{n} / \mathrm{a}$ & $\mathrm{n} / \mathrm{a}$ & $\mathrm{n} / \mathrm{a}$ & $2.00 \mathrm{e}-01$ & $n / a$ \\
\hline S97T000647 & D & Calciuns-ICP-Acid Dil. & $\mathrm{ug} / \mathrm{mL}$ & 99.20 & $<1.00 \mathrm{e}-01$ & 18.50 & 18.40 & 18.45 & 0.54 & $n / a$ & $2.00 \mathrm{e}-01$ & $\mathrm{n} / \mathrm{a}$ \\
\hline S971000647 & D & Cadmium-ICP-Acid Dil, & $\mathrm{ug} / \mathrm{mL}$ & 100.4 & $<5.00 \mathrm{e}-03$ & $<1.00 \mathrm{e}-02$ & $<1.00 \mathrm{e}-2$ & $\mathrm{n} / \mathrm{a}$ & $\mathrm{n} / \mathrm{a}$ & $n / a$ & $1.00 e-02$ & $\mathrm{n} / \mathrm{a}$ \\
\hline \$97T000647 & D & Cerium-ICP-Acid Dil. & $\mathrm{ug} / \mathrm{mL}$ & 99.00 & $<1.00 e-01$ & $<2.00 \mathrm{e}-01$ & $<2.00 e^{-1}$ & $\mathrm{n} / \mathrm{a}$ & $\mathrm{n} / \mathrm{a}$ & n/a & $2.00 \mathrm{e}-01$ & $\mathrm{n} / \mathrm{a}$ \\
\hline$\$ 97 T 000647$ & D & Cobalt-ICP-Acid Dil. & $\mathrm{ug} / \mathrm{mL}$ & 104.4 & $<2.00 e^{-02}$ & $<4.00 e-02$ & $<4.00 \mathrm{e}-2$ & $\mathrm{n} / \mathrm{a}$ & $\mathrm{n} / \mathrm{a}$ & $n / a$ & $4.00 \mathrm{e}-02$ & $\mathrm{n} / \mathrm{a}$ \\
\hline \$97T000647 & D & Chromium-ICP-Acid Dil. & $\mathrm{ug} / \mathrm{mL}$ & 99.80 & $<1.00 \mathrm{e}-02$ & $<2.00 \mathrm{e}-02$ & $<2.00 \mathrm{e}-2$ & $\mathrm{n} / \mathrm{a}$ & $\mathrm{n} / \mathrm{a}$ & $\mathrm{n} / \mathrm{a}$ & $2.00 \mathrm{e}-02$ & $\mathrm{n} / \mathrm{a}$ \\
\hline$\$ 977000647$ & D & Copper-ICP-Acid Díl. & $\mathrm{ug} / \mathrm{mL}$ & 101.8 & $<1.00 e^{-02}$ & $<2.00 e^{-02}$ & $<2.00 \mathrm{e}-2$ & $\mathrm{n} / \mathrm{a}$ & n/a & $\mathrm{n} / \mathrm{a}$ & $2.00 \mathrm{e}-02$ & $\mathrm{n} / \mathrm{a}$ \\
\hline s971000647 & $D$ & Iron-ICP-ACId Dil. & $\mathrm{ug} / \mathrm{mL}$ & 93.40 & $<5.00 \mathrm{e}-02$ & $1.83 e-01$ & $1.81 \mathrm{e}-01$ & $1.82 \mathrm{e}-01$ & 1.10 & $n / a$ & $1.00 \mathrm{e}-01$ & $\mathrm{n} / \mathrm{a}$ \\
\hline 597T000647 & D & Potassium-ICP-Acid Dil. & $\mathrm{ug} / \mathrm{mL}$ & 98.80 & $<5.00 e^{-01}$ & $<1.000$ & $<1.00 \mathrm{e} 0$ & $n / a$ & $\mathrm{n} / \mathrm{a}$ & $\mathrm{n} / \mathrm{a}$ & 1.000 & $\mathrm{n} / \mathrm{a}$ \\
\hline S97T000647 & D & Lanthanum-ICP-Acid Dil. & $\mathrm{ug} / \mathrm{mL}$ & 100.8 & $<5.00 e-02$ & $<1.00 e^{-01}$ & $<1.00 \mathrm{e}-1$ & $n / a$ & $n / a$ & $n / a$ & $1.00 e-01$ & $\mathrm{n} / \mathrm{a}$ \\
\hline S97T000647 & 0 & Lithium-ICP-Acid Dil. & $\mathrm{ug} / \mathrm{mL}$ & 99.00 & $<1.00 \mathrm{e}-02$ & $<2.00 \mathrm{e}-02$ & $<2.00 e^{-2}$ & $\mathrm{n} / \mathrm{a}$ & $\mathrm{n} / \mathrm{a}$ & $\mathrm{n} / \mathrm{a}$ & $2.00 \mathrm{e}-02$ & $\mathrm{n} / \mathrm{a}$ \\
\hline 5977000647 & D & Magnesium-ICP-Acid Dil. & $\mathrm{ug} / \mathrm{mL}$ & 100.8 & $<1.00 \mathrm{e}-01$ & 3.900 & 3.890 & 3.895 & 0.26 & $n / a$ & $2.00 \mathrm{e}-01$ & $\mathrm{n} / \mathrm{a}$ \\
\hline 5971000647 & D & Manganese-ICP-Acid Dil. & $\mathrm{ug} / \mathrm{mL}$ & 98.60 & $<1.00 \mathrm{e}-02$ & $8.95 e-02$ & $8.90 e-02$ & $8.92 e-02$ & 0.56 & $\mathrm{n} / \mathrm{a}$ & $2.00 e^{-02}$ & $\mathrm{n} / \mathrm{a}$ \\
\hline 5977000647 & D & Molybdenum-ICP-Acid Dil. & $\mathrm{ug} / \mathrm{mL}$ & 103.6 & $<5.00 \mathrm{e}-02$ & $<1: 00 e-01$ & $<1.00 \mathrm{e}-1$ & $n / a$ & $\mathrm{n} / \mathrm{a}$ & n/a & $1.00 \mathrm{e}-01$ & $\mathrm{n} / \mathrm{a}$ \\
\hline$\$ 97 T 000647$ & 0 & Sodium-ICP-Acid Dil. & $\mathrm{ug} / \mathrm{mL}$ & 103.0 & $<1.00 \mathrm{e}-01$ & 4.310 & 4.290 & 4.300 & 0.47 & $n / a$ & $2.00 \mathrm{e}-01$ & $\mathrm{n} / \mathrm{a}$ \\
\hline$\$ 977000647$ & $D$ & Neodymium-ICP-Acid Dil. & $\mathrm{ug} / \mathrm{mL}$ & 100.4 & $<1.00 \mathrm{e}-01$ & $<2.00 \mathrm{e}-01$ & $<2.00 \mathrm{e}-1$ & $\mathrm{n} / \mathrm{a}$ & $\mathrm{n} / \mathrm{a}$ & $n / a$ & $2.00 \mathrm{e}-01$ & $n / a$ \\
\hline S97T000647 & $D$ & Nickel-ICP-Acid Dil. & $\mathrm{ug} / \mathrm{mL}$ & 99.40 & $<2.00 \mathrm{e}-02$ & $<4.00 \mathrm{e}-02$ & $<4.00 \mathrm{e}-2$ & $\mathrm{n} / \mathrm{a}$ & $\mathrm{n} / \mathrm{a}$ & $\mathrm{n} / \mathrm{a}$ & $4.00 \mathrm{e}-02$ & $\mathrm{n} / \mathrm{a}$ \\
\hline \begin{tabular}{|l} 
S97TO \\
\end{tabular} & D & Phosphorus-ICP-Acid Dil. & $\mathrm{ug} / \mathrm{mL}$ & 104.0 & $<2.00 \mathrm{e}-01$ & $<4.00 \mathrm{e}-01$ & $<4.00 \mathrm{e}^{-1}$ & n/a & $n / a$ & n/a & $4.00 \mathrm{e}-01$ & $n / 2$ \\
\hline S97T000647 & D & Lead-ICP-Acid Dil. & ug/mL & 98.00 & $<7.00 \mathrm{e}-01$ & $<2.00 \mathrm{e}-01$ & $<2.00 e^{-1}$ & $\mathrm{n} / \mathrm{a}$ & $\mathrm{n} / \mathrm{a}$ & $n / a$ & $2.00 \mathrm{e}-01$ & $n / a$ \\
\hline \$97T000647 & $D$ & Sul fur-ICP-Acid Dil. & $\mathrm{ug} / \mathrm{mL}$ & 101.0 & $<1.00 \mathrm{e}^{-01}$ & 6.310 & 6.310 & 6.310 & 0.00 & $\mathrm{n} / \mathrm{a}$ & $2.00 \mathrm{e}-01$ & $n / \mathbf{a}$ \\
\hline S97T0 & D & Antimony-ICP-Acid Dil. & $\mathrm{ug} / \mathrm{mL}$ & 98.00 & $<6.00 \mathrm{e}-02$ & $<1.20 \mathrm{e}-01$ & $<1.20 \mathrm{e}^{-1}$ & $\mathrm{n} / \mathrm{a}$ & $\mathrm{n} / \mathrm{a}$ & $\mathrm{n} / \mathrm{a}$ & $1.20 \mathrm{e}-01$ & $\mathrm{n} / \mathrm{a}$ \\
\hline \$97T & D & Selenium-ICP-Acid Dil. & $\mathrm{ug} / \mathrm{mL}$ & 95.00 & $<1.00 e^{-01}$ & $<2.00 e-01$ & $<2.00 \mathrm{e}-1$ & $\mathrm{n} / \mathrm{a}$ & $n / a$ & $\mathrm{n} / \mathrm{a}$ & $2.00 \mathrm{e}-01$ & $n / a$ \\
\hline \$97T000647 & $D$ & Silicon-1CP-Acid Dil. & $\mathrm{ug} / \mathrm{mL}$ & 99.00 & $<5.00 \mathrm{e}-02$ & 5.350 & 5.350 & 5.350 & 0.00 & $\mathrm{n} / \mathrm{a}$ & $1.00 \mathrm{e}-01$ & $\mathrm{n} / \mathrm{a}$ \\
\hline S97T000647 & D & Samarium-ICP-Acid Dil. & $\underline{u g} / \mathrm{mL}^{\prime}$ & 98.60 & $<1.00 \mathrm{e}-01$ & $<2.00 e-01$ & $<2.00 \mathrm{e}-1$ & $\mathrm{n} / \mathrm{a}$ & n/a & $\mathrm{n} / \mathrm{a}$ & $2.00 \mathrm{e}-01$ & $n / a$ \\
\hline \$97T000647 & D & Stront ium-ICP-Acid Dil. & $\mathrm{ug} / \mathrm{mL}$ & 99.80 & $<1.00 \mathrm{e}-02$ & $8.86 e^{-02}$ & $8.81 e-02$ & $8.83 e-02$ & 0.57 & $\mathrm{n} / \mathrm{a}$ & $2.00 \mathrm{e}-02$ & $\mathrm{n} / \mathrm{a}$ \\
\hline S97T & $\bar{D}$ & Titanium-ICP-Acid Dil. & ug/mL & 100.2 & $<1.00 \mathrm{e}-02$ & $<2.00 e-02$ & $<2.00 \mathrm{e}-2$ & $n / a$ & $\mathrm{n} / \mathrm{a}$ & $n / a$ & $2.00 e^{-02}$ & $\mathrm{n} / \mathrm{a}$ \\
\hline S97T000647 & D & Thallium-ICP-Acid Dill. & $\mathrm{ug} / \mathrm{mL}$ & 96.40 & $<2.00 \mathrm{e}-01$ & $<4.00 \mathrm{e}-01$ & $<4.00 e^{-1}$ & $\mathrm{n} / \mathrm{a}$ & $\mathrm{n} / \mathrm{a}$ & $\mathrm{n} / \mathrm{a}$ & $4.00 e-01$ & $\mathrm{n} / \mathrm{a}$ \\
\hline 5977000647 & $D$ & Uranium-ICP-Acid Dil. & $\mathrm{ug} / \mathrm{mL}$ & 98.80 & $<5.00 \mathrm{e}-01$ & 1.000 & $<1.00 \mathrm{e} 0$ & $\mathrm{n} / \mathrm{a}$ & $\mathrm{n} / \mathrm{a}$ & $n / a$ & 1.000 & $n / a$ \\
\hline$\$ 977000647$ & $D$ & Vanadium-ICP-Acid Dil. & $\mathrm{ug} / \mathrm{mL}$ & 103.6 & $<5.00 \mathrm{e}-02$ & $<1.00 \mathrm{e}-01$ & $<1.00 \mathrm{e}-1$ & $n / a$ & $\mathrm{n} / \mathrm{a}$ & $n / a$ & $1.00 \mathrm{e}-01$ & $\mathrm{n} / \mathrm{a}$ \\
\hline S97TC & D & Zinc-ICP-Acid Dil. & ug/mL & 98.80 & $<1.00 \mathrm{e}-02$ & $1.04 \mathrm{e}-01$ & $1.01 \mathrm{e}-01$ & $1.03 e-01$ & 2.93 & $\mathrm{n} / \mathrm{a}$ & $2.00 e^{-02}$ & $\mathrm{n} / \mathrm{a}$ \\
\hline S97T0 & D & Zirconium-ICP-Acid Dil. & $\mathrm{ug} / \mathrm{mL}$ & 101.6 & $<1.00 \mathrm{e}-02$ & $<2.00 \mathrm{e}-02$ & $<2.00 \mathrm{e}-2$ & $\mathrm{n} / \mathrm{a}$ & $\mathrm{n} / \mathrm{a}$ & $\mathrm{n} / \mathrm{a}$ & $2.00 e-02$ & $n / a$ \\
\hline \$97T000647 & & Fluoride-IC-Dionex $4000 / 4500$ & $\mathrm{ug} / \mathrm{mL}$ & 90.51 & $<1.20 \mathrm{e}-02$ & $<1.92 \mathrm{e}-01$ & $<1.92 \mathrm{e}-1$ & $\mathrm{n} / \mathrm{a}$ & $\mathrm{n} / \mathrm{a}$ & $n / a$ & $1.92 e^{-01}$ & $\mathrm{n} / \mathrm{a}$ \\
\hline S97T000647 & & Chloride-IC-Dionex $4000 / 4500$ & $\underline{\mathrm{ug}} / \mathrm{mL}$ & 102.5 & $<1.70 \mathrm{e}-02$ & 7.933 & 8.360 & 8.146 & 5.28 & $\mathrm{n} / \mathrm{a}$ & $2.72 e-01$ & $n / a$ \\
\hline S97T000647 & & Mitrite-IC - Dionex $4000 / 4500$ & $\mathrm{ug} / \mathrm{mL}$ & 100.6 & $<1.08 \mathrm{e}-01$ & 2.114 & $<1.73 \mathrm{e} 0$ & $\mathrm{n} / \mathrm{a}$ & $\mathrm{n} / \mathrm{a}$ & $n / a$ & .728 & $n / a$ \\
\hline
\end{tabular}




\begin{tabular}{|c|c|c|c|c|c|c|c|c|c|c|c|c|}
\hline Sample\# & A\# & Analyte & Unit & Standard \% & B lank & Result & Dupl icate & Average & RPD \% & Spk Rec \% & Det Limit & Count Err\% \\
\hline$\$ 97 \mathrm{~T} 000647$ & & Bromide by Ion Chromatograph & $\mathrm{ug} / \mathrm{mL}$ & 97.62 & $<1.25 \mathrm{e}-01$ & 2.000 & $<2.00 \mathrm{e} 0$ & $n / a$ & $\mathrm{n} / \mathrm{a}$ & $n / a$ & 2.000 & $n / a$ \\
\hline S971000647 & & Nitrate by IC-Dionex $4000 / 4500$ & $\mathrm{ug} / \mathrm{mL}$ & 98.99 & $1.39 \mathrm{e}-01$ & 4.702 & 5.480 & 5.091 & 15.3 & $\mathrm{n} / \mathrm{a}$ & 2.224 & $n / a$ \\
\hline 5971000647 & & Phosphate-IC-Dionex $4000 / 4500$ & ug/mL & 98.90 & $<1.20 \mathrm{e}-01$ & 2.077 & $<1.92 \mathrm{e} 0$ & $\mathrm{n} / \mathrm{a}$ & $n / a$ & $n / a$ & 1.920 & $\mathrm{n} / \mathrm{a}$ \\
\hline $597 T 000647$ & & Sulfate by IC-Dionex $4000 / 4500$ & $\mathrm{ug} / \mathrm{mL}$ & 99.05 & $<1.38 \mathrm{e}-01$ & 17.80 & 18.20 & 18.00 & 2.22 & $\mathrm{n} / \mathrm{a}$ & 2.208 & $\mathrm{n} / \mathrm{a}$ \\
\hline 5971000647 & & Oxalate-IC-Dionex $4000 / 450$ & ug/mL & 103.2 & $<1.05 \mathrm{e}-01$ & $1 . \overline{32}$ & $<1.68 \mathrm{e} 0$ & $n / a$ & $n / a$ & n/a & 1.680 & $n / a$ \\
\hline S971000647 & & Alpha in Liquid Samples & uCi/mL & 90.00 & $<5.06 \mathrm{e}-07$ & $1.15 \mathrm{e}-06$ & $9.51 e-07$ & $1.05 \mathrm{e}-06$ & 18.9 & $\mathrm{n} / \mathrm{a}$ & $3.97 e-07$ & $4.83 E+01$ \\
\hline
\end{tabular}


Table 2. Data summary Table

T-204

CORE NUMBER: 188

SEGMENT \#:

SEGMENT PORTION: U Upper Half of Segmen

\begin{tabular}{|c|c|c|c|c|c|c|c|c|c|c|c|c|}
\hline Sample\# & $\mathrm{R} \mid$ A\# & Analyte & Unit & Standard \% & Blank & Result & Duplicate & Average & RPD \% & Spk Rec \% & Det Limit & Count Err\% \\
\hline $597 \mathrm{~T} 000499$ & & DSC Exotherm using Mettler & Joules/g & 90.33 & $\mathrm{n} / \mathrm{a}$ & $0.00 \mathrm{e}+00$ & $0.00 \mathrm{e}+00$ & $0.00 \mathrm{e}+00$ & 0.00 & $n / a$ & $n / a$ & $\mathrm{n} / \mathrm{a}$ \\
\hline S97T000499 & & DSC Exotherm Dry Calculated & Joules/g Dry & $n / a$ & $\mathrm{n} / \mathrm{a}$ & $0.00 \mathrm{e}+00$ & $0.00 e+00$ & $0.00 e+00$ & 0.00 & $n / a$ & $\mathrm{n} / \mathrm{a}$ & $\mathrm{n} / \mathrm{a}$ \\
\hline S97T000499 & & \% water by TGA using Mettler & $\%$ & 99.50 & $n / a$ & 88.58 & 80.95 & 84.77 & 9.00 & $n / a$ & $n / a$ & $n / a$ \\
\hline
\end{tabular}

L Lower Half of Segment: L Lower Half of Segment

\begin{tabular}{|c|c|c|c|c|c|c|c|c|c|c|c|c|}
\hline Sample\# & A\# & Analyte & Unit & Standard \% & Blank & Result & Duplicate & Average & $\mathrm{RPD} \%$ & Spk Rec \% & Det Limit & Count Err\% \\
\hline S97T000494 & & Bulk Density of Sample & $\mathrm{g} / \mathrm{mL}$ & $\mathrm{n} / \mathrm{a}$ & $n / a$ & 1.150 & $n / a$ & $n / a$ & $\mathrm{n} / \mathrm{a}$ & n/a & $5.00 \mathrm{e}-01$ & $n / a$ \\
\hline \$97T000498 & & DSC Exotherm using Mettler & Joules/g & 90.33 & $\mathrm{n} / \mathrm{a}$ & $0.00 \mathrm{e}+00$ & $0.00 \mathrm{e}+00$ & $0.00 \mathrm{e}+00$ & 0.00 & n/a & $n / a$ & $\mathrm{n} / \mathrm{a}$ \\
\hline S97T000498 & & DSC Exotherm Dry Calculated & Joules/g Dry & $\mathrm{n} / \mathrm{a}$ & $n / a$ & $0.00 e+00$ & $0.00 \mathrm{e}+00$ & $0.00 \mathrm{e}+00$ & 0.00 & $\mathrm{n} / \mathrm{a}$ & $\mathrm{n} / \mathrm{a}$ & $n / a$ \\
\hline \$97T000498 & & $\%$ Water by TGA using Mettler & $\%$ & 99.50 & $\mathrm{n} / \mathrm{a}$ & 79.53 & 78.89 & 79.21 & 0.81 & $\mathrm{n} / \mathrm{a}$ & $\mathrm{n} / \mathrm{a}$ & $\mathrm{n} / \mathrm{a}$ \\
\hline S97T000587 & $F$ & Alpha of Digested Solid & uci $/ \mathrm{g}$ & 86.00 & $<2.47 \mathrm{e}-03$ & $1.76 \mathrm{e}-01$ & $1: 51 \mathrm{e}-01$ & $1.63 e-01$ & 15.3 & $\mathrm{n} / \mathrm{a}$ & $3.00 e-03$ & $8.02 \mathrm{E}+00$ \\
\hline
\end{tabular}


Table 2. Data Surnary Table

$\mathrm{T}-204$

CORE NUMBER: 188

SEGMENI \#: 2

SEGMENT PORIION: L Lower Half of Segmen

\begin{tabular}{|c|c|c|c|c|c|c|c|c|c|c|c|c|}
\hline Somple\# & A\# & Analyte & Unit & Standard \% & Blank: & Result & Duplicate & Average & RPD \% & Spk Rec \% & Det Limit & Count Err\% \\
\hline S97T000570 & & Bulk Density of Sample & $\mathrm{g} / \mathrm{mL}$ & $\mathrm{n} / \mathrm{a}$ & $\mathrm{n} / \mathrm{a}$ & 1.170 & $n / a$ & $\mathrm{n} / \mathrm{a}$ & $\mathrm{n} / \mathrm{a}$ & $\mathrm{n} / \mathrm{a}$ & $5.00 e-01$ & $\mathrm{n} / \mathrm{a}$ \\
\hline$\$ 977000574$ & & DSC Exotherm Dry Calculated & Joules/g Dry & $n / a$ & $\mathrm{n} / \mathrm{a}$ & $0.00 \mathrm{e}+00$ & $0.00 \mathrm{e}+00$ & $0.00 \mathrm{e}+00$ & 0.00 & $n / a$ & $n / a$ & $\mathrm{n} / \mathrm{a}$ \\
\hline$\$ 977000574$ & & DSC Exatherm on Perkin Elmer & Joules/g & 93.36 & $\mathrm{n} / \mathrm{a}$ & $0.00 \mathrm{e}+00$ & $0.00 \mathrm{e}+00$ & $0.00 \mathrm{e}+00$ & 0.00 & $n / a$ & n/a & n/a \\
\hline S97T000574 & & \% Water by TGA on Perkin Elmer & $\%$ & 98.50 & $n / a$ & 75.56 & 79.43 & 77.50 & 4.99 & $\mathrm{n} / \mathrm{a}$ & $\mathrm{n} / \mathrm{a}$ & $n / a$ \\
\hline S97T000588 & $F$ & Alpha of Digested Solid & $\mathrm{uCj} / \mathrm{g}$ & 86.00 & $.47 \mathrm{e}-03$ & $1.38 \mathrm{e}-01$ & $1.49 e^{-01}$ & $1.44 \mathrm{e}-01$ & 7.67 & 75.29 & $3.00 e^{-03}$ & $9.17 E+00$ \\
\hline
\end{tabular}


Table 2. Data Summary Table

CORE MUMBER: 188

SEGMENT \#: 3

SEGMENT PORTION: \& LOWER Half of Segment

\begin{tabular}{|c|c|c|c|c|c|c|c|c|c|c|c|c|}
\hline Sample\# & R A\# & Analyte & Unit & Standard \% & Blank & Result & Duplicate & Average & RPD \% & Spk Rec \% & Det Limit & Count Err\% \\
\hline S97T000571 & & Bulk Density of sample & $\mathrm{g} / \mathrm{mL}$ & $n / a$ & $\mathrm{n} / \mathrm{a}$ & 1.160 & $\mathrm{n} / \mathrm{a}$ & $\mathrm{n} / \mathrm{a}$ & $n / a$ & n/a & $5.00 \mathrm{e}-01$ & $n / a$ \\
\hline$\$ 97 T 000575$ & & DSC Exotherm Dry Calculated & Joules/g Dry & $n / a$ & $n / a$ & $0.00 \mathrm{e}+00$ & $0.00 \mathrm{e}+00$ & $0.00 \mathrm{e}+00$ & 0.00 & $n / a$ & $n / a$ & $n / a$ \\
\hline S97T000575 & & DSC Exotherm on Perkin Elmer & Joules/g & 93.36 & $n / a$ & $0.00 \mathrm{e}+00$ & $0.00 \mathrm{e}+00$ & $0.00 \mathrm{e}+00$ & 0.00 & $n / a$ & $\mathrm{n} / \mathrm{a}$ & $\mathrm{n} / \mathrm{a}$ \\
\hline S97T000575 & & $\%$ Water by TGA on Perkin Elmer & $\%$ & 98.50 & $n / a$ & 79.82 & 79.52 & 79.67 & 0.38 & $n / a$ & $n / a$ & $n / a$ \\
\hline$\$ 97 \mathrm{~T} 000589$ & $F$ & Alpha of Digested Solid & uCi $/ \mathrm{g}$ & 86.00 & $<2.47 \mathrm{e}-03$ & $1.38 e-01$ & $1.16 \mathrm{e}-01$ & $1.27 e-01$ & 17.3 & $n / a$ & $3.00 e-03$ & $9.18 \mathrm{E}+00$ \\
\hline
\end{tabular}


Table 2. Data Summary Table

CORE NUMBER: 188

SEGMENT \#: 4

SEGMENT PORTION: U Upper Half of Segment

\begin{tabular}{|c|c|c|c|c|c|c|c|c|c|c|c|c|}
\hline Sample\# & A\#t & Analyte & Unit & Standard \% & Blank & Result & Dupl icate & Average & RPD \% & Spk Rec \% & Limit & Count Err\% \\
\hline \$97T000580 & & DSC Exotherm Dry Calculated & Joules/g Dry & $\mathrm{n} / \mathrm{a}$ & $\mathrm{n} / \mathrm{a}$ & $0.00 \mathrm{e}+00$ & $0.00 \mathrm{e}+00$ & $0.00 e+00$ & 0.00 & $n / a$ & $n / a$ & $n / a$ \\
\hline S9710 & & DSC Exotherm on Perkin Elmer & Joules/g & 94.90 & $n / a$ & $0.00 \mathrm{e}+00$ & $0.00 \mathrm{e}+00$ & $0.00 e+00$ & & $n / a$ & $n / a$ & $m a$ \\
\hline S97T000580 & & $\%$ Water by IGA on Perkin Elmer & $1 \%$ & 98.72 & $n / a$ & 72.48 & 71.39 & 71.94 & 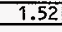 & $\mathrm{n} / \mathrm{a}$ & $n / a$ & \\
\hline
\end{tabular}

\begin{tabular}{|c|c|c|c|c|c|c|c|c|c|c|c|c|}
\hline Sample\# & A\# & Analyte & Unit & Standard \% & Blank & Result & Duplicate & Average & RPD $\%$ & Spk Rec \% & Det Limit & Count Err\% \\
\hline S97T000572 & & Bulk Densjity of Sample & $\mathrm{g} / \mathrm{mL}$ & $\mathrm{n} / \mathrm{a}$ & $\mathrm{n} / \mathrm{a}$ & 1.200 & $n / a$ & n/a & $\mathrm{n} / \mathrm{a}$ & $n / a$ & $5.00 e-01$ & $n / a$ \\
\hline$\$ 971000576$ & & DSC Exotherm Dry Calculated & Joules/g Dry & $\mathrm{n} / \mathrm{a}$ & $n / a$ & $0.00 \mathrm{e}+00$ & $0.00 \mathrm{e}+00$ & $0.00 e+00$ & 0.00 & $n / a$ & $n / a$ & $\mathrm{n} / \mathrm{a}$ \\
\hline S97T000576 & & DSC Exotherm on Perkin Elmer & Joules/g & 94.90 & $\mathrm{n} / \mathrm{a}$ & $0.00 \mathrm{e}+00$ & $0.00 \mathrm{e}+00$ & $0.00 \mathrm{e}+00$ & 0.00 & $\mathrm{n} / \mathrm{a}$ & $n / a$ & $n / a$ \\
\hline S97T000576 & & $\%$ water by TGA on Perkin Elmer & $\%$ & 98.72 & $n / a$ & 74.94 & 74.82 & 74.88 & 0.16 & $\mathrm{n} / \mathrm{a}$ & $n / a$ & $\mathrm{n} / \mathrm{a}$ \\
\hline S97T000590 & $F$ & Alpha of Digested Solid & $u \mathrm{Ci} / \mathrm{g}$ & 80.50 & $3.00 \mathrm{e}-03$ & $1.38 \mathrm{e}-01$ & $1.36 \mathrm{e}-01$ & $1.37 e-01$ & 1.46 & 77.57 & $2.00 \mathrm{e}-03$ & $8.68 \mathrm{E}+00$ \\
\hline
\end{tabular}


Table 2. Data Sumary Table

$T-204$

CORE NUMBER: 188

SEGMENT \#: 5

SEGMENT PORTION: U Upper Half of Segment

\begin{tabular}{|c|c|c|c|c|c|c|c|c|c|c|c|c|}
\hline Sample\# & A\# & Analyte & Unit & Standard \% & Blank & Result & Duplicate & Average & RPD $\%$ & Spk Rec \% & Det Limit & It Err \\
\hline S97T0 & & DSC Exotherm Dry Calculated & Joules/g Dry & $\mathrm{n} / \mathrm{a}$ & $\mathrm{n} / \mathrm{a}$ & $0.00 \mathrm{e}+00$ & $0.00 e+00$ & $0.00 e+00$ & 0.00 & $\mathrm{n} / \mathrm{a}$ & $\mathrm{n} / \mathrm{a}$ & $\mathrm{n}$ \\
\hline $597 T 000633$ & & DSC Exotherm on Perkin Elmer & Joules/g & 96.45 & $\mathrm{n} / \mathrm{a}$ & $0.00 \mathrm{e}+00$ & $0.00 \mathrm{e}+00$ & $0.00 e+00$ & 0.00 & $n / a$ & $\mathrm{n} / \mathrm{a}$ & 1 \\
\hline$\$ 971000633$ & & $\%$ Water by TGA on Perkin Elmer & $\%$ & 98.92 & $n / a$ & 76.90 & 76.18 & 76.54 & 0.94 & $\mathrm{n} / \mathrm{a}$ & $n / a$ & i \\
\hline
\end{tabular}

\begin{tabular}{|c|c|c|c|c|c|c|c|c|c|c|c|c|}
\hline Sample\# & $\mathrm{R}$ A\# & Analyte & Unit & Standard \% & Blank & Result & Duplicate & Average & RPD $\%$ & Spk Rec \% & Det Limit & Count Err\% \\
\hline 5977000603 & & DSC Exotherm Dry Calculated & Joules/g Dry & $\mathrm{n} / \mathrm{a}$ & $\mathrm{n} / \mathrm{a}$ & $0.00 \mathrm{e}+00$ & $0.00 e+00$ & $0.00 e+00$ & 0.00 & $\mathrm{n} / \mathrm{a}$ & $n / a$ & $n / a$ \\
\hline S97T000603 & & DSC Exotherm on Perkin Elmer & Joules/g & 97.08 & $\mathrm{n} / \mathrm{a}$ & $0.00 \mathrm{e}+00$ & $0.00 e+00$ & $0.00 \mathrm{e}+00$ & 0.00 & $\mathrm{n} / \mathrm{a}$ & $n / a$ & $n / a$ \\
\hline 5977000603 & & $\%$ water by TGA on Perkin Elmer & $\%$ & 98.72 & $\mathrm{n} / \mathrm{a}$ & 75.93 & 74.92 & 75.43 & 1.34 & $\mathrm{n} / \mathbf{a}$ & $\mathrm{n} / \mathrm{a}$ & $\mathrm{n} / \mathrm{a}$ \\
\hline S97T000609 & & Bulk Density of Sample & $\mathrm{g} / \mathrm{mL}$ & $n / a$ & $n / a$ & 1.210 & $\mathrm{n} / \mathrm{a}$ & $n / a$ & $\mathrm{n} / \mathrm{a}$ & $\mathrm{n} / \mathrm{a}$ & $5.00 e-01$ & $n / a$ \\
\hline S97T000621 & $\mathrm{F}$ & Alpha of bigested Solid & uci $/ g$ & 80.50 & $3.00 e-03$ & $1.27 \mathrm{e}-01$ & $1.65 e-01$ & $1.46 e^{-01}$ & 26.0 & $\mathrm{n} / \mathrm{a}$ & $2.00 e-03$ & $9.10 E+00$ \\
\hline
\end{tabular}


CORE NUMBER: 188

SEGMENT \#: 6

SEGMENT PORTION: $U$ Upper Half of Segment

\begin{tabular}{|c|c|c|c|c|c|c|c|c|c|c|c|c|}
\hline Sample & $\mathrm{R}$ A\# & Analyte & Unit & andard \% & Blank & $d t$ & Duplicate & Average & RPD \% & Spk Rec \% & Det Limit & Count Err\% \\
\hline 597T000634 & & DSC Exotherm Dry Calculated & Joules/g Dry & $\mathrm{n} / \mathrm{a}$ & $\mathrm{n} / \mathrm{a}$ & $0.00 \mathrm{e}+00$ & $0.00 e+00$ & $0.00 \mathrm{e}+00$ & 0.00 & $n / a$ & $n / a$ & $n / a$ \\
\hline S97T0 & & DSC Exotherm on Perkin ELmer & Joules/g & 96.45 & $\mathrm{n} / \mathrm{a}$ & $0.00 \mathrm{e}+00$ & $0.00 \mathrm{e}+00$ & $0.00 \mathrm{e}+00$ & 0.00 & $\mathrm{n} / \mathrm{a}$ & $n / a$ & $\mathrm{n} / \mathrm{a}$ \\
\hline \$9710 & & $\%$ Water by TGA on Perkin Elmer & & 98.92 & n/a & 75.64 & 77.96 & 76.80 & 3.02 & $n / a$ & $n / a$ & $n / a$ \\
\hline
\end{tabular}

\begin{tabular}{|c|c|c|c|c|c|c|c|c|c|c|c|c|}
\hline Sample\# & $\mathrm{R} \mid \mathrm{A \#}$ & Analyte & Unit & standard \% & Blank & Result & Duplicate & Average & RPD \% & Spk Rec \% & Det Limit & Count Err\% \\
\hline$\$ 977000604$ & & DSC Exotherm Dry Calculated & Joules/g Dry & n/a & $n / a$ & $0.00 \mathrm{e}+00$ & $0.00 \mathrm{e}+00$ & $0.00 \mathrm{e}+00$ & 0.00 & $\mathrm{n} / \mathrm{a}$ & $\mathrm{n} / \mathrm{a}$ & $\mathrm{n} / \mathrm{a}$ \\
\hline 5977000604 & & IDSC Exotherm on Perkin Elmer & Joules/g & 97.08 & $\mathrm{n} / \mathrm{a}$ & $0.00 \mathrm{e}+00$ & $0.00 \mathrm{e}+00$ & $0.00 \mathrm{e}+00$ & 0.00 & $\mathrm{n} / \mathrm{a}$ & $n / a$ & $n / a$ \\
\hline 5971000604 & & $\%$ Water by TGA on Perkin Elmer & $\%$ & 98.17 & $\mathrm{n} / \mathrm{a}$ & 76.81 & 75.92 & 76.37 & 1.17 & $n / a$ & $n / a$ & $n / a$ \\
\hline $597 \mathrm{~T} 000610$ & & Bulk Density of Sample & $\mathrm{g} / \mathrm{mL}$ & $\mathrm{n} / \mathrm{a}$ & $\mathrm{n} / \mathrm{a}$ & 1.170 & $\mathrm{n} / \mathrm{a}$ & $\mathrm{n} / \mathrm{a}$ & $n / a$ & $\mathrm{n} / \mathrm{a}$ & $5.00 e^{-01}$ & $\mathrm{n} / \mathrm{a}$ \\
\hline 5971000622 & $F$ & Alpha of Digested Solid & $\mathrm{uCi} / \mathrm{g}$ & 80.50 & $3.00 e-03$ & $1.29 e-01$ & $1.38 \mathrm{e}-01$ & $1.34 \mathrm{e}-01$ & 6.74 & $n / a$ & $3.00 e-03$ & $9.06 \mathrm{E}+00$ \\
\hline
\end{tabular}


Table 2. Data sumary rable

CORE NUMBER: 188

SEGMENT \#:

SEGMENT PORTION: U Upper Half of Segment

\begin{tabular}{|c|c|c|c|c|c|c|c|c|c|c|c|c|}
\hline & & & & & & & & & & & & \\
\hline S97T000635 & & DSC Exotherm Dry Calculated & Joules/g Dry & $\mathrm{n} / \mathrm{a}$ & $n / a$ & $0.00 \mathrm{e}+00$ & $0.00 \mathrm{e}+00$ & $0.00 \mathrm{e}+00$ & 0.00 & $n / a$ & $n / a$ & n/ \\
\hline
\end{tabular}

\begin{tabular}{|c|c|c|c|c|c|c|c|c|c|c|c|c|}
\hline Samp & A\# & Analyt & Unit & Standard $\%$ & Blank & Result & Duplicate & Average & RPD \% & Spk Rec \% & Det Limit & Count Err\% \\
\hline S97T000605 & & DSC Exotherm Dry Calculated & Joules/g Dry & $\mathrm{n} / \mathrm{a}$ & $\mathrm{n} / \mathrm{a}$ & $0.00 e+00$ & $0.00 \mathrm{e}+00$ & $0.00 \mathrm{e}+00$ & 0.00 & $\mathrm{n} / \mathrm{a}$ & $n / a$ & $\mathrm{n} / \mathrm{a}$ \\
\hline S971000605 & & DSC Exotherm on Perkin Elmer & Joules/g & 94.94 & $n / a$ & $0.00 \mathrm{e}+00$ & $0.00 \mathrm{e}+00$ & $0.00 \mathrm{e}+00$ & 0.00 & $\mathrm{n} / \mathrm{a}$ & n/a & $\mathrm{n} / \mathrm{a}$ \\
\hline 605 & & \% water by TGA on Perkin Elmer & & 98.82 & $\mathrm{n} / \mathrm{a}$ & 76.21 & 74.04 & 75.12 & 2.89 & $\mathrm{n} / \mathrm{a}$ & $\mathrm{n} / \mathrm{a}$ & $\mathrm{n} / \mathrm{a}$ \\
\hline S97T000611 & & Bulk Density of Sample & $\mathrm{g} / \mathrm{mL}$ & $n / a$ & n/a & 1.180 & $n / a$ & n/a & $\mathrm{n} / \mathrm{a}$ & $\mathrm{n} / \mathrm{a}$ & $5.00 \mathrm{e}-01$ & $\mathrm{n} / \mathrm{a}$ \\
\hline 5977000623 & & Alpha of Digested Solid & uCi/g & 88.50 & $<1.92 \mathrm{e}-03$ & $<3.1$ & $1.46 e^{-01}$ & n/a & $\mathrm{n} / \mathrm{a}$ & $n / a$ & $5.00 e^{-03}$ & $5.00 E+02$ \\
\hline
\end{tabular}


Table 2. Data Summary Table

CORE NUMBER: 188

SEGHENT \#:

SEGMENT PORTION: $U$ Upper Hal $f$ of Segment

\begin{tabular}{|c|c|c|c|c|c|c|c|c|c|c|c|c|}
\hline Sample\# & $\mathbf{R}$ A\# & Analyte & Unit & Standard \% & Blank & Result & Duplicate & Average & RPD \% & Spk Rec \% & Det Limit & Count Err\% \\
\hline S97T000636 & & DSC Exotherm Dry Calculated & Joules/g Dry & $n / a$ & $n / a$ & $0.00 e+00$ & $0.00 e+00$ & $0.00 \mathrm{e}+00$ & 0.00 & $n / a$ & $\mathrm{n} / \mathrm{a}$ & $\mathrm{n} / \mathrm{a}$ \\
\hline 36 & & DSC Exotherm on Perkin Elmer & Joules/g & 92.90 & $\mathrm{n} / \mathrm{a}$ & $0.00 \mathrm{e}+00$ & $0.00 \mathrm{e}+00$ & $0.00 \mathrm{e}+00$ & 0.00 & $\mathrm{n} / \mathrm{a}$ & $n / a$ & $\mathrm{n} / \mathrm{a}$ \\
\hline$\$ 977000636$ & & $\%$ Water by TGA on Perkin Elmer & $\%$ & 98.89 & $n / a$ & 72.05 & 72.78 & 72.41 & 1.01 & $n / a$ & $\mathrm{n} / \mathrm{a}$ & n/a \\
\hline
\end{tabular}

\begin{tabular}{|c|c|c|c|c|c|c|c|c|c|c|c|c|}
\hline Sample\# & A\# & Analyte & Unit & standard \% & Blank & Result & Duplicate & Average & RPD \% & Spk Rec \% & Det Limit & Count Err\% \\
\hline S97T000606 & & DSC Exotherm Dry Calculated & Joules/g Dry & $n / a$ & $n / a$ & $0.00 e+00$ & $0.00 \mathrm{e}^{4}+00$ & $0.00 \mathrm{e}+00$ & 0.00 & $n / a$ & $n / a$ & $n / a$ \\
\hline $597 T 000606$ & & DSC Exotherm on Perkin Elmer & Joules/g & 94.94 & $\mathrm{n} / \mathrm{a}$ & $0.00 e+00$ & $0.00 \mathrm{e}+00$ & $0.00 \mathrm{e}+00$ & 0.00 & n/a & $n / a$ & $n / a$ \\
\hline S977000606 & & $\%$ Water by TGA on Perkin Elmer & $\%$ & 98.82 & n/a & 73.21 & 69.64 & 71.42 & 5.00 & $\mathrm{n} / \mathrm{a}$ & $n / a$ & $\mathrm{n} / \mathrm{a}$ \\
\hline S97T000612 & & Bulk Density of sample & $\mathrm{g} / \mathrm{mL}$ & $\mathrm{n} / \mathrm{a}$ & $\mathrm{n} / \mathrm{a}$ & 1.200 & $n / a$ & $\mathrm{n} / \mathrm{a}$ & $n / a$ & $n / a$ & $5.00 \mathrm{e}-01$ & $n / a$ \\
\hline$\$ 977000624$ & F & Alpha of Digested Solid & $\mathrm{uCi} / \mathrm{g}$ & 88.50 & $<1.92 e^{-03}$ & $1.84 \mathrm{e}-01$ & $1.58 \mathrm{e}-01$ & $1.71 e^{-01}$ & 15.2 & $\mathrm{n} / \mathrm{a}$ & $5.00 \mathrm{e}-03$ & $8.39 E+00$ \\
\hline
\end{tabular}

$\stackrel{20}{a}$ 
Table 2. Data Sumary Table

$\mathrm{T}-204$

CORE NUMBER: 188

SEGNENT \#: 9

SEGMENT PORTION: U Upper Half of Segment

\begin{tabular}{|c|c|c|c|c|c|c|c|c|c|c|c|c|}
\hline Sam: & A\# & te & Unit & standard \% & Blank & Result & Duplicate & Average & RPD \% & Spk Rec \% & Det Limit & ant Err\% \\
\hline 0637 & & Exotherm Dry Calculated & Joules/g Dry & $n / a$ & $n / a$ & $0.00 \mathrm{e}+00$ & $0.00 \mathrm{e}+00$ & $0.00 \mathrm{e}+00$ & 0.00 & $n / a$ & $n / a$ & $\mathrm{n} / \mathrm{s}$ \\
\hline S97T & & DSC Exotherm on Perkin Elmer & Joules/g & 94.90 & $n / a$ & $0.00 \mathrm{e}+00$ & $0.00 \mathrm{e}+00$ & $0.00 \mathrm{e}+00$ & 0.00 & $\mathrm{n} / \mathrm{a}$ & $n / a$ & $n / a$ \\
\hline S97T0 & & $\%$ Water by TGA on Perkin Elme & $\%$ & 98.17 & $n / a$ & 71.04 & 75.10 & 73.07 & 5.56 & $n / a$ & $\mathrm{n} / \mathrm{a}$ & $n / a$ \\
\hline
\end{tabular}

\begin{tabular}{|c|c|c|c|c|c|c|c|c|c|c|c|c|}
\hline Sample\# & R $\mid$ A\# & Analyte & Unit & Standard \% & Blank & Result & Dupl icate & Average & RPD \% & Spk Rec \% & Det Limit & Count Err\% \\
\hline S97T000607 & & DSC Exotherm Dry Calculated & Joules/g Dry & $n / a$ & $n / a$ & $0.00 \mathrm{e}+00$ & $0.00 \mathrm{e}+00$ & $0.00 \mathrm{e}+00$ & 0.00 & $\mathrm{n} / \mathrm{a}$ & $n / a$ & $n / a$ \\
\hline S97T000607 & & DSC Exotherm on Perkin Elmer & Joules/g & 94.52 & $n / a$ & $0.00 \mathrm{e}+00$ & $0.00 \mathrm{e}+00$ & $0.00 \mathrm{e}+00$ & 0.00 & n/a & $\mathrm{n} / \mathrm{a}$ & $\mathrm{n} / \mathrm{a}$ \\
\hline S971000607 & & $\%$ water by TGA on Perkin Elmer & $\%$ & 98.05 & $\mathrm{n} / \mathrm{a}$ & 74.42 & 75.99 & 75.20 & 2.09 & $\mathrm{n} / \mathrm{a}$ & $\mathrm{n} / \mathrm{a}$ & $n / a$ \\
\hline S97T000613 & & Bulk Density of Sample & $\mathrm{g} / \mathrm{mL}^{\mathrm{L}}$ & $\mathrm{n} / \mathrm{a}$ & n/a & 1.190 & $\mathrm{n} / \mathrm{a}$ & $\mathrm{n} / \mathrm{a}$ & $\mathrm{n} / \mathrm{a}$ & $\mathrm{n} / \mathrm{a}$ & $5.00 \mathrm{e}-01$ & $\mathrm{n} / \mathrm{a}$ \\
\hline$\$ 977000625$ & $F$ & Alpha of Digested Solid & uCi/g & 88.50 & $<1.92 \mathrm{e}-03$ & $2.08 \mathrm{e}-01$ & $1.89 e-01$ & $1.99 \mathrm{e}-01$ & 9.57 & $\mathrm{n} / \mathrm{a}$ & $5.00 \mathrm{e}-03$ & $7.52 \mathrm{E}+00$ \\
\hline
\end{tabular}


SEGMENT PORTION: U Upper Half of Segment

\begin{tabular}{|c|c|c|c|c|c|c|c|c|c|c|c|c|}
\hline Sample\# & A\# & Analyte & Unit & Standard \% & Blank & Result & Duplicate & Average & RPD \% & Spk Rec \% & Det Limit & Count Err\% \\
\hline 5977000638 & & DSC Exotherm Dry Calculated & Joules/g Dry & $n / a$ & $n / a$ & $0.00 \mathrm{e}+00$ & $0.00 \mathrm{e}+00$ & $0.00 \mathrm{e}+00$ & 0.00 & $\mathrm{n} / \mathrm{a}$ & $n / a$ & $n / a$ \\
\hline$\frac{100000}{597000638}$ & & DSC Exotherm on Perkin Elmer & Joules/g & 94.90 & $\mathrm{n} / \mathrm{a}$ & $0.00 e+00$ & $0.00 \mathrm{e}+00$ & $0.00 e+00$ & 0.00 & $n / a$ & $\mathrm{n} / \mathrm{a}$ & $n / a$ \\
\hline \$971000638 & & \% Water by TGA on Perkin Elmer & $\%$ & 98.17 & $\mathrm{n} / \mathrm{a}$ & 53.74 & 73.28 & 63.51 & 30.8 & $\mathrm{n} / \mathrm{a}$ & $n / a$ & $\mathrm{n} / \mathrm{a}$ \\
\hline
\end{tabular}

\begin{tabular}{|c|c|c|c|c|c|c|c|c|c|c|c|c|}
\hline Sample\# & R & Analyte & Unit & Standard \% & Blank & Result & Duplicate & Average & RPD \% & Spk Rec \% & Det Limit & Count Err\% \\
\hline 9977000608 & & DSC Exotherm Dry Calculated & Joules/g Dry & $\mathrm{n} / \mathrm{a}$ & n/a & $0.00 \mathrm{e}+00$ & $0.00 \mathrm{e}+00$ & $0.00 \mathrm{e}+00$ & 0.00 & $n / a$ & $\mathrm{n} / \mathrm{a}$ & $n / a$ \\
\hline S97T000608 & & DSC Exotherm on Perkin Elmer & Joules/g & 94.52 & $n / a$ & $0.00 \mathrm{e}+00$ & $0.00 e+00$ & $0.00 \mathrm{e}+00$ & 0.00 & $n / a$ & $n / a$ & $\mathrm{n} / \mathrm{a}$ \\
\hline$\$ 97 T 000608$ & & $\%$ Water by TGA on Perkin Elmer & $\%$ & 98.05 & $n / a$ & 73.89 & 74.98 & 74.44 & 1.46 & $n / a$ & $\mathrm{n} / \mathrm{a}$ & $n / a$ \\
\hline S97T000614 & & Bulk Density of Sample & $\mathrm{g} / \mathrm{mL}$ & $\mathrm{n} / \mathrm{a}$ & $\mathrm{n} / \mathrm{a}$ & 1.210 & $\mathrm{n} / \mathrm{a}$ & $\mathrm{n} / \mathrm{a}$ & $\mathrm{n} / \mathrm{a}$ & $n / a$ & $5.00 e-01$ & $n / a$ \\
\hline S971000626 & $F$ & Alpha of Digested Solid & $\mathrm{uCi} / \mathrm{g}$ & 88.50 & $<1.92 \mathrm{e}-03$ & $1.57 \mathrm{e}-01$ & $1.41 e^{-01}$ & $1.49 \mathrm{e}-01$ & 10.7 & $n / a$ & $4.00 \mathrm{e}-03$ & $8.46 \mathrm{E}+00$ \\
\hline
\end{tabular}


HNF-SD-WM-DP-255, REV. 0

CHAIN OF CUSTODY FORMS 
HNF-SD-WM-DP-255, REV. 0

THIS PAGE INTENTIONALLY LEFT BLANK

44 


\section{CHAIN-OF-CUSTODY RECORD FOA CORE SAMPLING}

(4) Tonk $720 \%$

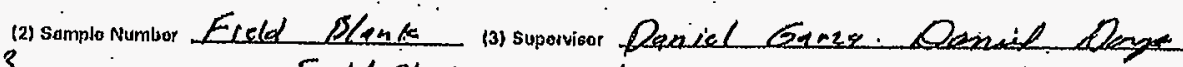

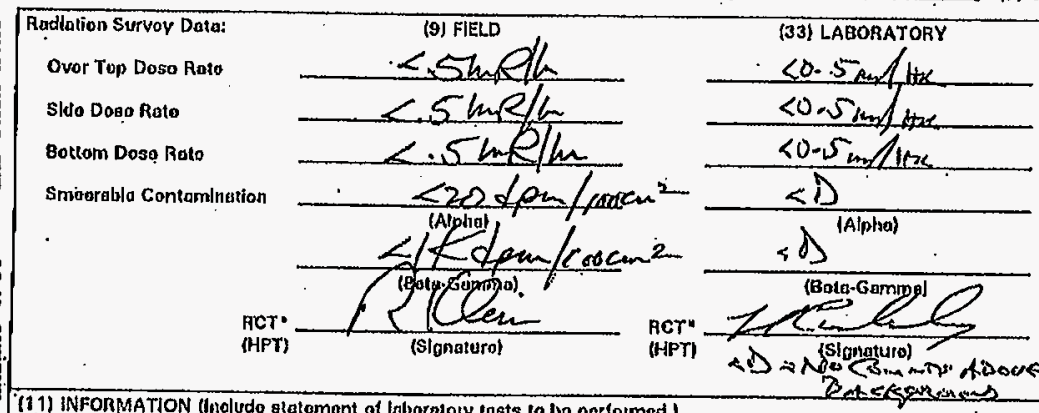

(6) sagmont Ereld Blaik 71 coro $188^{\circ}$

(8) Cenk Sorlal Numbar 1013 .c.

(11) INFORMATION (Inaludo slatament of laboratory tosts to bo porformed.)

Ratesgenes

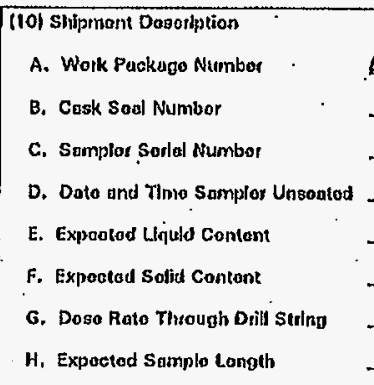

$\mu 5-96-00306$ 12204

q4- Of 7

$4 / 11 / 47$

10070

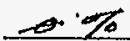

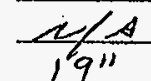

in

DeTionized Hzo used for Field Blank

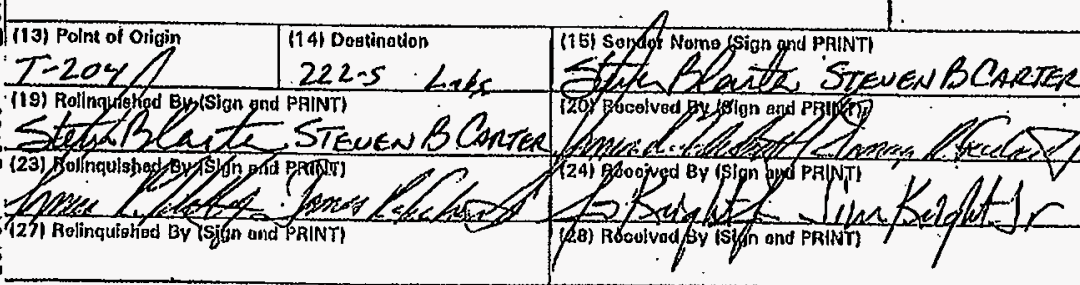

(10) Seal Intaot Upon Rolonso? adYos

口No
(32) Sool Data Constatont with thla Record?

Shipmont No,

\section{(16) Dofortima (17) Sondor Commonts \\ (22) Recoiver Commonts \\ (21) Dalg) livo}

$0 x-15-97$

(25) Datedifag- (26) Rocolvor Commante

$x / 5-17$

(29) Oato/Tlmo (30) Rovolvar Commonts
(311 Soat Intrat Upon Rocolpt? A Yos $\square$ No

?.

No $\quad X_{\text {Yos }}^{\text {Nomont }}$


CHAIN-OF-CUSTODY RECORD FOR CORE SAMPLING

Shipment Number Doow-08-7TF (2) Sample Number $97-60$ $T=20 y$ diation Survey Data: Jver Top Oose Rato ide Dose Rate lottom Dose Rate imoarable Contamination

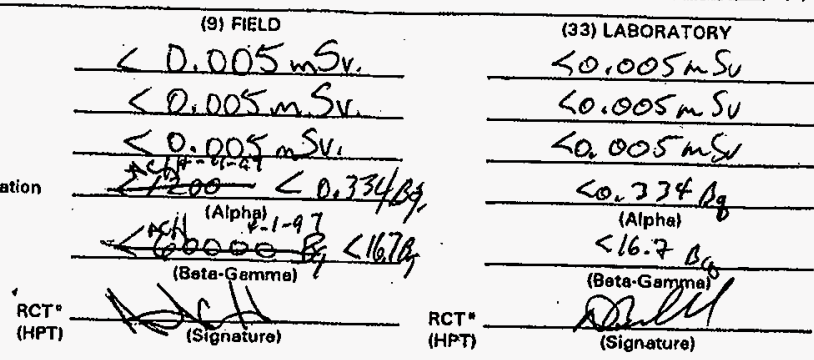
(9) FIELD

INFORMATION (Include statement of leboratory tests to bo parformod.)

(6) Sogment 1

(3) Supervisor Orail Nonge.

(7) Cora 188

(8) Cask Serial Number $f(0 / 3-C$

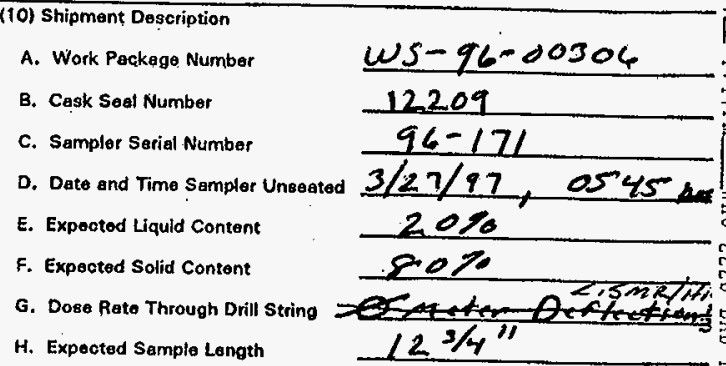

\section{$\infty$} 2LL-5 labs. Blipquishod By/sign gnd pRINT)

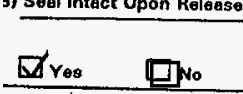

(31) Seal Intect Upon Receipt? (28) Received By (Sign and PRINT)

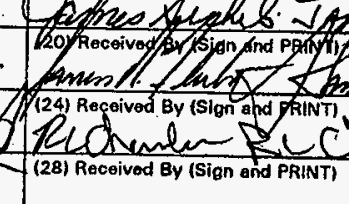 \\ (34) Leboratory Comments}

\section{(16) Regertimo $4-1 \rightarrow 0$ (21) Data/Time (21) Data/Timiz (25) DatorTimo $t_{m} /-97 / 339$ (29) Dato/Time}

(17) Sonder Comments (22) Receiver Commente (26) Receiver Comments (30) Recoivor Commonts

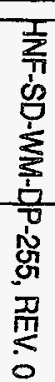
Xos Shipment No. (32) Seal Date Consistent with this Rocord?

$\square$ No $\quad \underbrace{\text { Shipment No. }}_{\text {Shos }} \square$ No
Cask Soal No. ¿Yos $\square$ No Sampla No, UBUTION: Whiten - Ortition at Sammin Mannanamant 
CHAIN-OF-CUSTODY RECORD FOR CORE SAMIPLING (2) Samplo Numbar $97+6 z$.

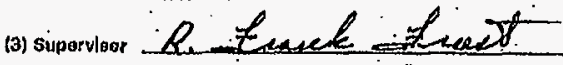

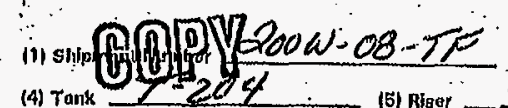
3 (B) Sagmont 3 (7) coro 188 (D) Caiks sartal Numbar -76

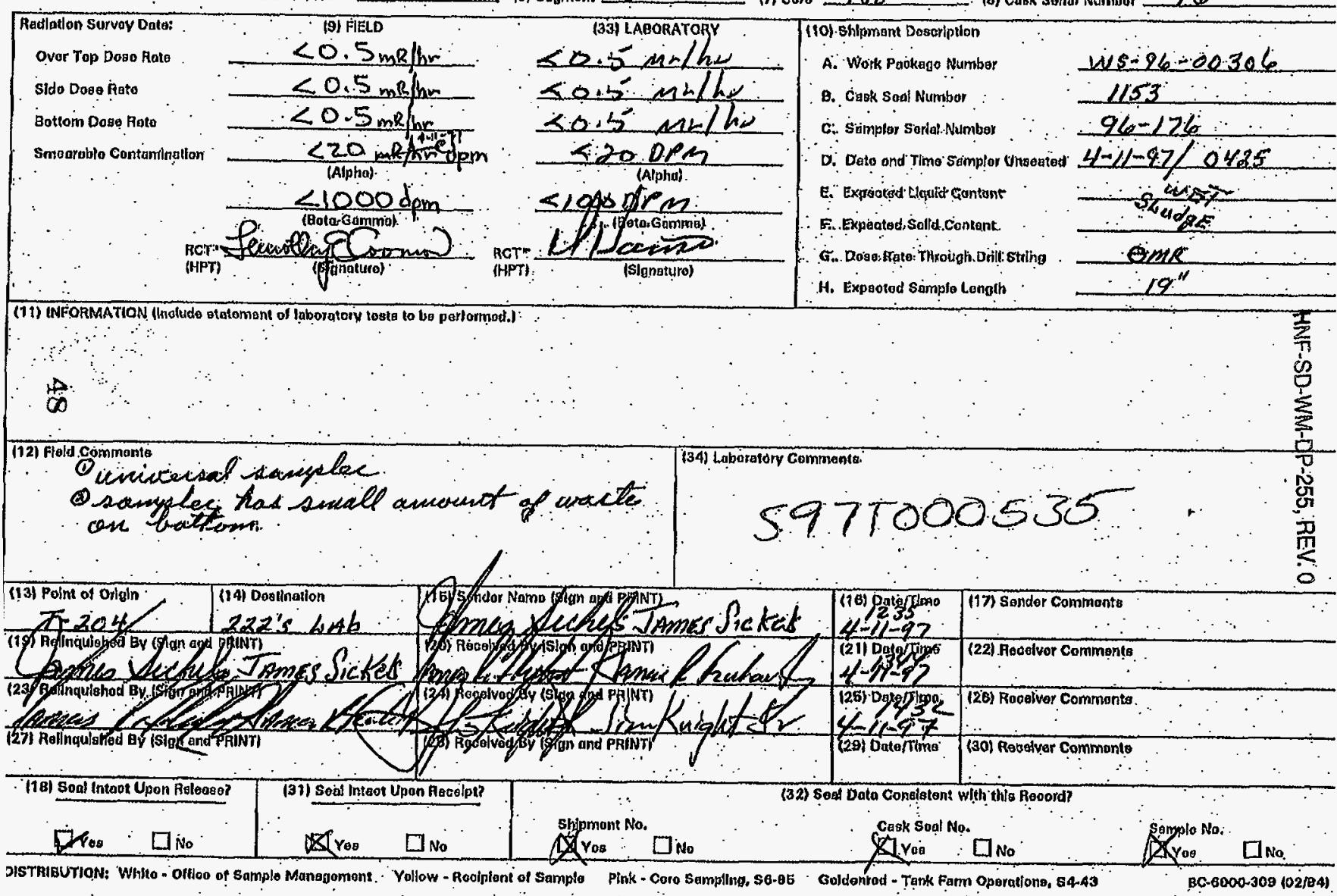




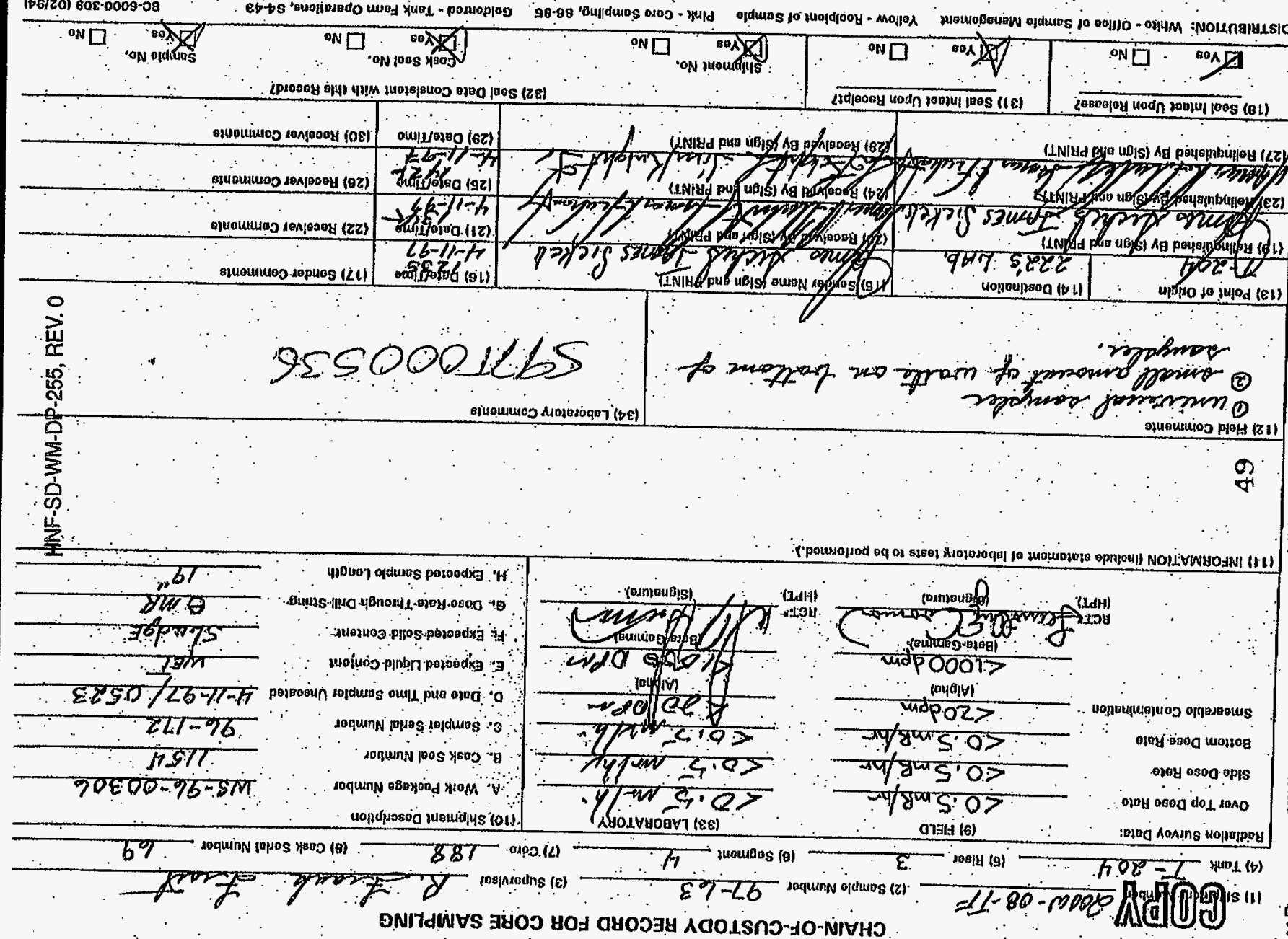


(bo/20) 602-0009.08.

E6 †s 'eltopolodo unef YuOL : postoploo

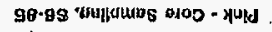

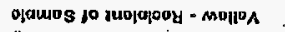

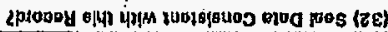

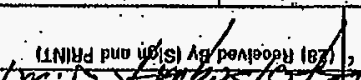
$\angle 6=5-30$

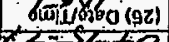
$>\sqrt{1}$

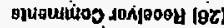

opuruiuog.

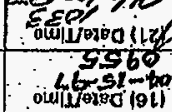

,

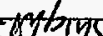

\section{1} Nisd 10 ob st arou $(6 z)$

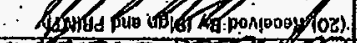

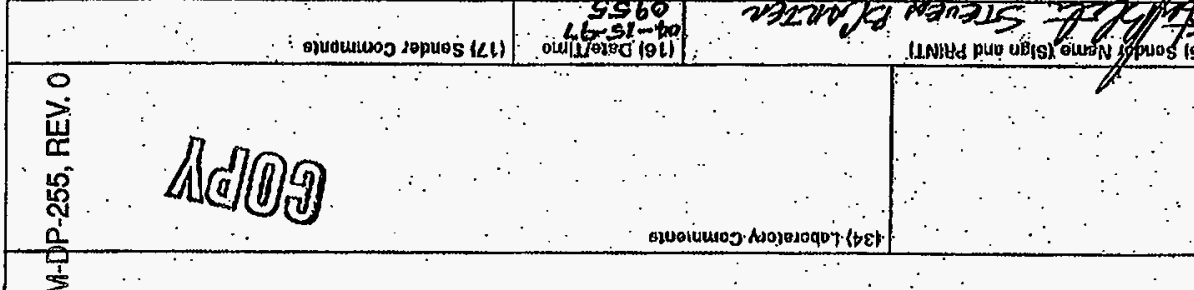
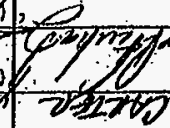

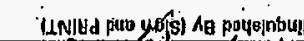
$\mathbb{Z}_{\operatorname{sen}}^{\prime} \sum_{(b)}^{\prime \prime}$

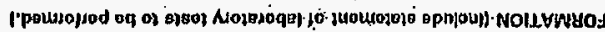

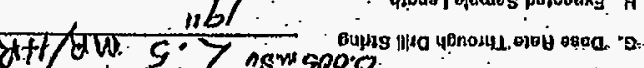

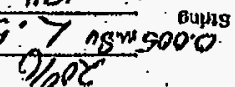

OTPS.

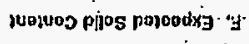
Mugiuos p|nb|7 posoodxa "a

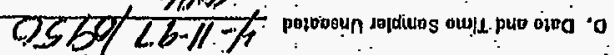
bs $7-2 b$

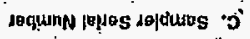
द्SGET Jequinn obos Yo8s o 908OO-9b-SM

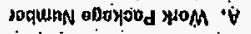
Mondjeseo Juedudius (ol)

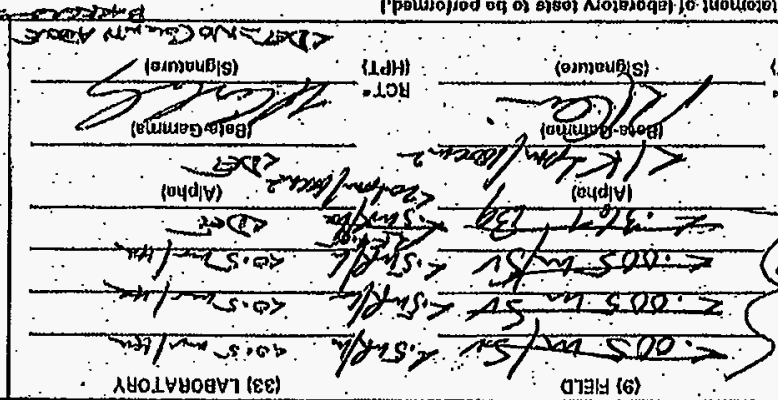

4.51.<smiles>c1ccccc1</smiles>
got etoc u olvy ag0o expy esood dol ioliog houns uo

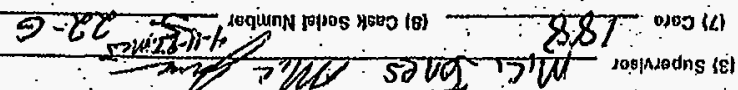
$108=1$ 


\section{CHAIN-OF-CUSTODY RECORD FOR CORE SAMPLING}

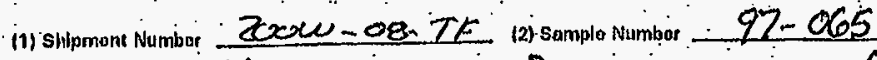
(4) Tank T2204 (6) nisor 3 (6) sogmont . 6 (3) șuporvisor M.c

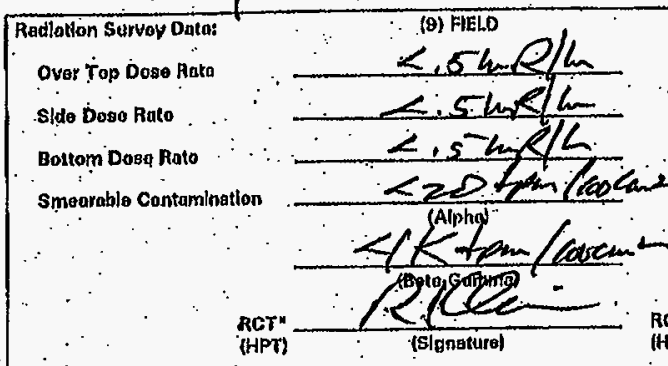

111) WFORMATION IInolude - elatomant of -laboratory teats to bo porformed.)
(33) LABORATORY $\because$ ) (10) Shlpment Dogerlption

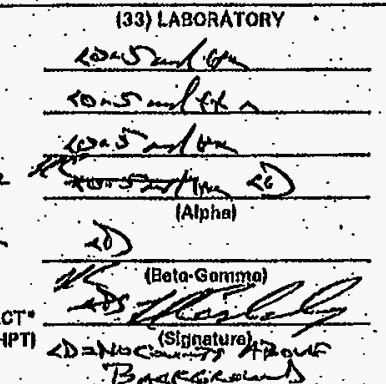

A. Wark Packago Numbar

B. Cask Sàai Numbar

C. Semplor Sorlal Number

D. Doto and Mina Samplap Unseatod

E. Expootad Llquld Contant

F. Expojtad Solla Contont

opos mS.

c. Doso Rato Through Dellit string -

H. Exparted Sample Longth
(8) Cosk serial Numbar $27 \div 0$

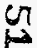

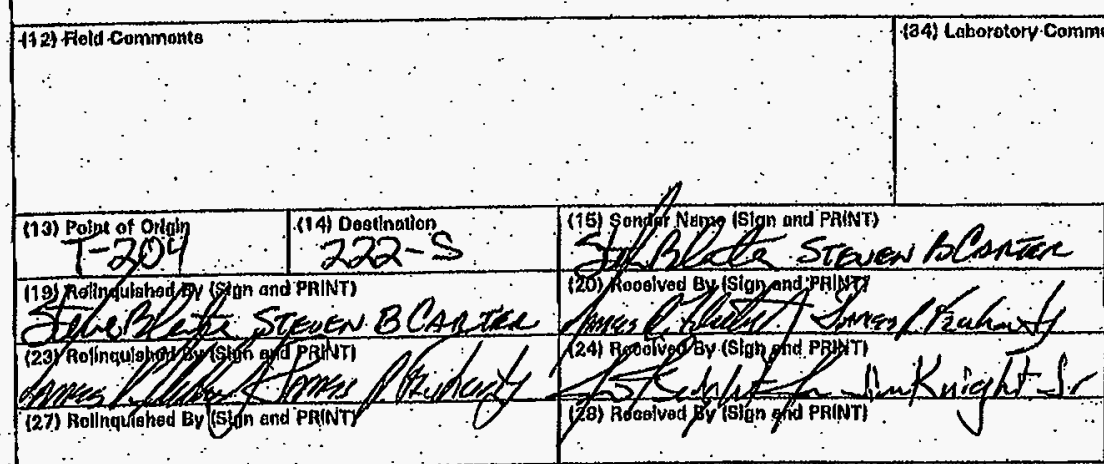

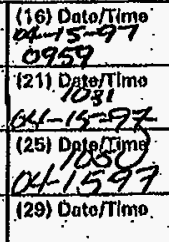

$45-96-00306$ 12453 $96-174$ $\$ 00 \%$ 27 $20 \% 20$

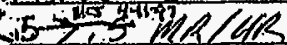
$\because 1 q^{\prime \prime}$

$$
\text { . }
$$

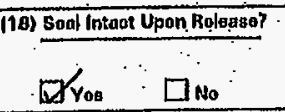

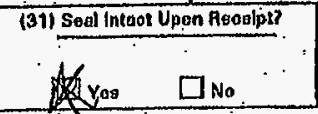

Nono.

Stymant No.

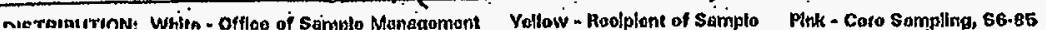

Goldenrod - Tảk Faim Opoyatlons, $\mathbf{5 4 - 4 3}$

Goots sad No. $\square$ No
(17). Sonder Comments

\section{(22) Recolver Commonte}

(26) Rocolvar Commanto.

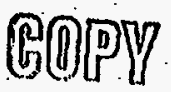

(aO) Reosivar Cammonts
32) Soof Data Conotstont with.this flecord?

Y 


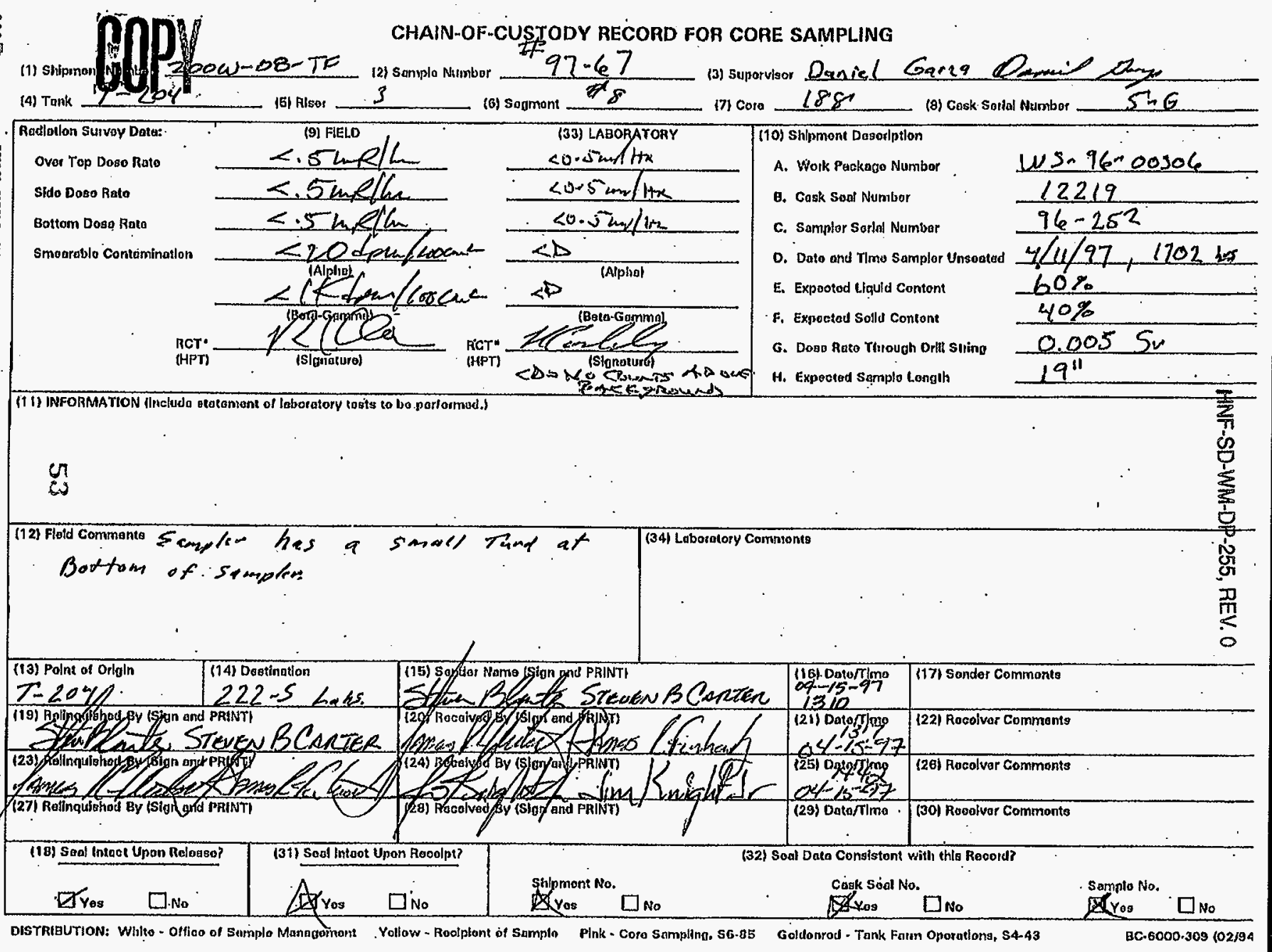




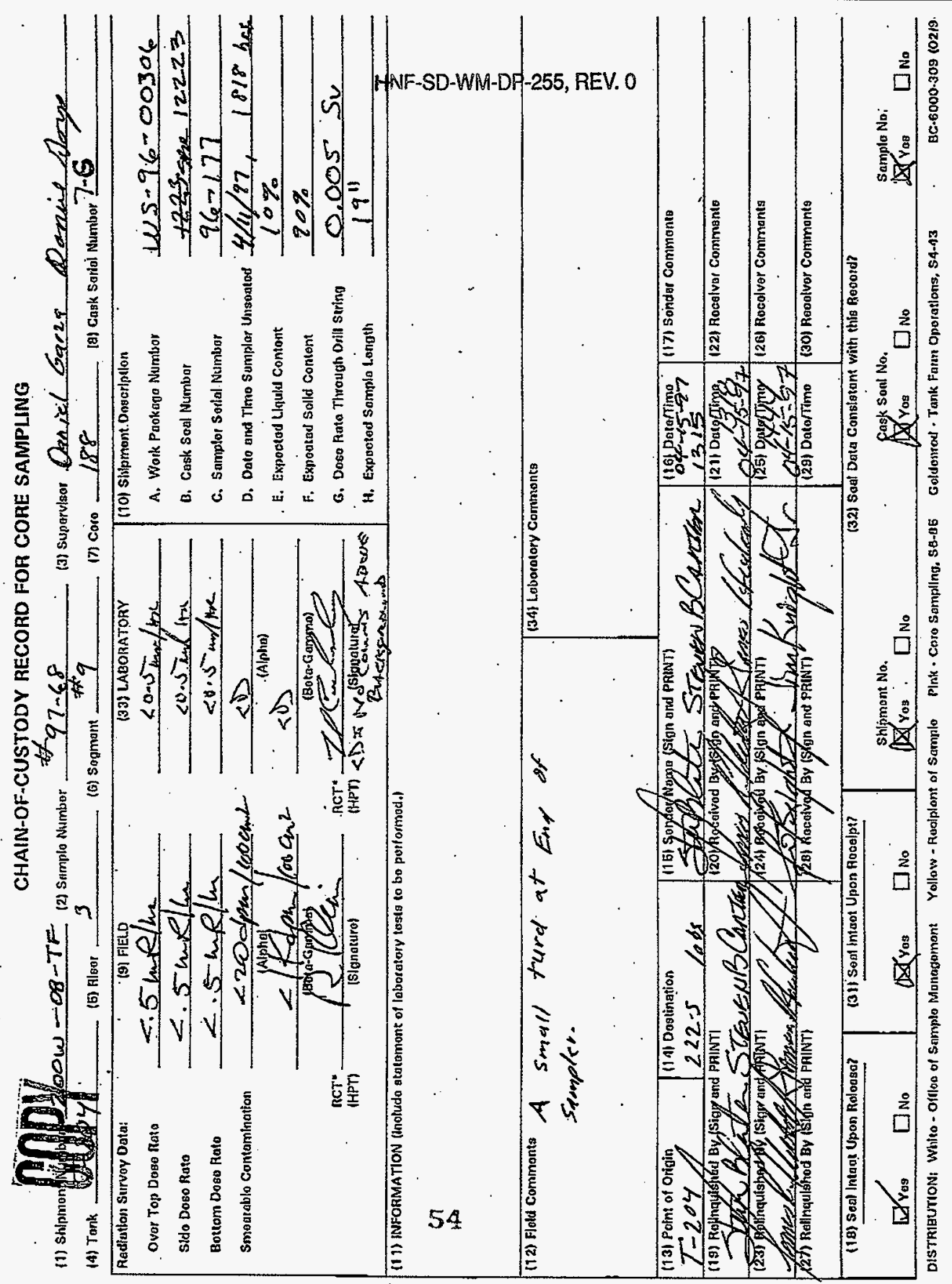




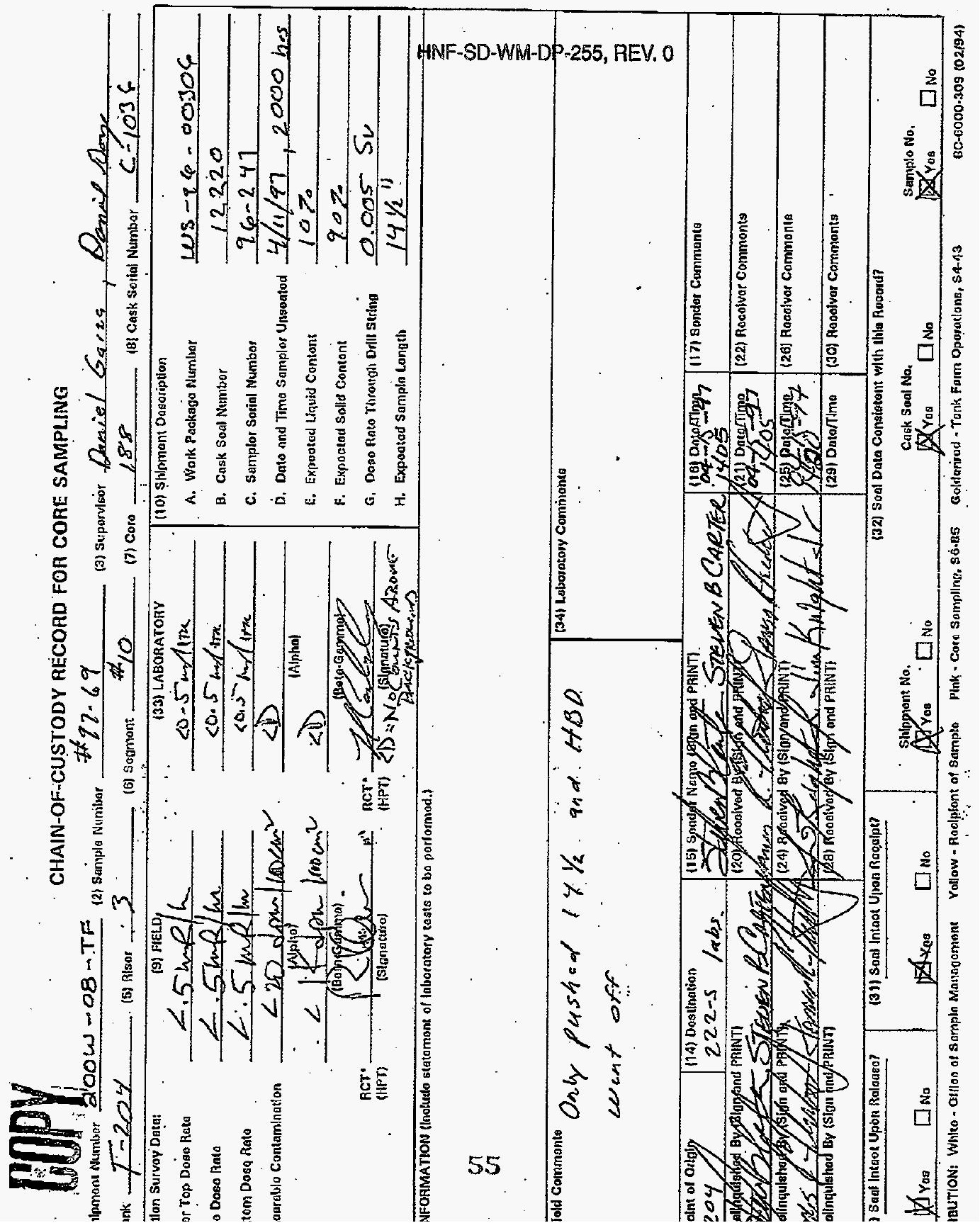


HNF-SD-WM-DP-255, REV. 0

PHOTOGRAPHS

56 
HNF-SD-WM-DP-255, REV. 0

THIS PAGE INTENTIONALLY LEFT BLANK 


\section{HNF-SD-WM-DP-255, REV. 0}

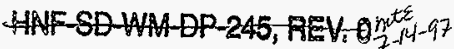

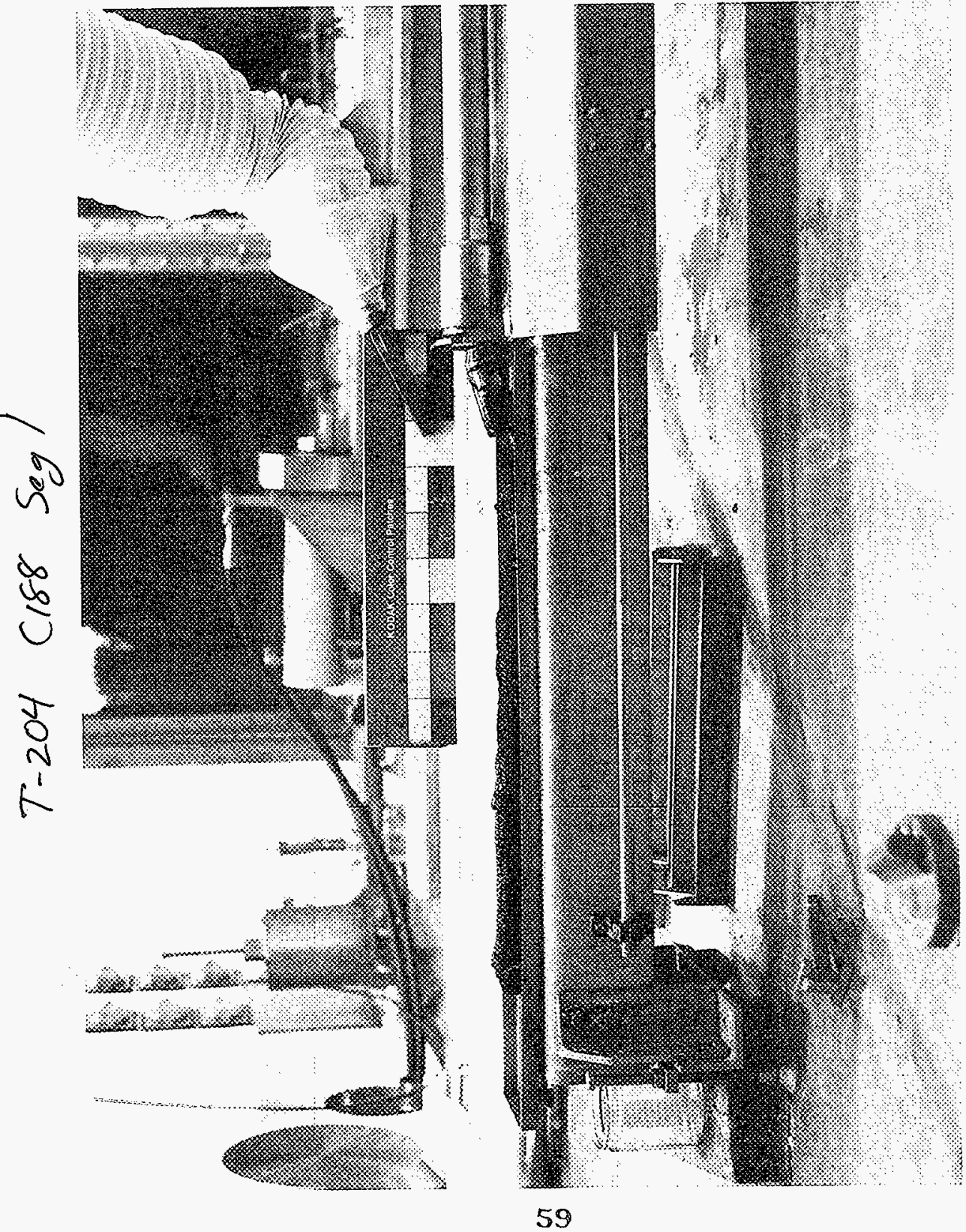




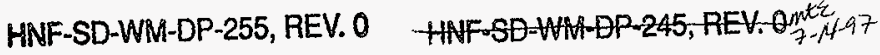

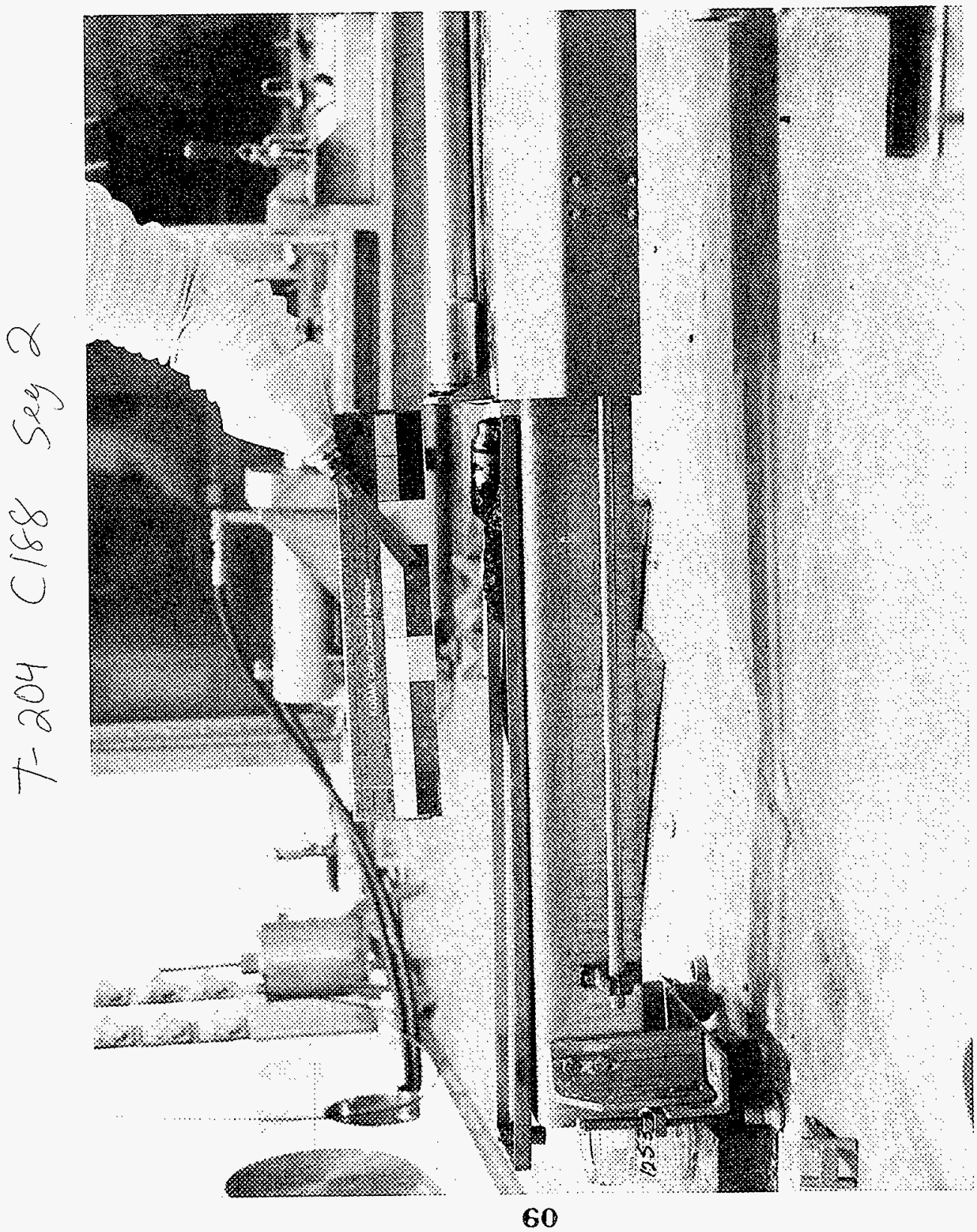




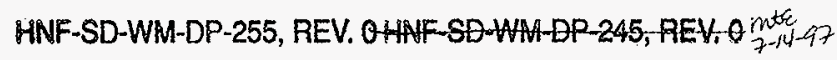

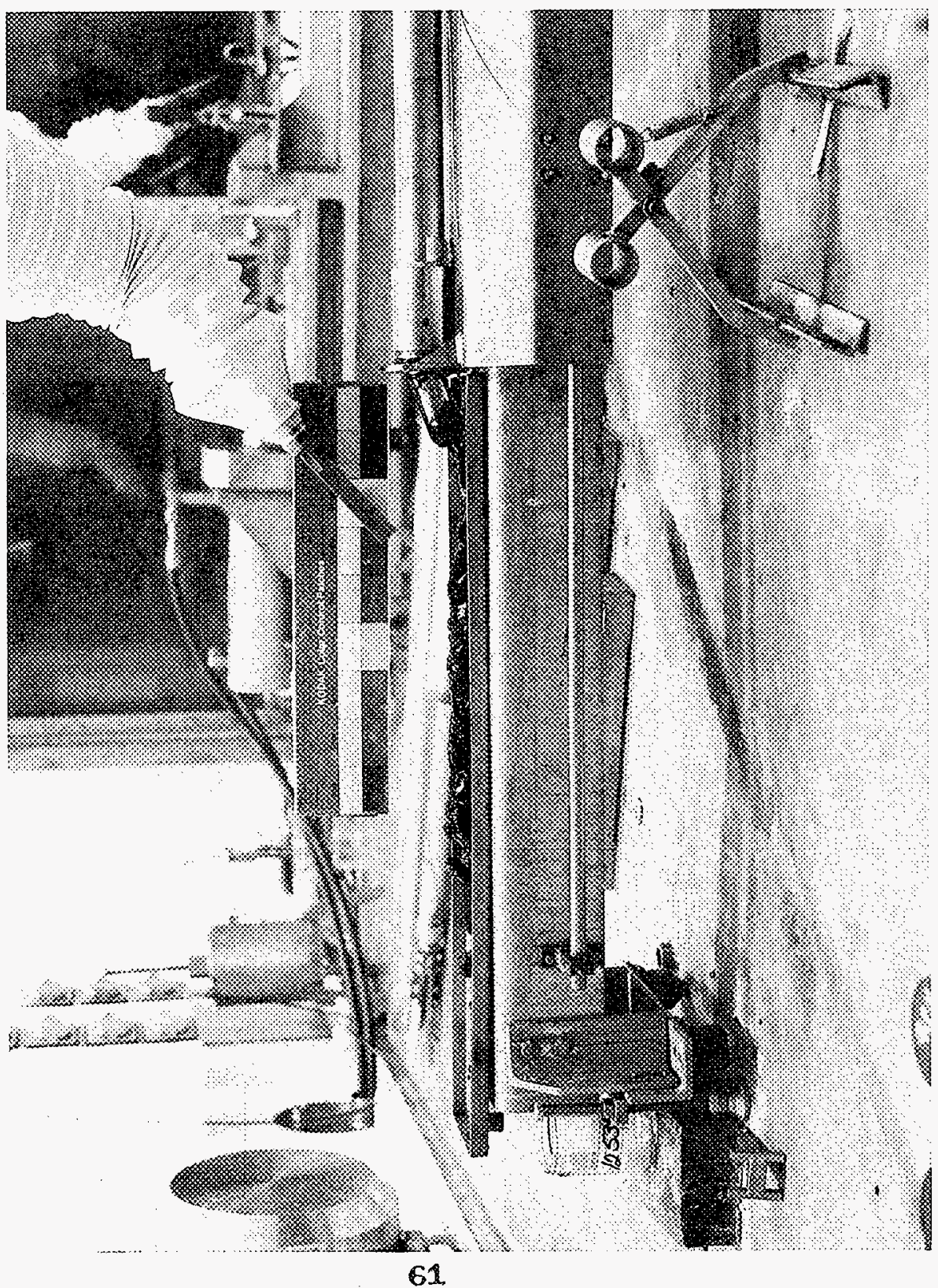




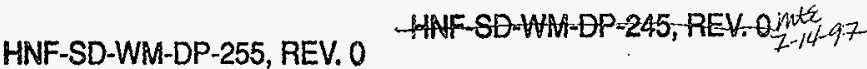

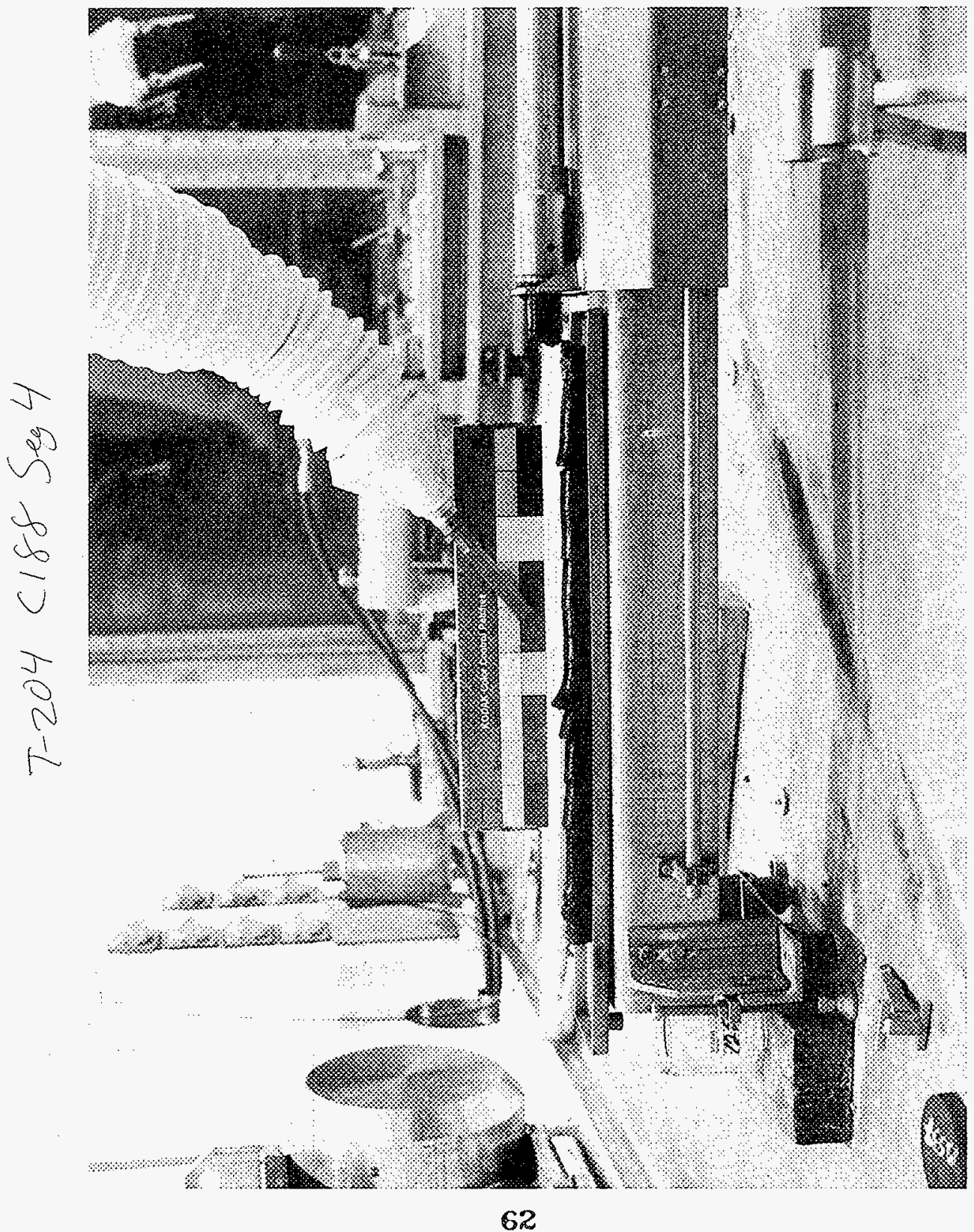




\section{HNF-SD-WM-DP-255, REV. 0 HAF-SQWAA-DP-245,REV.01nte}

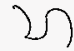
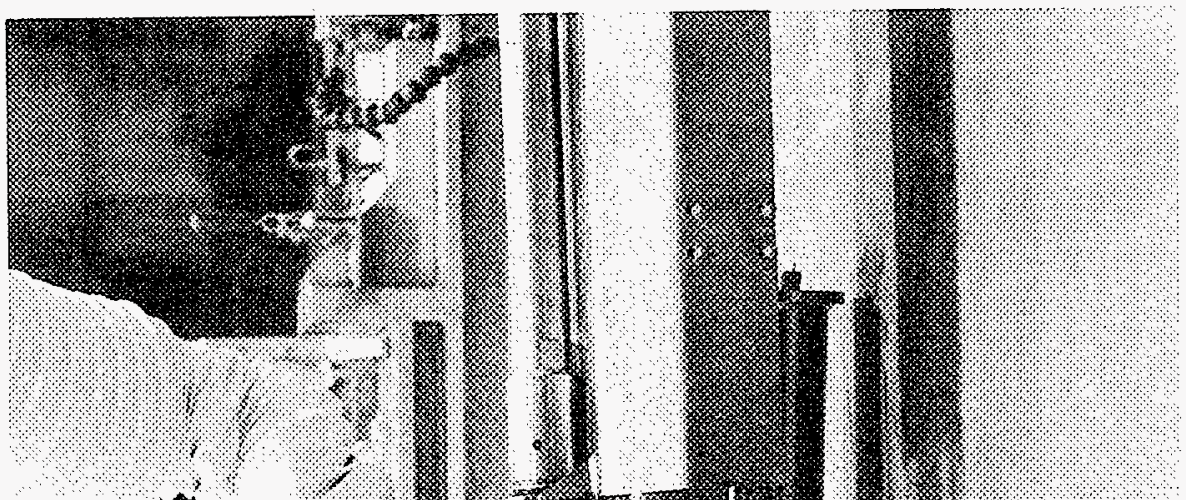

$\frac{2}{6}$
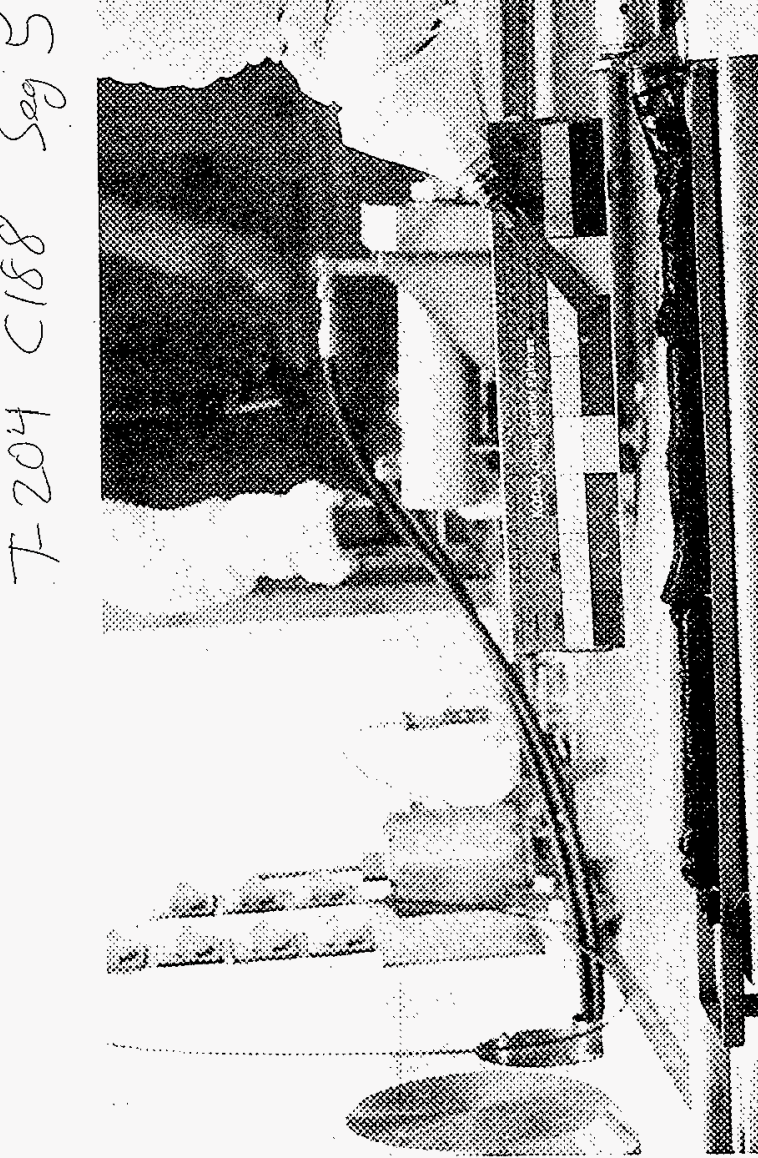

63 


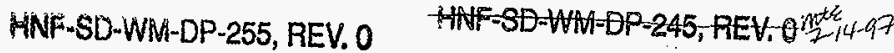
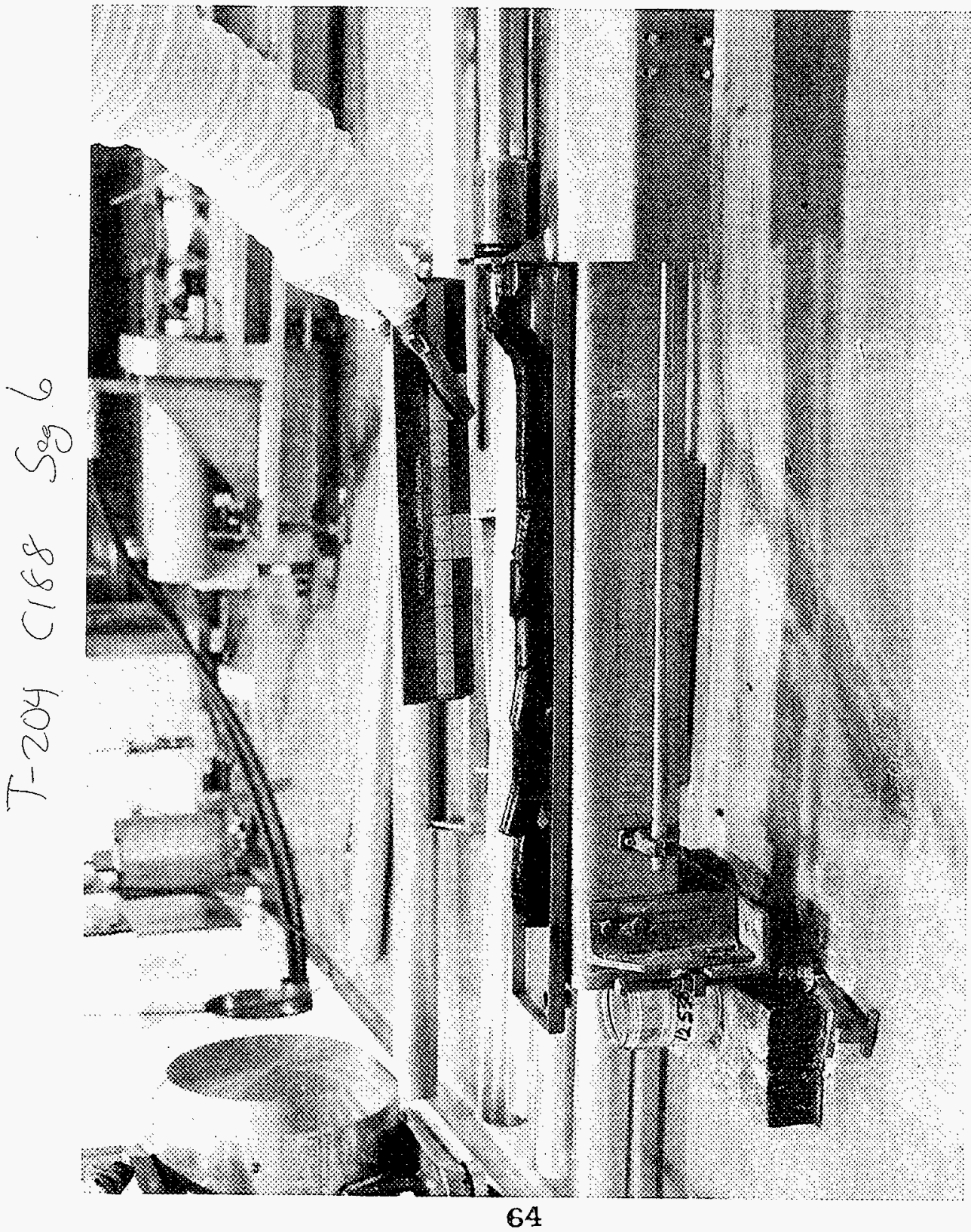


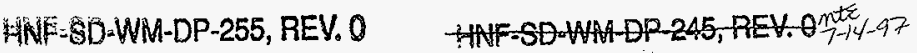

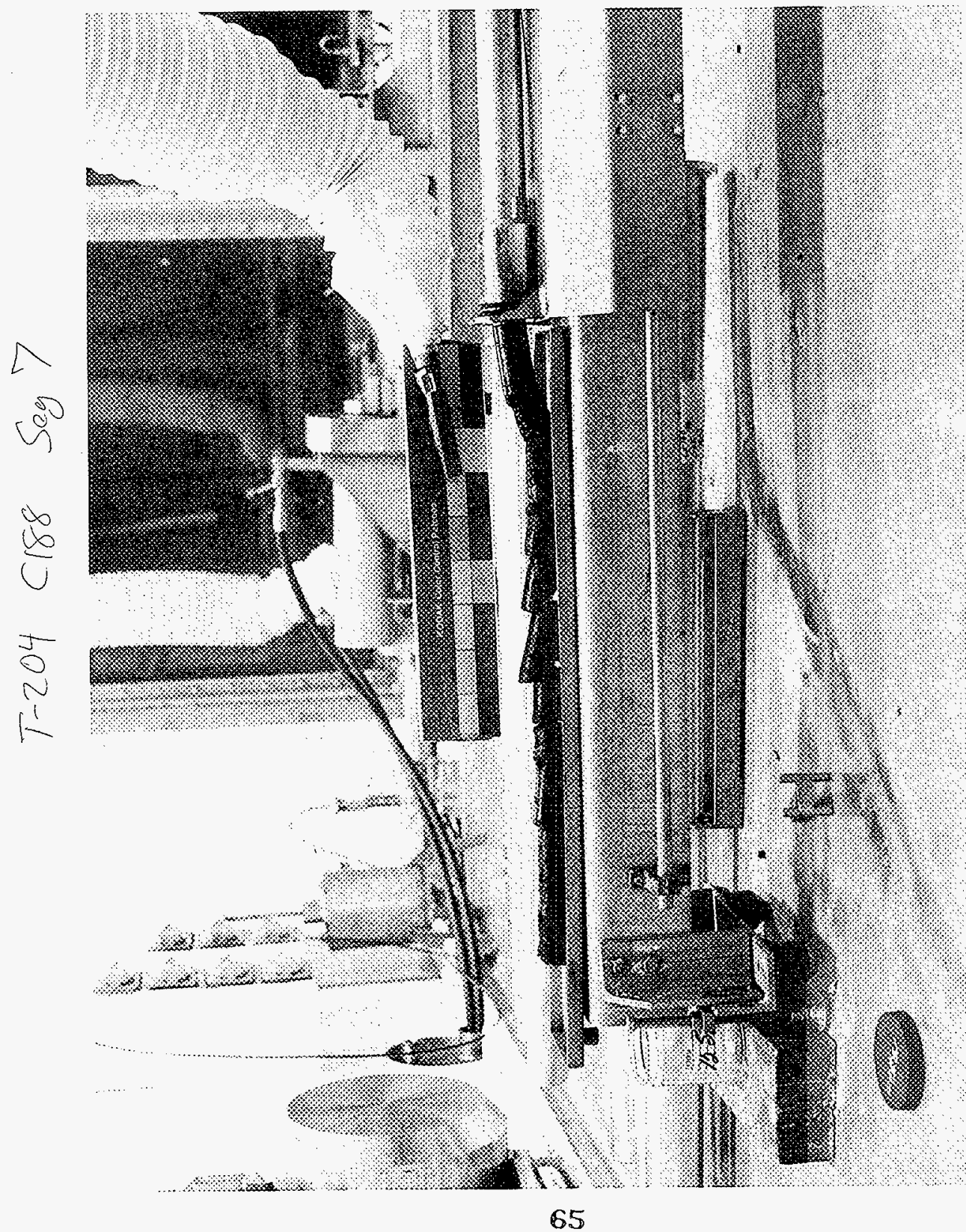




\section{A}

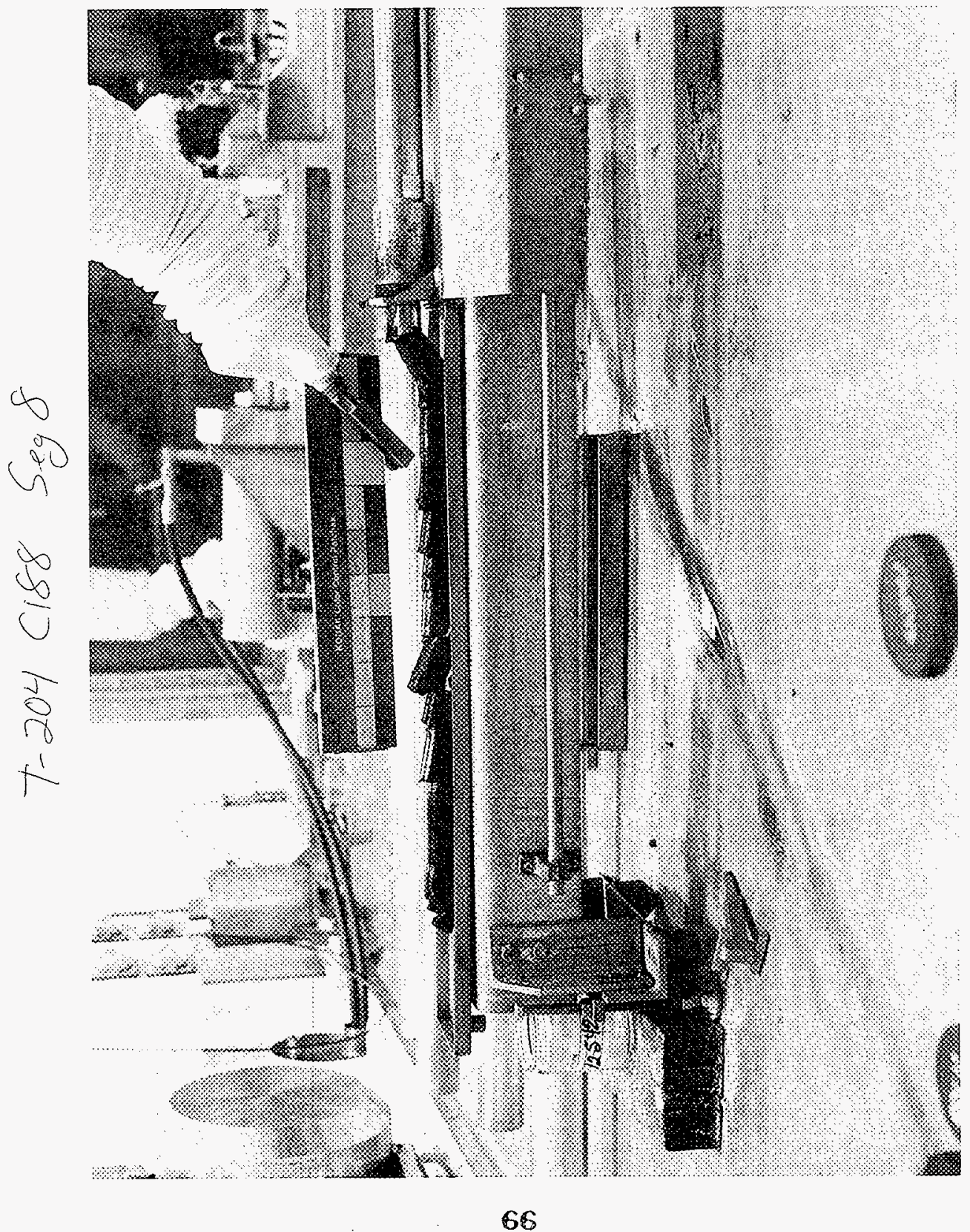




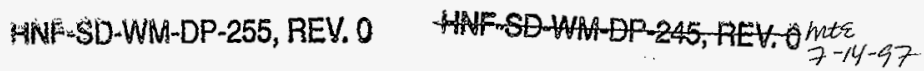

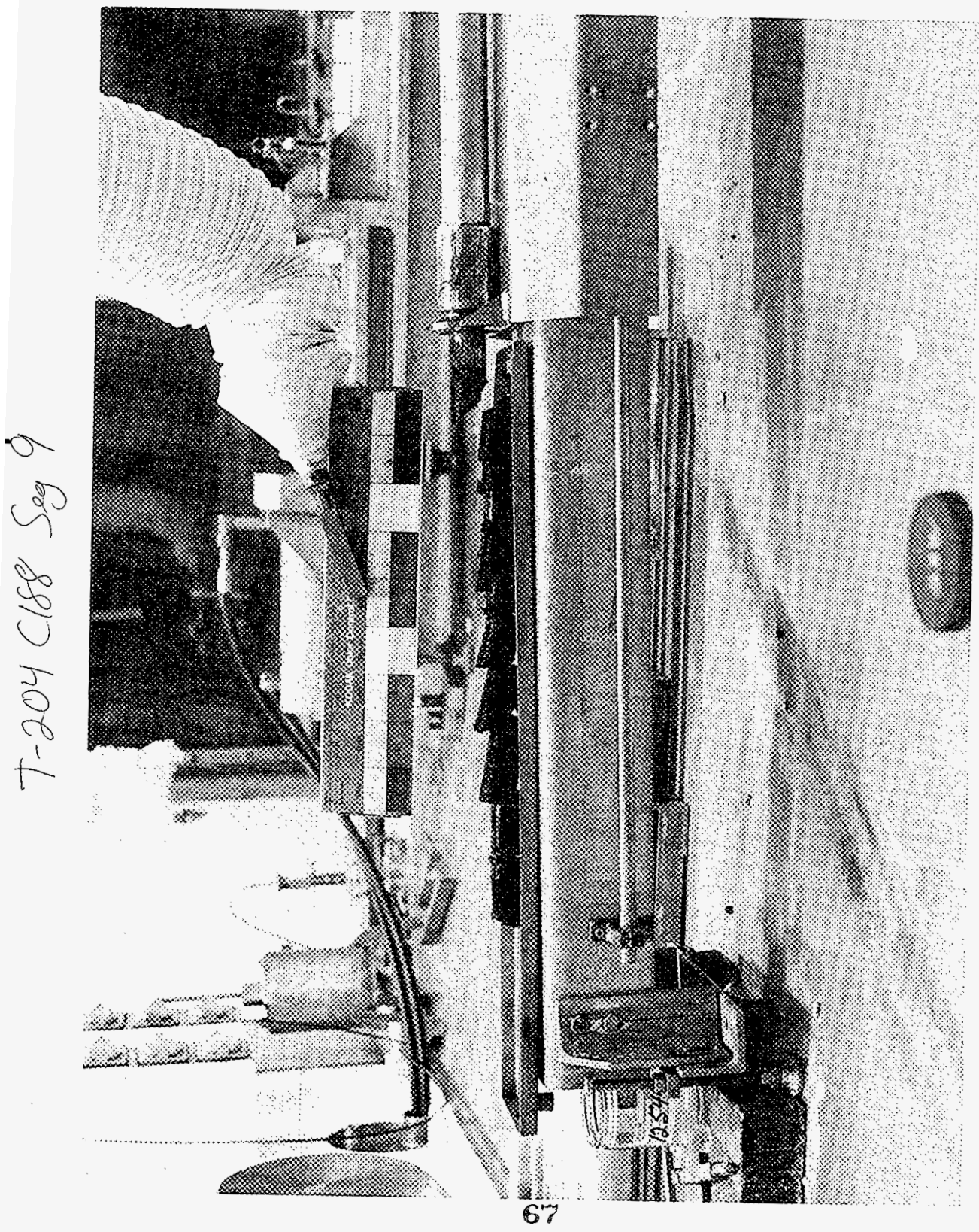




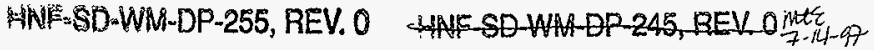

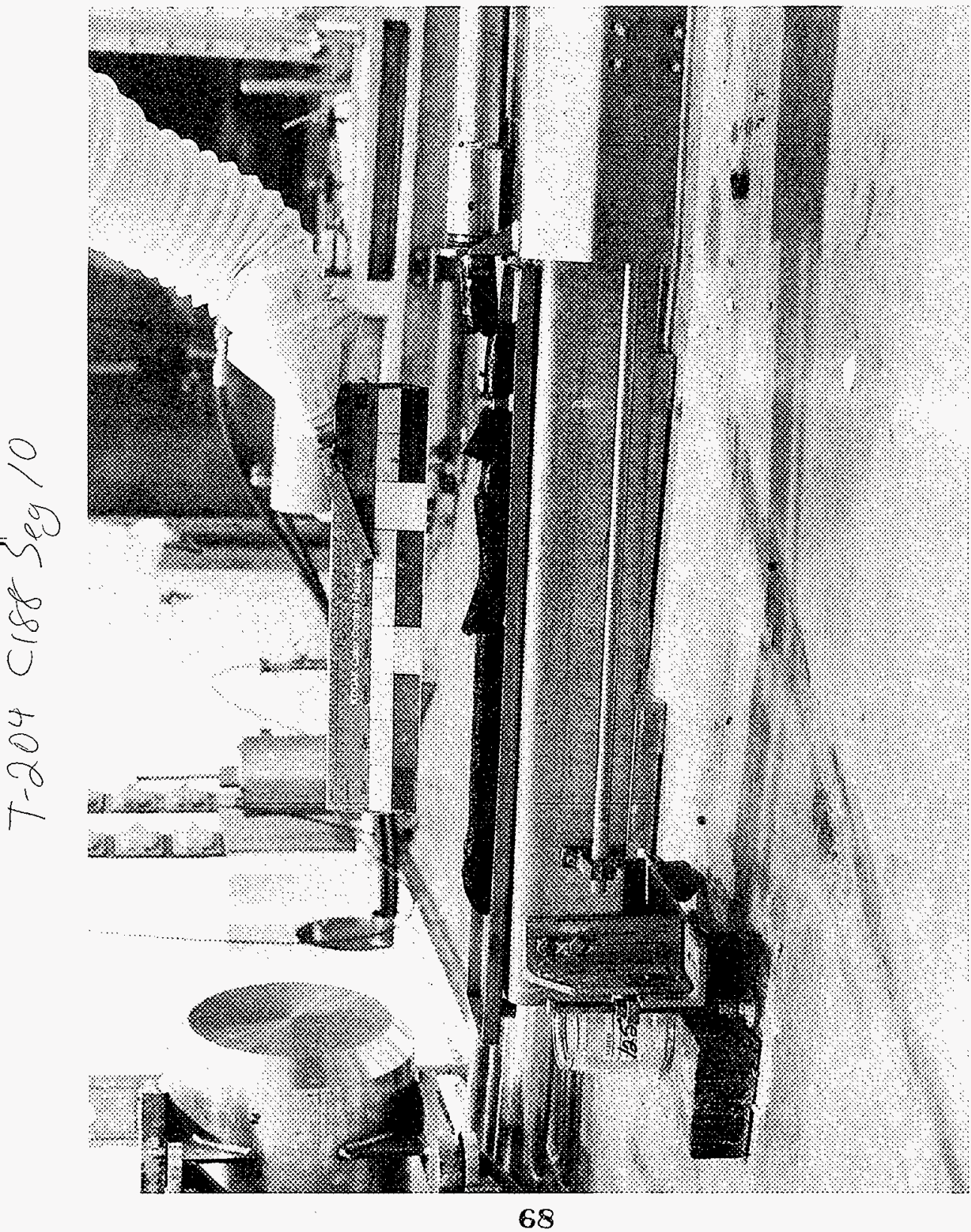


HNF-SD-WM-DP-255, REV. 0

COMPOSITE WORKSHEETS 
HNF-SD-WM-DP-255, REV. 0

THIS PAGE INTENTIONALLY LEFT BLANK 


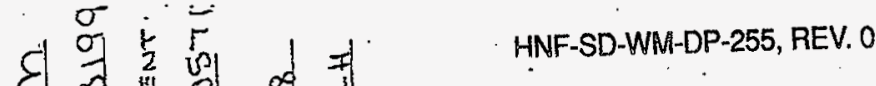

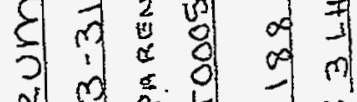

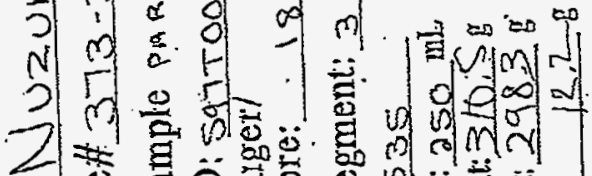

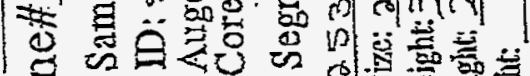

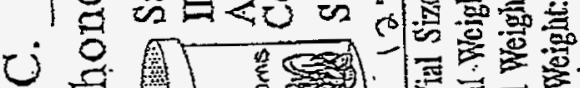

a. 合

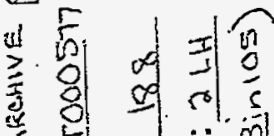

2

(1)

릴

0

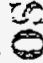

.

.

邹

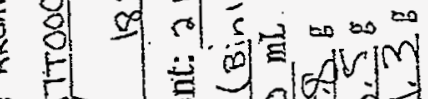

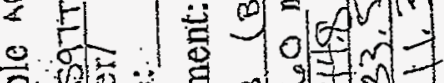

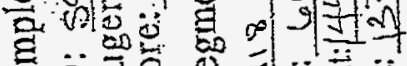

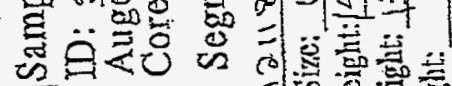

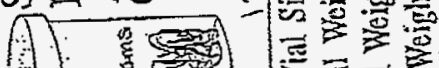

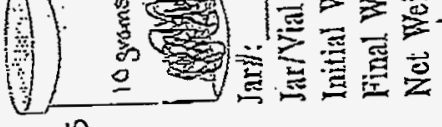

in

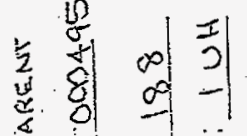

¿

告 की 药

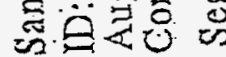

U

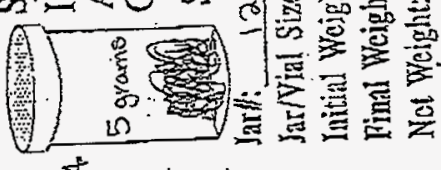

$r \infty$ 获

$1)$ 중

政

.

(1)

क्ष

$-i$

6

s.

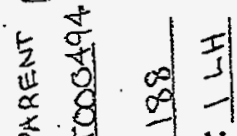

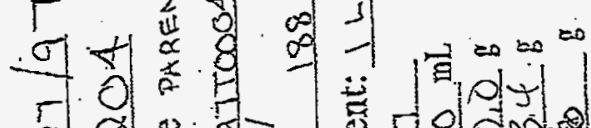

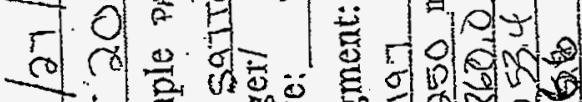

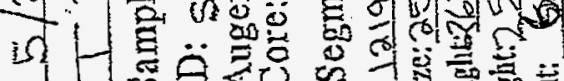

$\rightarrow$ on 00

(1 1 -

42

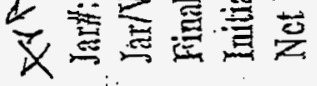

se

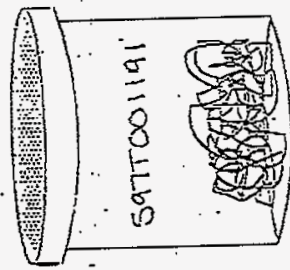

के

11 कुष्य

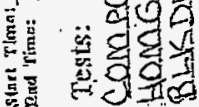

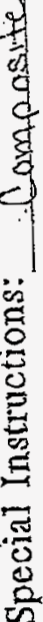

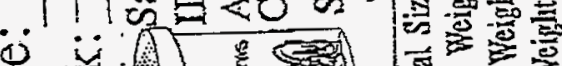

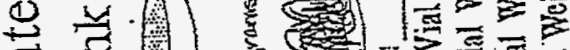

त 


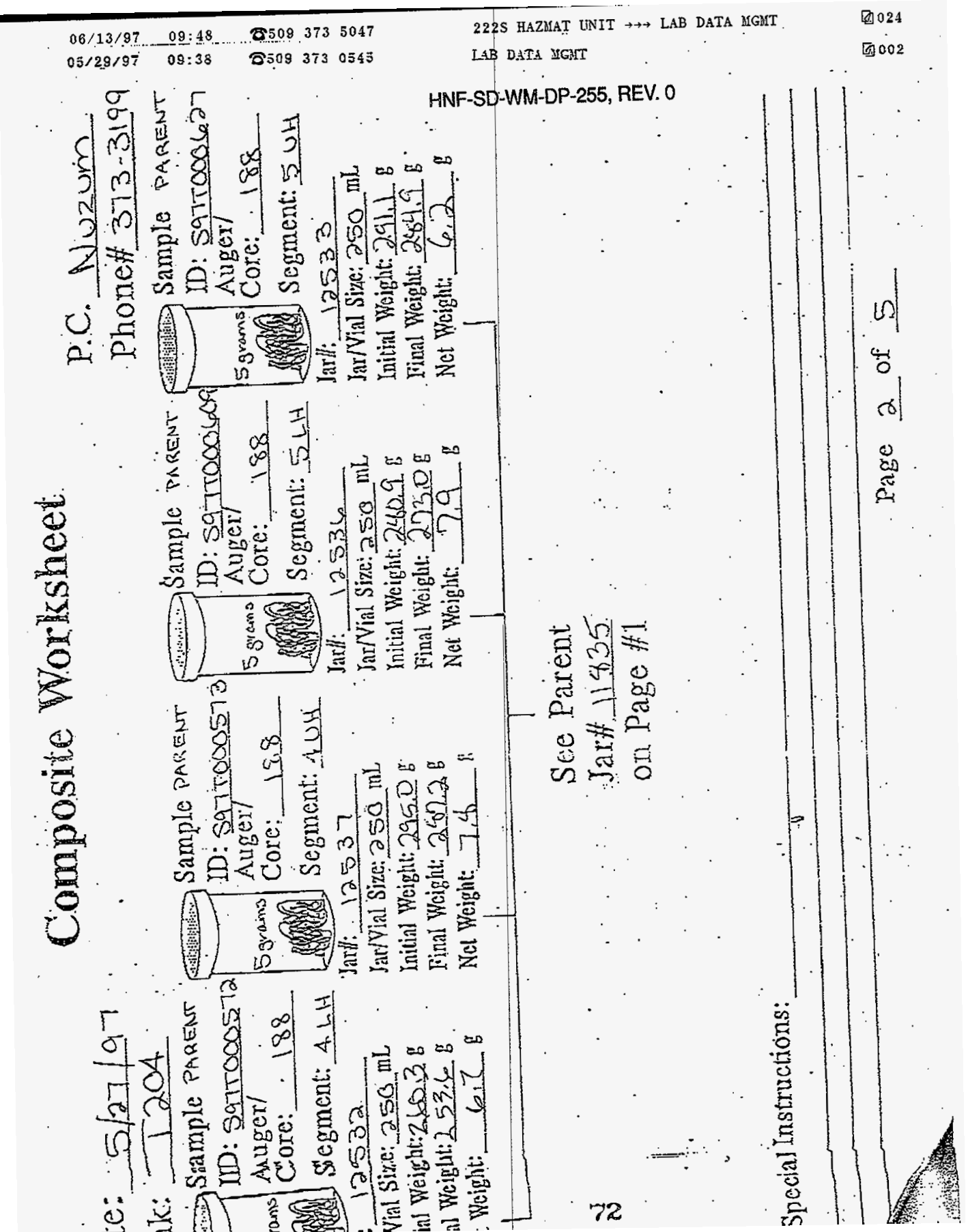



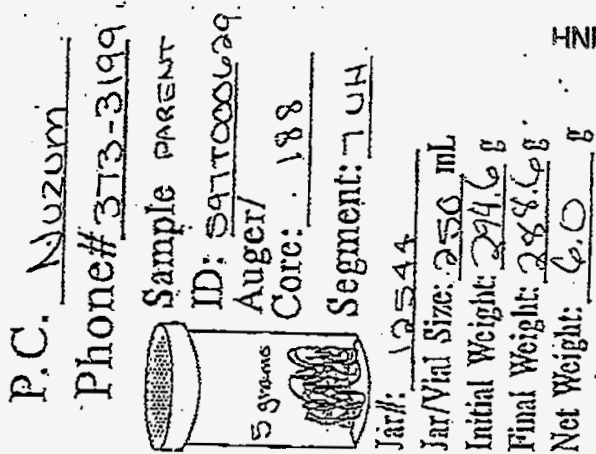

Q 0 Q

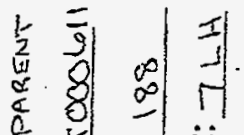

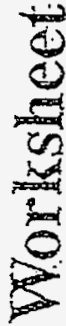<smiles>CC(C)C</smiles>

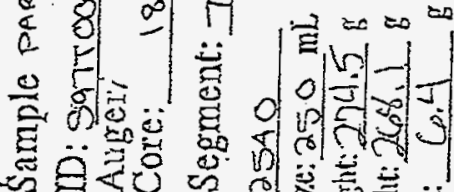

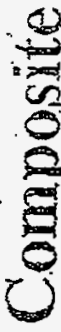

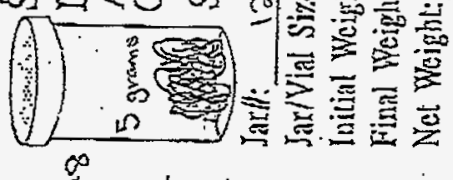

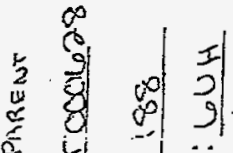

c.

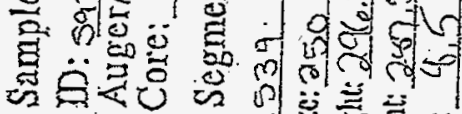

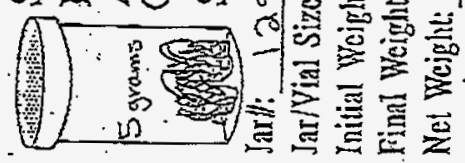

至行

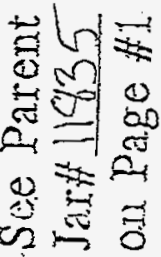

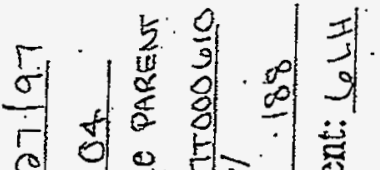

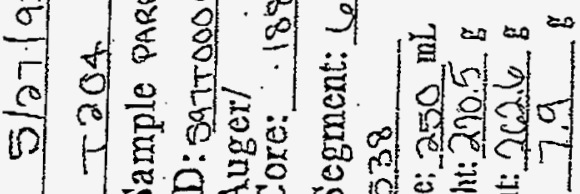

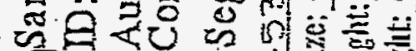

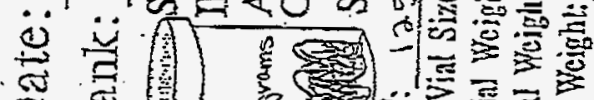

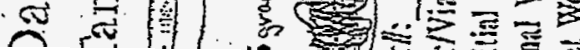




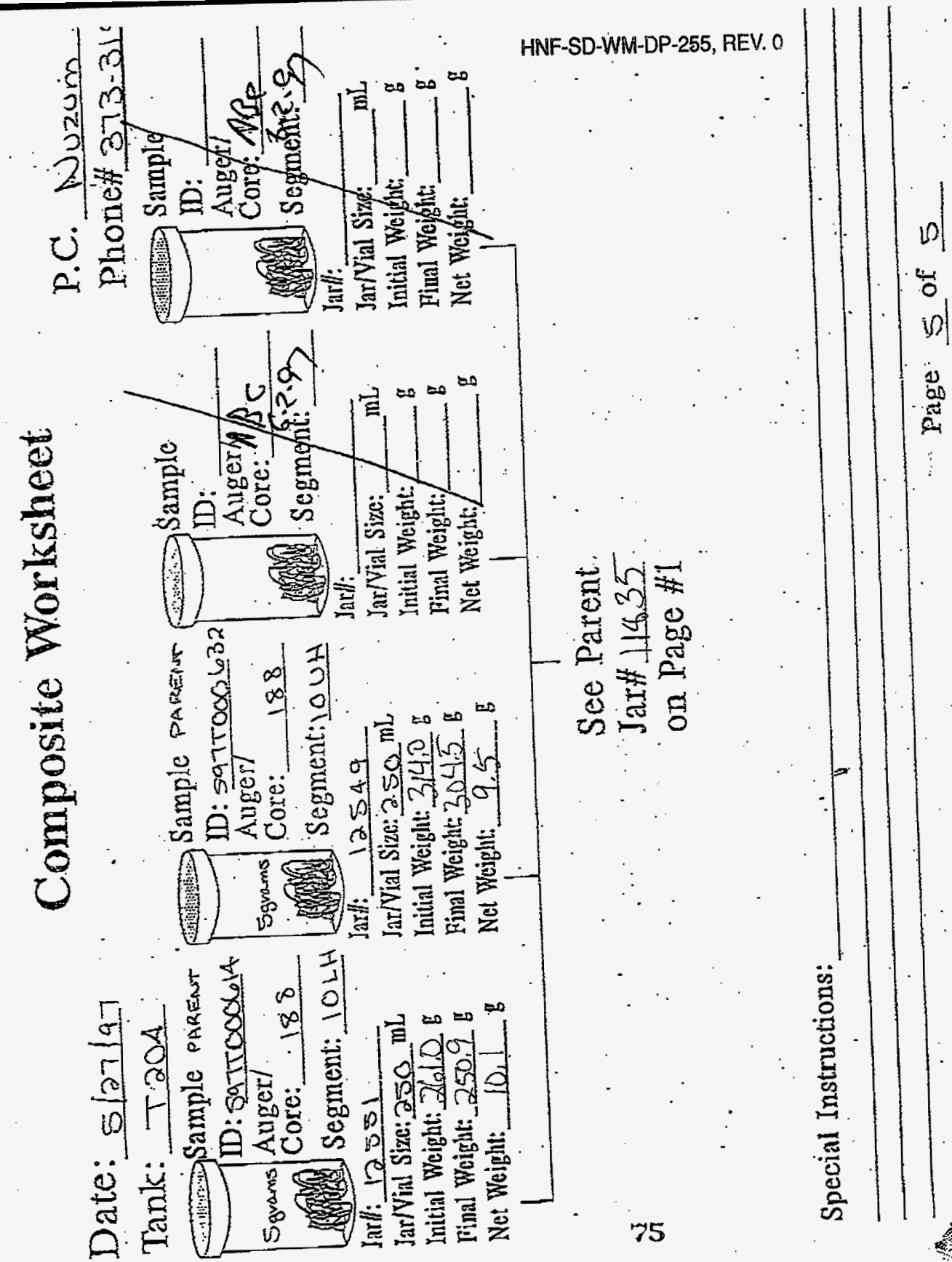


06/13/97 09:38_2509 3735047

222S HAZMAT INIT $\rightarrow \rightarrow \rightarrow$ LAB DATA MGM

005

HNF-SD-WM-DP-255, REV. 0

Final Composite Calculation Shat

Date: 6-1.97 Technician: A CAmplahl_____

Total number of jars used to bund the composite: 18 .

Composite Jar number: 11835 Jar size: 125

1) Sample from segment jar 12197 to composite jar $=3.0$ grams

2) Sample from segment jar 12189 to composite jar = 5.0 grams

3) Sample from segment jar 121.18 to composite. jar = 1.0 .0 grams

4) Sample from segment jar $12535^{\circ}$ io composite jar $=10,0$ grans

5) Sample from segment jar 2532 to composite jar. +5.0 grams

6) Sample from segment jar 12537 to composite jar $=5.1$ crams

7) Sample from segment jar 12536 to composite jor $=6.2$ grams

8) Sample iron segment jar 12533 to composite jar $=5,0$ grams

9) Sample from segment jar 12538 to composite jar $=5.0$ grams

10) Sample iron segment jor 12539 to composite jar $=5.0$ grams

11). Sample ir om segment jar 12540 to composite jar $=5,0$ grams

12) Sample from segment jar 2544 to composite jar $=4.9$ grins

13) Sample from segment jar 12542 to composite jar $=7.9$ grans

14) Sample from segment jar 1254 i to composite jar $=\frac{5,9}{5}$ gris

15) " $"$ " $12541 "$ " $" 11=\frac{5}{50}$

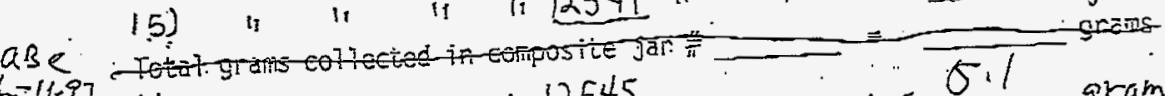

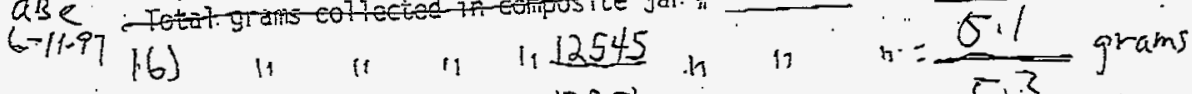

17) " $" 11,12551 " 11.5 .3$ grams

ib) " 111212549 in $11,1.5 .2$ grams

Total grams elected in Commit Jar $\frac{}{76}=105.6$ grams 
HNF-SD-WM-DP-255, REV. 0

SAMPLE HANDLING 
HNF-SD-WM-DP-255, REV. 0

THIS PAGE INTENTIONALLY LEFT BLANK 


\section{LABCORE Data Entry Template for Worklist\# 17438}

Analyst: JLE

Method: LO-160-103 Rev/Mod
Instrument: $\mathrm{BA} 000$

Book \#

HNF-SD-WM-DP-255, REV. 0

Worklist Comment: T-204 C188 SEG\# 1 RISER 3 EXTRUSION

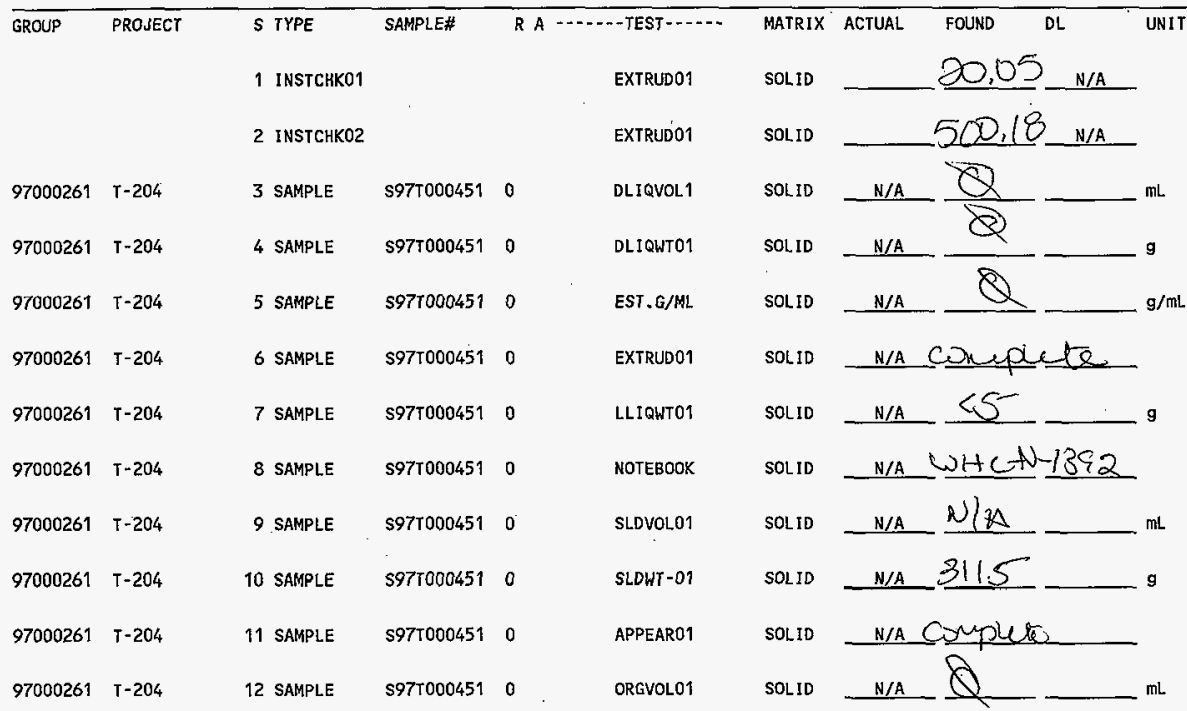

Final page for worklist \# 17438
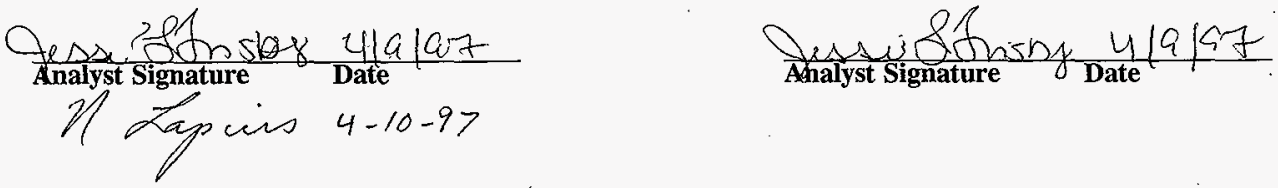

Data Entry Comments:

Units shown for $Q C$ (SPK \& STD) may not reflect the actual units. $D L=$ Detection Limit, $S=$ Worklist Slot Number,

$R=$ Replicate Number, $A=$ Aliquot Code. 


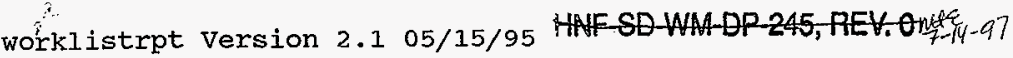

04/15/97 14:17

LABCORE Data Entry Template for Worklist\# 17566

\section{Analyst:}

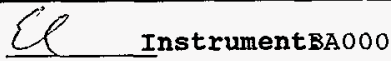

Method: LO-160-103 Rev/Mod

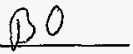

\section{Worklist comment 2204 C188 SEG \# 2 RISER 3 EXTRUSION}

\section{HNF-SD-WM-DP-255, REV. 0}

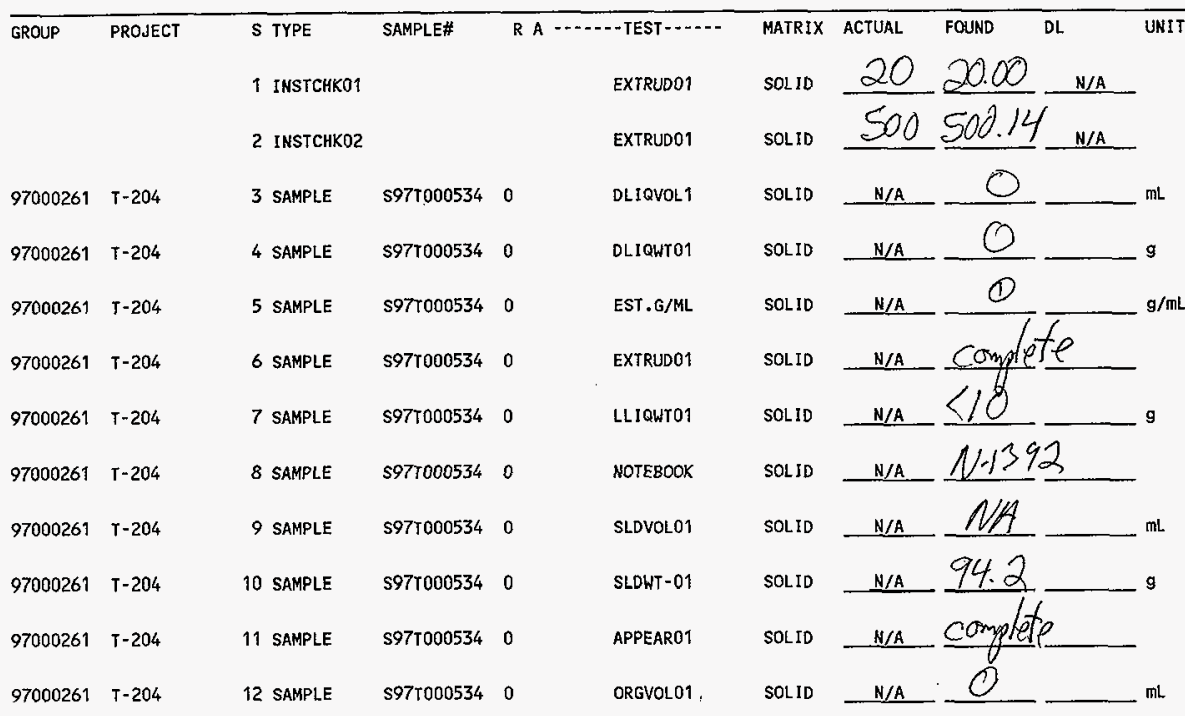

Final page for worklist \# 17566
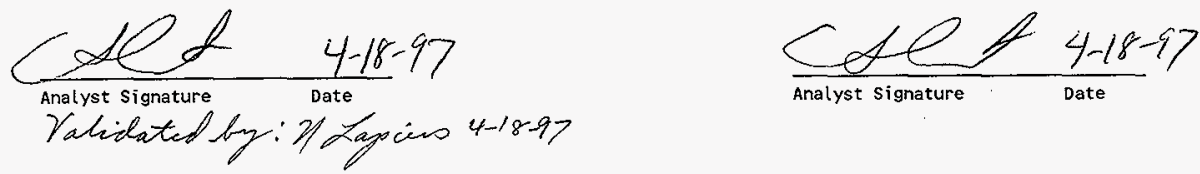

Data Entry Comments:

Units shown for QC (SPK \& STD) may not reflect the actual units. DL = Detection Limit, $S=$ Worklist Slot Number, $R=$ Repl icate Number, $A=A l$ iquot Code. 
HAF-SD-WAP-245, REV. Ond $74-97$

worklistrpt version $2.105 / 15 / 95$

HNF-SD-WM-DP-255, REV. 0

Page:

LABCORE Data Entry Template for Worklist\# 17567

Analyst:

E. InstrumentBAO00

Book \# UA

Method: LO-160-103 Rev/Mod DO

Worklist Comment:204 C188 SEG \# 3 RISER 3 EXTRUSION

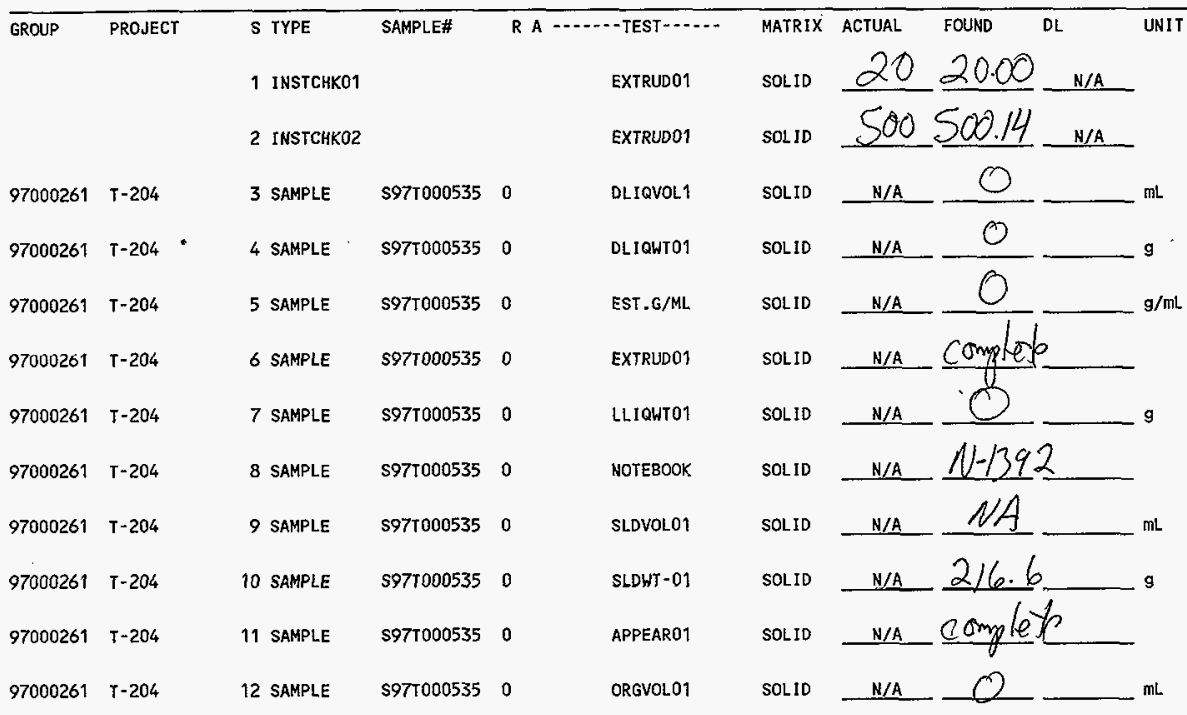

Final page for worklist \# 17567
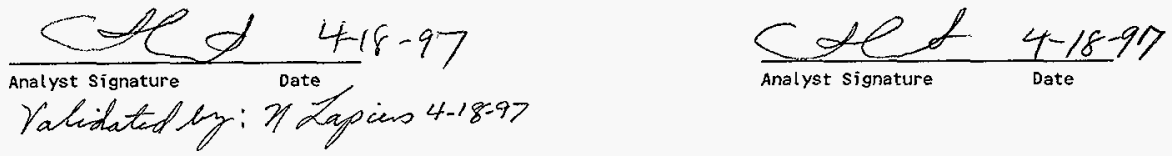

Data Entry Comments:

Units shown for aC (SPK \& STD) may not reflect the actual units. DL = Detection Limit, $S=$ Worklist $s$ lot Number, $R=$ Repl icate Number, $A=A l$ iquot Code. 
worklistrpt Version $2.105 / 15 / 95$

HNF-SD-WAHEP-245, REV.,0nte $74-97$

Page:

$04 / 15 / 97$ 14:27

\section{LABCORE Data Entry Template for Worklist\# 17568}

Analyst:

InstrumentBA000

Method: LO-160-103 Rev/Mod
Book \# NA

HNF-SD-WM-DP-255, REV. 0

Work1ist Comment $\div 204$ C188 SEG \# 4 RISER 3 EXTRUSION

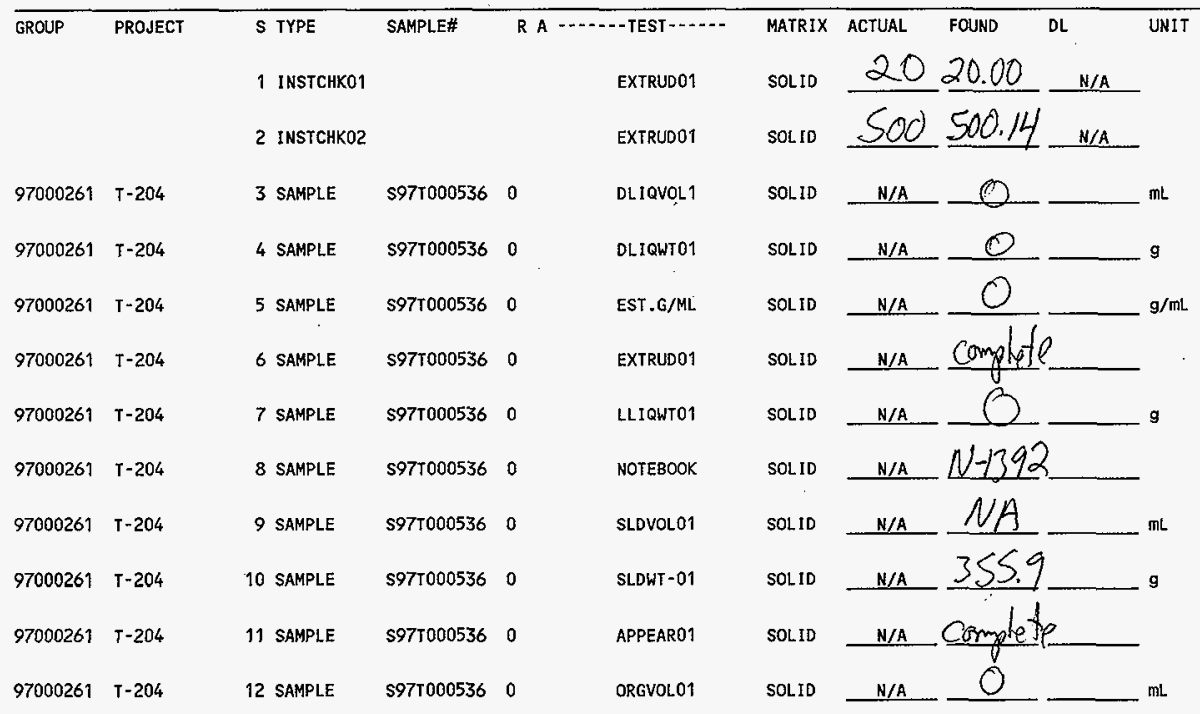

Finat page for worklist \# 17568
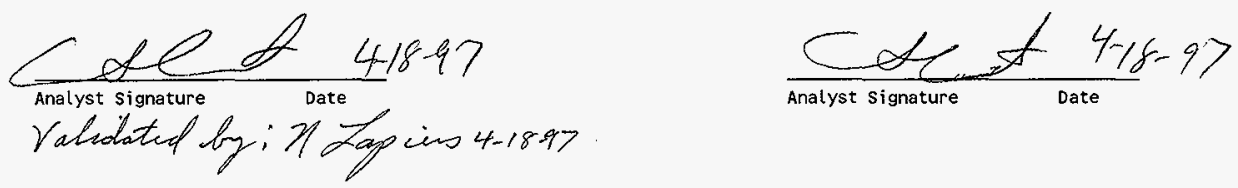

Data Entry Comments:

Units shown for QC (SPK \& STD) may not reflect the actual units. DL = Detection Limit, $s=$ Workl ist slot Nunber, $R=$ Replicate Number, $A=A$ iquot code. 
worklistrpt version $2.105 / 15 / 95$

HAF-SE-WAT-OP-245,REV Ont

Page: 04/15/97 14:29

LABCORE Data Entry Template for Worklist\# 17569

Analyst: $\quad$ Be InstrumentBA000

Method: Lo-160-103 Rev/Mod $\beta($
Book \# NA

HNF-SD-WM-DP-255, REV. 0

Worklist comment:T-204 Cl88 SEG \# 5 RISER 3 EXTRUSION

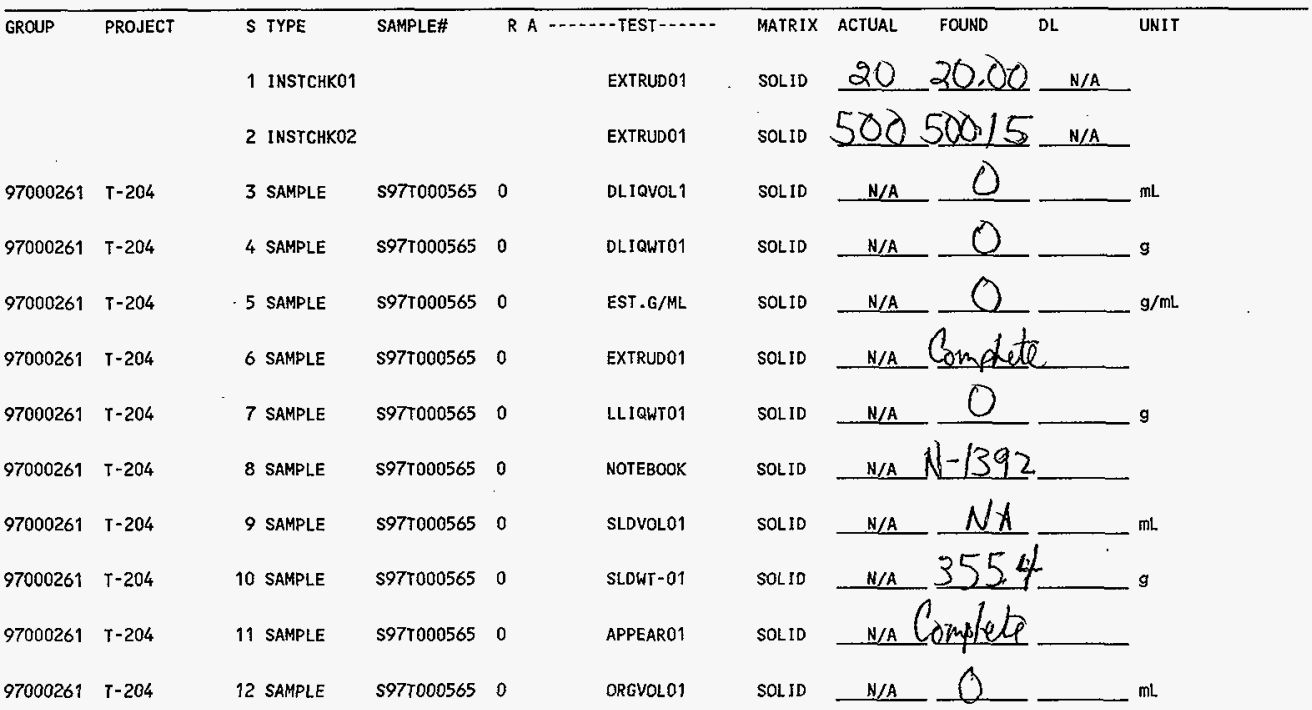

Final page for worklist \# 17569

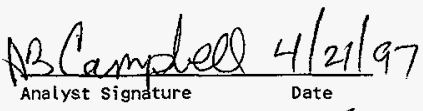

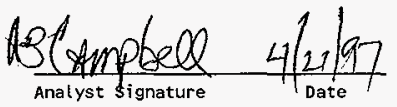

Validated By NILapiers 4-21-97

Data Entry Comments:

Units shown for QC (SPK \& STD) may not reflect the actual units. DL = Detection Limit, $S=$ Worklist slot Number, $R=$ Replicate Number, $A=$ Al iquot Code.

83 
LABCORE Data Entry Template for Worklist\# 17570

Analyst:

AB InstrumentBA000

Method: Lo-160-103 Rev/Mod

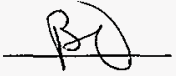

Book \# $0 / 9$

HNF-SD-WM-DP-255, REV. 0

Worklist comment: T-204 C188 SEG \# 6 RISER 3 EXTRUSION

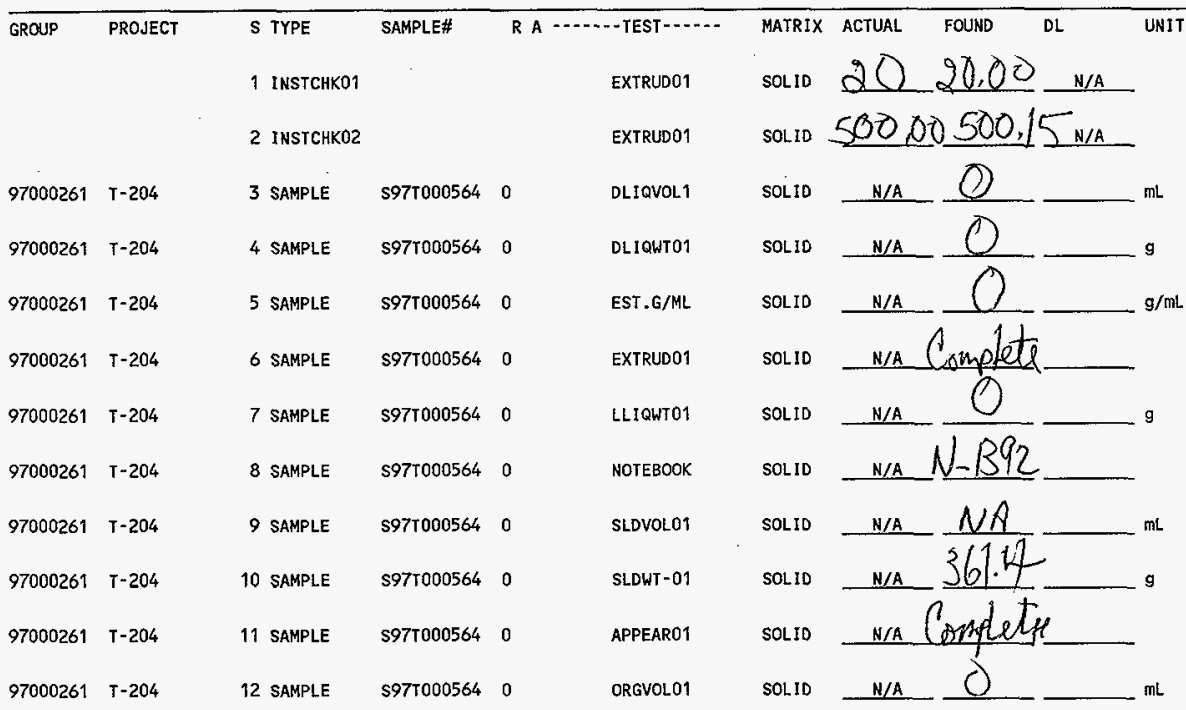

Final page for worklist \#17570
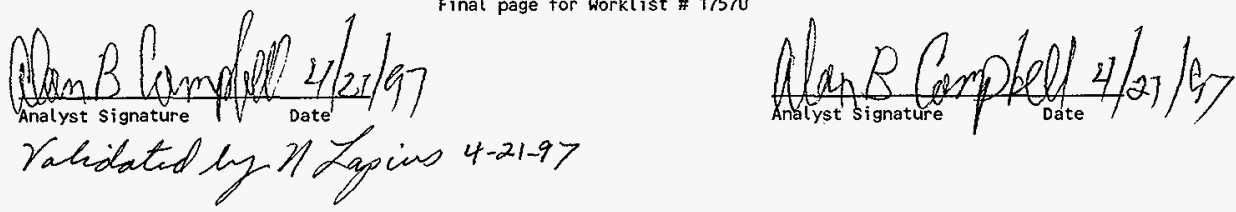

Data Entry Comments:

Units shown for QC (SPK \& STO) nay not reflect the actual units. DL = Detection Limit, $S=$ Worklist slot Number, $R=$ Repl icate Number, $A=A$ liquot Code. 
worklistrpt Version $2.105 / 15 / 95$

IABCORE Data Entry Template for worklist\# 17571

Analyst:

ABC InstrumentBAO0O

Book \#NA

Method: Lo-160-103 Rev/Mod

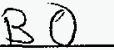

HNF=SD-WM-DP-255, REV. 0

Worklist Comment:T-204 C188 SEG \# 7 RISER 3 EXTRUSION

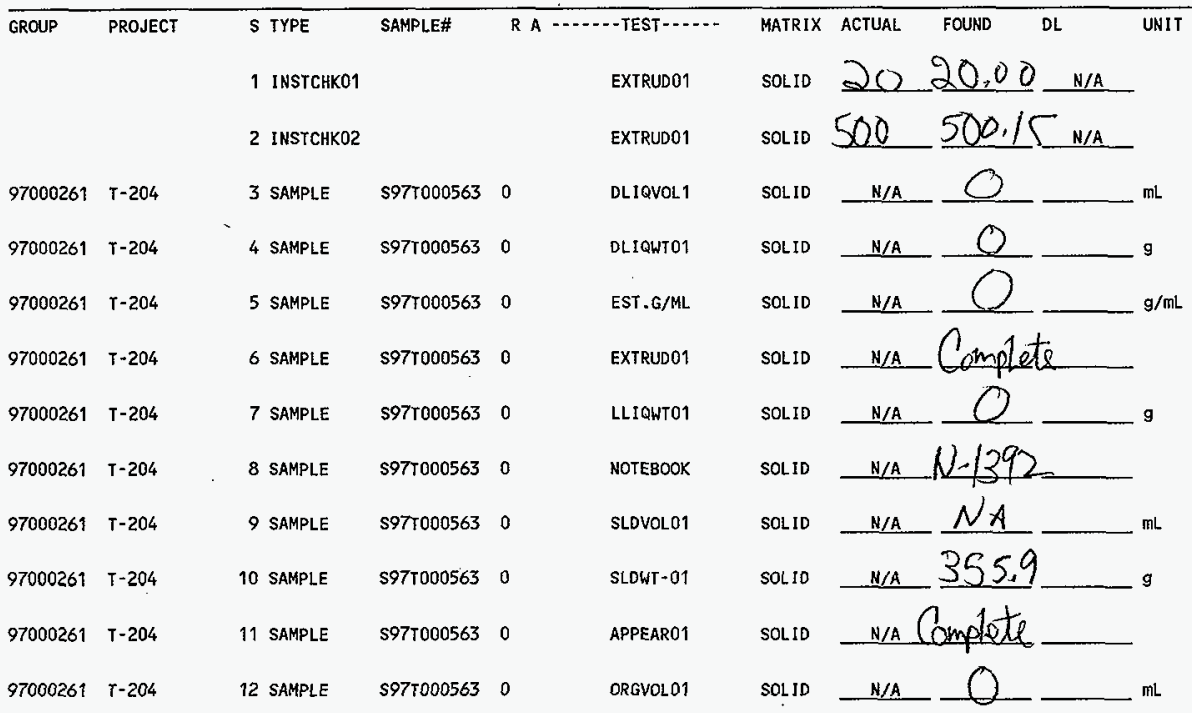
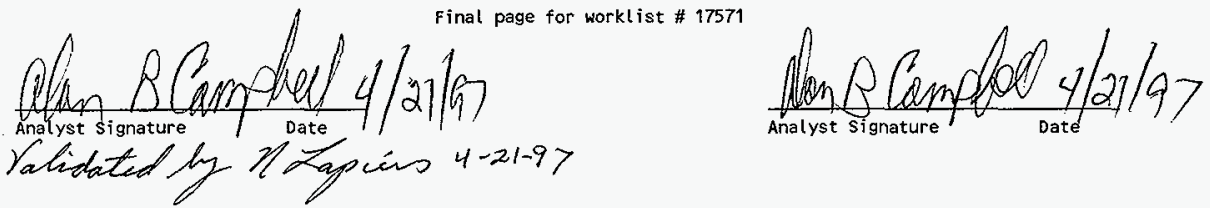

Data Entry Comments:

Units shown for QC (SPK \& STD) may not reflect the actual units. DL = Detection Limit, $\mathbf{S}=$ Worklist $\mathrm{Slot}$ Number, $\mathrm{R}=$ Replicate Number, $\mathrm{A}=\mathrm{Aliquot}$ Code. 


\section{LABCORE Data Entry Template for Worklist\# 17602}

Analyst: $\quad$ ASe Instrument: BA000 _ Book\# WA

Method: LO-160-103 Rev/Mod BO

HNF-SD-WM-DP-255, REV. 0

Worklist Comment: T-204 C188 SEG \# 8 RISER 3 EXTRUSION

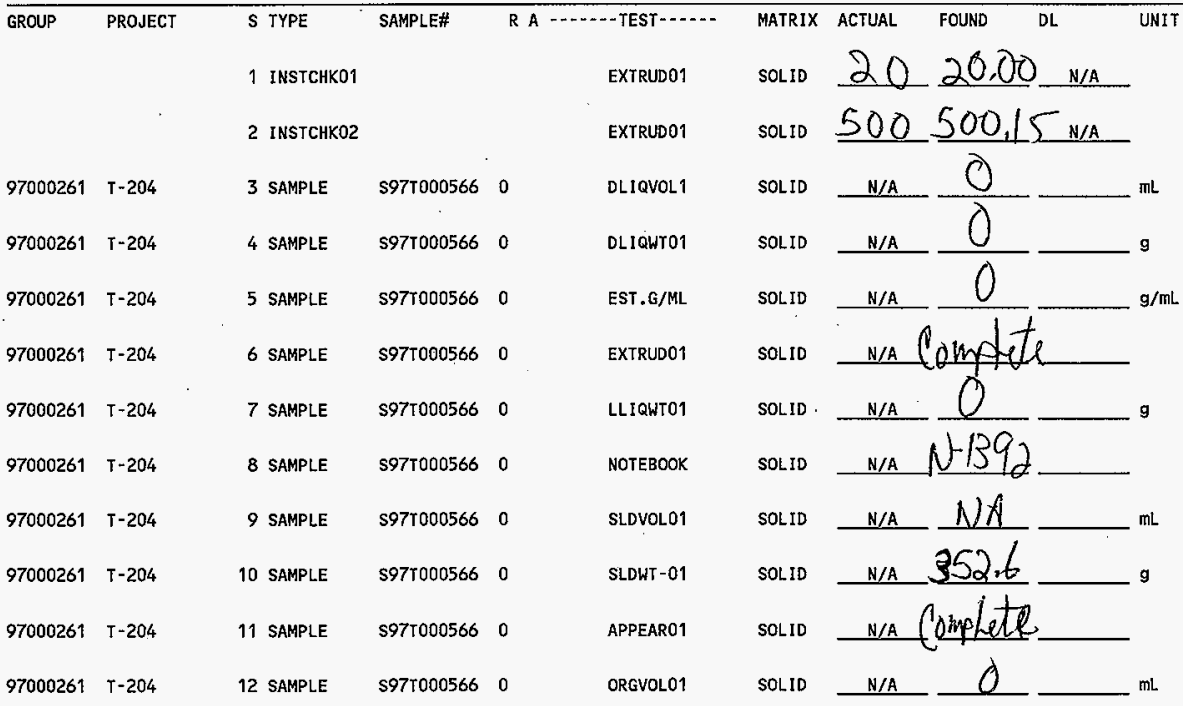

\section{Final page for worklist \# 17602}
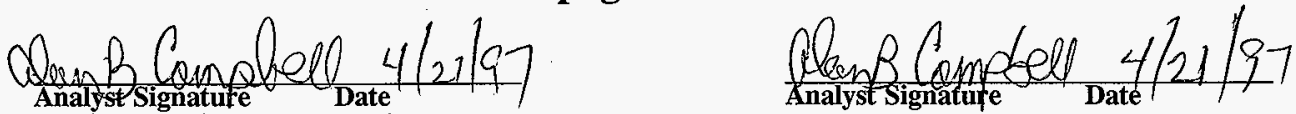

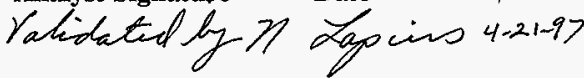

Data Entry Comments:

Units shown for $Q C$ (SPK \& STD) may not reflect the actual units. $D L=$ Detection Limit, $S=$ Worklist Slot Number,

$R=$ Replicate Number, $A=$ Aliquot Code. 


\section{LABCORE Data Entry Template for Worklist\# 17603}

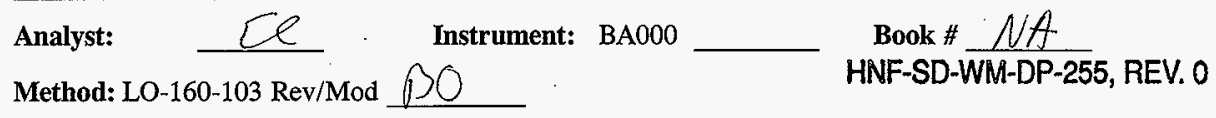

Worklist Comment: T-204 C188 FIELD BLANK RISER 3 EXTRUSION

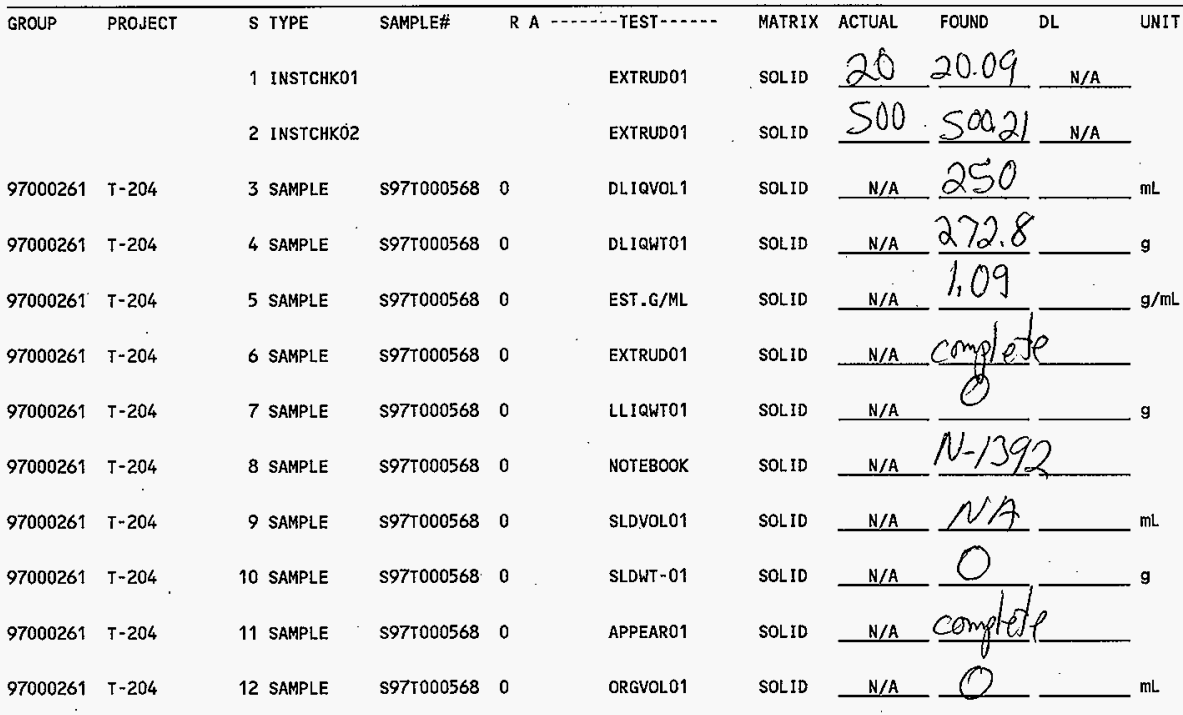

\section{Final page for worklist \# 17603}
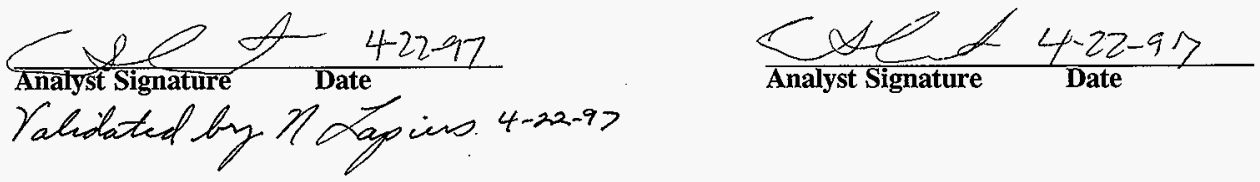

Data Entry Comments:

Units shown for $Q C$ (SPK \& STD) may not reflect the actual units. $D L=$ Detection Limit, $S=$ Worklist Slot Number, $R=$ Replicate Number, $A=$ Aliquot Code. 


\section{LABCORE Data Entry Template for Worklist\# 17604}

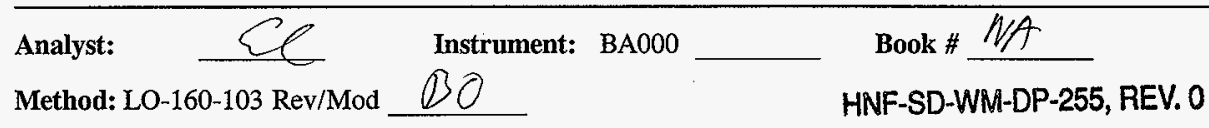

Worklist Comment: T-204 C188 SEG \# 9 RISER 3 EXTRUSION

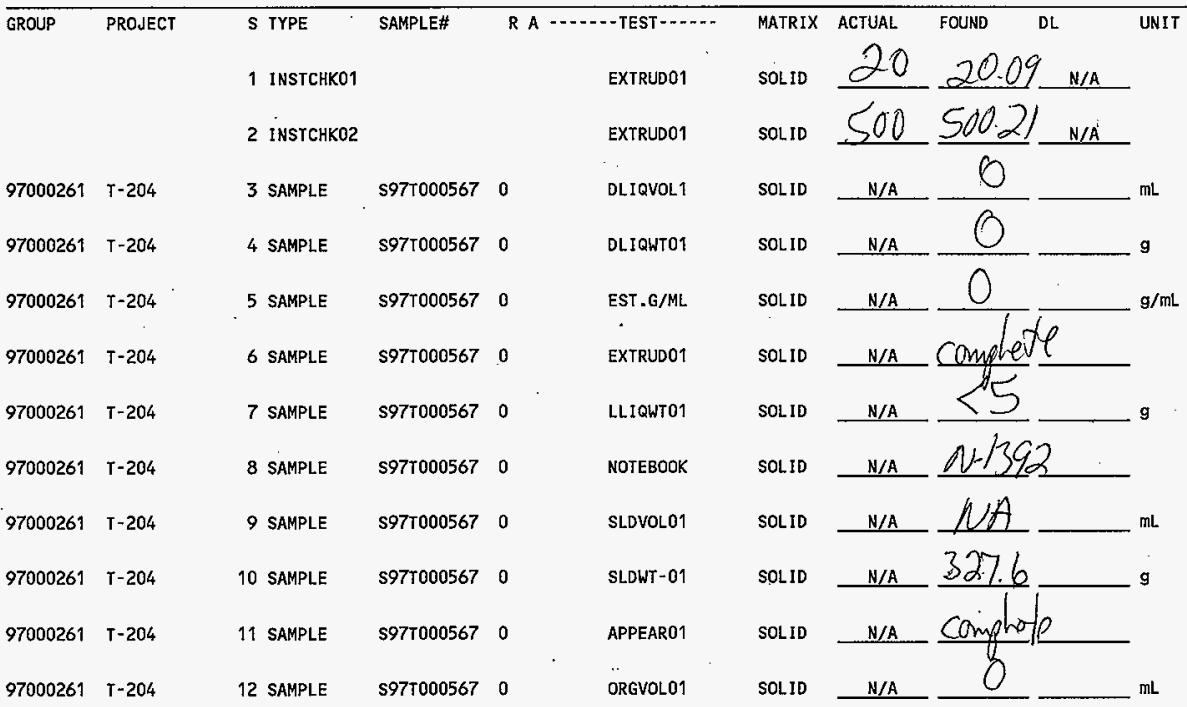

\section{Final page for worklist \# 17604}
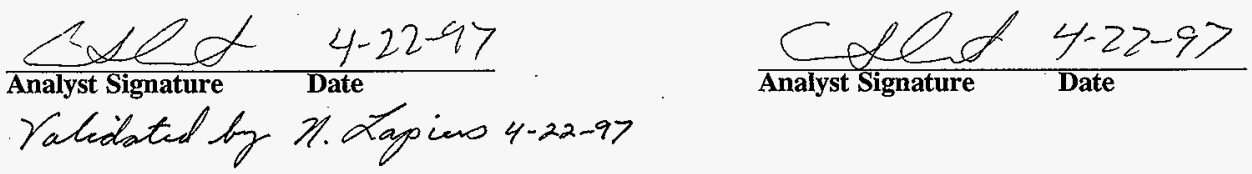

Data Entry Comments:

Units shown for $Q C$ (SPK \& STD) may not reflect the actual units. $D L=$ Detection Limit, $S=$ Worklist Slot Number, $R=$ Replicate Number, $A=$ Aliquot Code. 


\section{LABCORE Data Entry Template for Worklist\# 17605}

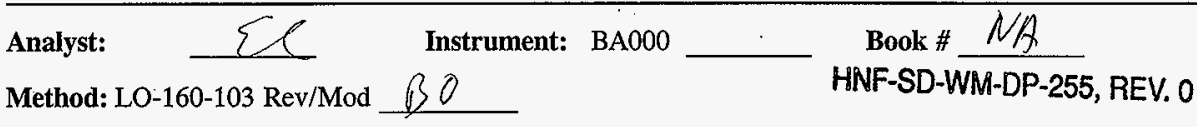

Worklist Comment: T-204 C188 SEG \# 10 RISER 3 EXTRUSION

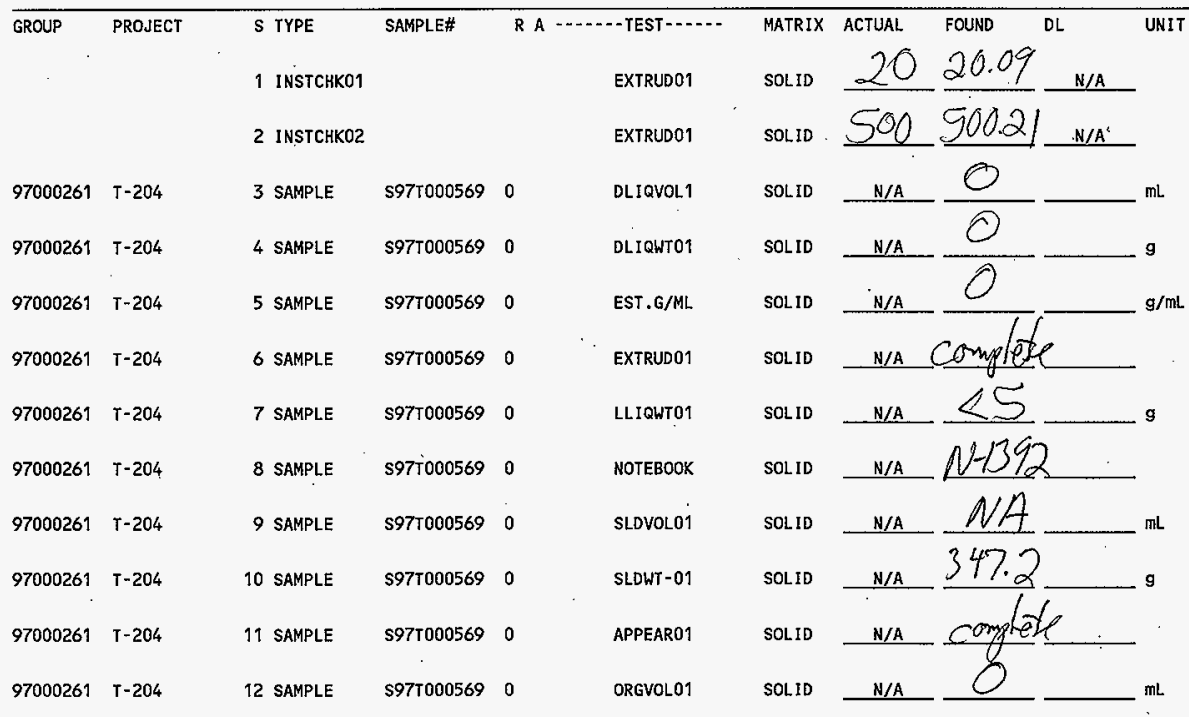

\section{Final page for worklist \# 17605}

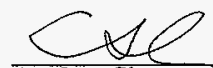

Analyst Signature

Yaludated biz 27 . Zapiens $4-22-97$

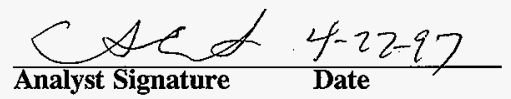

Data Entry Comments:

Units shown for $Q C$ (SPK \& STD) may not reflect the actual units. $D L=$ Detection Limit, $S=$ Worklist Slot Number, $R=$ Replicate Number, $A=$ Aliquot Code. 
HNF-SD-WM-DP-255, REV. 0

SAMPLE PREPARATIONS 
HNF-SD-WM-DP-255, REV. 0

THIS PAGE INTENTIONALLY LEFT BLANK

91 
A.pr.30.1997 12:59M WHC 222S LAB ROOK 2A BACKSIDE

No. 2571 P. $2 / 2$

warklistrpt Version 2.1 05/15/95

04/29/97 19:43

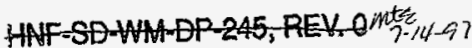

Pags

\section{LABCORE Data Entry Template for Worklist\# 17711}

Analyst: $K N T$ Instrument: FUSO1

Method: LA-549-141 Rev/Mod F-O

Book \#

HNF-SD-WM-DP-255, REV. 0

Worklist Comment: T-204 FUSION DIGEST C188S1LH, 2LH, 3LH lad

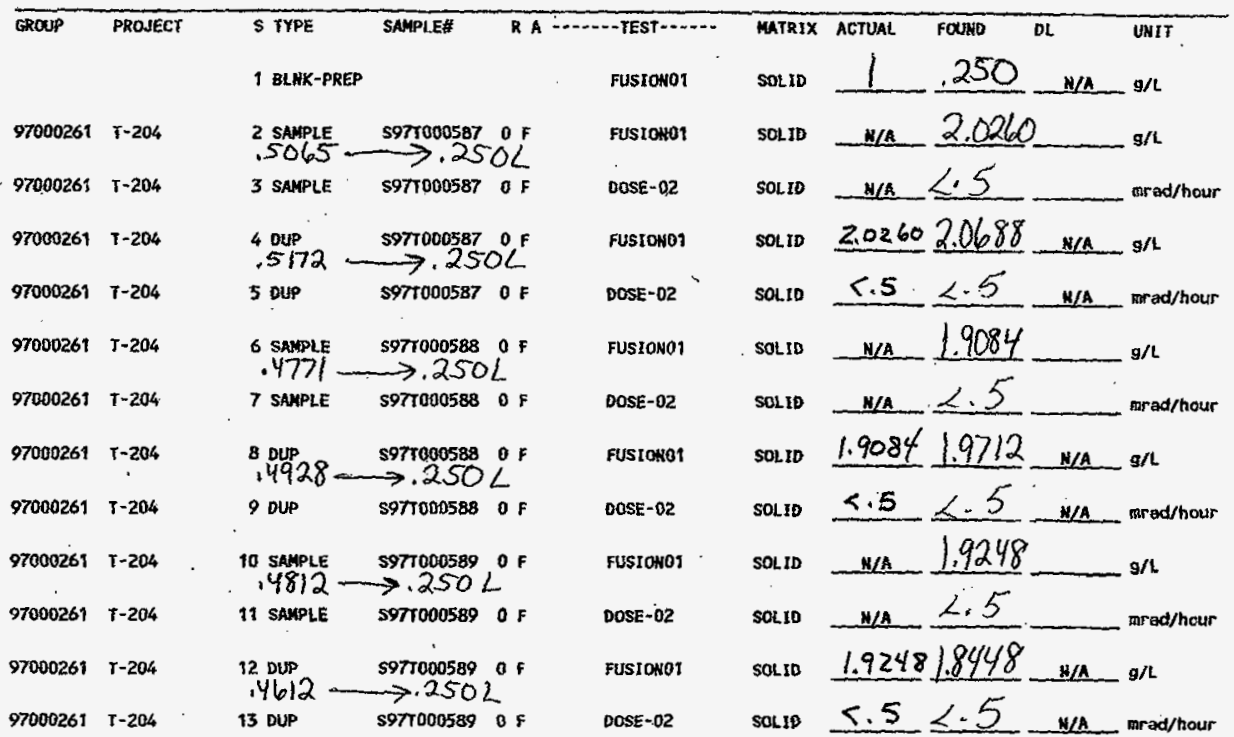

\section{Final page for worklist \# 17711}

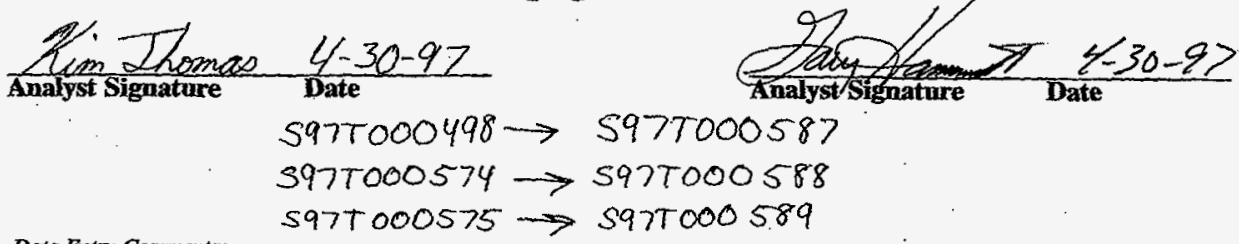

Data Entry Comments:

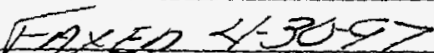

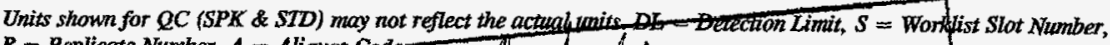

$R=$ Replicate Number, $A=$ Aliquot Code

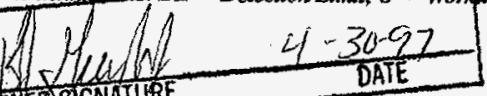

REVITHERSIGNATURE 


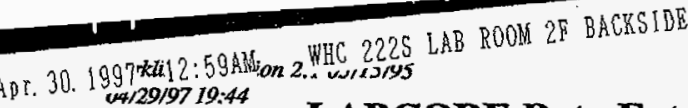

\section{LABCORE Data Entry Template for Worklist\# 17712}

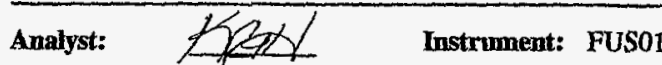

Method: LA-549-141 Rev/Mod F-O
Book \#

HNF-SD-WN-DP-255, REV. 0

Worklist Comment: T-204 FUSION DIGEST C188S4LH, 5LH, 6LH, lad

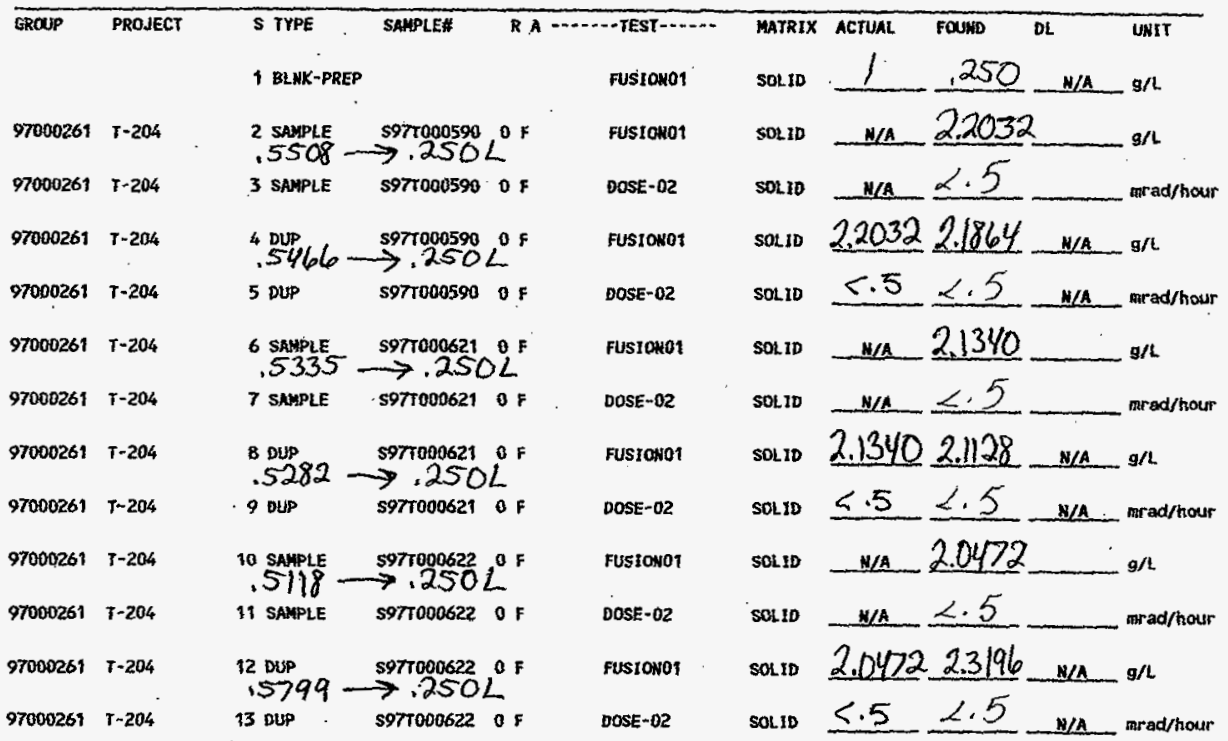

\section{Final page for worklist \# 17712}

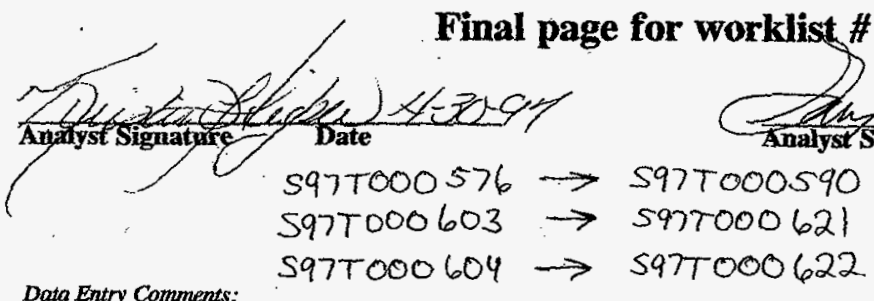

Dota Entry Comments:

$$
\begin{aligned}
& 597 T 000576 \rightarrow 597 T 000590 \\
& 597 T 000603 \rightarrow 5977000621 \\
& 597 T 000604 \rightarrow 5977000622
\end{aligned}
$$

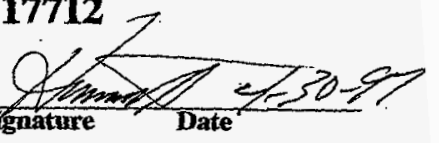

Fever $\alpha+20.22$

Units shown for QC (SPK \& STD) may not reflect the actual units. $D L=$ Detection Limit, $S=$ Weatlist Slot Number, $\boldsymbol{R}=$ Replicate Number, $\boldsymbol{A}=$ Aliguat Code.

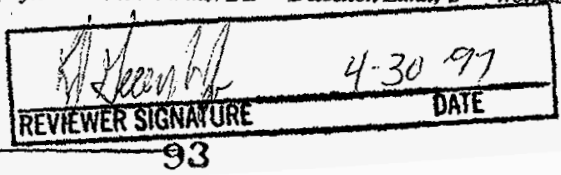




\section{LABCORE Data Entry Template for Worklist\# 17713}

\section{Analyst:}

Method: LA-549-141 Rev/Mod
Instrument: FUS01

Book \# CUA

Worklist Comment: T-204 FUSION DIGEST C188S7LH, 8LH, 9LH, 10LH lad

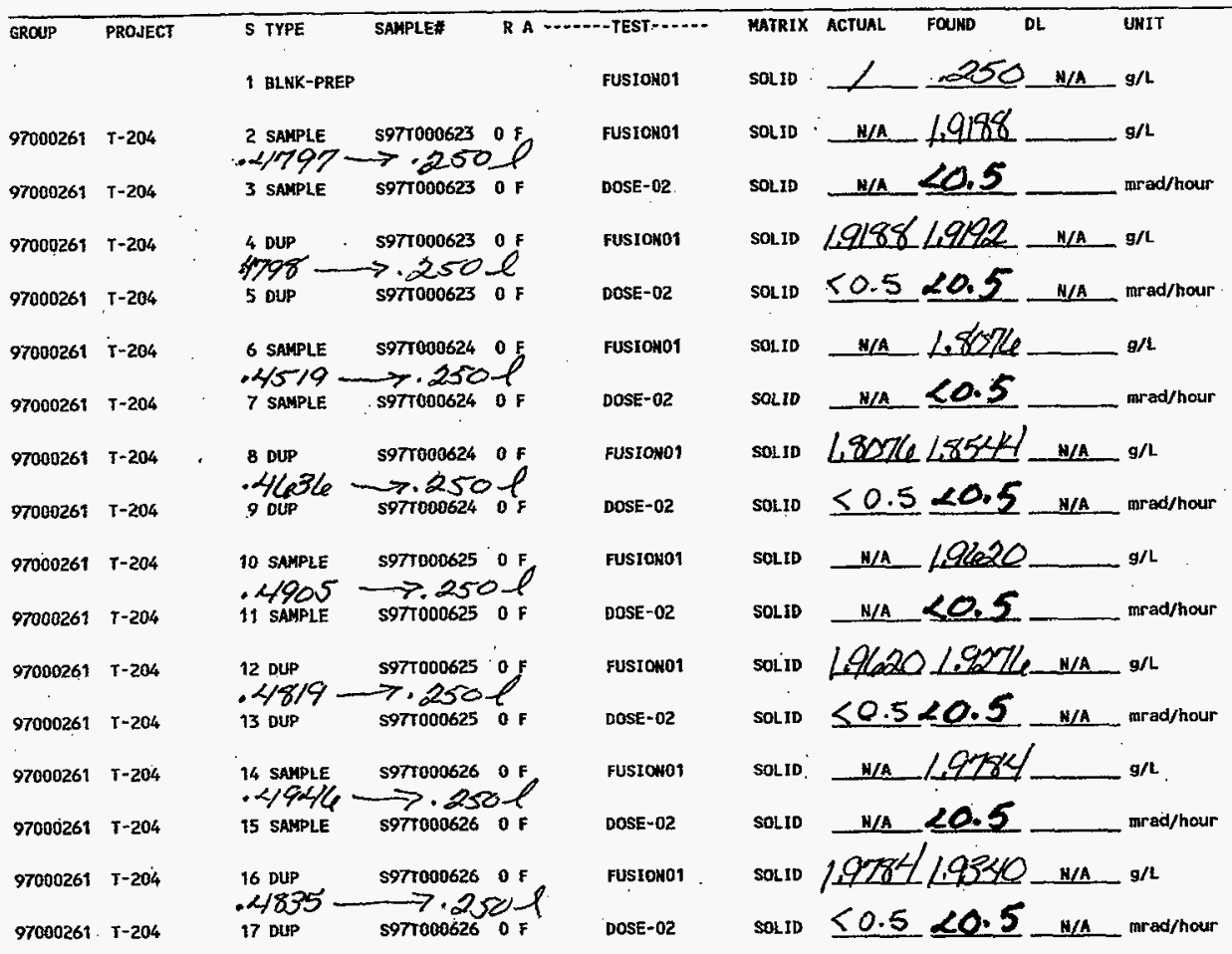

Data Entry Comments:

$52-97$

Units shonn for QC (SPK \& STD) may not reflect the actual wits. $D L=$ Detection Limit, $S=$ Worklist Slot Number,

$R=$ Replicate Number, $A=$ Aliquot Code. 


GROUP PROJECT S TYPE SAMPLE\# R A $\cdots . . .-$ TEST-... MATRIX ACTUAL FOUND DL. UNIT
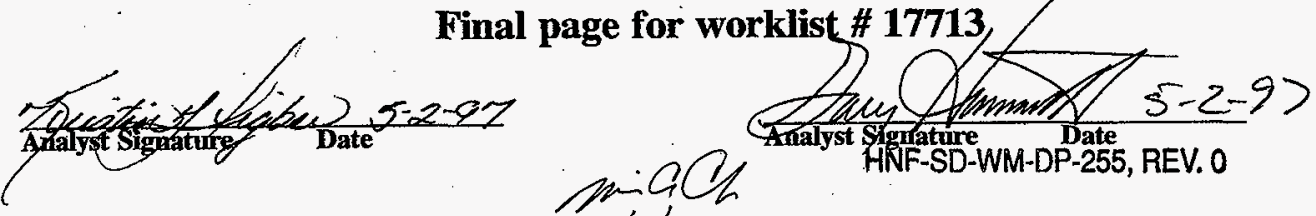

$5 / 3 / 97$

Units shown for QC (SPK \& STD) may not reflect the actual units. $D L=$ Detection Limit, $S=$ Worklist Slot Number, $R=$ Replicate Number, $A=$ Aliquot Code. 


\section{LABCORE Data Entry Template for Worklist\# 18345}

\begin{tabular}{lll}
\hline Analyst: $\frac{\mathscr{K} / \mathcal{X}}{\text { Instrument: FUS01 }}$ & Book $\#$ \\
Method: LA-549-141 Rev/Mod & & HNF-SD-WM-DP-255, REV. 0
\end{tabular}

Worklist Comment: T-204 FUSION DIGEST CORE COMPOSITE C188 lad

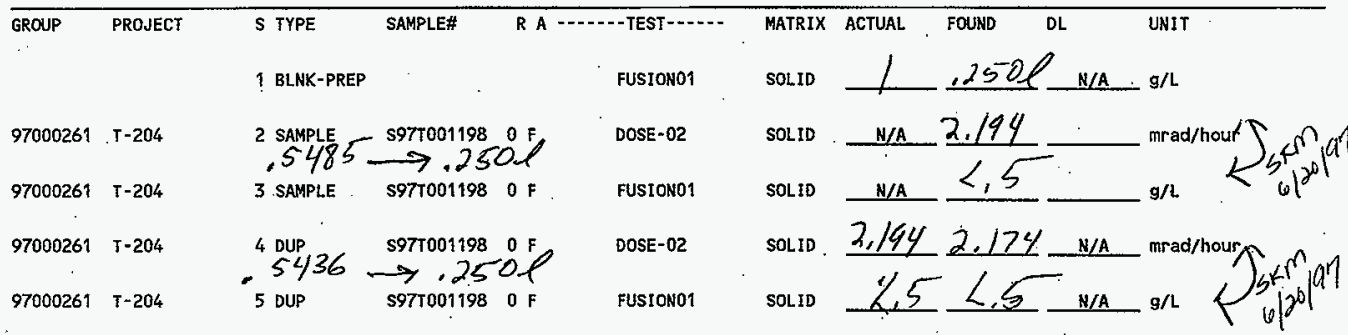

\section{Final page for worklist \# 18345}

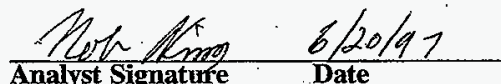

Analyst Signature Date

Analyst Signature Date

$S 97 T 001197 \rightarrow 547 T 00149$

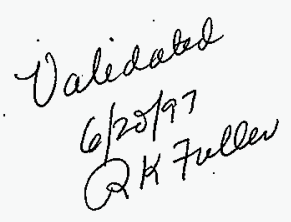

Data Entry Comments:

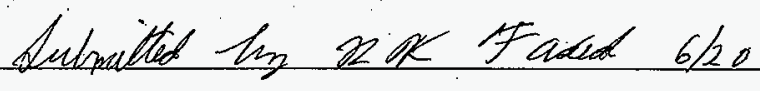

Units shown for QC (SPK \& STD) may not reflect the actual units. $D L=$ Detection Limit, $S=$ Worklist Slot Number, $R=$ Replicate Number, $A=$ Aliquot Code. 


\section{LABCORE Data Entry Template for Worklist\# 18346}

Analyst: PfN Instrument: $\mathrm{H} 2 \mathrm{OO}$

Method: LA-504-101 Rev/Mod

Book \#

HNF-SD-WM-DP-255, REV. 0

Worklist Comment: T-204 H2O DIGEST CORE COMPOSITE C188 lad

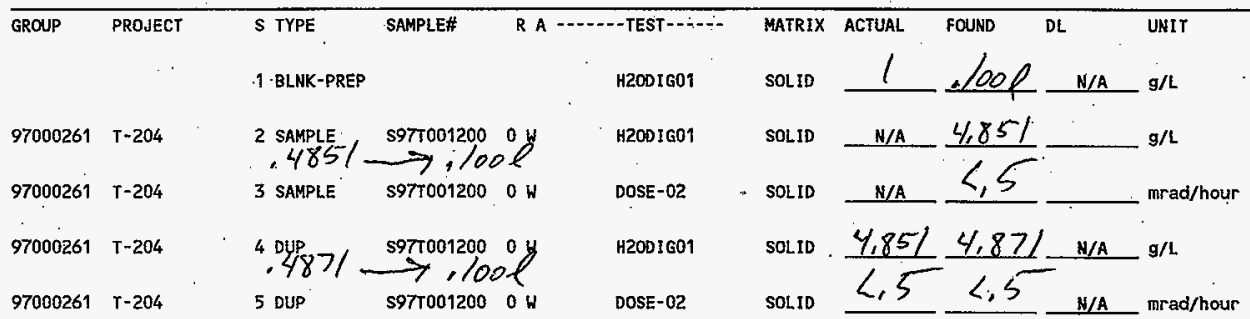

\section{Final page for worklist \# 18346}

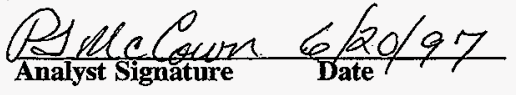

Analyst Signature

$597 T 001197 \longrightarrow$ S97T 1200

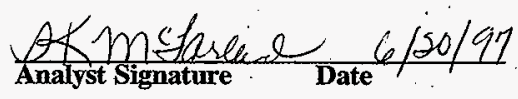

Data Entry Comments:

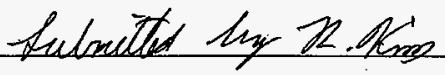

Units shown for QC (SPK \& STD) may not reflect the actual units. $D L=$ Detection Limit, $S=$ Worklist Slot Number, $R=$ Replicate Number, $A=$ Aliquot Code. 
HNF-SD-WM-DP-255, REV. 0

BULK DENSITY WORKSHEETS 
HNF-SD-WM-DP-255, REV. 0

THIS PAGE INTENTIONALLY LEFT BLANK 


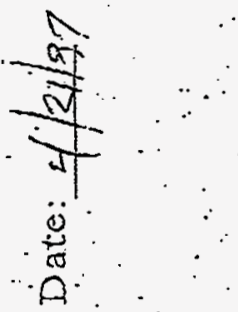

\section{HNF-SD-WN-DP-255, REV. 0}

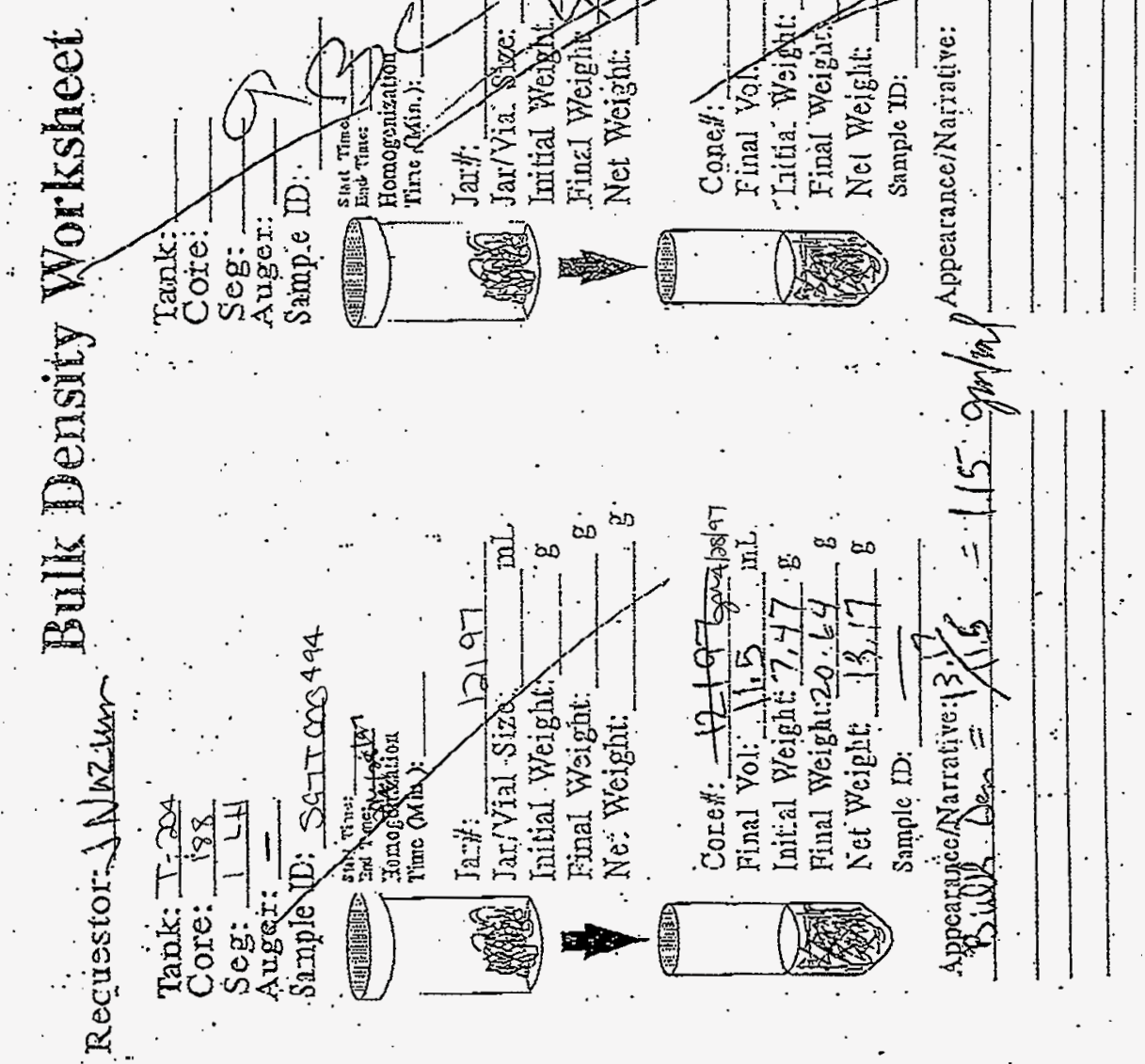




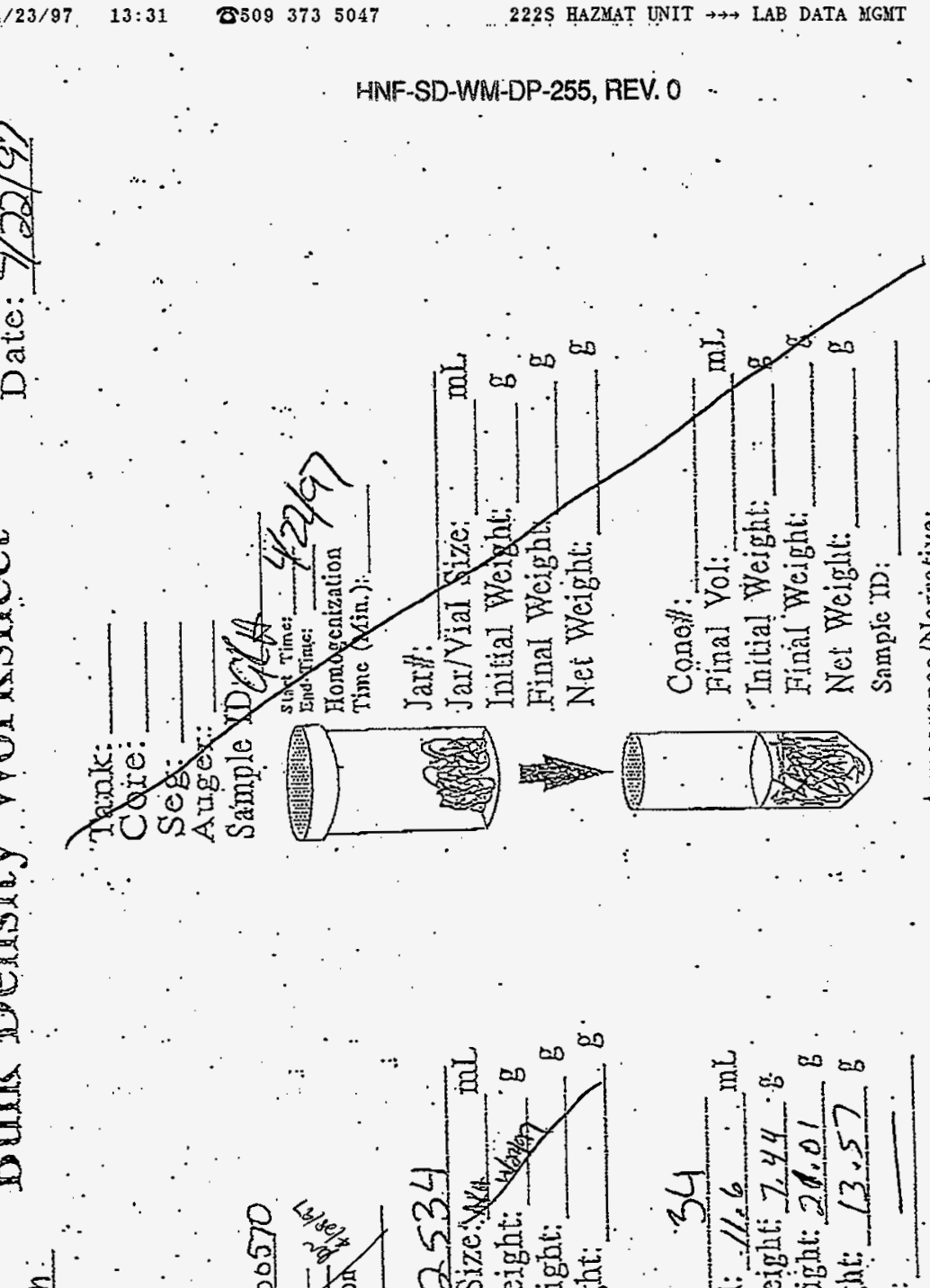
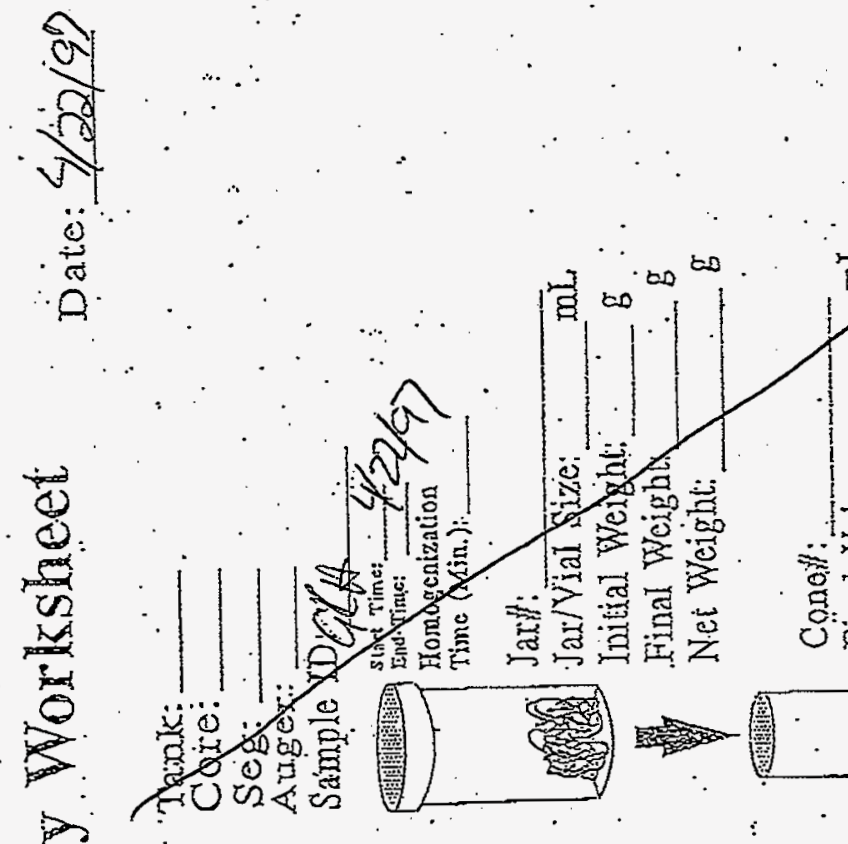

(1)

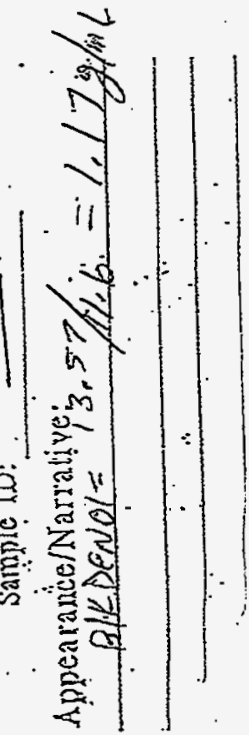

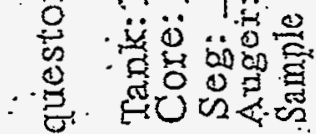



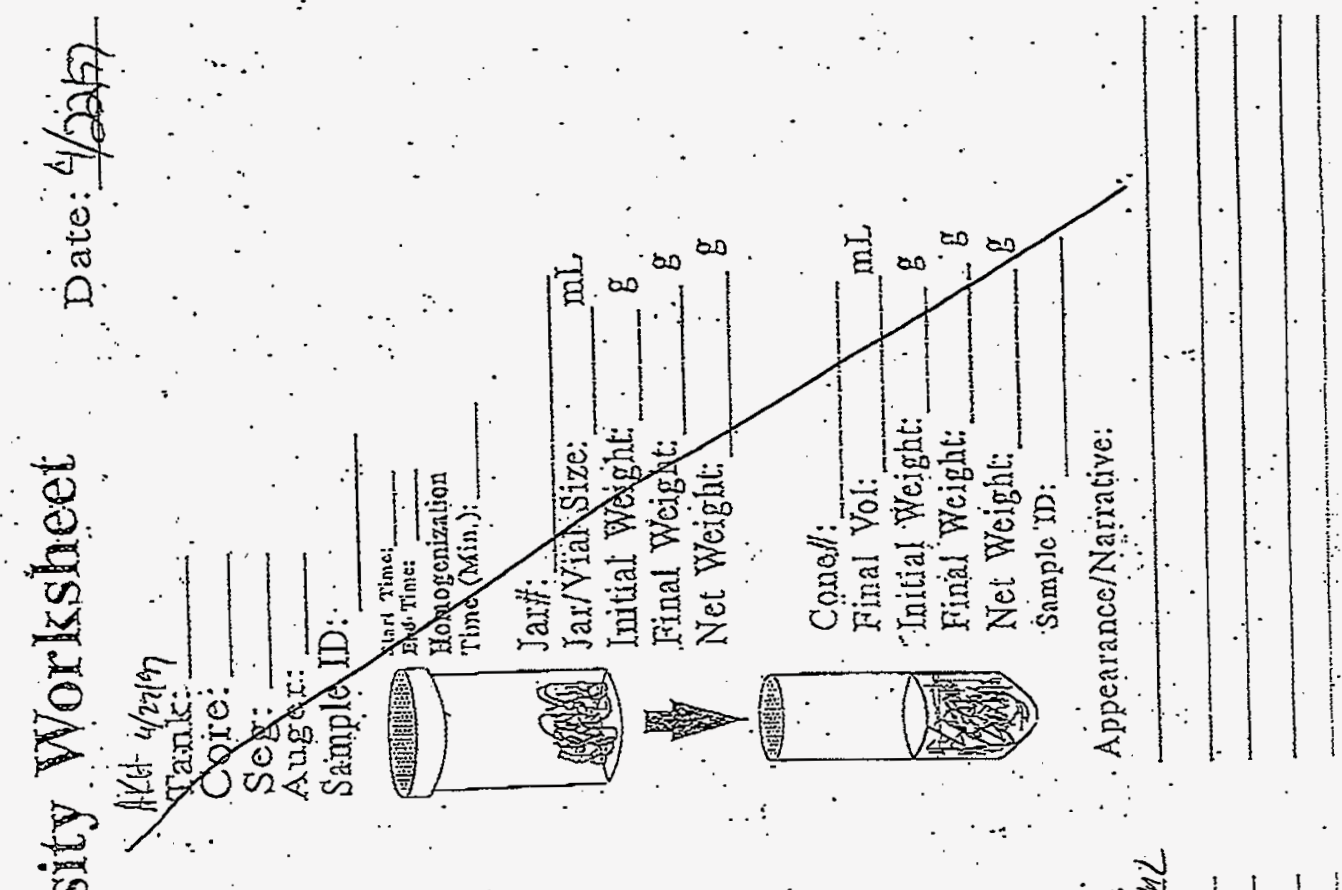
Requestot: Rizum:

\section{Bulk Density Worksheet}

Tank: \pm-204

Core: 188

Seg: 414

Augex:

Sample ID: $597+000572$

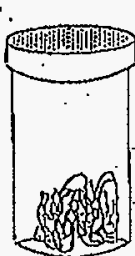

sinf! tryat:

Homogcuizatioto of â

nimc (Min.):

Jar:f: $\angle 2-532$ Jar/Vial Size:" ml Initial Weight: Final Weight:

Net Weight:

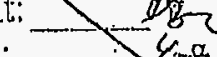

Conell: 32

Final Yol: $1120 \ldots$ Int

Initial Weight: $7.45 \cdot 8$

Final Weight: 20.69 :

Net Weight: 13.248

Sample ID:

Appearance/Narrative

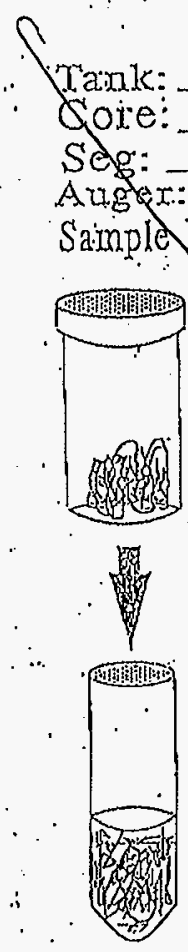

D:-

shyl Timic:

IIINAtisine:

Trombacnization

T'ime (xin.):

Jarit:

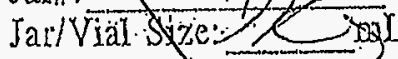

Initial Weigh:

Final Weight:

Nel Weighl:

Cone:l:

Final Vol:

Initial Weight:

Final Weight:

Net Weight:

Sampic 'TD:

Appearance/Narrative:

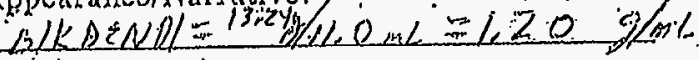


Requestor: puturn.

\section{Bullk Density Worksheet}

Tank: T-204

Cote: 188

Seg: $5 \div 1$

Aragex:

Sample 1D: s970000609

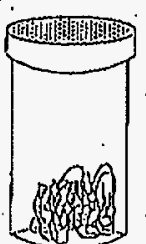

in

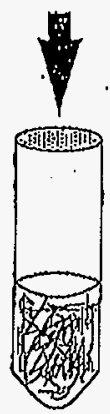

Strst Tinze:

Lind Time:

Homogenizatidn of

lime (Miu.):

Jarit:

Jax/Vial Size: Initial Weight: Final Weight: Net Weight:

Conell: $\quad \frac{36}{\text { Final Vol: } 9,5}$ Initial Weight: 7, $46 \cdot \mathrm{g}$ Pinal Weight: Kh 92 g Net Weight: $\| L_{1} \mathrm{~g}$

Sample ro: Appearance/Narralive:

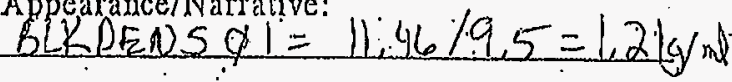

'taiok:

core:

S\&g:

Auger:

Sainple ID:-

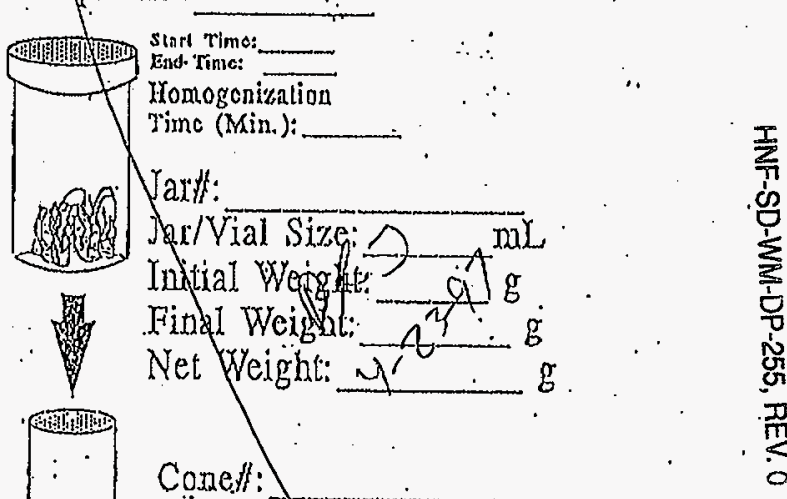

Final Vol mL "Initial Weight: g Final Weighn Net Weight: Sample $\times D$ :

Appearance/Narrative: 


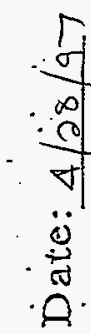

HNF-SD-WM-DP-255, REV. 0 - :

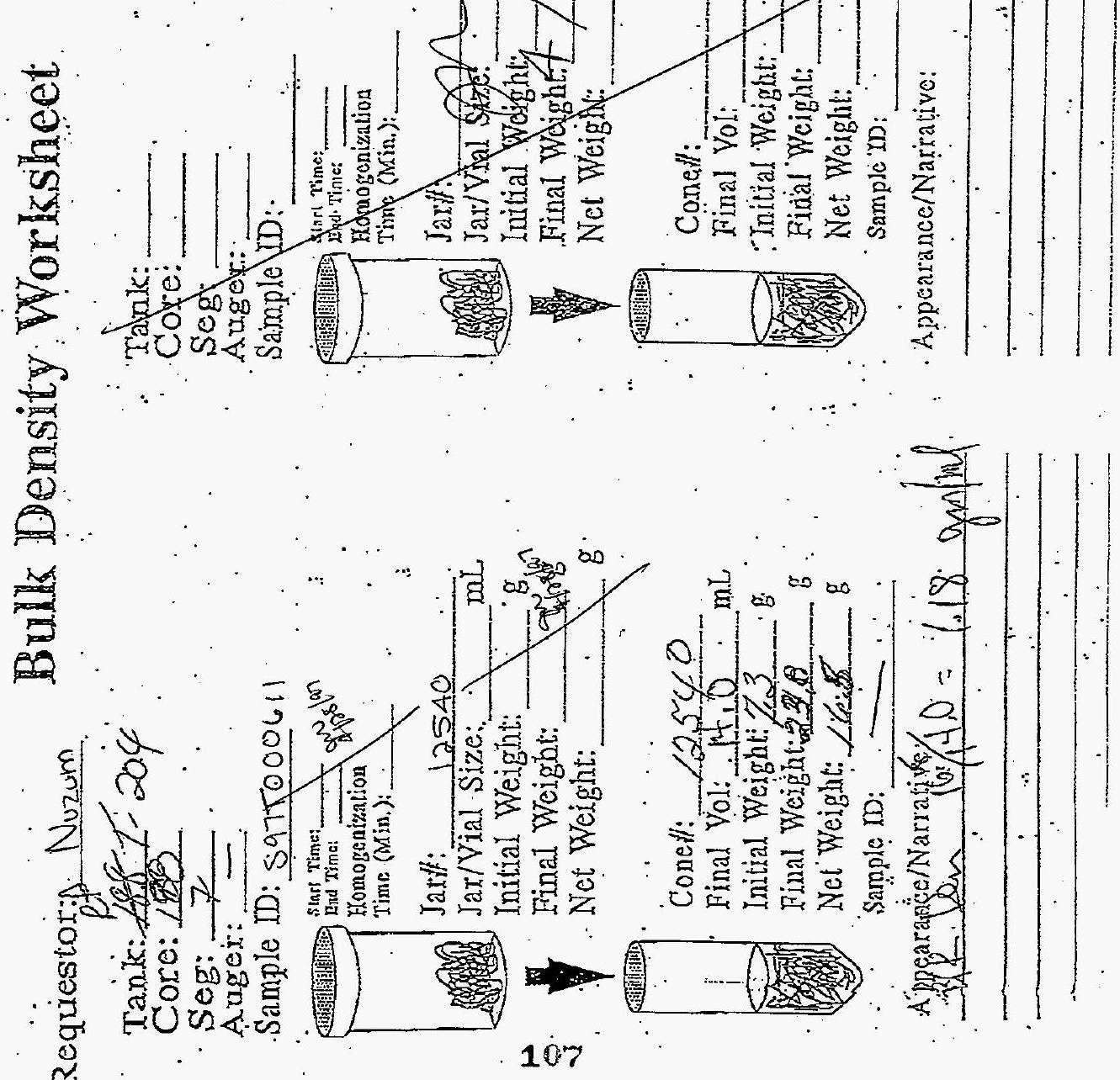




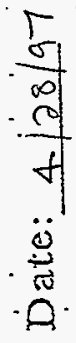
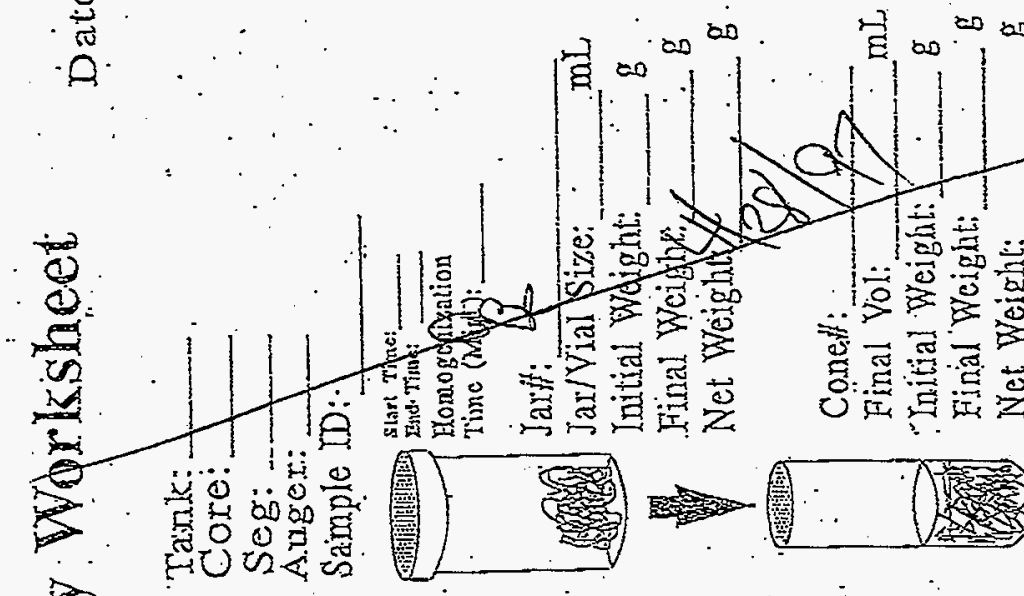


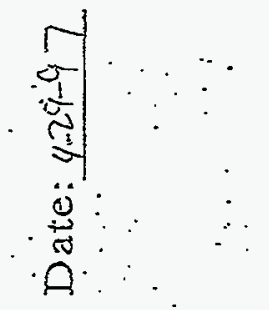

HNF-SD-WM-DP-255, REV. O 


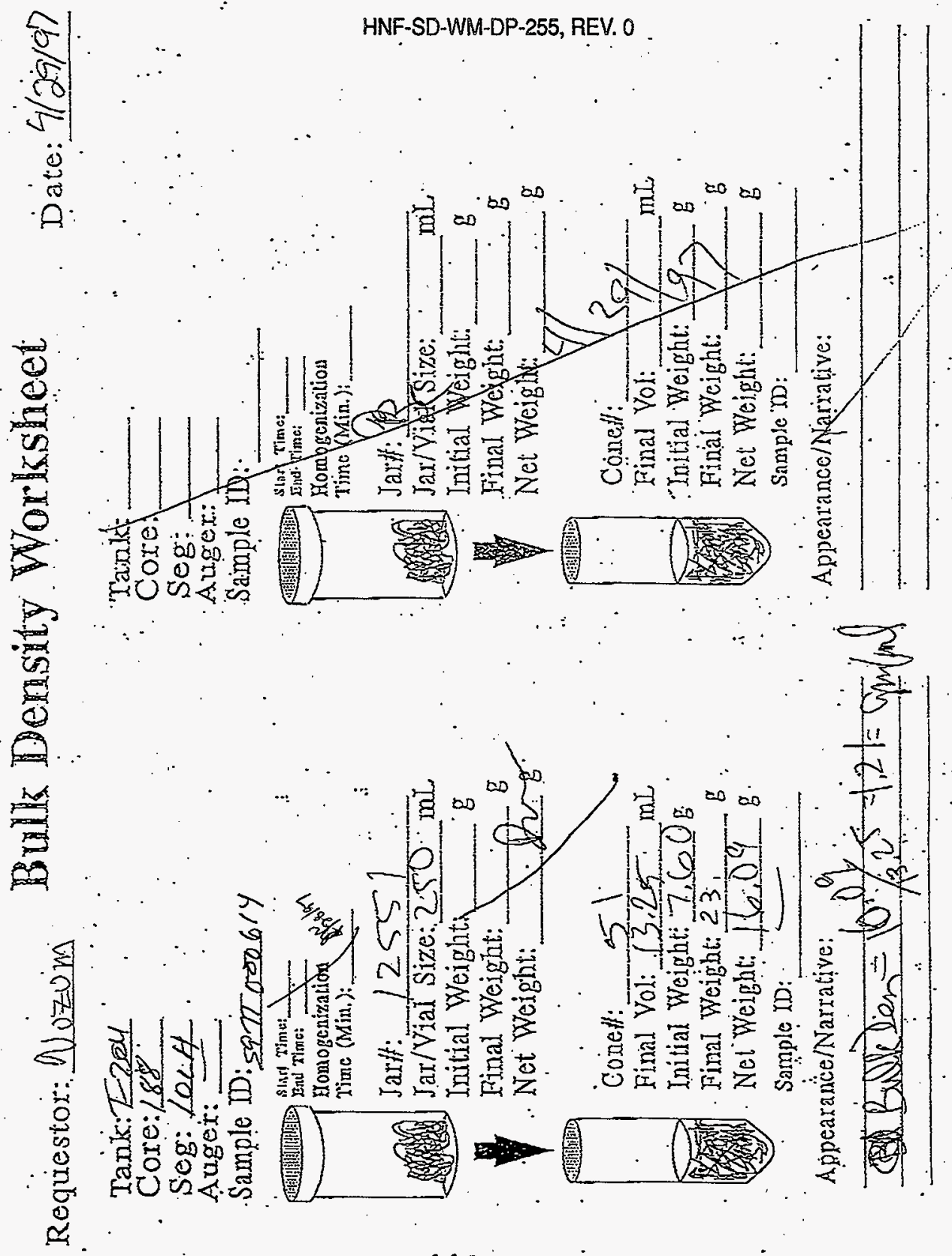




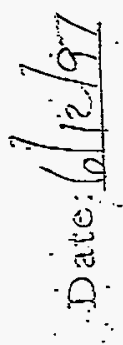

HNF-SD-WM-DP-255, REV. 0

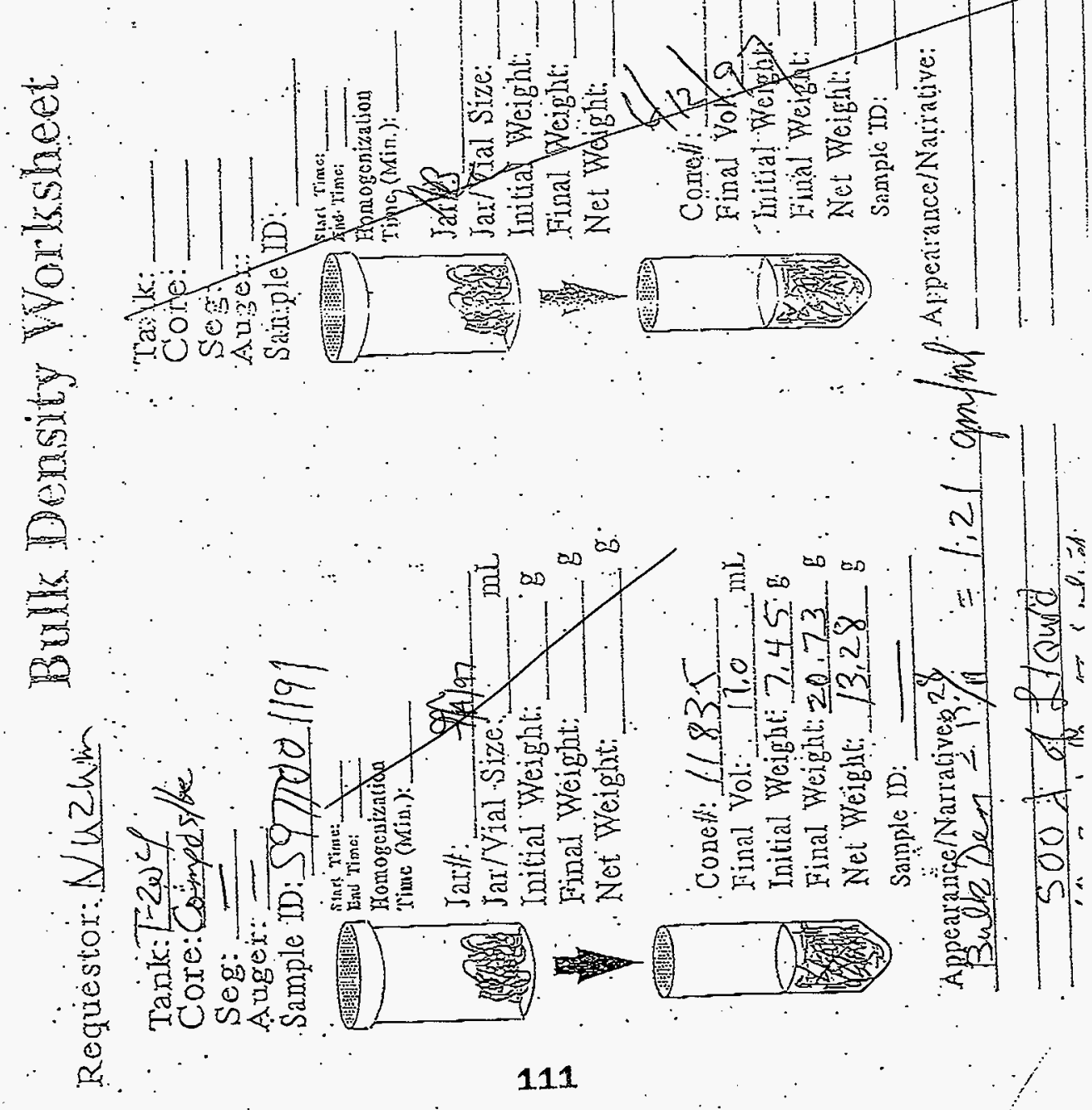


HNF-SD-WM-DP-255, REV. 0

INORGANIC ANALYSES 
HNF-SD-WM-DP-255, REV. 0

\section{THIS PAGE INTENTIONALLY LEFT BLANK}




\section{LABCORE Data Entry Template for Worklist\# 17819}

Analyst: SAGF Instrument: DSC0 1 Book\#12N14 - B

Method: LA-514-113 Rev/Mod D-O HNF-SD-WM-DP-255, REV. 0

Worklist Comment: T204, DSC-01 Run under Nitrogen. skm

\begin{tabular}{|c|c|c|c|c|c|c|c|c|c|c|}
\hline GROUP & PROJEC̣T & $\begin{array}{l}S \text { TYPE } \\
1 \text { STD }\end{array}$ & SAMPLE\# & R A & DSC-01 & $\begin{array}{l}\text { MATRIX } \\
\text { LIQUID }\end{array}$ & $\begin{array}{l}\text { ACTUAL } \\
28.45 \\
\end{array}$ & $\begin{array}{l}\text { FOUND } \\
27.3\end{array}$ & $\begin{array}{l}\text { DL } \\
\text { N/A } \\
\end{array}$ & $\begin{array}{l}\text { UNIT } \\
\text { Joules }\end{array}$ \\
\hline 97000261 & $T-204$ & 2 SAMPLE & s97T000647 & 0 & DSC-01 & LIQUID & N/A. & 0 & & Joules, \\
\hline 7000261 & $T-204$ & 3 DUP & s971000647 & 0 & osc-01 & LIQUID & 0 & 0 & $N / A$ & oules/s \\
\hline
\end{tabular}

Surie $h$ Dultion 5-3.97

Analyst Signature Date

Validated 5/8197\$3ache lor 


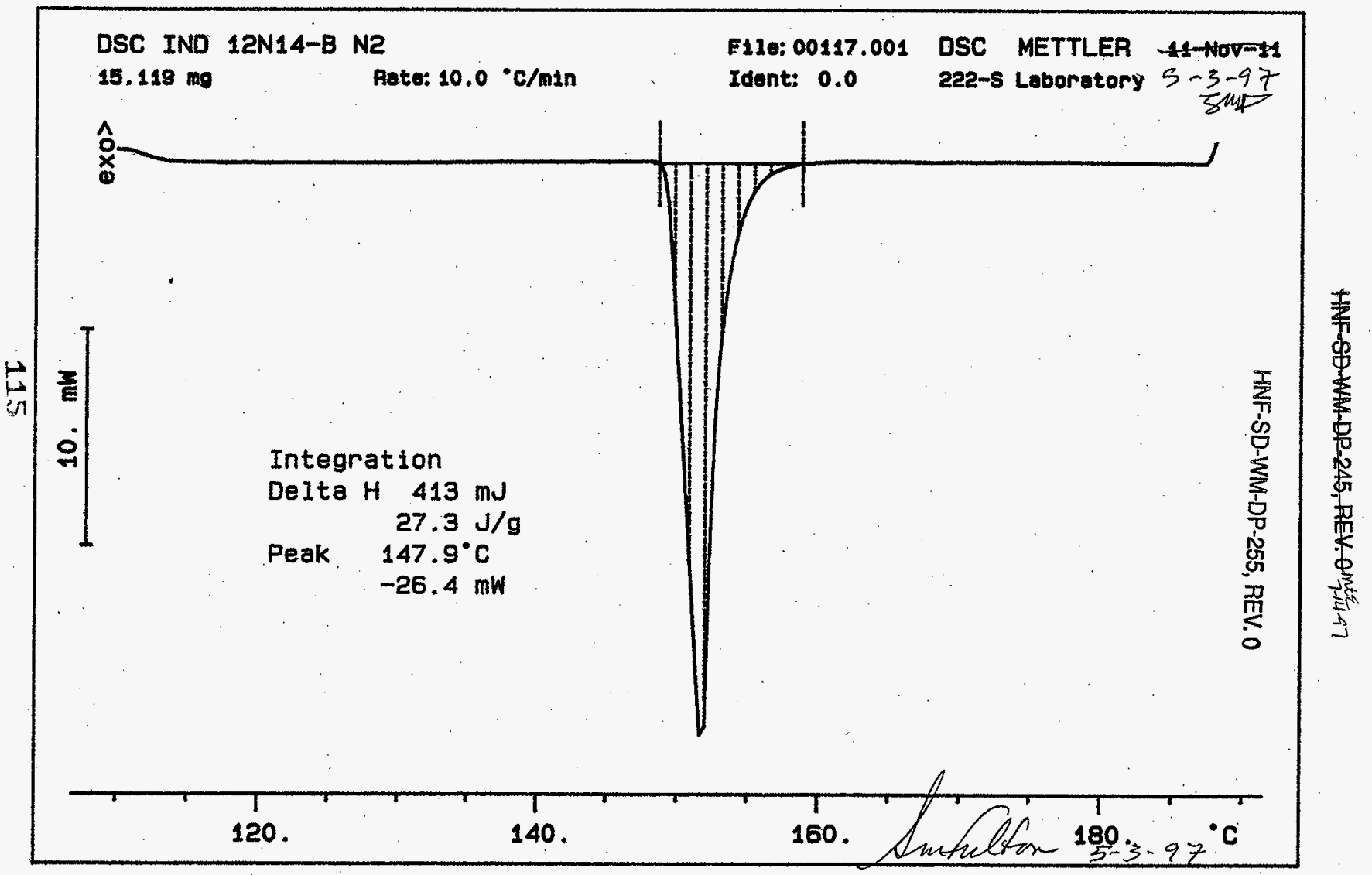




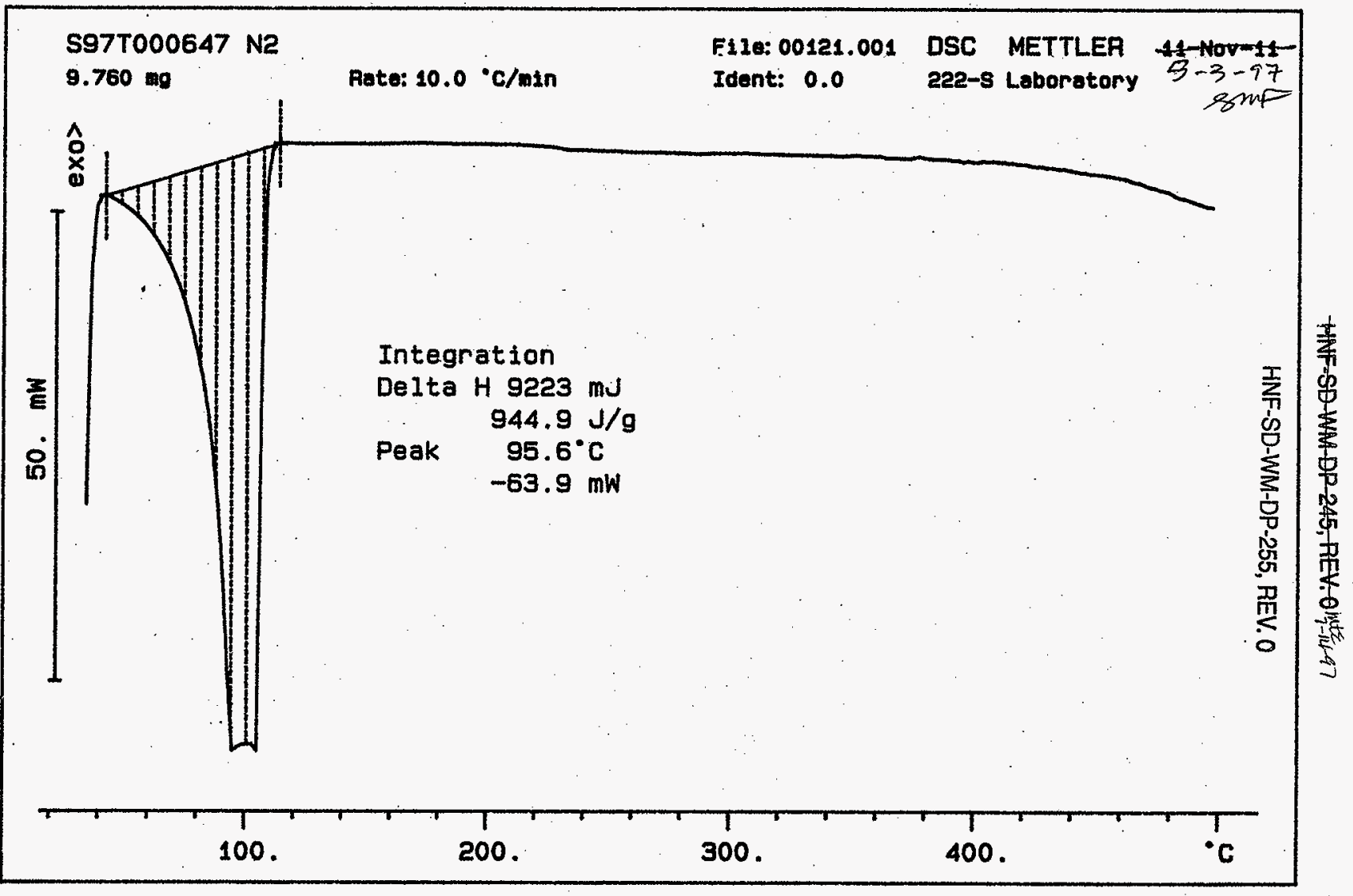




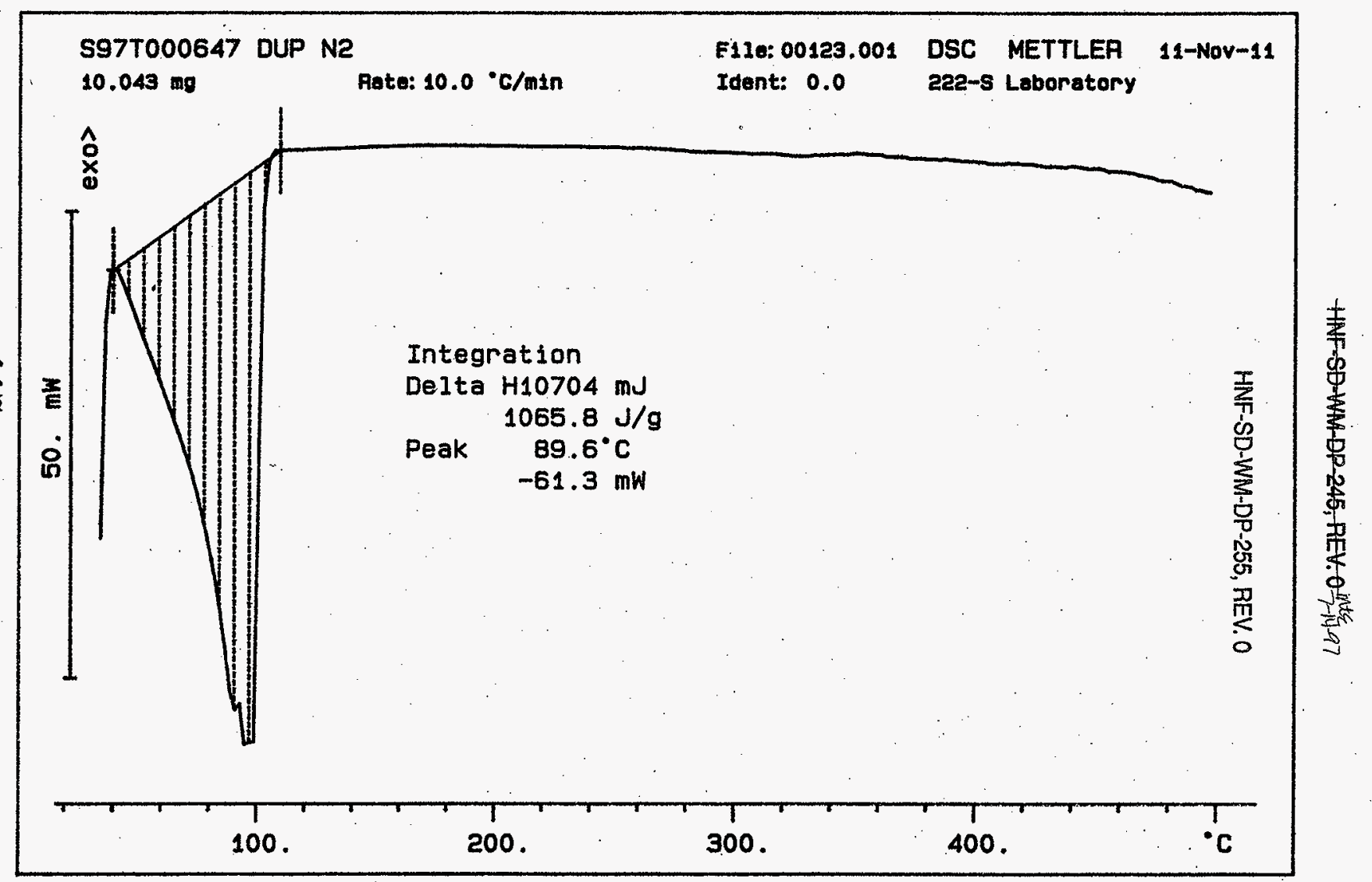




\section{LABCORE Data Entry Template for Worklist\# 17828}

Analyst: $\quad A D P$ Instrument: $D S C 0 \perp$ Book $\#(2 N) /=B$.

Method: LA-514-113 Rev/Mod D-O

HNF-SD-WM-DP-255, REV. 0

Worklist Comment: T204, DSC-01 Run under nitrogen. skm

\begin{tabular}{|c|c|c|c|c|c|c|c|c|c|c|}
\hline GROUP & PROJECT & $\begin{array}{l}\text { S TYPE } \\
1 \text { STD }\end{array}$ & SAMPLE\# & & $\begin{array}{l}\text { DSC-01 } \\
\text { DEST }-\cdots . . . .-\end{array}$ & $\begin{array}{l}\text { MATRIX } \\
\text { SOLID }\end{array}$ & $\begin{array}{l}\text { ACTUAL } \\
28.4 / 5 \\
\end{array}$ & $\begin{array}{l}\text { FOUND } \\
25.7 \\
\end{array}$ & $\begin{array}{l}D L \\
N / A \\
\end{array}$ & $\begin{array}{l}\text { UNIT } \\
\text { Joules/g }\end{array}$ \\
\hline 97000261 & $T-204$ & 2 SAMPLE & S97T000498 & 0 & DSC-01 & SOLID & N/A & 0 & & Joules $/ g$ \\
\hline 97000261 & $T-204$ & 3 DuP & S97T000498 & 0 & DSC-01 & SOEID & 0 & 0 & $\mathbb{N} / \mathrm{A}$ & Joules/ \\
\hline 97000261 & $T-204$ & 4 SAMPLE & S977008499 & 0 & DSC-01 & SOLID & N/A & 0 & & Joules/g \\
\hline 97000261 & $r-204$ & 5 DUP & s97T000499 & 0 & DSC-01 & SOLID & 0 & 0 & N/A & Soules/g \\
\hline
\end{tabular}

Final page for worklist \# 17828

Anthom Turumta 05-03-97.

Analyst Sighature: Date

validated $5 / 8 / 97$ Sachelor

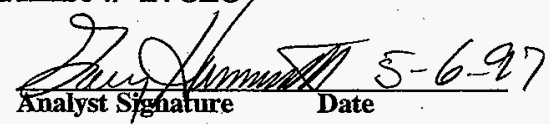

Data Entry Comments:

Units shown for $Q C$ (SPK \& STD) may not reflect the actual units. $D L=$ Detection Limit, $S=$ Worklist Slot Number, $R=$ Replicate Number, $A=$ Aliquot Code. 
HAF-SD-WMA-DP-245,REV.0MEE

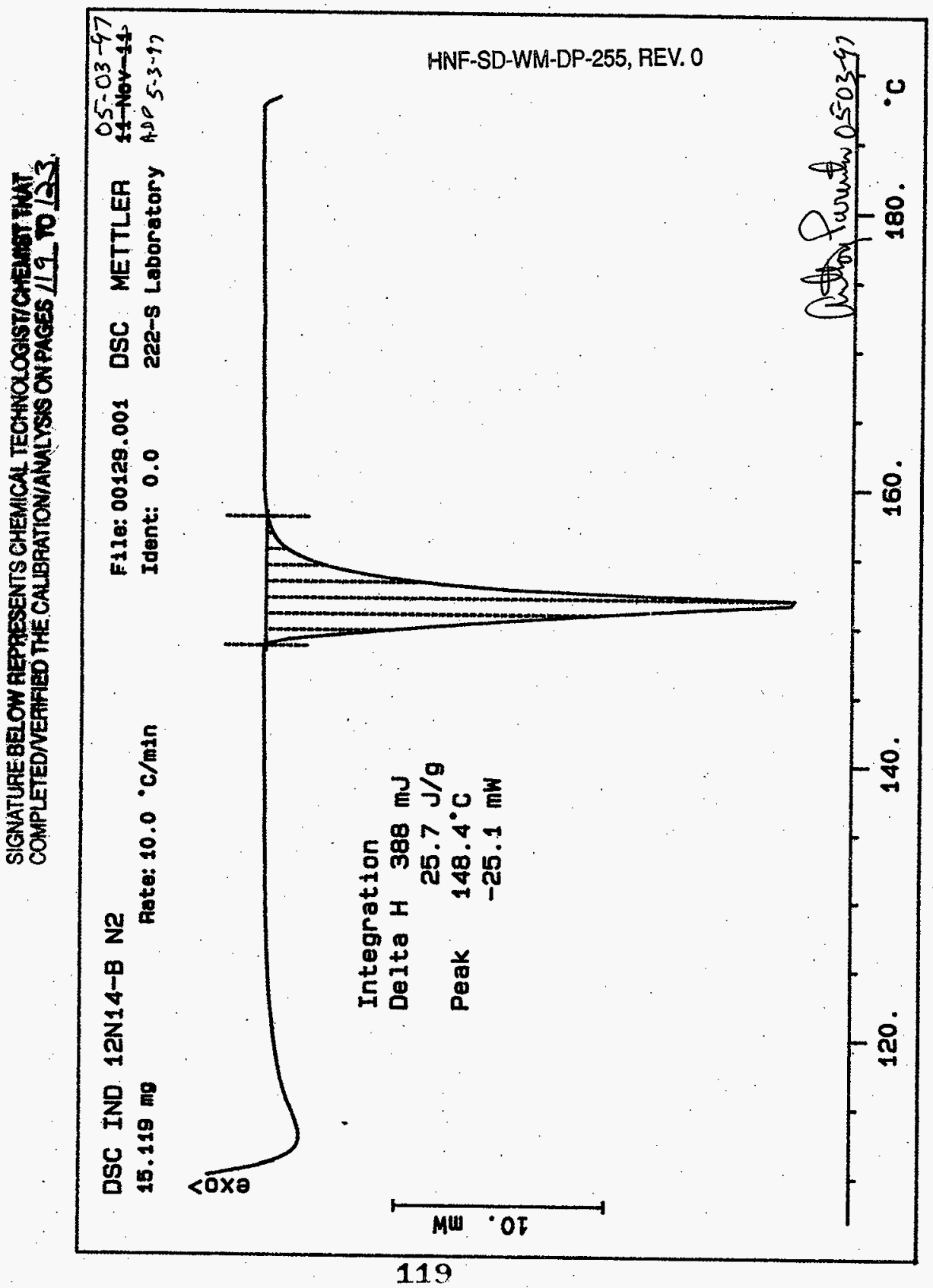


HNF-SD-WM-DP-245, REV. 0 ME/ $74-97$

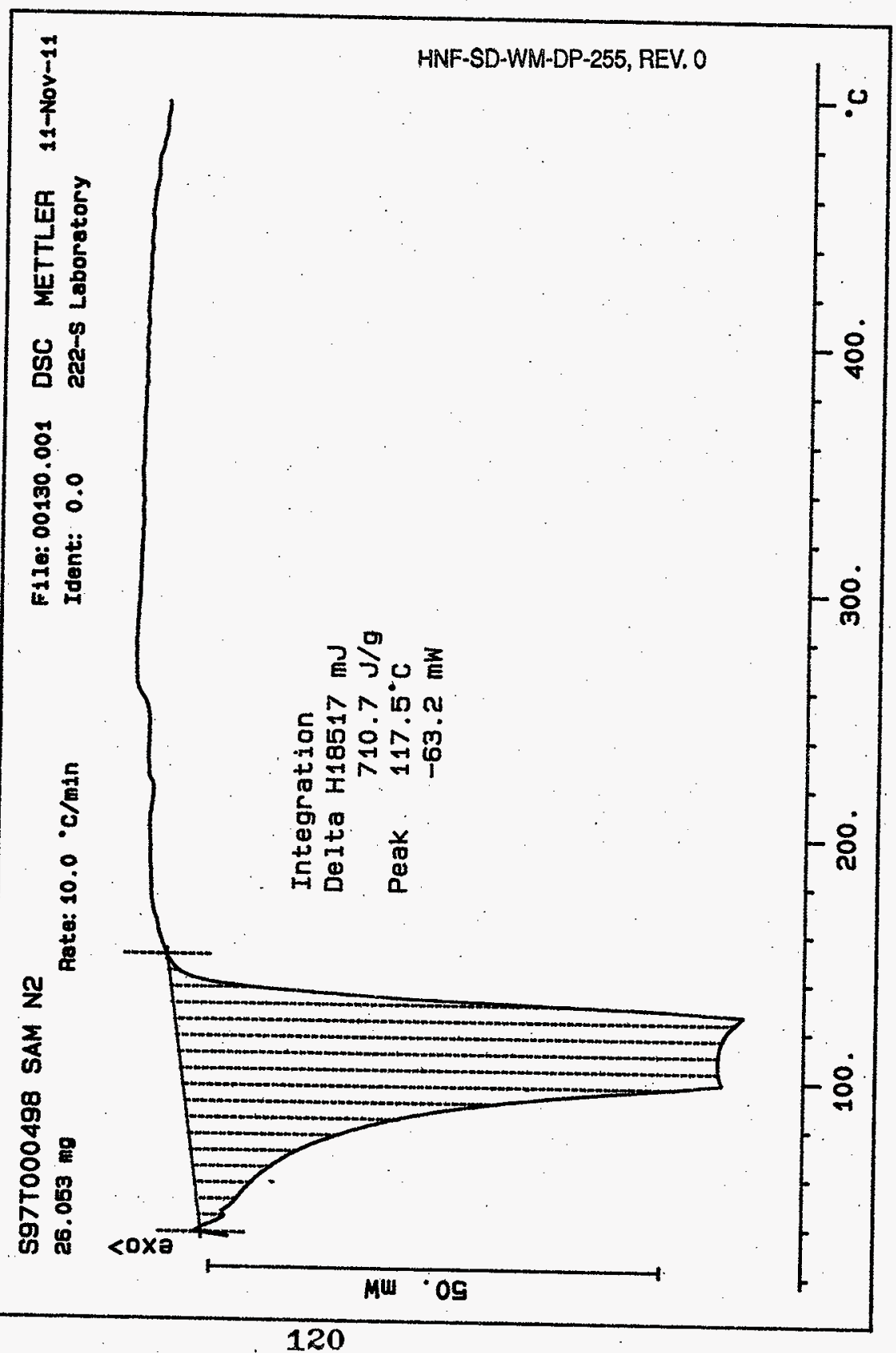




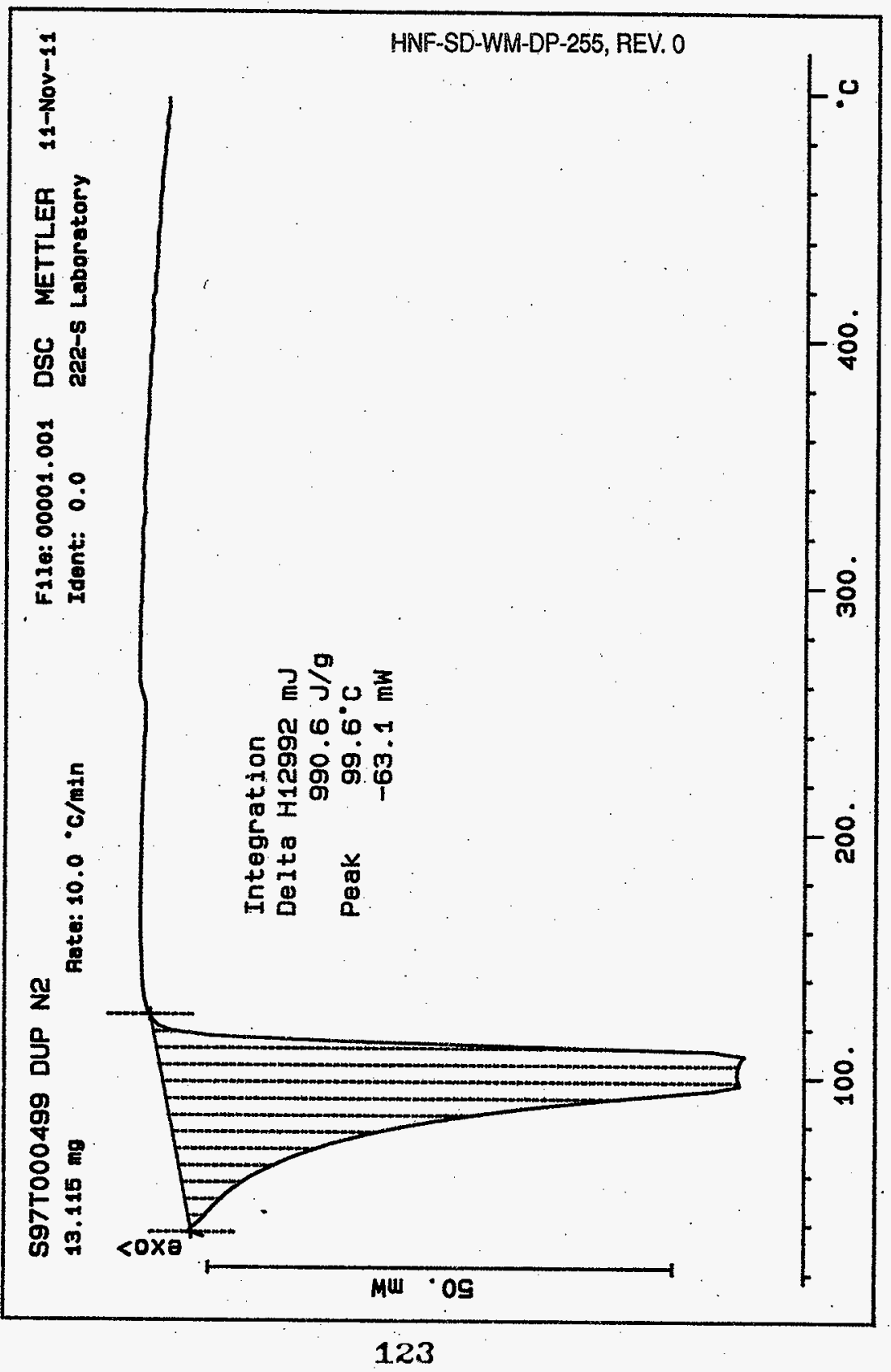




\section{LABCORE Data Entry Template for Worklist\# 17829}

Analyst: $\quad$ ADP $\quad$ Instrument: $D S C 0 \div 3$ Book\# $12 N 14 B$

Method: LA-514-114 Rev/Mod__ D-D

HNF-SD-WM-DP-255, REV. 0

Worklist Comment: T204, DSC-01 Run under nitrogen: škm

\begin{tabular}{|c|c|c|c|c|c|c|c|c|c|c|c|}
\hline GROUP & PROJECT & $\begin{array}{l}S \text { TYPE } \\
1 \text { STD }\end{array}$ & SAMPLE\# & R A & $\cdots \cdots$ & $\begin{array}{l}\text { TEST } \cdots \cdots \\
\text { DSC-03 }\end{array}$ & $\begin{array}{l}\text { MATRIX } \\
\text { SOLID }\end{array}$ & $\begin{array}{l}\text { ACTUAL } \\
28.45 \\
\end{array}$ & $\begin{array}{l}\text { FOUND } \\
26.56 *\end{array}$ & $\begin{array}{l}O L \\
\times \quad N / A \\
\end{array}$ & $\begin{array}{l}\text { UNIT } \\
\text { Joules }\end{array}$ \\
\hline 97000261 & $\mathrm{~T}-204$ & 2 SAMPLE & s97r000574 & 0 & & DSC-03 & SOLID & N/A & 0 & & Joules \\
\hline 97000261 & $T-204$ & 3 DUP & S97T000574 & o & $\because$ & DSC-03 & SOLID & 0 & 0 & N/A & Joules/s \\
\hline 97000261 & $\mathrm{~T}-204$ & 4 SAMPLE & S97T000575 & 0 & & DSC-03 & SOLID & N/A & 0 & & Joules/g \\
\hline 97000261 & $T-204$ & 5 DUP & s97T000575 & 0 & & DSC-03 & SOLID & 0 & 0 & N/A & Joules, \\
\hline
\end{tabular}

\section{Final page for worklist \# 17829}

See Aftached for Additional Signatures Analyst' Signature

turee $\%$

validated s/15/97 Bhachelor
Fincluo thack 5-15-97

Data Entry Comments:

Units shown for $Q C$ (SPK \& STD) may not reflect the actual units. $D L=$ Detection Limit, $S=$ Worklist Slot Number, $R=$ Replicate Number, $A=$ Aliquot Code. 


\section{LABCORE Data Entry Template for Worklist\# 17829}

Analyst: $\quad$ ANP Instrument: DSCO Book\# $12 N 14-B$

Method: LA-514-113 Rev/Mod D-O

HNF-SD-WAA-DP-255, REV. 0

Worklist Comment: T204, DSC-01 Run under nitrogen. skm

\begin{tabular}{|c|c|c|c|c|c|c|c|c|c|c|}
\hline \multirow[t]{2}{*}{ GROUP } & \multirow[t]{2}{*}{ PROJECT } & \multirow{2}{*}{$\begin{array}{l}\text { S TYPE } \\
1 \text { \$TD }\end{array}$} & \multirow[t]{2}{*}{ SAMPLE\# } & \multicolumn{2}{|r|}{ R A $\cdots \cdots$ TEST $\ldots \ldots$} & \multirow{2}{*}{$\begin{array}{l}\text { MATRIX } \\
\text { SOLID }\end{array}$} & \multirow[t]{2}{*}{ ACTUAL } & \multirow[t]{2}{*}{ FOUND } & \multirow{2}{*}{$\begin{array}{l}\text { DL } \\
\quad \text { N/A }\end{array}$} & \multirow{2}{*}{$\begin{array}{l}\text { UNIT } \\
\text { Joules/g }\end{array}$} \\
\hline & & & & & DSC-01 & & & & & \\
\hline 97000261 & $T-204$ & 2 SAMPLE & s97T000574 & 0 & $\mathrm{DSC}-01$ & SOLID & N/A & & & Joutes/g \\
\hline 97000261 & $T-204$ & 3 DUP & s97T000574 & 0 & DSC-01 & SOLID & & & $N / A$ & Joules/g \\
\hline 97000261 & $T-204$ & 4 SAMPLE & s97T000575 & 0 & DSC-01 & SOLID & $N / A$ & & & Joules/g \\
\hline 97000261 & $T-204$ & 5 DUP & s97T000575 & 0 & DSC-01 & SOLID & & & $N / A$ & Joules/g \\
\hline
\end{tabular}

\section{Final page for worklist \# 17829}

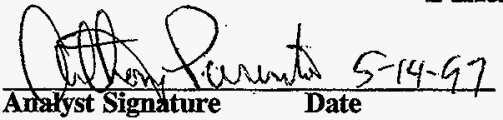

Antrost Signature Date

Analyșt Signature Date

Data Entry Comments:

Samples Rin in DSC-03.883 5/i4/17

Units shown for QC (SPK \& STD) may not reflect the actual units. $D L=$ Detection Limit, $S=$ Worklist Slot Number, $R=$ Replicate Number, $A=$ Aliquot Code. 
Curve 1: DSC

File info: INMDO10513 Tue May 13 20: 40:17 1997

Sample Weight: 8.850 ing

12N14-B

SIGNATUREBELOW REPRESENTS CHEMICAL TEOHNOLOGISTHCHEMST WHAT CONPLETEDNERAFIED THE CALBBRATION/ANALYSIS ONPAGES 126 . 10120

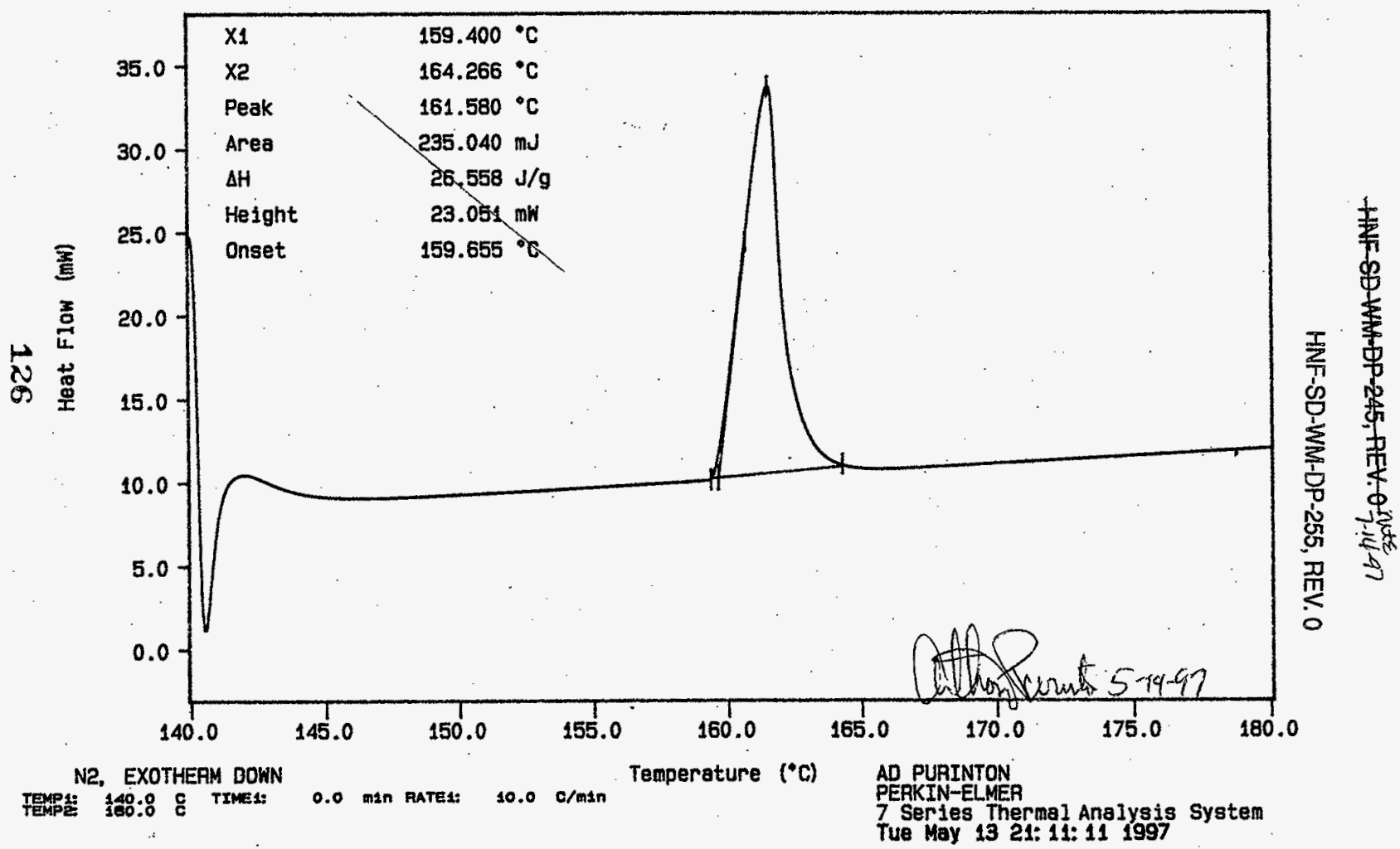


Curve 1: DSC

File infa: SAM050614 Wed May 14 03: 49: 571997

Sample Weight: $38.380 \mathrm{mg}$

S97T000574 SAM

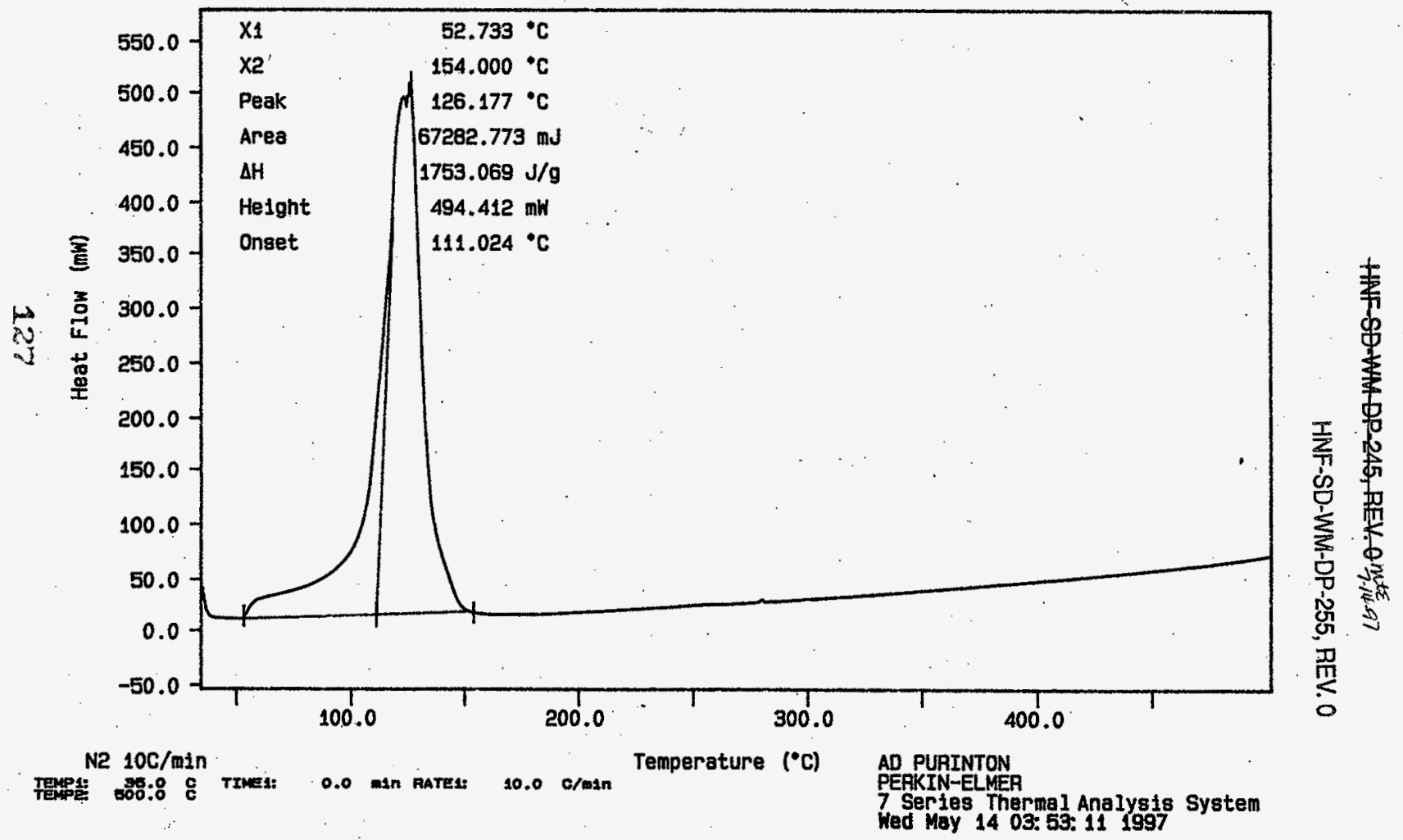


Curve 1: DSC

File info: SAM070514 Wed May 14 04: 44: 461997

Sample Weight: $32.350 \cdot \mathrm{mg}$

S97T000574 DUP

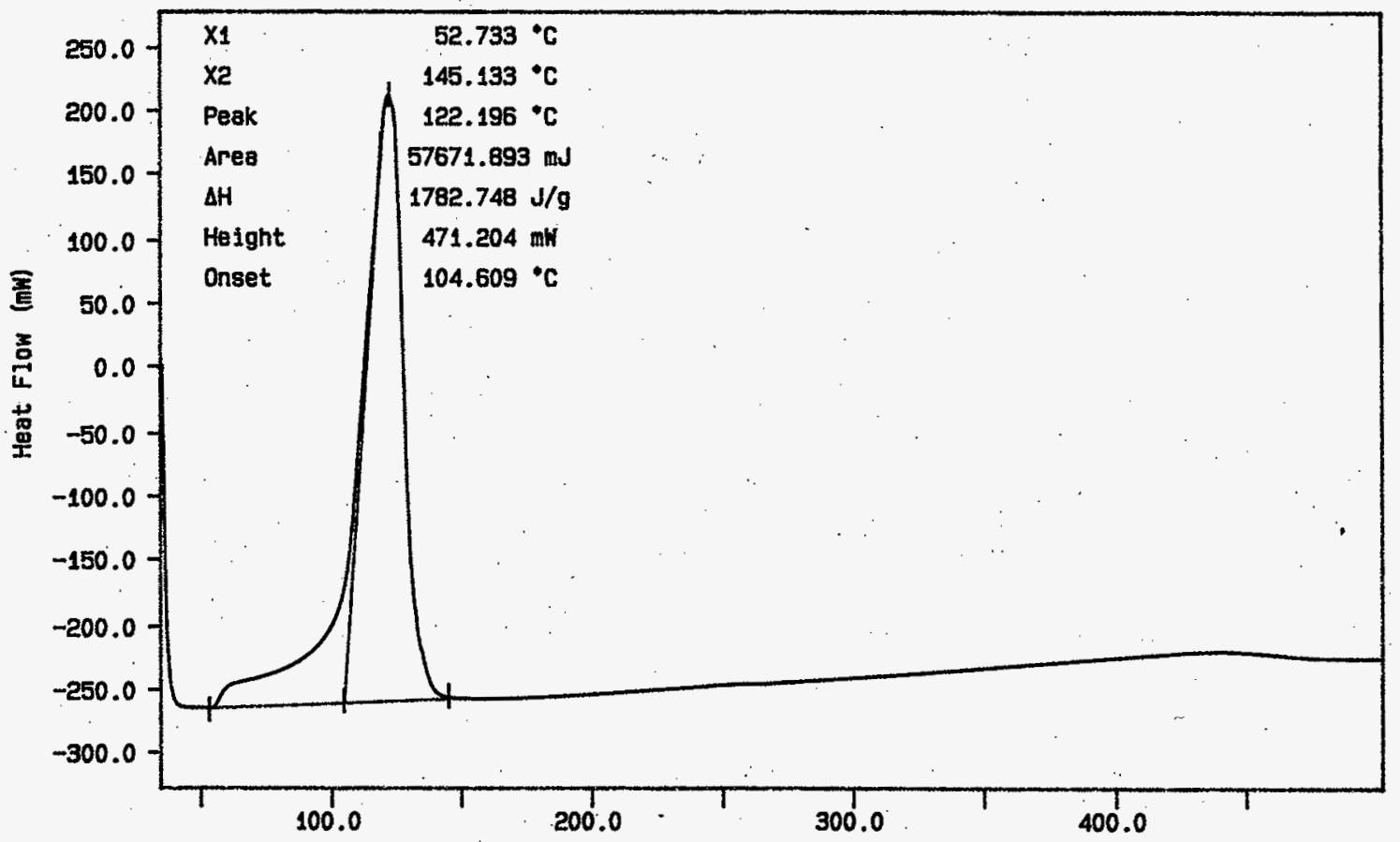

N2 $10 \mathrm{C} / \mathrm{min}$ 
Curve 1: DSC

File info: SAM051401 Wed May 14 06: 35: 26 1997

Sample Weight: $12.720 \mathrm{mg}$

5977000575

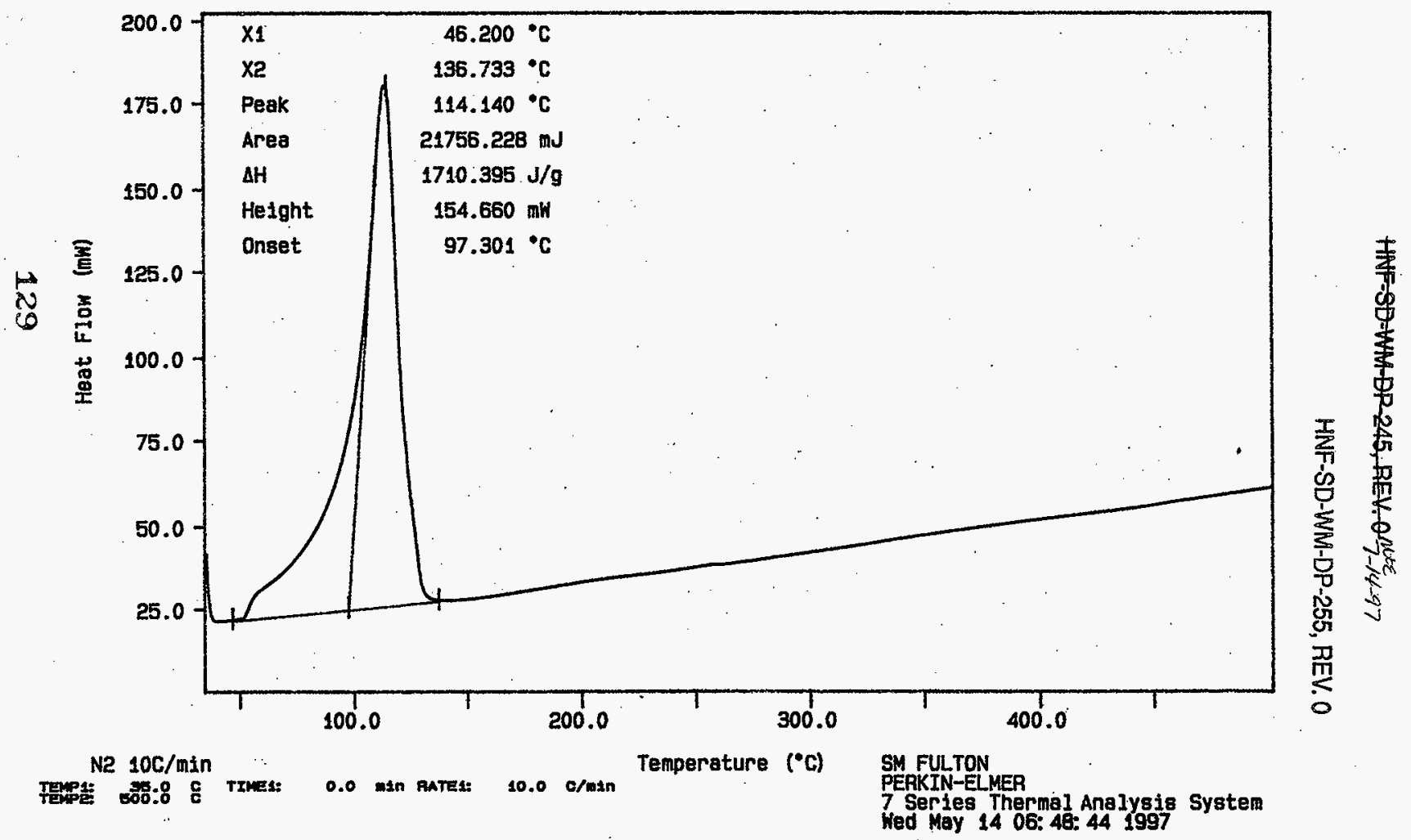


Curve 1: DSC

File info: SAM051402 Wed May 14 07: 39: 361997

Sample Weight: 10.280

S97T000575 DUP

$\mathrm{mg}$

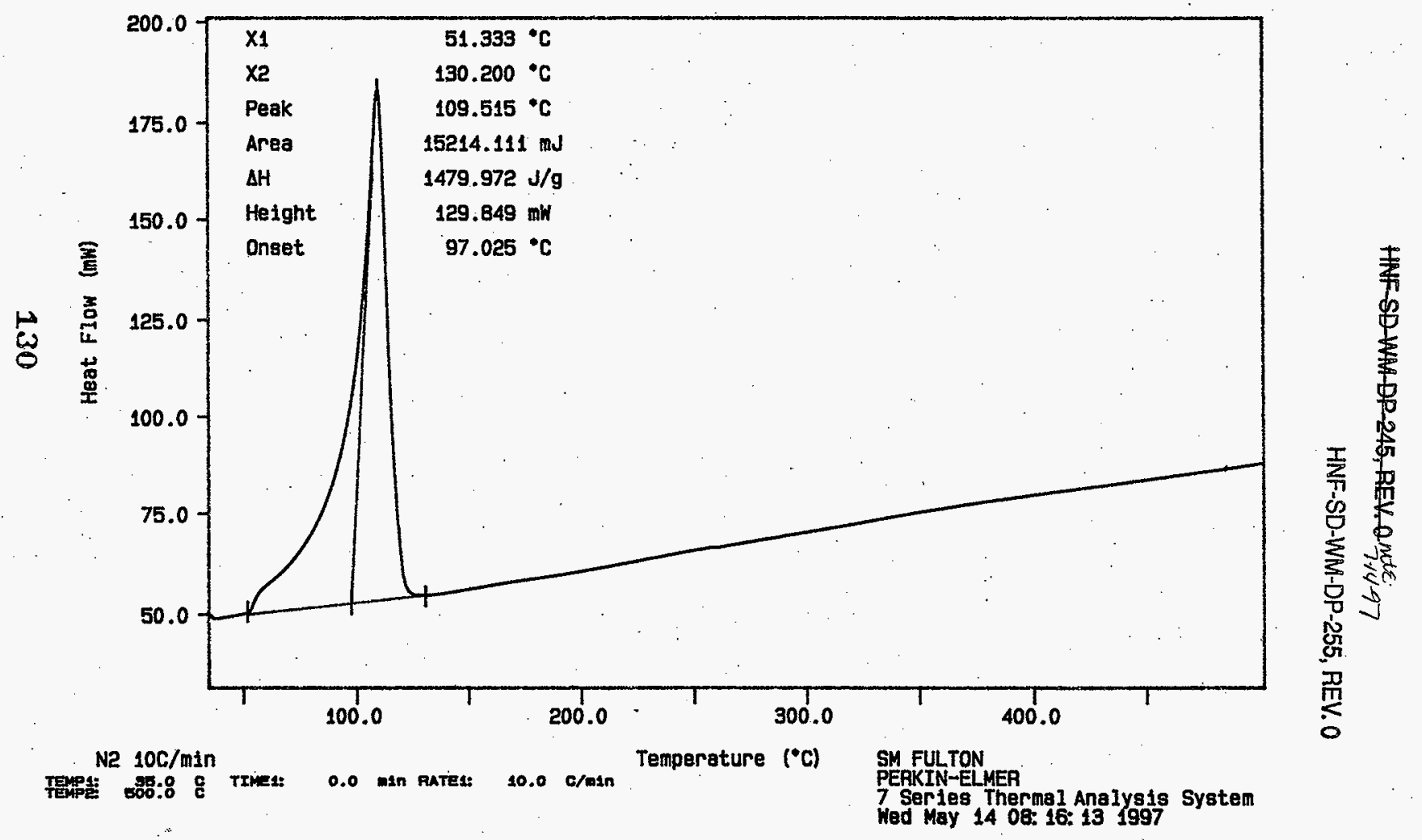




Analyst: $\quad$ SMF Instrument: $\mathrm{DSCO} 3$ Book \# $12 \mathrm{~N} / 4 \mathrm{~B}$

Method: LA-514-114 Rev/Mod $D-O$ HNF-SD-WM-DP-255, REV. 0

Worklist Comment: T204, DSC-01 Run under nitrogen. skm

\begin{tabular}{|c|c|c|c|c|c|c|c|c|c|c|}
\hline GROUP & $\begin{array}{l}\text { PROJECT } \\
\vdots \\
\end{array}$ & $\begin{array}{l}\text { S TYPE } \\
1 \text { STD }\end{array}$ & SAMPLE\#. & & $\begin{array}{c}\text { A } \\
\text { DSC }-03\end{array}$ & $\begin{array}{l}\text { MATRIX } \\
\text { SOL } 10\end{array}$ & $\begin{array}{l}\text { ACTUAL } \\
28.45 \\
\end{array}$ & $\begin{array}{l}\text { FouNd } \\
27.00 * \\
\end{array}$ & N/A & $\begin{array}{l}\text { UNIT } \\
\text { - Joules/g }\end{array}$ \\
\hline 97000261 & $T-204$ & 2 SAMPLE & 5977000576 & 0 & DSC-03 & SOLID & $N / A$ & 0 & & Joutes/g \\
\hline 97000261 & $T-204$ & 3 DUP & s97T000576 & 0 & $2 \times 2 \times D S c-03$ & SOLID & 0 & 0 & N/A & Joules/g \\
\hline 97000261 & $T-204$ & 4 SAMPLE & \$97T000580. & 0 & DSC -03 & SOLID & N/A & 0 & & Joules/g \\
\hline 97000261 & $T-204$ & 5 DUP & S97T000580 & 0 & Dsc- 03 & SOLID & 0 & 0 & N/A & Joules/g \\
\hline
\end{tabular}

\section{Final page for worklist \# 17830} $\frac{\text { See Attachod for Signature }}{\text { Analyst Signature }}$

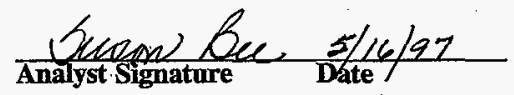

Data Entry Comments:

Onits shown for $Q C$ (SPK \& STD) may not reflect the actual units. $D L=$ Detection Limit, $S=$ Worklist Slot Number, $R=$ Replicate Number, $A=$ Aliquot Code. 


\section{LABCORE Data Entry Template for Worklist\# 17830}

Analyst: $\quad$ SMF

Method: LA-514-113 Rev/Mod
Instrument: DSCO

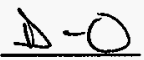

Book \# $12 \cup 14-B$

HNF-SD-WM-DP-255, REV. 0

Worklist Comment: T204, DSC-01 Run under nitrogen. skm

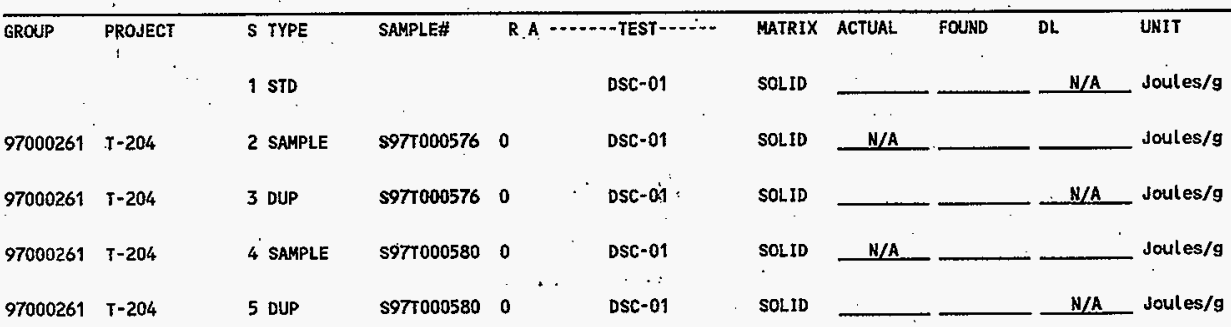

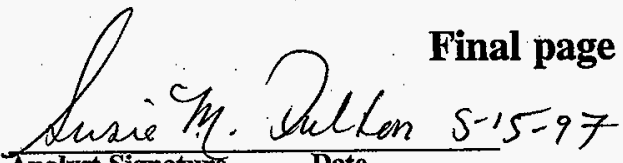

Analyst Signature

Date

Analyst-Signature

Date

Data Entry Comments:

Units shown for $Q C$ (SPK \& STD) may not reflect the actual units. $D L=$ Detection Limit, $S=$ Worklist Slot Number, $R=$ Replicate Number, $A=$ Aliquot Code. 
Curve 1: DSC

File Info: IND051501 Thu May 15 02: 36: 271997 Sample Meight: 10.940

mg

12N14-B

SIGNATURE BELOW REPRESENTS CHEMICAL TECHNOLOGIST/CHEMIST THAT COMPLETED/VERTFIED THE CALLBRATION/ANALYSIS ON PAGES 133 TO 137

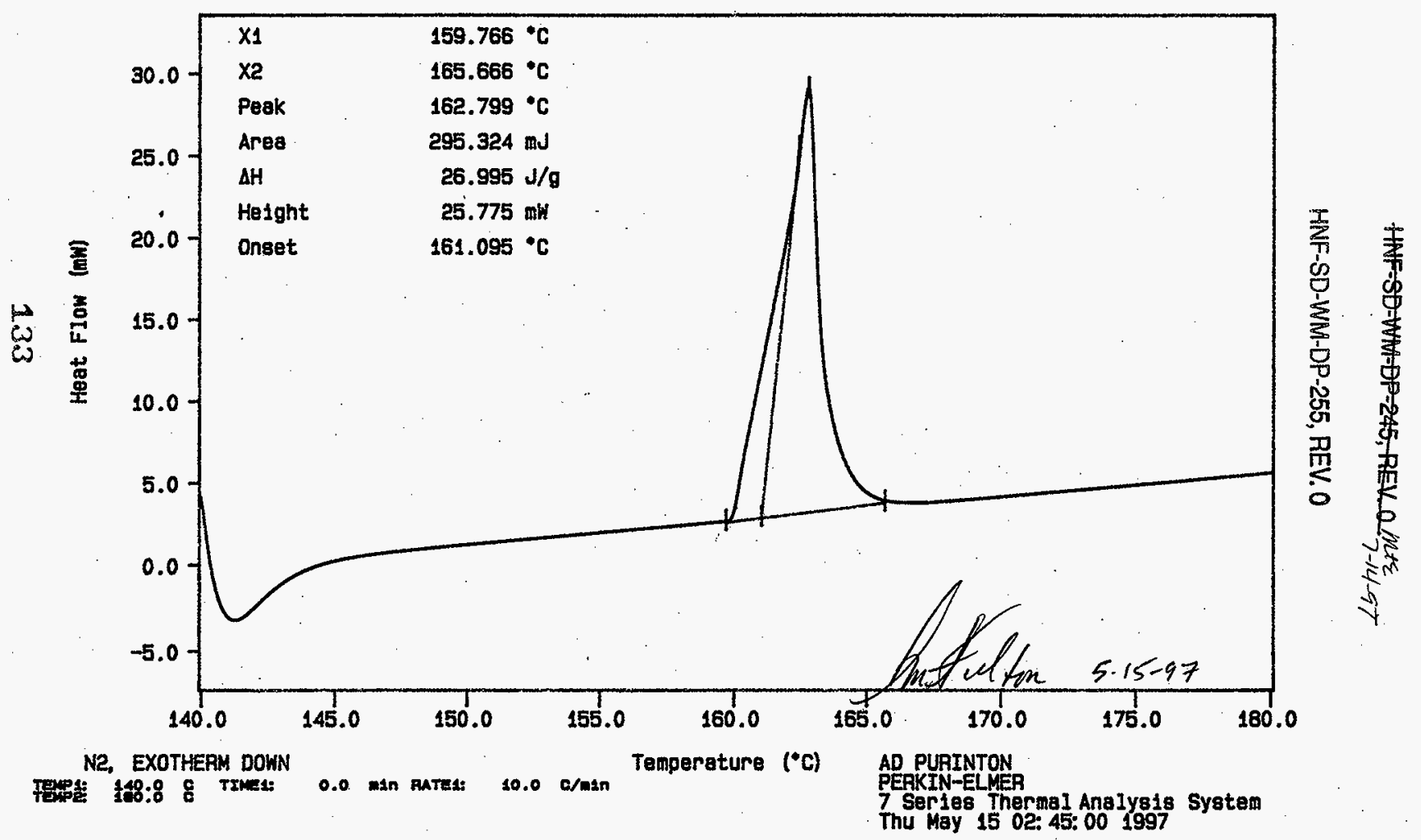


Curve 1: DSC

F11e info: SAM051505 Thu May 15 13:52: 151997

Sample Weight: 24.410 mg

S97T000576

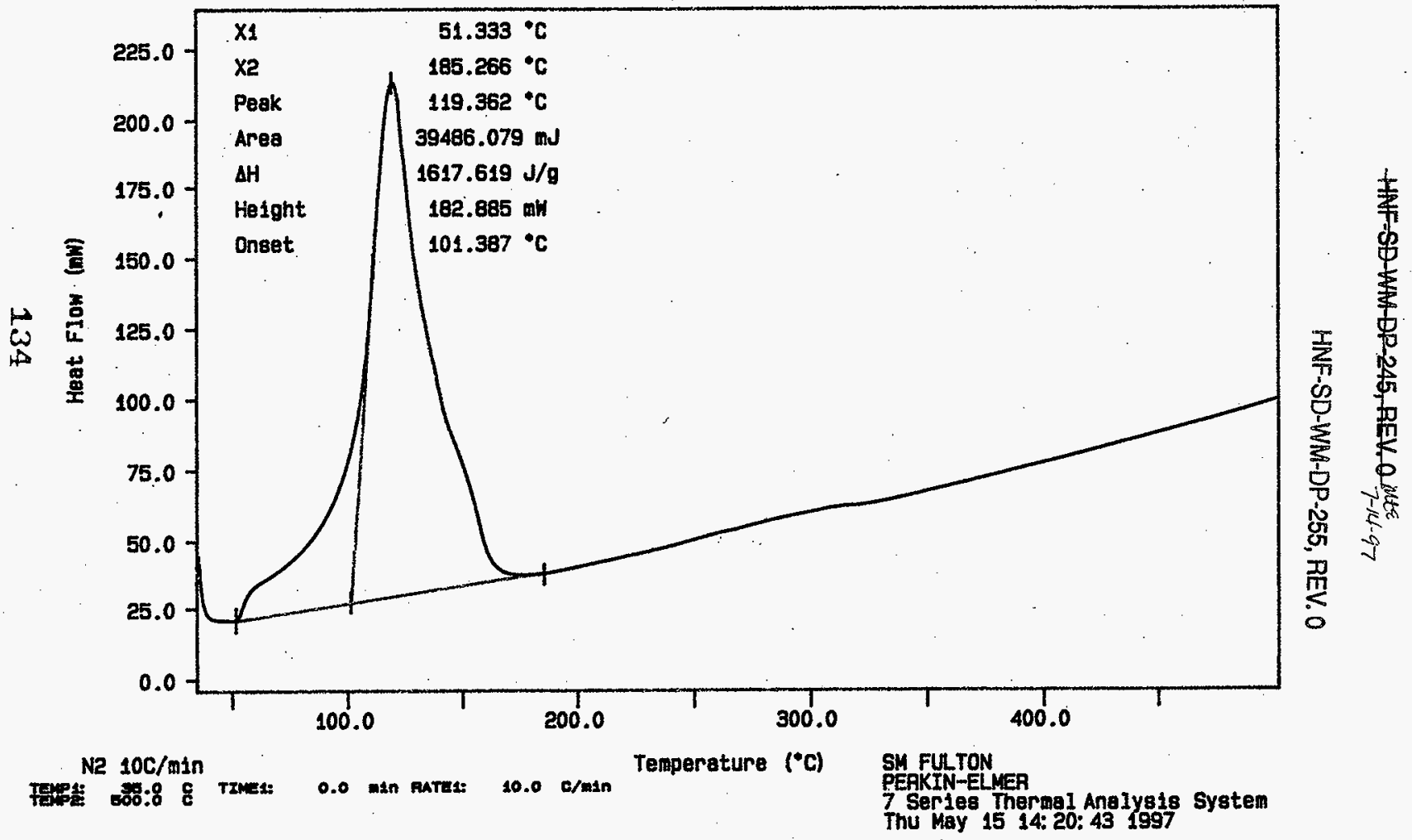


Curve 1: DSC

F1le Info: SAM051506 Thu Hay 15 15: $13: 511997$

Sample Neight: 27.350

S97T000576 DUP

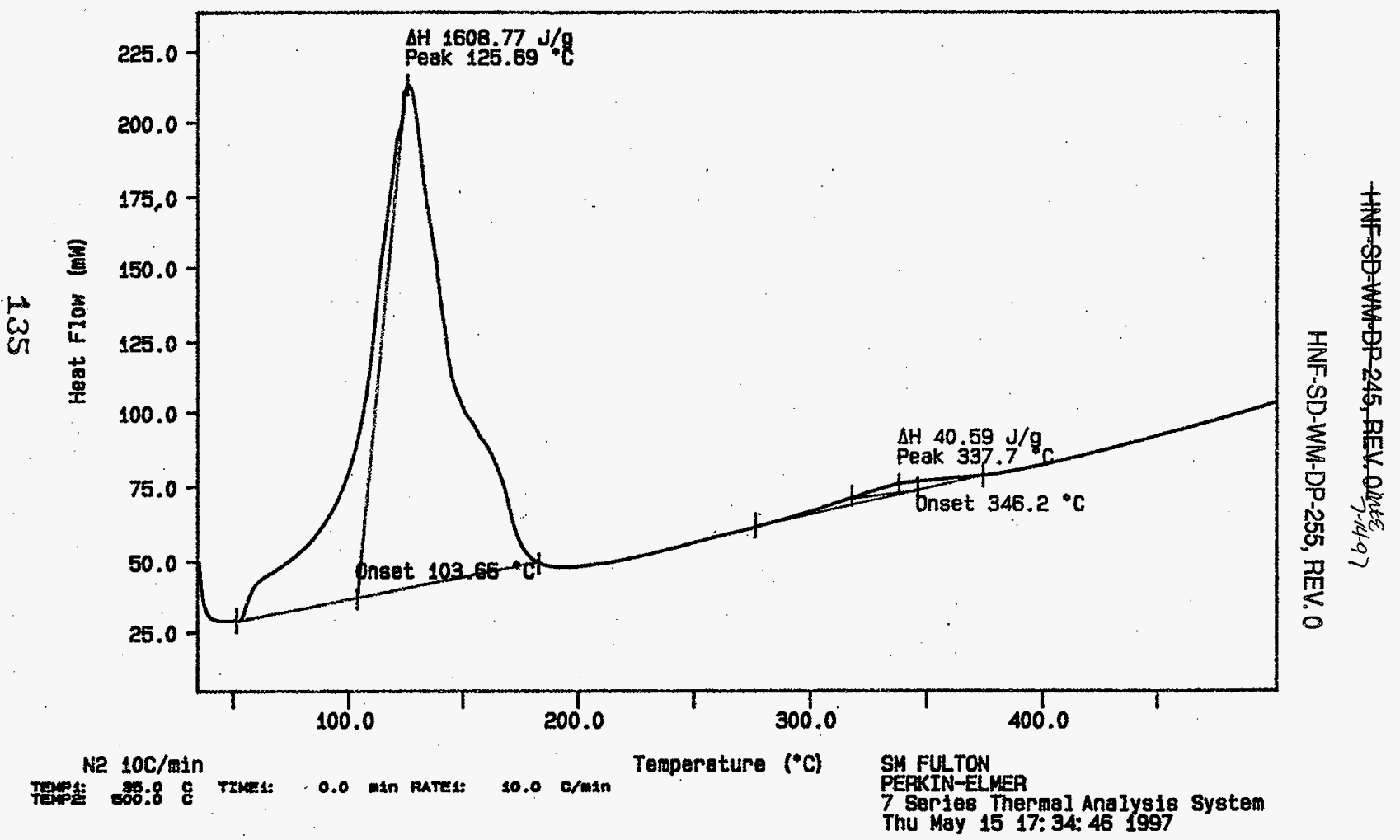


Curve 1: DSC

File Info: SAM051507 Thu May 15 16: 38: 491997

Sample Weight: 16.260 ag

S97TO00580 SAM

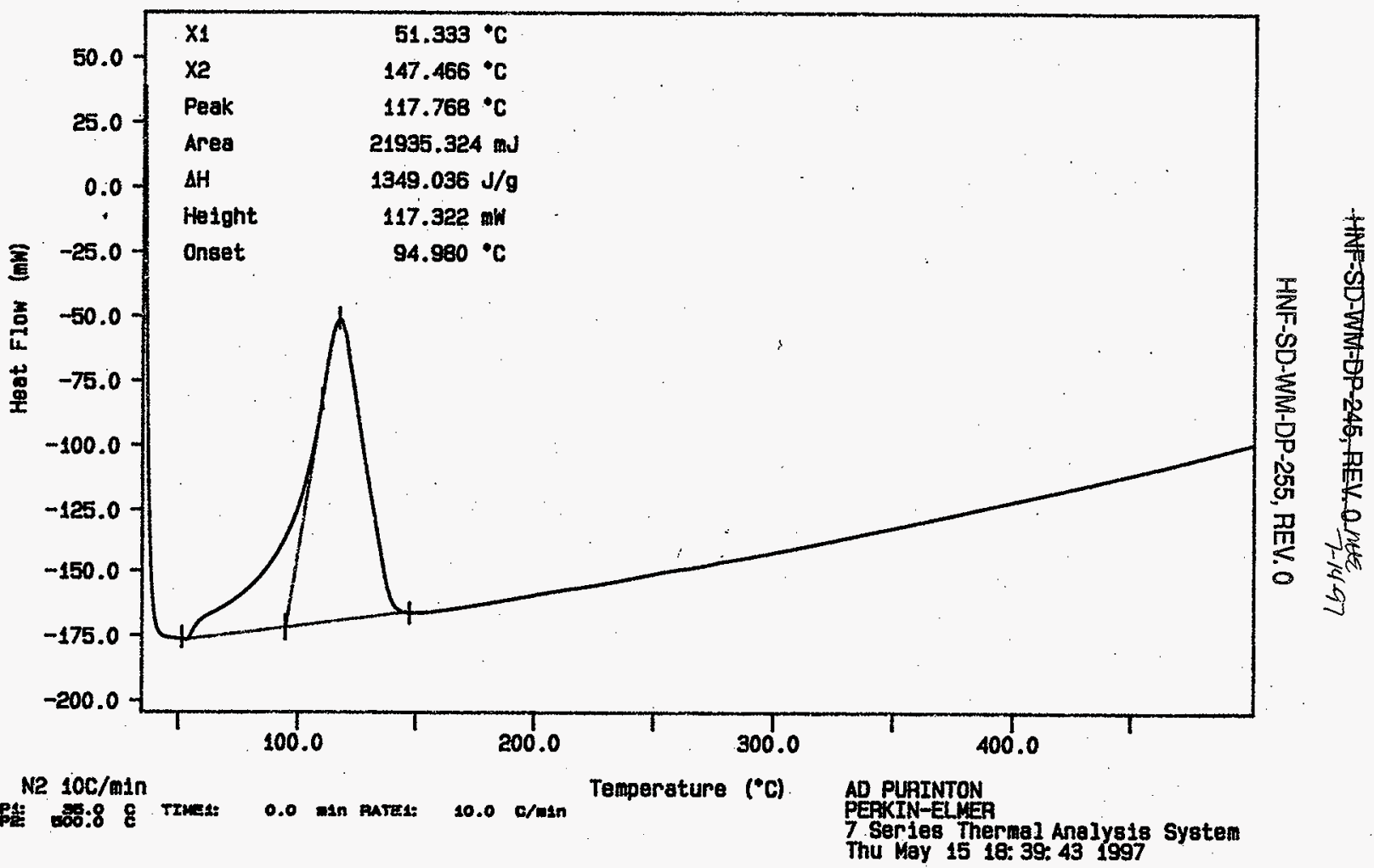


HNF-SD-WM-DP-255, REV. 0

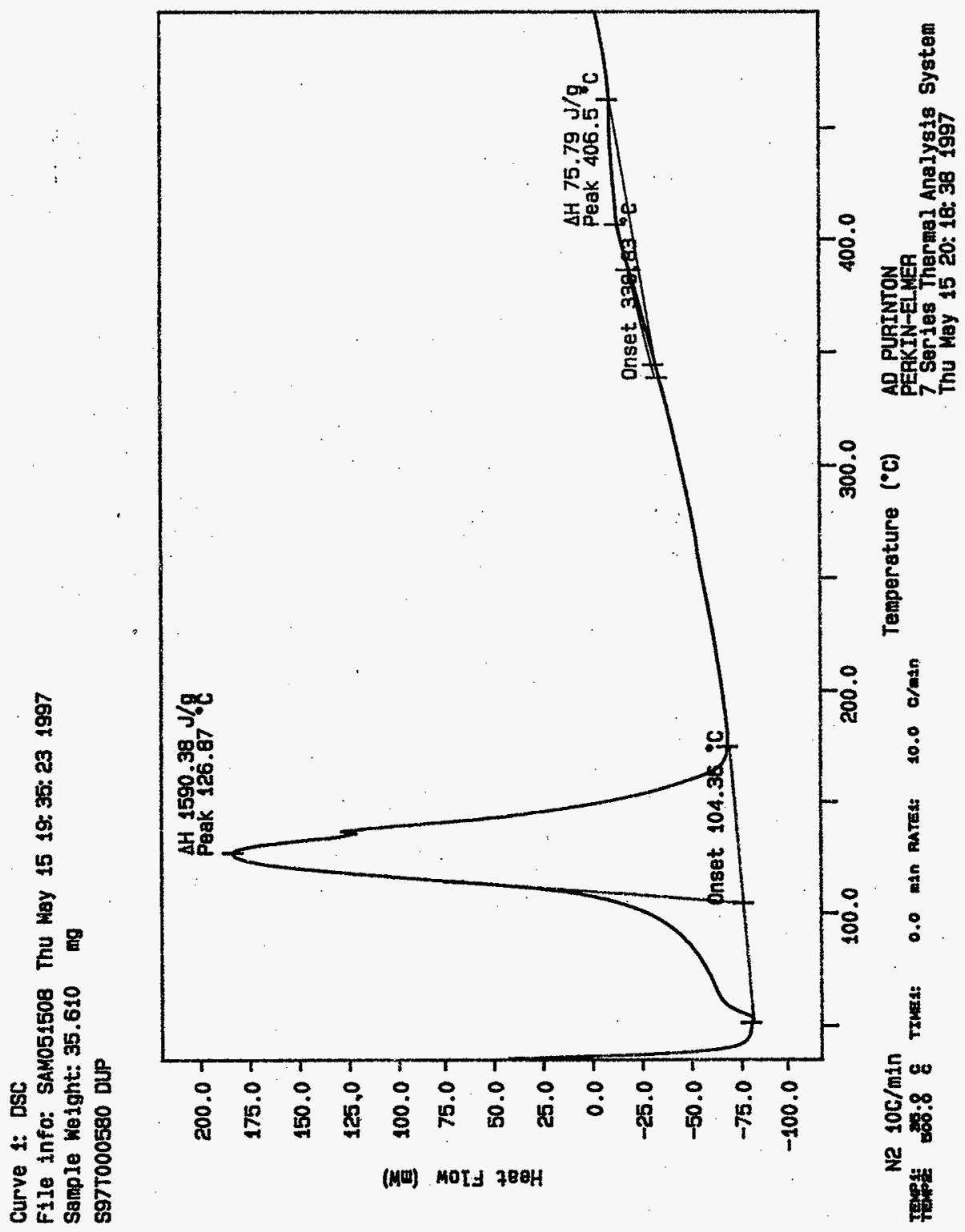




\section{LABCORE Data Entry Template for Worklist\# 17831}

Page: 1

Analyst: $A D P$ Instrument: $D S C 0-3 \quad$ Book \# $/ 2 N / 4 B$

Method: LA-514-114 Rev/Mod D-O

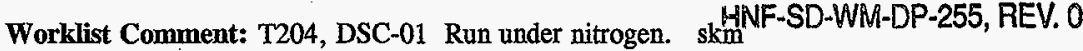

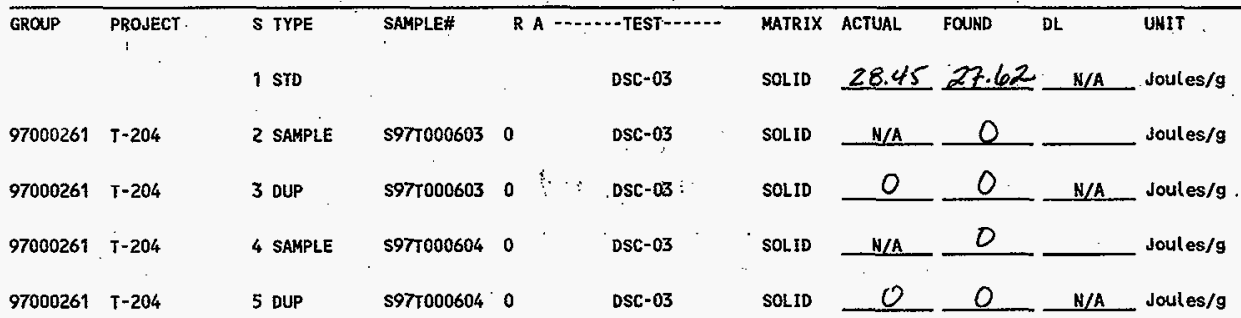

\section{Final page for worklist \# 17831}

See Attached. for Signature. Analyst Signature Date

validated 5/21/97\%onaclelor

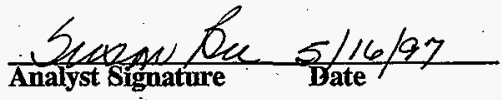

Data Entry Comments:

Units shown for $Q C$ (SPK \& STD) may not reflect the actual units. $D L=$ Detection Limit, $S=$ Worklist Slot Number, $R=$ Replicate Number, $A=$ Aliquot Code. 


\section{LABCORE Data Entry Template for Worklist\# 17831}

Analyst: $\therefore$ Instrument: $\mathrm{DSCO} 3 \quad$ Book $12 \mathrm{~N} / 4 \mathrm{~B}$

Method: LA-514-113 Rev/Mod

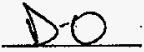

HNF-SD-WM-DP-255, REV. 0

Worklist Comment: T204, DSC-01 Run under nitrogen. skm

\begin{tabular}{|c|c|c|c|c|c|c|c|c|c|c|}
\hline \multirow[t]{2}{*}{ GROUP } & PROJECT & S TYPE & SAMPLE\# & R A & -....... TEST & MATRIX & ACTUAL & FOLND & $\mathrm{DL}$ & UNIT \\
\hline & & 1 STD & & & DSC-01 & SOLID & & & $\mathbf{N} / \mathbf{A}$ & Joules/g \\
\hline 97000261 & $T-204$ & 2 SAMPLE & $\$ 97 T 000603$ & 0 & DSC-01 & SOLID & $\mathrm{N} / \mathrm{A}$ & & & Joules/g \\
\hline 97000261 & $T-204$ & 3 DUP & s97T000603 & 0 & DSC-01 & SOLID & & & $N / A$ & Joules/g \\
\hline 97000261 & $T-204$ & .4 SAMPLE & s97r000604 & 0 & DSC-01 & SOLID & $N / A$ & & & Joutes/g \\
\hline 97000261 & $T-204$ & 5 DUP & \$975000604 & 0 & DSC-01 & SOLID & & & $N / A$ & Joules/g \\
\hline
\end{tabular}

\section{Final page for worklist \# 17831}

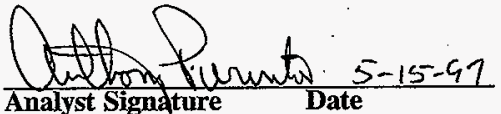

\section{Analyst Signature Date}

Data Entry Comments:

Samples run on DSC-03. $5 / 16 / 17$

Units shown for $Q C$ (SPK \& STD) may not reflect the actual units. $D L=$ Detection Limit, $S=$ Worklist Slot Number, $R=$ Replicate Nimber, $A=$ Aliquot Code. 
Curve 1: DSC

F11e info: IND051501 Thu May 15 02: 36: 271997

Sample Weight: $10.940 \mathrm{mg}$

12N14-B

SIGNATURE BEL OW REPRESENTS CHEMICAL TECHNOLOGIST/CHEMIST THAT COMPLETED/VERHEO THE CALLBATIONANMLYSIS ON PAGES 140 TO 144

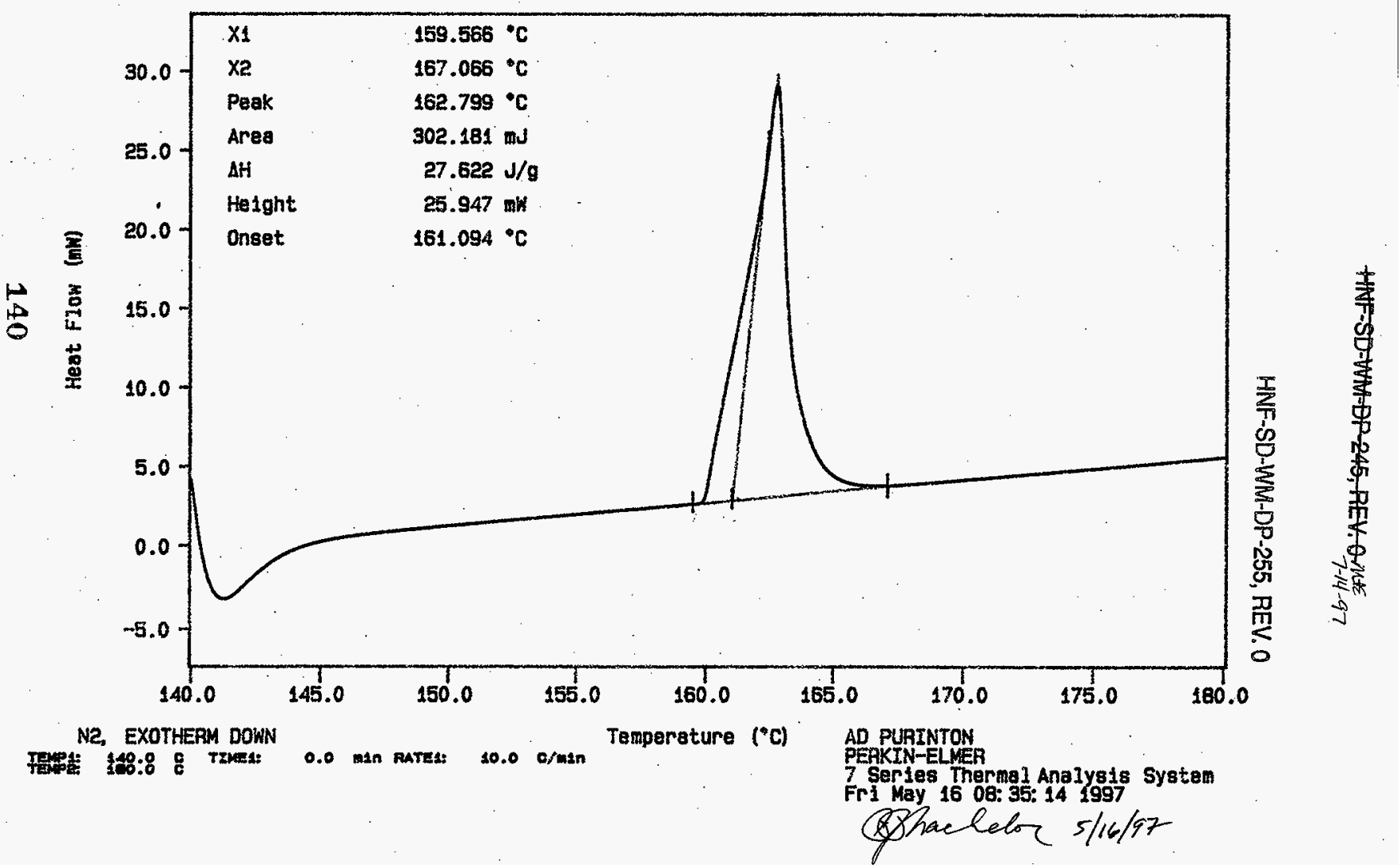


Curve 1: DSC

F1le info: SAM051509 Thu May 15 23: 26: 091997

Sample Weight: $24.200 \mathrm{mg}$

S97T000603 SAM
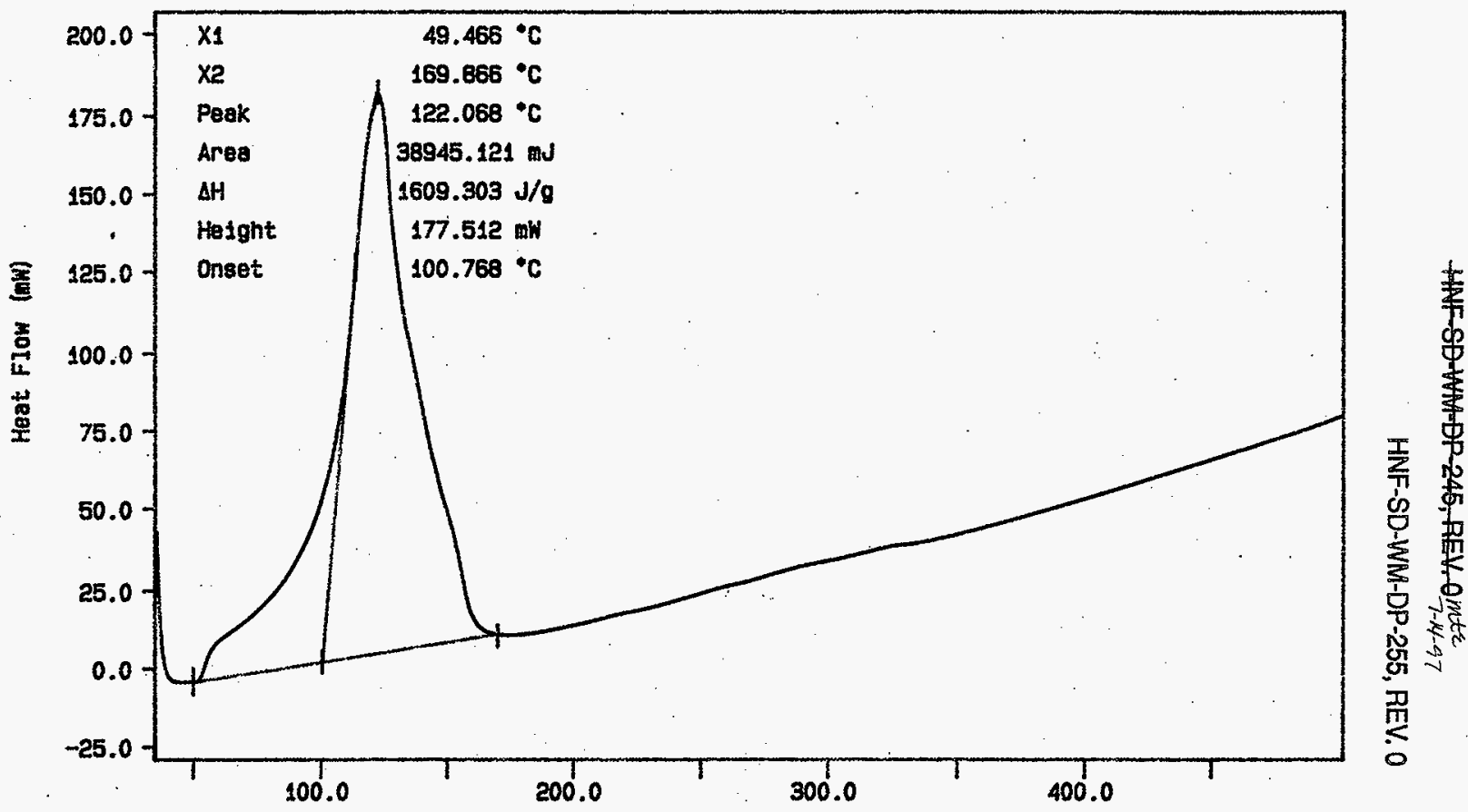

N2 $40 \mathrm{c} / \mathrm{min}$

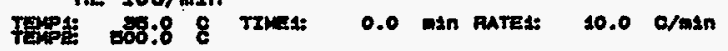

Temperature ('C) AD PURINTON

PERKIN-ELNER

7 Serjes thernal Anolysis System

Thu May 15 23: 58: 37 1997 
Curve i: DSC

F110 Info: SAM051510 Fr1 May 16 00:48:01 1997

Sample Wetght: $19.620 \mathrm{ng}$

S97T000603 DUP

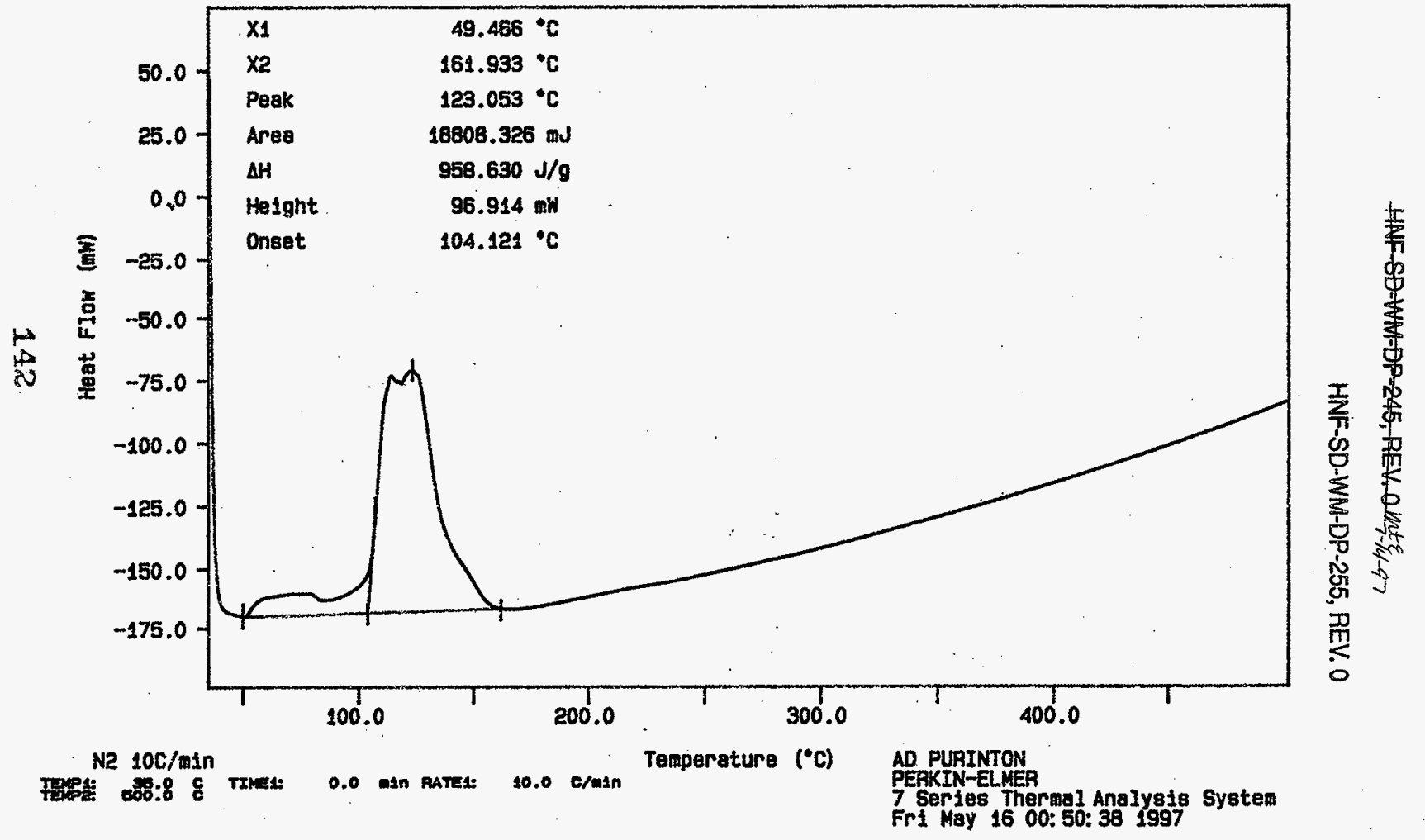


Curve 1: DSC

File Info: SAN051511 Fri Hay 16 01: 45: 371997

Sample Neight: 37.770

mg

597T000604 SAM

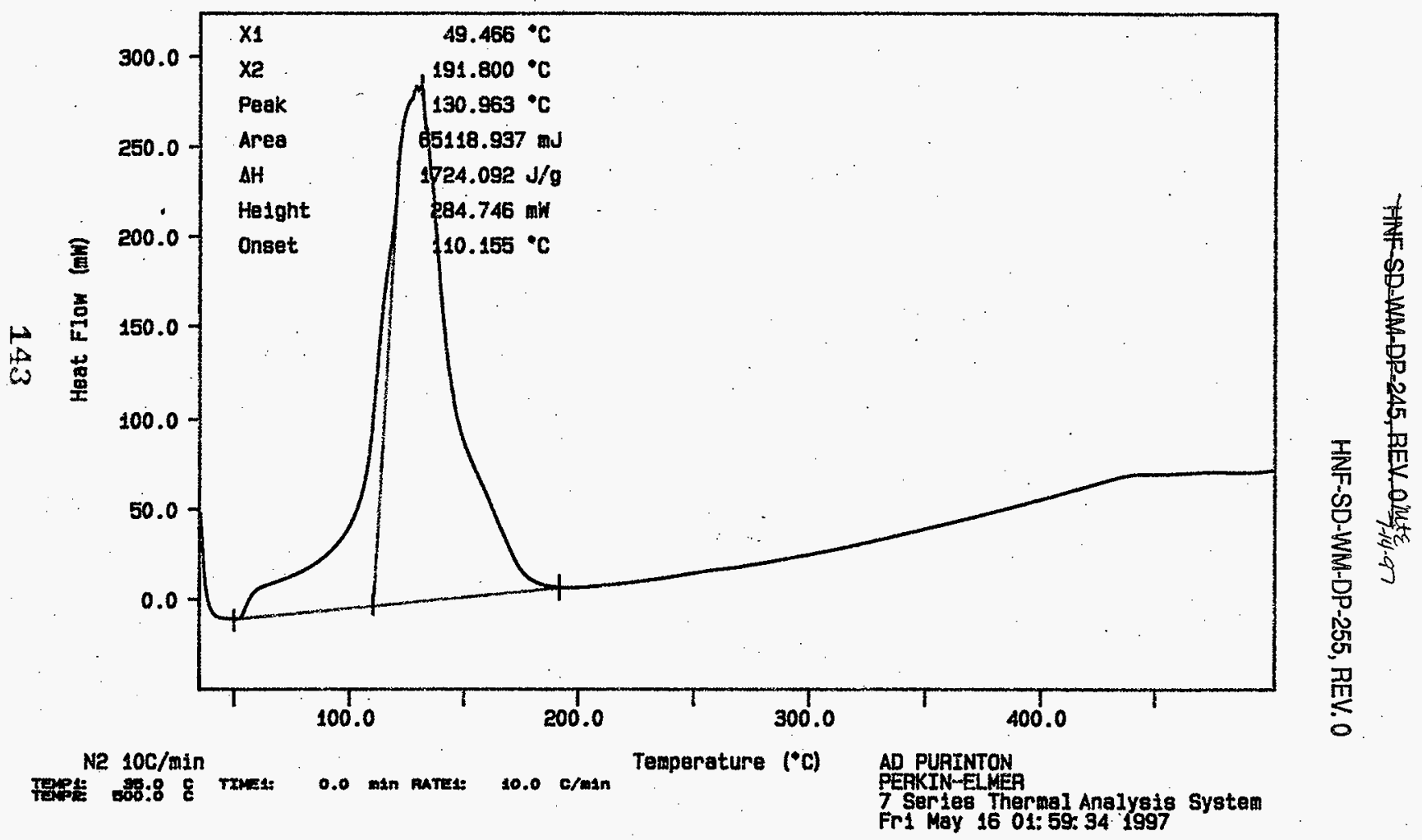


Curve 1: DSC

F1le Info: SAM051513 Frt May 16 02:54: 171997

Sample Height: $24.970 \mathrm{mg}$

S97T000604 DUP
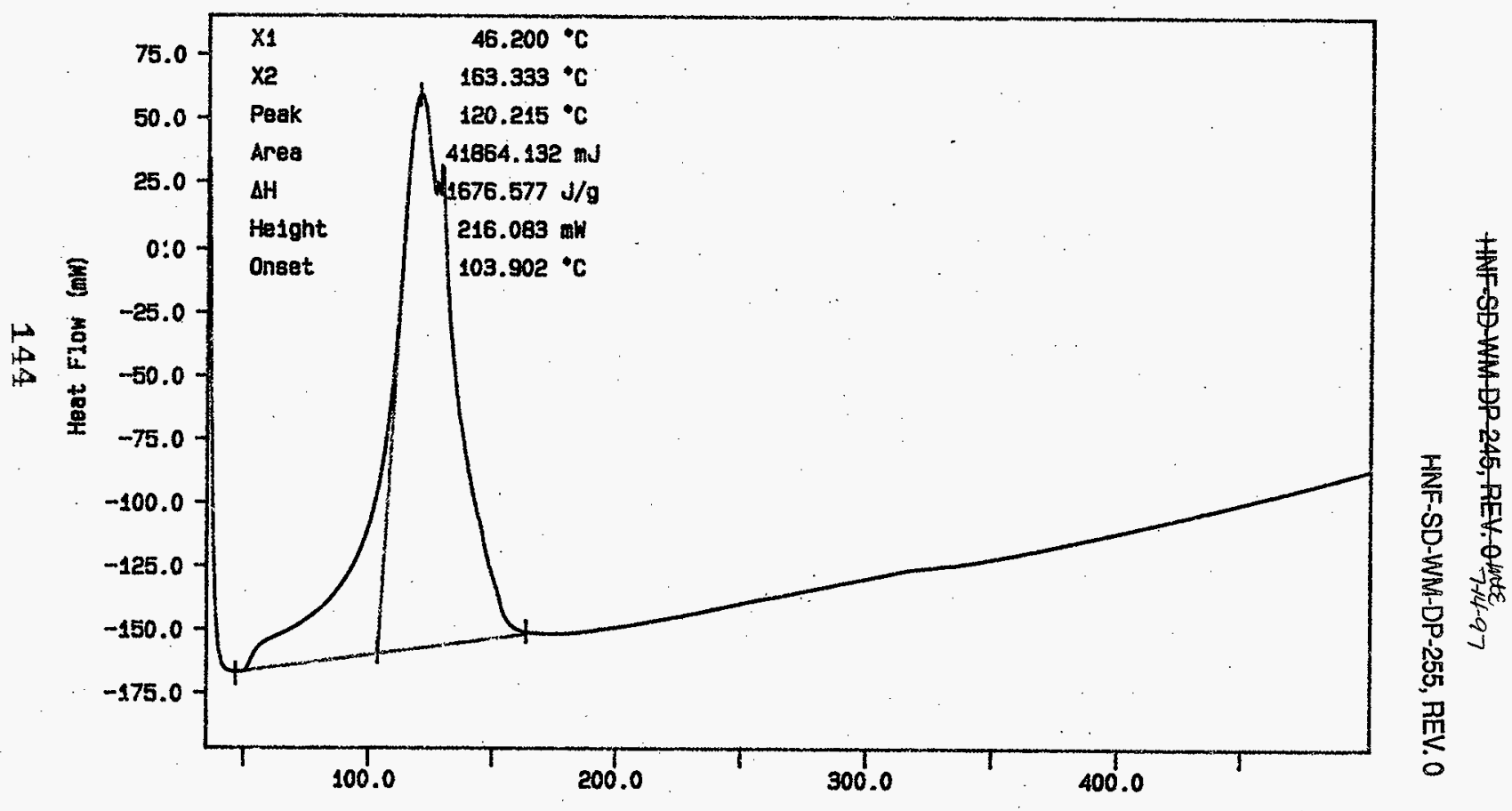

N2 10c/ain

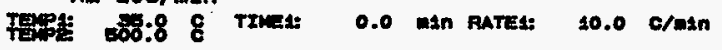

Temperature $\left({ }^{\circ} \mathrm{C}\right)$

AD PURINTON

7 Sertes Thermal Anelysis System

Fri May 16 03: 12: 221997 


\section{LABCORE Data Entry Template for Worklist\# 17832}

Analyst: $\quad$ ADP $\quad$ Instrument: $D S C 0 \quad 3 \quad$ Book \# $12 \times 14 B$

Method: LA-514-114 Rev/Mod D-O

Worklist Comment: T204, DSC-01 Run under nitrogen. skm

HNF-SD-WM-DP-255, REV. 0

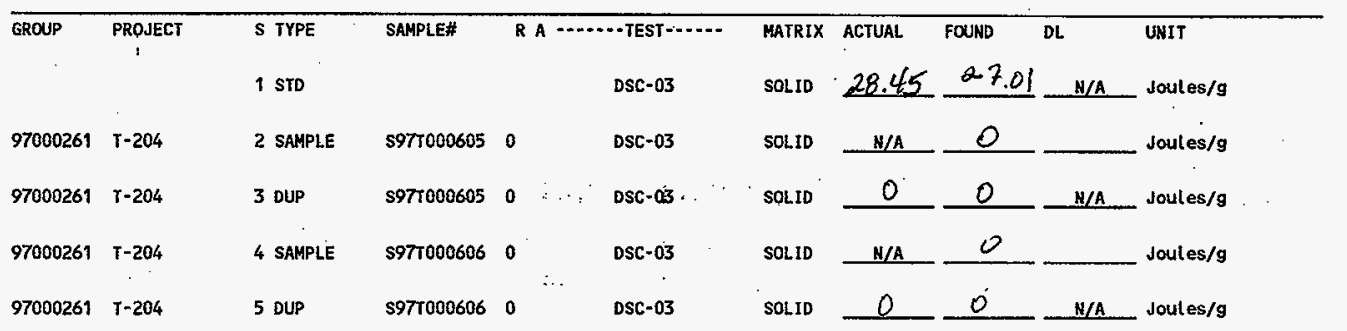

\section{Final page for worklist \# 17832}

See Attached for Signature

Analyst Signature

Validated
Date

$5 / 22 / 97 \$ 3 a c h e b r$

Data Entry Comments:

Units shown for QC (SPK \& STD) may not reflect the actual units. $D L=$ Detection Limit, $S=$ Worklist Slot Number, $R=$ Replicate Number, $A=$ Aliquot Code. 
Analyst: APP Instrument: DSCO 3 Book \# 12 N $14 B$

Method: LA-514-113 Rev/Mod DO

Worklist Comment: T204, DSC-01 Run under nitrogen. skm
HNF-SD-WM-DP-255, REV. 0

\begin{tabular}{|c|c|c|c|c|c|c|c|c|c|c|}
\hline GROUP & $\begin{array}{c}\text { PRQJECT } \\
: 1\end{array}$ & $\begin{array}{l}\text { S TYPE } \\
1 \text {. STD }\end{array}$ & SAMPLE\# & R A & $\begin{array}{l}\text { DSC }-01 \\
\end{array}$ & $\begin{array}{l}\text { MATRIX } \\
\text { SOL IO }\end{array}$ & ACTUAL & FOUND & $\mathrm{N} / \mathrm{A}$ & $\begin{array}{l}\text { UNIT } \\
\text { Joules/g }\end{array}$ \\
\hline 97000261 & $T-204$ & 2 SAMPLE & s977000605 & 0 & DSC-01 & SOLID & $N / A$ & & & Joules/g \\
\hline 97000261 & $T-204$ & 3 DUP & s977000605 & 0 & $\therefore$ DSC-01 & SOLID & & & $N / A$ & Joules/g \\
\hline 97000261 & $T-204$ & 4 SAMPLE & s97r000606 & 0 & $\begin{array}{l}\text { Dsc-ö1 } \\
\vdots\end{array}$ & SOLID & N/A & & & Joules/g \\
\hline 97000261 & $T-204$ & 5 DUP & \$97T000606 & 0 & DSC-01 & SOLID & & & $N / A$ & Joutes/g \\
\hline
\end{tabular}

\section{Final page for worklist \# 17832}

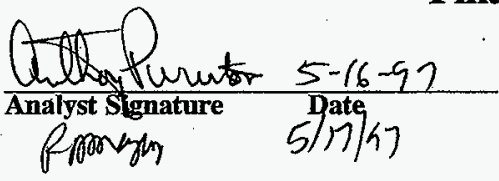

Analyst Siguature Date

Data Entry Comments:

Samples run on DSC-03. 833 S/16/97

Units shown for QC (SPK \& STD) may not reflect the actual units. DL = Detection Limit, $S=$ Worklist Slot Number, $R=$ Replicate Number, $A=$ Aliquot Code. 
Curve 1: DSC

File info: IND051601 Fri May 16 03: 24:36 1997

Sample Vejght: 10.940 mg

12N14-B

SIGNATURE BELOW REPAESENTS CHEMICAL TECHNOLOGIST/CHEMIST THAT

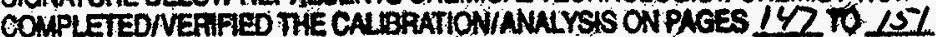

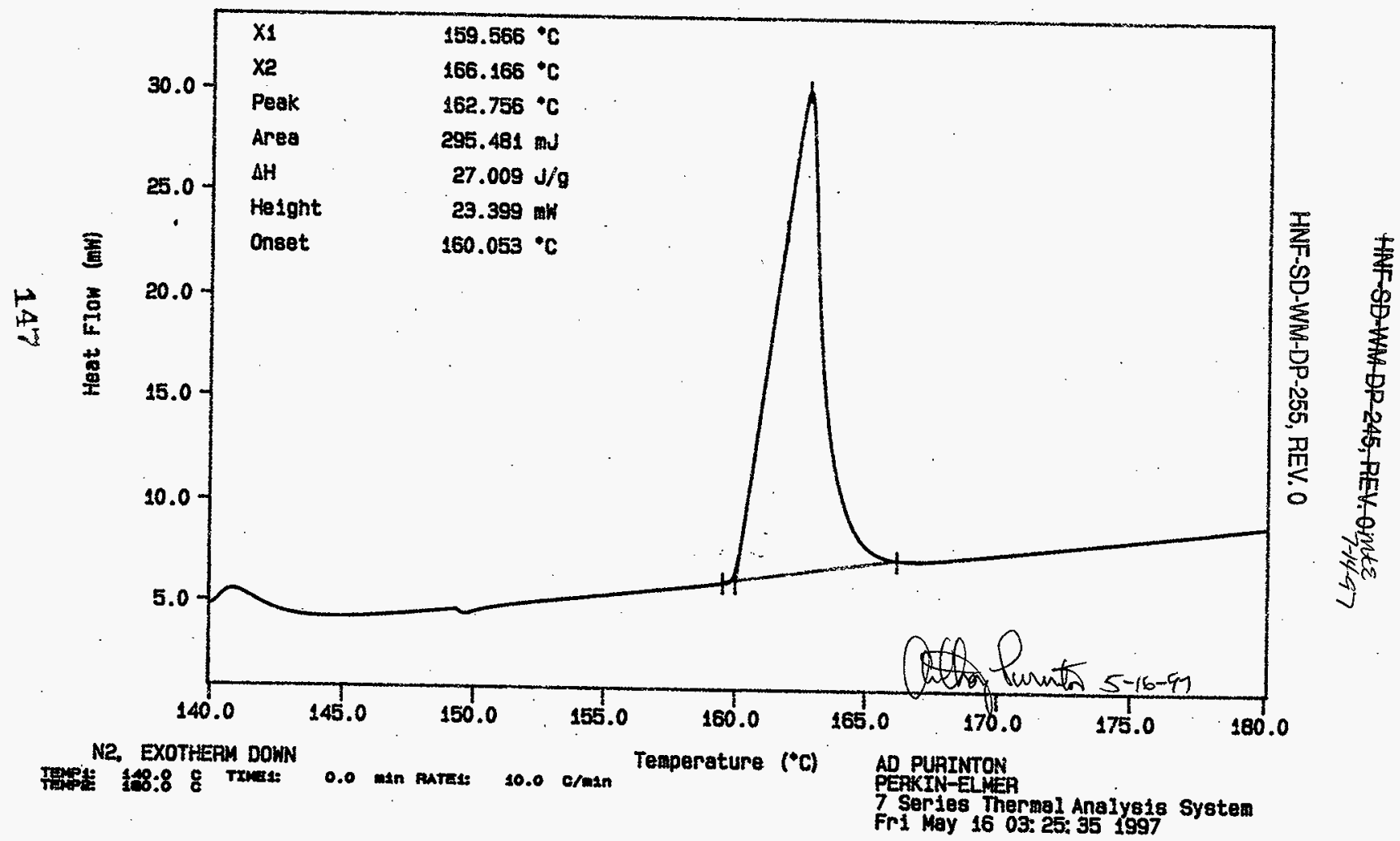


Curve 1: DSC

File info: SAM051601 Fri May 16 04: 23:55 1997

Sample Neight: $18.940 \mathrm{mg}$

S97T000605 SAM

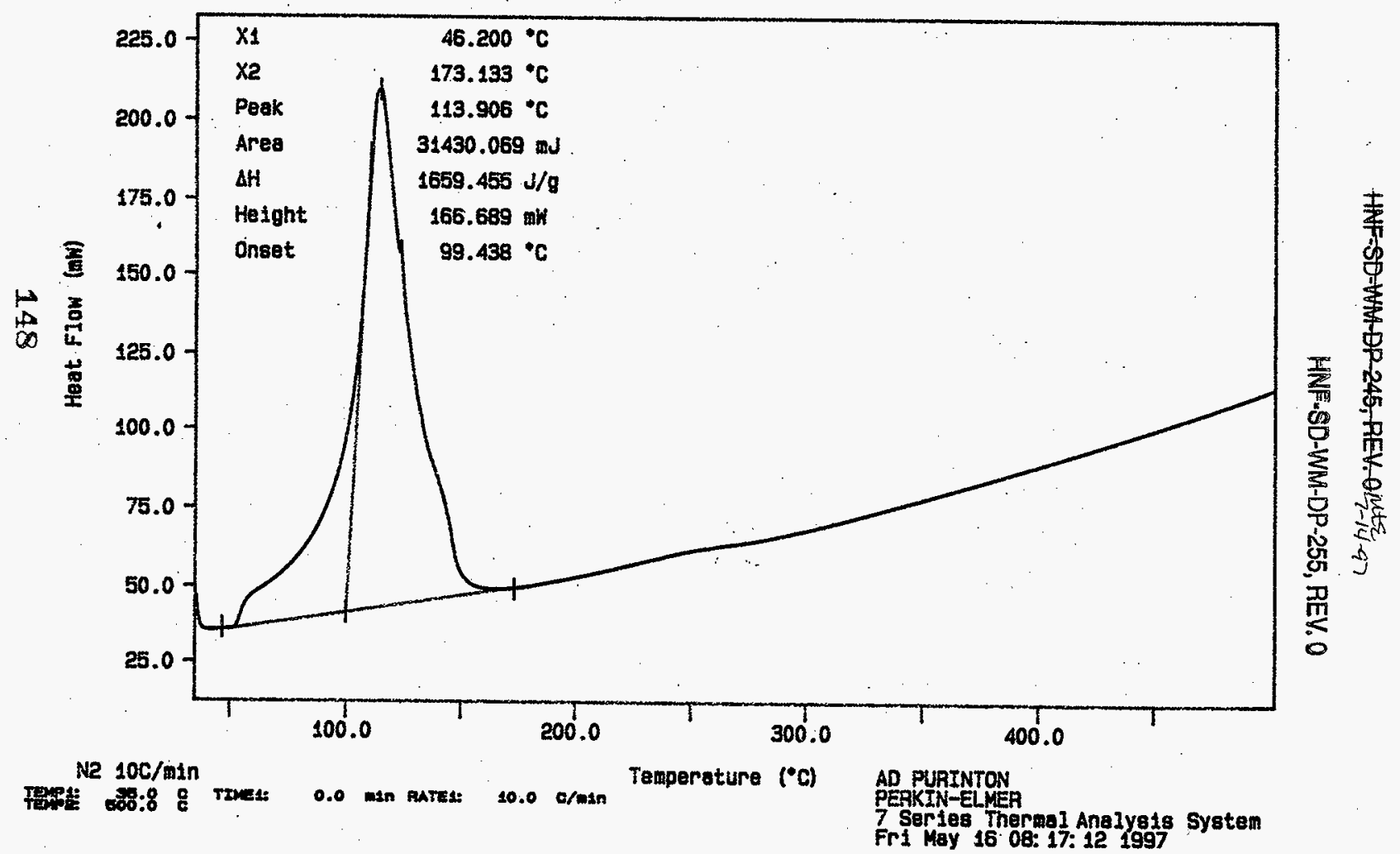


Curve 1: DSC

File info: SAM051602 Frt Hay 16 19: 36:05 1997

Sample Weight: 49.440 ang

s97T000605Dup

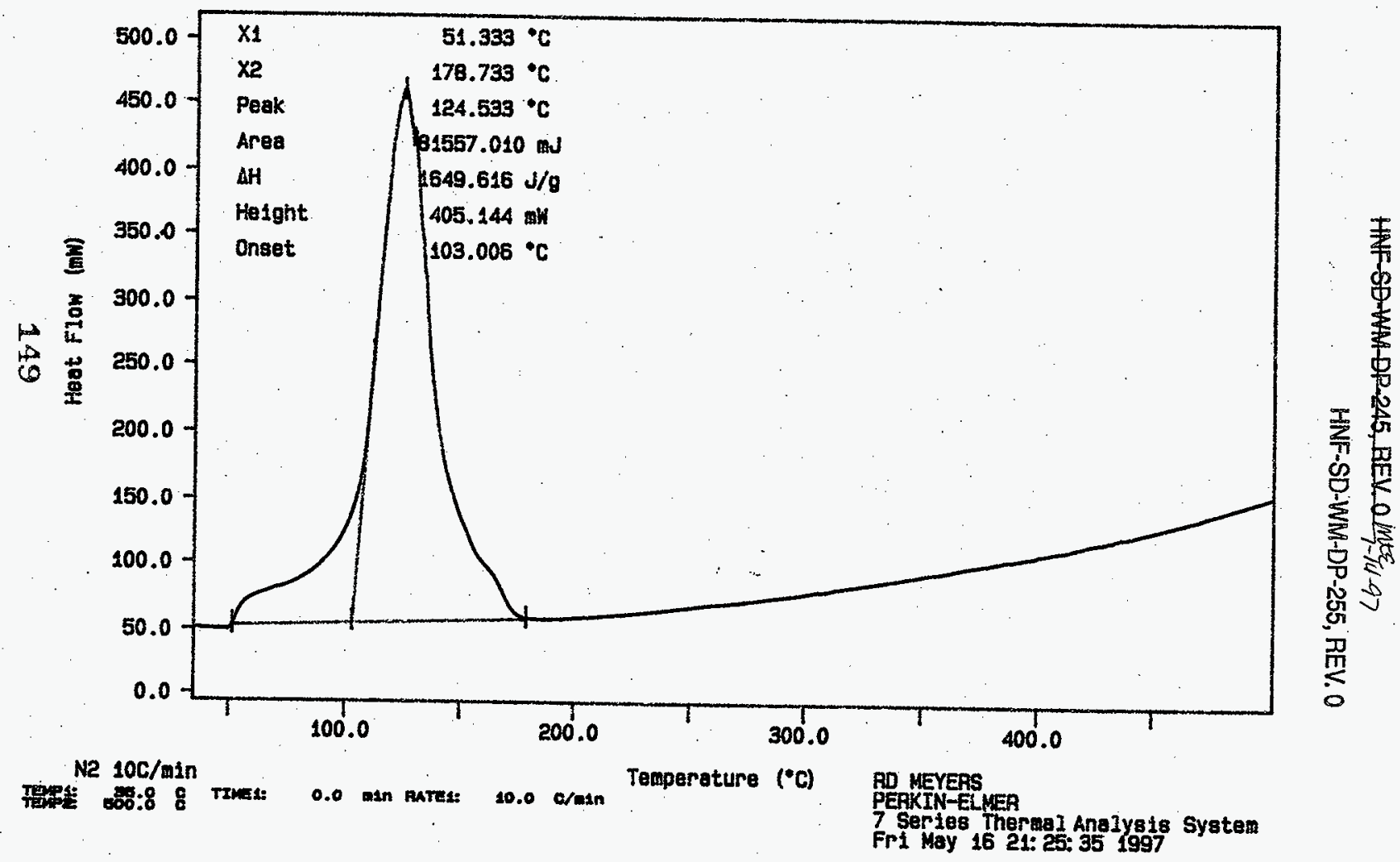


Curve 1: DSC

File info: saM051603 Frd May i6 22: 16:35 1997

Sample Weight: 29.680 mg

S97T000605

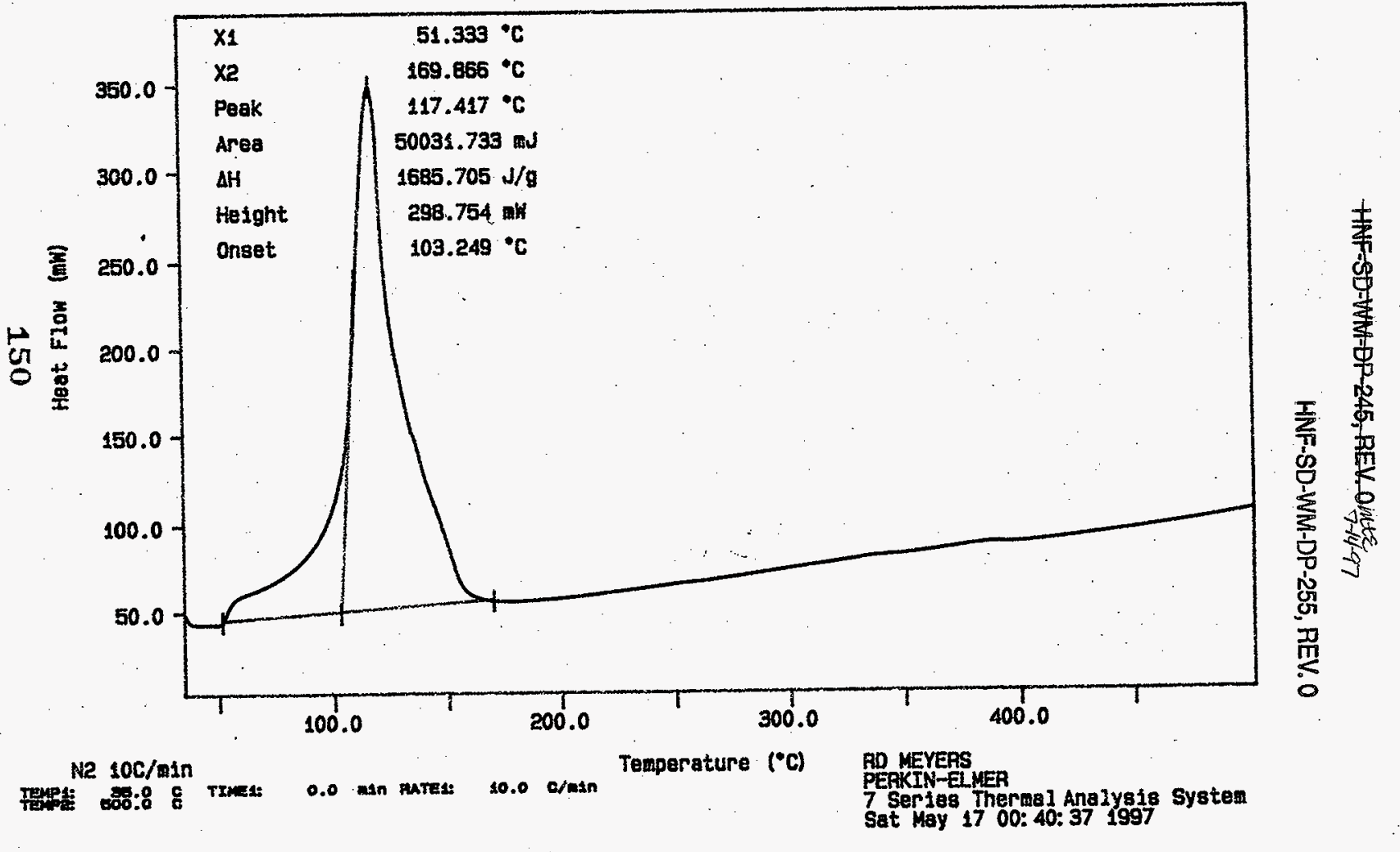


Curve 1: DSC

File info: SAM055701 Sat May 17 01: 41:32 1997

Samplo Ketght: 35.030

ag

s97T000606Dup

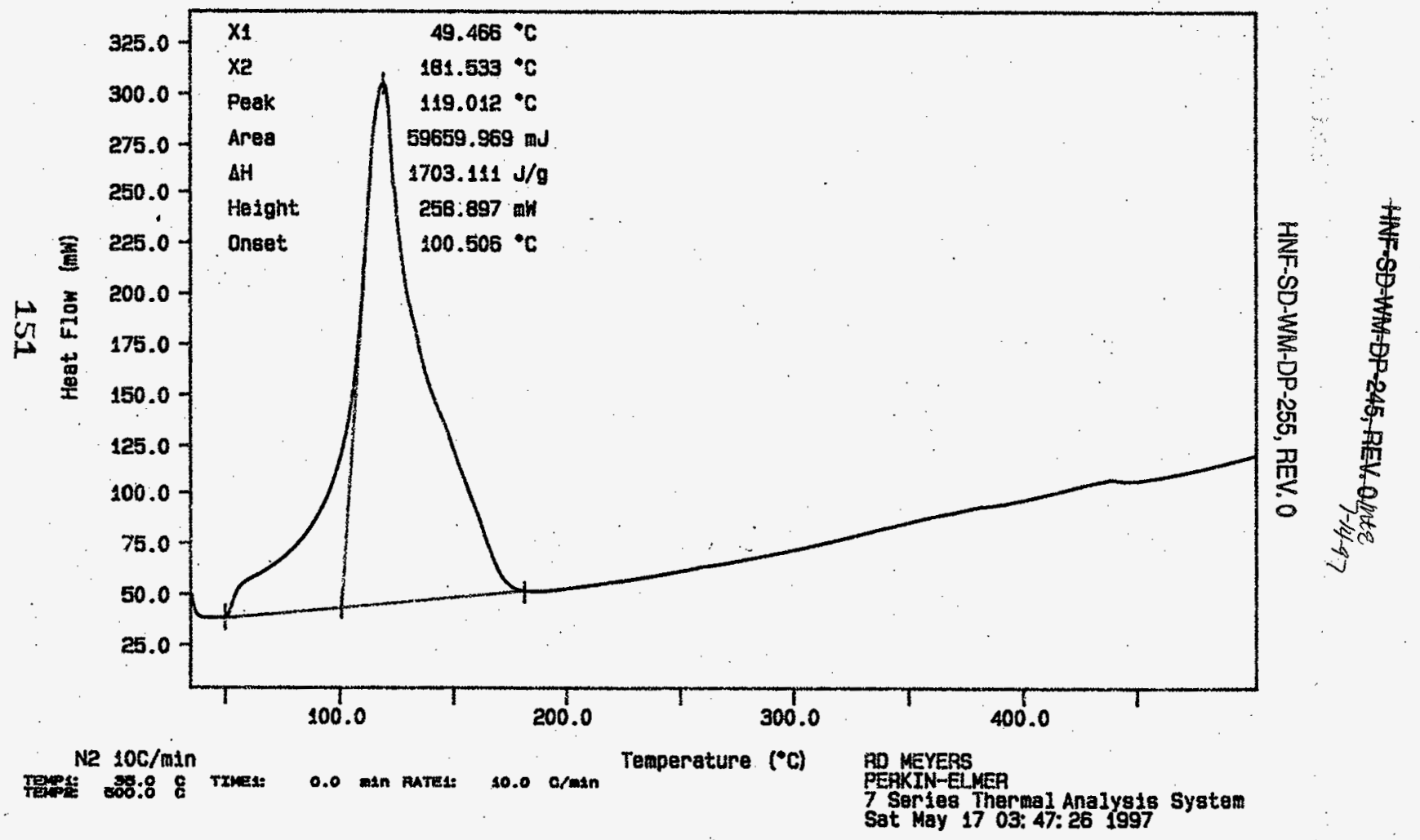




\section{LABCORE Data Entry Template for Worklist\# 17833}

Page: 1

Analyst: $\quad$ RDM Instrument: DSCO $3 \cdot$ Book\# $12 N 14 B$

Method: LA-514-114 Rev/Mod D-O

Worklist Comment: T204, DSC-01 Run under nitrogen. skm

\begin{tabular}{|c|c|c|c|c|c|c|c|c|c|c|}
\hline \multirow[t]{2}{*}{ GROUP } & PROJECT & S TYPE & SAMPLE\# & RA & $\ldots+\ldots$ & MATRIX & ACTUAL & FOUND & $\mathrm{DL}$ & UNIT \\
\hline & & I STD & & & DSC-03 & SOLID & 28.45 & 26.89 & $N / A$ & Joules/g \\
\hline 97000261 & $T-204$ & 2 SAMPLE & s97T000607 & 0 & DSC-03 & SOLID & N/A & 0 & & Joules/g \\
\hline 97000261 & $T-204$ & 3 DUP & \$97T000607 & 0 & DSC-03 & SOL ID & 0 & 0 & $N / A$ & Joules/g \\
\hline 97000261 & $T-204$ & 4 SAMPLE & s97T000608 & 0 & DSC-03 & SOLID. & $\mathrm{N} / \mathrm{A}$ & 0 & & Joules/g \\
\hline 97000261 & $\underset{i+}{T-204}$ & 5 DUP & 5977000608 & 0 & DSC-03 & SOLID & 0 & 0 & N/A & Joules/g \\
\hline
\end{tabular}

\section{Final page for worklist \# 17833}

\section{See Attached for Signature
Analyst Signature
Validated $5 / 22 / 97 \& 8$ hachelor \\ See Attached for fignature
Analyst Signature
Validated $5 / 22 / 97 \& 8$ machelor}

HNF-SD-WM-DP-255, REV, 0

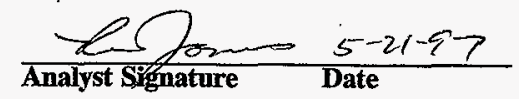

Data Entry Comments:

Units shown for $Q C$ (SPK \& STD) may not reflect the actual units. $D L=$ Detection Limit, $S=$ Worklist Slot Number, $R=$ Replicate Number, $A=$ Aliquot Code. 


\section{LABCORE Data Entry Template for Worklist\# 17833}

Page: 1

Analyst: $\quad$ Instrument: DSC0.3 Book \# $12 N 14 B$

Method: LA-514-113 Rev/Mod_D - D

Worklist Comment: T204, DSC-01 Run under nitrogen. skm

HNF-SD-WVM-DP-255, REV. 0

\begin{tabular}{|c|c|c|c|c|c|c|c|c|c|c|}
\hline \multirow[t]{2}{*}{ GROUP } & \multirow[t]{2}{*}{ PROJECT } & \multirow{2}{*}{$\begin{array}{l}\text { S TYPE } \\
\text { \& STD }\end{array}$} & \multirow[t]{2}{*}{ SAMPLE\# } & \multicolumn{2}{|c|}{ R A - - IEST-N.... } & \multirow{2}{*}{$\begin{array}{l}\text { MATRIX } \\
\text { SOLID }\end{array}$} & \multirow[t]{2}{*}{ ACTUAL } & \multirow[t]{2}{*}{ FOUND } & \multirow[b]{2}{*}{$N / A$} & \multirow{2}{*}{$\begin{array}{l}\text { UNIT } \\
\text { Joules/g }\end{array}$} \\
\hline & & & & & DSC-01 & & & & & \\
\hline 97000261 & $\mathrm{~T}-204$ & 2 SAMPLE & s977000607 & 0 & DSC-01 & SOLID & N/A & & & Joules/g \\
\hline 97000261 & $T-204$ & 3 DUP & s97T000607 & 0 & DSE-01 & SOLID & & & N/A & Joules/9 \\
\hline 97000261 & $T-204$ & 4 SAMPLE & S97T000608 & 0 & DSC-01 & SOLID. & N/A. & & & Joules/g \\
\hline 97000261 & $T-204$ & 5 DUP & S97T000608 & 0 & DSC-01 & SOLID & & & N/A & Joules/g \\
\hline
\end{tabular}

\section{Final page for worklist \# 17833}

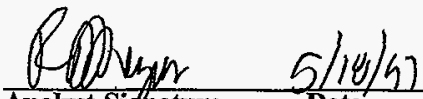

Analyst Sighature

\section{Date}

Analyst Signature

Date

Data Entry Comments:
Samples rau on DSC-03 Q $5 / 20 / 97$

Units shown for $Q C$ (SPK \& STD) may not reflect the actual units. $D L=$ Detection Limit, $S=$ Worklist Slot Number, $R=$ Replicate Number, $A=$ Aliquot Code. 
Curve 1: DSC

F11e info: TEA05170? Sat Mey 17 20:57: 321997

Sample Neight: 8.850

12N14-B

SIGNATURE BELOW REPAESENTS CHEMICAL TECHNOLOGIST/CHEMIST THAT COMPLEIEDNEEAFIED THE CALLBPATIONAANALYSIS ON PAGES 154 TO 158

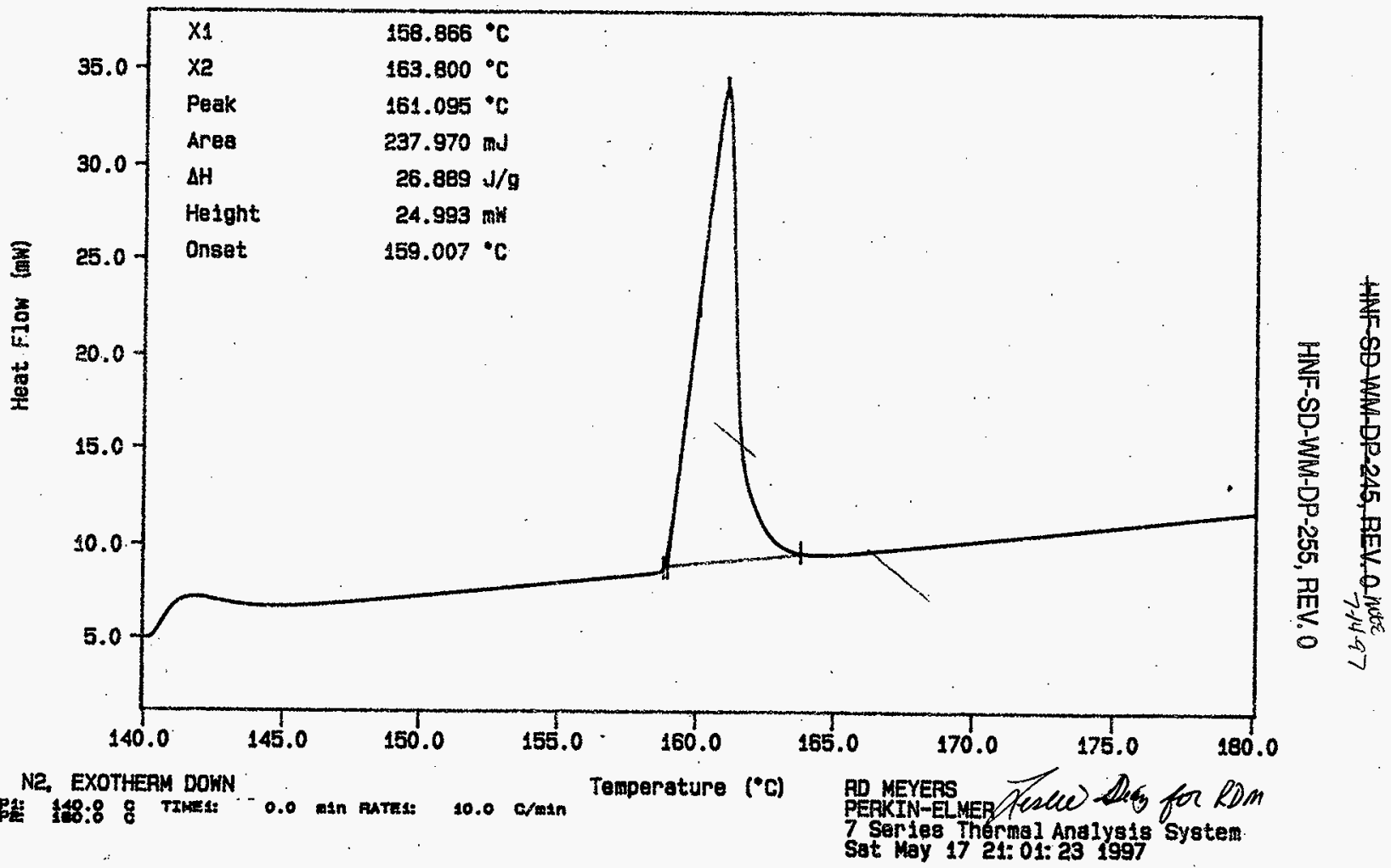


HAF-SO-WA-QP-245, PEV.

ite $7-14-77$

HNF-SD-WM-DP-255, REV. 0

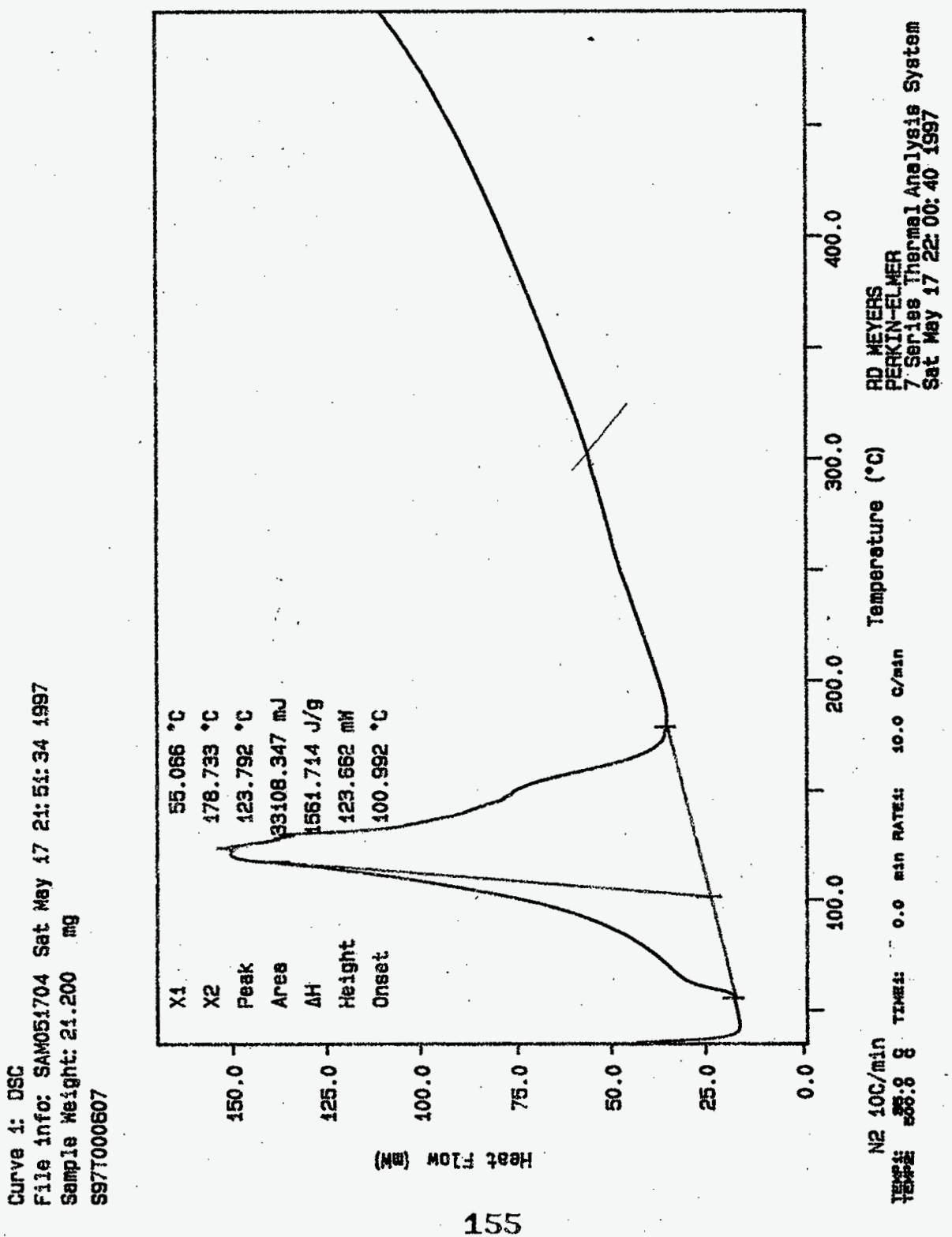


curve 1: DSC

Fila info: SAM051705 Sat May 17 22: 54:09 1997

Sample Height: $23.250 \mathrm{mg}$

S97T000607DUP

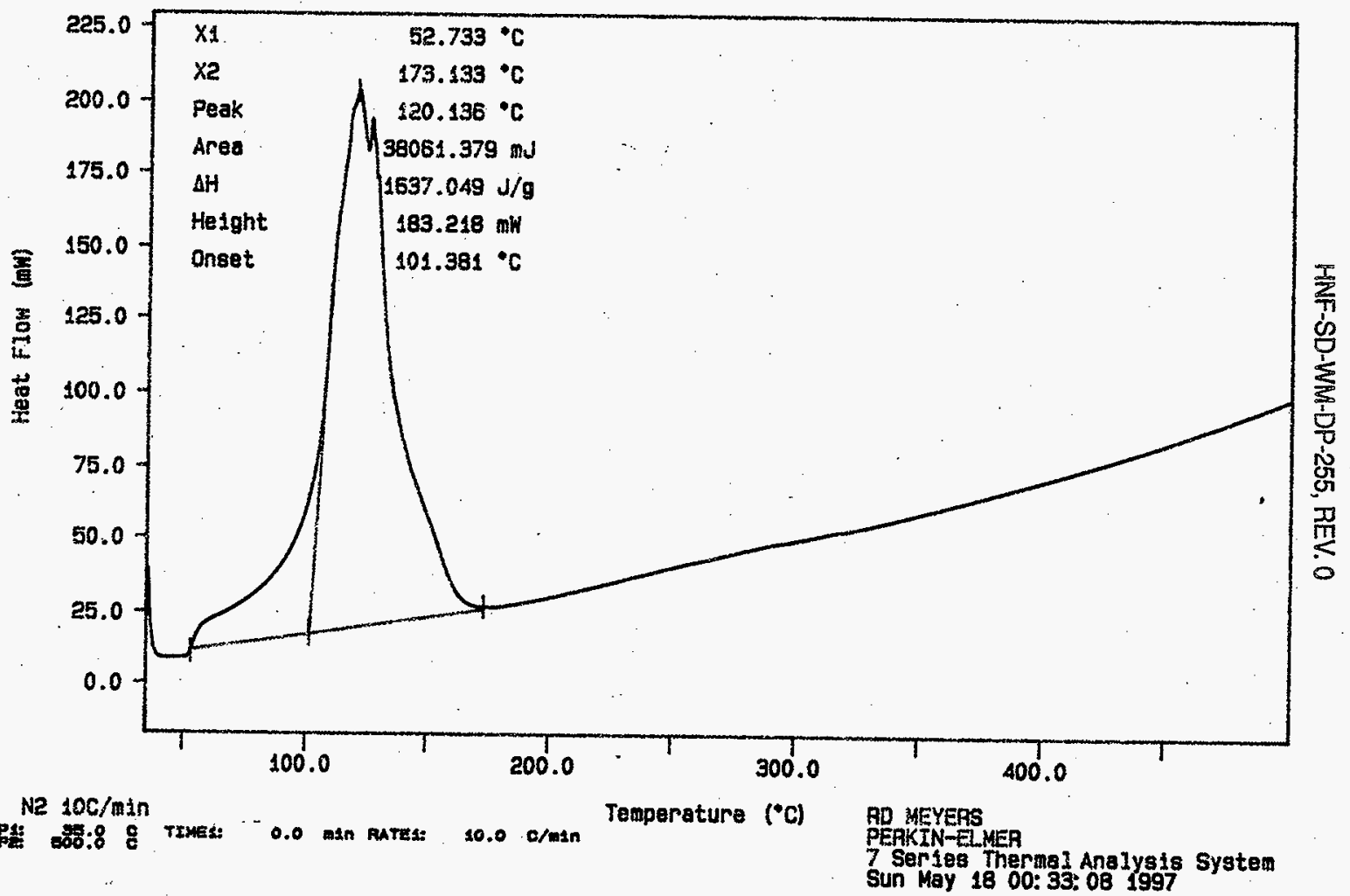


Curve 1: DSC

File info: SAM051801 Sun May 18 01: 25: 431997

Sanple Wetght: 26.950 .ng

\$97T000608

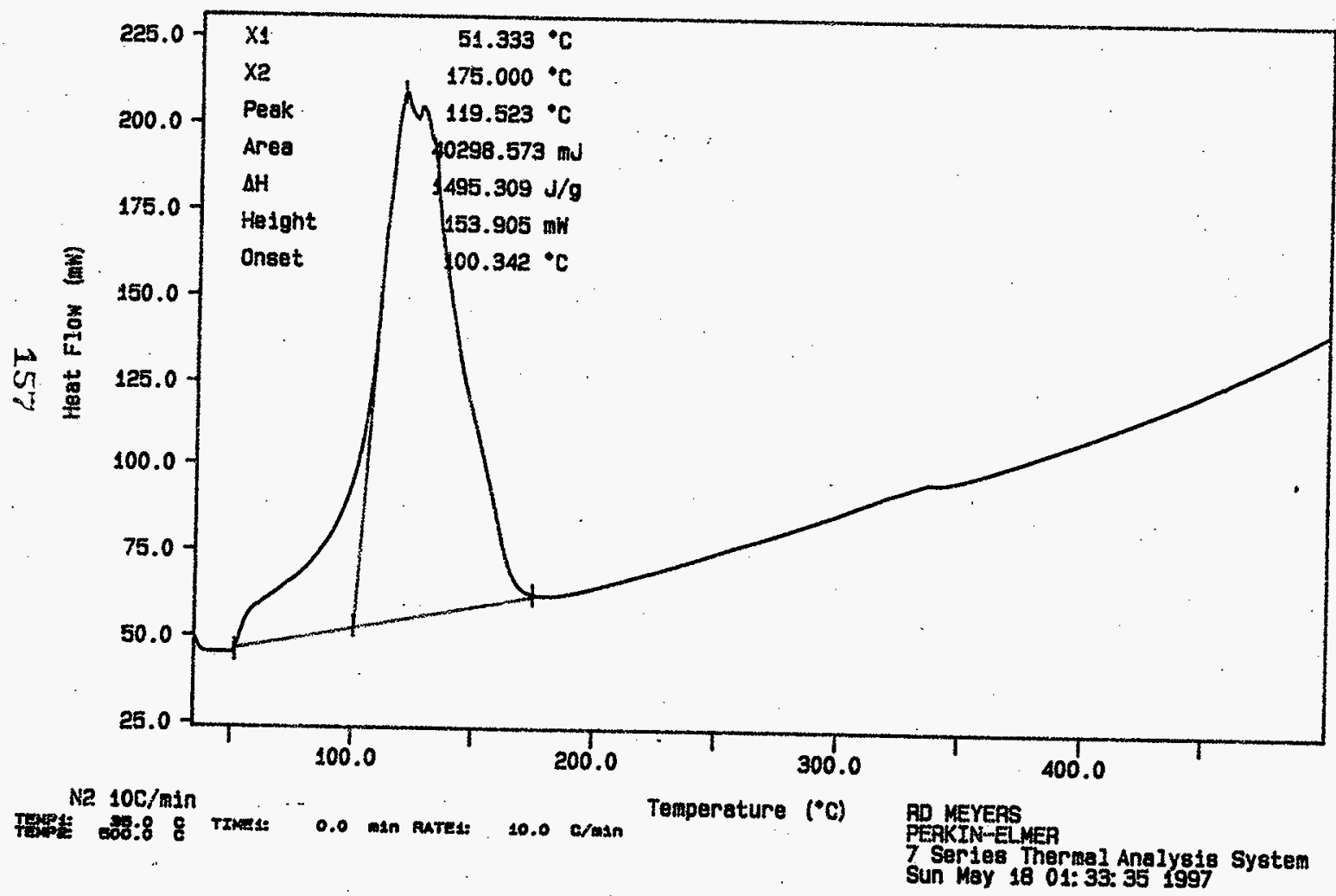


HAF-SO-WAM-DP-245, REV.0 Ont

HNF-SD-WRI-DP-255, REV. 0

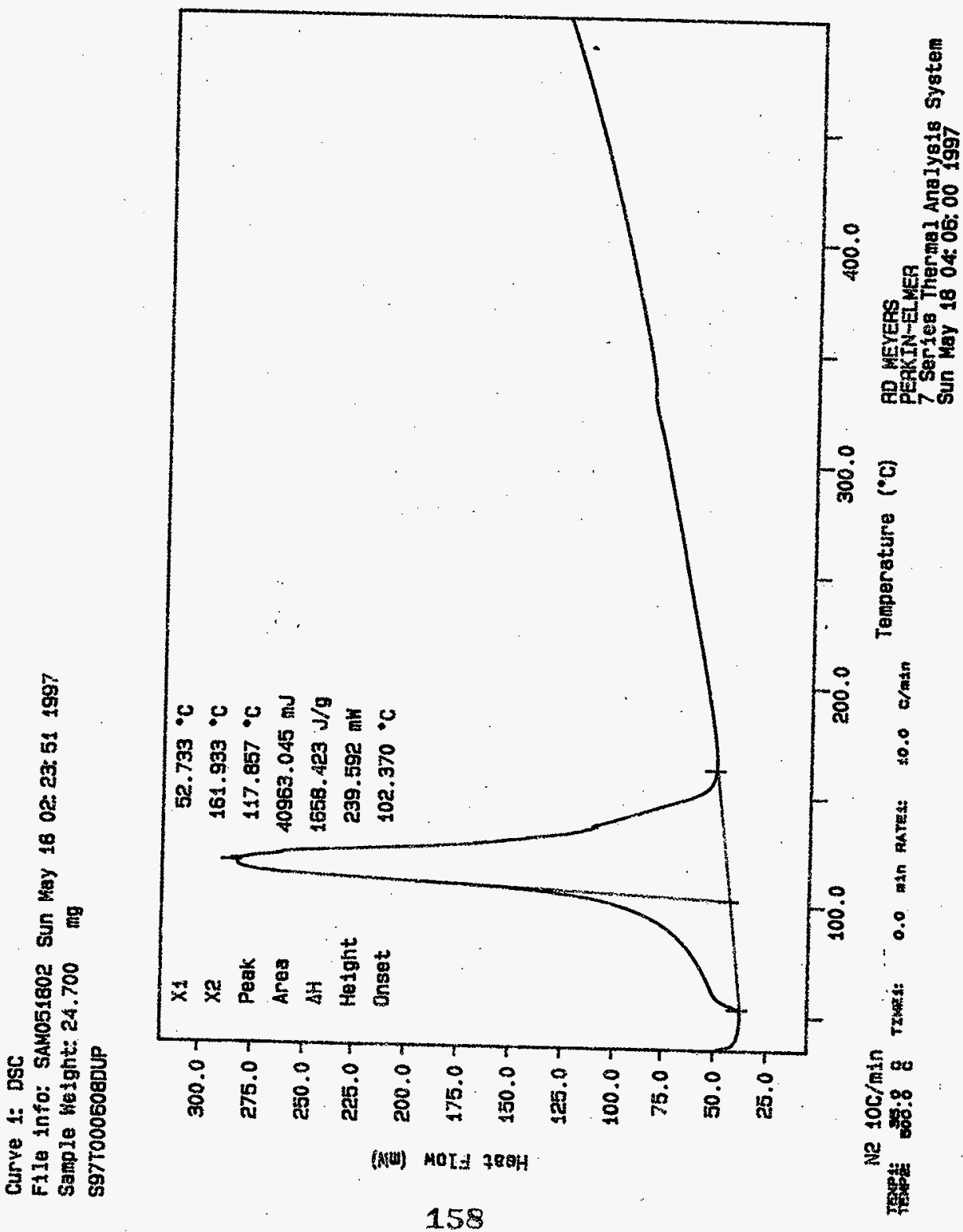




\section{LABCORE Data Entry Template for Worklist\# 17834}

Analyst: IDS Instrument: DSCO 3 Book \# $12 N 14$ B

Method: LA-514-114 Rev/Mod D-O

HNF-SD-WR-DP-255, REV. 0

Worklist Comment: T204, DSC-01 Run under nitrogen. skm

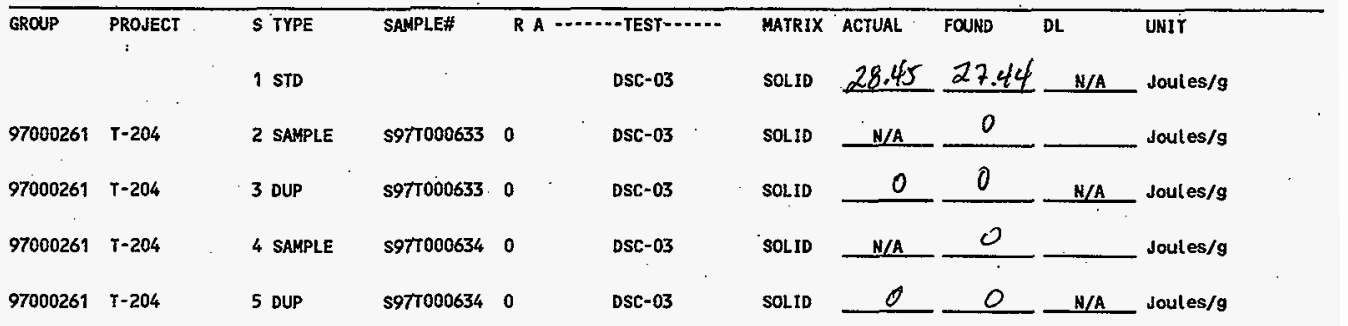

\section{Final page for worklist \# 17834}

See Affached. for Signature

Analyst Signature Date

validated 5/30/978rachelor

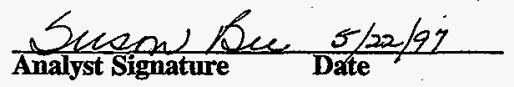

Data Entry Comments:

Units shown for $Q C$ (SPK \& STD) may not reflect the actual units. $D L=$ Detection Limit, $S=$ Worklist Slot Number, $R=$ Replicate Number, $A=$ Aliquot Code. 


\section{LABCORE Data Entry Template for Worklist\# 17834}

Analyst: $\quad J d 5$ Instrument: DSC0 3 Book\# $12 N / 4 B$

Method: LA-514-113 Rev/Mod D-O

HNF-SD-WM-DP-255, REV.0

Worklist Comment: T204, DSC-01 Run under nitrogen. skm

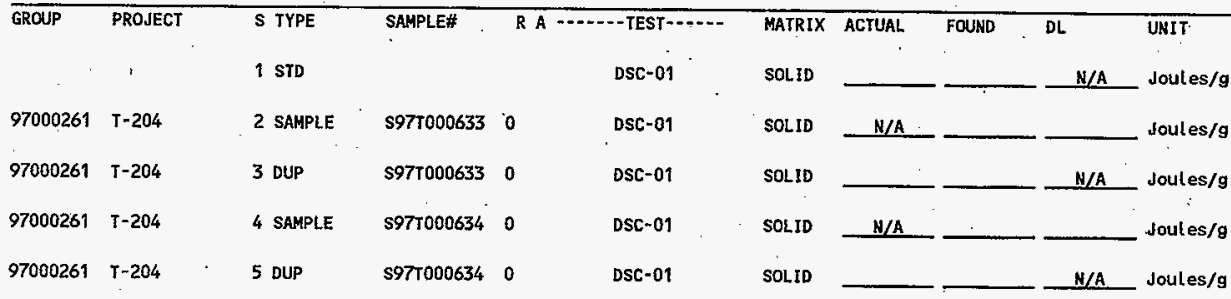

\section{Final page for worklist \# 17834}

5 SD $5 / 19 / 67$

Analyst Signatire|. Date

\section{Analyst Signatire Date}

Data Entry Comments:

Units shown for $Q C$ (SPK \& STD) moy not reflect the actual units. $D L=$ Detection Limit, $S=$ Worklist Slot Number, $R=$ Replicate Number, $A=$ Aliquot Code. 


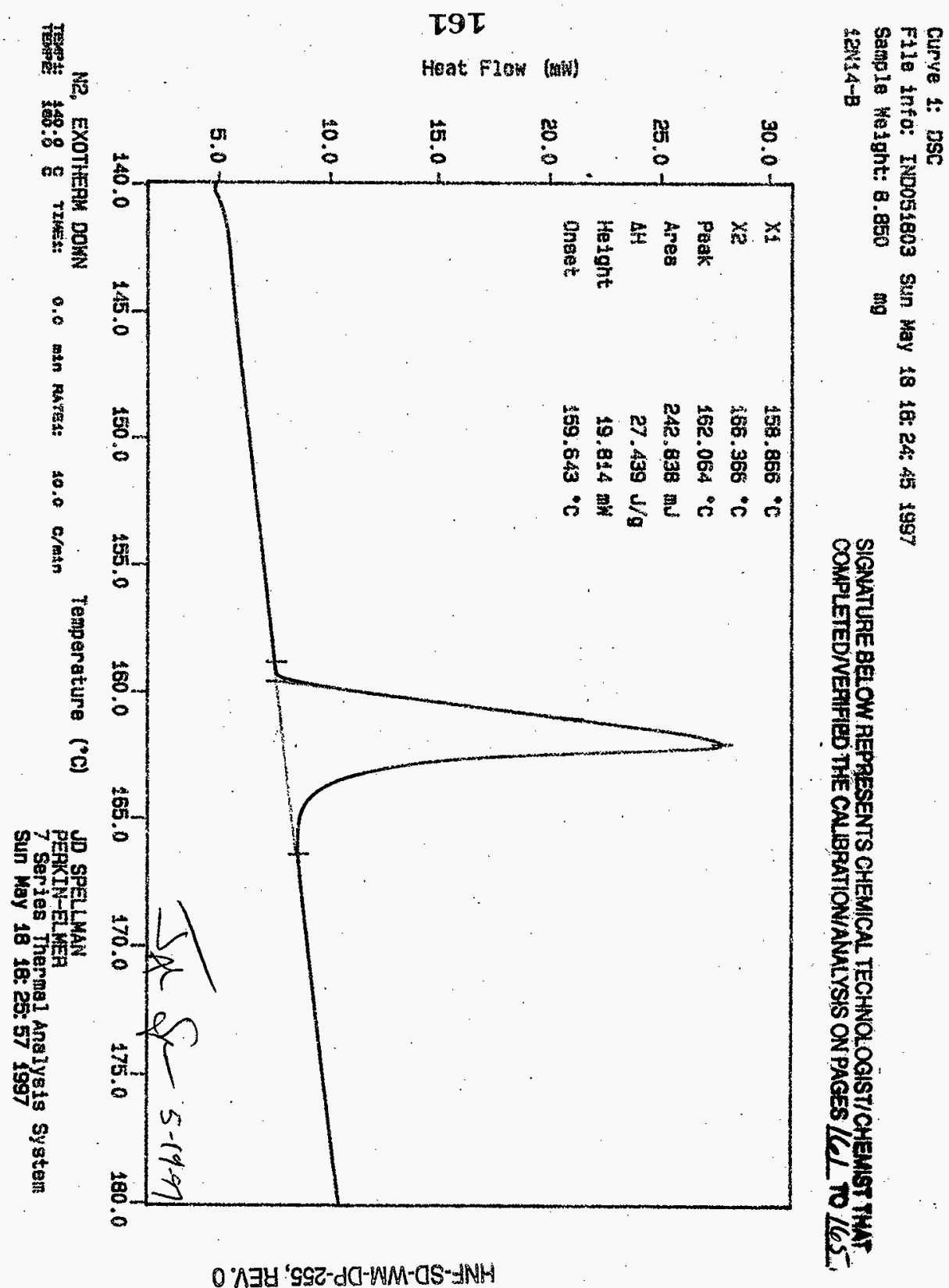

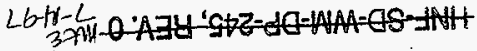




\section{Curve 1: DSC}

File info: SAM051803 Sun May 18 19: 35: 451997

Semple weight: 32.790

ang

\section{s97T000633}

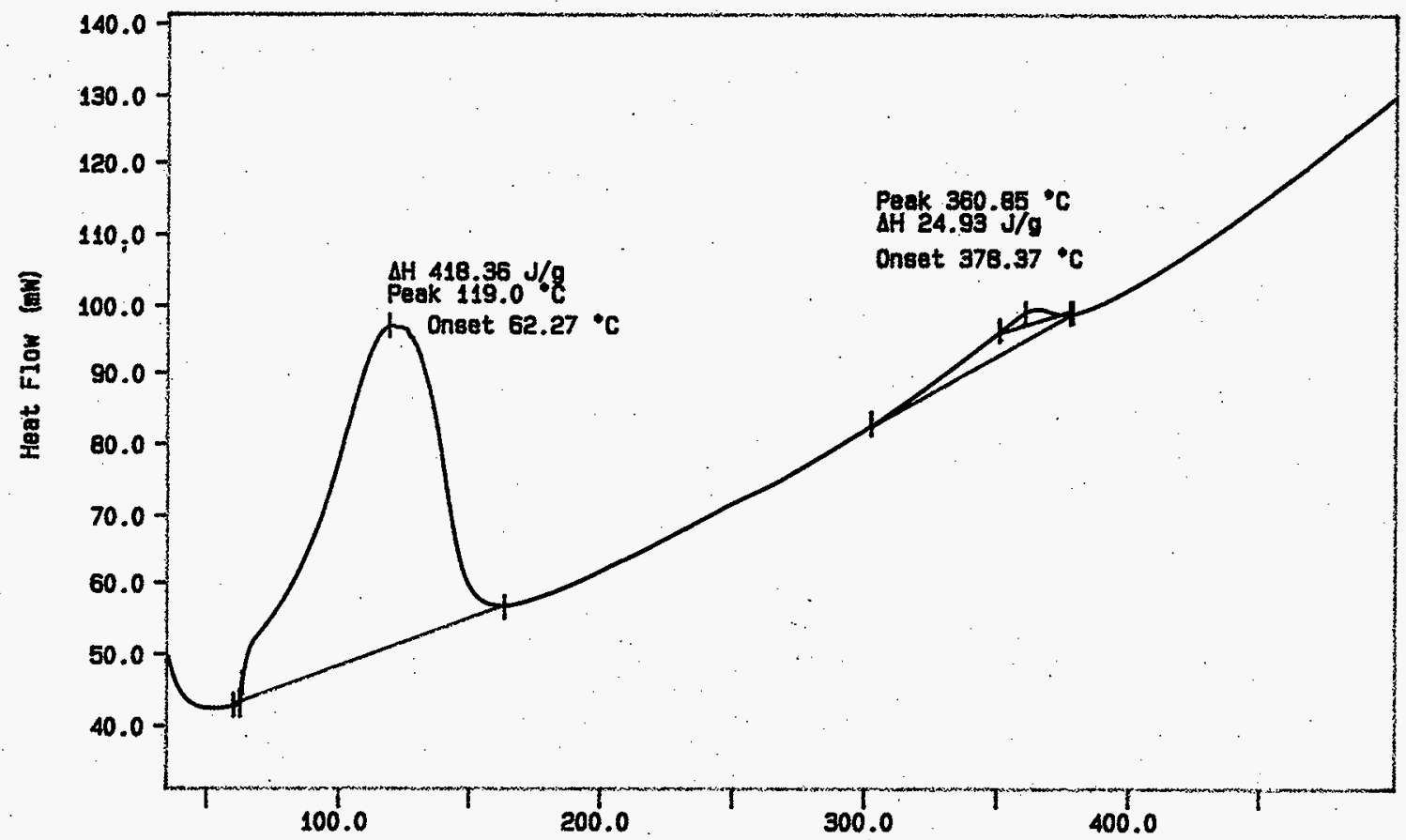

N2 10C/min

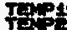

30.88
Trans:
Temperature ("C)

JD SPELLMAN

PERKIN-ELMER 7 Series thermel Anolysis System
Hed May 21 08: 12 : 24 1997 
Curve 1: DSC

File info: SAM051804 Sun May 18 22: 17:01 1997

Sample Weight: 38.950

mg

S97T000633DUP

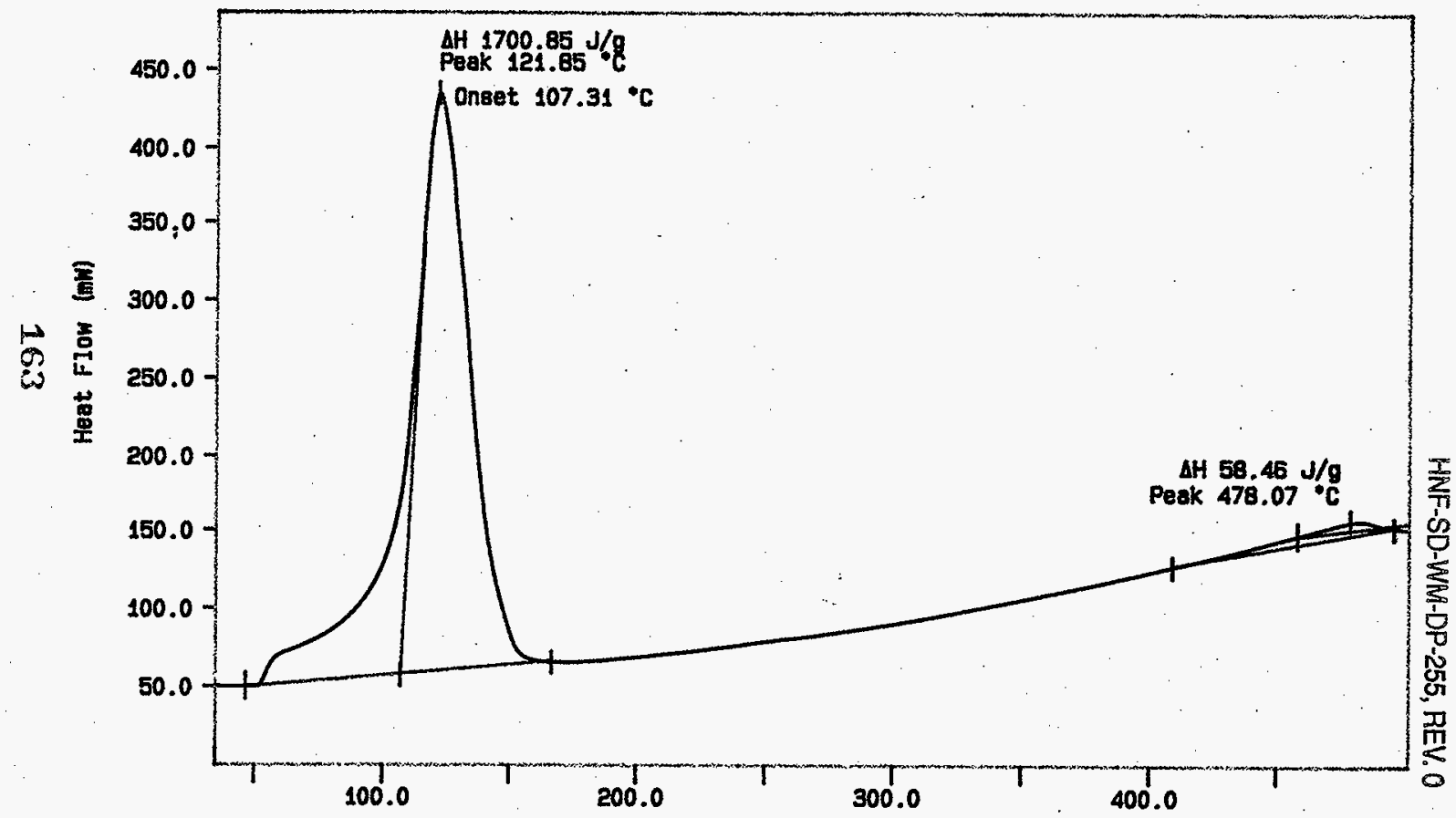

N2 $10 \mathrm{C} / \mathrm{min}$ 
Curve 1: DSC

File info: SAM051805 Mon May 19 01: 23: 381997

Sample Weight: 35.880

$\mathrm{mg}$

S97T000634

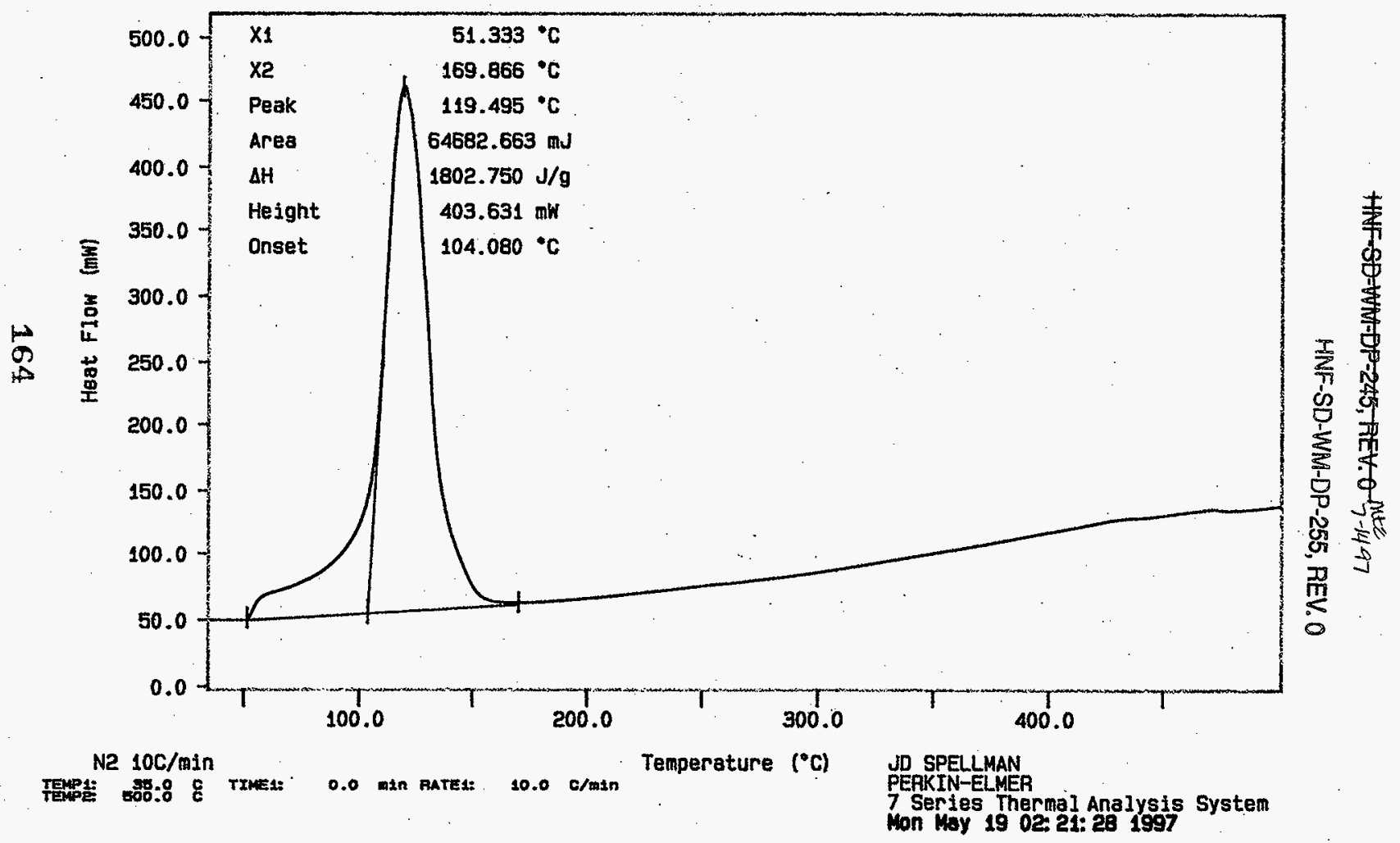




\section{LABCORE Data Entry Template for Worklist\# 17835}

Analyst: PJM Instrument: DSCO 3 Book\# 12 N $14 B$

Method: LA-514-114 Rev/Mod D - O

HNF-SD-WM-DP-255, REV.O

Worklist Comment: T204, DSC-01 Run under nitrogen. skm.

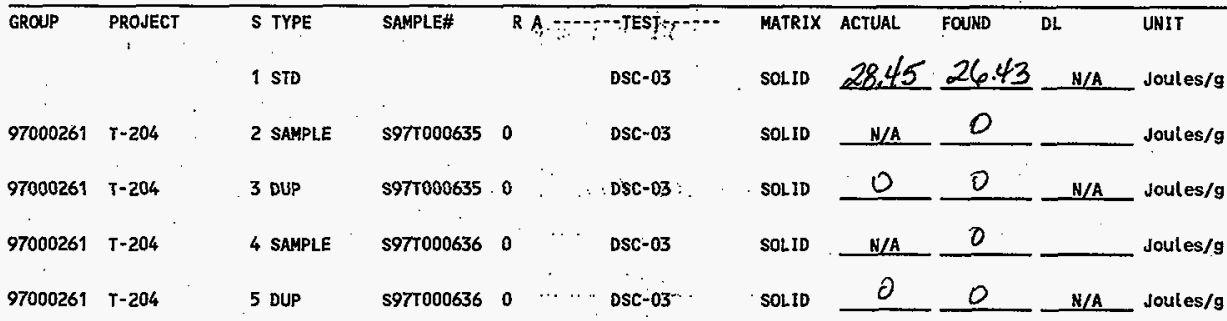

\section{Final page for worklist \# 17835}

See Attached for Signature Validated 6/2/97\% Pracheloz

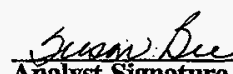

$5 / 22 / 97$ Analyst Signature
Bate

Data Entry Comments:

Units shown for $Q C$ (SPK \& STD) may not reflect the actiual inits. $R=$ Replicate Number, $A=$ Aliquot Code. 


\section{LABCORE Data Entry Template for Worklist\# 17835}

Analyst: $\operatorname{Asm}$ Instrument: DSC0 3 Book $12 N / 4 B$

Method: LA-514-113 Rev/Mod D-O

HNF-SD-WM-DP-255, REV.O

Worklist Comment: T204, DSC-01 Run under nitrogen. skm

\begin{tabular}{|c|c|c|c|c|c|c|c|c|c|c|}
\hline \multirow[t]{2}{*}{ GROUP } & \multirow{2}{*}{$\begin{array}{l}\text { PROJECT } \\
\quad:\end{array}$} & \multirow{2}{*}{$\begin{array}{l}\text { S TYPE } \\
1 \text { STD }\end{array}$} & \multirow[t]{2}{*}{ SAMPLE\# } & \multicolumn{2}{|c|}{ R A } & \multirow{2}{*}{$\begin{array}{l}\text { MATRIX } \\
\text { SOLID }\end{array}$} & \multirow{2}{*}{ ACTUAL } & \multirow[t]{2}{*}{ FOUND } & \multirow{2}{*}{$\begin{array}{l}D L \\
N / A \\
N\end{array}$} & \multirow{2}{*}{$\begin{array}{l}\text { UNIT } \\
\text { Joules/g }\end{array}$} \\
\hline & & & & & DSC-01 & & & & & \\
\hline 97000261 & $T-204$ & 2 SAMPLE & s97t000635 & 0. & $\mathrm{DSC}-01$ & SOLID & $N / A$ & - & & Joules/g \\
\hline 97000261 & $T-204$ & 3 DUP & S97T000635 & 0 & DSC-01 & SOL ID & & & N/A & Joules/g \\
\hline $9700026 t$ & $T-204$ & 4 SAMPLE & s971000636 & 0 & DSC-01 & SOL ID & $\mathrm{N} / \mathrm{A}$ & & & Joules/g \\
\hline 97000261 & $T-204$ & 5 DUP & \$97T000636 & 0 & DSC-01 & SOL ID & & & N/A & Joules/g \\
\hline
\end{tabular}

Final page for worklist \# 17835

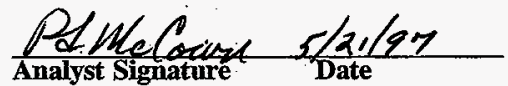

Analyst Signature Date

Data Entry Comments:
Ran samples on DSC-03. P3 $5 / 21 / 97$

Units shown for QC (SPK \& STD) may not reflect the actual units. $D L=$ Detection Limit, $S=$ Worklist Slot Number, $R=$ Replicate Number, $A=$ Aliquot Code. 
Curve 1: DSC

F1le info: InD052003 Tue May 20 22: 56: 391997

Sample Neight: 6.140

mg

12N14-B

SIGNATURE BELOW REPRESENTS CHEMICAL TECHNOLOGIST/CHEMIST THAF COMFLETEDNERIFIED THE CALBBRATIONANALYSIS ON PACES 16870172

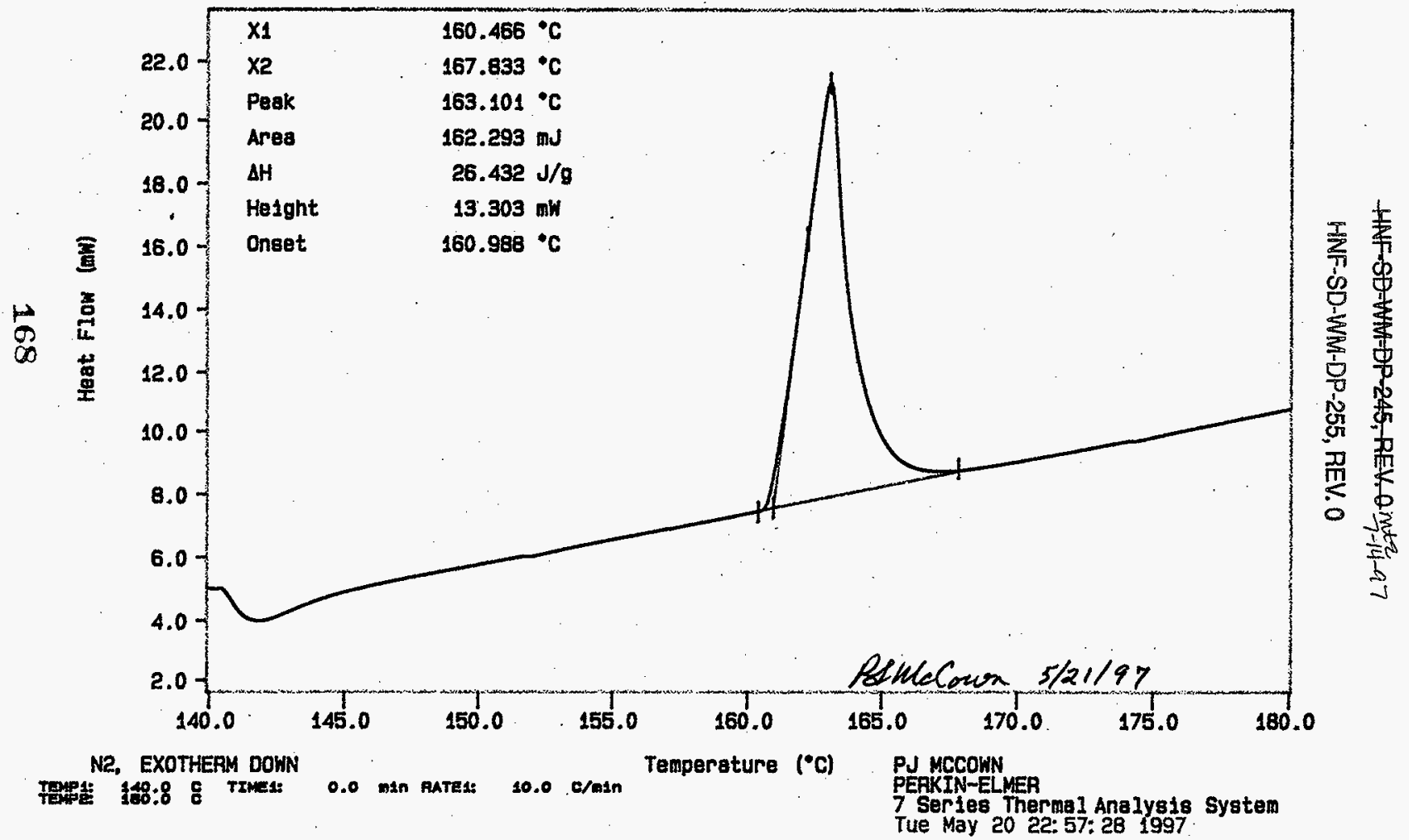


Curve 1: DSC

File Info: 5AM052101 Wed May 21 00:55: 231997

Sample Weight: 14.940 ing

597T000635

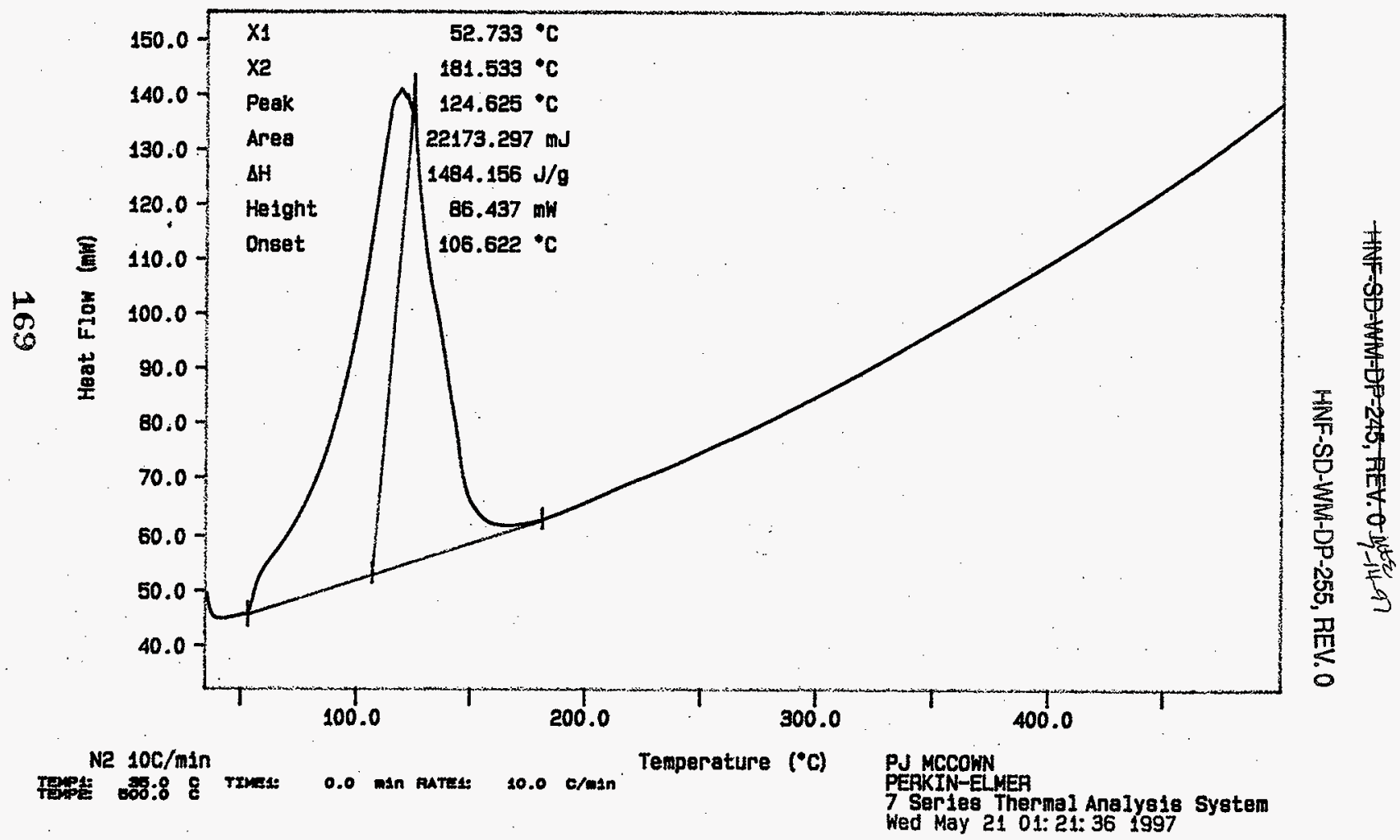


Curve 1: DSC

F11e info: SAM052102. Wed May 21 02: 16: 131997

Sample Neight: 13.860

mg

S97T000635 DUP

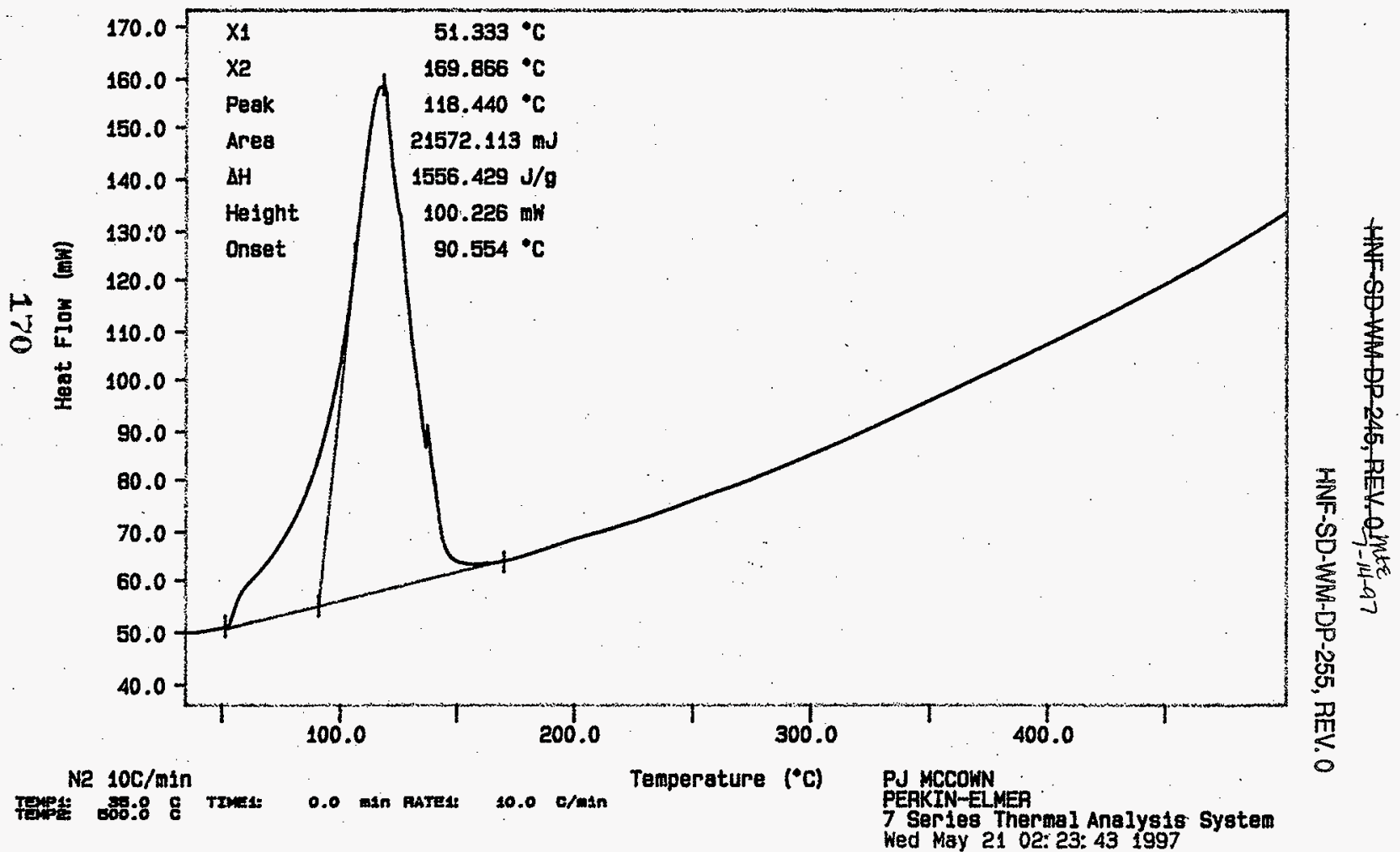


Curve 1: DSC

File info: SAM052103 Wed May 21 03: 25: 031997

Sample Veight: 11.940 mg

5977000635

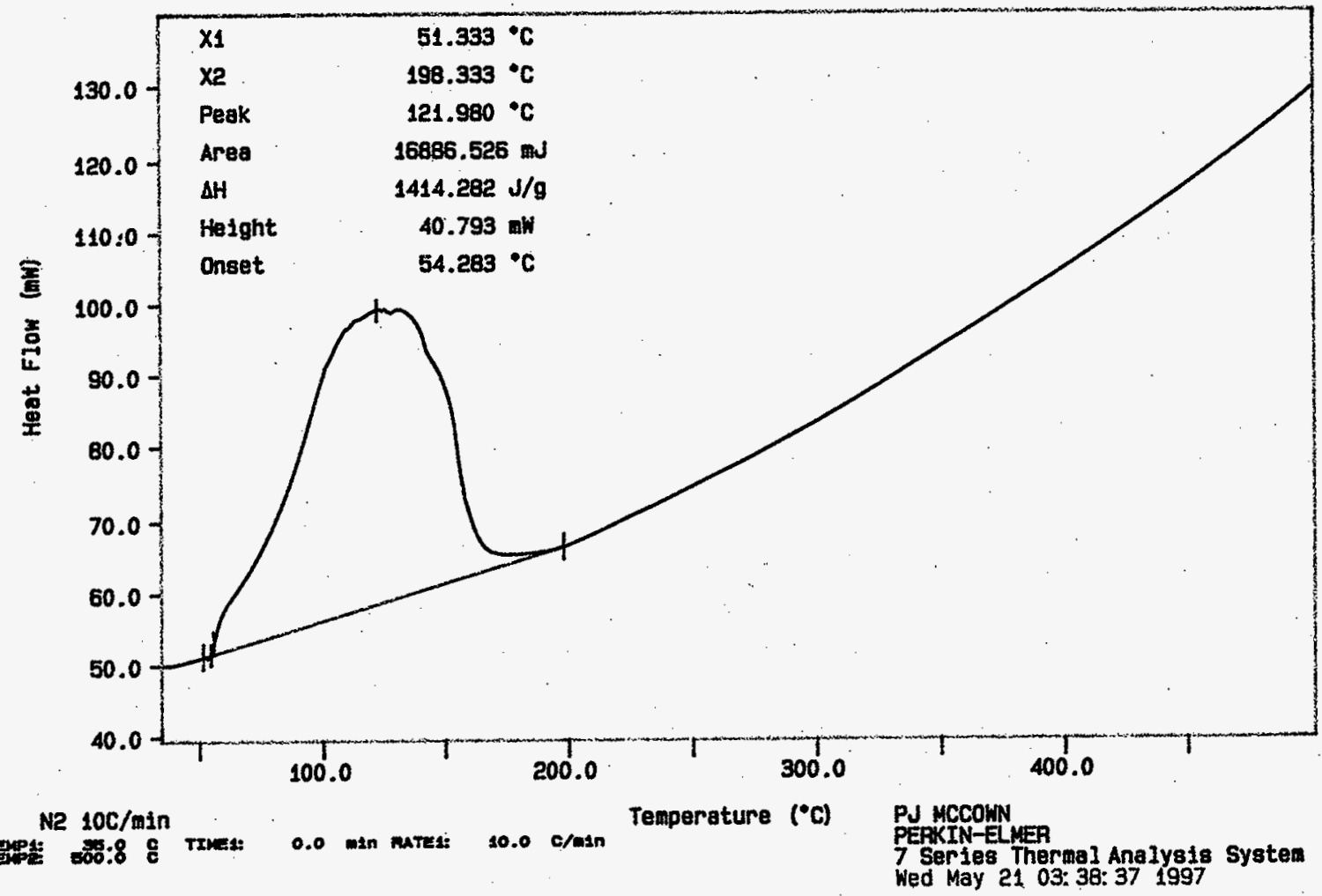

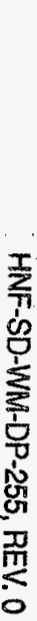


HAF-SD-WA-DP-245, REV.O 7 - $14-97$

HNF-SD-WM-DP-255, REV. 0

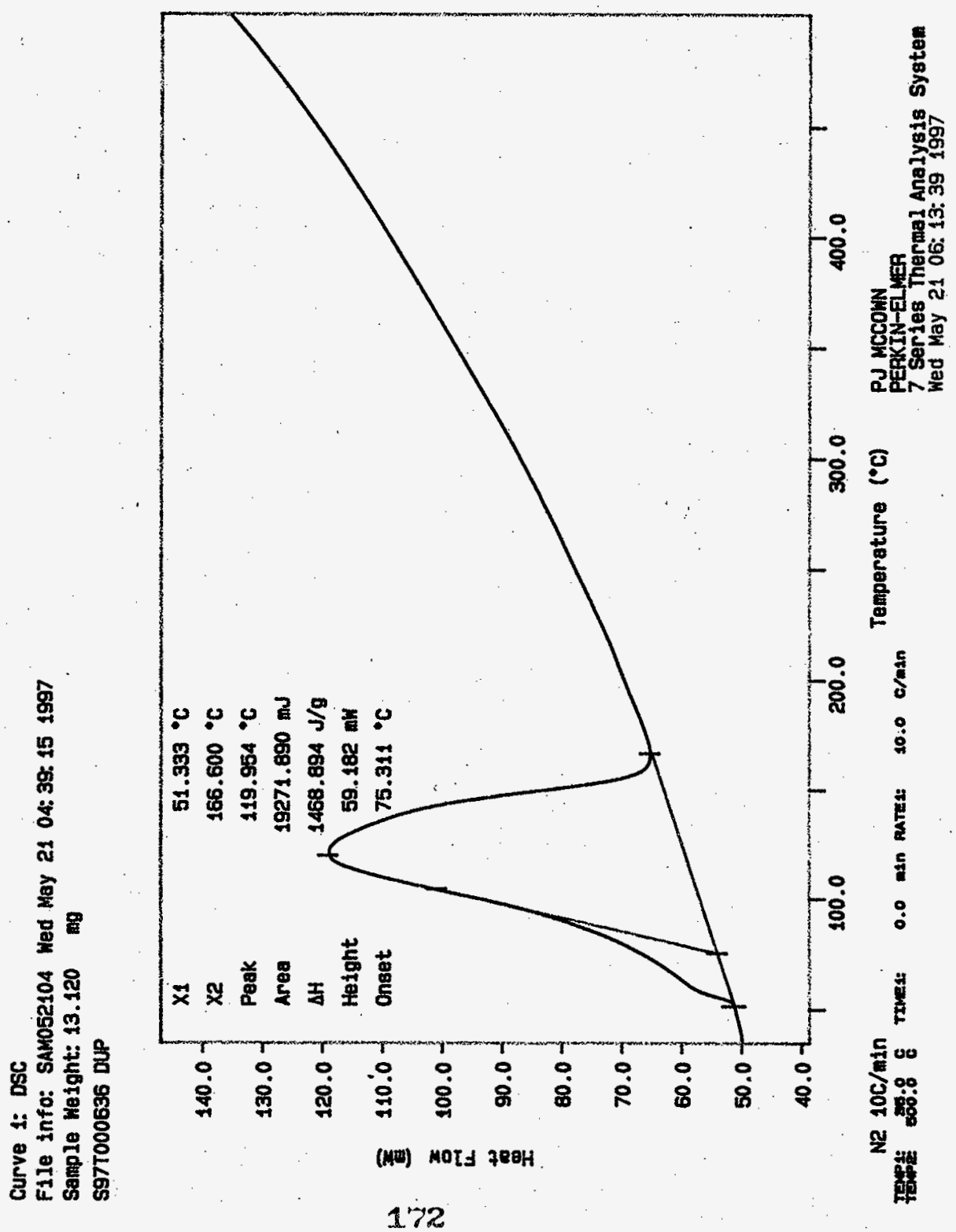




\section{LABCORE Data Entry Template for Worklist\# 17836}

Page: 1

\section{Analyst: $A D P$ Instrument: $D S C 0 \quad 3 \quad$ Book \# $12 N 14 B$}

Method: LA-514-114 Rev/Mod DO

Worklist Comment: T204, DSC-01 Run under nitrogen. skm HNF-SD-WM-DP-255, REV. 0

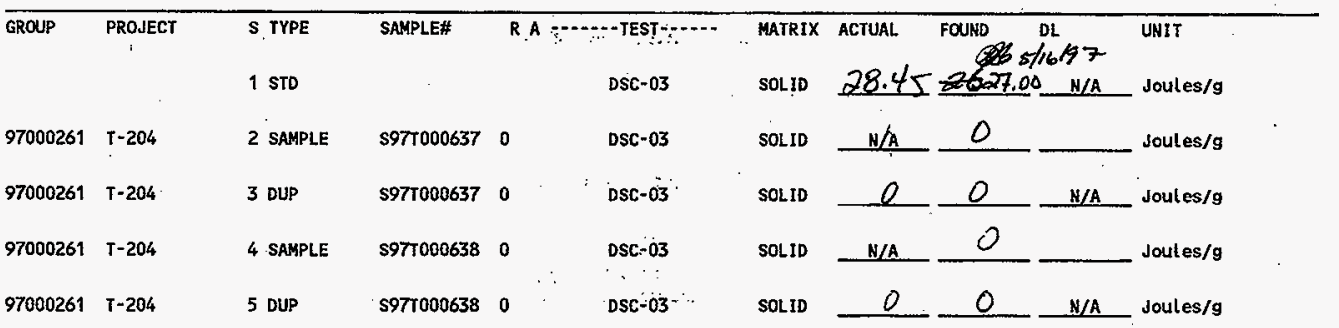

\section{Final page for worklist \# 17836}

See Attached for Signature

Analyst Signature Date

Validated sko/978 hachelor

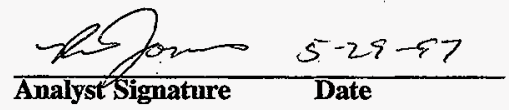

Data Entry Comments:

Units shown for QC (SPK \& STD) may not reflect the actual units. $D L=$ Detection Limit, $S=$ Worklist Slot Number, $R=$ Replicate Number, $A=$ Aliquot Code. 


\section{LABCORE Data Entry Template for. Worklist\# 17836}

Analyst: ADP Instrument: DSC0 3 Book \# 12N14B

Method: LA-514-113 Rev/Mod $D-O$

Worklist Comment: T204, DSC-01 Run under nitrogen. skm

\begin{tabular}{|c|c|c|c|c|c|c|c|c|c|c|}
\hline \multirow[t]{2}{*}{ GROIP } & \multirow{2}{*}{ PROJECT } & \multirow{2}{*}{$\begin{array}{l}\text { S TYPE } \\
1 \text { STD }\end{array}$} & \multirow[t]{2}{*}{ SAMPLE\# } & \multicolumn{2}{|c|}{$\begin{array}{lll}\text { R A } & \cdots & \text { - } \\
\end{array}$} & \multirow{2}{*}{$\begin{array}{l}\text { MATRIX } \\
\text { SOLID }\end{array}$} & \multirow{2}{*}{\multicolumn{2}{|c|}{$\begin{array}{l}\text { ACTUAL FOUND } \\
\text { 25/NGP }\end{array}$}} & \multirow{2}{*}{$\mathrm{DL}$} & \multirow{2}{*}{$\begin{array}{l}\text { UNIT } \\
\text { Joules/g }\end{array}$} \\
\hline & & & & & DSC-01 & & & & & \\
\hline 97000261 & $T-204$ & 2 SAMPLE & s97T000637 & 0 & DSC-01 & SOLID & N/A & & & Joules/g \\
\hline 97000261 & $T-204$ & 3 DUP & \$97r000637 & 0 & DSC-01 & SOLID & & & & Joules $/ g$ \\
\hline 97000261 & $r-204$ & 4 SAMPLE & s971000638 & 0 & DSC-01 & SOLID & N/A & & & Joules/g \\
\hline 97000261 & $\mathrm{r}-204$ & 5 DUP & s97T000638 & 0 & DSC-01 & SOLID & & & & Joules/g \\
\hline
\end{tabular}

\section{Final page for worklist \# 17836}

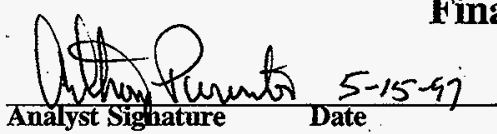

Data Entry Comments:

Samples run on DSC-03. $53 / 16 / 97$

Units shown for $Q C$ (SPK \& STD) may not reflect the actual units. $D L=$ Detection Limit, $S=$ Worklist Slot Number, $R=$ Replicate Number, $A=$ Aliquot Code. 


\section{Curve 1: DSC}

F1le info: IND051501. Thu May 15 02: 36: 271997

Sample Welght: 10.940

ing

12N14-B

SIGNATURE BEIOW REPRESENTS CHEMICAL TECHNOLOGISTICHEMST THAT

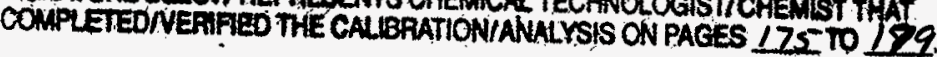

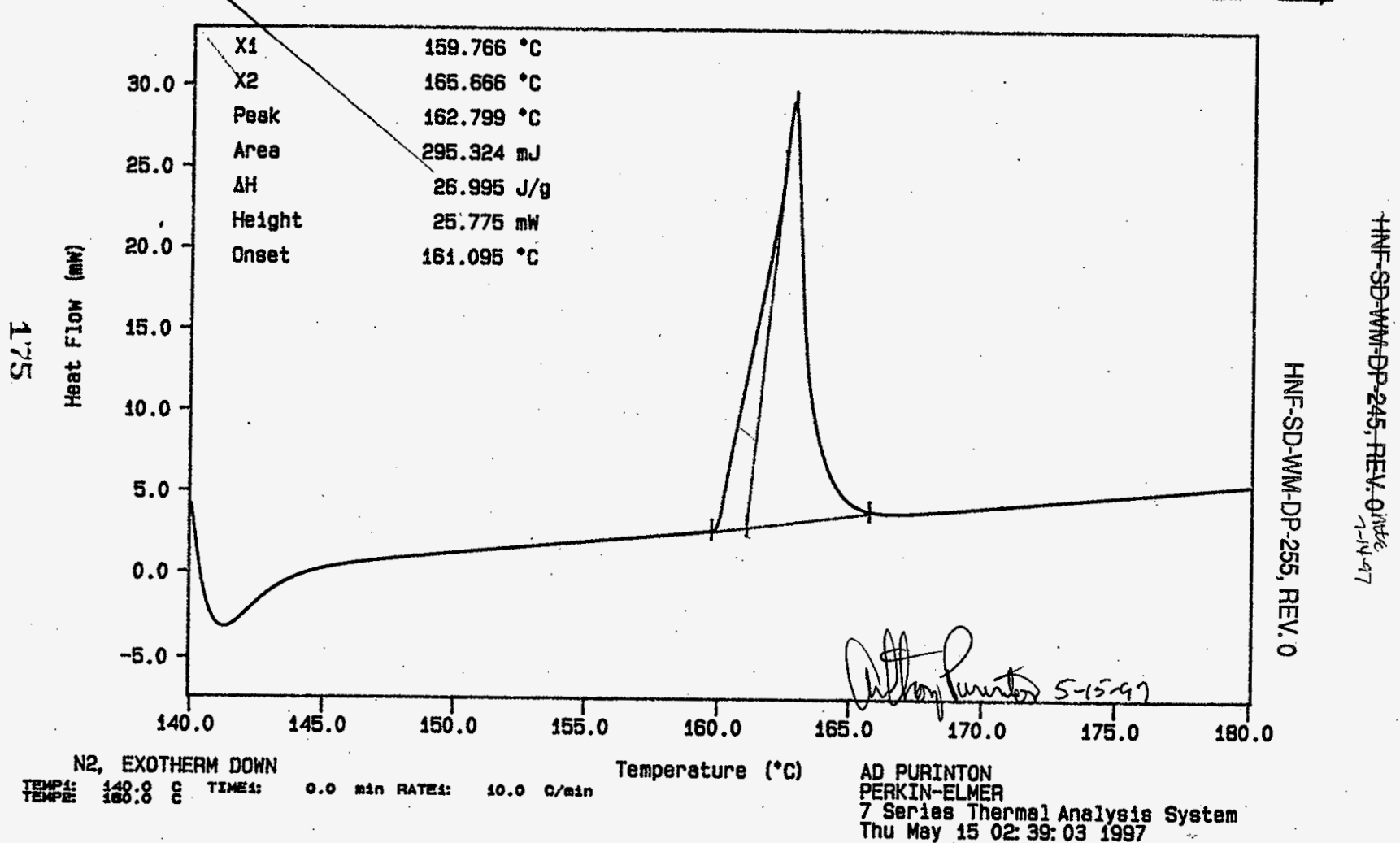


Curve 1: DSC

File info: SAM051501 Thu May 15 03:50: 121997

Sample Weight: $23.620 \mathrm{mg}$

S97T000637 SAM

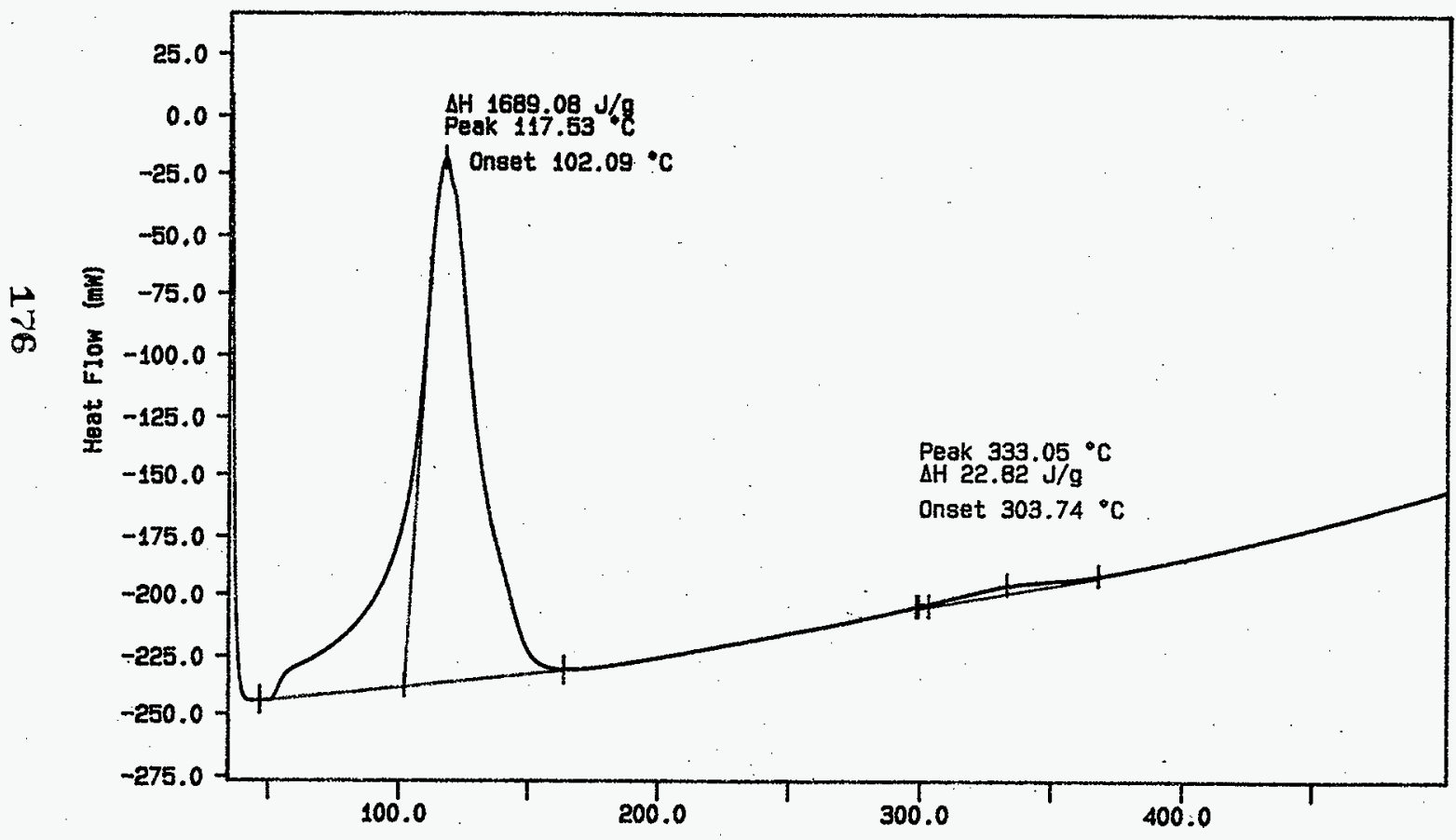

N2 $10 \mathrm{c} / \mathrm{min}$

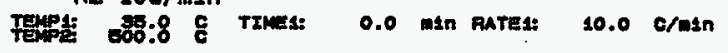

Temperature $(* \mathrm{C})$

AD PURINTON

PERKIN-ELMER

7 Series thermal Analysis System Thu May 15 08: 02: 251997 
Curve 1: DSC

File info: SAM051502 Thu May 15 09: 24: 281997

Sample Welght: 10.870

$597 T 000637$ DUP

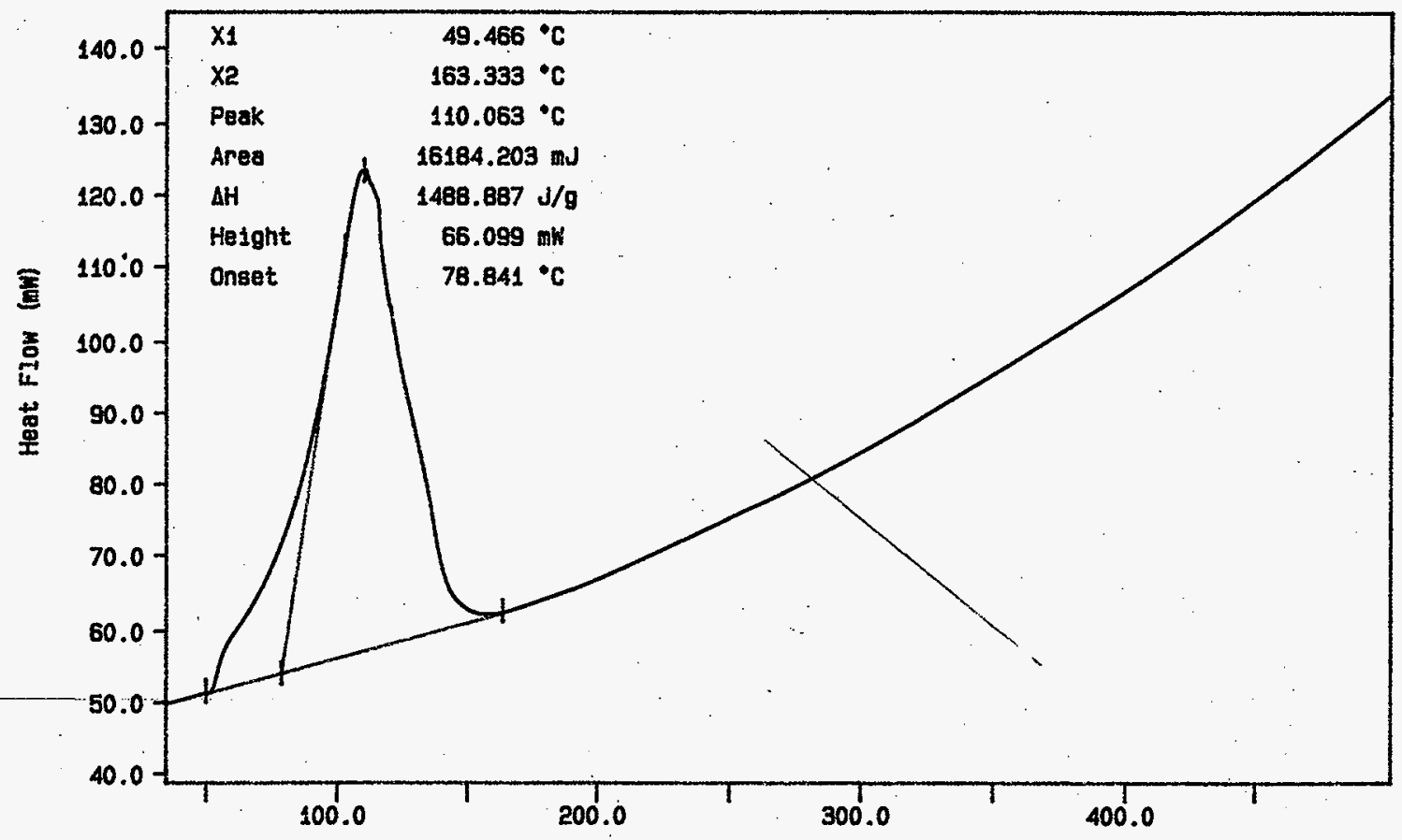

N2 10C/min

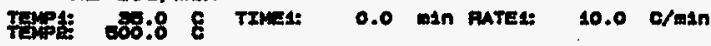

Temperature $\left({ }^{\circ} \mathrm{C}\right)$

KA MONTEITH

7 Series Thermal Analysis System Thu May 15 09: 47: 141997 
Curve 1: DSC

F1le Info: SAM051503 Thu May 15 11: 18: 261997

Sample Weight: 34.860 ang

S97T000638 SAM

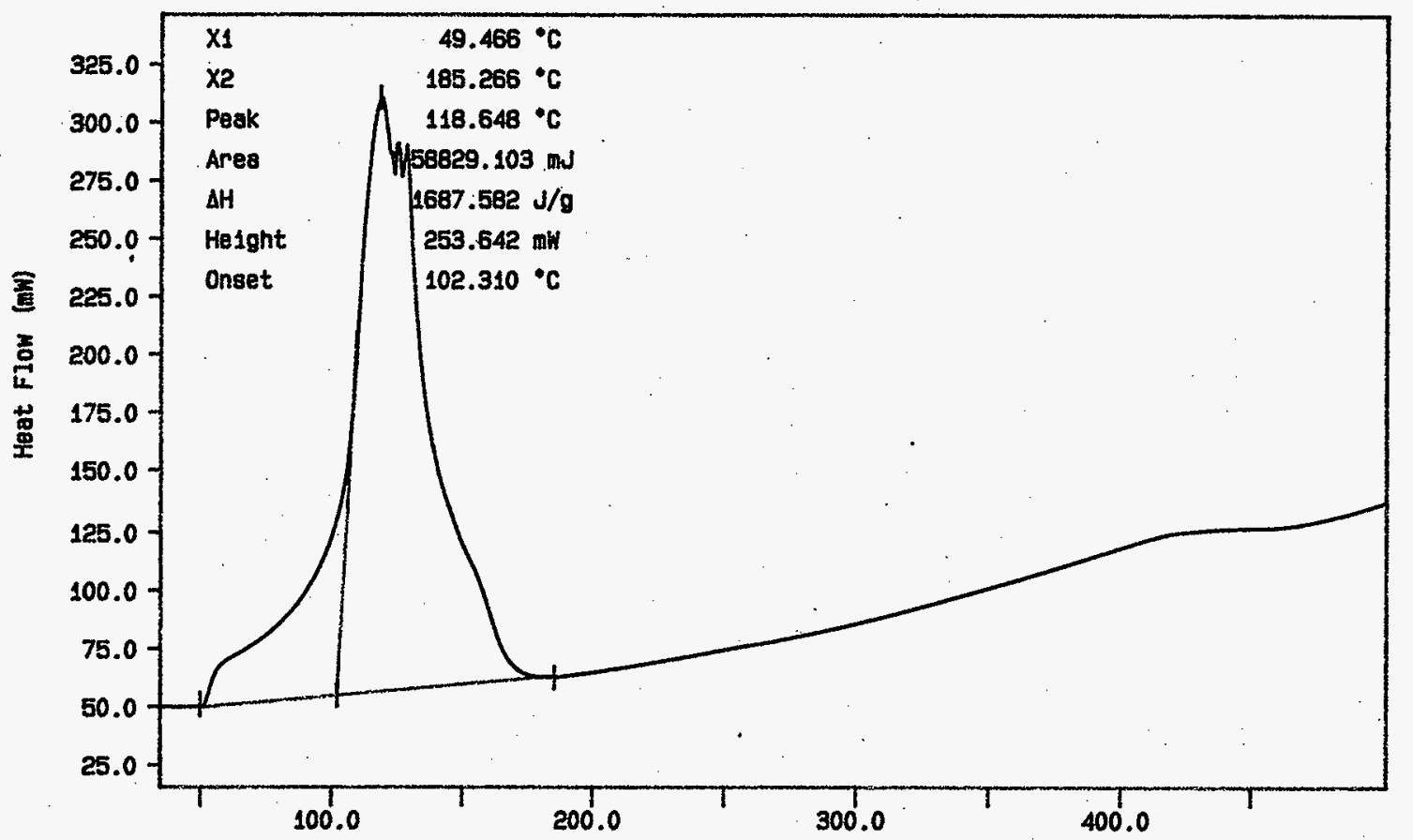

N2 $10 \mathrm{c} / \mathrm{min}$

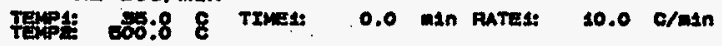

Temperature $\left({ }^{\circ} \mathrm{C}\right)$ KR MONTEITH

PEAKIN-ELMER

7 Sertes Thermal Analysis Systen

Thu May 15 11: 46: 20 1997 
Curve 1: DSC

F1le info: SAM051504 Thu May 15 12:39:54 1997

Sample Weight: 18.250 ing

s97T000638 DUP

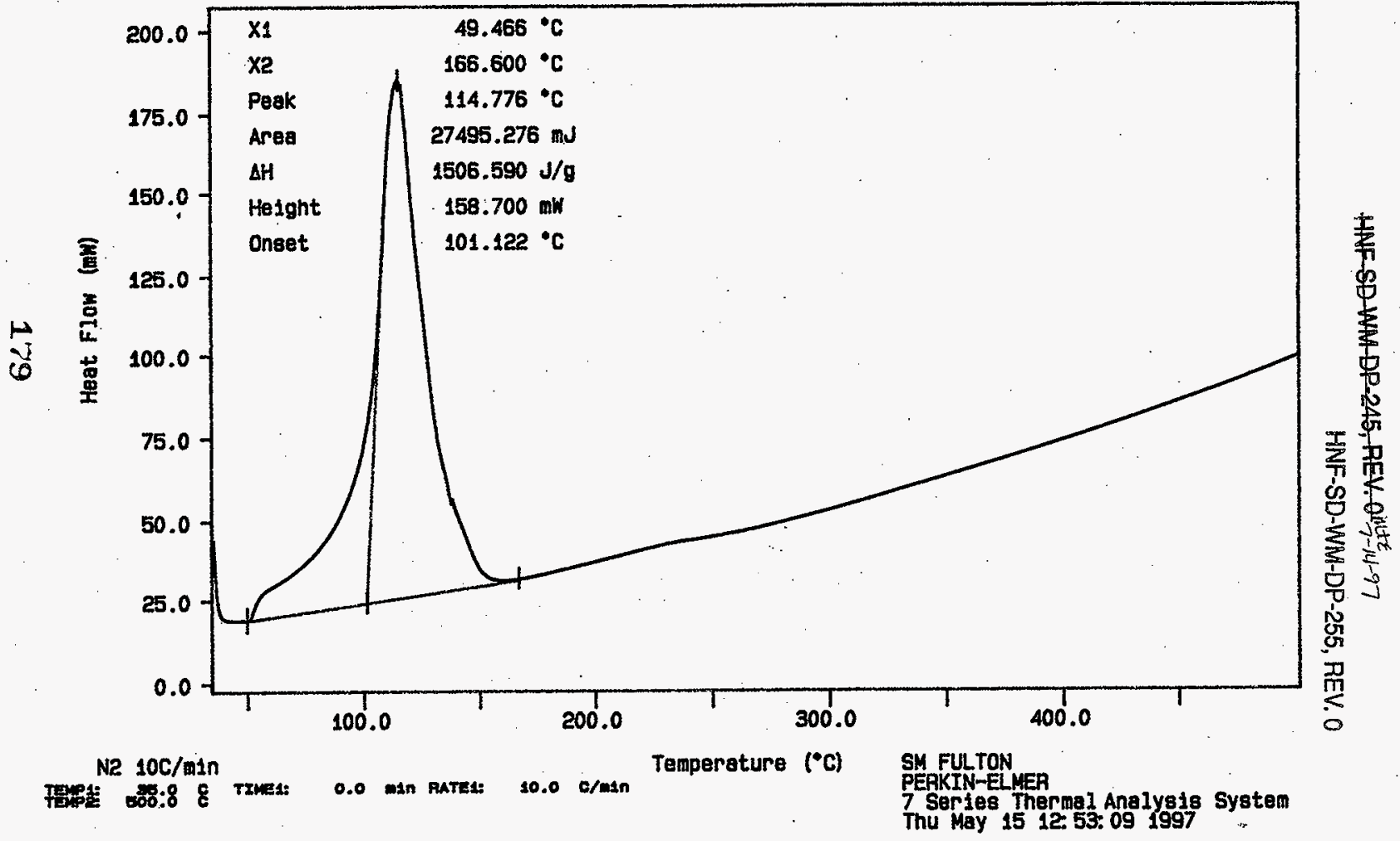




\section{LABCORE Data Entry Template for Worklist\# 18349}

\section{Analyst: \\ ppb \\ Book \# NA}

Method: LA-514-113-RevAAtod 5/29/97

Worklist Comment: DSC-02 T-204 solid. -ppb

\begin{tabular}{|c|c|c|c|c|c|c|c|c|c|c|}
\hline GROUP & PROJECT & $S$ TYPE & SAMPLE\# & R A & - TEST -...... & MATRIX & ACTUAL & FOUND & DL & UNIT \\
\hline 97000261 & $T-204$ & 1 SAMPLE & S97T000498 & 0 & DSC-02 & SOLID & N/A & 0 & & Joules/g Dry \\
\hline 97000261 & $T-204$ & 2 DuP & S97T000498 & 0 & DSC-02 & SOLID & 0 & 0 & $N / A$ & Joules/g Dry \\
\hline 97000261 & $\mathrm{~T}-204$ & 3 SAMPLE & S97T000499 & 0 & DSC-02 & SOLID & $N / A$ & 0 & & Joules/g Dry \\
\hline 97000261 & $T-204$ & 4 DUP & S97T000499 & 0 & DSC-02 & SOLID & 0 & 0 & N/A & Joules/g Dry \\
\hline 97000261 & $T-204$ & 5 SAMPLE & S971000574 & 0 & DSC-02 & SOLID & N/A & 0 & 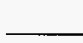 & Joules/g Dry \\
\hline 97000261 & $T-204$ & 6 DUP & S97T000574 & 0 & DSC-02 & SOLID & 0 & 0 & N/A & Joules/g Dry \\
\hline 97000261 & $T-204$ & 7 SAMPLE & S97T000575 & 0 & DSC-02 & SOL.ID & N/A & 0 & & Joules/g Dry \\
\hline 97000261 & $T-204$ & 8 DUP & S97T000575. & 0 & DSC-02 & SOLID & 0 & 0 & M/A & Joules/g Dry \\
\hline 97000261 & $\mathrm{~T}-204$ & 9 SAMPLE & S97T000576 & 0 & DSC-02 & SOL ID & N/A & 0 & & Joules/g Dry \\
\hline 97000261 & $T-204$ & 10 DUP & s97r000576 & 0 & DSC-02 & SOLID & 0 & 0 & $\mathrm{~N} / \mathrm{A}$ & Joules/g Dry \\
\hline 97000261 & $T-204$ & 11 SAMPLE & s971000580 & 0 & DSC-02 & SOLID & N/A & 0 & & Joules/g Dry \\
\hline 97000261 & $T-204$ & 12 DUP & S97T000580 & 0 & DSC-02 & SOLID & 0 & 0 & N/A & Joules/g Dry \\
\hline 97000261 & $T-204$ & 13 SAMPLE & s971000603 & 0 & DSC-02 & SOLID & N/A & 0 & & Joules/g Dry \\
\hline 97000261 & $T-204$ & 14 DUP & S97T000603 & 0 & DSC-02 & SOLID & 0 & 0 & N/A & Joules/g Dry \\
\hline 97000261 & $T-204$ & 15 SAMPLE & s97r000604 & 0 & DSC-02 & SOLID & N/A & 0 & - & Joules/g Dry \\
\hline 97000261 & $T-204$ & 16 DUP & S97T000604 & 0 & DSC-02 & SOLID & 0 & 0 & N/A & Joules/g Dry \\
\hline 97000261 & $T-204$ & 17 SAMPLE & S97T000605 & 0 & DSC-02 & SOLID & N/A & 0 & & Joules/g Dry \\
\hline 97000261 & $T-204$ & 18 DUP & S97T000605 & 0 & DSC-02 & SOLID & 0 & 0 & N/A & Joules/g Dry \\
\hline
\end{tabular}

\section{Data Entry Comments:}

\section{HNF-SD-WM-DP-255, REV. 0}




\section{LABCORE Data Entry Template for Worklist\# 18349}

GROUP PROJECT $S$ TYPE SAMPLE\# RA $\ldots$.....TEST $\cdots \ldots$ MATRIX ACTUAL FOUND DL UNIT

\section{Final page for worklist \# 18349}

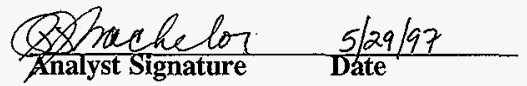

\author{
Analyst Signature Date
}

HNF-SD-WM-DP-255, REV. 0 


\section{LABCORE Data Entry Template for Worklist\# 18350}

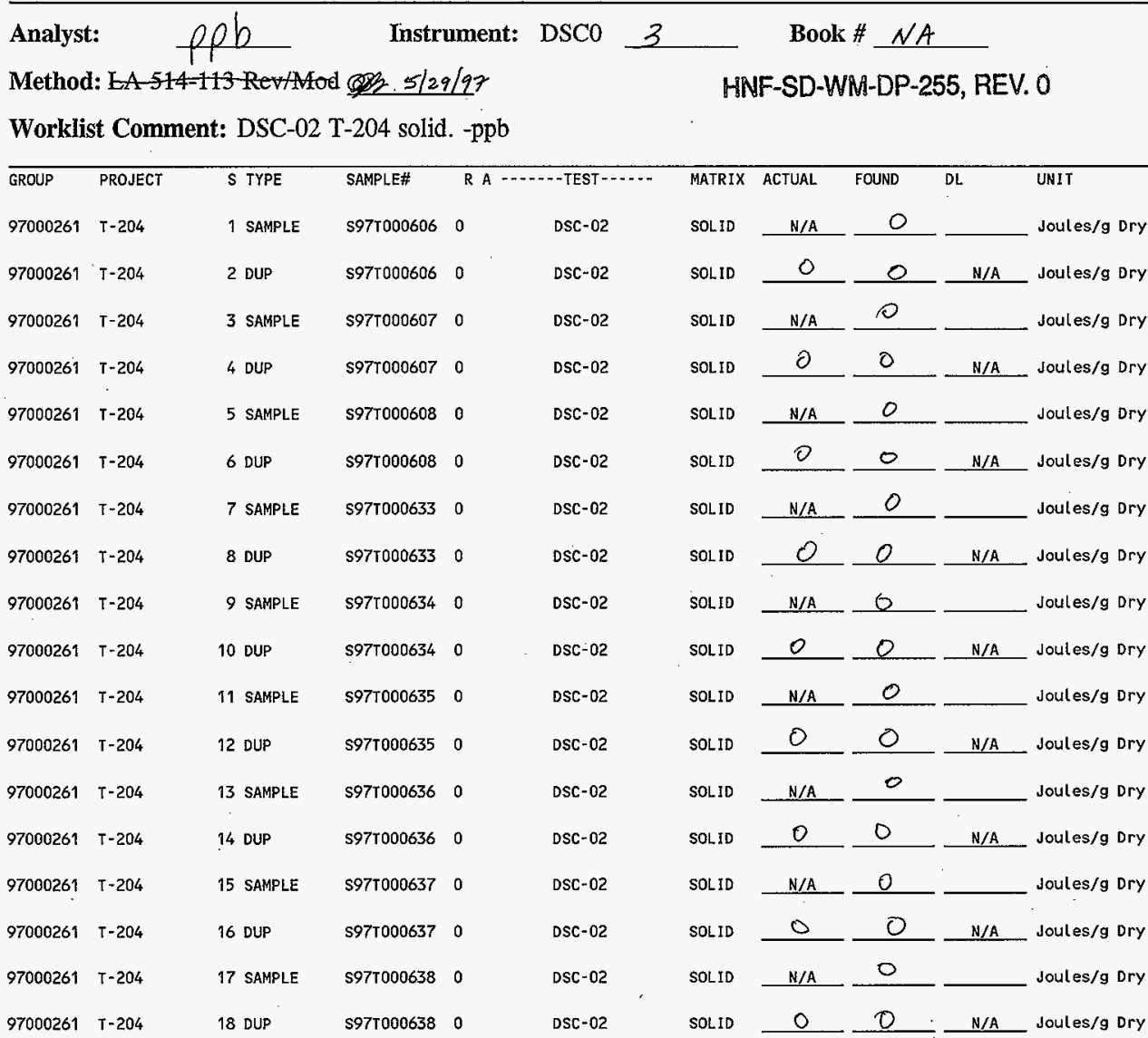

Data Entry Comments:

Units shown for $Q C$ (SPK \& STD) may not reflect the actual units. $D L=$ Detection Limit, $S=$ Worklist Slot Number,

$R=$ Replicate Number, $A=$ Aliquot Code. 


\section{LABCORE Data Entry Template for Worklist\# 18350}

\section{Final page for worklist \# 18350}

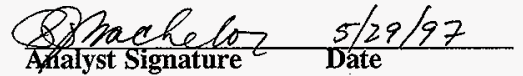

\author{
Analyst Signatire \\ Date
}

HNF-SD-WM-DP-255, REV: 0

Units shown for $Q C$ (SPK \& STD) may not reflect the actual units. $D L=$ Detection Limit, $S=$ Worklist Slot Number, $R=$ Replicate Number, $A=$ Aliquot Code. 


\section{LABCORE Data Entry Template for Worklist\# 18351}

Analyst: $\frac{p b}{1}$ Instrument:

Worklist Comment: DSC-02 T-204 liquid. -ppb

Book \# NA

HNF-SD-WM-DP-255, REV. 0

\begin{tabular}{|c|c|c|c|c|c|c|c|c|c|c|}
\hline$\overline{\text { GROUP }}$ & PROJECT & S TYPE & SAMPLE\# & $\mathrm{RA}$ & - TEST - - - & MATRIX & ACTUAL & FOUND & $\overline{\mathrm{DL}}$ & UNIT \\
\hline 97000261 & $T-204$ & 1 SAMPLE & s97T000647 & 0 & DSC-02 & LIQUID & $N / A$ & 0 & & Joules/g Dry \\
\hline 97000261 & $T-204$ & $2 \cdot$ DUP & s971000847 & 0 & DSC-02 & LIQUid & 0 & 0 & $\mathrm{~N} / \mathrm{A}$ & Joules/g Dry \\
\hline
\end{tabular}

\section{Final page for worklist \# 18351}

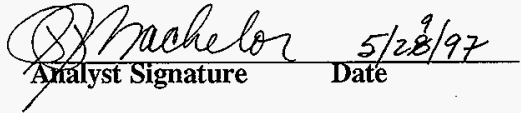

Analyst Signature Date

Data Entry Comments:

Units shown for $Q C$ (SPK \& STD) may not reflect the actual units. $D L=$ Detection Limit, $S=$ Worklist Slot Number, $R=$ Replicate Number, $A=$ Aliquot Code. 


\section{LABCORE Data Entry Template for Worklist\# 18689}

Analyst:

Instrument: DSCO

3

Book \# $12 N / 4 B$

Method: LA-514-114 Rev/Mod D-O

HNF-SD-WM-DP-255, REV. 0

Worklist Comment: T-204 DSC, RUN UNDER N2. RCJ

\begin{tabular}{|c|c|c|c|c|c|c|c|c|c|c|}
\hline GROUP & PROJECT & $\begin{array}{l}\text { S TYPE } \\
1 \text { STO }\end{array}$ & SAMPLE\# & RA & DSC-03 & $\begin{array}{l}\text { MATRIX } \\
\text { SOLID }\end{array}$ & $\begin{array}{l}\text { ACTUAL } \\
28.45 \\
\end{array}$ & $\begin{array}{l}\text { FOUND } \\
27.58 \\
\end{array}$ & $\begin{array}{l}D L \\
N / A \\
\end{array}$ & $\begin{array}{l}\text { UNIT } \\
\text { Joules/g }\end{array}$ \\
\hline 97000261 & $T-204$ & 2 SAMPLE & S97T001197 & 0 & DSC-03 & SOLID & N/A & 0 & & Joules/g \\
\hline 97000261 & $T-204$ & 3 DUP & \$971001197 & 0 & DSC-03 & SOLID & 0 & 0 & N/A & Joules/g \\
\hline
\end{tabular}

\section{Final page for worklist \# 18689}

See Attached for Signature

Analyst Signature Date

validated 6pis/47\%3achelor

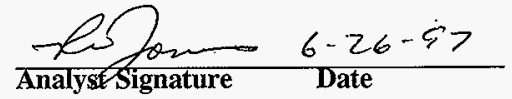

Data Entry Comments:

Units shown for $Q C$ (SPK \& STD) may not reflect the actual units. $D L=$ Detection Limit, $S=$ Worklist Slot Number,

$R=$ Replicate Number, $A=$ Aliquot Code. 


\section{LABCORE Data Entry Template for Worklist\# 18689}

\section{Analyst:

ADP \\ Instrument: $\mathrm{DSC} 0$ \\ Book \# $12 N|4|-B$}

Method: LA-514-113 Rev/Mod D.O

Worklist Comment: T-204 DSC, RUN UNDER N2. RCJ

HNF-SD-WMI-DP-255, REV. 0

\begin{tabular}{|c|c|c|c|c|c|c|c|c|c|c|}
\hline GROUP & PROJECT & S TYPE & SAMPLE\# & $\mathrm{RA}$ & $\therefore-1$ TEST $\cdots$ & MATRIX & ACTUAL. & FOUND & $\mathrm{DL}$ & UNIT \\
\hline & & 1 STD & & & DSC-01 & SOLID & & & $N / A$ & Joules/g \\
\hline 97000261 & $T-204$ & 2 SAMPLE & s97T001197 & 0 & DSC-01 & SOLID & $N / A$ & - & & Joutes/g \\
\hline 97000261 & $T-204$ & 3 DUP & s97T001197 & 0 & DSC-01 & SOLID & - & & $\mathrm{N} / \mathrm{A}$ & Joules/g \\
\hline
\end{tabular}

Outranturenta 6-19-97

Analyst Signature Date

\section{Final page for worklist \# 18689}

Data Entry Comments: on DSC-03. $8836 / 26 / 97$

Units shown for QC (SPK \& STD) may not reflect the actual units. $D L=$ Detection Limit, $S=$ Worklist Slot Number, $R=$ Replicate Number, $A=$ Aliquot Code. 
Curve 1: DSC

File info: InD061901 Thu wn 19 08:09:50 1997

Sample Neight: 12.160 ag 12N14-B

SIGUATURE OFIOW REPRESENTS CHEMICAL TECHNOLOGHST/CHEMET THAT

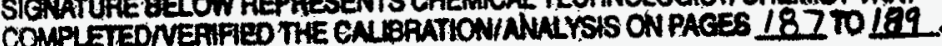

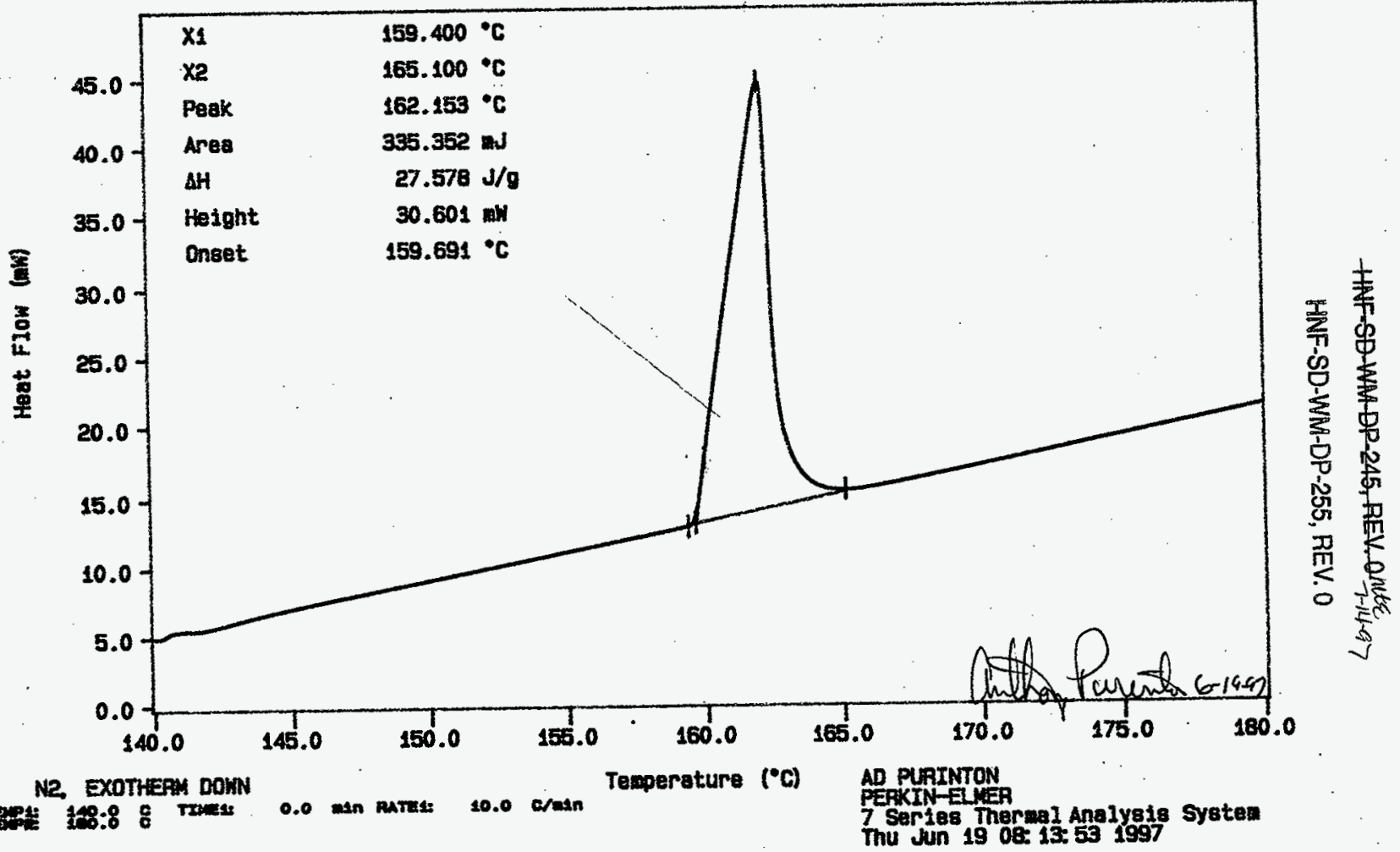


Curve 1: DSC

File info: SAM061901 Thu Jun 19 10: 27: 181997

Sample Weight: 43.070

mg

S97T001197 SAM

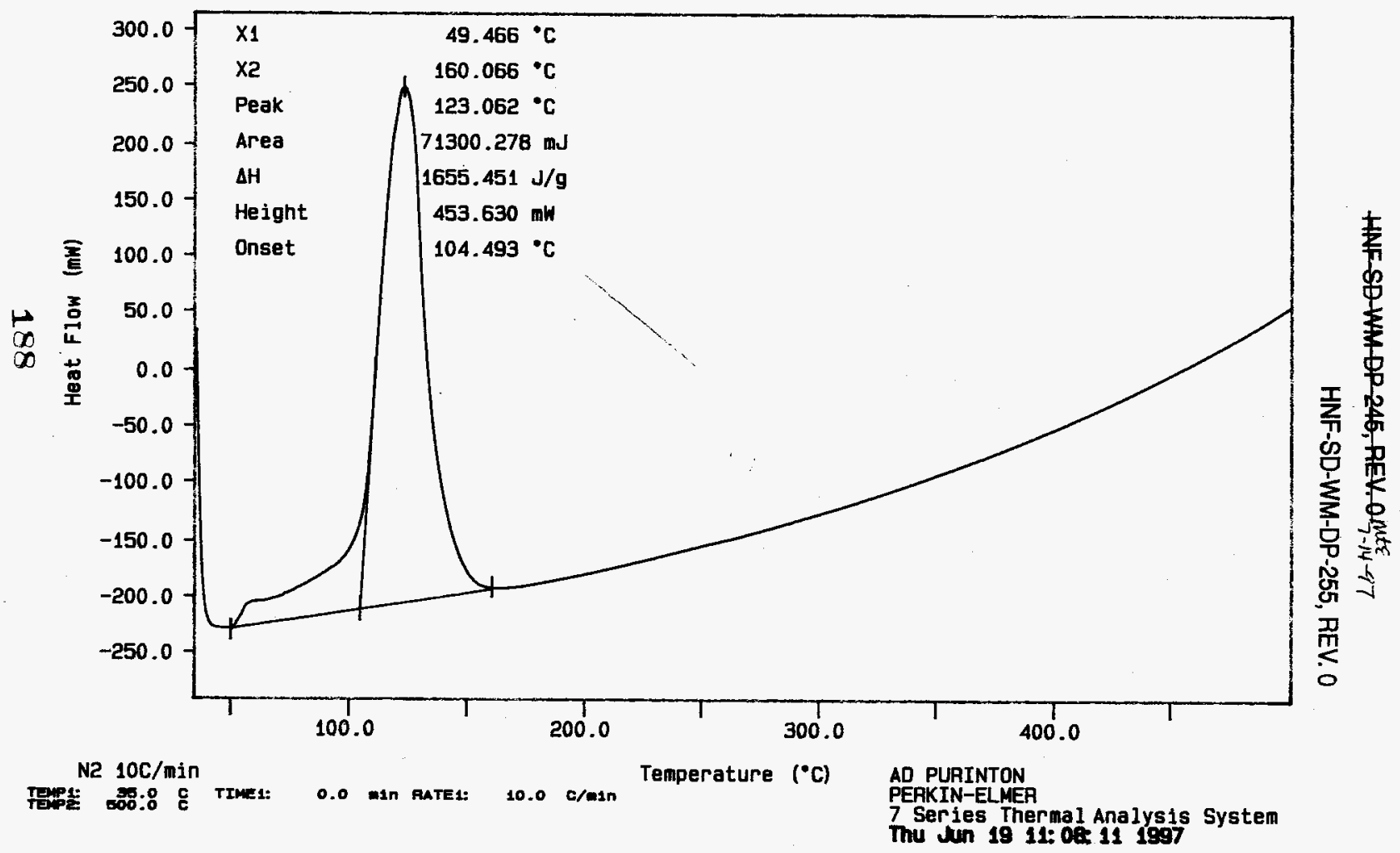


Curve 1: DSC

File info: SAM061902 Thu Jun 19 12:05: 111997

Sample Weight: 37.710 ing

s97T001197 DUP

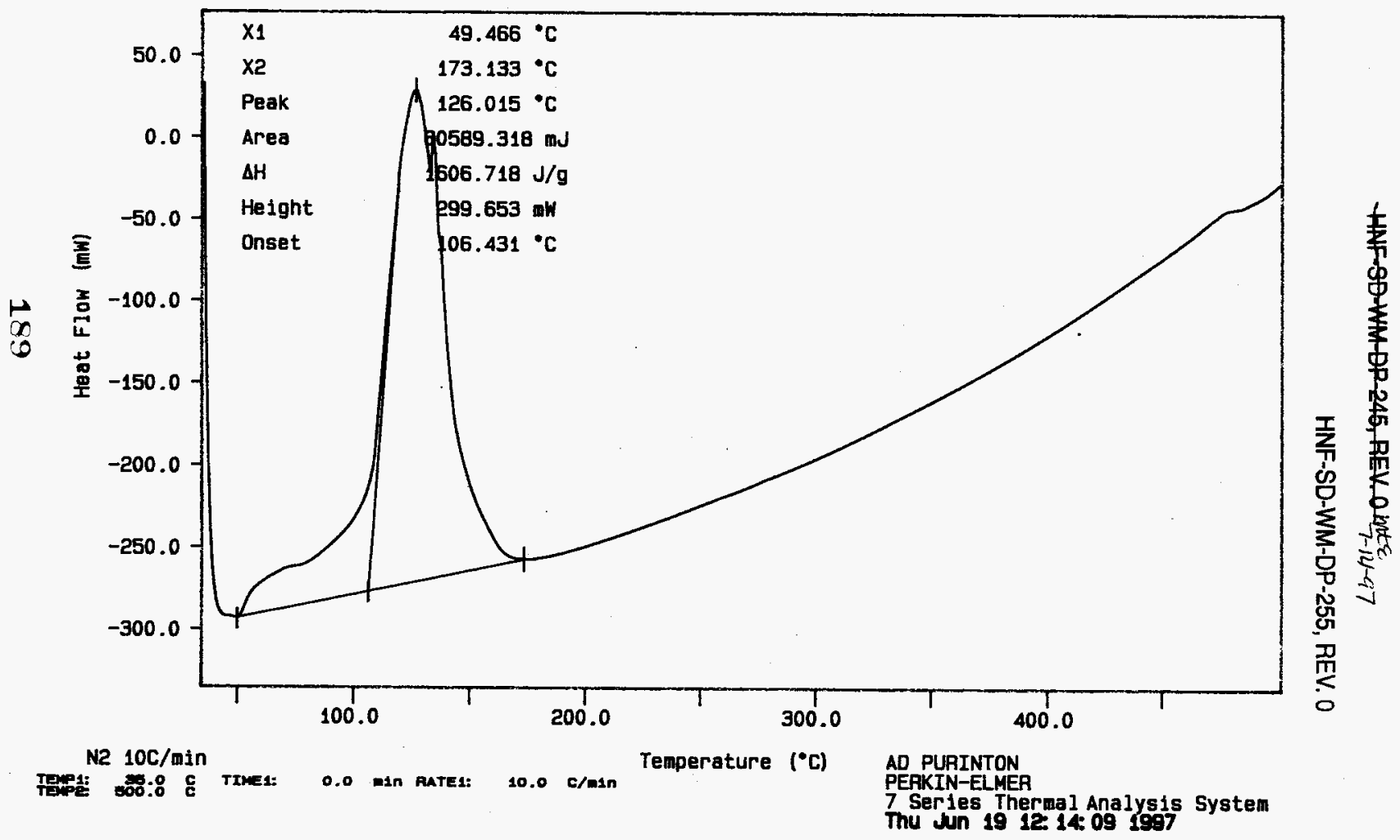




\section{LABCORE Data Entry Template for Worklist\# 19020}

\section{Analyst: $\rho \rho b$ Instrument: $\mathrm{DSCO} 2$ Book \# NA}

Method: 1 -514-113 7/,197

Worklist Comment: DSC-02 T-204 Comp solid. -ppb

HNF-SD-WMA-DP-255, REV. 0

\begin{tabular}{|c|c|c|c|c|c|c|c|c|c|c|}
\hline GROUP & PROJECT & S TYPE & SAMPLE\# & & -TEST-N..... & MATRIX & ACTUAL & FOUND & $\mathrm{DL}$ & UNIT \\
\hline 97000261 & $T-204$ & 1 SAMPLE & s97T001197 & 0 & DSC-02 & SOLID & N/A & 0 & & Joules/g Dry \\
\hline 97000261 & $T-204$ & 2 DUP & \$97T001197 & 0 & DSC-02 & SOLID & 0 & 0 & $\mathrm{~N} / \mathrm{A}$ & - Joules/g Dry \\
\hline
\end{tabular}

\section{Final page for worklist \# 19020}

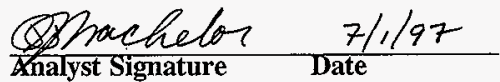

Analyst Signature Date

Data Entry Comments:

Units shown for $Q C$ (SPK \& STD) may not reflect the actual units. $D L=$ Detection Limit, $S=$ Worklist Slot Number, $R=$ Replicate Number, $A=$ Aliquot Code. 


\section{LABCORE Data Entry Template for Worklist\# 17838}

\section{Analyst: ADP Instrument: TGAO 1 Book $91 \mathrm{~N} 8-\mathrm{A}$ \\ Method: LA-560-112 Rev/Mod C-O \\ HNF-SD-WM-DP-255, REV. 0}

Worklist Comment: T204, TGA-01 Run under nitrogen. skm

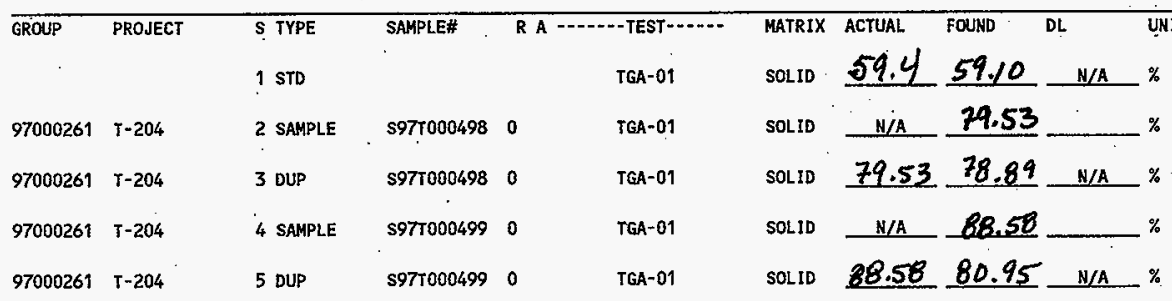

\section{Final page for worklist \# 17838;}

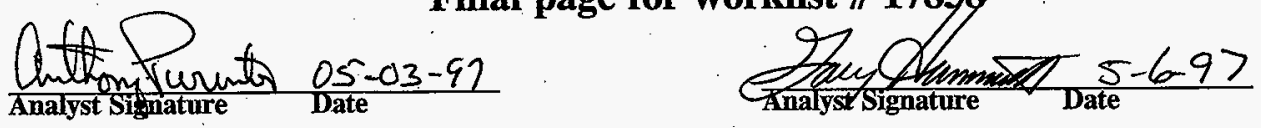
validated $5 / 8 / 97837 a c h$ lor

Data Entry Comments:

Units shown for QC (SPK \& STD) may not reflect the actual units. $D L=$ Detection Limit, $S=$ Worklist Slot Number, $R=$ Replicate Number, $A=$ Aliquot Code. 


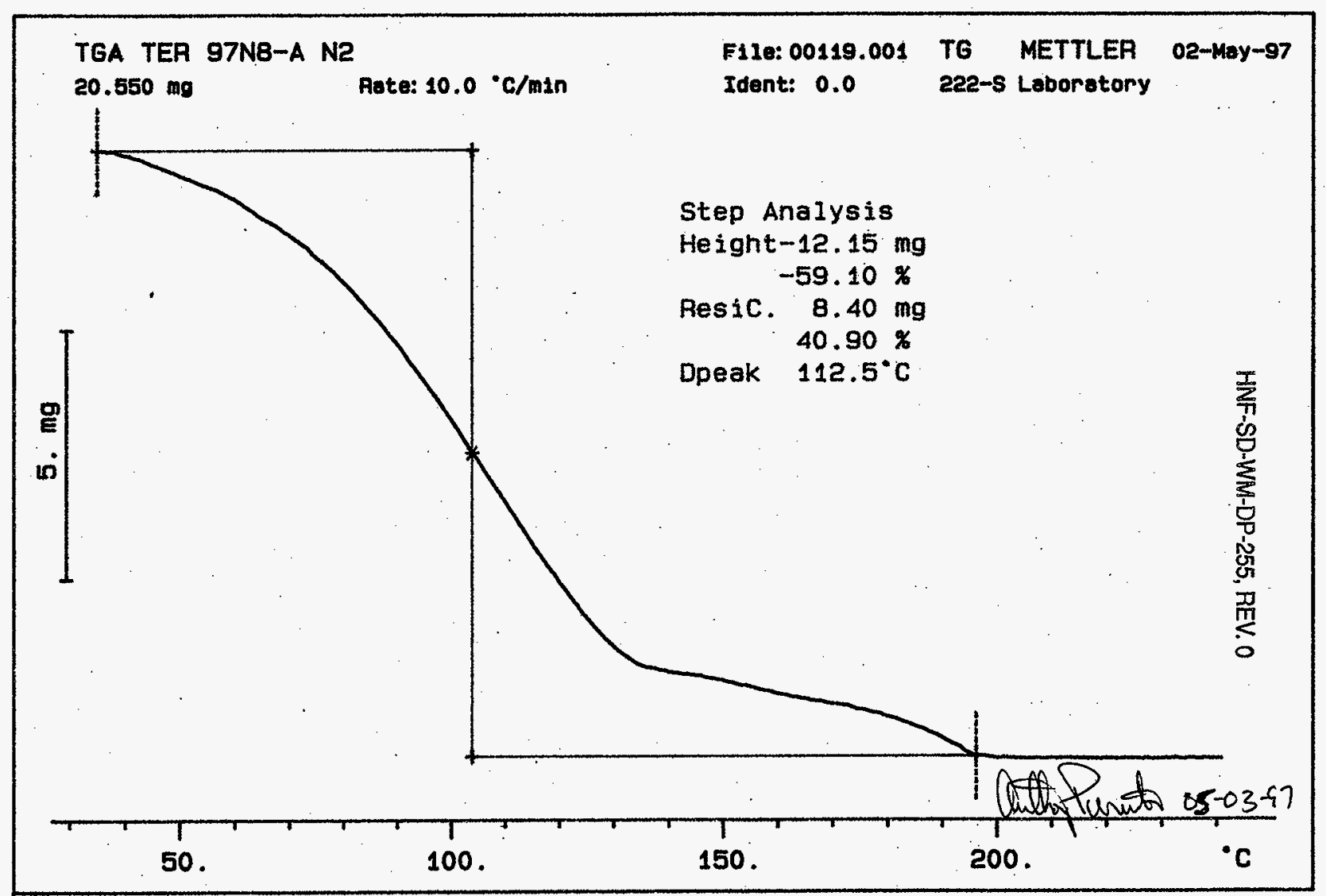




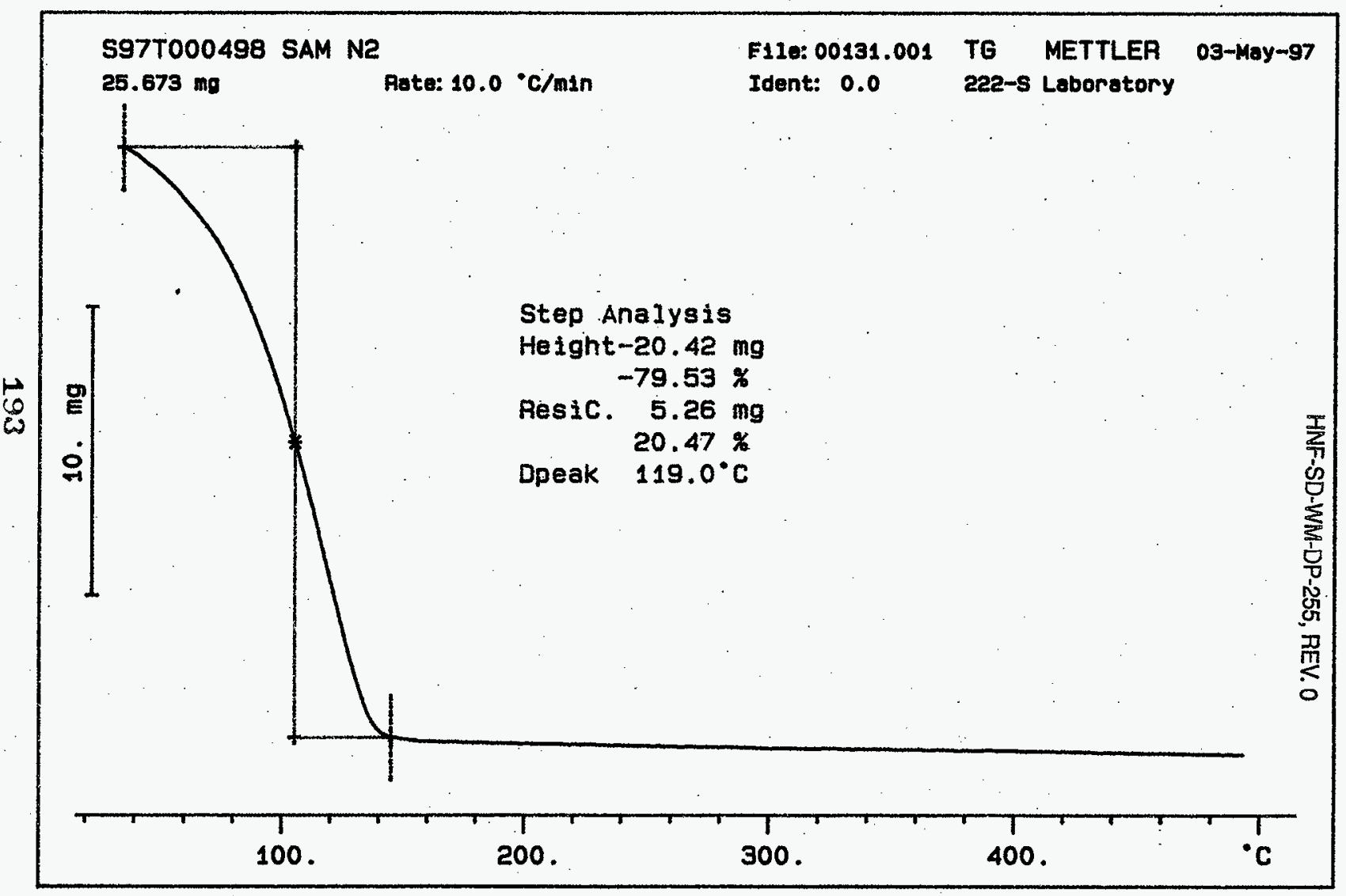




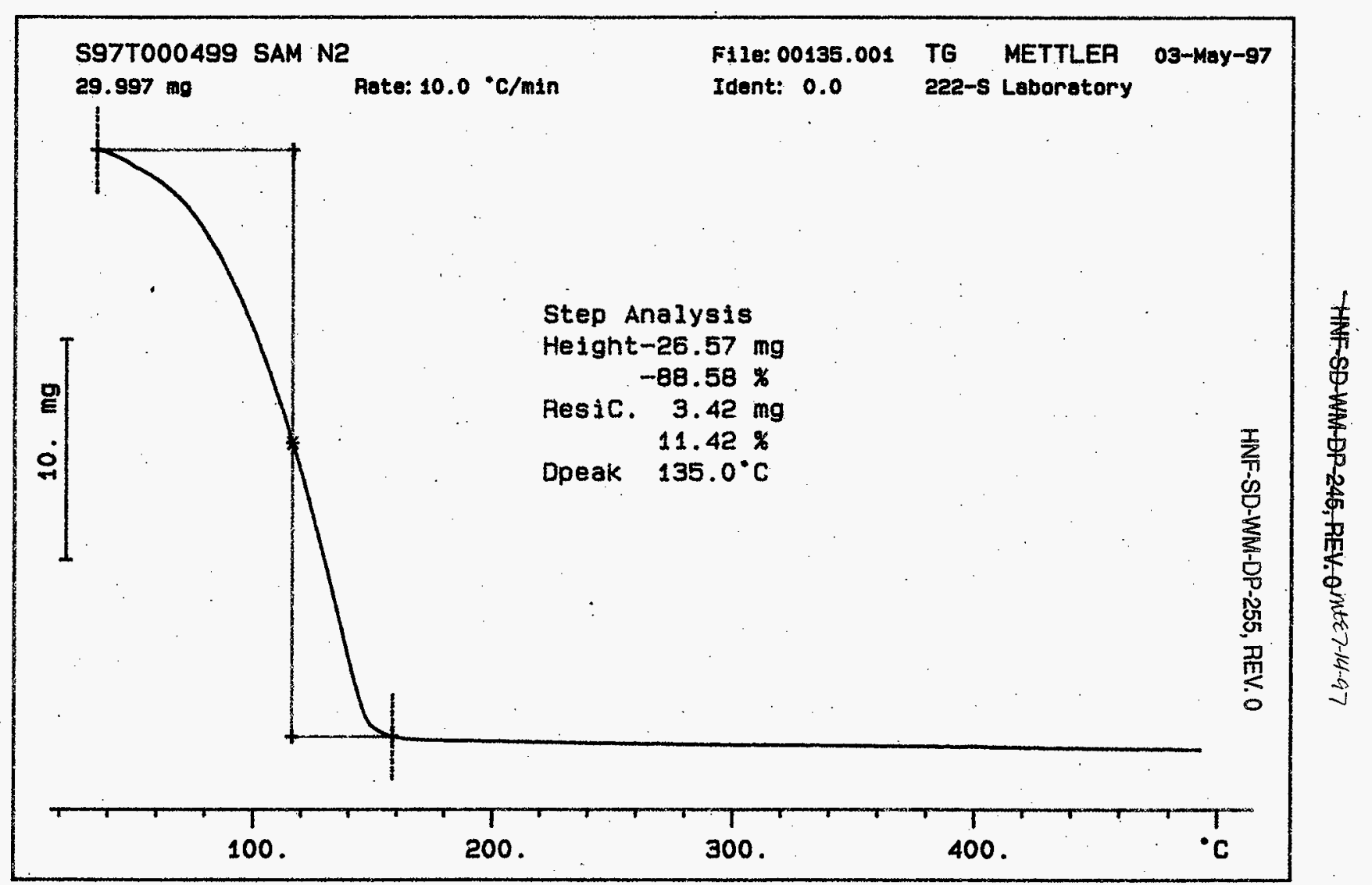




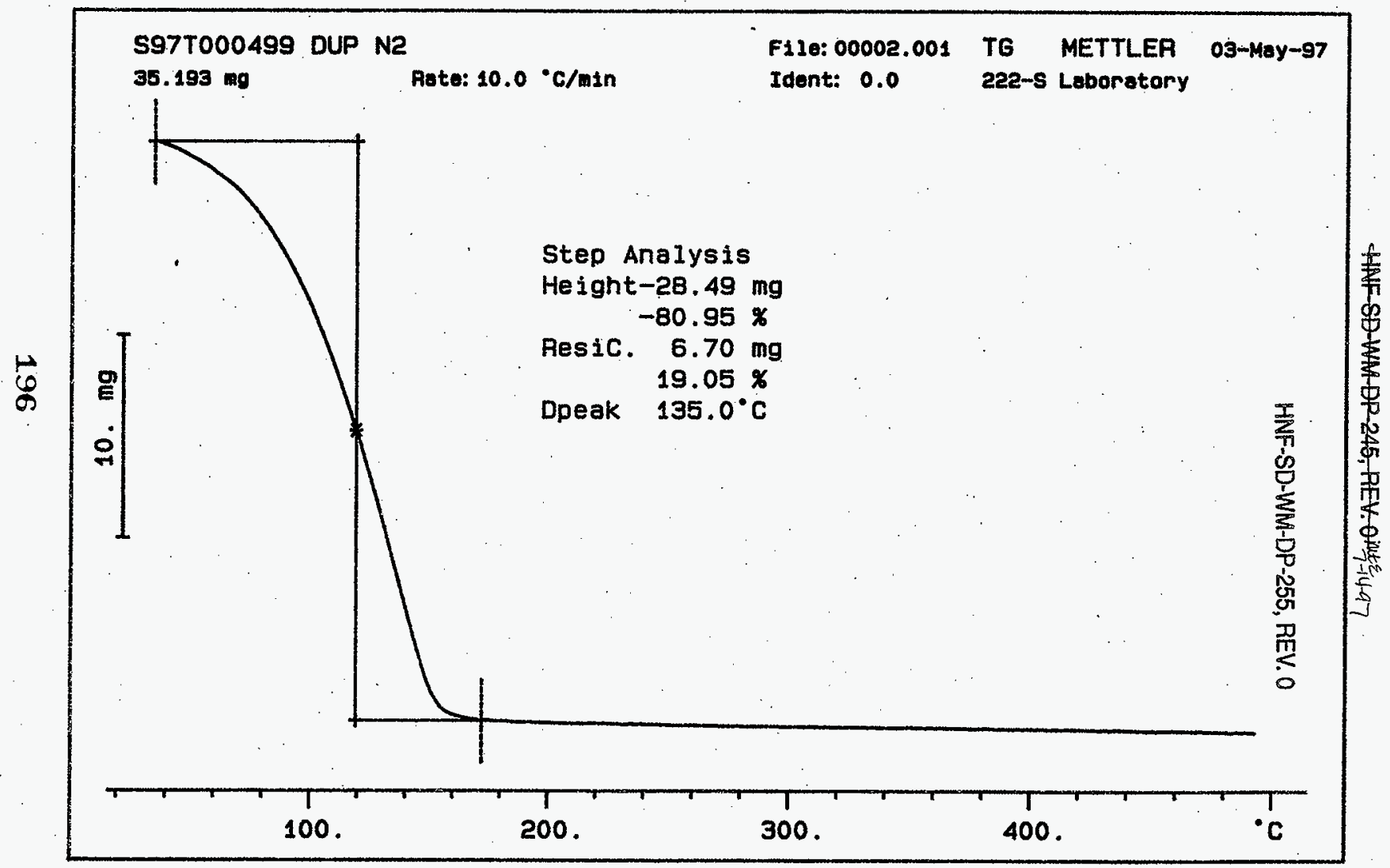




\section{LABCORE Data Entry Template for Worklist\# 17839}

Analyst: $\quad$ ADP Instrument: TGA0 3 Book \# 97 N8.A

Method: LA-514-114 Rev/Mod D-O HNF-SD-WH-DP-255, REV. 0

Worklist Comment: T204, TGA-01 Run under nitrogen. skm

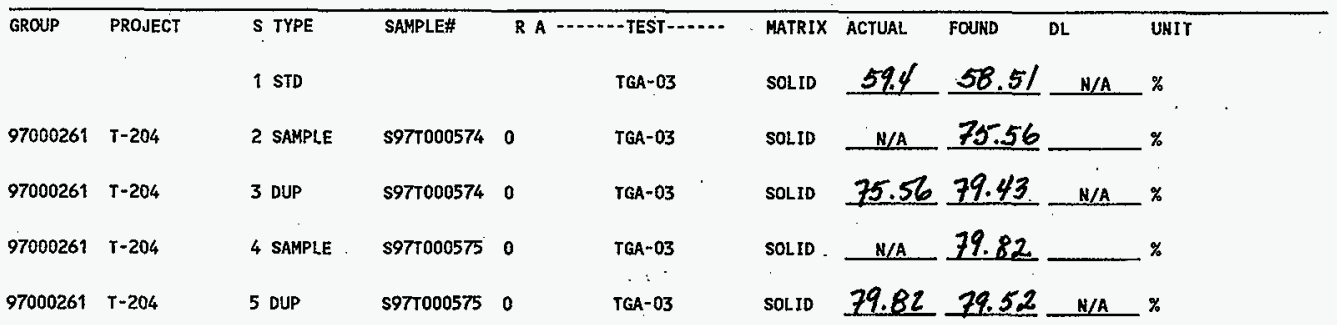

\section{Final page for worklist \# 17839} See Attached for Additiona/ Signatures

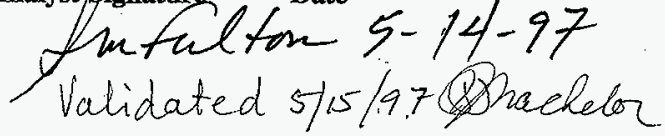

Thand onaele $5-15-97$

Analyst Signature Date

Data Entry Comments:

Units shown for $Q C$ (SPK \& STD) may not reflect the actual units. $D L=$ Detection Limit, $S=$ Worklist Slot Number, $R=$ Replicate Number, $A=$ Aliquot Code. 
Analyst: ADP Instrument: TGA0

Book $\# 97 N 8-1$ Method: LA-560-112 Rev/Mod $D-O$

Worklist Comment: T204, TGA-01 Run under nitrogen. skm

HNF-SD-WM-DP-255, REV. 0

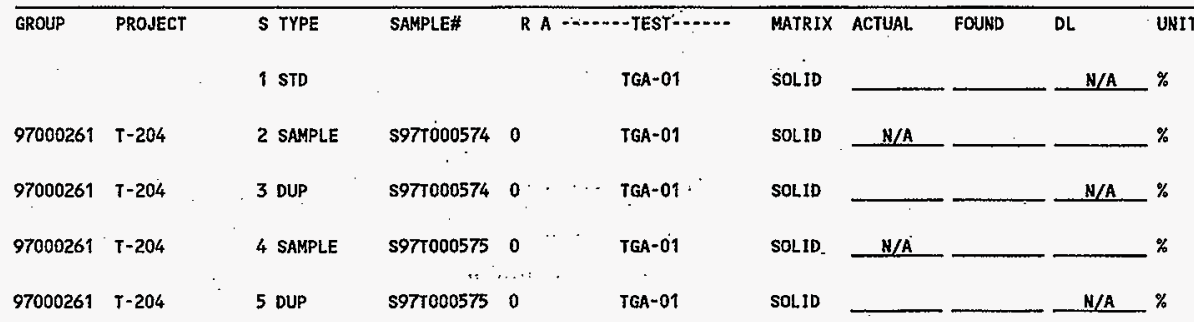

\section{Final page for worklist \# 17839}

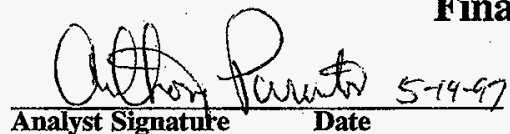

Analyst Signature Date

\section{Data Entry Comments:}

Units shown for $Q C$ (SPK \& STD) may not reflect the actual units. $D L=$ Detection Limit, $S=$ Worklist Slot Number, $R=$ Replicate Number, $A=$ Aliquot Code. 
Curve 1: TGA

File info: TERO10513 Tue May 13 22: 09:38 1997

Sample Weight: $13.546 \mathrm{mg}$

TGA STD 97NE-A

SIGNATURE BELOW REPRESENTS CHEMICAL TECHNOLOGIST/CHEMHST HWT COMPLETEDNEFIFIED THE CALLBRATION/ANALYSIS ON PAGES 199 TO 203.

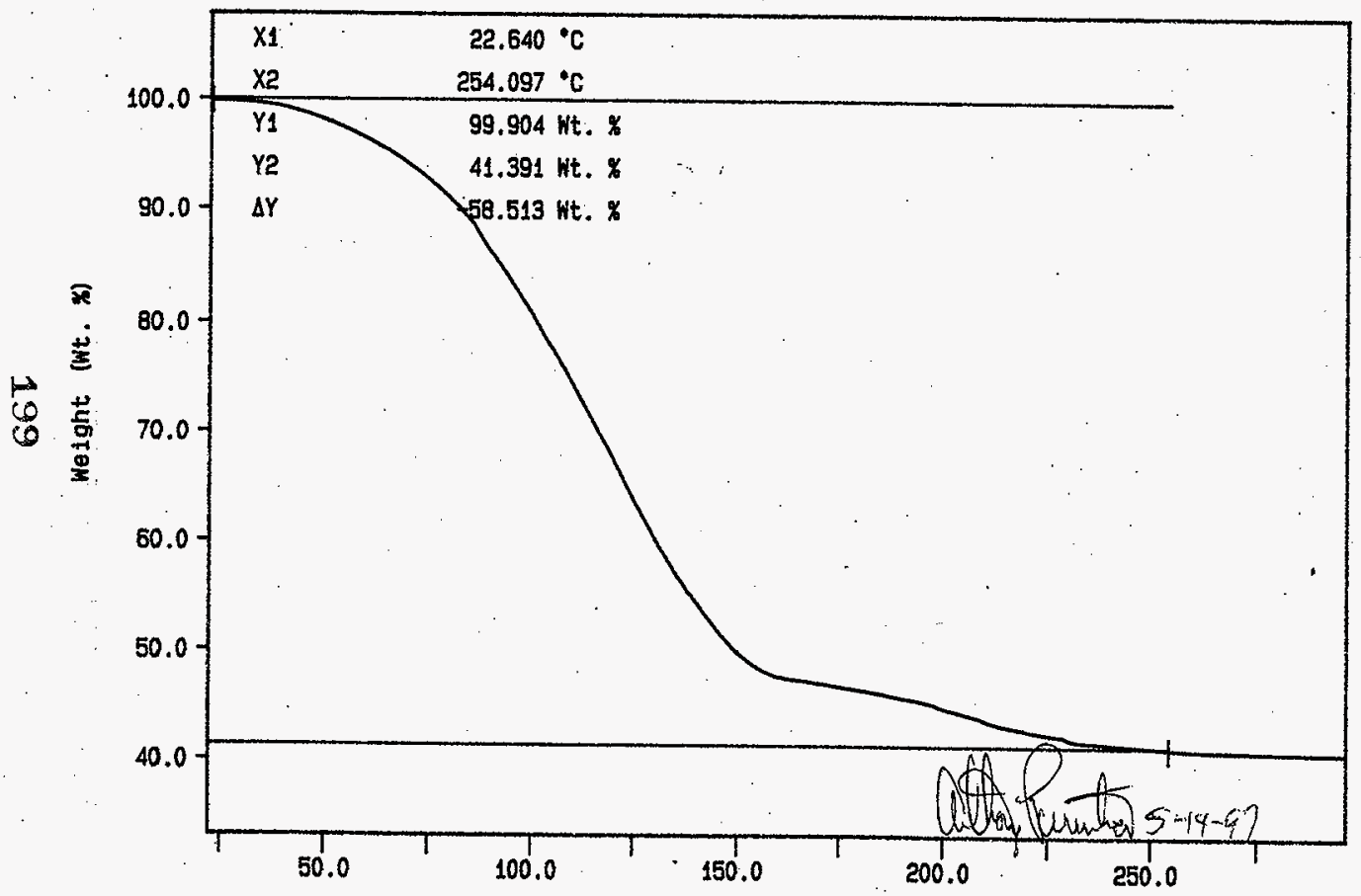

N2 $10 \mathrm{C} / \mathrm{MIN}$

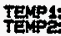

Temperature $\left({ }^{\circ} \mathrm{C}\right)$

$10.0 \mathrm{c} / \mathrm{man}$
A PURINTON PEAKIN-ELMER

7 Series Thermal Analysis Systen Tue May 13 22: 32:08 1997 
Curve 1: TGA

File info: SAM050514 Hed May 14 03: 46: 291997

Sample Weight: 9.154

$\mathrm{mg}$

\section{S97TO00574 SAM}

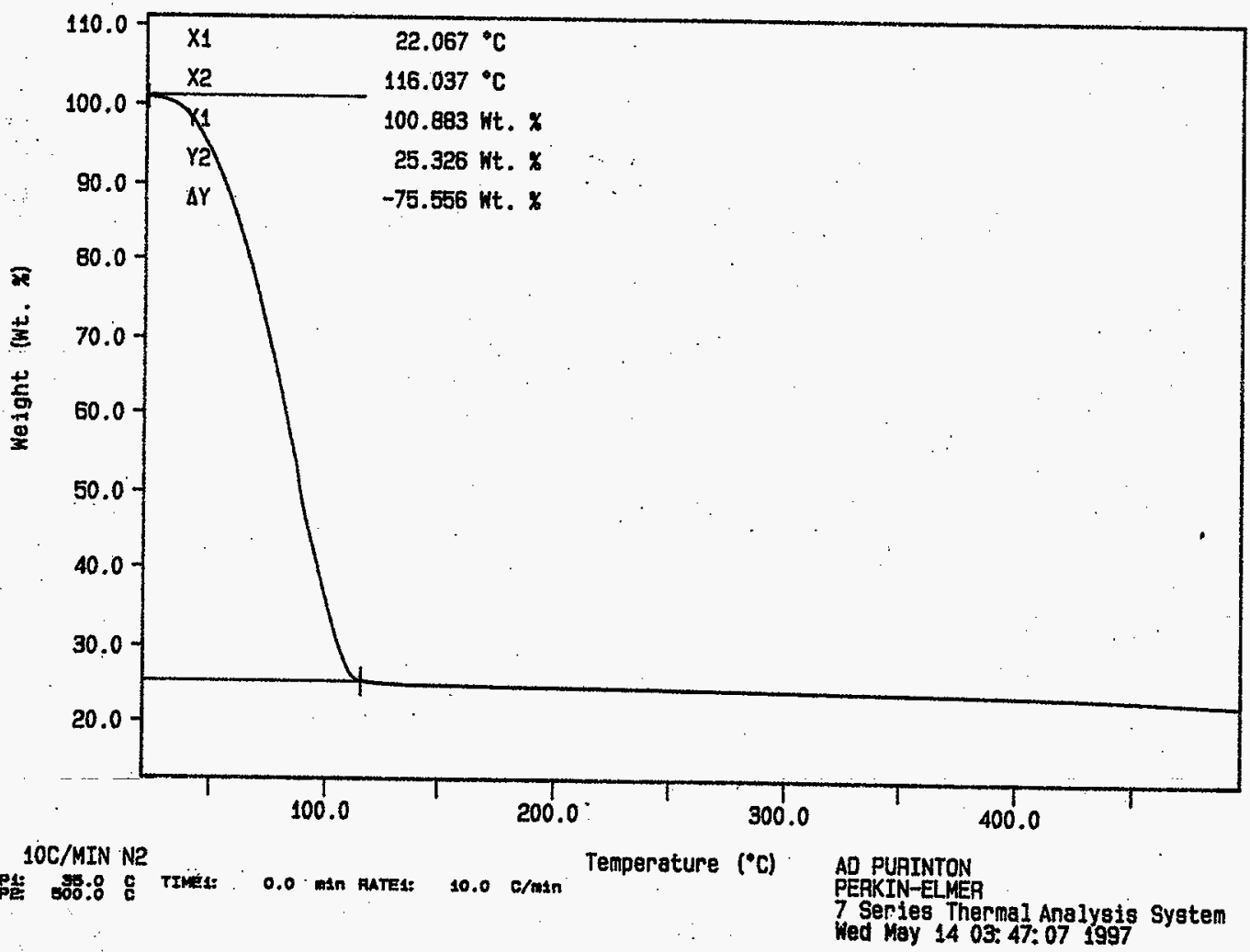


Curve 1: TGA

F1le info: SAM020514 Ned May 14 04:51:54 1997

Sample Weight: $11.481 \mathrm{mg}$

S97T000574 DUP

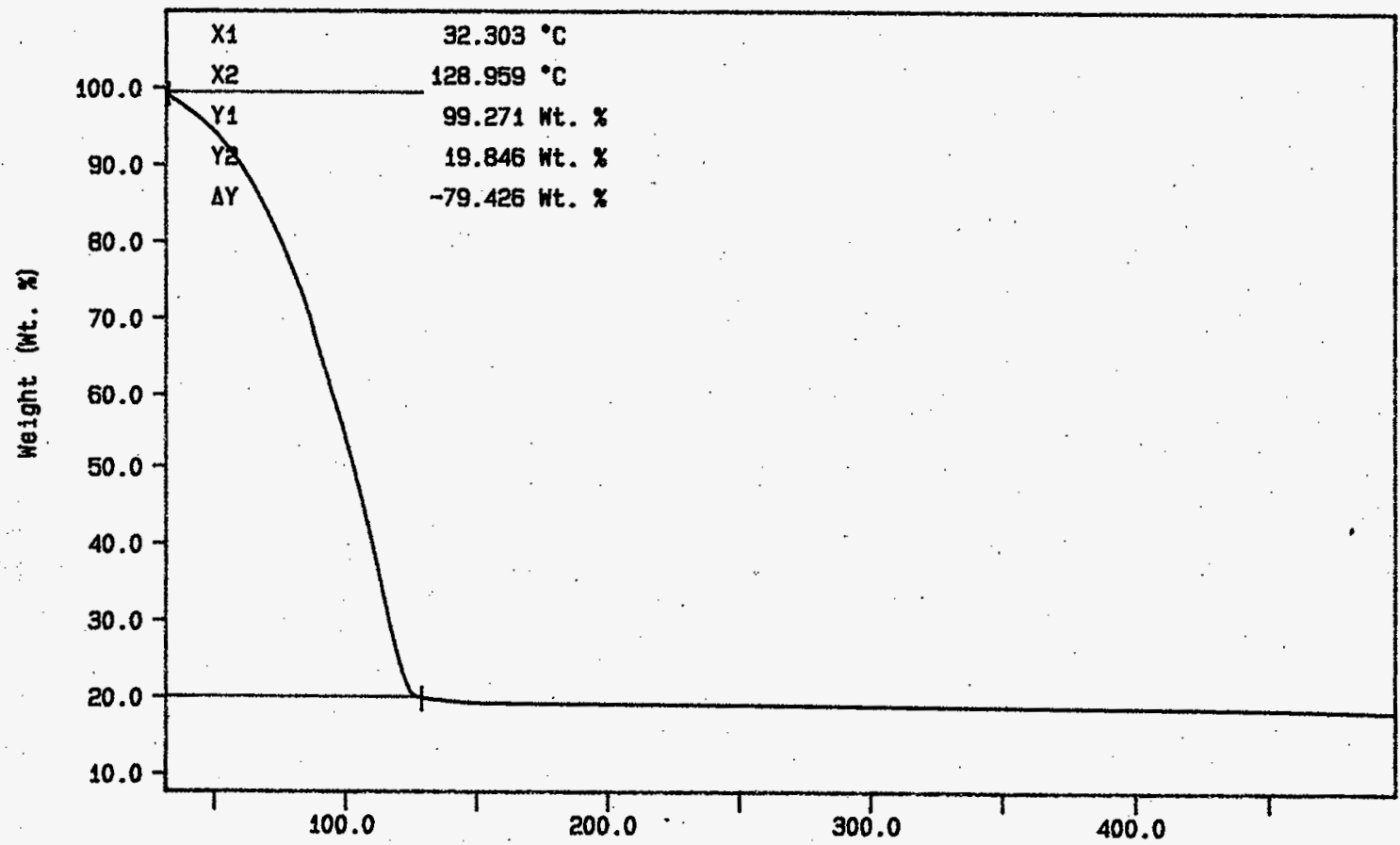

10C/MIN N2

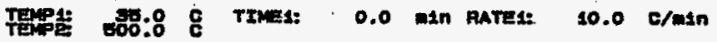

Temperature $\left({ }^{\circ} \mathrm{C}\right)$

AD PURINTON

PEAKIN-ELMER

7 Series Thermal Anelysis System

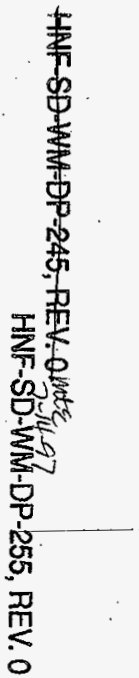


Curve 1: TGA

File info: SAM051401 Hed May 14 06: 36:53 1997

Sample Neight: $15.065 \mathrm{mg}$

597T000575

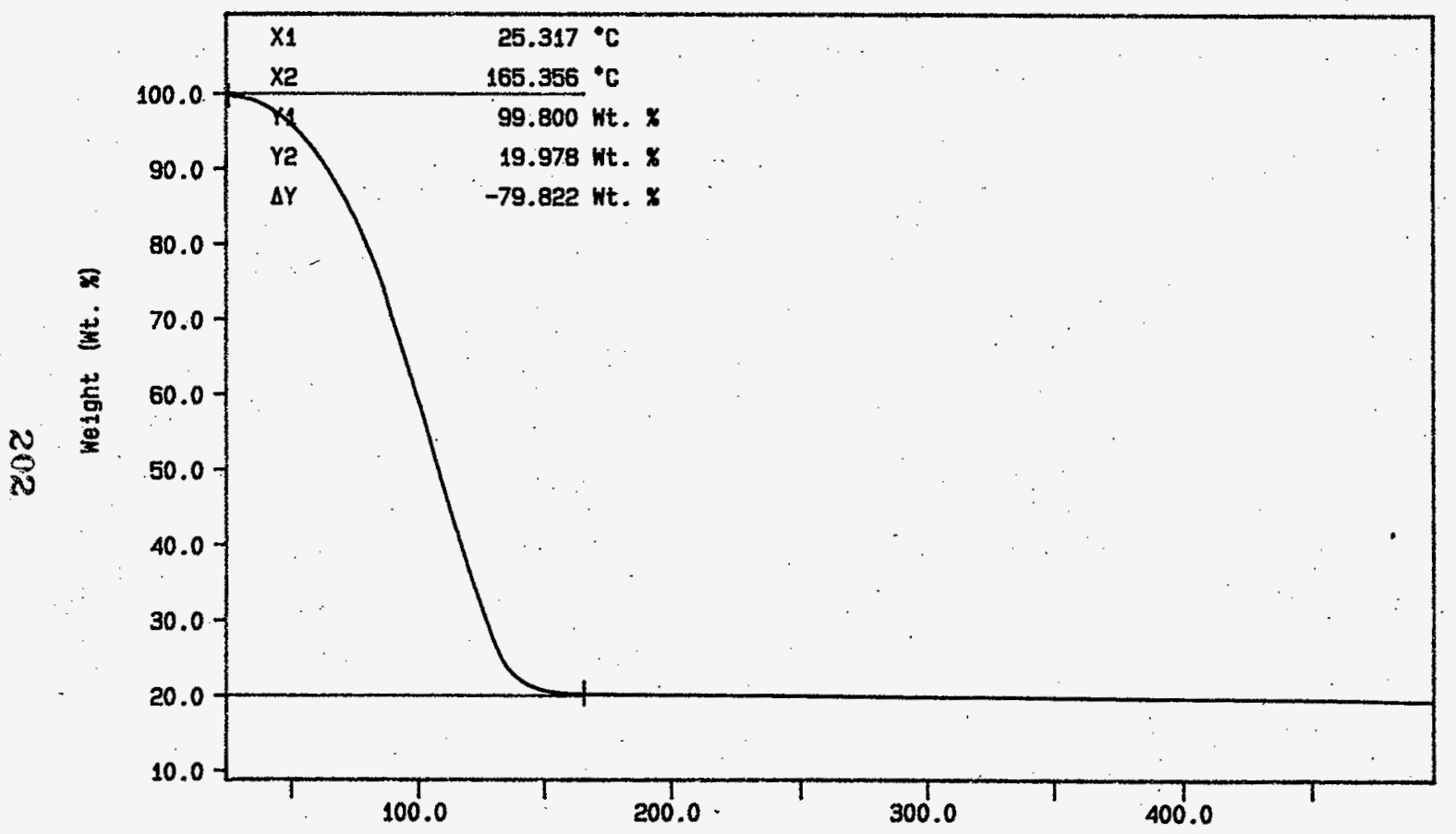

10C/MIN N2

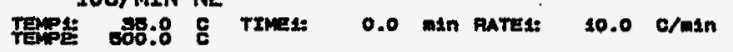

Temperature $\left({ }^{\circ} \mathrm{C}\right)$

SM FULTON

PERKIN-ELMEA

7 Serles Thermaj Analysis System

Wed May 14 06: 54:02 1997

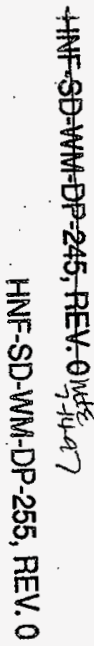


Curve 1: TGA

File info: SAM051402 Wed May 14 07: 45: 281997

Sample Welght: $\mathbf{8 . 6 6 5}$

ing

597T000575 DUP
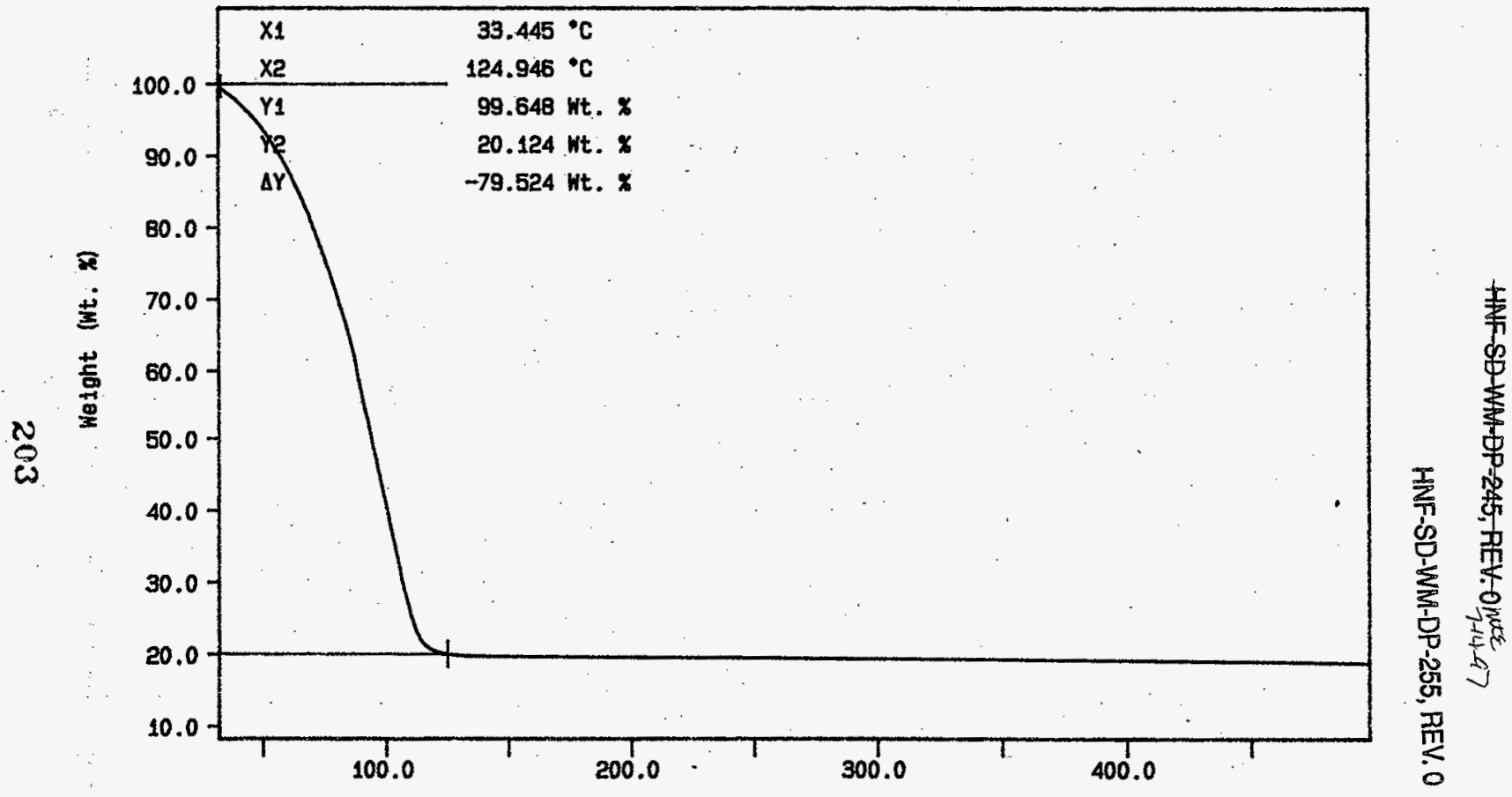

10C/MIN Ne TEEPRA

Temperature $\left({ }^{\circ} \mathrm{C}\right)$

SU FULTON

PEAKIN-ELMER

7 Sertes Thermal Analysis System Wed May 14 08: 11:09 1997 


\section{LABCORE Data Entry Template for Worklist\# 17842}

Analyst: $\quad$ ADP $\quad$ Instrument: TGA0 $3 \quad$ Book \# $97 N 8 \mathrm{~A}$

Method: LA-514-114 Rev/Mod D-O

HNF-SD-WM-DP-255, REV. 0

Worklist Comment: T204, TGA-01 Run under nitrogen. skm

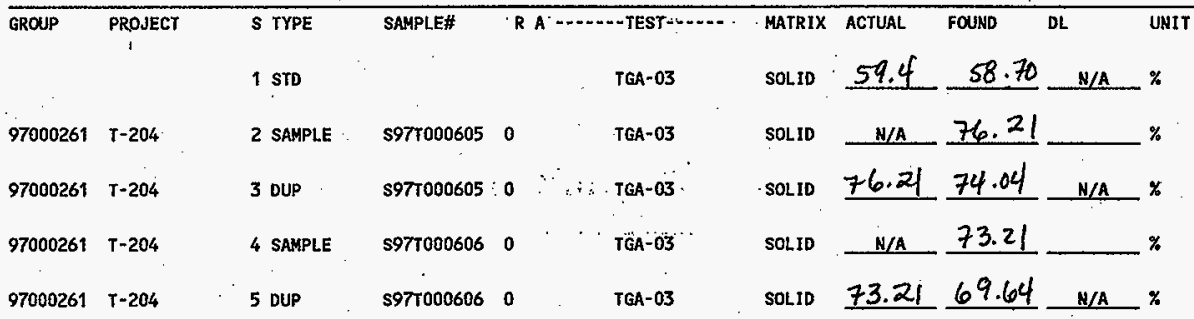

\section{Final page for worklist \# 17842}

$\frac{\text { See Aftacked for Signature }}{\text { Analyst Signature }}$

Validated 5/22/97\$8hache bo

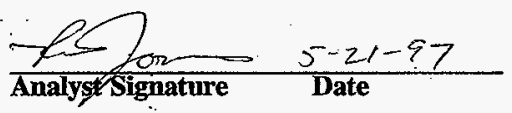

Data Entry Comments:

Units shown for $Q C$ (SPK \& STD) may not reflect the actual units. $D L=$ Detection Limit, $S=$ Worklist Slot Number, $R=$ Replicate Number, $A=$ Aliquot Code. 


\section{LABCORE Data Entry Template for Worklist\# 17842}

Page: 1

Analyst: ADO Instrument: TGA0 3 Book\# 97N8A

Method: LA-560-112 Rev/Mod D-O

Worklist Comment: T204, TGA-01 Run under nitrogen. skm HNF-SD-WM-DP-255, REV. 0

GROUP PRQJECT S TYPE SAMPLE\# R A $\ldots$ MATRIX ACTUAL FOUDD DL UEST

1 STD $\quad \because$ TGA-01 SOLID $\div$

$97000261 \quad T-204$

2 SAMPLE \$97T000605 0

TEA-01

SOL.ID N/A $\%$

$97000261 T-204$

3 DUP

$\$ 977000605 \cdot 0$

TGA-OA

SOLID W/A $\%$

$97000261 \quad T-204$

4 SAMPLE

\$97T000606 0

TGA-01

SOLID

$\mathrm{N} / \mathrm{A}$ $\%$

$97000261 T-204$

5 DUP

$597 T 000606 \quad 0$

TCA-01

SOLID

N/A $\%$

\section{Final page for worklist \# 17842}

\section{Chetron Puint 5-16-97}

Analyst Signature RODayn
Date

$5 / 17 / 57$

\author{
Analyst Signature Date
}

Data Entry Comments:

samples run on TGA-03. Q8 $5 / 16 / 97$

Units shown for $Q C$ (SPK \& STD) may not reflect the actual units. $D L=$ Detection Limit, $S=$ Worklist Slot Number, $R=$ Replicate Number, $A=$ Aliquot Code. 
Curve 1: TGA

F1le info: TERO51601 Fri May 16 03: 43: 38.1997

Sample Height: 14.009 mg

TGA STD 97NB-A

SIGNATURE BELOW REPRESENTS CHEMICAL TECHNOLOGST/CHEMIST THN COMPLETEDNERIFIED THE CALBRATIONIANALYSIS ON PAGES2OC 6 TO 210.

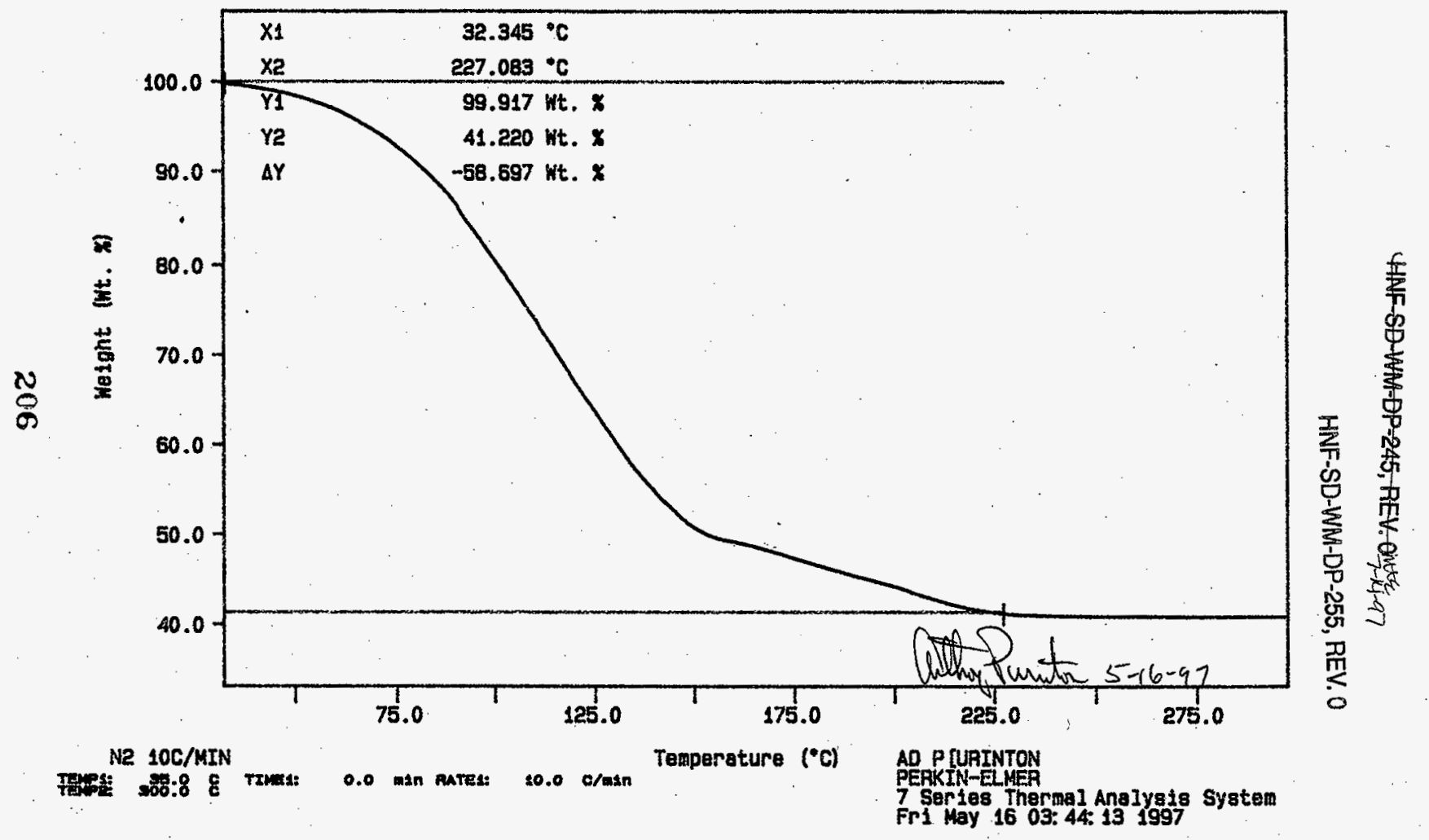


Curve 1: TGA

File Info: SAM051601 Frt May 16 04: 44:58 1997

Sample Weight: 37.374 ag

597T000605 SAM

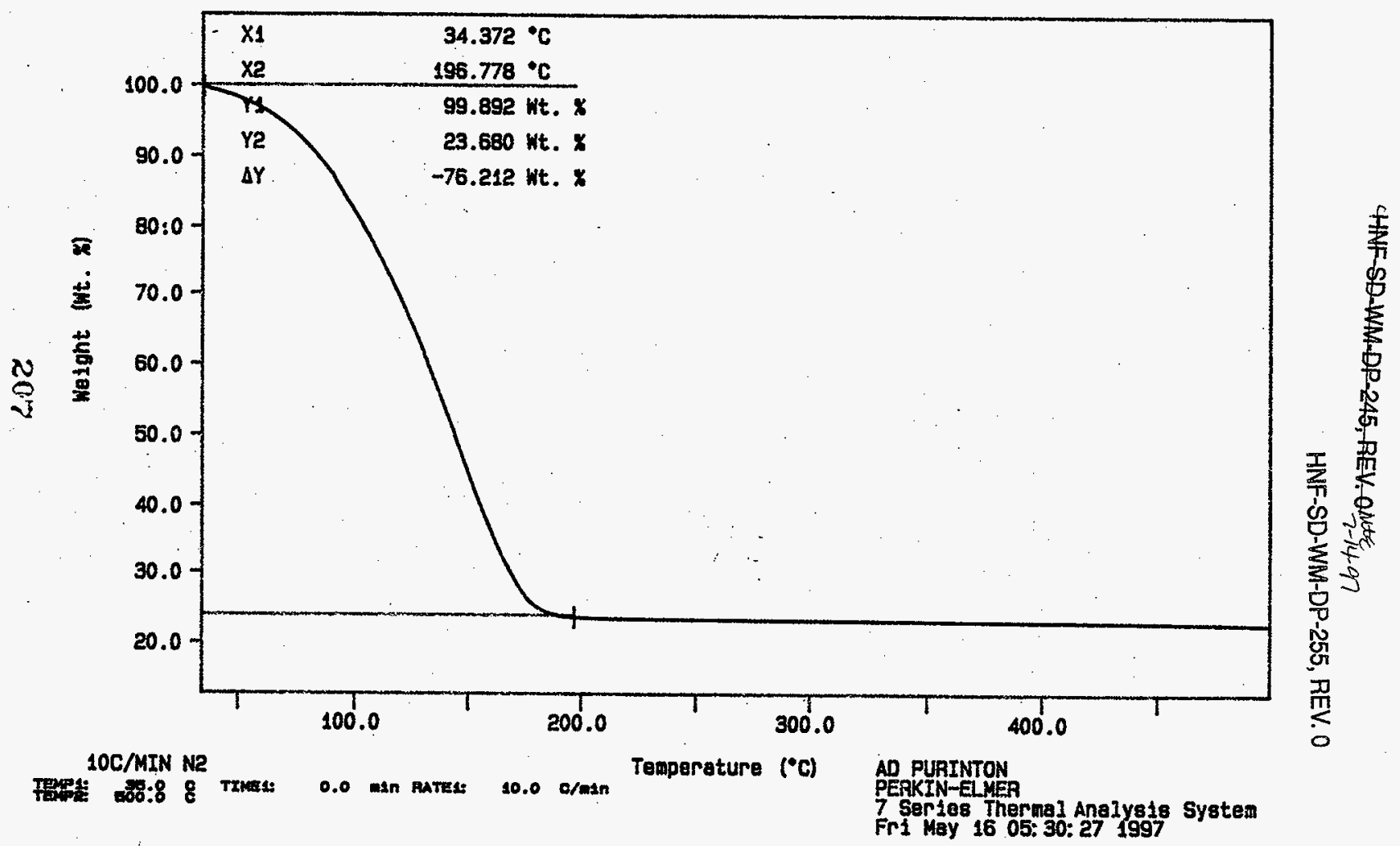


4NF-SD-WM-DP-245, REV.QMUt/4I-97

HNF-SD-WM-DP-255, REV. 0

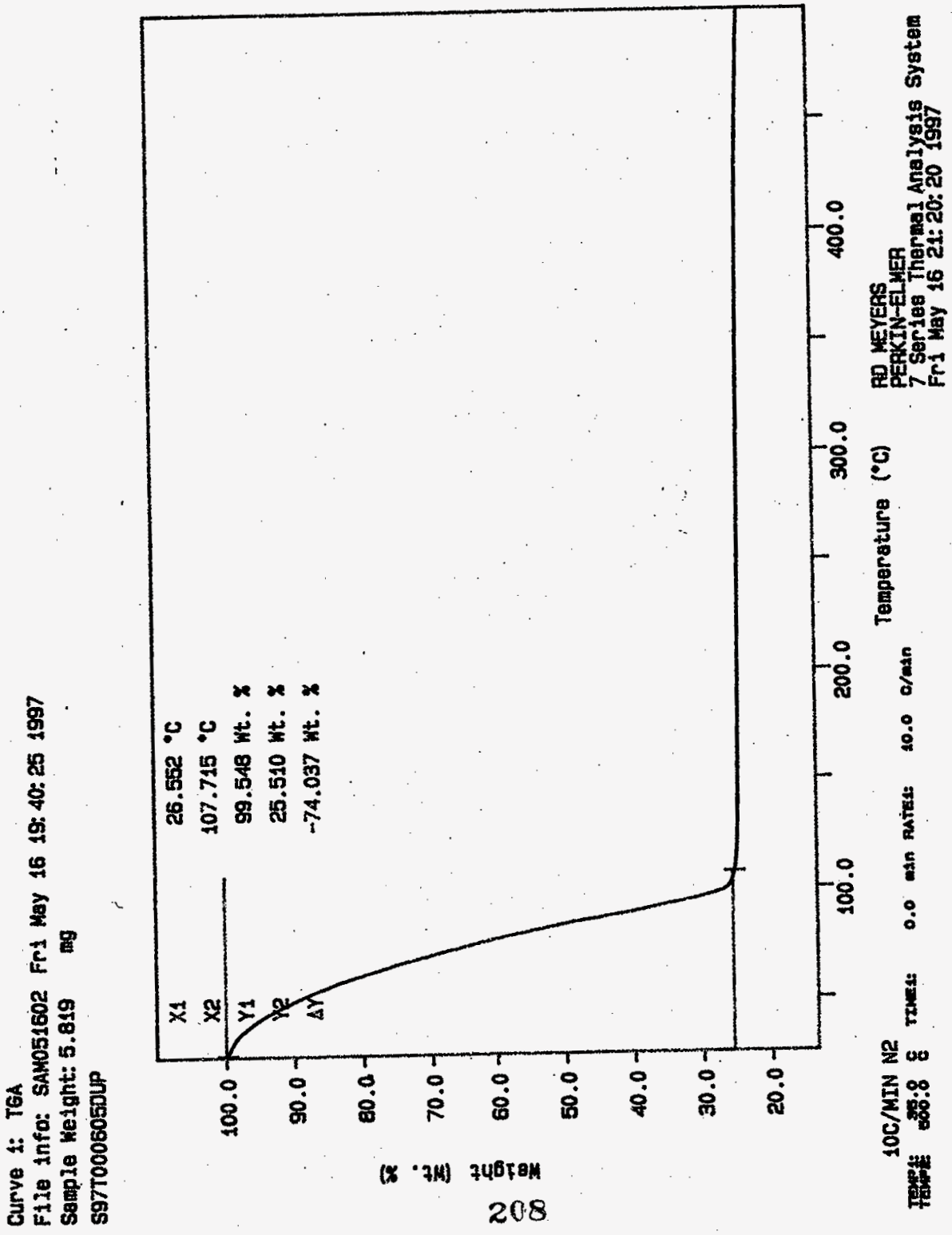


HANF-SD-WAM-DP-245,REV-0 $7-14-97$

HNF-SD-WM-DP-255, REV. 0

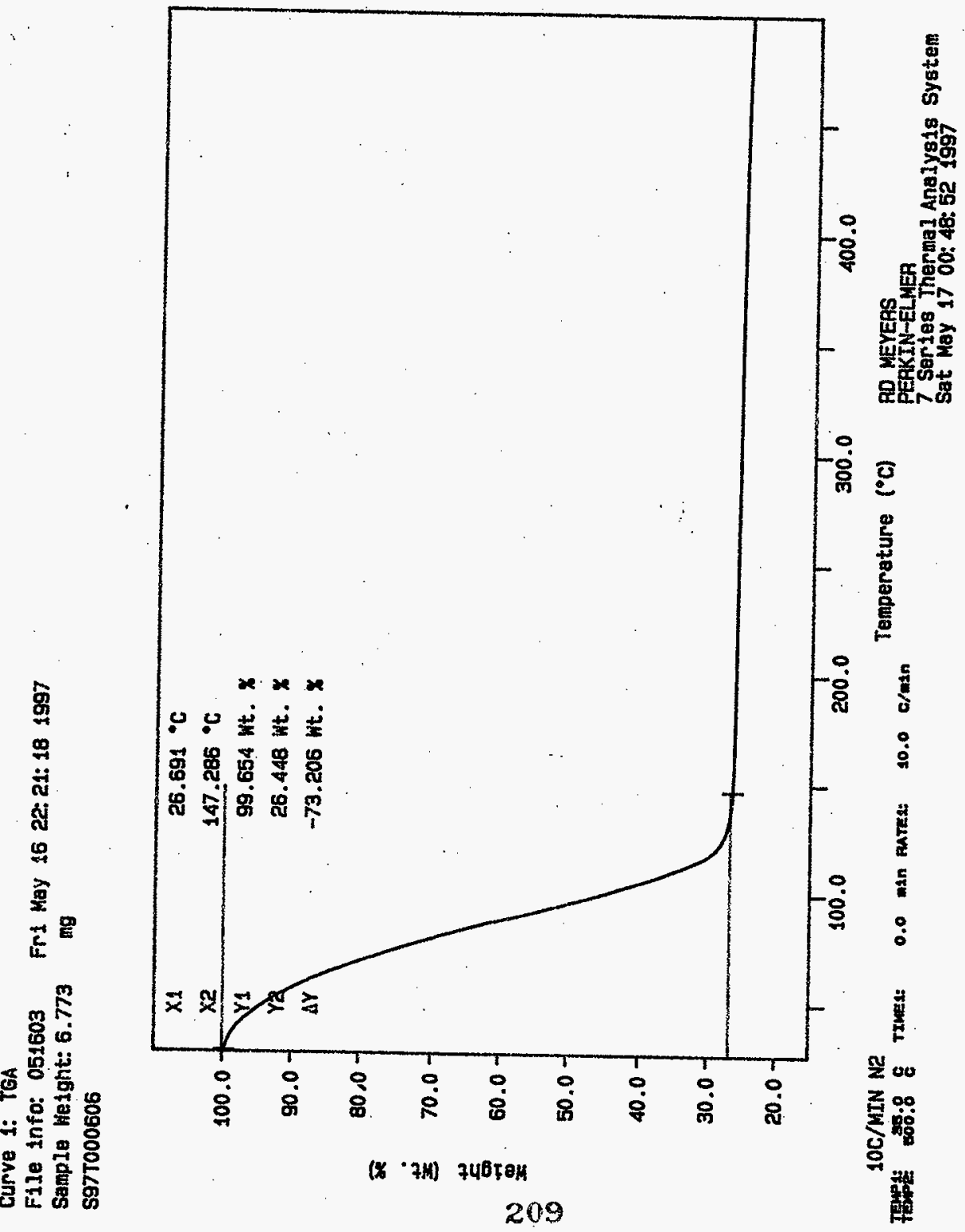


HNF-SD-WAM-DP-245, REV. OMEE $7-1497$

HNF-SD-WM-DP-255, REV. 0

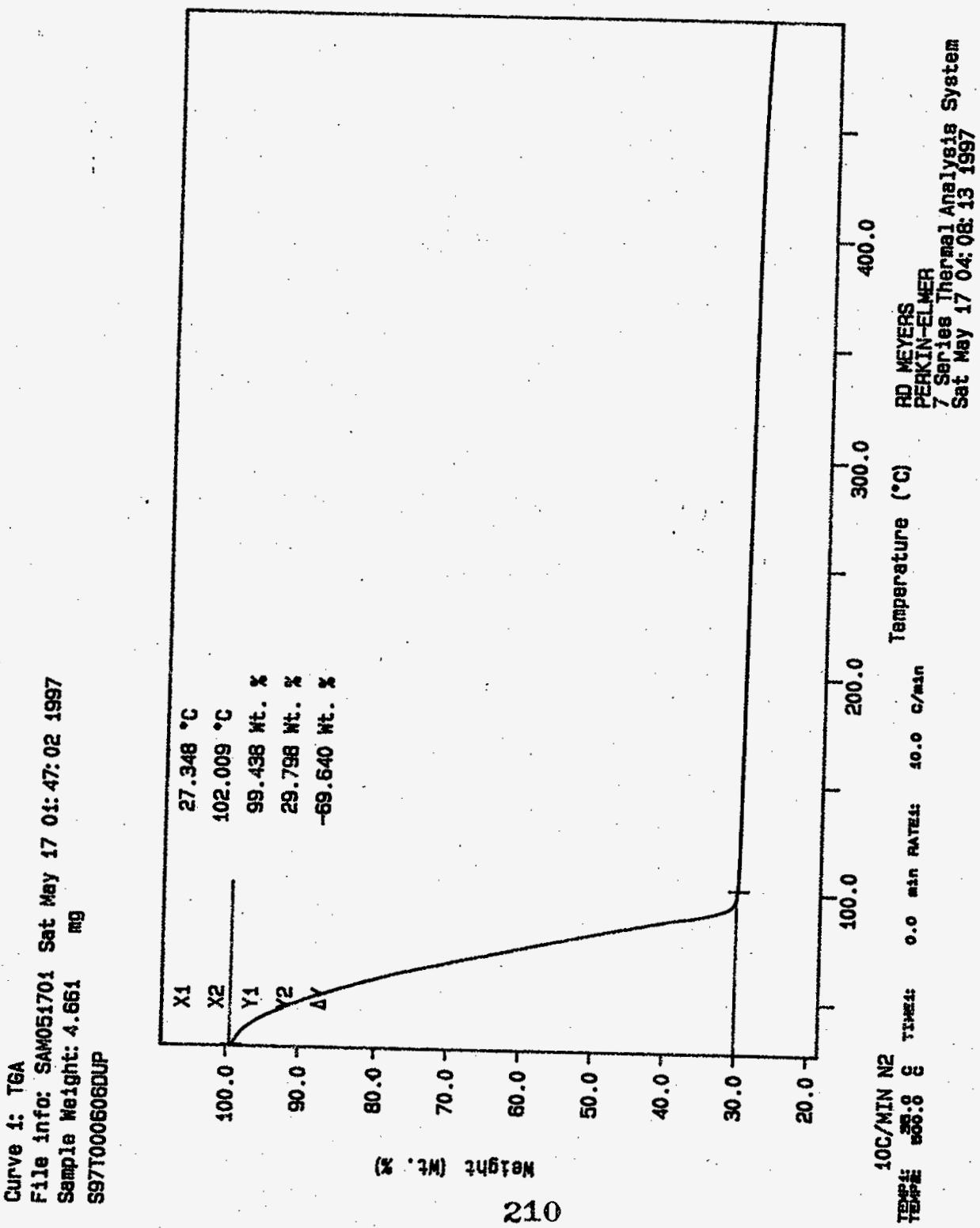




\section{LABCORE Data Entry Template for Worklist\# 17843}

Page: $\quad 1$

\section{Analyst: $\quad$ RDM Instrument: TGA0 3 3 Book \# 97 N8A}

Method: LA-514-114 Rev/Mod D-O

Worklist Comment: T204, TGA-01 Run under nitrogen. skm

HNF-SD-WM-DP-255, REV. 0

\begin{tabular}{|c|c|c|c|c|c|c|c|c|c|c|}
\hline GROUP & PROJECT & $\begin{array}{l}\text { S TYPE } \\
1 \text { STD }\end{array}$ & SAMPLE\# & $\mathrm{RA}$ & TEA-03 & $\begin{array}{l}\text { MATR1X } \\
\text { SOL10 }\end{array}$ & $\begin{array}{l}\text { ACTUAL } \\
59.4\end{array}$ & $\begin{array}{l}\text { FOUND } \\
58.24 \\
\end{array}$ & $\begin{array}{l}\mathrm{DL} \\
\mathrm{N} / \mathrm{A} \\
\end{array}$ & UHI \\
\hline 97000261 & $T \div 204$ & 2 SAMPLE & $\$ 977000607$ & 0 & TGA-03 & SOLID & N/A & 74.42 & & - \% \\
\hline 97000261 & $T-204$ & 3 DUP & 5971000607 & 0 & TGA-03 & SOLIO & 24.5 & 75.99 & $N / A$ & $\%$ \\
\hline 97000261 & $T-204$ & 4 SAMPLE & s97T000608 & 0 & TGA-03. & SOLID & N/A & 73.89 & & $\%$ \\
\hline 97000261 & $T-204$ & 5 DUP & S97T000608 & 0 & TEA-03 & SOLID & 23.89 & 74.98 & & $\%$ \\
\hline
\end{tabular}

\section{Final page for worklist \# $\mathbf{1 7 8 4 3}$}

Seel Attached for Signature.

Analyst Signature Date

validated 5/22/97\% \$hacheloz

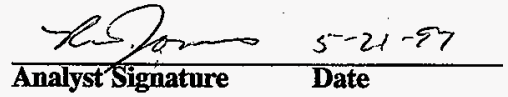

Analyst Signature Date

Data Entry Comments:

Units shown for. $Q C$ (SPK \& STD) may not reflect the actual units. $D L=$ Detection Limit, $S=$ Worklist Slot Number, $R=$ Replicate Number, $A=$ Aliquot Code. 


\section{LABCORE Data Entry Template for Worklist\# 17843}

\section{Analyst: $\mathrm{EDN}$ Instriment: TGA0 3 Book \# I INGA \\ Method: LA-560-112 Rev/Mod D D D \\ HNF-SD-WM-DP-255, REV. 0}

Worklist Comment: T204, TGA-01 Run under nitrogen. skm

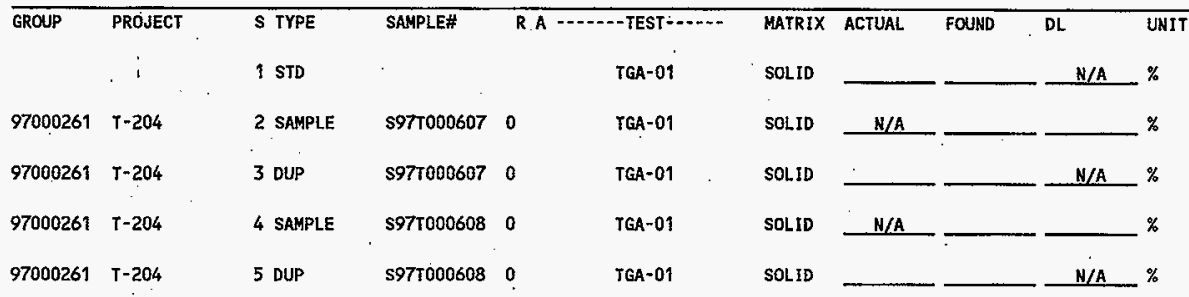

\section{Final page for worklist \# 17843}

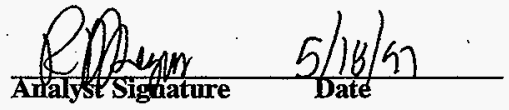

Analyst Signature Date

Data Entry Comments:

Units shown for $Q C$ (SPK \& STD) may not reflect the actual units. $D L=$ Detection Limit, $S=$ Worklist Slot Number, $R=$ Replicate Number, $A=$ Aliquot Code. 
Curve 1: TGA

File info: TER051701 Sat May 17 18:10:12 1997

Sample Netght: 14.239

ng

TGA STD 97NB-A

SIQNATURE BELOW REPRESENTS CHEMICAL TECHNOLOGIST/CHEMIST THAT

CONPLEISONERIFIEO THE CALLRATION/ANALYSIS ON PAGES313 T0217.

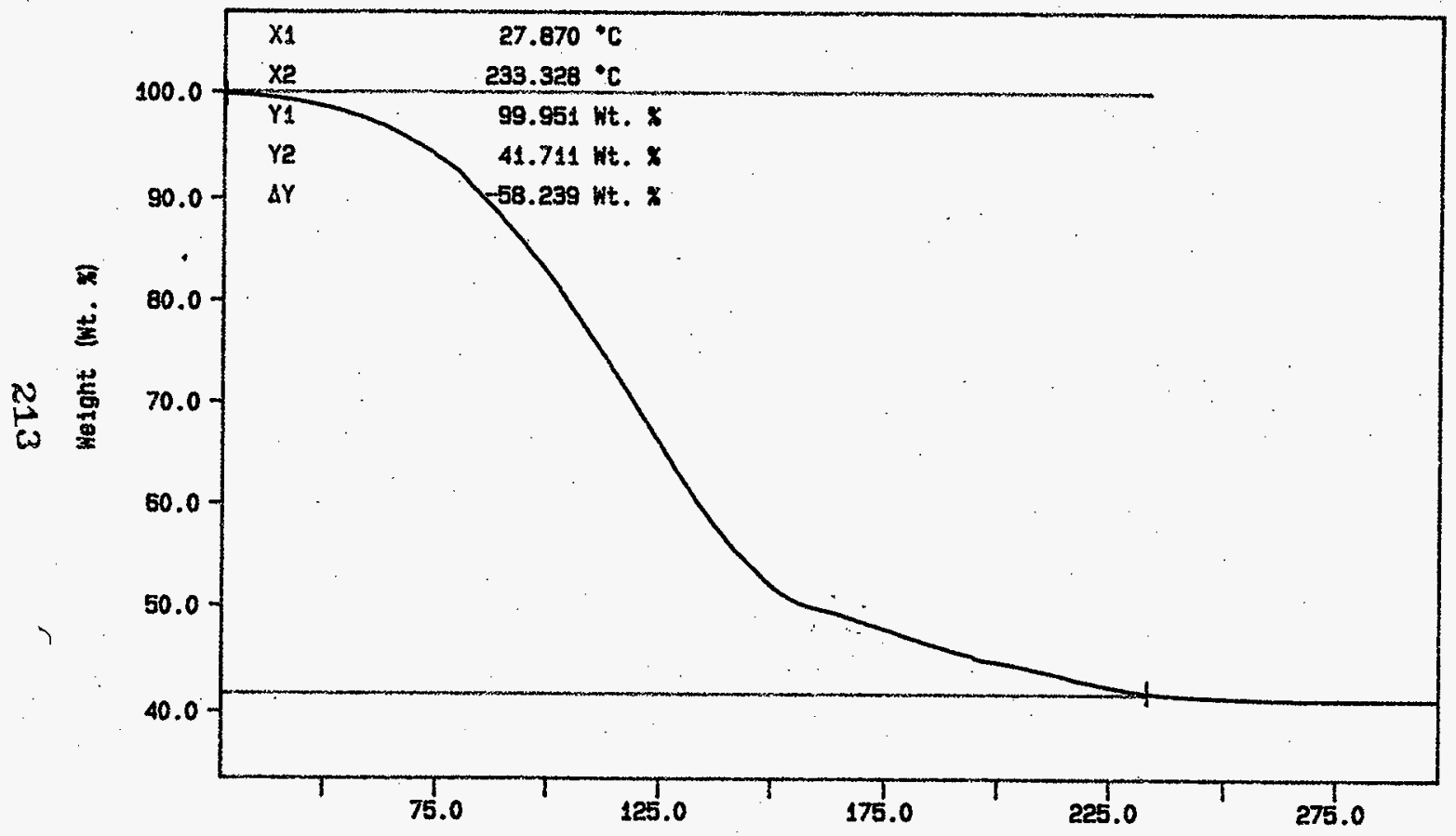

Ne $10 \mathrm{C} / \mathrm{MIN}$

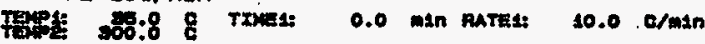

Teapereture $\left(^{\circ} \mathrm{Cl}\right.$ RD NEYERS

PERKIN-ELMER hesue Deag.for RDM 7 Series Thermel Analysis System Sat May 17 20:51: 42 1997 
Curve 1: TGA

F1le info: SAM051704. Sat May 17 21: $44: 541997$

Semple Wetght: 7.724

s977000607

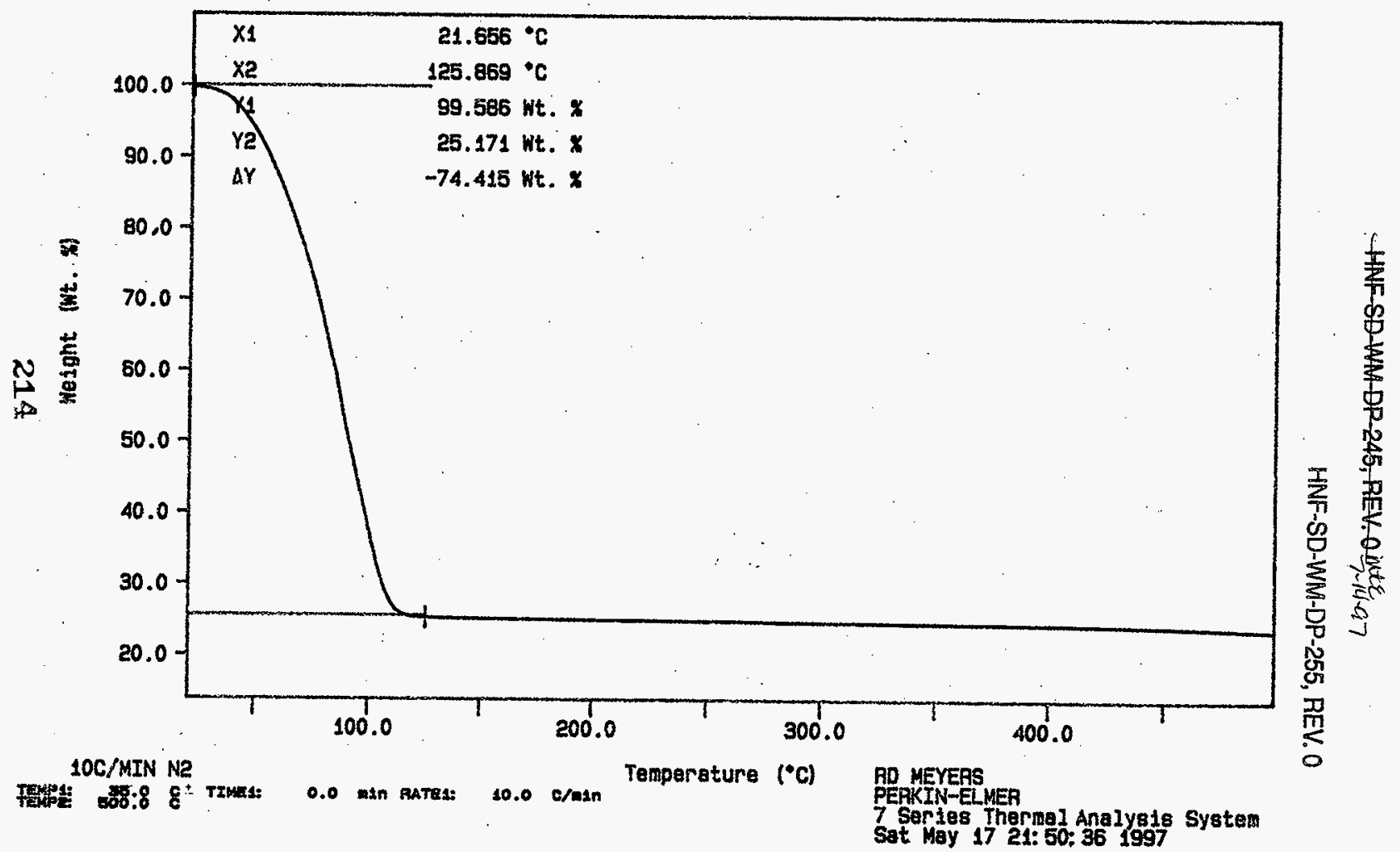


HNF-SD-WAM-DP-245, REV.0int

HNF-SD-WMI-DP-255, REV. 0

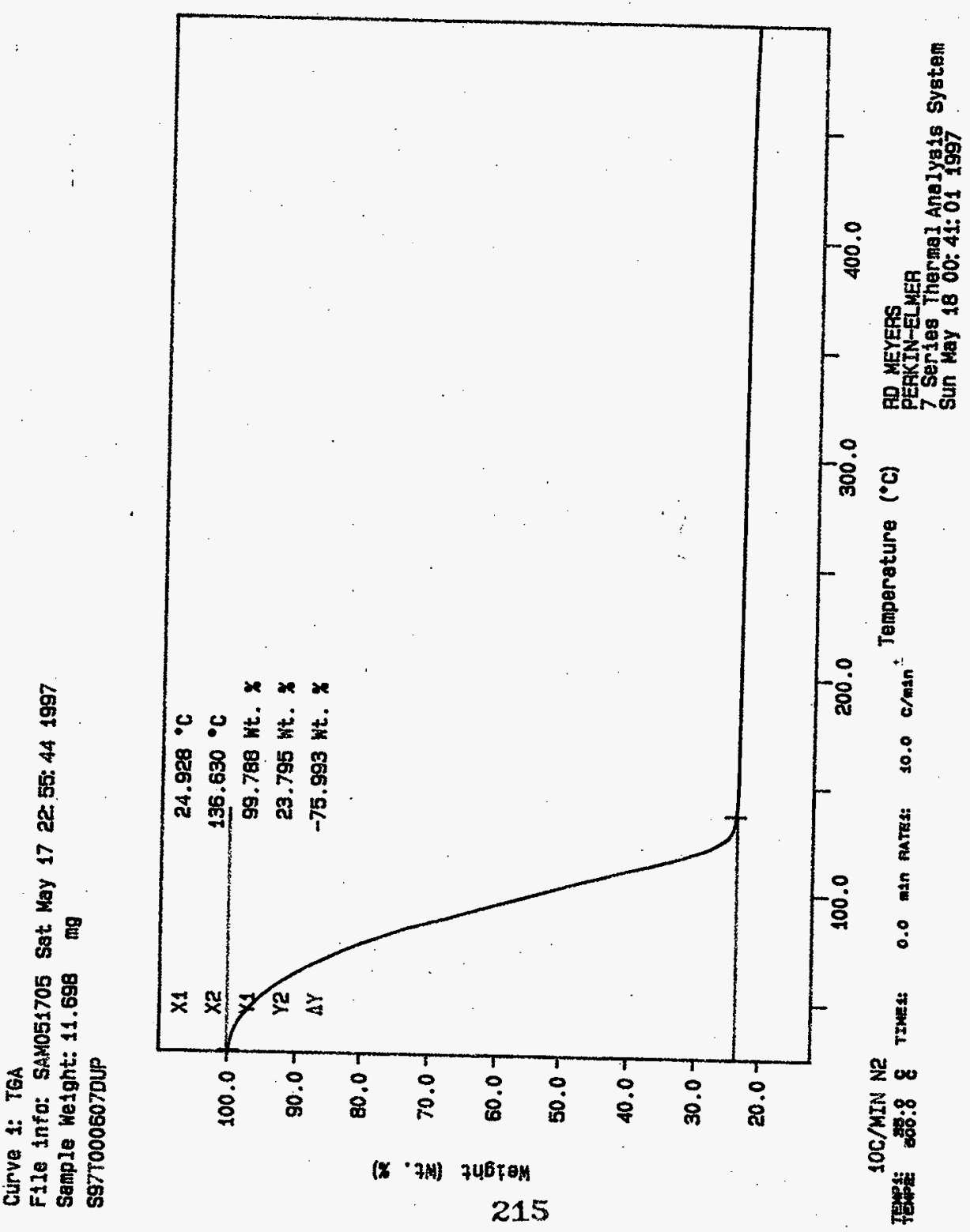


HAF-SD-WA-DP-245,REV.0int $7-14-97$

HNF-SD-WHA-DP-255, REV. 0

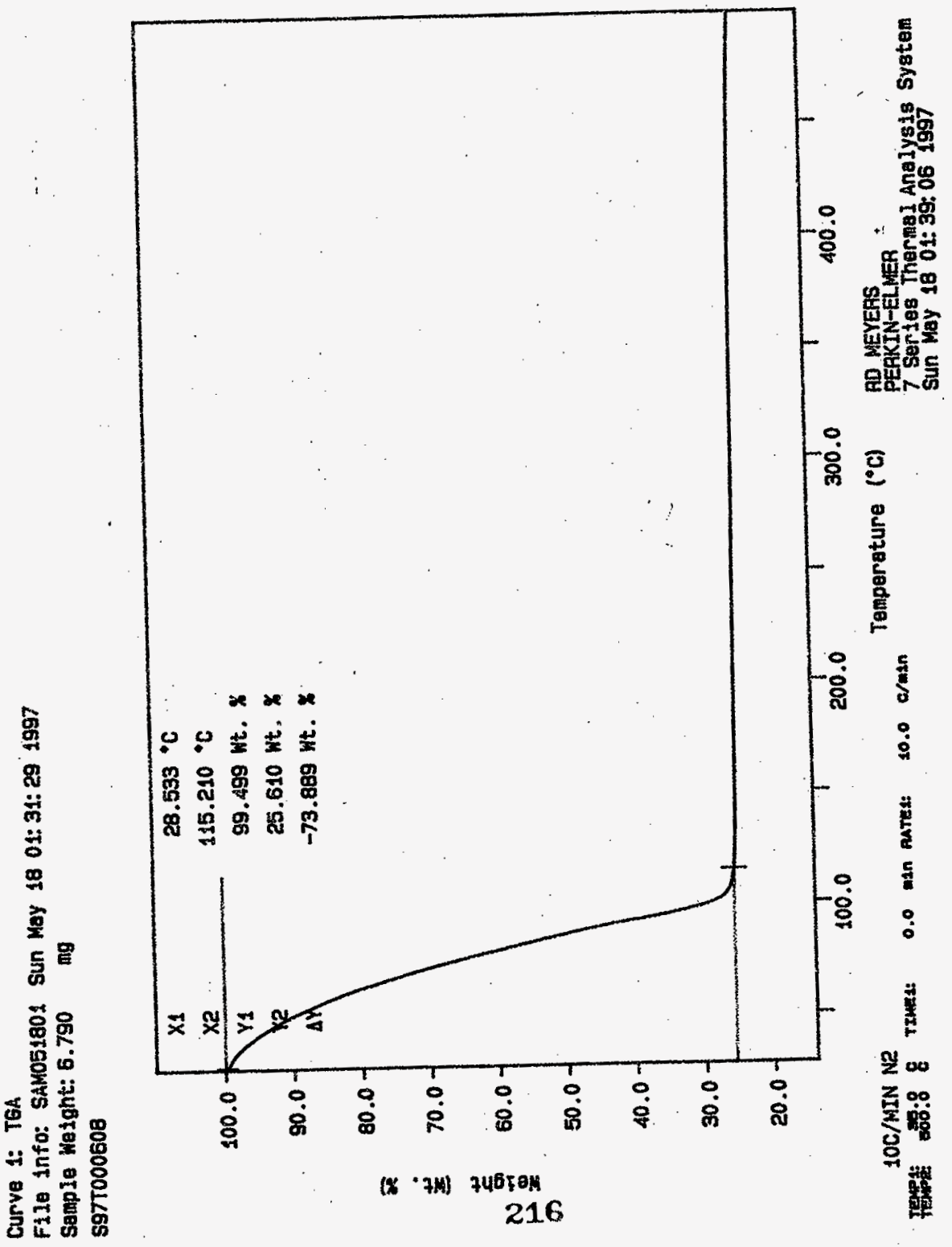


Curve 1: TEA

File info: SAM051802 sun May 18 02: $38: 29199 \%$

Sample Nelght: $9.511 \mathrm{mg}$

s97T0006080uP

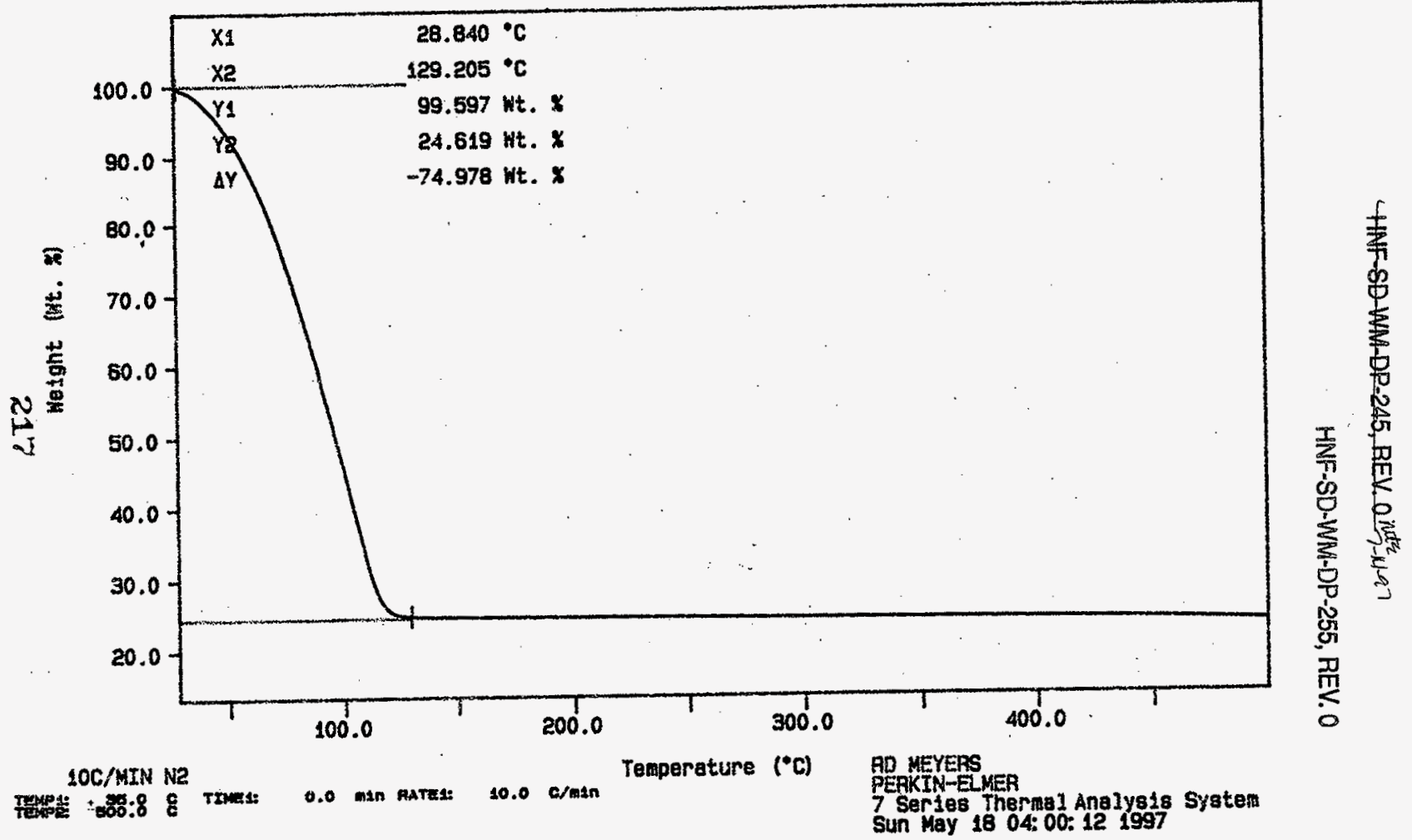


HNF-SD-WM-DP-245,REV.OMtE 7 -1497

HNF-SD-WM-DP-255, REV. 0

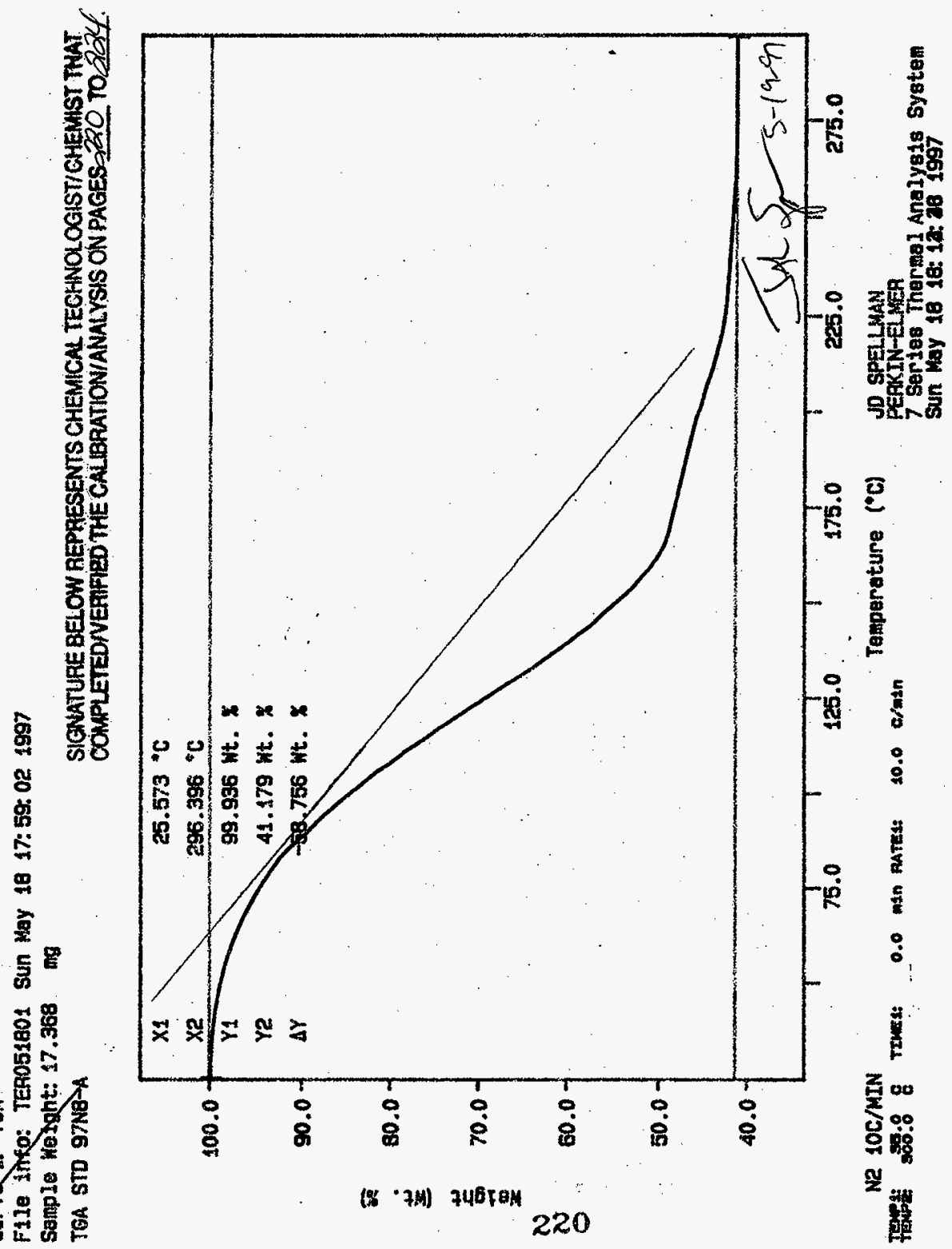


Curve 1: TGA

File info: SAM051803 Sun May 18 19: 30:29 1997

Sample Height: 24:624 ing

S97T000633

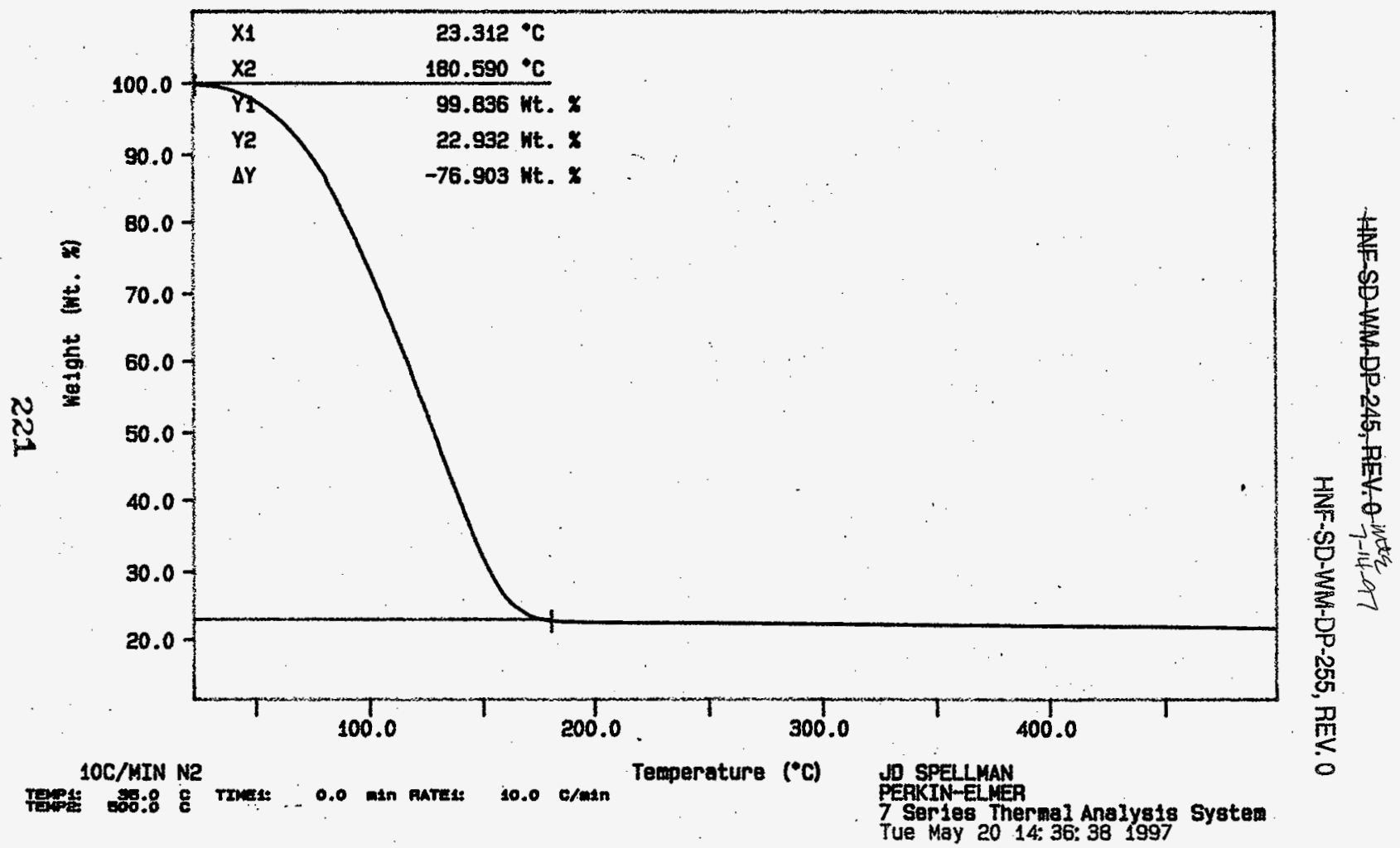


HNF-SD-WRA-DP-255, REV. 0

HAF-SD-WAAPP-245, REV Dint5 $-14-a 1$

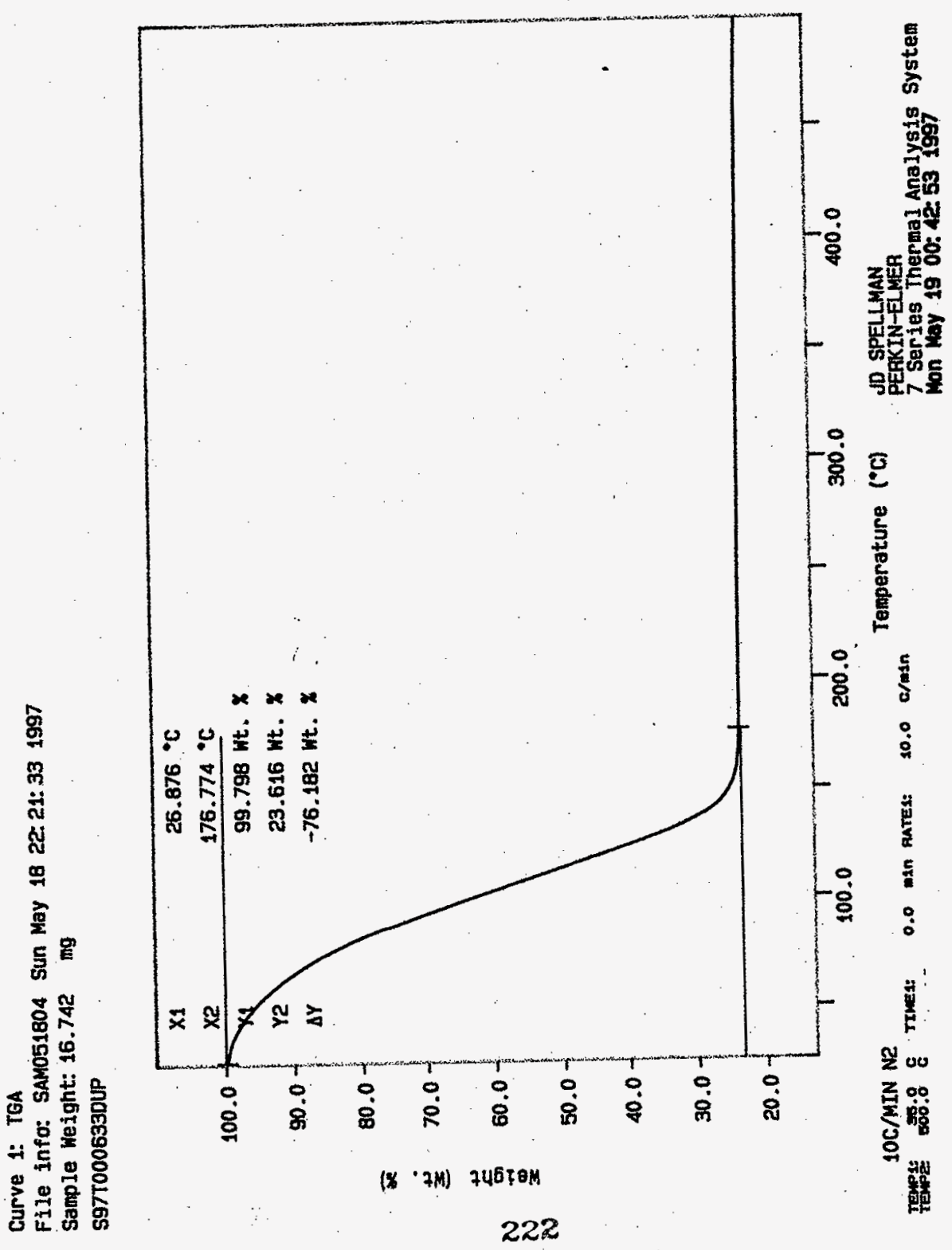


Curve 1: TGA

File info: SAM051605 Mon May 19 01: 35: 141997

Sample Keight: $16.615 \mathrm{mg}$

\$97T000634

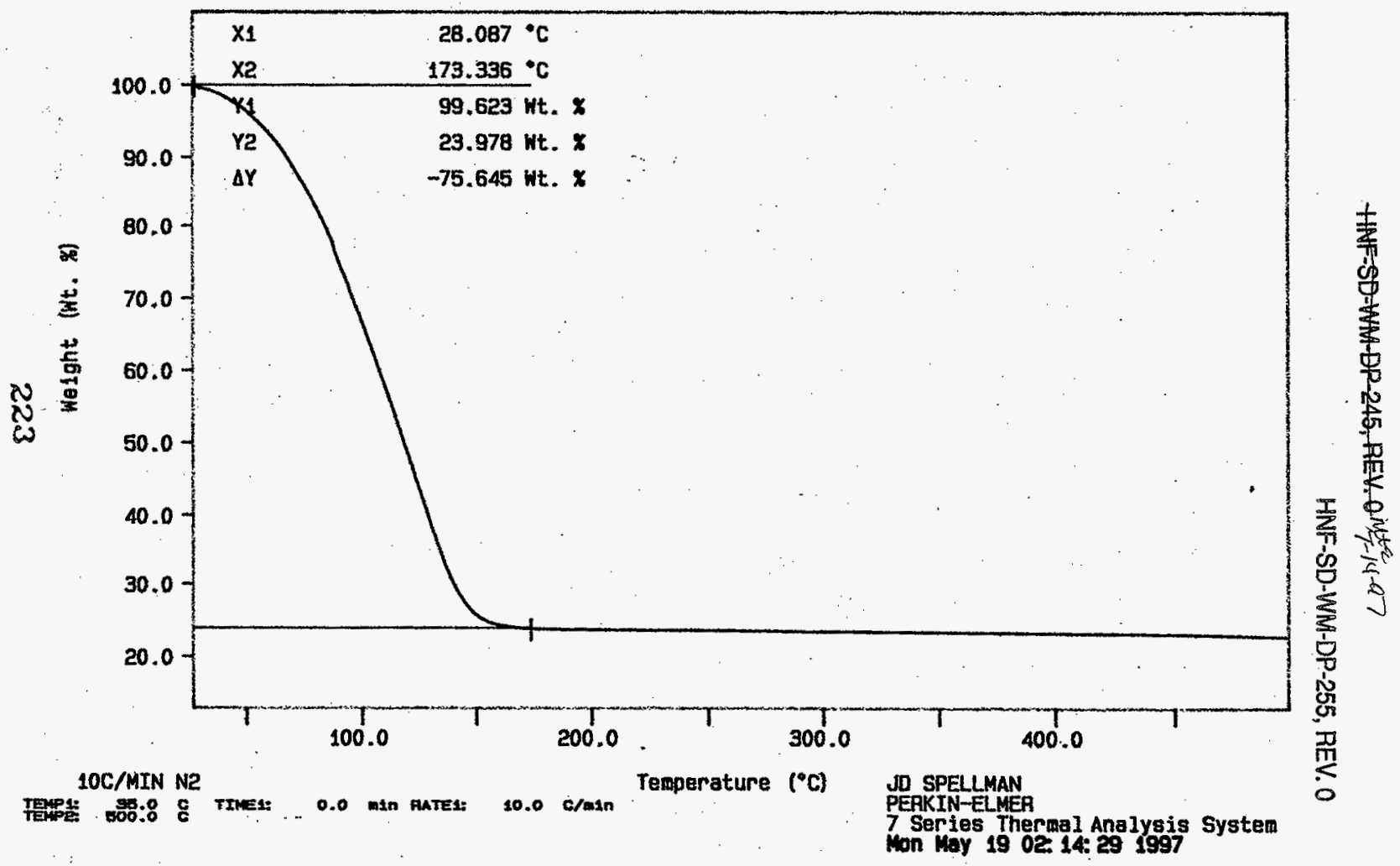


Curve 1: TGA

File info: SAM051806 Mon May. 19 03:07: 321997

Sample Weight: 22.823

S97T000634DUP

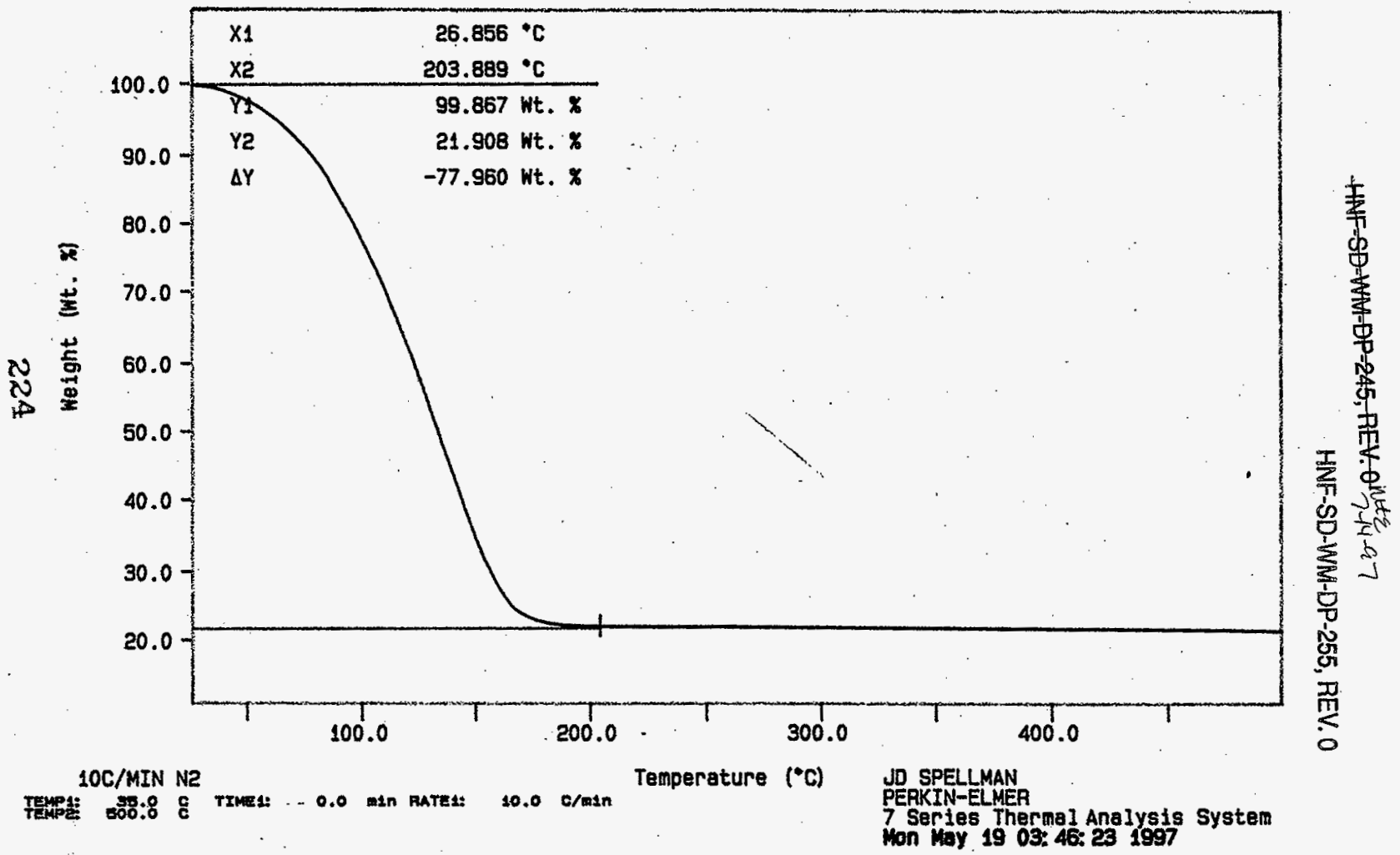




\section{LABCORE Data Entry Template for Worklist\# 17845}

Analyst: $\quad$ PJM Instrument: TGA0 3 300 _ $97 \mathrm{~N} 8 \mathrm{~A}$

Method: LA-514-114 Rev/Mod D-O

HNF-SD-WM-DP-255, REV. 0

Workdist Comment: T204, TGA-01 Run under nitrogen. skm

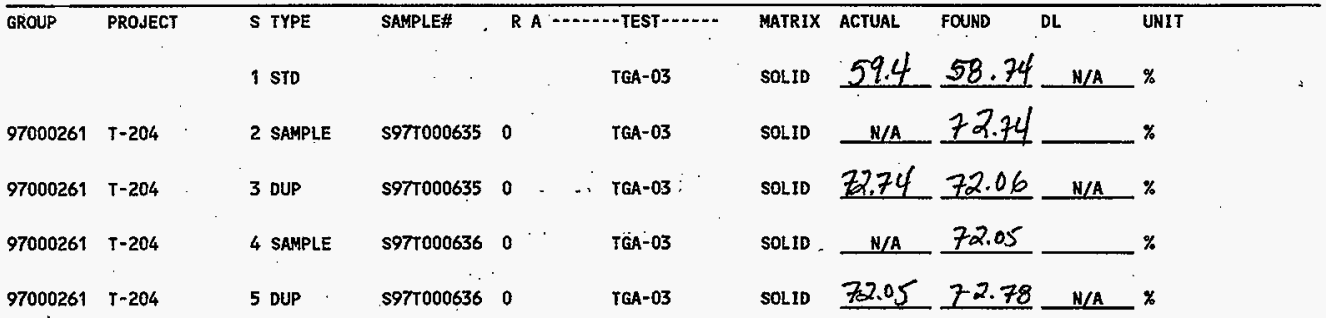

\section{Final page for worklist \# 17845}

See Attached for Signature Analyst Signature Bate Validated $6 / 2 / 97$ Bachelor

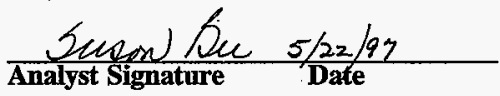

Data Entry Comments:

Units shown for $Q C$ (SPK \& STD) may not reflect the actual units. $D L=$ Detection Limit, $S=$ Worklist Slot Number, $R=$ Replicate Number, $A=$ Aliquot Code. 


\section{LABCORE Data Entry Template for Worklist\# 17845}

Analyst: MetM

Method: LA-560-112 Rev/Mod

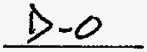

Book.\# 97N8A

Worklist Comment: T204, TGA-01 Run under nitrogen. skm

\begin{tabular}{|c|c|c|c|c|c|c|c|c|c|}
\hline GROUP & PROAECT & $\begin{array}{l}\text { S TYPE } \\
\text { I STD }\end{array}$ & SAMPLE\# & RA & $\begin{array}{l}\text { TEA }-01 \\
\text { TEST }\end{array}$ & $\begin{array}{l}\text { MATRIX } \\
\text { SOL ID }\end{array}$ & ACTUAL & FOUND & $\begin{array}{l}\mathrm{DL} \cdot \\
\mathrm{N} / \mathrm{A} \\
\end{array}$ \\
\hline 97000261 & $T-204$ & 2 SANPLE & S97T000635 & 0 & TGA-01 & SOLID & N/A & & \\
\hline 97000261 & $T-204$ & 3 DUP & \$97T000635 & 0 & TGA-0T & SOLID & & & $N / A$ \\
\hline 97000261 & $T-204$ & 4 SAMPLE & S97T000636. & 0 & TGA-01 & SOLID & N/A & & $\therefore$ \\
\hline 97000261 & $T-204$ & 5 DUP & S97T000636 & 0 & TGA-01 & SOLID. & & & N/A \\
\hline
\end{tabular}

\section{Final page for worklist \# 17845}

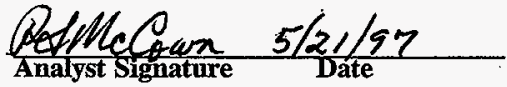

\section{Analyst Siguature Date}

Data Entry Comments:

Ran samples on TGA-03. Q83 5/21/97

Units shown for QC (SPK \& STD) may not reflect the actual units. $D L=$ Detection Limit, $S=$ Worklist Slot Number, $R=$ Replicate Number, $A=$ Aliquot Code. 
Curve 1: TGA

F1le info: TER052003 Tue May 20 23: 17:02 1997

Semple Wejght: 27.987

97NB-A

SIGNATURE BELOW REPRESENTS CHEMICN TECHNOLOGIST/CHEMIST IMAT

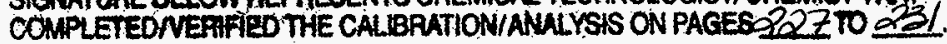

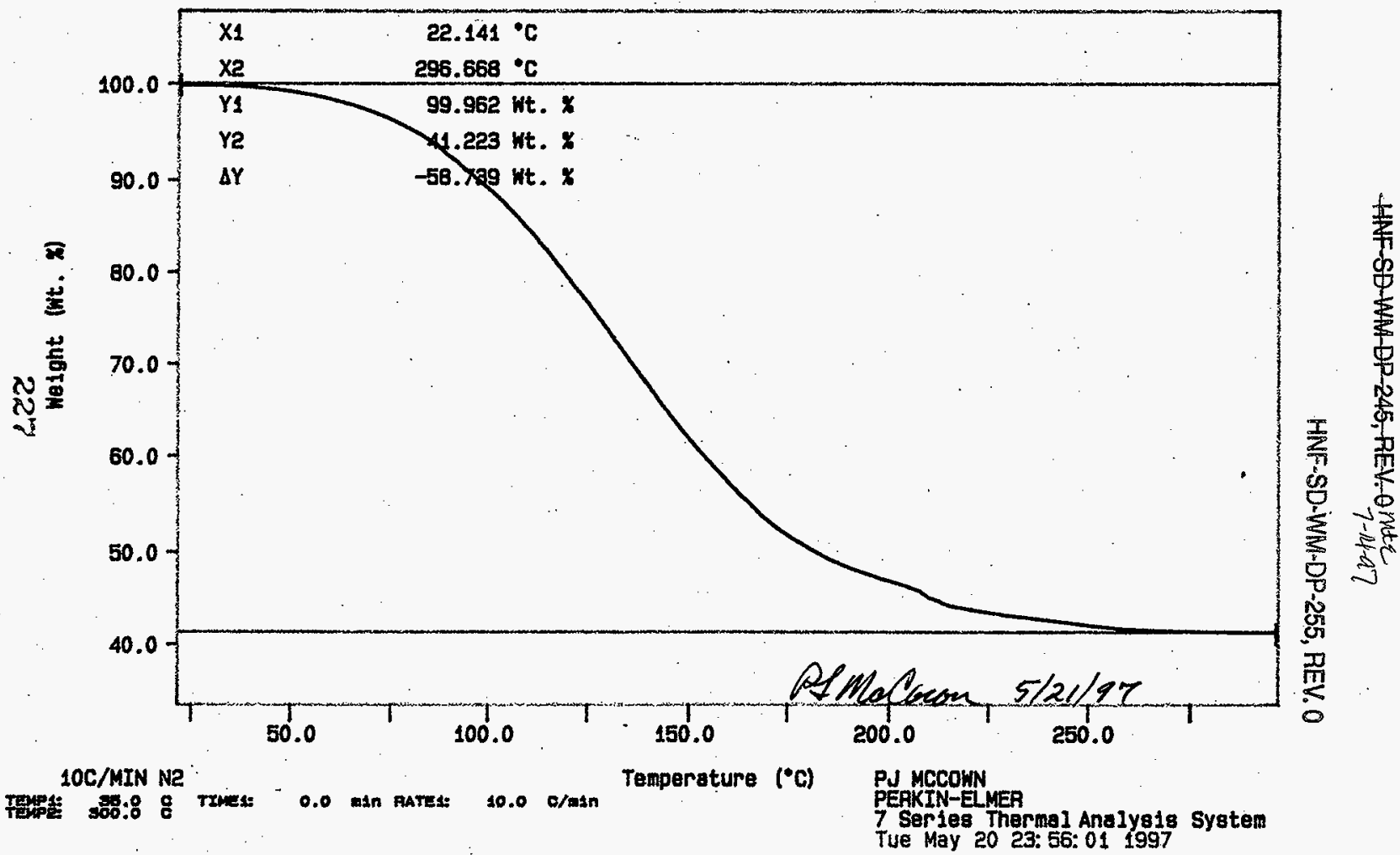


Curve 1: TGA

F1le Info: SAM052101 Wed May 21 00:56: 531997

Sample Weight: 8.761

\$977000635

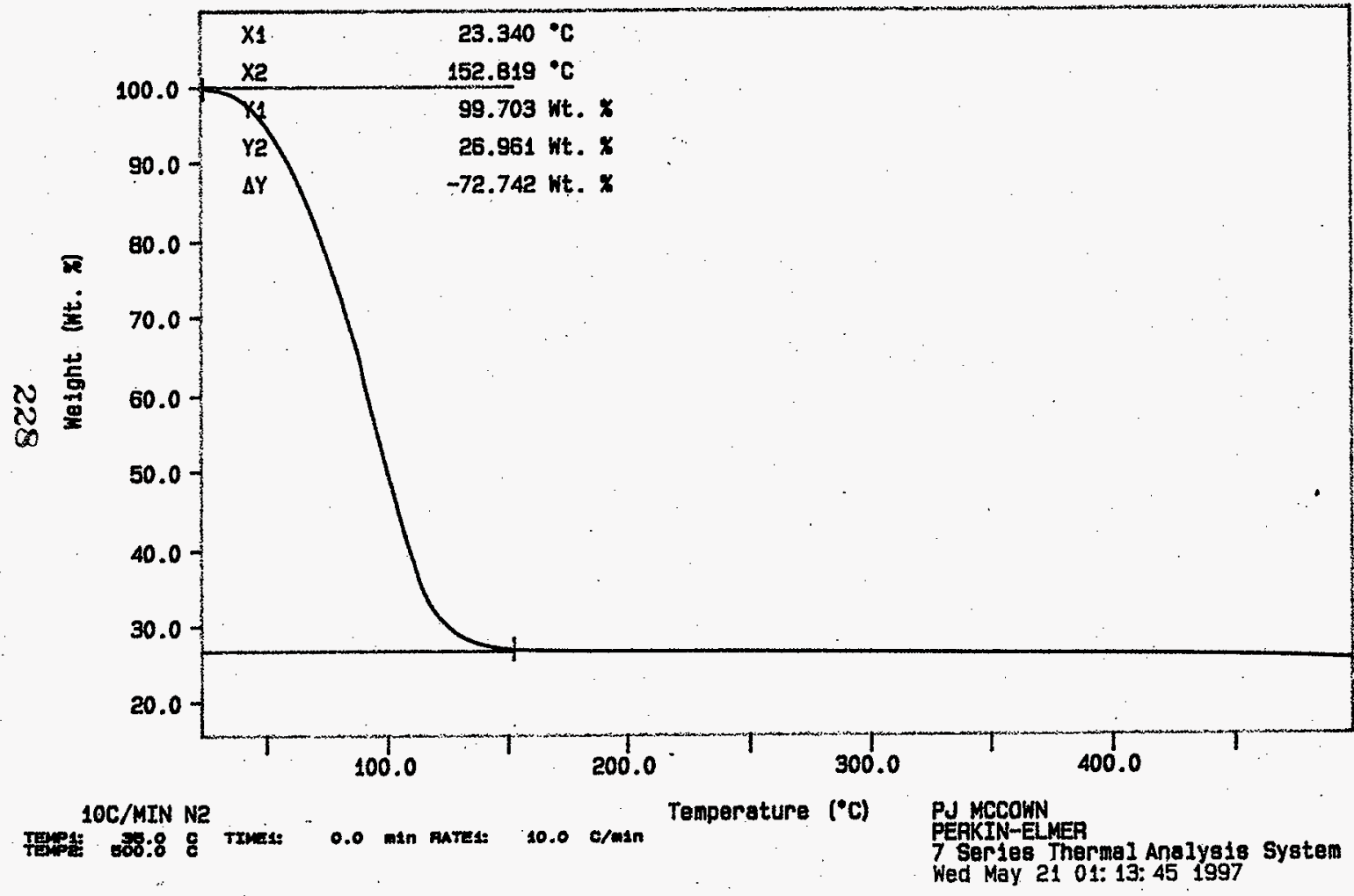


Curve 1: TGA

File info: SAM052102 Wed May 21 02: 16: 121997

Sample Weight: $12.983 \mathrm{mg}$

S97T000635 DUP

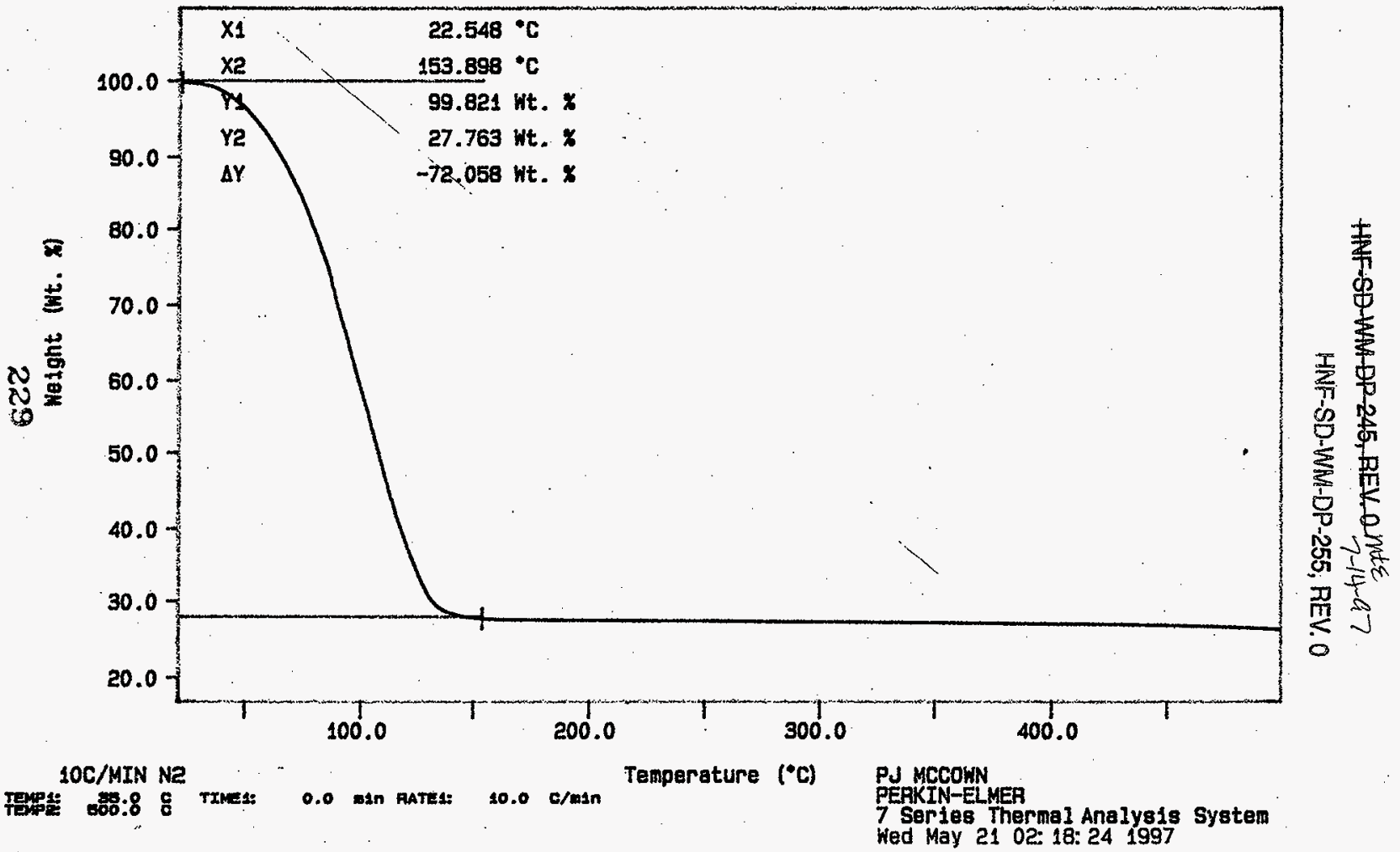


Curve 1: TGA

File info: SAM052103 Hed May 21 03: 25:56 1997

Sample Weight: $\mathbf{B . 2 6 4}$

S97T000636

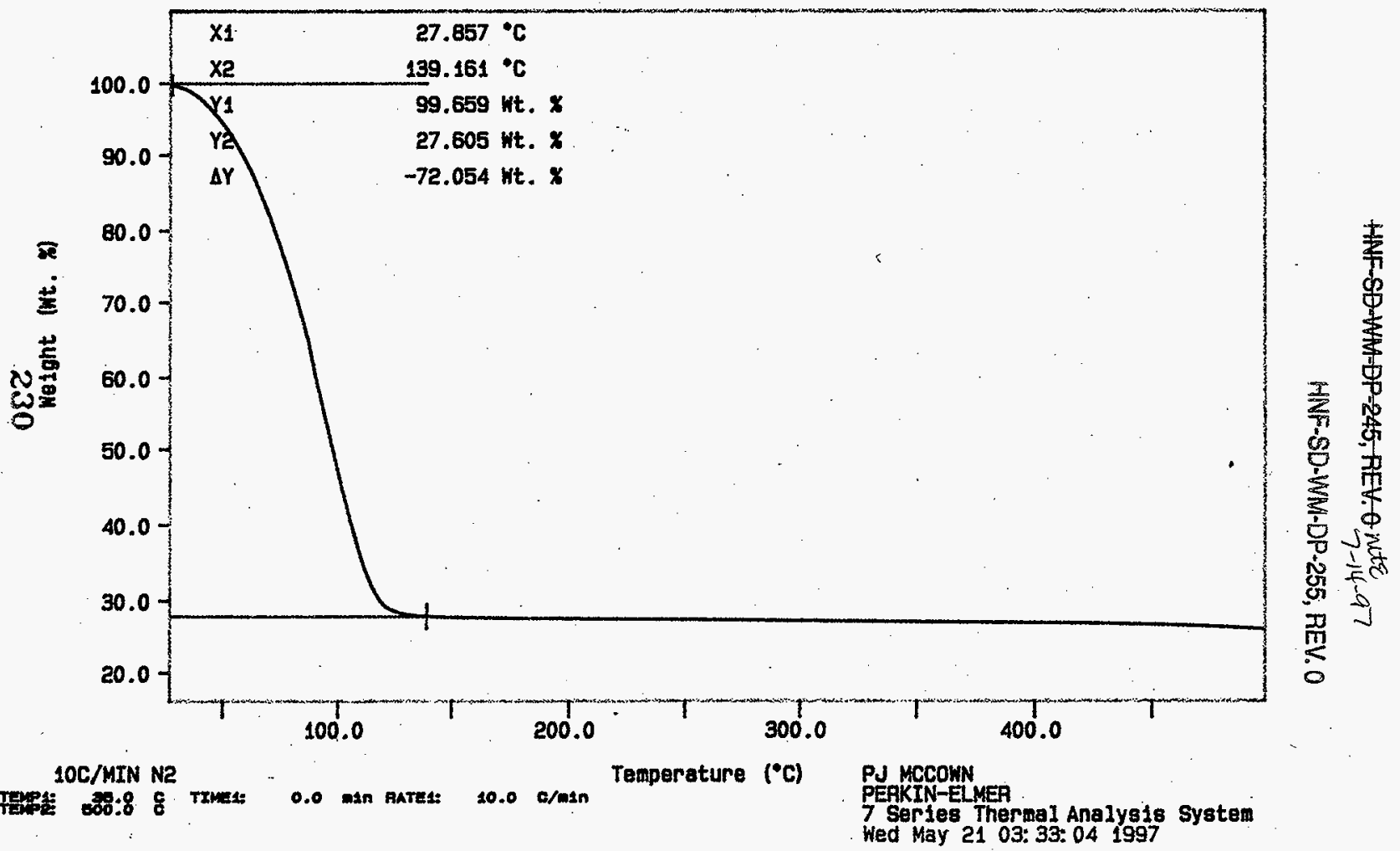


Curve 1: TEA

F士le info: SAM052104 Med May 21 04:38:54 1997

Sample Neight: 14.953 mg

S97T000636 DUP

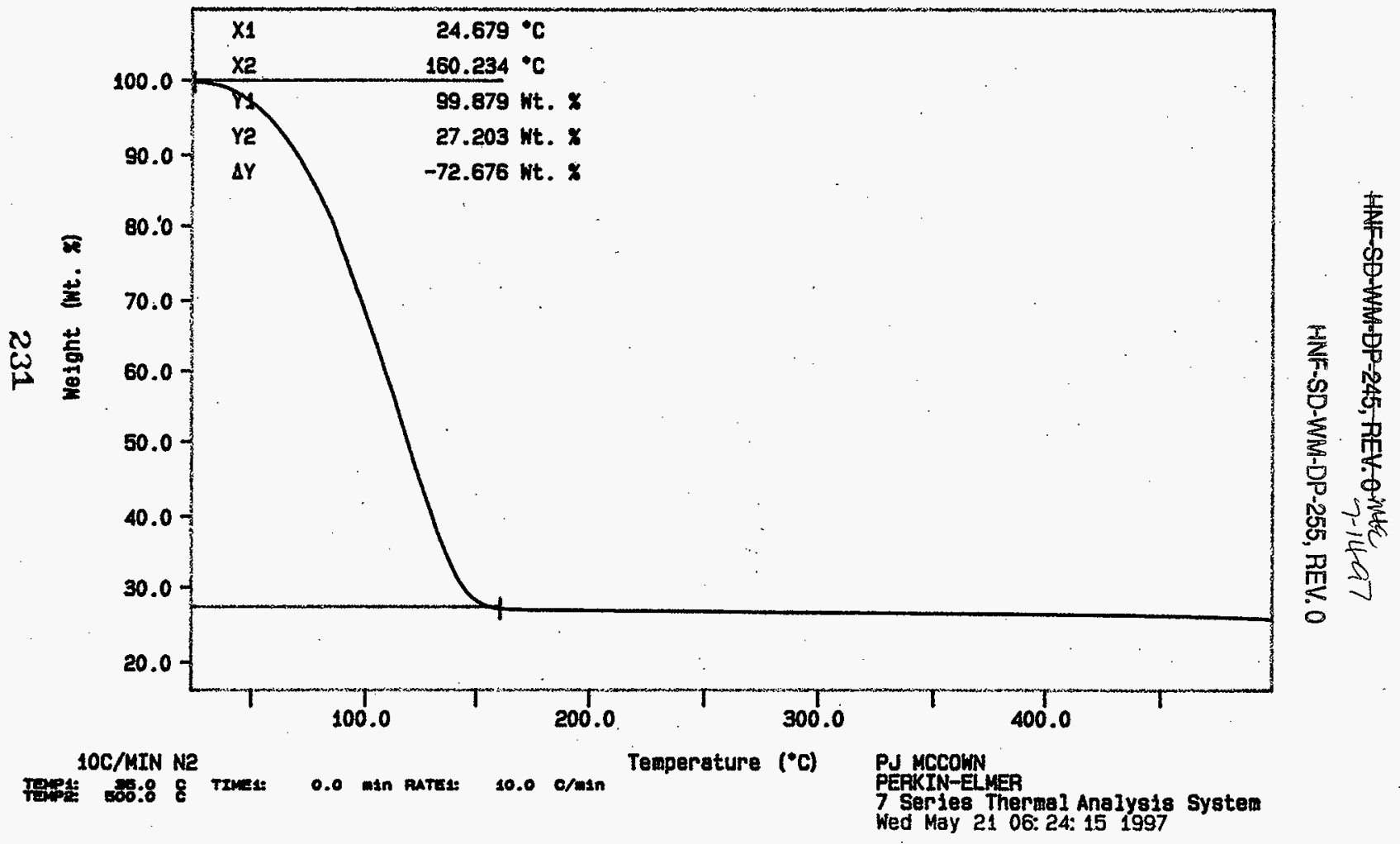




\section{LABCORE Data Entry Template for Worklist\# 17846}

\section{Analyst: $\quad$ ADP Instrument: TGA0 3 Book \# 97 N89}

Method: LA-514-114 Rev/Mod D-O

Worklist Comment: T204, TGA-01 Run under nitrogen. skm

\begin{tabular}{|c|c|c|c|c|c|c|c|c|c|c|}
\hline GROUP & $\begin{array}{l}\text { PROJECT } \\
:\end{array}$ & $\begin{array}{l}\text { S TYPE } \\
1 \text { STO }\end{array}$ & SAMPLE\# & & $\begin{array}{c}\text { A } 1 \text { TEST } \\
\text { TGA- } 03\end{array}$ & $\begin{array}{l}\text { MATRIX } \\
\text { SOLID }\end{array}$ & $\begin{array}{l}\text { ACTUAL } \\
59.4 \\
\end{array}$ & $\begin{array}{l}\text { FOUND } \\
58.10 \\
\end{array}$ & $\begin{array}{l}\mathrm{OL} \\
\mathrm{N} / \mathrm{A} \\
\end{array}$ & $\begin{array}{l}\text { UNIT } \\
\%\end{array}$ \\
\hline 97000261 & $\tau-204$ & 2 SAMPLE & S97T000637 & 0 & TGA-03 & SOLID & N/A & 75.63 & & $\%$ \\
\hline 97000261 & $T-204$ & 3 DUP & $597 T 000637$ & 0. & TGA-03 & SOLID & 75.63 & 75.63 & $\mathrm{~N} / \mathrm{A}$ & $\%$ \\
\hline 97000261 & $T-204$ & 4 SAMPLE & 5971000638 & 0 & TGA-03 & SOLID & $N / A$ & 75.24 & & $\%$ \\
\hline 97000261 & $r-204$ & 5 DUP & s97T000638 & 0 & TEA-03 & SOLID & 75.2 & 74.74 & N/A & $\%$ \\
\hline
\end{tabular}

\section{Final page for worklist \# 17846}

See Aftached for Signature Analyst Signature . Date

Rejected 5/21/97\$rachelor

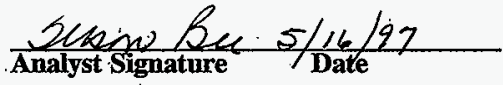

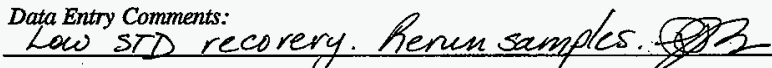

Units shown for $Q C$ (SPK \& STD) may not reflect the actual units. $D L=$ Detection Limit, $S=$ Worklist Slot Number,

$R=$ Replicate Number, $A=$ Aliquot Code. 


\section{LABCORE Data Entry Template for Worklist\# 17846}

Analyst: ADP Instrument: TGA0 3 Book \# 97N8A

Method: LA-560-112 Rev/Mod

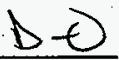

HNF-SD-WAM-DP-255, REV. 0

Worklist Comment: T204, TGA-01 Run under nitrogen. skm

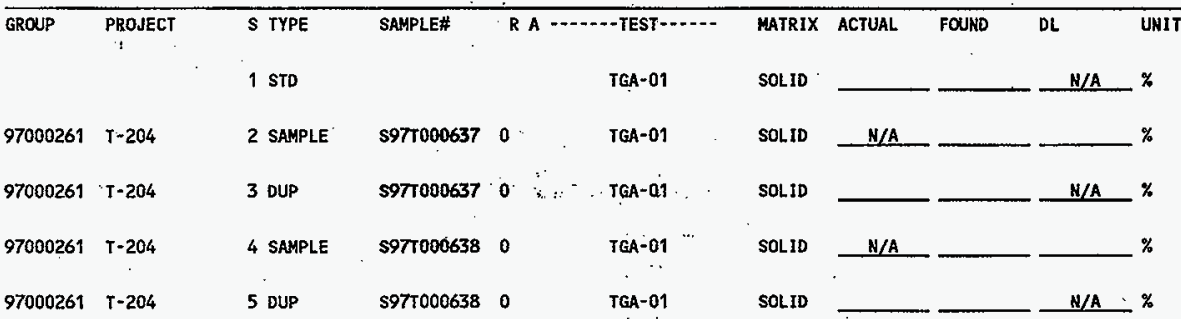

\section{Final page for worklist \# 17846}

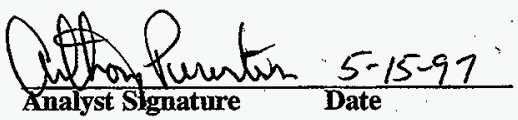

Analyst Signature Date

Data Entry Comments:
Samples rum on TGA-03. 30 . $5 / 16 / 97$

Units shown for QC (SPK \& STD) may not reflect the actual units. $D L=$ Detection Limit, $S=$ Worklist Slot Number,

$R=$ Replicate Number, $A=$ Aliquot Code. 
Curve 1: TGA

F1le info: TER051501 Thu May 15 02: 57:04 1997

Sample Weight: 14.820 mg

TGA STD 97NB-A

SICNATURE BELOW REPRESENTS CHEMCAL TECHNOLOGST/CHEMIST THAT COMPLETED/VETIEDTHE CALBRATIONIANALYSIS ON PACES 234 10 238

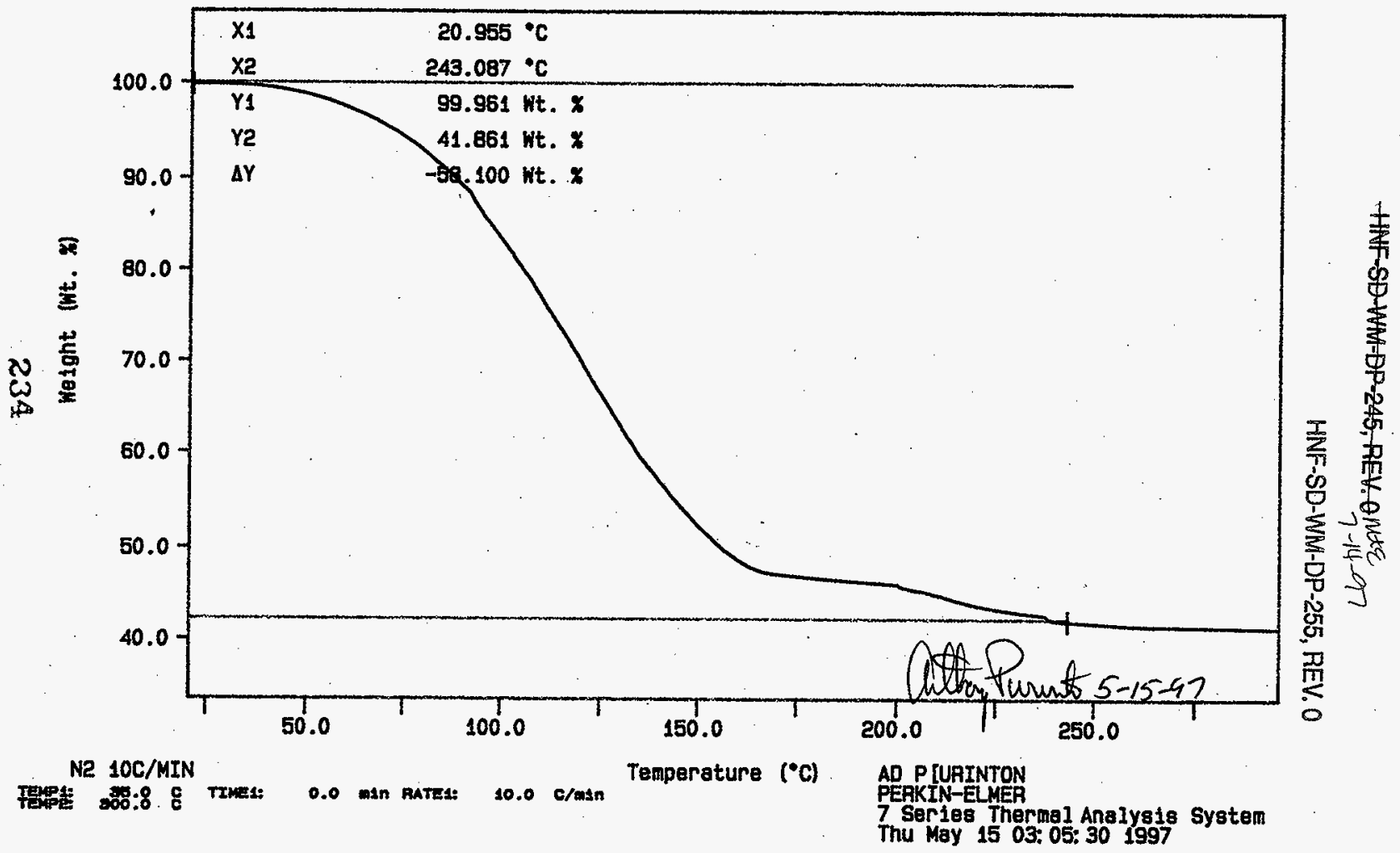


Curve 1: TGA

File info: SAM051501 Thu Nay 15 04: 10: 161997

Sample Weight: 12.397 mg

S97T000637 SAM

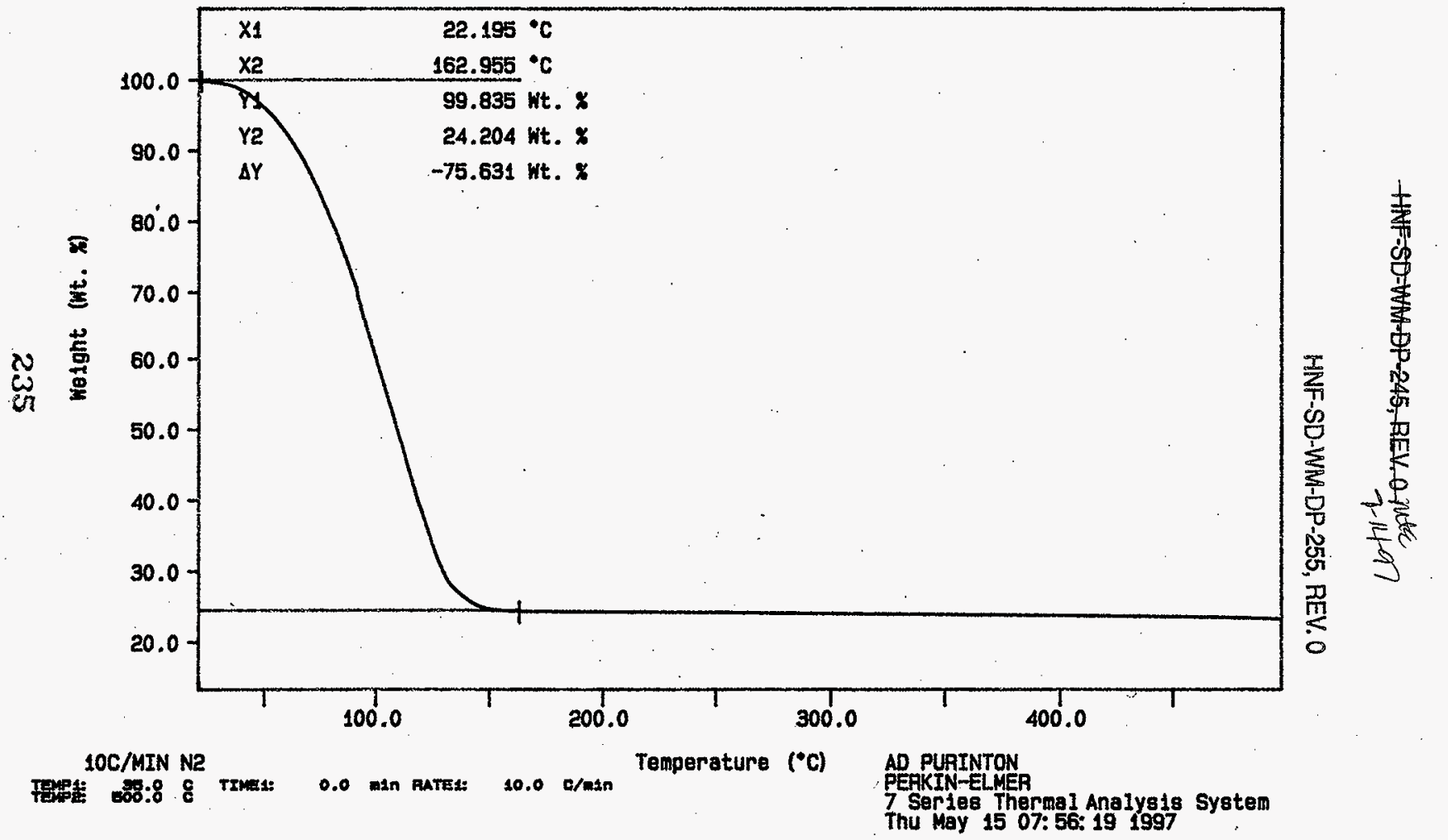


Curve 1: TGA

F1le info: SAM051502 Thu May 15 09: 24: 491997

Sample Wigight: $21.443 \mathrm{mg}$

\$97T000637 DUP

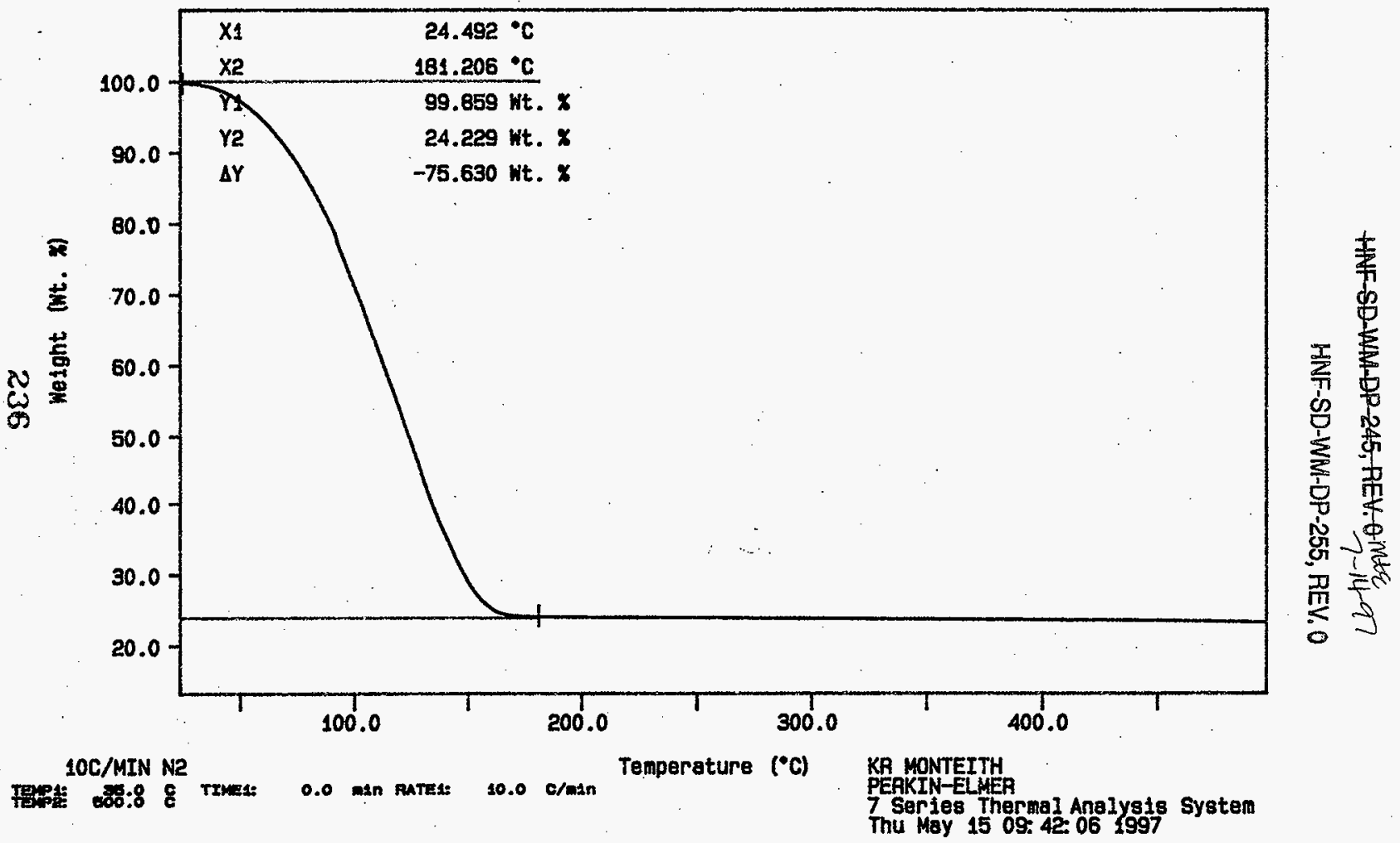


Curve 1: TGA

F1le info: SAM051503 Thu May 15 11: $16: 441997$

Sample Neight: 16.905

S97T000638 SAM

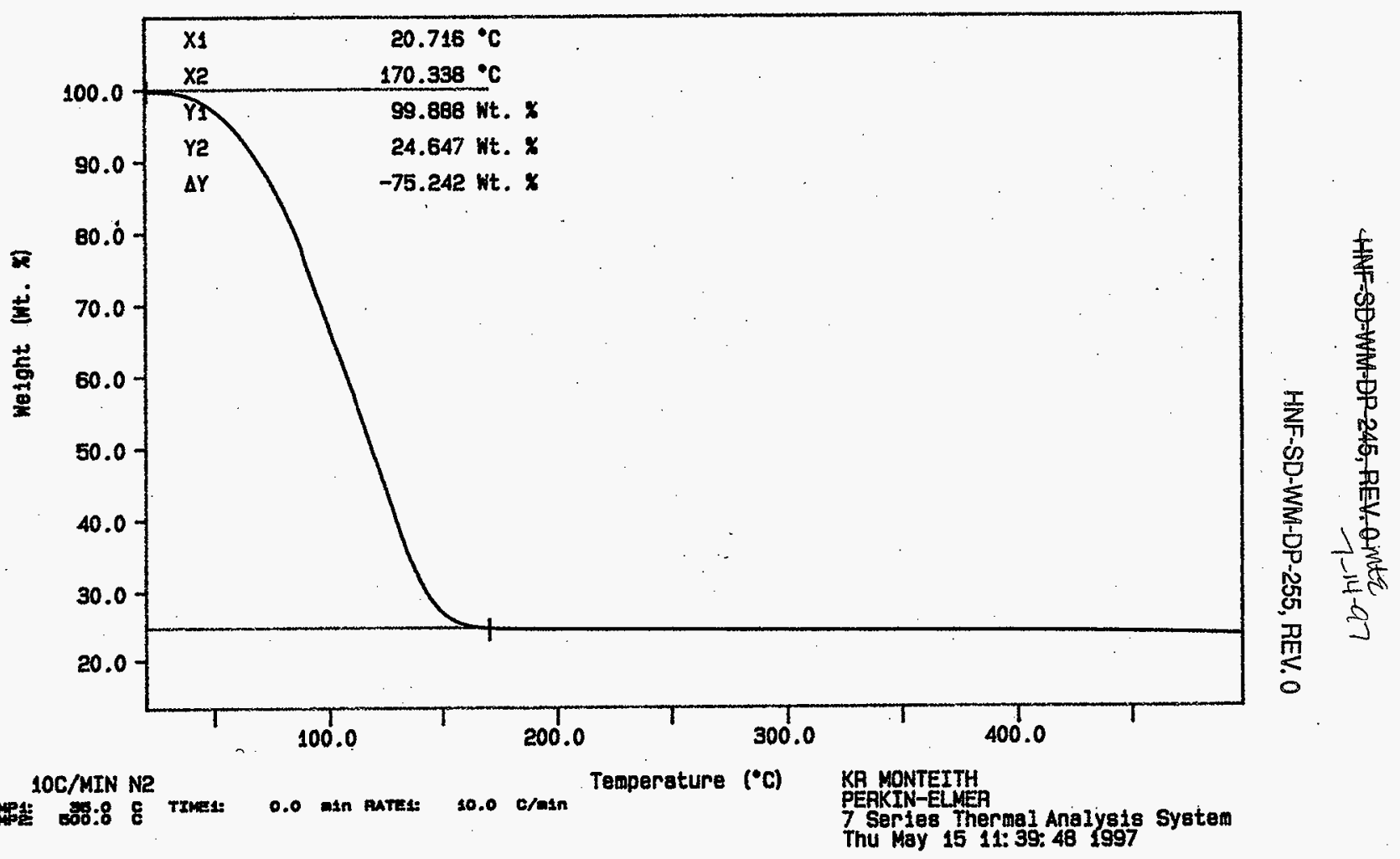


Curve 1: TGA

F110 Info: SAM051504 Thu May 15 12: 43: 131997

Sample Weight: 14.848 mg

S97T000638 DUP

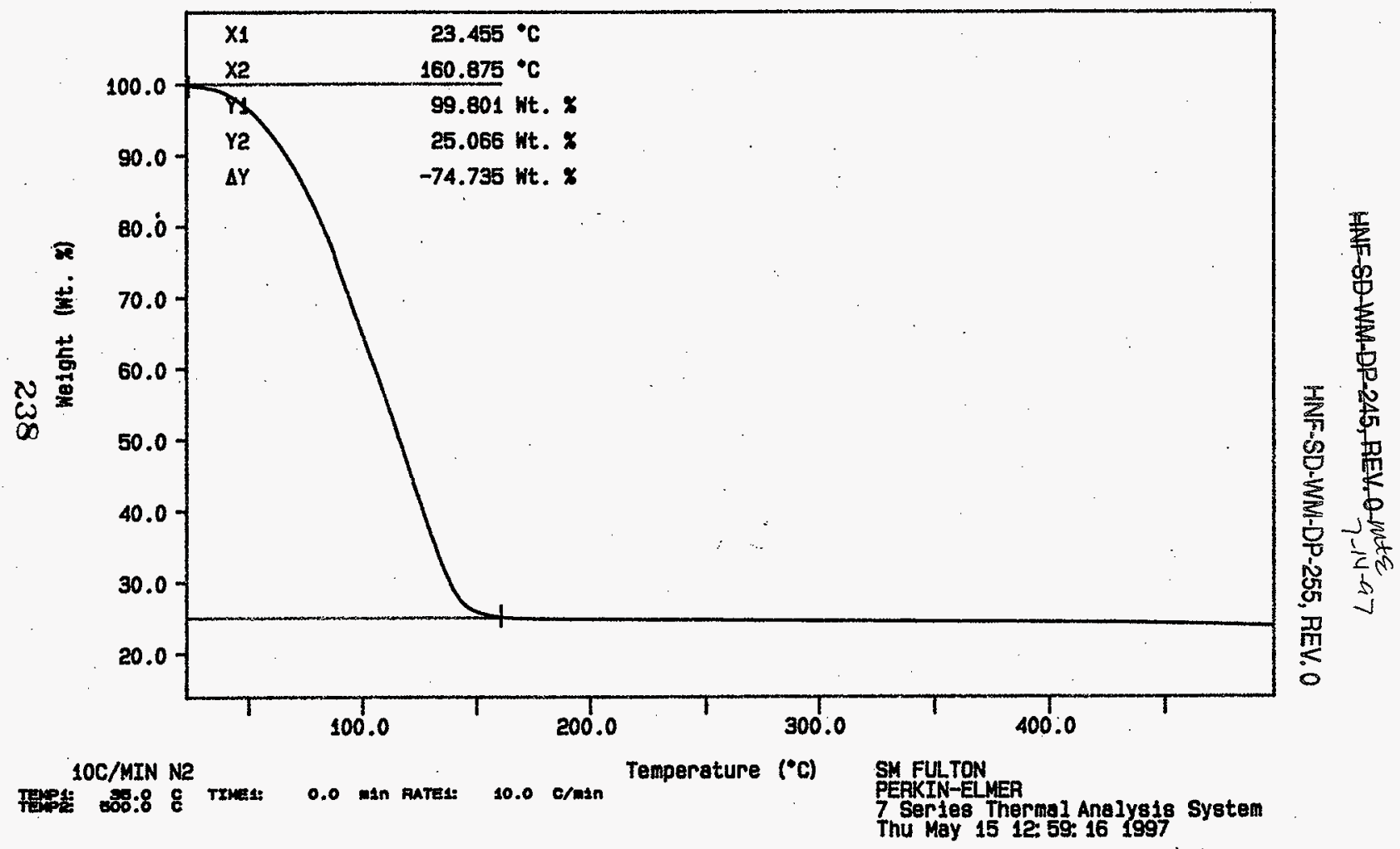


worklistrpt.Version 2:1 05/15/95

HAF-SD-WAAPP-245, REV-Onete $04 / 30 / 9712: 27$

LABCORE Data Entry Template for Worklist\# 17847

Analyst: SMF Instrument: TGA0 1 Book $99708-4$

Method: LA -560-112 Rev/Mod C - O

HNF-SD-WM-DP-255, REV. 0

Worklist Comment: T204, TGA-01 Run under nitrogen. km

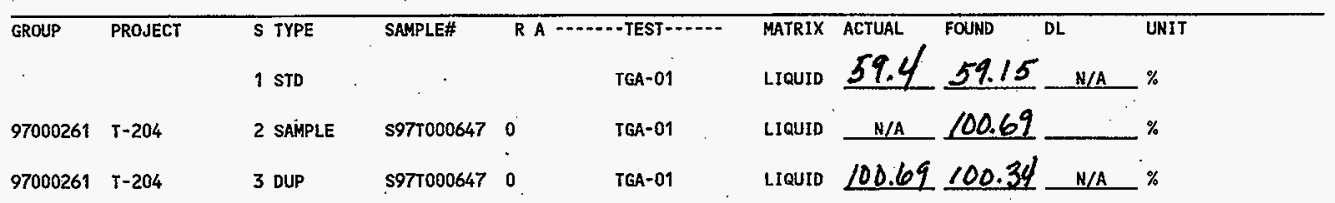

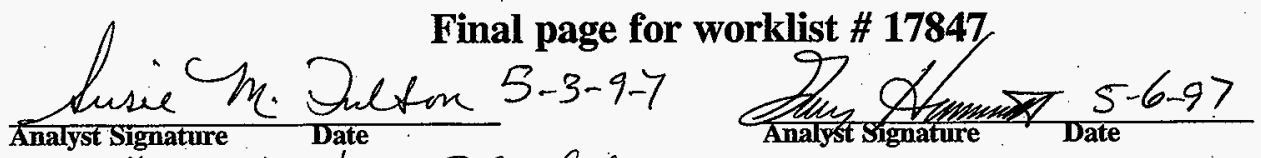

validated 5/8/97 Bachelor

Data Entry Comments:

Units shown for $Q C$ (SPK \& STD) may not reflect the actual units. $D L=$ Detection Limit, $S=$ Worklist Slot Number, $R=$ Replicate Number, $A=$ Aliquot Code.

239 


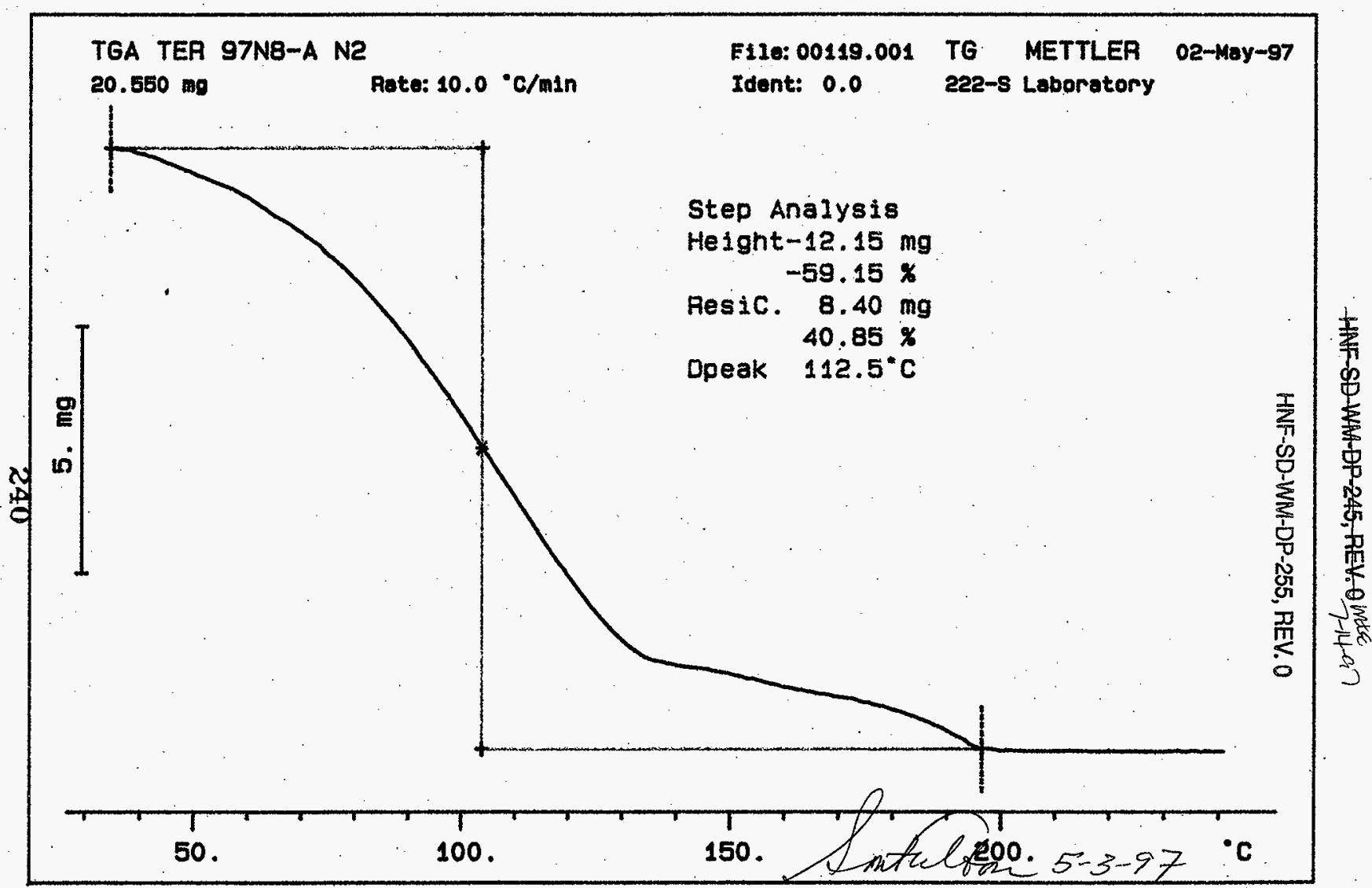




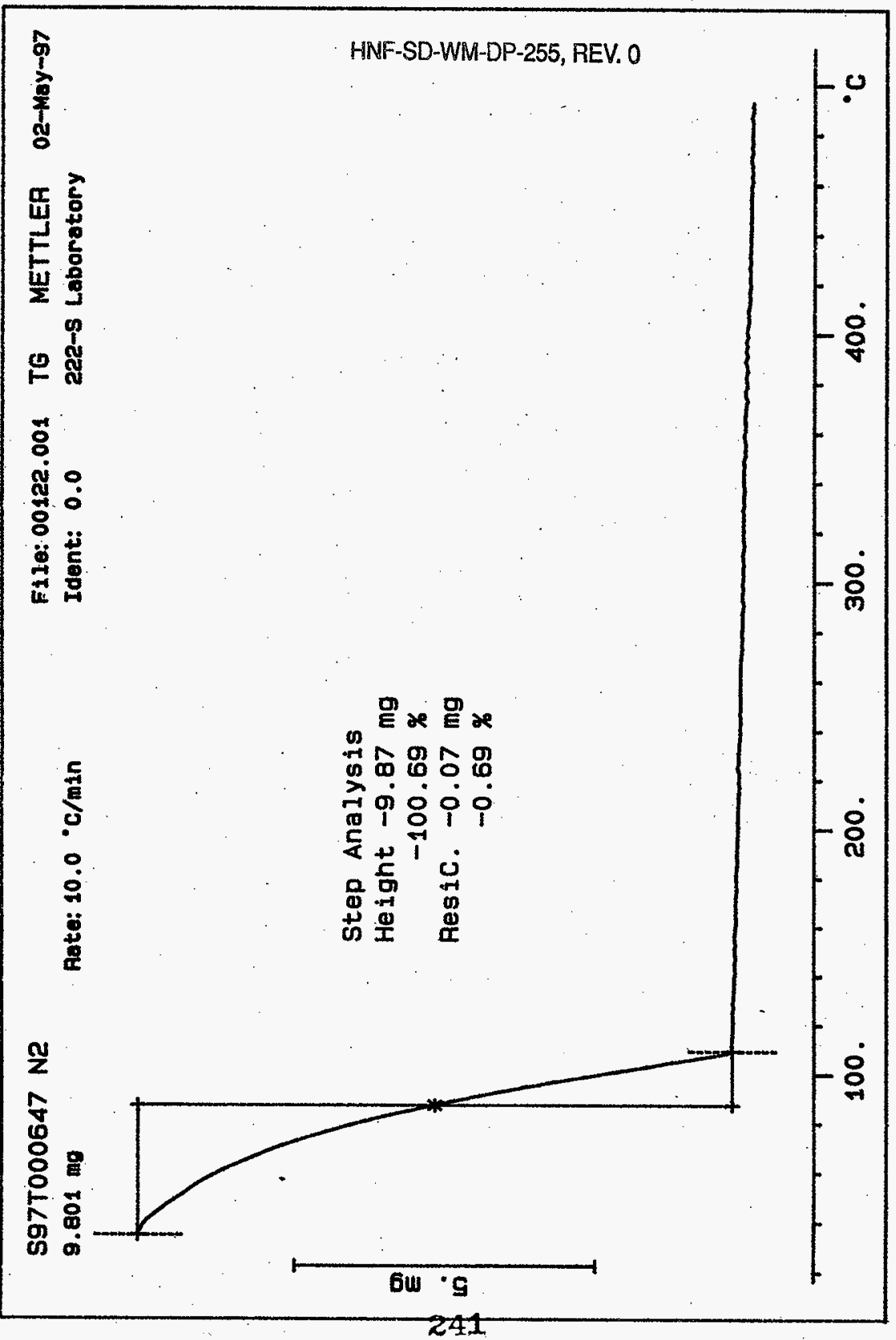




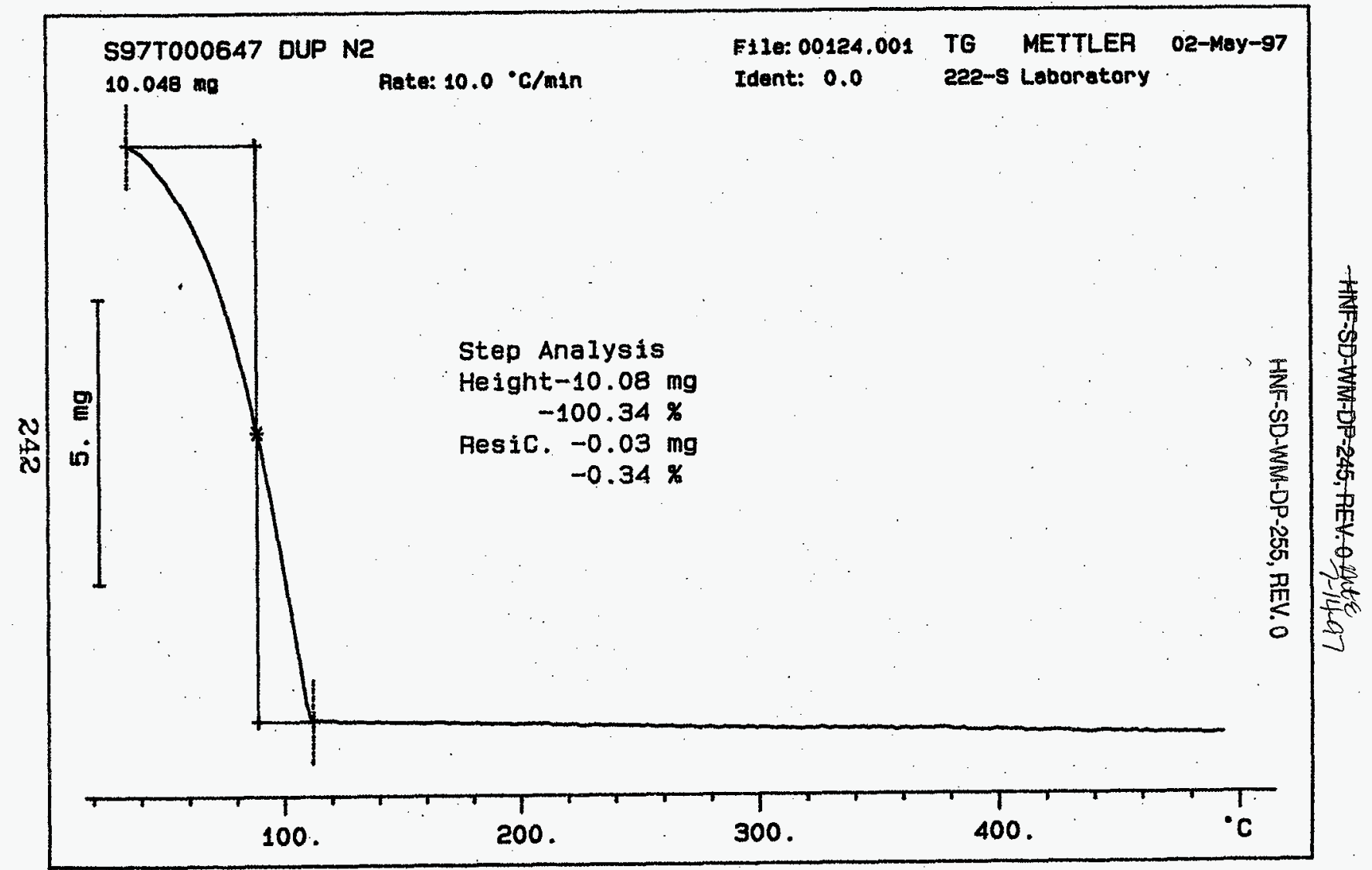




\section{LABCORE Data Entry Template for Worklist\# 18239}

Page: $\quad 1$

Analyst: Instrument: TGAO 3. Book\#97N8A

Method: LA-514-114 Rev/Mod $\mathrm{D}-\mathrm{O}$

HNF-SD-WM-DP-255, REV.O

Worklist Comment: Rerun due to high RPD, low STD. Rund under N2. -PPB

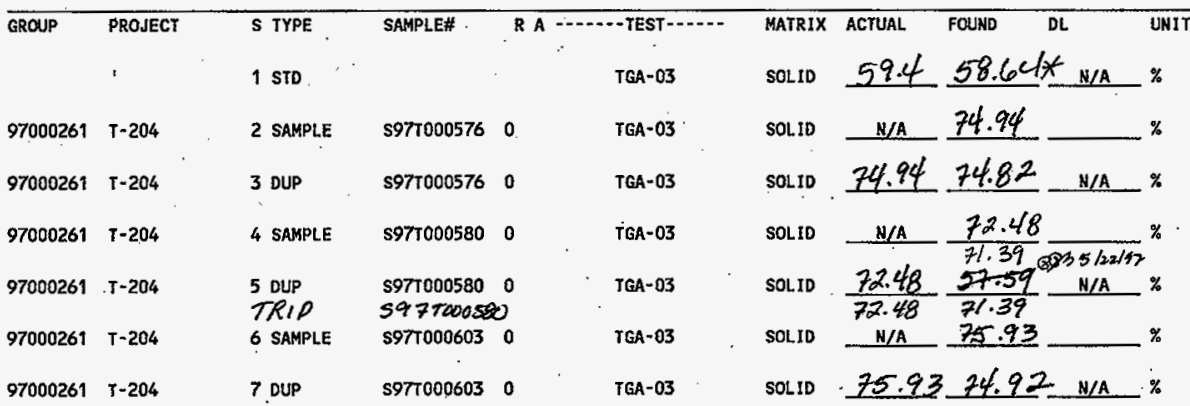

Final page for worklist \# 18239
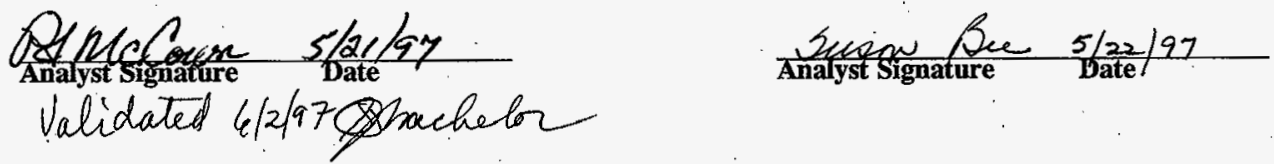

Data Entry Comments:

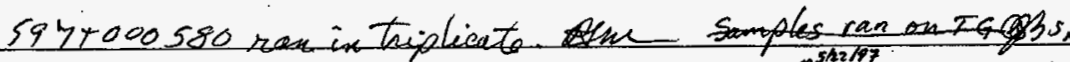

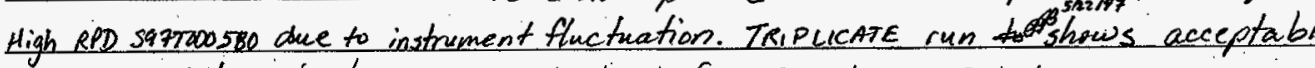

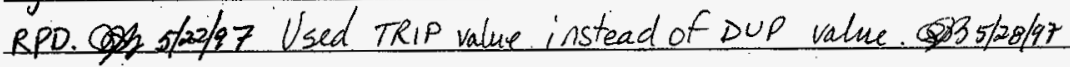

Units shown for QC (SPK \& STD) may not reflect the actual units. $D L=$ Detection Limit, $S=$ Worklist Slot Number, $R=$ Replicate Number, $A=$ Aliquot Code. 
Curve 1: TOA

F1le info: TERO52101 Ned May 21 07: 08:54 1997

Sample Veight: $22.268 \mathrm{ng}$

SIGNATURE BSTOW REPRESENTS CHEMICAL TECHNOLOGIST/CHEMIST TMII

97NB-A

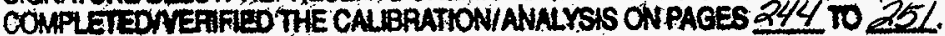

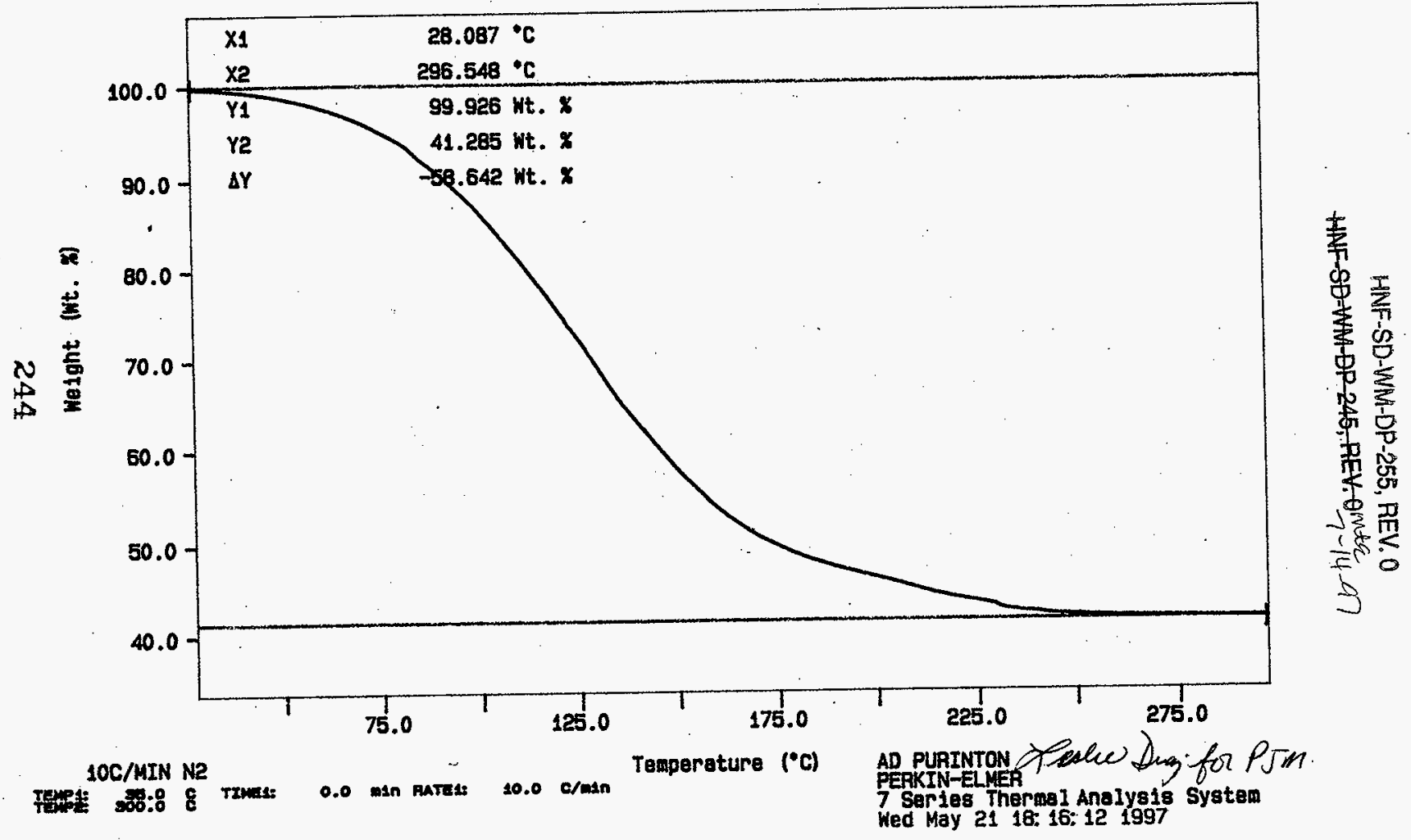


Cunve 1: TGA

F11e Info: SAM052113 Wed May 21 20:03: 281997

Semple Neight: 17.934 . ing

s977000576

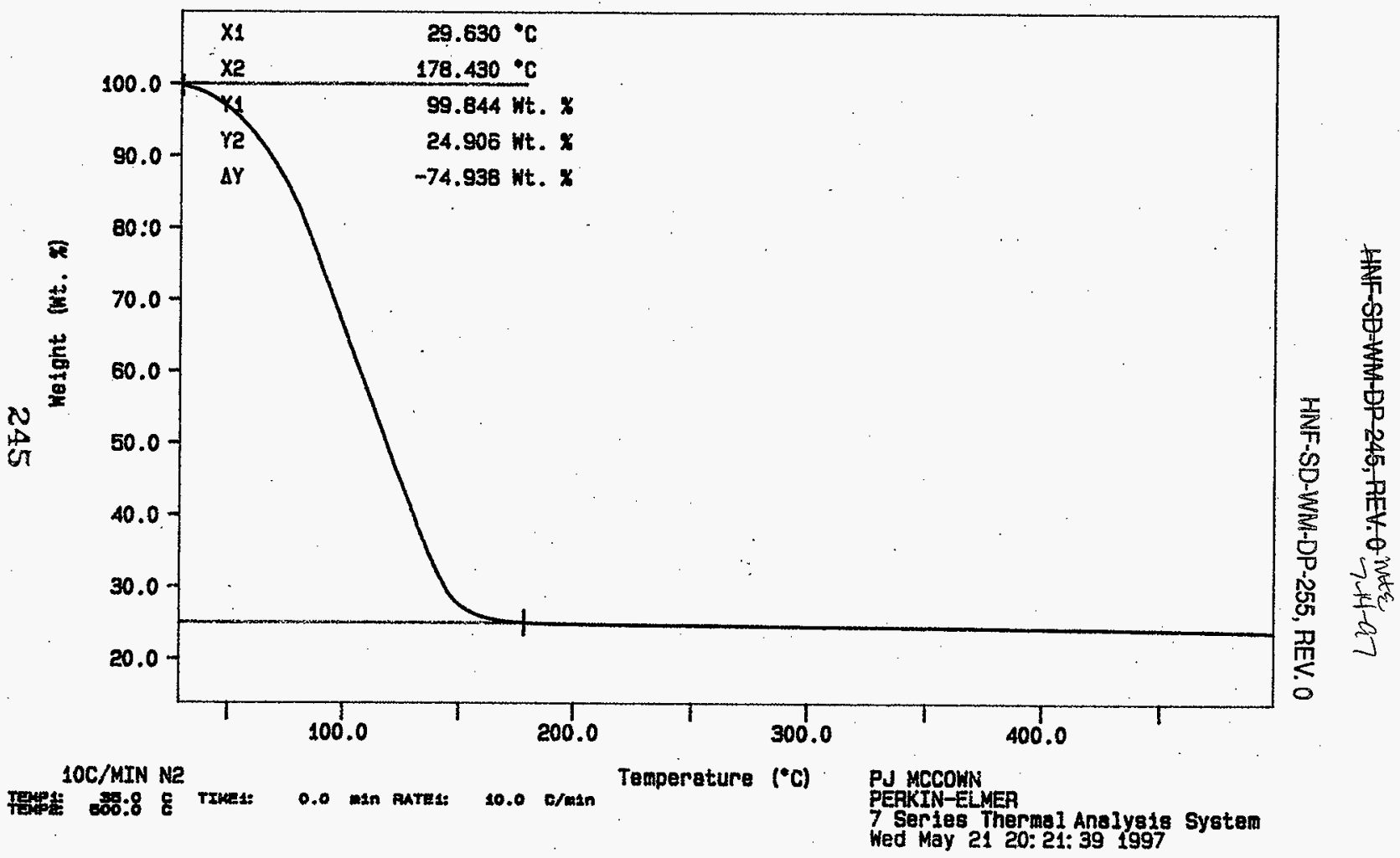




\section{Curve 1: TGA}

File tnfo: SAM052114 Wed May 21 21: 16: 331997

Somple Meight: 17.903

S97T000576 DUP

ang

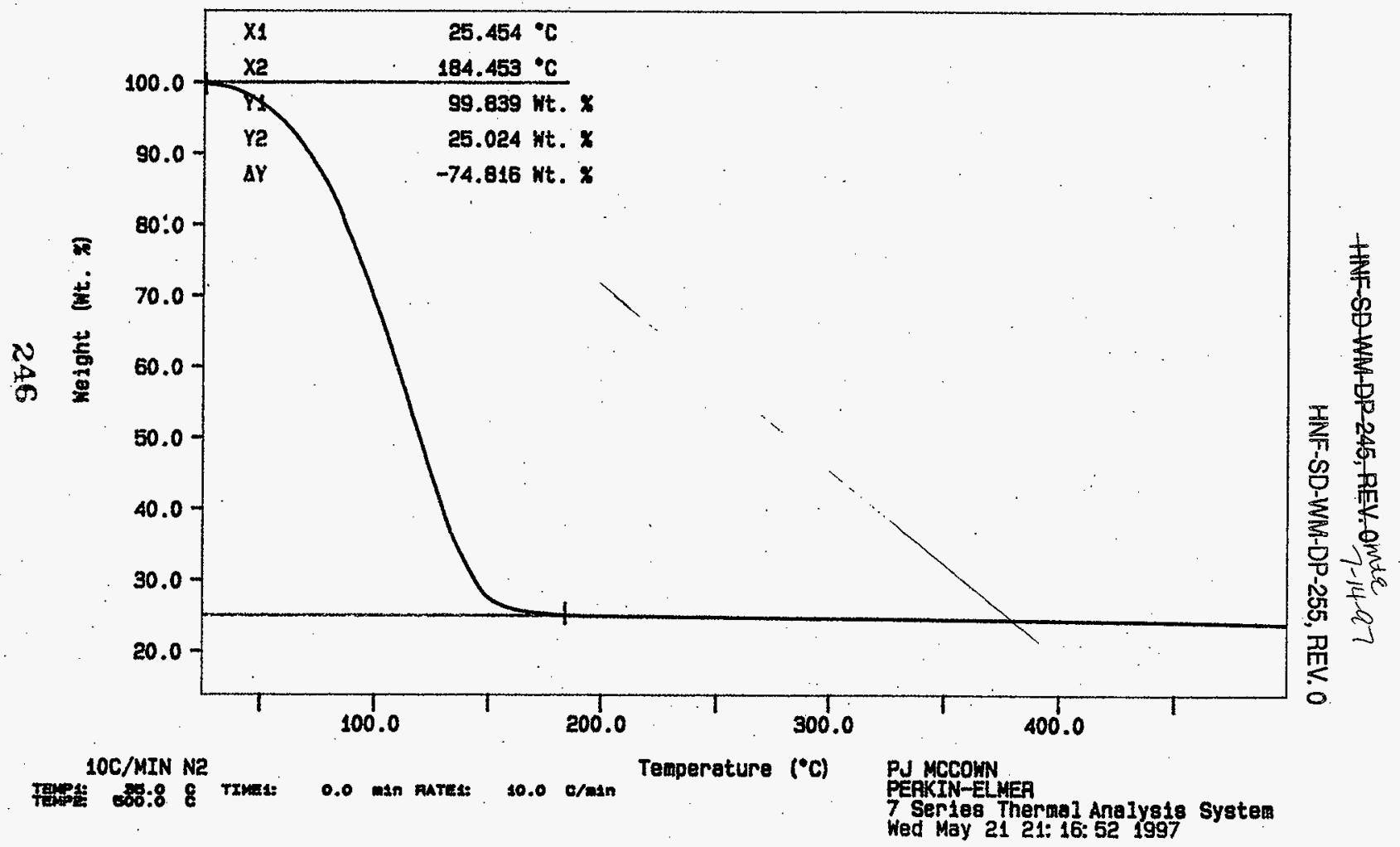


Curve 1: TGA

File Info: SAM052115 Hed May 21 22: 29: 461997

Sample We1ght: 13.248

5977000580

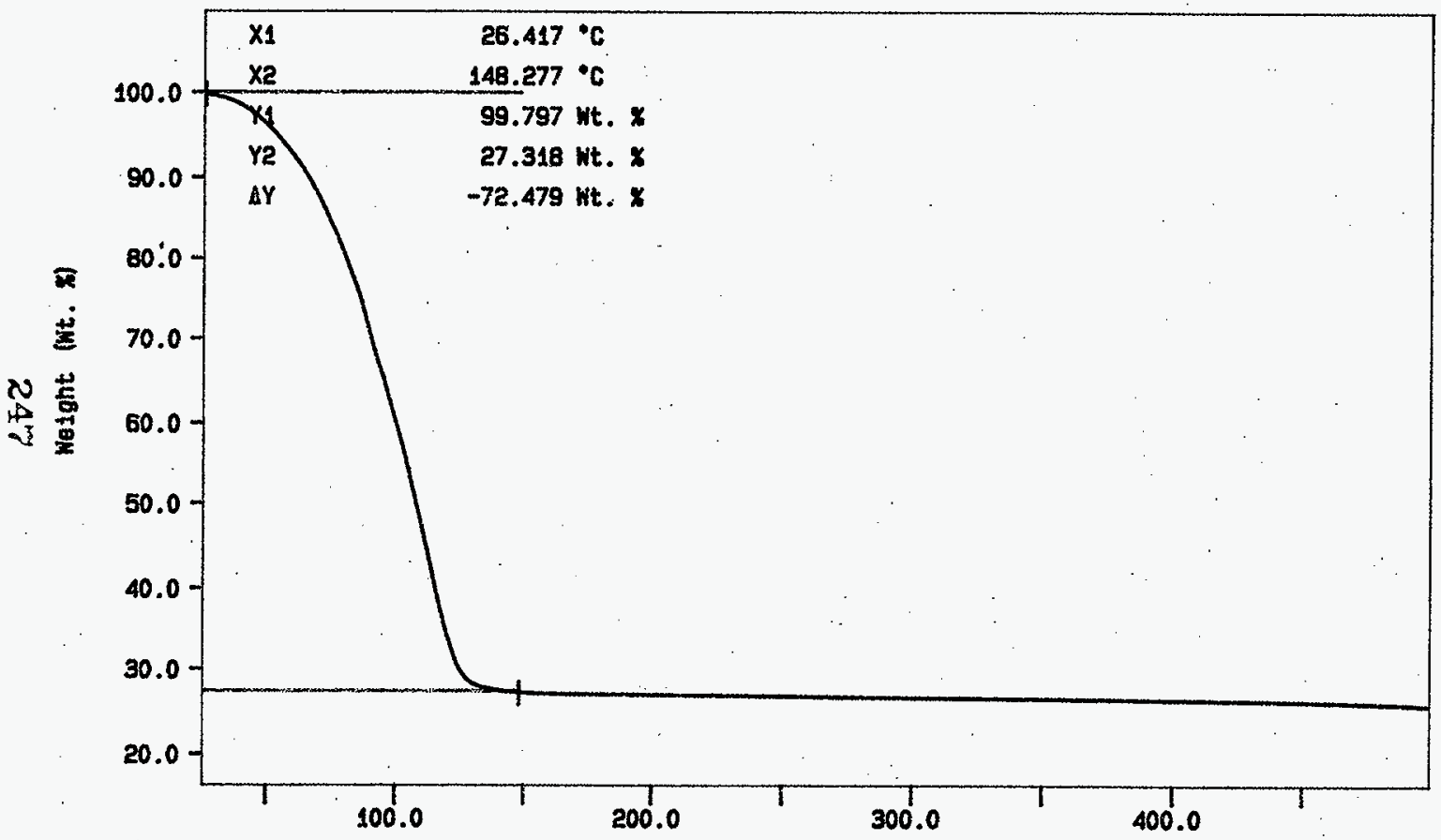

10C/MIN N2

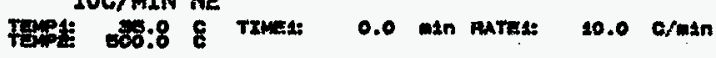

Temperature $\left({ }^{\circ} \mathrm{C}\right)$

PJ MCCONN

PERKIN-ELMER

7 Series Thermal Analysis System Wed May 21 22: 30: 541997

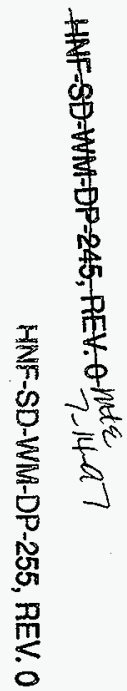


Curve 1: TGA

File info: SAM052201 Wed May 21 23:54: 151997

Sample Weight: $18.480 \mathrm{mg}$

$597 T 000580$ DUP

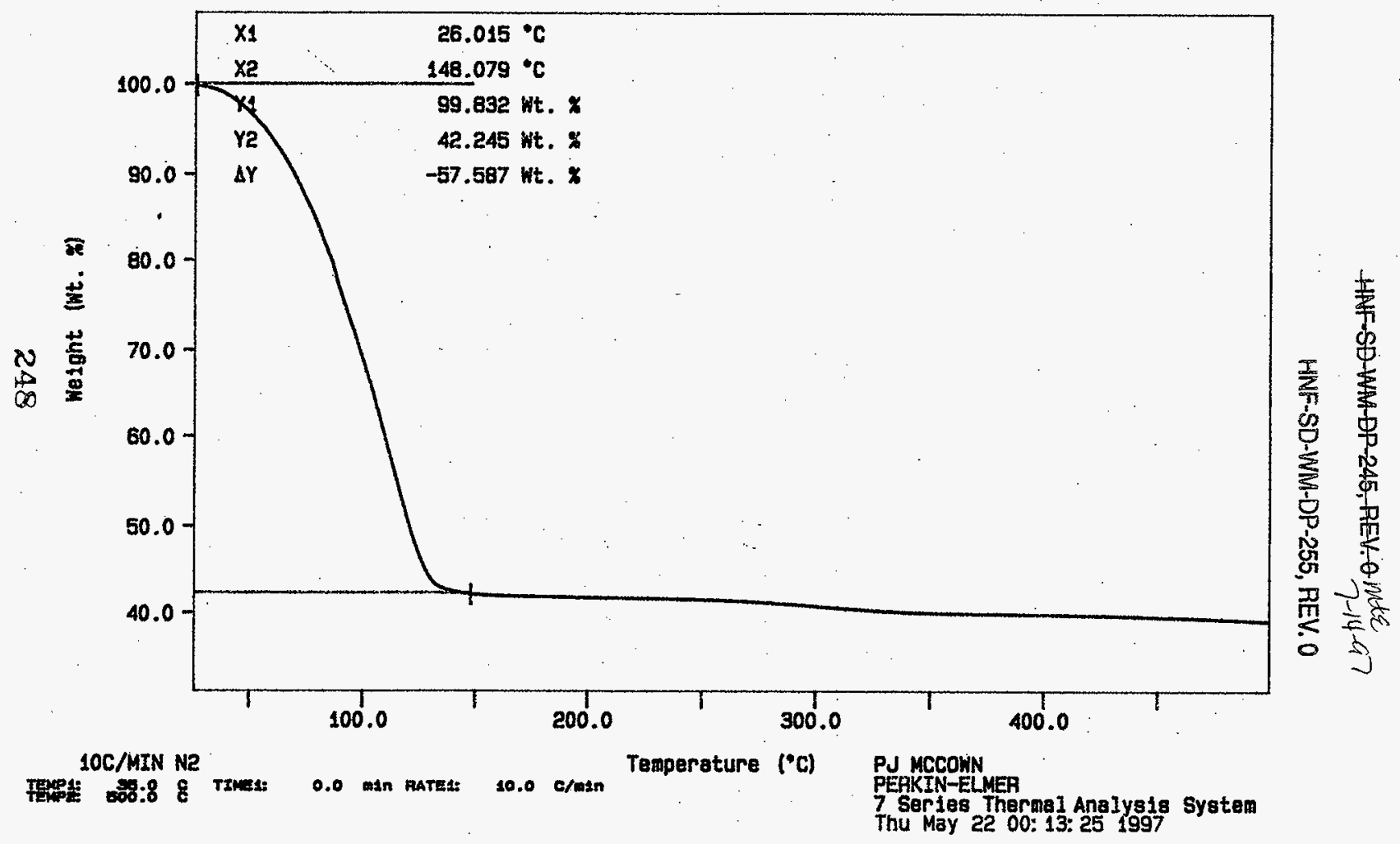


Curve 1: TEA

File info: SAM052202 Thu May 22 01: 06:52 1997

Sample Veight: 14.400

S97T000580 TAIP

nig

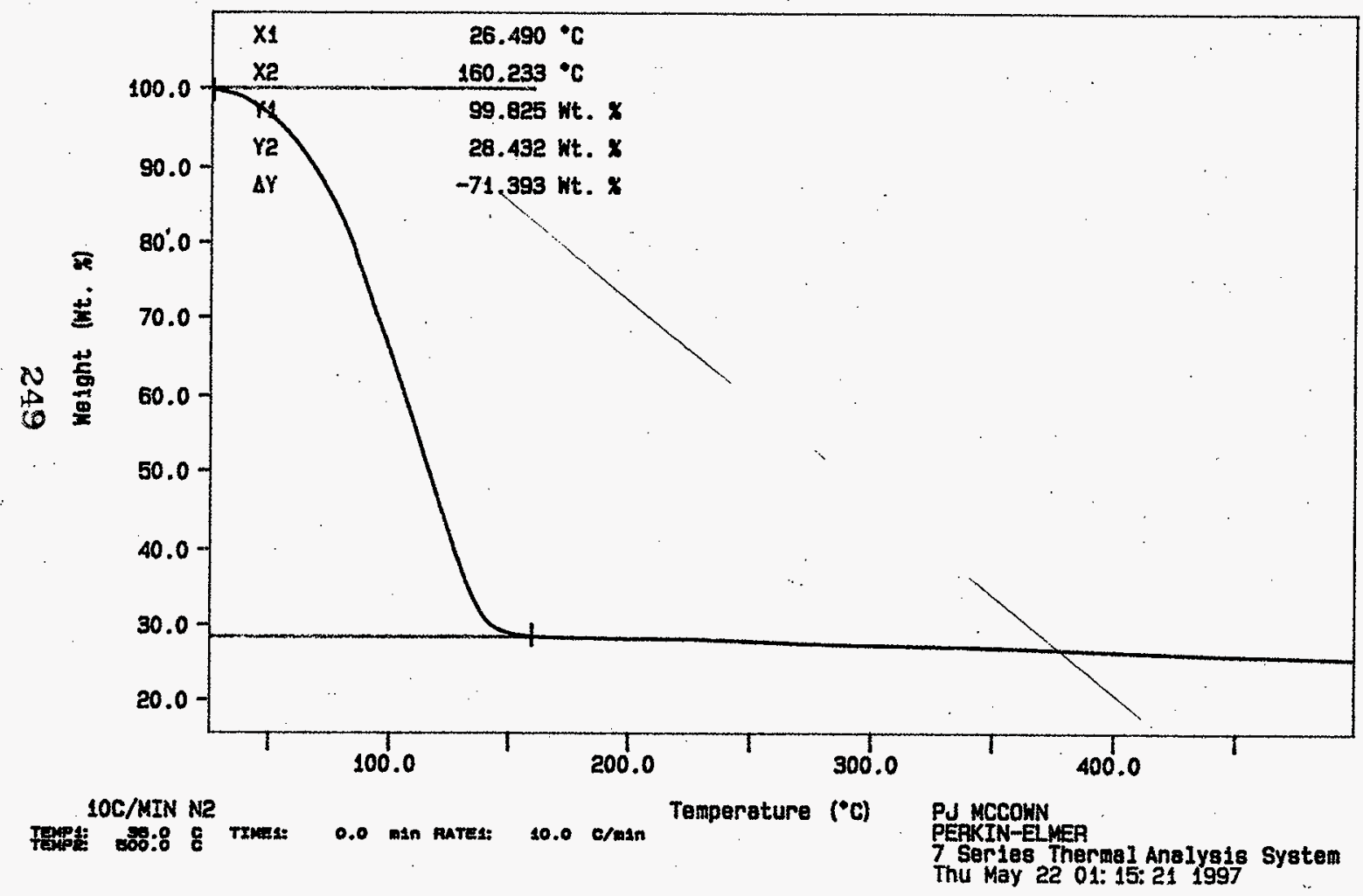

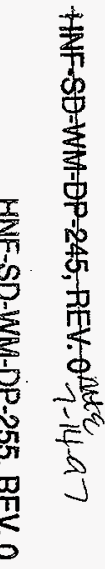


Curve 1: TGA

F1le info: SAM052203 Thu May 22 02: 16: 481997

Sample Weight: 16.693 ag

S977000603

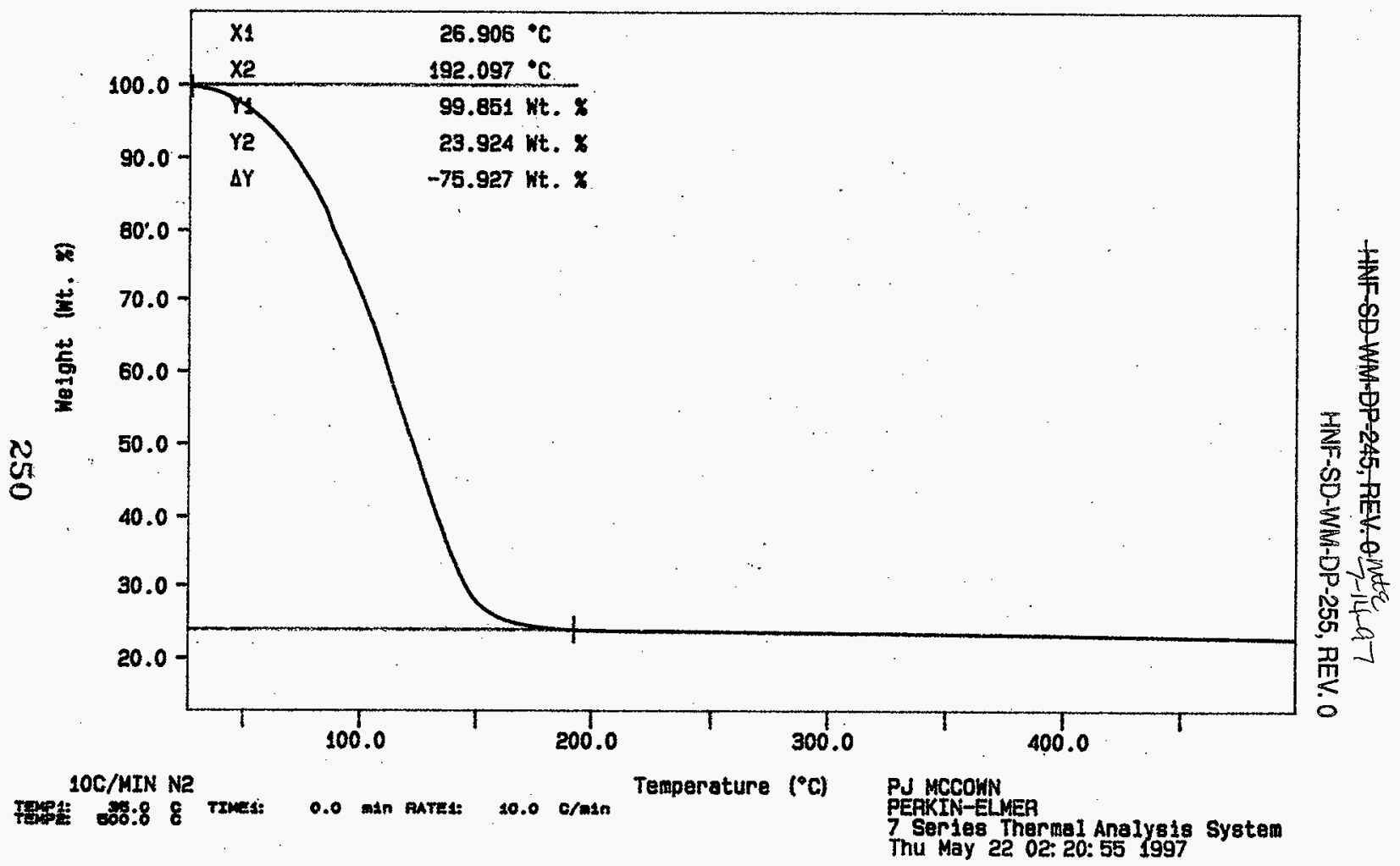


Curve 1: TGA

File info: SAM052204 Thu May 22 03: 27:02 1997

Sanple veight: 17.267

S97T000603 DuP

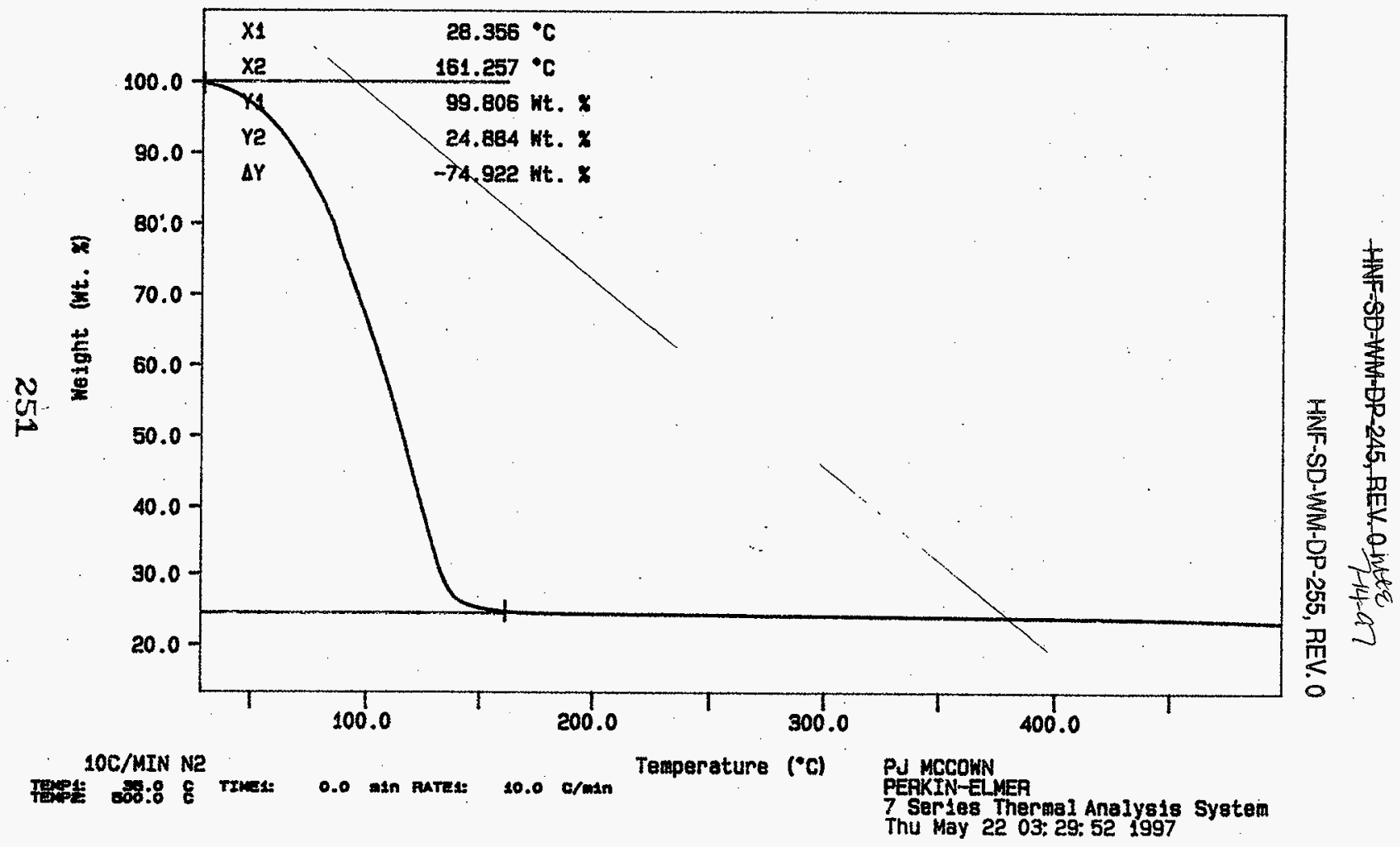




\section{LABCORE Data Entry Template for Worklist\# 18240}

Analyst: $\quad A D P$ Instrument: TGA0

Method: LA-514-114 Rev/Mod D-O

Book \# $97 \sqrt{8} \mathrm{~A}$

HNF-SD-WM-DP-255, REV. 0

Worklist Comment: Rerun due to high RPD, low STD. Run under N2. -PPB

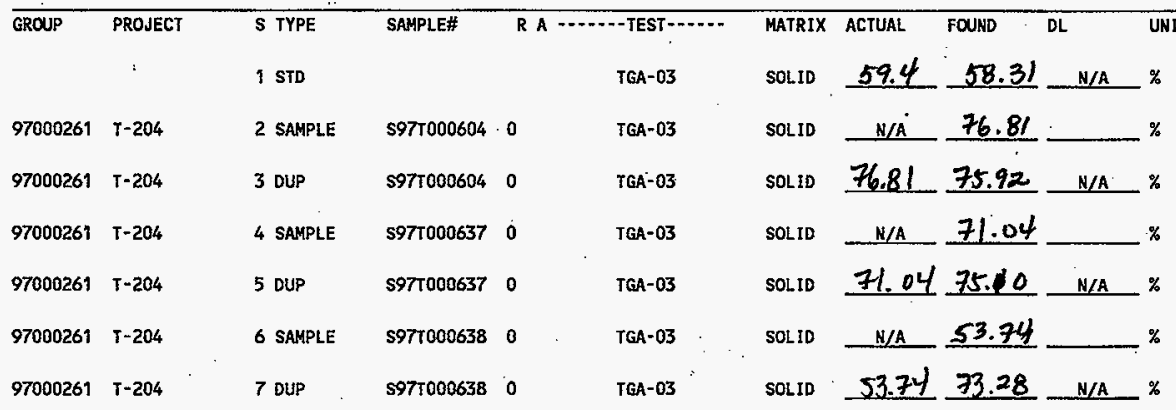

\section{Final page for worklist \# 18240}

Wh twuta 5-22-97

Analyst Sigu ature

validated 6/2/97 Bochebr

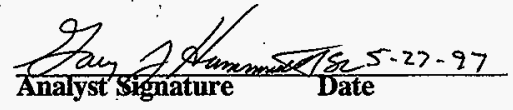

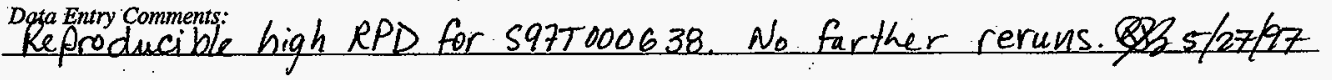

Units shown for $Q C$ (SPK \& STD) may not reflect the actual units. $D L=$ Detection Limit, $S=$ Worklist Slot Number,

$R=$ Replicate Number, $A=$ Aliquot Code. 
Curve 1: TGA

File Infa: TER052201 Thu May 22 06: 27: 161997 Sample We1ght: $24.197 \mathrm{mg}$

97NB-A

SIGNATURE BELOWREPRESENTS CHENCAL TECHNOLOGISTICHEMIST THN OOWHETSONERIMED THE CALIBRATIONIANALYSIS ON PAGES2 33 to 258

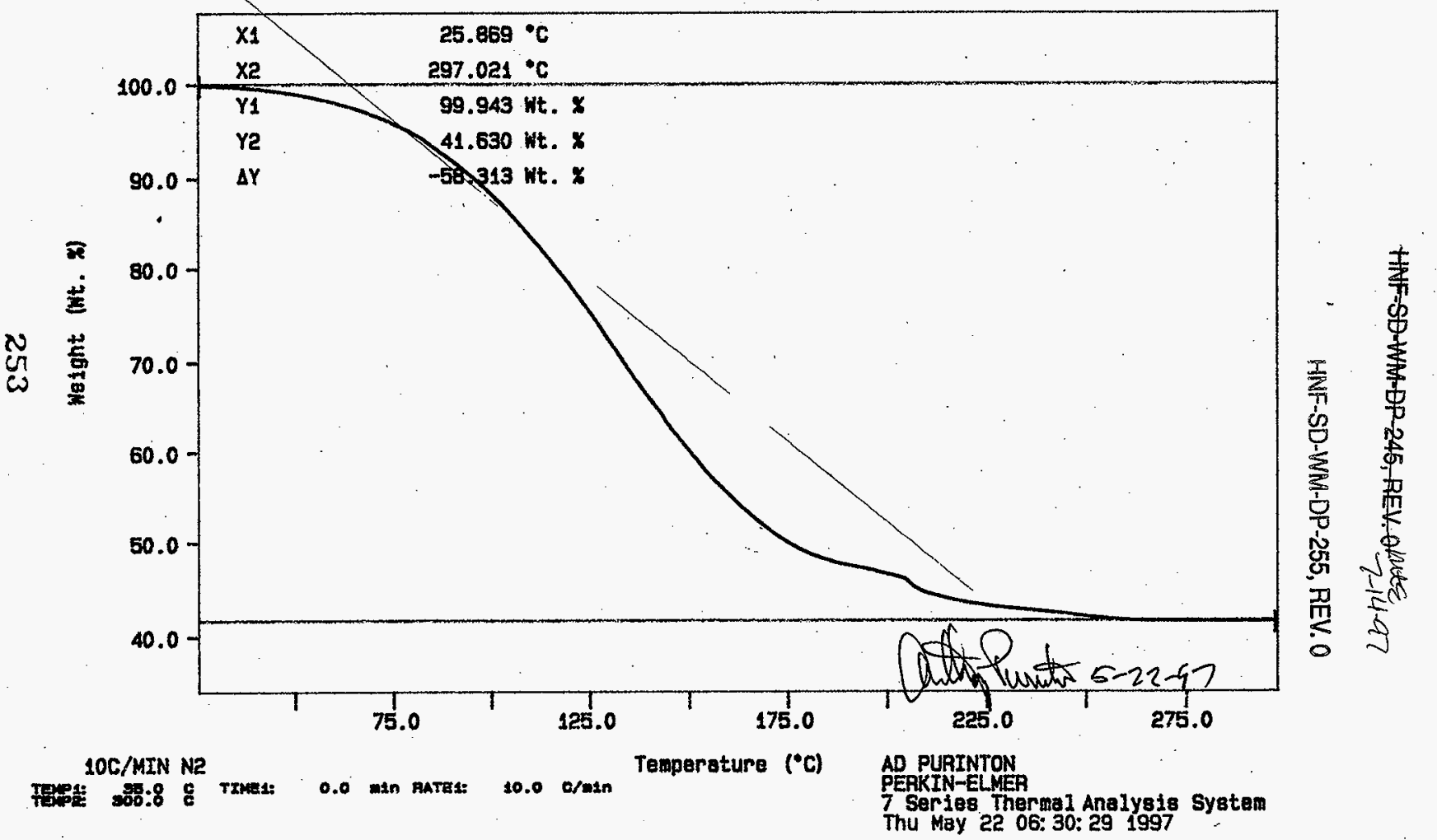


Curve 2: TGA

File info: SAM052206 Thu Hay 22 07:35: 361997

Semple Veight: 14.756

S977000604 SAM

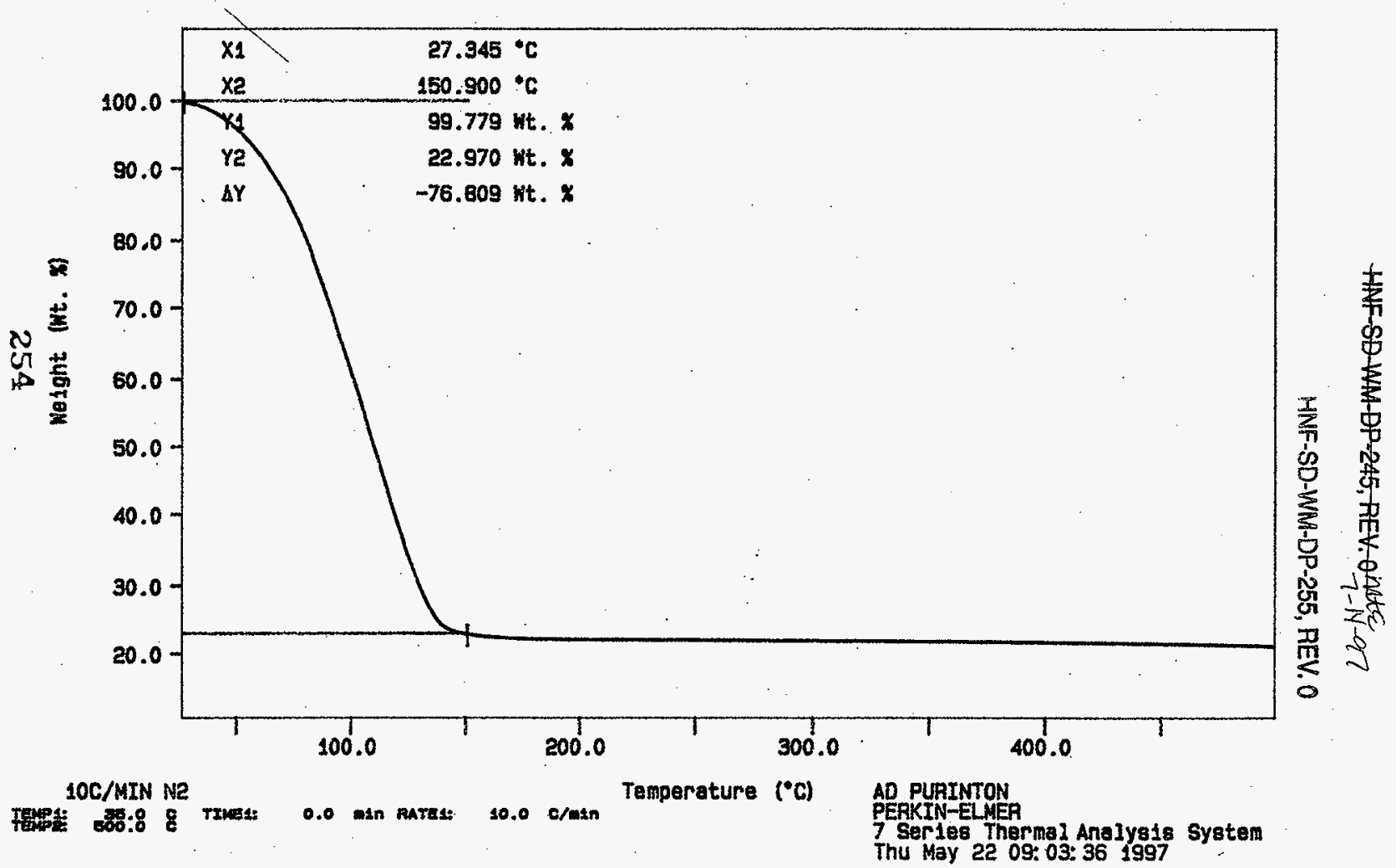


HAF-SD-WAA-DP-245, REV.0 7nth $7-1497$

HNF-SD-WMM-DP-255, REV. 0

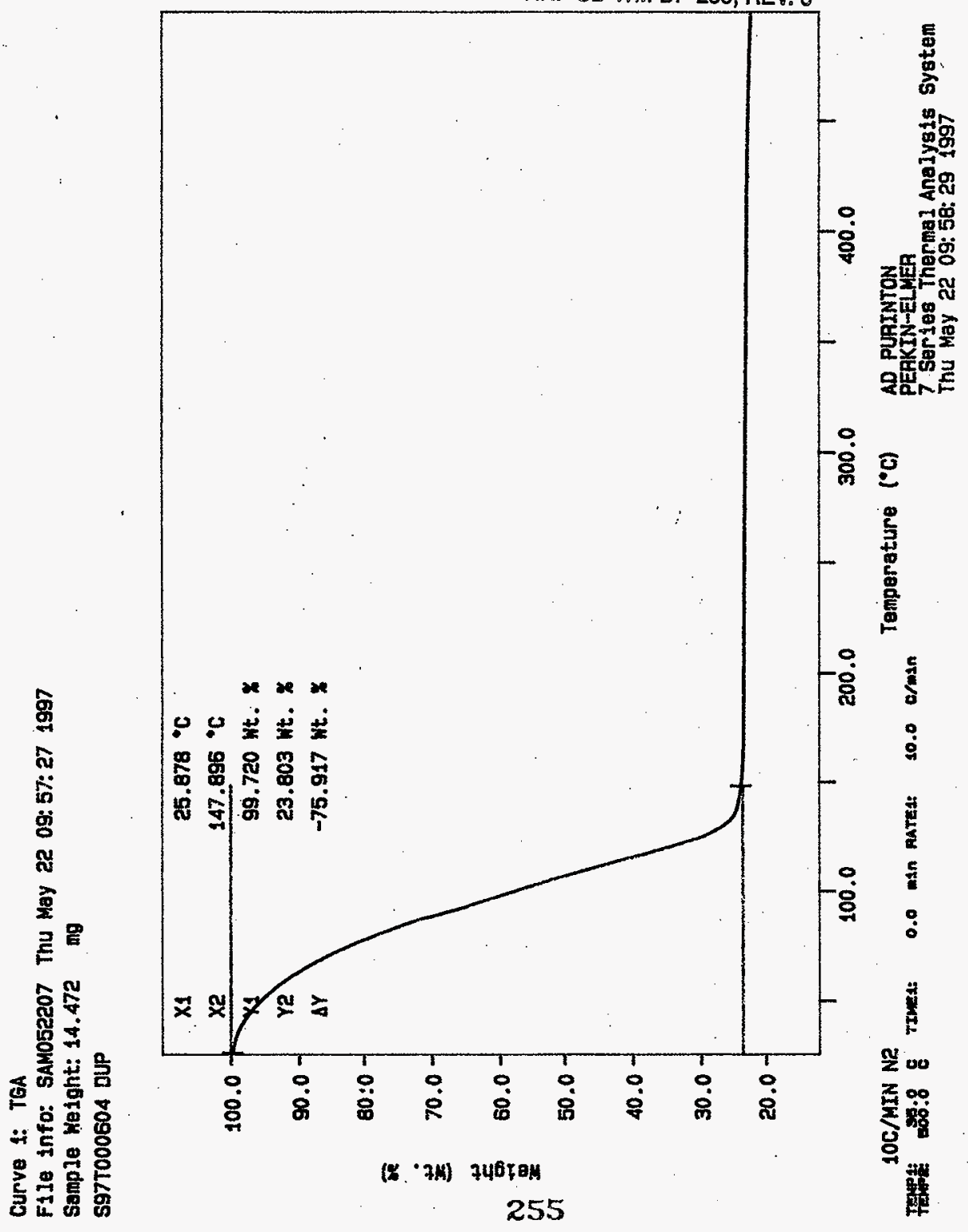


Curve 1: TGA

File info: 5AM052208 Thu May 22 11:05: 28.1997

Sample Welght: 20.778

S97T000637 SAM

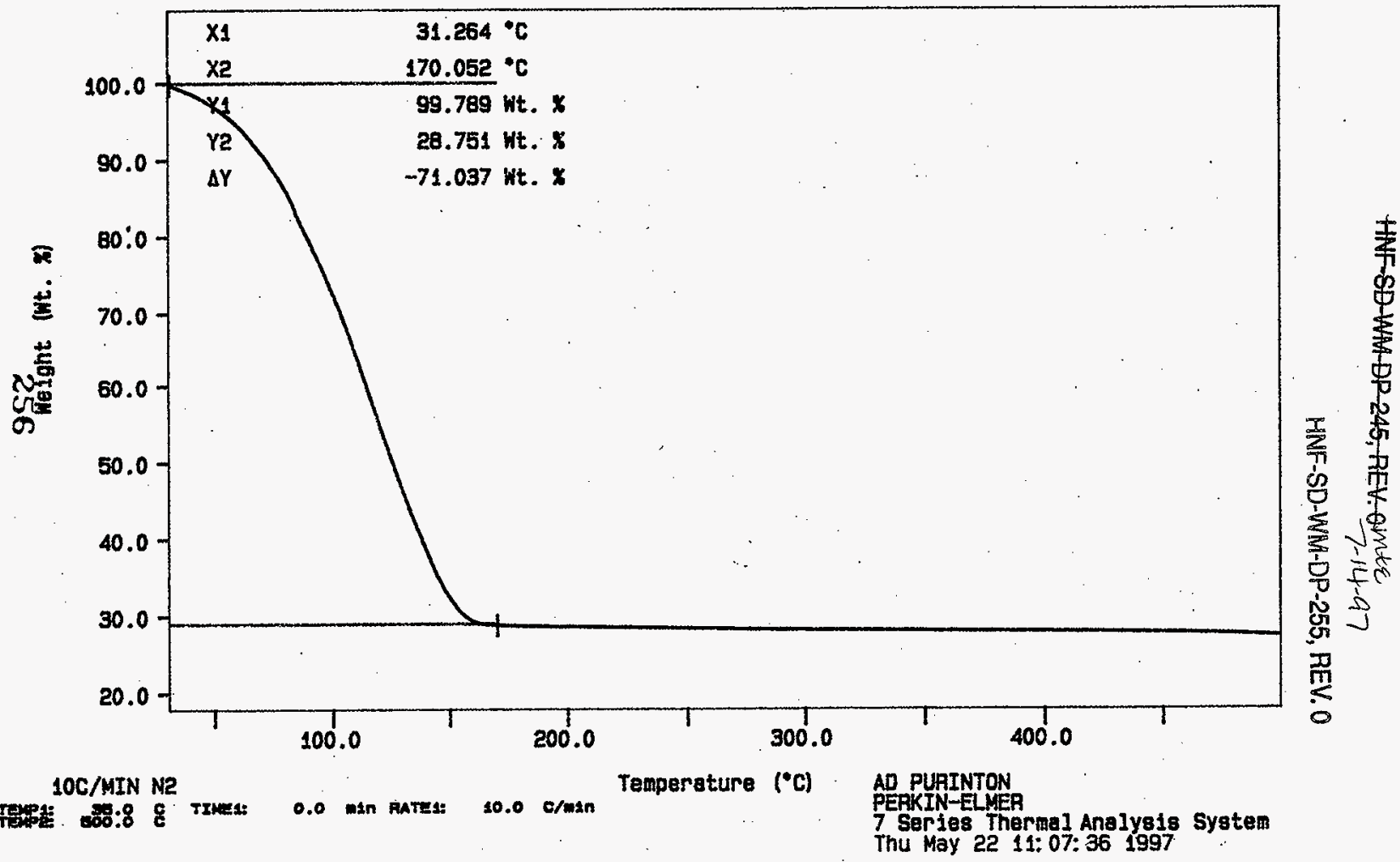


Curve s: TGA

F1le Info: SAM052209 Thu May 22 12: 11:09 1997

Sample Weight: 14.932

5977000637 Dup

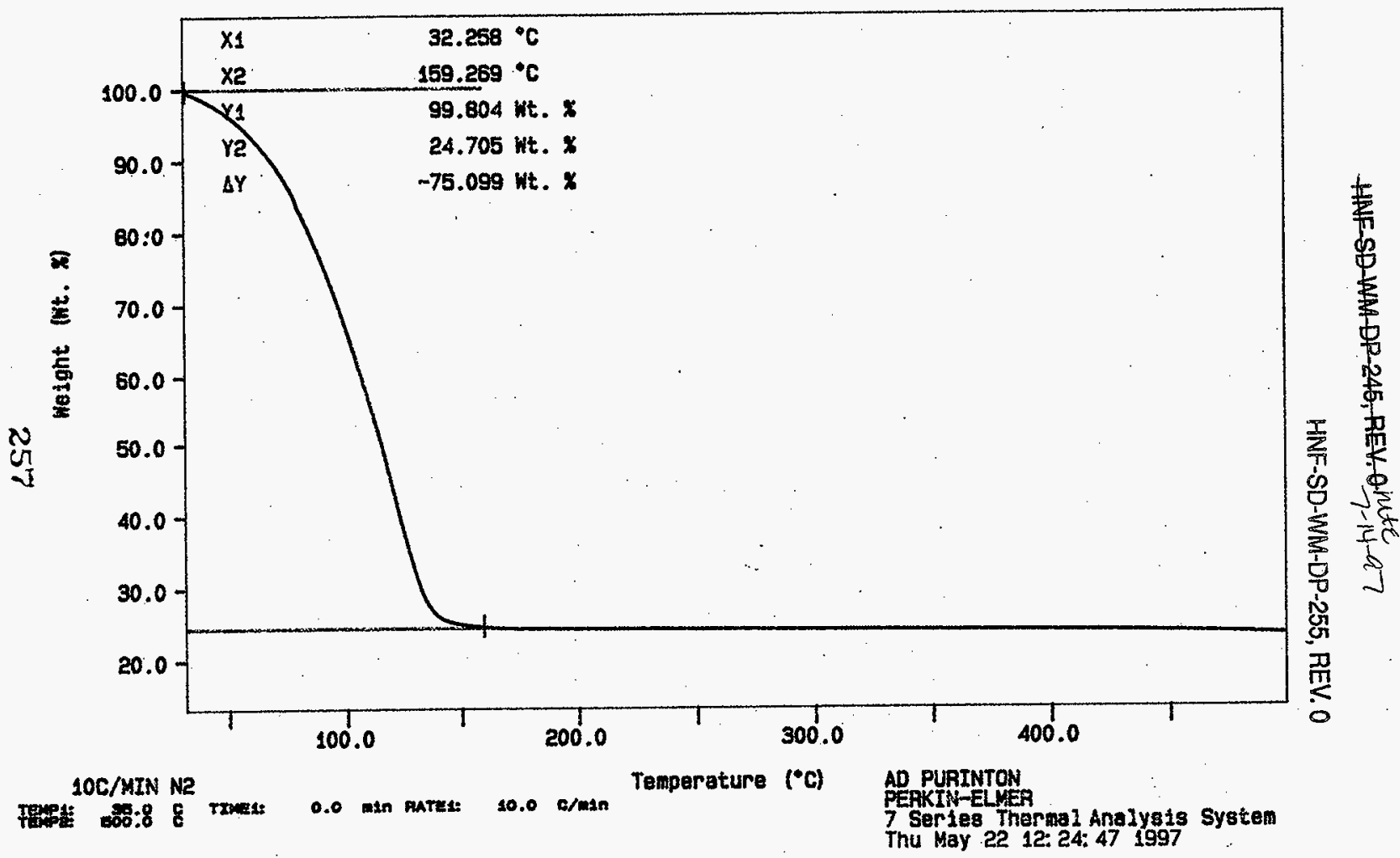


Curve 1: TGA

F1le info: SAM052210 The May 22 13:18; 201997

Sample Weight: 34.752 ng

s97T000638 SAM

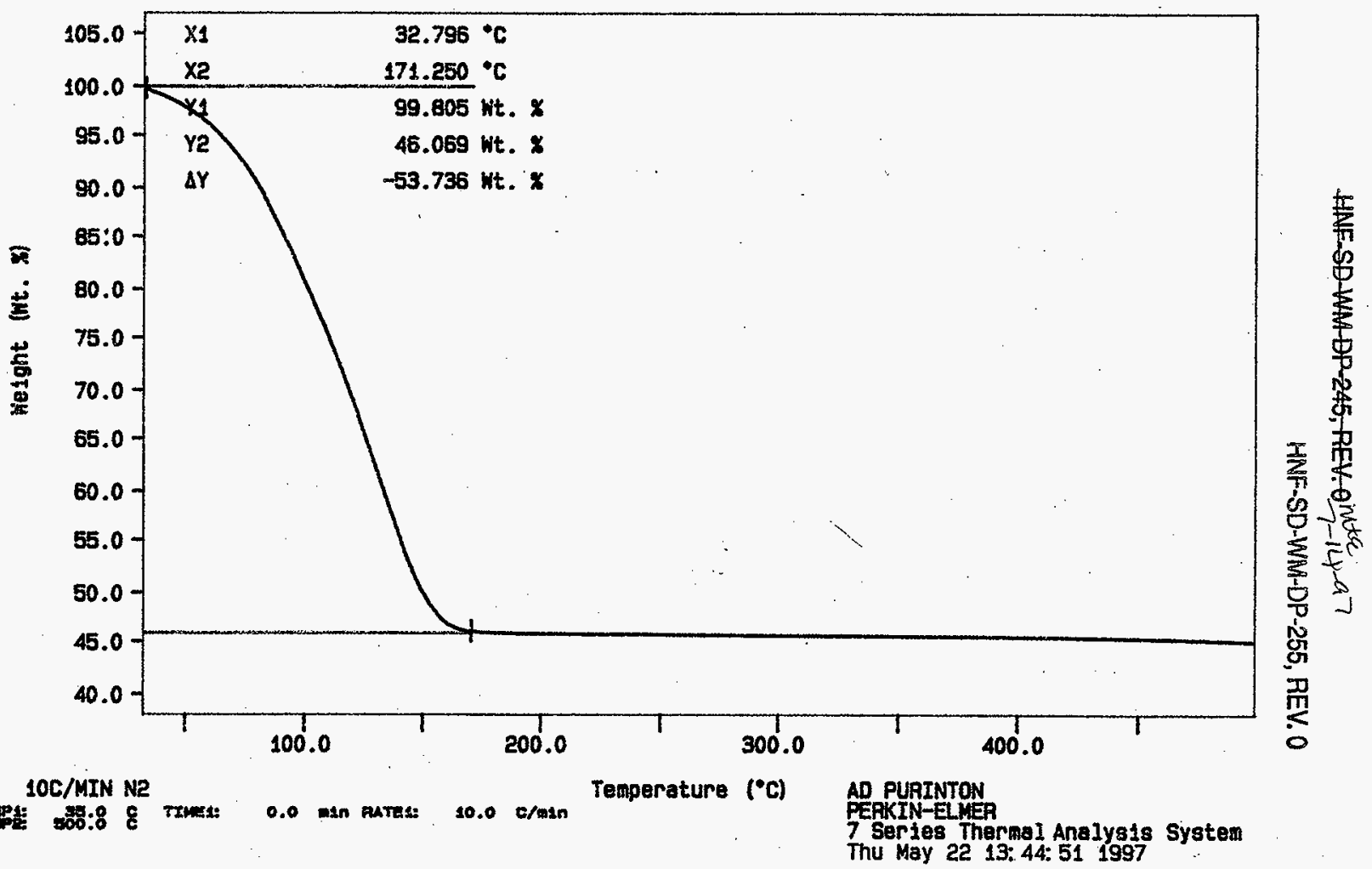


Curve 1: TGA

F1le info: SAk052211 Thu May 22 14: 37: 281997

Somple Height: $22.898 \mathrm{mg}$

s97T000638 DUP

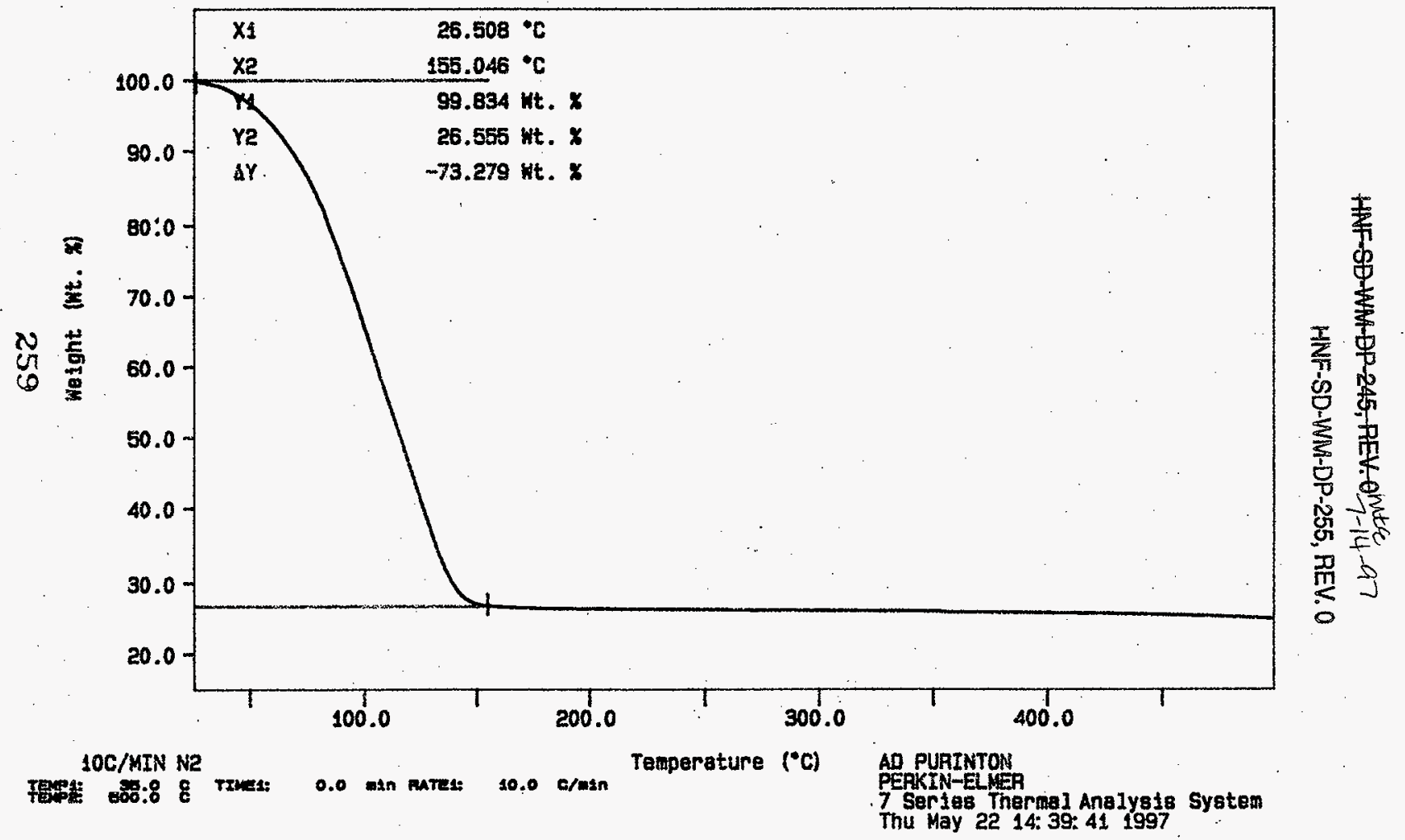




\section{LABCORE Completed Worklist Report for Worklist\# 18685}

Analyst: jds

Instrument: TGA03

Book\#

Method: Rev/Mod

Worklist Comment: T-204 TGA, RUN UNDER N2. RCJ

\begin{tabular}{lllllll}
\hline Seq Type & Sample\# R A & Test & Matrix & Actual & Found & DL or Yield Unit
\end{tabular}

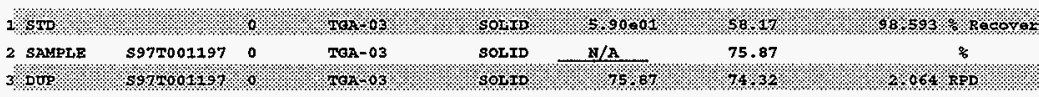

\section{Final page for worklist\# 18685}

Analyst Signature Date

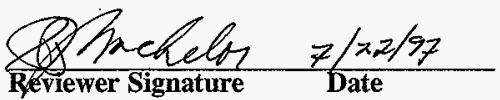




\section{LABCORE Data Entry Template for Worklist\# 18685}

Analyst: Id Instrument: TGAO 3 Book \# 103 NOA

Method: LA-560-112 Rev/Mod D-O

Worklist Comment: T-204 TGA, RUN UNDER N2. RCJ

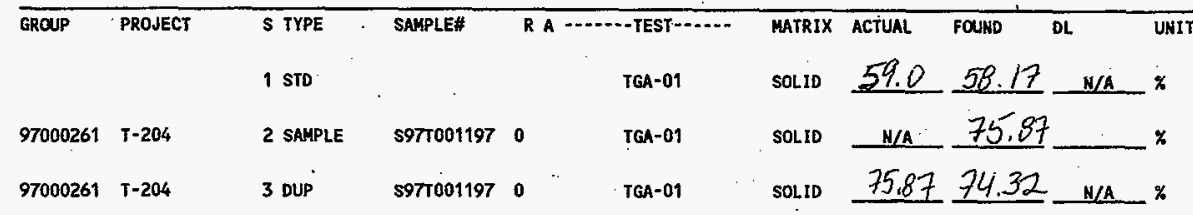

\section{Final page for worklist \# 18685}

Sh

Analyst Signatime $1 / 22197$ Date

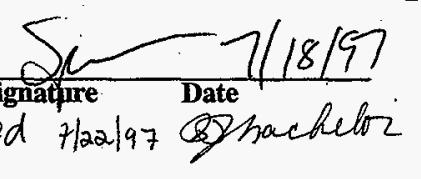

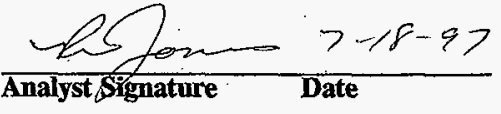

Data Entry Comments:
Samples run on TGA-03. $8837 / 22 / 17$

Units shown for $Q C$ (SPK \& STD) may not reflect the actual units. $D L=$ Detection Limit, $S=$ Worklist Slot Number, $\boldsymbol{R}=$ Replicate Number, $A=$ Aliquot Code. 


\section{Curve 1: TGA}

F110 Info: TER061901 Thu Jun 19 0a: $27: 571997$

Sanple Ne1ght: 14.280

103NB-A

ing

ก
S!GNATURE BELOW REPRESENTS CHEMICAL TECHNOLOGIST/CHENIST THAT COMPLETED/VEATFIED THE CALIBAATIONANALYSIS ON PAGESOZLOZTO 264

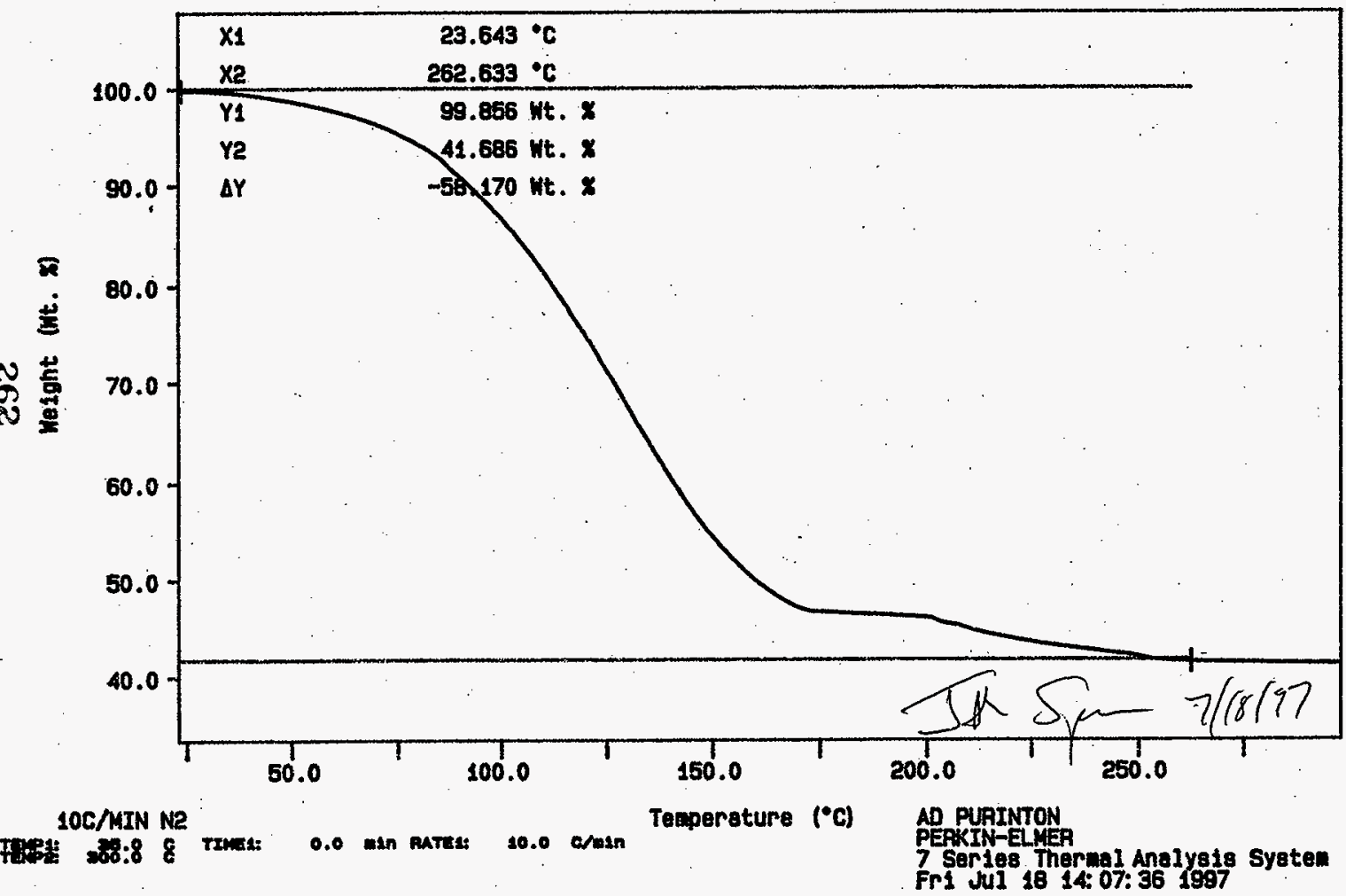


Curve 1: TGA

File Info: SAM061901 Thu Jun 19 10:25: 281997

Sample Weight: 25.501 .ng

\$97T001197 SAM

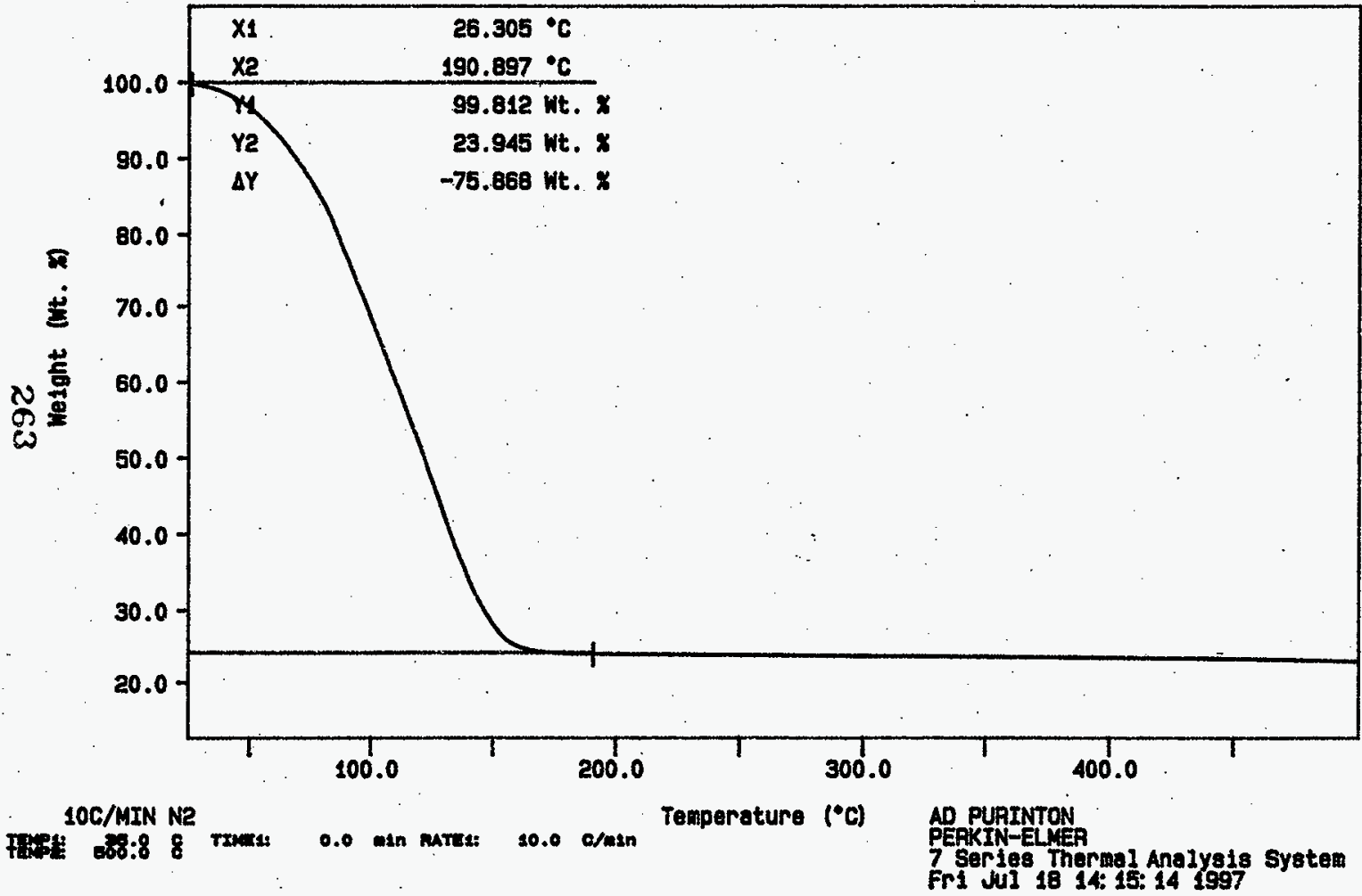


Curve 1: TEA

File info: SAM061902 Thu Jun 19 12: 01:56 1997

Sample Weight: $23.601 \mathrm{mg}$

S97T001197 DUP

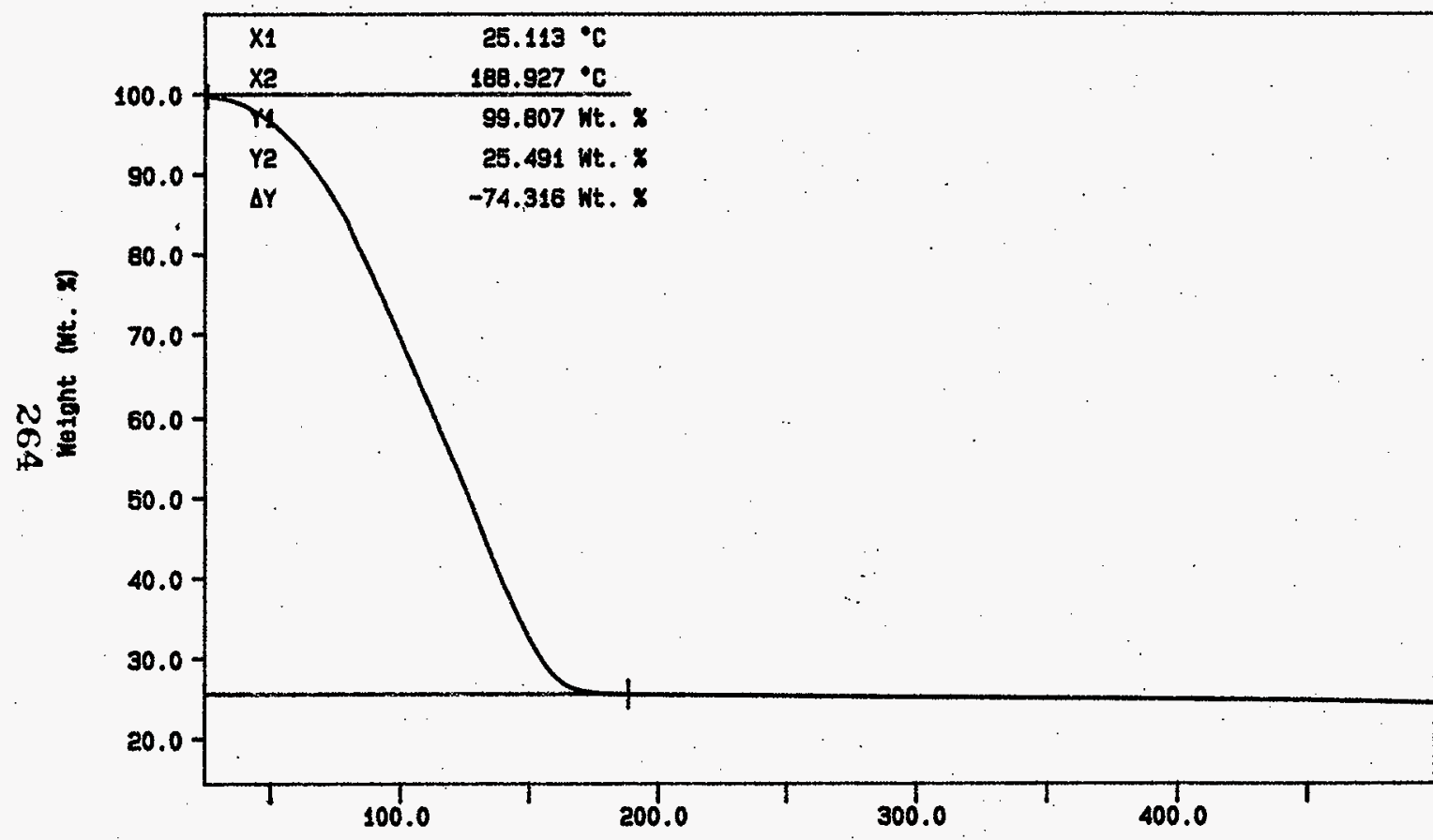

10C/MIN N2

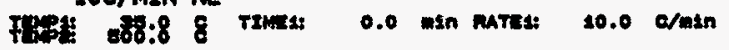

Temperature $\left({ }^{\circ} \mathrm{C}\right)$ AD PUAINTON 


\section{LABCORE Data Entry Template for Worklist\# 17820}

Analyst: RS Instrument: BA001_ Book 133 N $16-A$

Method: LA-510-112 Rev/Mod $\square-0$

Worklist Comment: T204, SPG-01 Use a cal. pipette. skm

MNF.SD-WM-DP-255, REV. 0

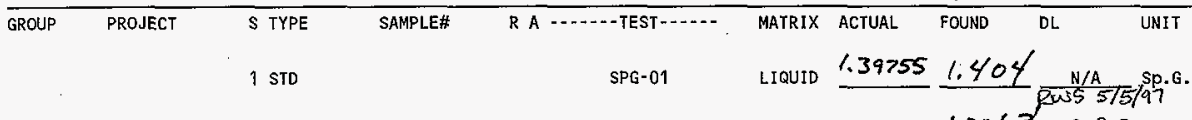

$97000261 \quad T-204$

2 SAMPLE $\quad$ S97T000647 0

SPG-01

LiQuid N/A 1.006Z 0.010 sp.G. RuSS $5 / 519^{\circ}$

$97000261 \quad T-204$

3 DUP

S97T000647 0

SPG-01

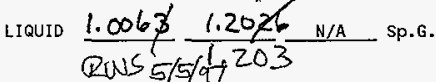

\section{Final page for worklist \# 17820}

Mae Run Sreen 4-30-97

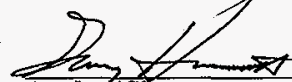

Analyst Signature

Date

Analyst Signature

Date

approved Rus onviden 5/5/97

Data Entry Comments:

Units shown for $Q C$ (SPK \& STD) may not reflect the actual units. $D L=$ Detection Limit, $S=$ Worklist Slot Number, $R=$ Replicate Number, $A=$ Aliquot Code. 


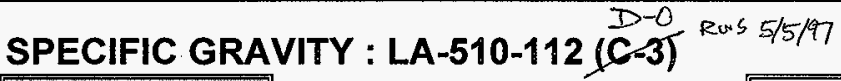

\begin{tabular}{|c|c|c|c|}
\hline \multirow[b]{2}{*}{ W6 } & & \multirow[b]{2}{*}{ STANDARD } & \multirow[b]{2}{*}{ STANDARD } \\
\hline & & & \\
\hline STANDARD & Gross Weight (W2) & 1.4036 & 1.4354 \\
\hline Wholom & Tare Weight (W1) & 1.2972 & 1.3309 \\
\hline 17820 & Weight of Solution (W2-W1) & 0.10638 & 0.10457 \\
\hline r. & Volume of Solution $\mu \mathrm{L}$ & 75.1400 & 75.1400 \\
\hline SPG-0:1 & Specific Gravity & 1.4158 & 1.3917 \\
\hline S & Specific Gravity (Average) & 1.4037 & \\
\hline \multicolumn{4}{|c|}{ 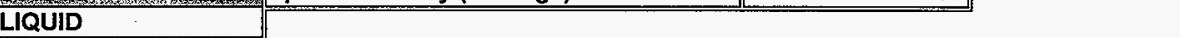 } \\
\hline \multicolumn{4}{|l|}{ 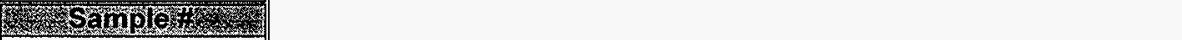 } \\
\hline \multicolumn{4}{|l|}{ STD } \\
\hline 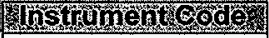 & \multirow{3}{*}{\multicolumn{3}{|c|}{$\begin{array}{l}\text { Gross Weight }(W 2)=W t \text {. of vial + cap + cotton + solution } \\
\text { Tare Weight }(W 1)=W t \text {. of vial + cap + cotton }\end{array}$}} \\
\hline BA001 & & & \\
\hline 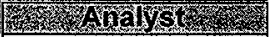 & & & \\
\hline RAG & \multirow{2}{*}{\multicolumn{3}{|c|}{ Specific Gravity $=[(\mathrm{W} 2-\mathrm{W} 1) * 1000 \mu \mathrm{L} / \mathrm{mL}] /[$ Vol. of Solution $\mu \mathrm{L} * 1.000 \mathrm{~g} / \mathrm{mL}]$}} \\
\hline 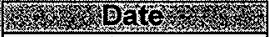 & & & \\
\hline $04 / 30 / 97$ & \multicolumn{3}{|l|}{ v RESULT $v$} \\
\hline 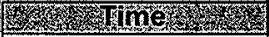 & Specific Gravity Average = & 4.404 & \\
\hline 02:00 PM & & & \\
\hline
\end{tabular}

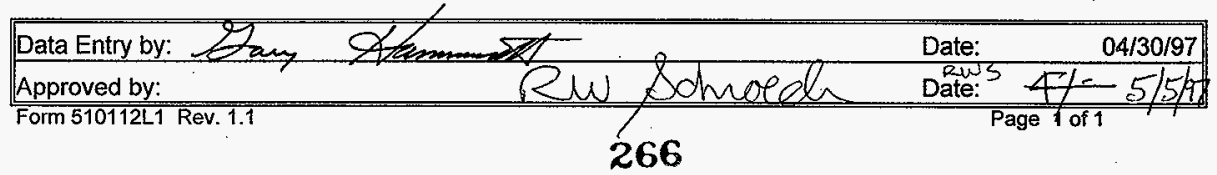


SPECIFIC GRAVITY : LA-510-112 $(\mathrm{D}-3)^{\text {POW }} / 5 / 17$

\begin{tabular}{|c|c|c|c|}
\hline 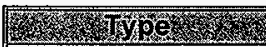 & & SAMPLE & REPLICATE \\
\hline SAMPLE & Gross Weight (W2) & 1.4007 & 1,3753 \\
\hline 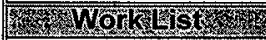 & Tare Weight (W1) & 1.3251 & 1.2850 \\
\hline 17820 & Weight of Solution (W2-W1) & 0.07561 & 0.09036 \\
\hline \% & Volume of Solution $\mu \mathrm{L}$ & 75.1400 & 75.1400 \\
\hline SPG-01 & Specific Gravity & 1.0063 & 1.2026 \\
\hline 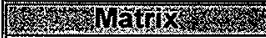 & Specific Gravity (Average) & 1.1044 & \\
\hline
\end{tabular}

LIQUID

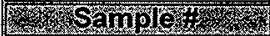

S97T000647

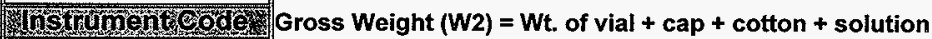

BA001

3.

RAG

Tare Weight $(W 1)=W t$. of vial + cap + cotton

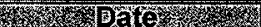

$04 / 30 / 97$

Specific Gravity $=[(W 2-W 1) * 1000 \mu \mathrm{L} / \mathrm{mL}] /[$ Vol. of Solution $\mu \mathrm{L} * 1.000 \mathrm{~g} / \mathrm{mL}]$

V RESULT V

6xis

Specific Gravity Average =

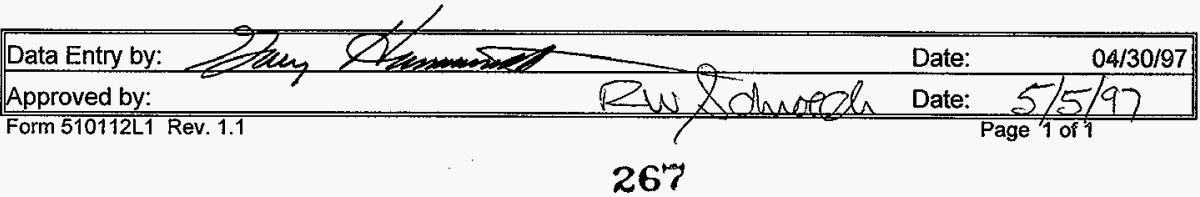




\section{LABCORE Completed Worklist Report for Worklist\# 17900}

\section{Analyst: smf}

Method: $\angle 4-533 \%$ OSRev/Mod
Book\# 50N20D

HNF-SD-WR-DP-255, REV. 0

Worklist Comment: T204 @IC-01 rerun. Try 11 or 21 df.jmf

\begin{tabular}{|c|c|c|c|c|c|c|c|c|c|}
\hline$\overline{\text { Seq Type }}$ & Sample & $\mathbf{R ~ A}$ & & Test & Matrix & Actual & Found DL or Yield & Unit & \\
\hline $1 \mathrm{CcB}$ & & 0 & $19 I C-0 \%$ & \% & oc & 1 & $1<2002$ & 4g/pu & \\
\hline $1 \mathrm{cCB}$ & & 0 & $\triangle I C-Q C$ & CL & Qc & 1 & $<1.700-2$ & $\mathrm{ug} / \mathrm{mr}$ & \\
\hline $1 \mathrm{CPB}$ & & 0 & $0 \mathrm{IO} \alpha \mathrm{C}$ & Hot & 00 & $1 \%$ & $41080-1$ & $10 \mathrm{~g} / \mathrm{mi}$ & ঋ/ \\
\hline $\mathrm{I} \mathrm{CCB}$ & & 0 & ac- $Q c$ & BR & $8 c$ & 1 & $<1.25 e-1$ & $u g / m L$ & \\
\hline $1<c B$ & & 0 & $010-0$ & 403 & oc & x & 1339001 & ig/ & \\
\hline $1 \mathrm{CCB}$ & & 0 & aIc-oc & $\mathrm{PO4}$ & 20 & $I$ & $<1.200-1$ & $\mathrm{ug} / \mathrm{mL}$ & \\
\hline $10 \mathrm{cos}$ & & 0 & $810 \% 0$ & 804 & 20 & 1 & $1438-1$ & $19 \%$ m & \\
\hline $1 \mathrm{CCB}$ & & 0 & OIC-QC & OXALATE2 & ec & 1 & $<1.05 \theta-1$ & $\mathrm{ug} / \mathrm{mr}$ & \\
\hline $2 \mathrm{col}$ & & 0 & $\sigma \mathrm{O} O \mathrm{O}$ & 2 & oc & 590001 & $5 \times 340+01$ & Wo-orox & \% \\
\hline $2 \mathrm{ccv}$ & & 0 & IC-QC & os & QO & 7.90001 & $8.10 e+01$ & \& Recovery & \\
\hline $2 \mathrm{cov}$ & & 0 & $\mathrm{Src}_{\mathrm{\alpha C}}$ & ro2 & $0 \%$ & 543602 & 5460102 & Hecover & \\
\hline $2 \mathrm{ccv}$ & & 0 & OIC-QC & $\mathrm{BR}$ & QC & 5.89002 & $5.750+02$ & $\%$ Recovery & \\
\hline $2 / 6 \mathrm{~V}$ & & 0 & $0 \times C 00$ & No3 & 80 & $504-02$ & $5088-02$ & $\%$ Recovory & \\
\hline $2 \mathrm{ccv}$ & & 0 & PIC-QC & PO4 & Qd & 5.45002 & $5.390+02$ & \% Recovery & \\
\hline $2 \% c v$ & & 0 & $810 \% 6$ & $1504 \%$ & $0 \%$ & $6 \times 1002$ & $625 \cdot 02$ & 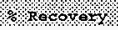 & \\
\hline $2 \mathrm{ccv}$ & & 0 & - $I C-Q C$ & OXALATE2 & ec & 5.32002 & $5.490+02$ & \& Recovery & \\
\hline 3 SAMPtS & $597=000647$ & 0 & 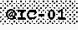 & 102 & Lxoutp & 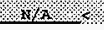 & $14270-01$ & $100.9 / \mathrm{mr} / 2$ & $\%$ \\
\hline 3 SAMPLE & $\$ 97 T 000647$ & 0 & OIC-01 & $C L-02$ & IIQDID & $\mathrm{N} / \mathrm{A}$ & $7.933 e+00$ & ug/mL & \\
\hline 3 SAMPA & 8974000647 & 0 & $0 \mathrm{r} \sigma \mathrm{OL}$ & 402002 & Yroud & NA & $2,1-40100$ & ongm & \\
\hline 3 SAMPLE & s97T000647 & 0 & GIC-01 & $B R-02$ & IIQUID & $N / A<$ & $2.0000+00$ & $\mathrm{ug} / \mathrm{mr}$ & $\therefore$ \\
\hline 3 SAMPLE & 5879000647 & 00 & QIC 01 & 203702 & - Topso & $\mathrm{N} / \mathrm{s}$ & $4702=00$ & ug: $/$ nt & \% \\
\hline 3 SAMPLE & s97T000647 & 0 & $0 \mathrm{IC}-01$ & PO4-02 & IIQUID & N/A & $2.077 a+00$ & $\mathrm{ug} / \mathrm{mI}$ & \\
\hline 3 SAMPRA & $597>000647$ & \% & $810-01$ & $80 \times 02$ & $4 \pi 00 \mathrm{PD}$ & n/ & $17800+01$ & 19/rim & $\%$ \\
\hline 3 SAMPLE & \$97T000647 & 0 & OIC-01 & OXALATE2 & IIQOID & $N / A$ & $1.7320+00$ & $\mathrm{ug} / \mathrm{mI}$ & \\
\hline 4 pup & $597 \% 000647$ & 0 & orC 01 & 802 & Lrouto & $4-1,9201$ & 410201 & $\mathrm{RPD}$ & $\%$ \\
\hline $4 D J P$ & $\$ 975000647$ & 0 & OIC-01 & $\mathrm{CL}-02$ & ITQUID & $7.930+00$ & $8.360+00$ & RPD & \\
\hline $400 P$ & 5972000647 & 0 & olc-o1 & 102802 & YIOPID & $2110 \% 00$ & $1 / 1 \% 300$ & RPD & \\
\hline 4 DUP & 5977000647 & 0 & OIC-01 & $\mathrm{BR}-02$ & LIQUID & $<2.0000$ & $<2.0000$ & RPD & \\
\hline 4 DUP: & 5970000647 & 0 & $610-01$ & 10302 & - 20012 & 170000 & $1488+00$ & RPD & 11 \% \\
\hline $4 D U P$ & $\$ 975000647$ & 0 & अIC-01 & $F 04-02$ & IIQDID & $2.080+00$ & $<1.9200$ & RPD & \\
\hline $4 \mathrm{pOH}$ & 592000064 & $0 \%$ & 01001 & $\mathrm{sol}_{02}$ & Mrouxp & $1780+0$ & $18026+01$ & RDP & \%स\% \\
\hline 4 DUP & S97T000647 & 0 & aIc-01 & OXALATE2 & IIQUID & $1.73 e+00$ & $<1.68 \mathrm{ea}$ & RPD & \\
\hline
\end{tabular}

\section{Final page for worklist\# 17900}

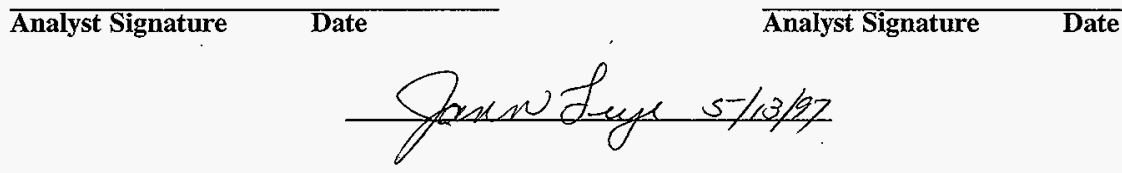

Units shown for $Q C(B L K / B K G)$ may not reflect the actual units. 


\section{LABCORE Data Entry Template for Worklist\# 17900}

Analyst: $\quad$ SMF Instrument: ICO 1 Book\# 50N20-1)

Method: LA-533-105 Rev/Mod $D-1$

Worklist Comment: T204 @IC-01 rerun. Try 11 or 21 df.jmf

HNF-SD-WM-DP-255, REV. 0

\begin{tabular}{|c|c|c|c|c|c|c|}
\hline S Type & Sample\# & $\mathbf{R A}$ & Test & Natrix & Project & \\
\hline $1 \mathrm{CCB}$ & v & & @IC:QC & $Q C$ & & \\
\hline $2 \mathrm{CCV}$ & & & @IC-QC & QC & & \\
\hline 3 SAMPLE & S97T000647 & 0 & @IC-01 & IIQUID & 97000261 T-204 & \\
\hline & Analytes Reque & ested: & $\begin{array}{l}\text { BR-02 } \\
\text { OXALATE }\end{array}$ & $2, \begin{array}{l}\text { CL-02 } \\
2, \quad \text { PO4-02 }\end{array}$ & $, \begin{array}{l}\mathrm{F}-02 \\
\mathrm{SO}-02\end{array}, \mathrm{NO} 2-02$, & , NO3-02 \\
\hline
\end{tabular}

4 DUP $\quad$ S97T000647 0 QIC-01 LIQUID

\section{Final page for worklist \# 17900}

\section{Analyst Signature Date}
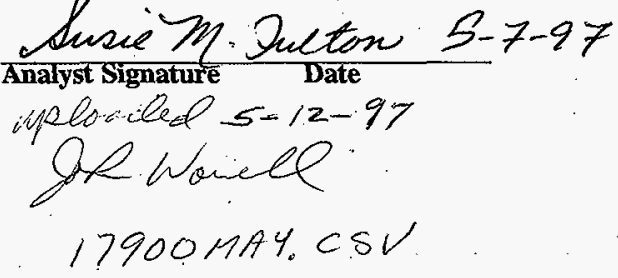

$1790019 A 4.0 .5 V$

iplowiled $5+12-97$

Data Entry Comments:

PF.16 $=.200 \mathrm{me} \operatorname{san} \rightarrow 3.0 \mathrm{ml}$ Eluent. Insufent sample (ale that was left

$\bar{S}=$ Worklist Slot Number, $R=$ Replicate Number, $A=$ Aliquot Code.

\section{was}

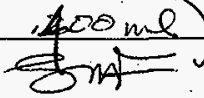




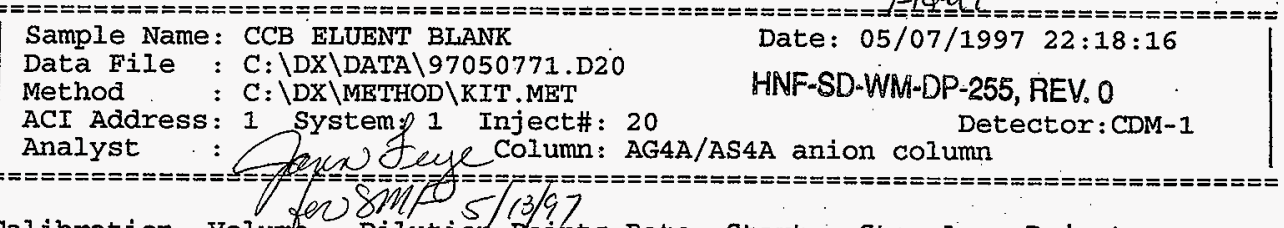

Calibration Volum Dilution Points Rate start Stop Area Reject External 1

$13000 \quad 5 \mathrm{~Hz} \quad 0.00 \quad 10.00$ 30

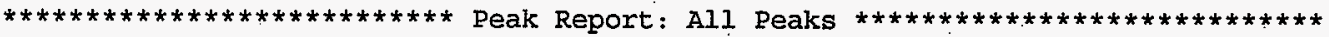

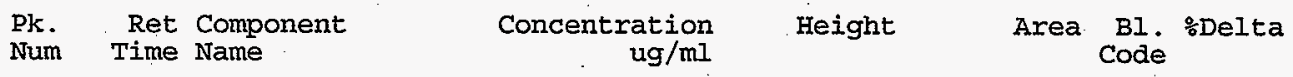

\begin{tabular}{|c|c|c|c|c|c|c|}
\hline $\begin{array}{l}1 \\
2 \\
3 \\
4\end{array}$ & $\begin{array}{l}1.43 \\
1.66 \text { chloride } \\
3.33 \text { nitrate } \\
6.29 \text { sulfate }\end{array}$ & $\begin{array}{l}0.000 \\
0.011 \\
0.139 \\
0.018\end{array}$ & $\begin{array}{l}60 \\
46 \\
80 \\
47\end{array}$ & $\begin{array}{r}465 \\
205 \\
535 \\
1002\end{array}$ & $\begin{array}{l}1 \\
1 \\
1 \\
1\end{array}$ & $\begin{array}{l}4.18 \\
2.36 \\
4.42\end{array}$ \\
\hline & & 0.168 & 232 & 2206 & & \\
\hline
\end{tabular}

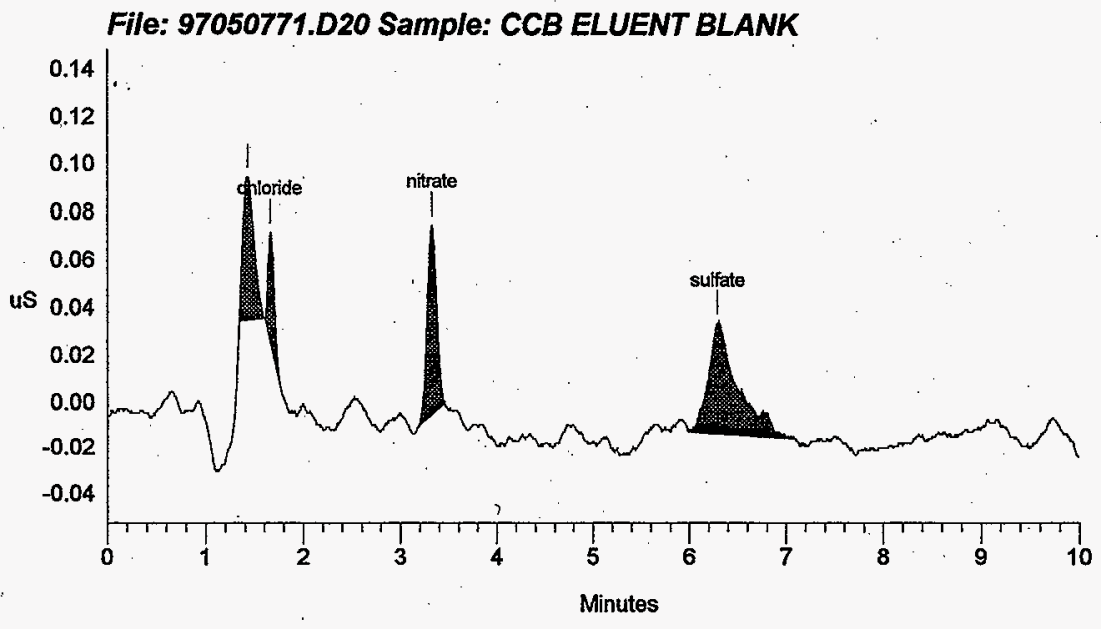


HAF-SQWAM-DP-245, REV. 0 TUEC

Data Reprocessed On 05/07/1997 22:38:40

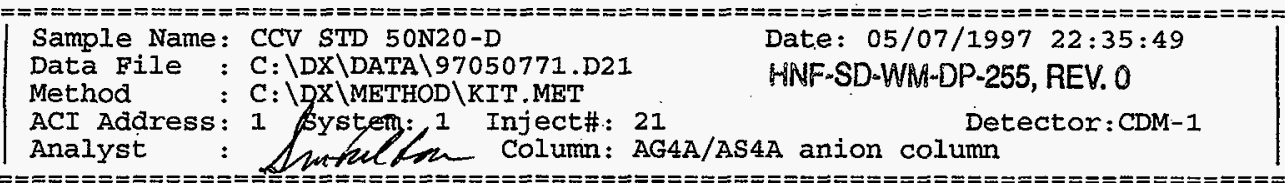

Calibration Volume Dilution Points Rate start stop Area Reject

External $\quad 1 \quad 101 \quad 3000$.5Hz $0.00 \quad 10.00 \quad 30$

$\star * * * * * * * * \star * * * * * * * * * * * * * * * * * *$ Peak Report : AlI Peaks $* * * * * * * * * * * * * * * * * * * * * * * * * * * *$

Pk. Ret Component Concentration Height Area Bl. $\%$ Delta

Num Time Name $\mathrm{ug} / \mathrm{ml}$ Code

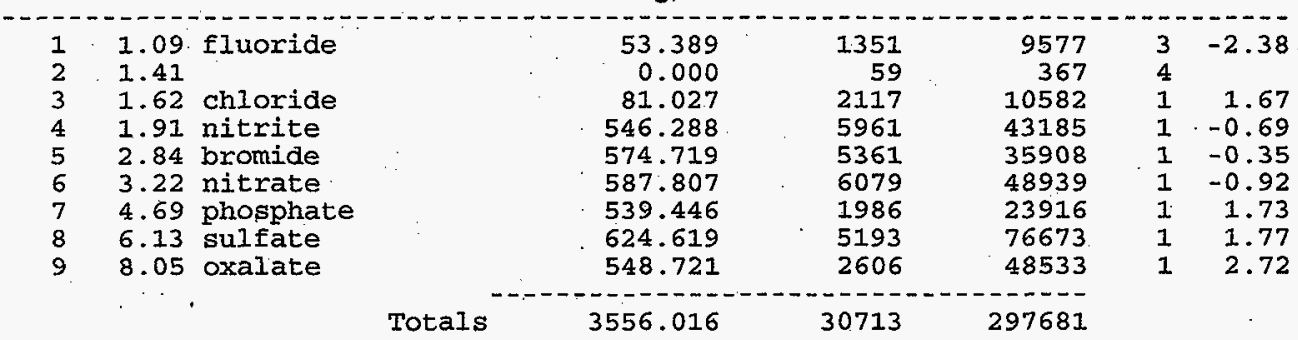

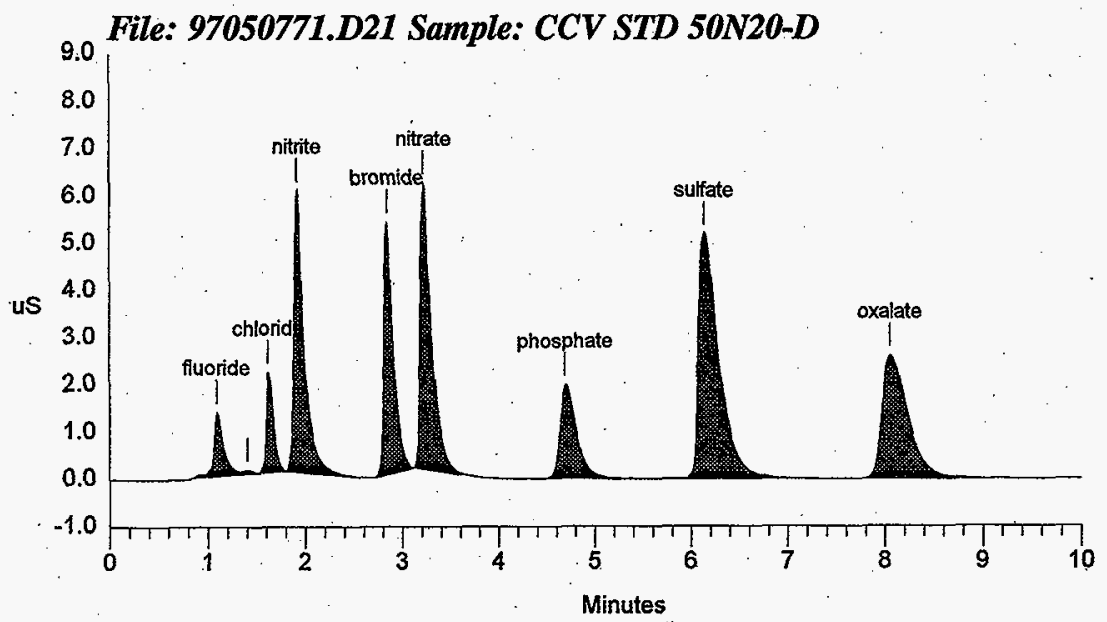




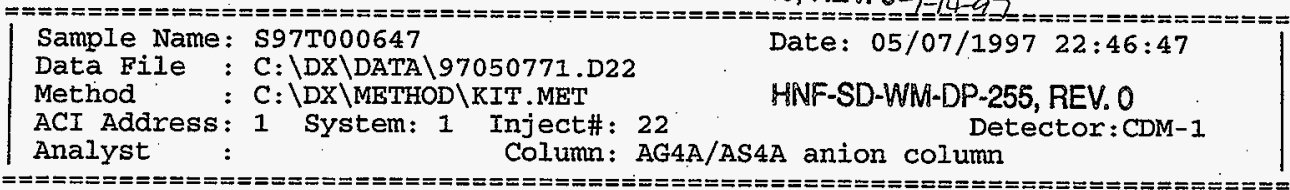

Calibration Volume Dilution Points Rate start stop Area Reject

Calioration

External

1

16. $3000 \quad 5 \mathrm{~Hz} \quad 0.00 \quad 10.00$

30

$\star * * * * * * * * * * * * * * * * * * * * * * * * * *$ Peak Report: AlI Peaks $* * * * * * * * * * * * * * * * * * * * * * * * * * * *$

Pk. Ret Component Concentration Height Area Bl. :Delta

Num Time Name $\mathrm{ug} / \mathrm{ml}$

$\begin{array}{lll}1 & 1.25 \\ 2 & 1.43 \\ 3 & 1.63 \text { chloride } \\ 4 & 1.97 \text { nitrite } \\ 5 & 2.92 \text { bromide } \\ 6 & 3.32 \text { nitrate } \\ 7 & 4.75 \text { phosphate } \\ 8 & 6.19 \text { sulfate } \\ 9 & 8.16 \text { oxalate }\end{array}$

$\begin{array}{rrr}0.000 & 31 \\ 0.000 & 61 \\ 7.933 & 1279 \\ 2.114 & 56 \\ 1.906 & 61 \\ 4.702 & 246 \\ 2.077 & 25 \\ 17.802 & 865 \\ 1.732 & 39\end{array}$

Totals

38.265

2664

31

61

79

56

61

246

25

865

39

39.

Code

$\begin{array}{rrr}106 & 1 & \\ 393 & 1 & \\ 6522 & 1 & 2.51 \\ 290 & 1 & 2.42 \\ 372 & 1 & 2.46 \\ 1839 & 1 & 2.15 \\ 296 & 1 & 2.89 \\ 14251 & 1 & 2.65 \\ 725 & 1 & 4.08\end{array}$

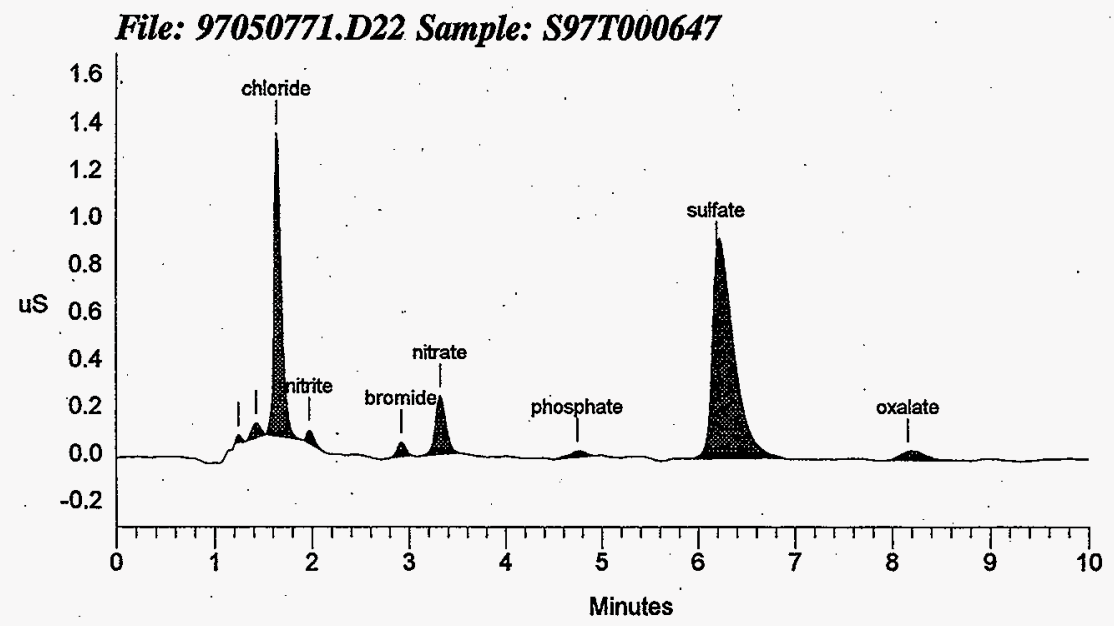




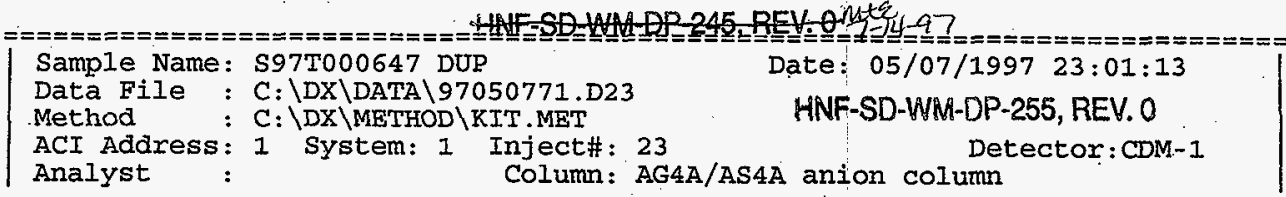
Sample Name: S97T000647 DUP
Data File : C: \DX\DATA $97050771 . D 23$
Method : C: $\backslash D X \backslash M E T H O D \backslash K I T . M E T$
Date: $05 / 07 / 1997$ 23:01:13
ACI Address: 1 System: 1 Inject\# : 23

Calibration Volume Dilution Points Rate Start Stop Area Reject

External $163000 \quad 5 \mathrm{~Hz} 0.00 \quad 10.00 \quad 30$

$\star * * * * * * * * * * * * * * * * * * * * * * * * * *$ Peak Report: All Peaks $* * * * * * * * * * * * * * * * * * * * * * * * * * * *$

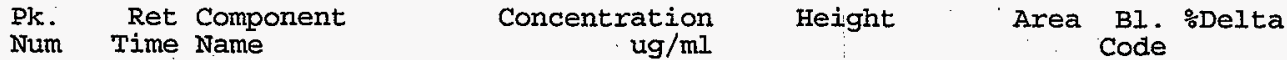

Num Time Name ug/mi

$\begin{array}{ll}1 & 1.25 \\ 2 & 1.43 \\ 3 & 1.63 \text { chloride } \\ 4 & 3.31 \text { nitrate } \\ 5 & 6.19 \text { sulfate }\end{array}$

Thes

Totals
0.000

0.000

8.355

5.478

32
81
1346
305
857

32.016

2620

$\begin{array}{rrr}99 & 1 & \\ 526 & 1 & \\ 6869 & 1 & 2.51 \\ 2247 & 1 & 1.95 \\ 14540 & 1 & 2.65\end{array}$

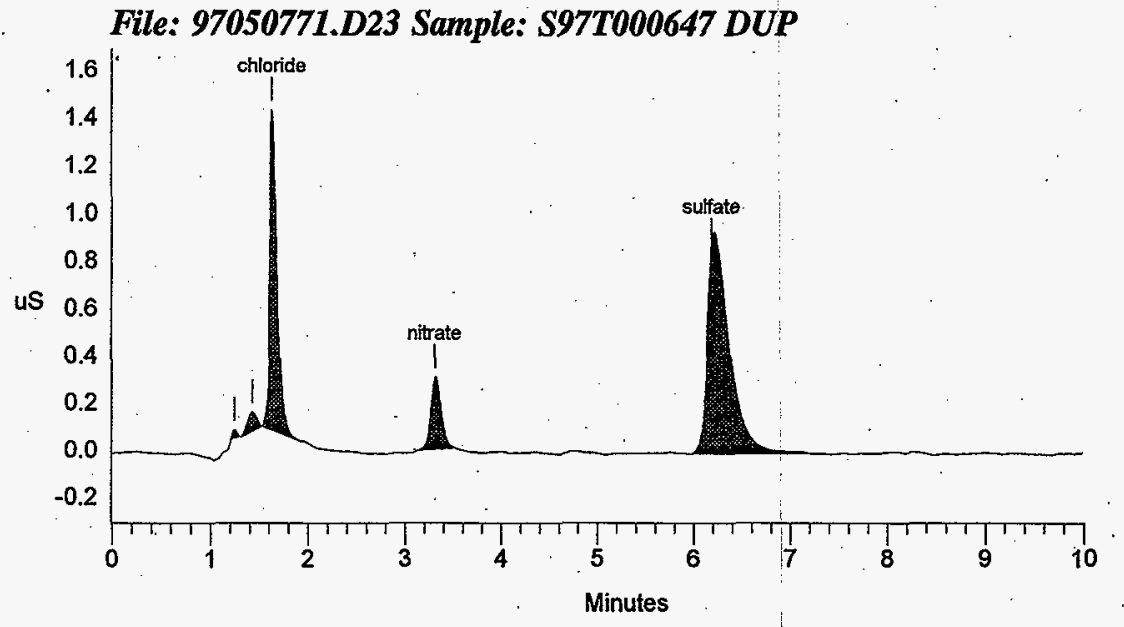




\section{LABCORE Completed Worklist Report for Worklist\# 19440}

\section{Analyst: kgh}

\author{
Instrument: IC01
}

Book\# $72020 A$

Method: $\angle A-533-105 \mathrm{Rev} / \mathrm{Mod} D-1$

Worklist Comment: T204, @IC-01 skm

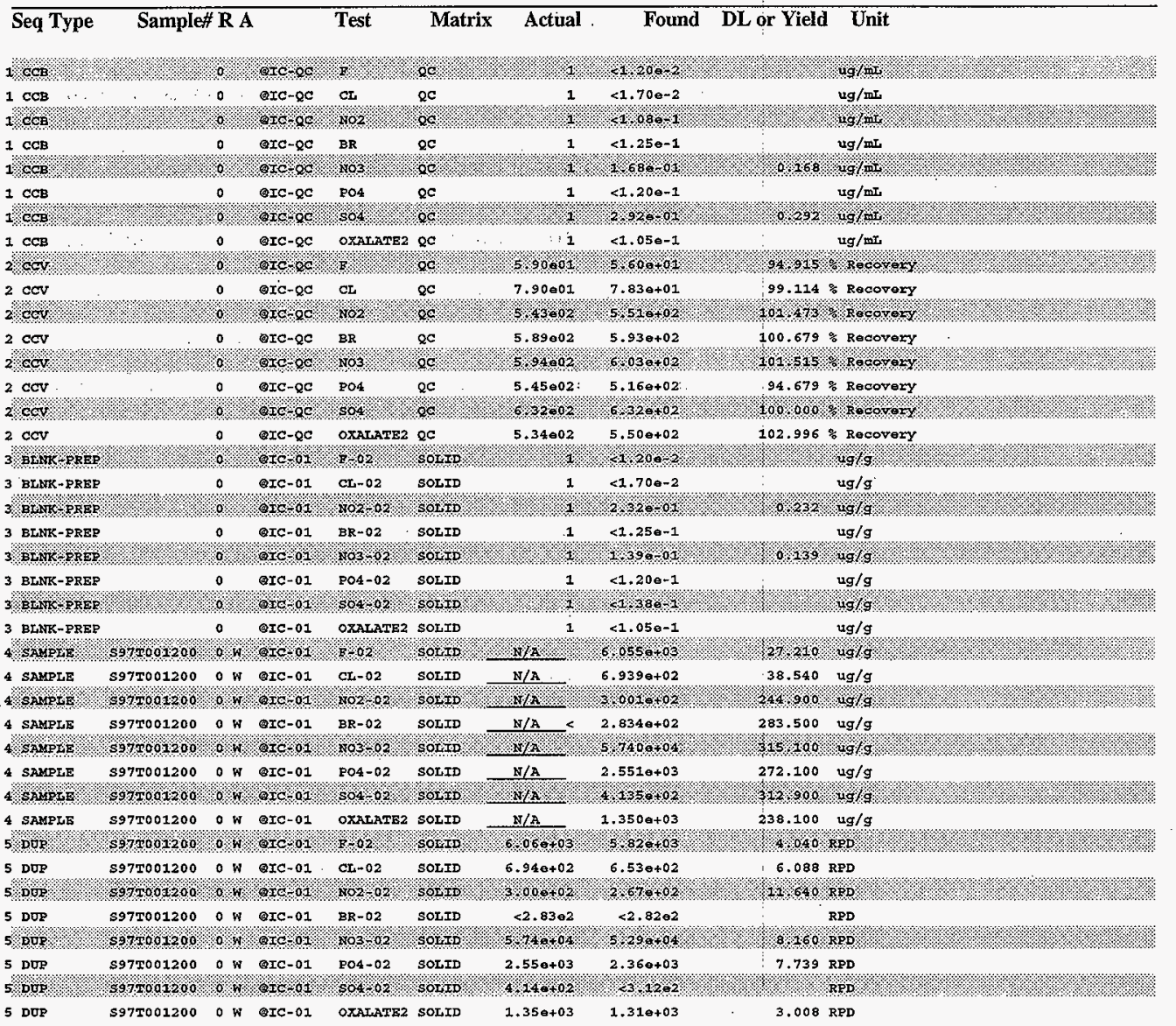




\section{LABCORE Completed Worklist Report for Worklist\# 19440}

Seq Type Sample\# A Test Matrix Actual Found DL or Yield Onit

\section{Final page for worklist\# 19440}

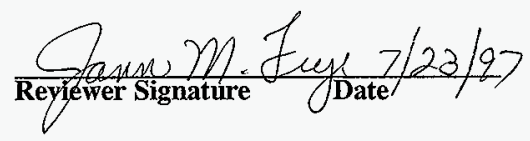




$$
H N F-S \Delta-W M-O P-255, \text { ReV. } 0
$$

$07 / 23 / 9708: 24$

Page: 1

$A-0004-1$

LABCORE Data Entry Template for Worklist\# 19440

Analyst: $\quad \mathrm{K} 6 \mathrm{H}$ Instrument: $\mathrm{ICO} \perp$ Book\# $72 \mathrm{~N} 20 \mathrm{~A}$

Method: LA-533-105 Rev/Mod D-1

Worklist Comment: T204, @IC-01 skm

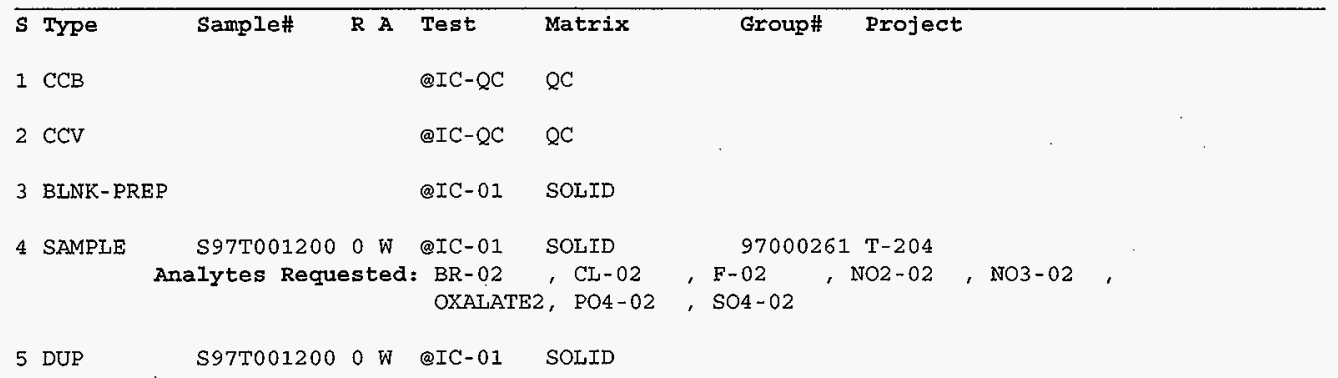

Final page for worklist \# 19440

$\frac{7 m f \text { cuefwe } 647 / 23 / 37}{\text { Date }}$

$\overline{\text { Analyst Signature Date }}$

Re-uploaded 7-23-97

19440 TuL.CSV

Validated $>123 / 979$ mage

Data Entry Comments: Data fran 18822 was re uploaded to 19440 . No real rerun un as dene.

Ph ad wanted spike ran, tut re sun

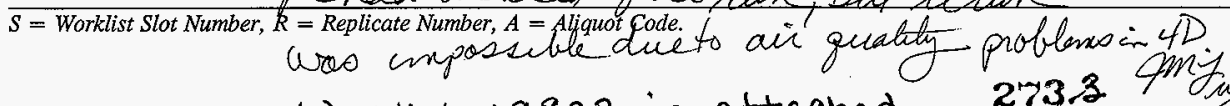




\section{LABCORE Completed Worklist Report for Worklist\# 18822}

Analyst: kgh

Method:

Worklist Comment: T-204 IC. RCJ
Book\# 72N20A

HNF-SD-WM-DP-255, REV. 0

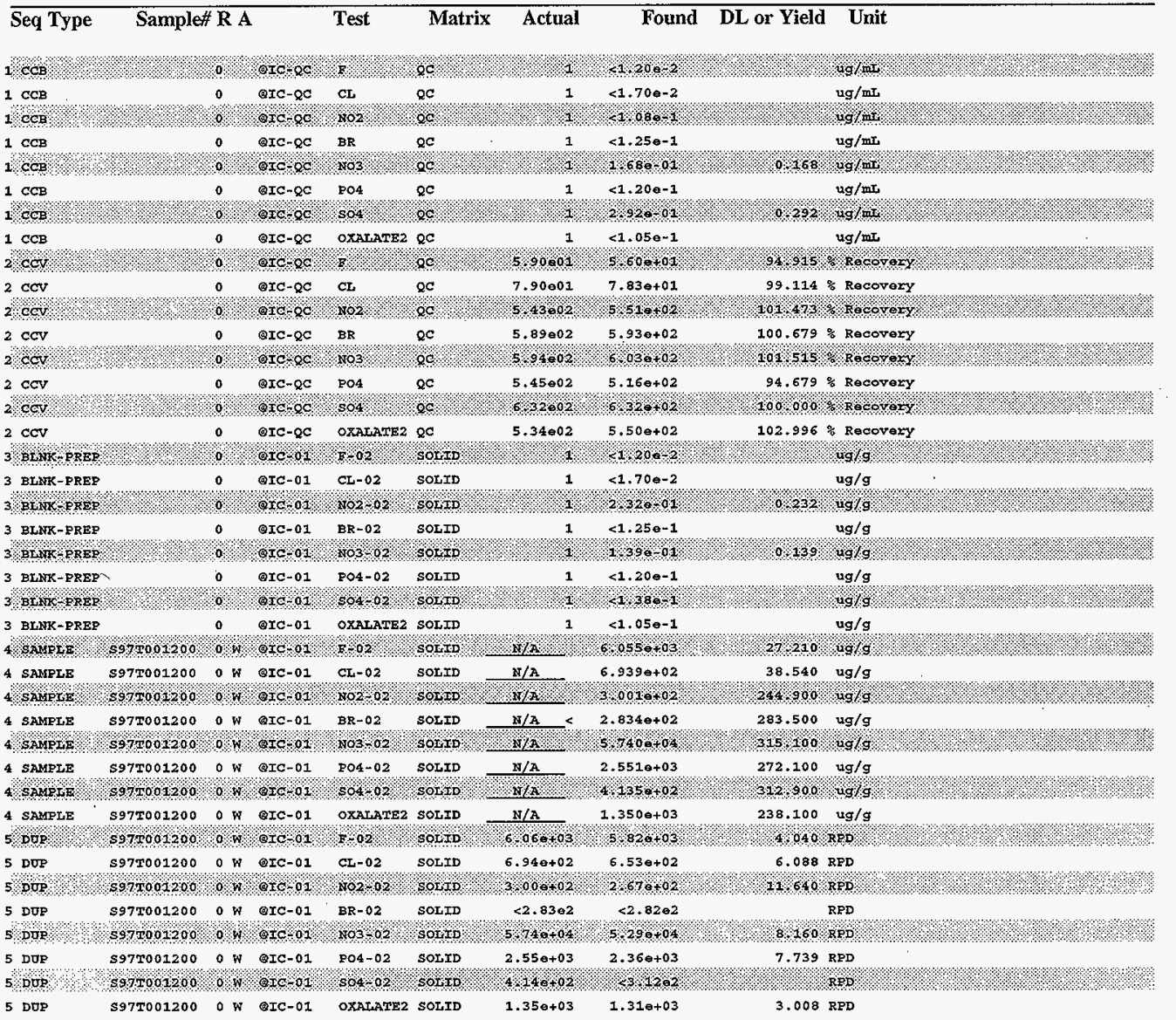


HNF-SD-WMA-DP-255, REV 0

worklistdata Version 1.0 05/15/96

06/26/97 13:52

HAF-SD-WMA-QP-245, REV.QU, $7-14-97$

Page: 2

\section{LABCORE Completed Worklist Report for Worklist\# 18822}

Seq Type Sample\#RA Test Matrix Actual Found DL or Yield Unit

\section{Final page for worklist\# 18822}

Analyst Signature Date

Analyst Signature Date

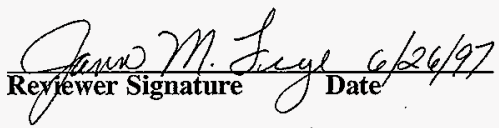




\section{LABCORE Data Entry Template for Worklist\# 18822}

Analyst:

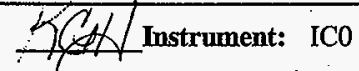

Method: LA-533-105 R Rev/Mod D-l
Book\# $62120 \%$

HNF-SD-WR-DP-255, REV. 0

Worklist Comment: T-204 IC. RCJ

\begin{tabular}{|c|c|c|c|c|c|c|c|c|}
\hline$\overline{\mathbf{s}}$ & Type & Sample\# & RA & Test & Matrix & Group\# & Project & \\
\hline 1 & $\mathrm{CCB}$ & & & $@ I C-Q C$ & $\mathrm{QC}$ & & & . \\
\hline 2 & $\mathrm{CCV}$ & & & $@ I C-Q C$ & $\mathrm{QC}$ & & & \\
\hline 3 & BLNK-PREP & & & @IC-01 & SOLID & & & \\
\hline 4 & SAMPLE AI & $\begin{array}{r}\text { 597T001200 } \\
\text { nalytes Reque }\end{array}$ & $\begin{array}{l}0 W \\
\text { ested: }\end{array}$ & $\begin{array}{l}\text { QIC-01 } \\
=\text { BR-02 } \\
\text { OXATATE2 }\end{array}$ & $\begin{array}{l}\text { SOLID } \\
\text { CL-02 } \\
\text { 2. PO4-02 }\end{array}$ & $\begin{array}{l}97000261 \\
, \mathrm{~F}-02 \\
\mathrm{SO}-02\end{array}$ & $\begin{array}{r}\mathrm{T}-204 \\
\mathrm{NO}-02\end{array}$ & NO3-02 \\
\hline
\end{tabular}

5 DUP

S97T001200 0 W QIC-01 SOLID

\section{Final page for worklist \# $\mathbf{1 8 8 2 2}$}

Anatyst sign

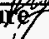

Bate

uploaded 6-25-97

Gth wowall

$018022 \pi 0 k \cdot \operatorname{cs} \mathrm{V}$

Walidated $6 / 25 / 97$ qm

Data Entry Comments:

$S=$ Worklist Slot Number, $R=$ Replicate Number, $A=$ Aliquot Code. 


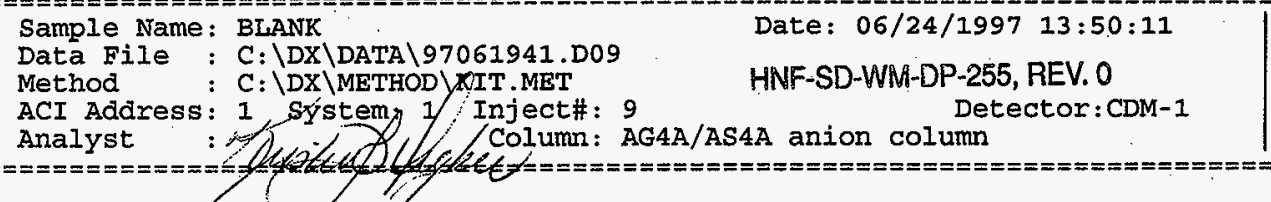

Calibration volume Dilution Points Rate start Stop Area Reject External 1

$13000 \quad 5 \mathrm{~Hz} \quad 0.00 \quad 10.00$

30

$* * * * * * * * * * * * * * * * * * * * * * * * * * *$ Peak Report: All Peaks $* * * * * * * * * * * * * * * * * * * * * * * * * * * *$

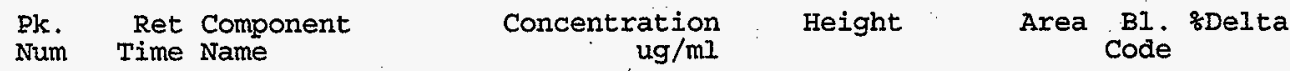

$\begin{array}{lll}2 & 1.46 & \\ 3 & 1.65 & \text { chloride } \\ 4 & 3.25 & \text { nitrate } \\ 5 & 5.92 & \text { sulfate }\end{array}$

.

0.000

27

41

0.009

102

0.292

153

209

180

1

Totals

-...-

781

4312

1

3.77

$1 \quad 0.10$

$\begin{array}{ll}1 & -1.77\end{array}$

0.469

324

5482

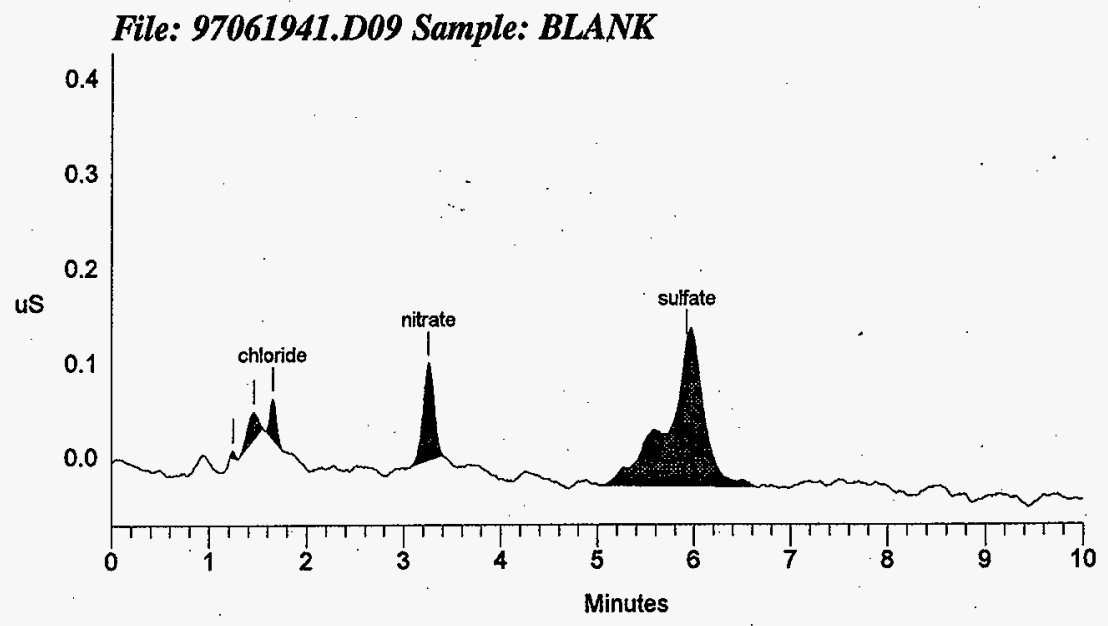

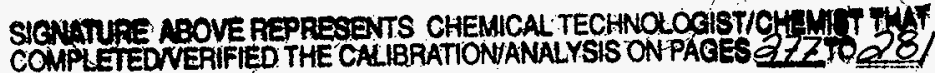




\section{HNF-SD-WA-OP-245, REV.OMU:

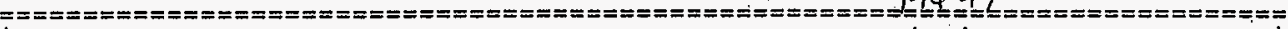

Sample Name: STD 72N20-A

Data File: C: DX\DATA\97061941.D10

Method

: C: \DX\METHOD \KTT.MET

ACI Address: 1 System: 01 Inject\# : 10
Date: 06/24/1997 14:01:42

HNF-SD-WM-DP-255, REV. 0

Detector : $C D M-1$

Analyst

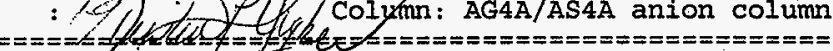

Calibration Volume Difution Points Rate start stop Area Reject

External $\quad 1 \quad 30130005 \mathrm{~Hz} 0.000 .000$

$* * * * * * * * * * * * * * * * * * * * * * * * * * *$ Peak Report: All Peaks $* * * * * * * * * * * * * * * * * * * * * * * * * * * *$ Pk. Ret Component $\quad \begin{array}{r}\text { Concentration } \\ \text { ug/ml }\end{array}$
Num. Time Name

$\begin{array}{lll}1 & 1.09 & \text { fluoride } \\ 2 & 1.60 \text { chloride } \\ 3 & 1.88 \text { nitrite } \\ 4 & 2.78 \text { bromide } \\ 5 & 3.14 \text { nitrate } \\ 6 & 4.51 \text { phosphate } \\ 7 & 5.76 \text { sulfate } \\ 8 & 7.57 \text { oxalate }\end{array}$

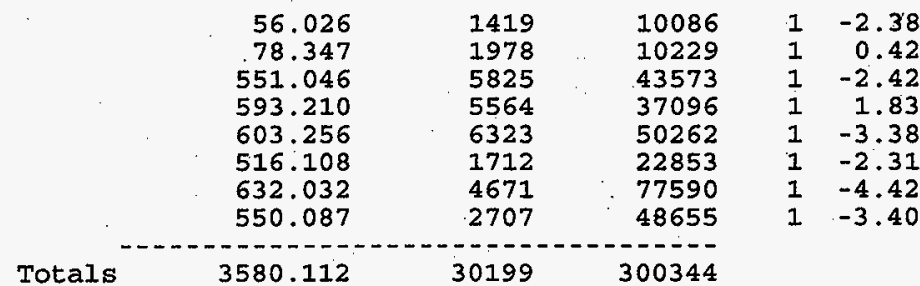

File: 97061941.D10 Sample: STD 72N20-A

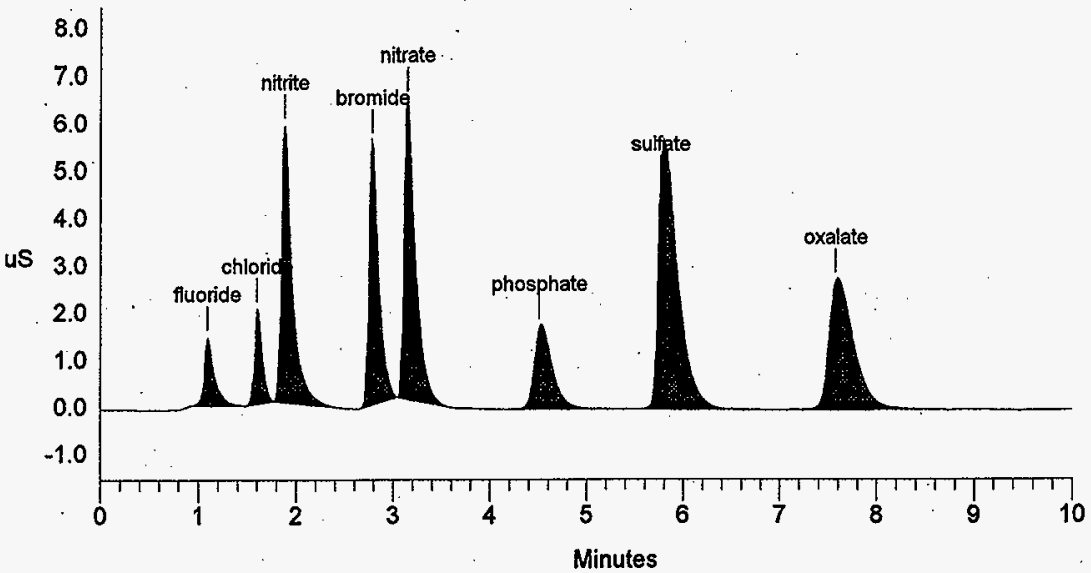




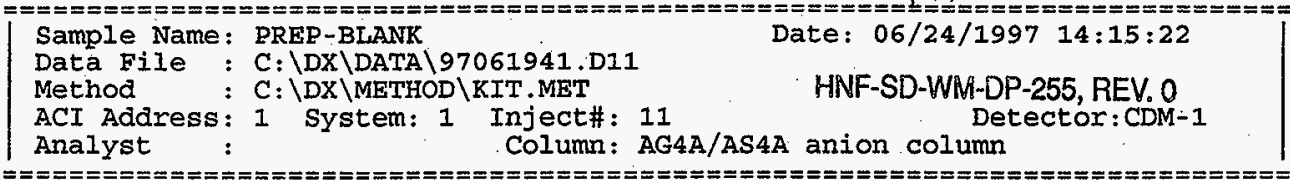

Calibration Volume Dilution Points Rate start Stop Area Reject

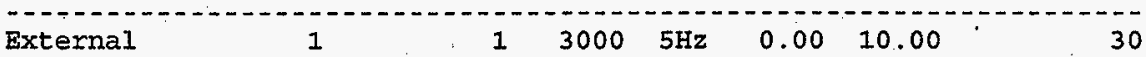

$\star * * * * * * * * * * * * * * * * * * * * * * * * * *$ Peak Report: All Peaks $* * * * * * * * * * * * * * * * * * * * * * * * * * * *$ Pk. Ret Component $\begin{gathered}\text { Concentration } \\ \text { ug/ml }\end{gathered}$ Num Time Name
Nught Area Bl.
Code

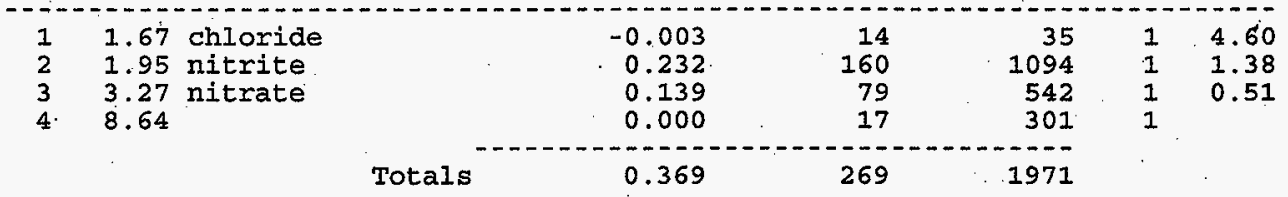

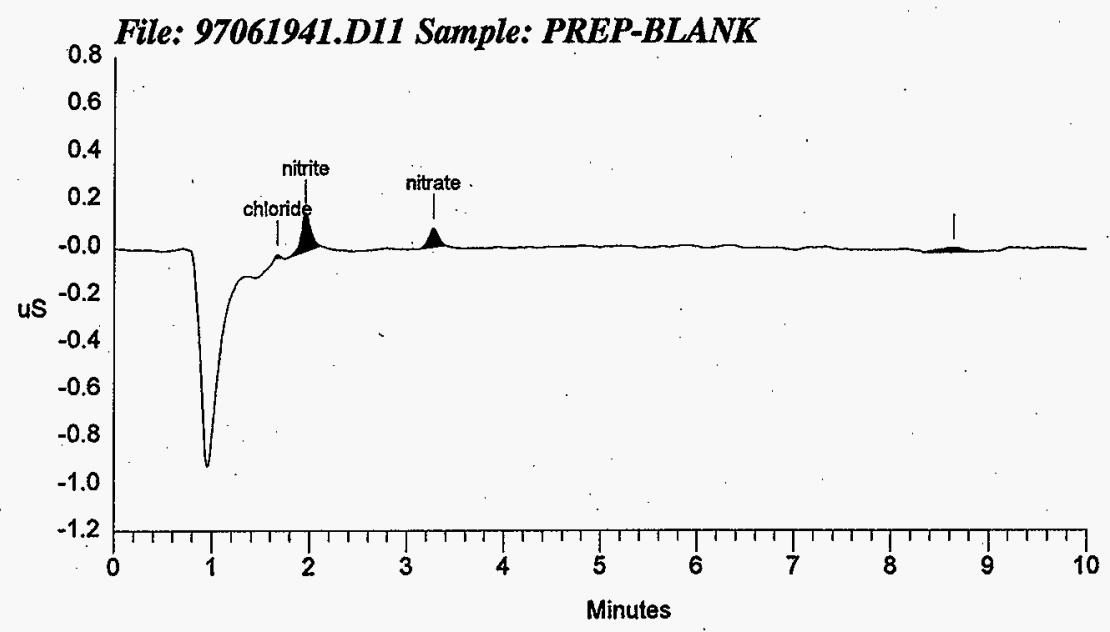




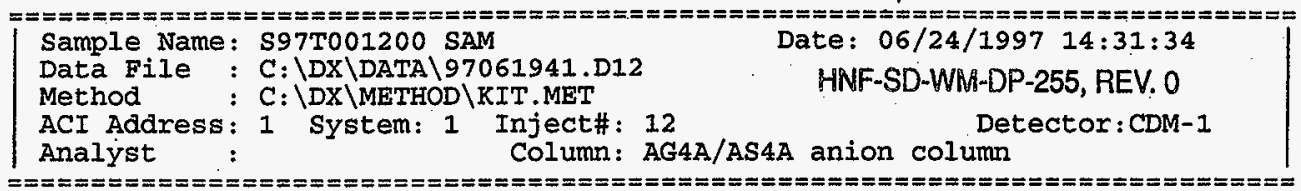

Calibration Volume Dilution Points Rate start stop Area Reject

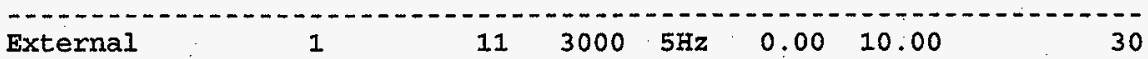

$* * * * * * * * * * * * * * * * * * * * * * * * * * *$ Peak Report: All Peaks $* * * * * * * * * * * * * * * * * * * * * * * * * * * *$

Pk. Ret Component Concentration
$\mathrm{ug} / \mathrm{mI}$ Height Area $\begin{gathered}\text { Bl. } \\ \text { Nodelta }\end{gathered}$

Num Time Name $\mathrm{ug} / \mathrm{mI}$ Code

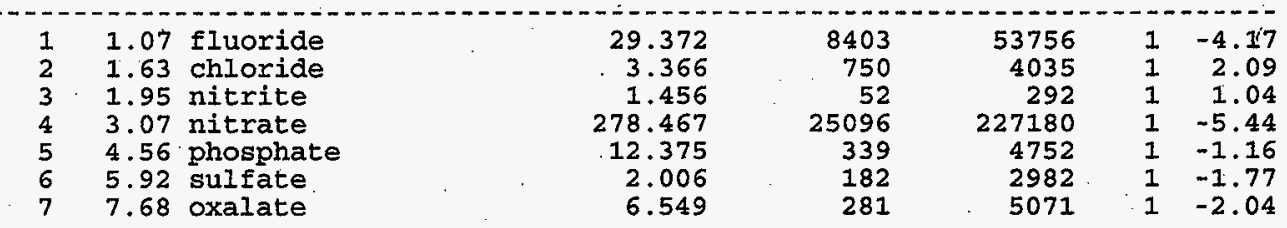

Totals

333.590

35103

298068

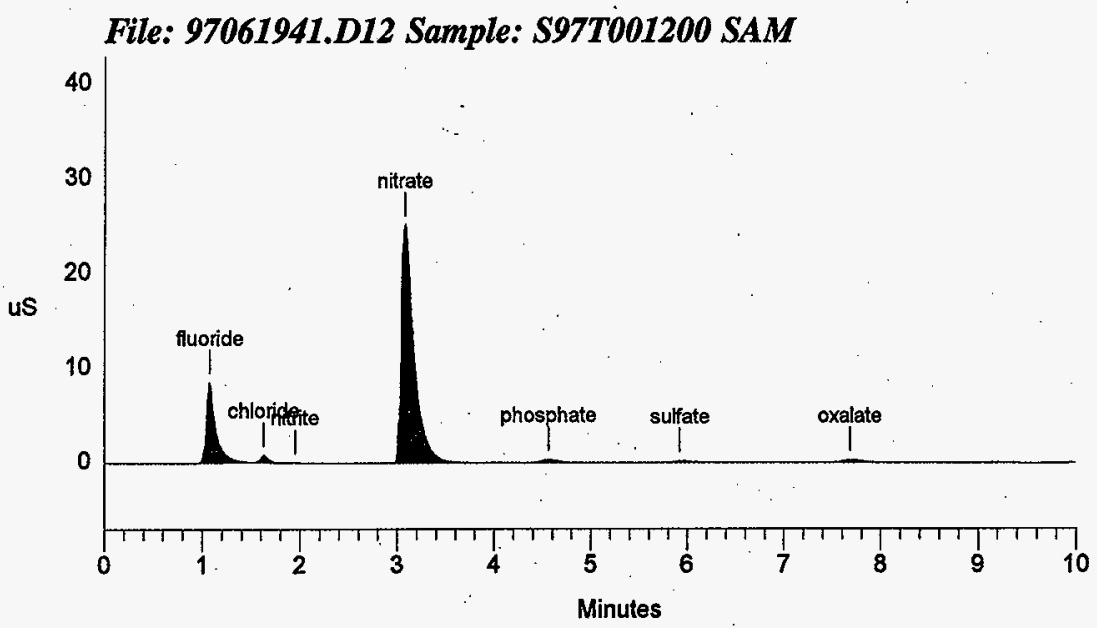


Data Reprocessed on 06/25/1997 $13: 36: 13$

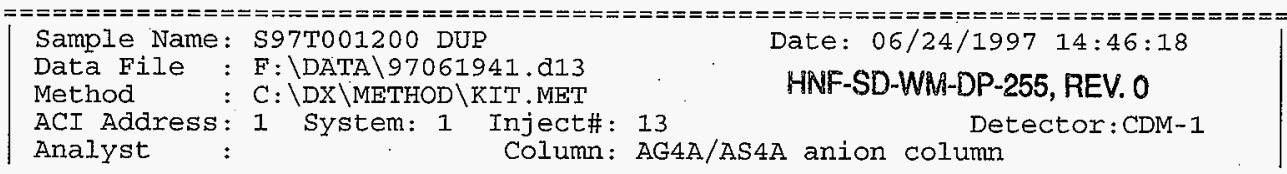

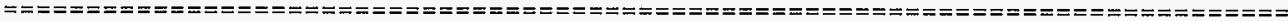

Calibration Volume Dilution Points Rate start stop Area Reject

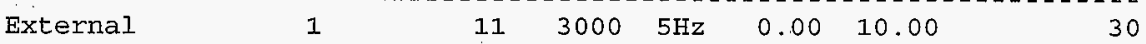

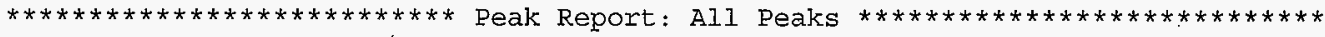

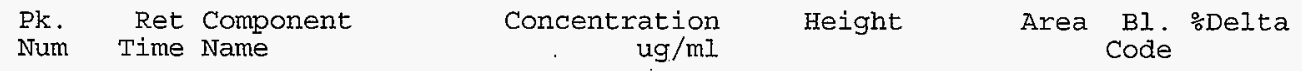

$\begin{array}{lll}1 & 1.07 & \text { fluoride } \\ 2 & 1.62 & \text { chloride } \\ 3 & 1.95 & \text { nitrite } \\ 4 & 3.07 & \text { nitrate } \\ 5 & 4.56 & \text { phosphate } \\ 6 & 5.92 & \text { sulfate } \\ 7 & 7.68 \text { oxalate }\end{array}$

\begin{tabular}{rrrr}
28.327 & 7795 & 51681 \\
3.182 & 720 & 3817 \\
1.300 & 37 & 178 \\
257.475 & 23638 & 208784 \\
& 11.477 & 346 & 4385 \\
& 0.957 & 122 & 1829 \\
Totals & 6.378 & 269 & 4933 \\
\hline & 309.095 & 32926 & 275607
\end{tabular}

File: 97061941.d13 Sample: S97T001200 DUP

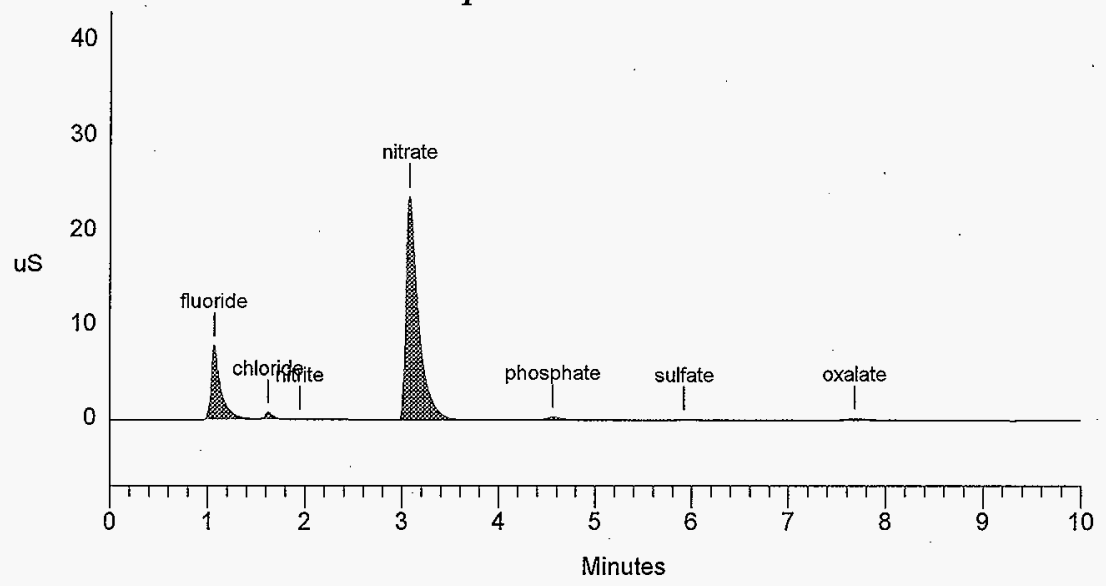




\section{LABCORE Data Entry Template for Worklist\# 17851}

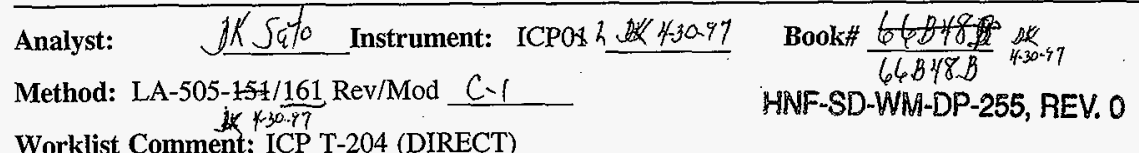

Worklist Comment: ICP T-204 (DIRECT)

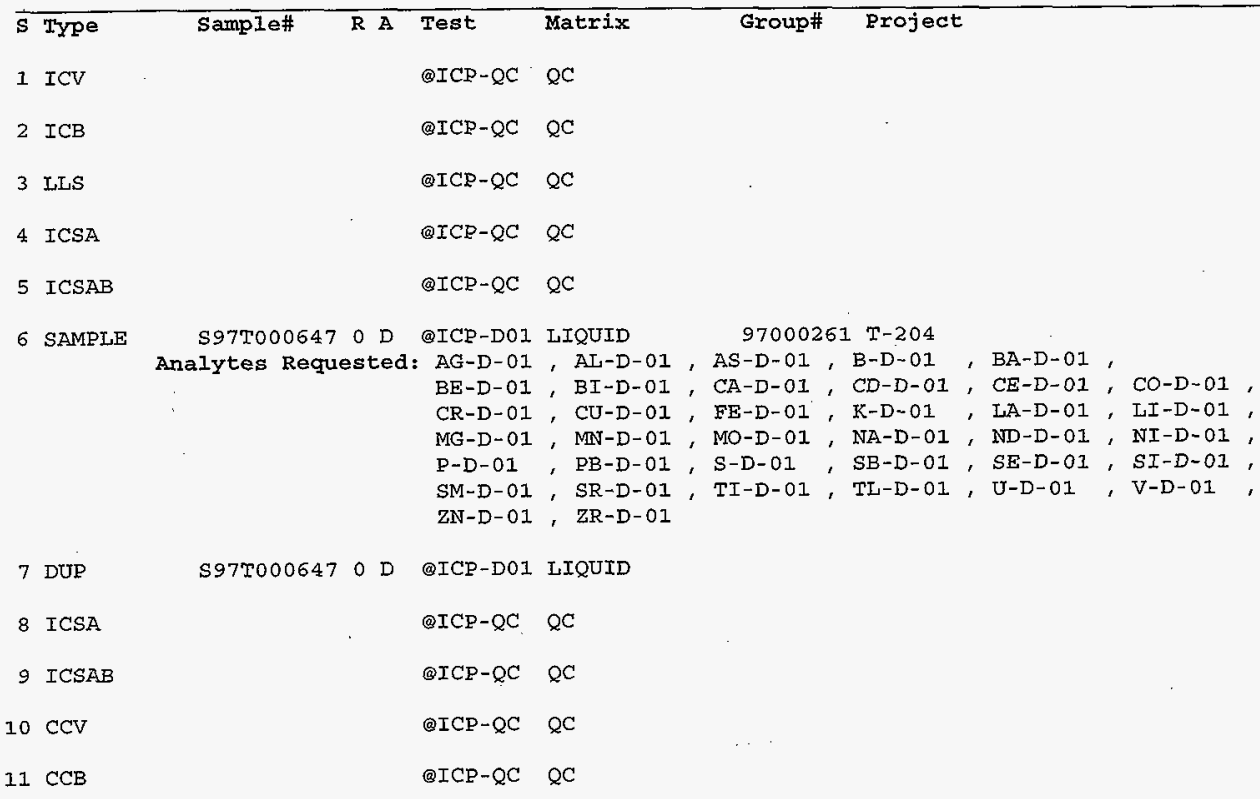

\section{Final page for worklist \# 17851}

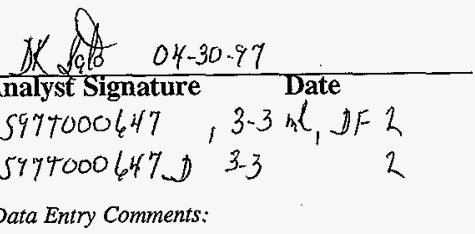

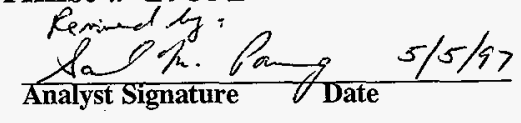

$\bar{S}=$ Worklist Slot Number, $R=$ Replicate Number, $A=$ Aliquot Code. 


\begin{tabular}{|c|c|c|c|c|c|c|c|c|}
\hline 将 & Sample Name & File & Hethod & Date & Time & OpID & Type & Mode \\
\hline $\begin{array}{l}1 \\
2 \\
3 \\
4 \\
5 \\
6 \\
7 \\
8 \\
9 \\
10 \\
11 \\
12 \\
13 \\
14 \\
15 \\
16 \\
17 \\
18 \\
19 \\
20\end{array}$ & $\begin{array}{l}\text { ICV } \\
\text { ICB } \\
\text { ILS } \\
\text { ICSA } \\
\text { ICSAB } \\
\text { PREPBLKSJA } \\
\text { S97TO00518_ D } \\
\text { S97TO00518_D } \\
\text { GGV } \\
\text { GCB } \\
\text { S97T000523 } \\
\text { S97T000523_D } \\
\text { S97T000524- } \\
\text { S97T000524_D } \\
\text { S97T000647- } \\
\text { S97T000647_D } \\
\text { ICSA } \\
\text { ICSAB } \\
\text { CCV I } \\
\text { CCB I }\end{array}$ & $\begin{array}{l}970430 \mathrm{~A} \\
970430 \mathrm{~A} \\
970430 \mathrm{~A} \\
970430 \mathrm{~A} \\
970430 \mathrm{~A} \\
970430 \mathrm{~A} \\
970430 \mathrm{~A} \\
970430 \mathrm{~A} \\
970430 \mathrm{~A} \\
970430 \mathrm{~A} \\
970430 \mathrm{~A} \\
970430 \mathrm{~A} \\
970430 \mathrm{~A} \\
970430 \mathrm{~A} \\
970430 \mathrm{~A} \\
970430 \mathrm{~A} \\
970430 \mathrm{~A} \\
970430 \mathrm{~A} \\
970430 \mathrm{~A} \\
970430 \mathrm{~A}\end{array}$ & $\begin{array}{l}\text { ICP2 } \\
\text { ICP2 } \\
\text { ICP2 } \\
\text { ICP2 } \\
\text { ICP2 } \\
\text { ICP2 } \\
\text { ICP2 } \\
\text { ICP2 } \\
\text { ICP2 } \\
\text { ICP2 } \\
\text { ICP2 } \\
\text { ICP2 } \\
\text { ICP2 } \\
\text { ICP2 } \\
\text { ICP2 } \\
\text { ICP2 } \\
\text { ICP2 } \\
\text { ICP2 } \\
\text { ICP2 } \\
\text { ICP2 }\end{array}$ & $\begin{array}{l}04 / 30 / 97 \\
04 / 30 / 97 \\
04 / 30 / 97 \\
04 / 30 / 97 \\
04 / 30 / 97 \\
04 / 30 / 97 \\
04 / 30 / 97 \\
04 / 30 / 97 \\
04 / 30 / 97 \\
04 / 30 / 97 \\
04 / 30 / 97 \\
04 / 30 / 97 \\
04 / 30 / 97 \\
04 / 30 / 97 \\
04 / 30 / 97 \\
04 / 30 / 97 \\
04 / 30 / 97 \\
04 / 30 / 97 \\
04 / 30 / 97 \\
04 / 30 / 97\end{array}$ & $\begin{array}{l}09: 54 \\
09: 57 \\
10: 03 \\
10: 06 \\
10: 09 \\
10: 13 \\
10: 16 \\
10: 19 \\
10: 23 \\
10: 27 \\
10: 30 \\
10: 33 \\
10: 36 \\
10: 39 \\
10: 56 \\
11: 02 \\
11: 05 \\
11: 08 \\
11: 11 \\
11: 14\end{array}$ & $\begin{array}{l}\text { DKS } \\
\text { DKS } \\
\text { DKS } \\
\text { DKS } \\
\text { DKS } \\
\text { DKS } \\
\text { DKS } \\
\text { DKS } \\
\text { DKS } \\
\text { DKS } \\
\text { DKS } \\
\text { DKS } \\
\text { DKS } \\
\text { DKS } \\
\text { DKS } \\
\text { DKS } \\
\text { DKS } \\
\text { DKS } \\
\text { DKS } \\
\text { DKS }\end{array}$ & $\begin{array}{l}Q \\
Q \\
Q \\
Q \\
Q \\
Q \\
S \\
S \\
Q \\
Q \\
S \\
S \\
S \\
S \\
S \\
S \\
Q \\
Q \\
Q \\
Q\end{array}$ & $\begin{array}{l}\text { CONG } \\
\text { CONC } \\
\text { CONC } \\
\text { CONC } \\
\text { CONC } \\
\text { CONC } \\
\text { CONC } \\
\text { CONC } \\
\text { CONC } \\
\text { CONC } \\
\text { CONC } \\
\text { CONC } \\
\text { CONC } \\
\text { GONC } \\
\text { CONC } \\
\text { CONC } \\
\text { GONC } \\
\text { GONC } . \\
\text { CONC } \\
\text { CONC }\end{array}$ \\
\hline
\end{tabular}

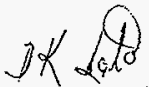

$84-30.97$

Q. $103 \quad 5977000518$

5977000523

5477000524

$7.204 \quad 5977000647$ 
Wed $04-30-9711: 17: 34$ AM page 2

\# Sample Name

$1 \mathrm{ICV}$

2 ICB

3 LLS

4 ICSA

5 ICSAB

6 PREPBIKTJA

7 S97T000518

8 597T000518 D

$9 \mathrm{CCV}$

$10 \mathrm{CCB}$

11 S97T000523

12 S97T000523 D

13 S97T000524

14 S97T000524 D

15 \$97T000647

16 \$97T000647_D

17 ICSA

18 ICSAB

19 CCV 1

$20 \mathrm{CCB}^{-1}$

非 Sample Name B

1. $\mathrm{IGV}$

$2 \mathrm{ICB}$

3 LLS

4 ICSA

5 ICSAB

6 PREPBIKTJA

7 S97T000518

8 S97T000518 D

$9 \mathrm{CCV}$

$20 \mathrm{CCB}$

11 \$97T000523

12 \$97T000523 D

13 S97TO00524-

14 S97T000524 D

15 S97T000647

16 597T000647 D

17 ICSA

18 ICSAB

$19 \mathrm{CCV} 1$

$20 \mathrm{CCB}_{-1}^{-1}$

J) Sample Name

$1 \mathrm{IGV}$

2 ICB

3 LLS

4 ICSA

5 ICSAB
Ag

Al

As

4. 928

.0004

.0234

.0010

.9685

.0006

.0557

.0496

4.928

.0012

.0360

.0545

.0424

.0495

$-.0003$

.0003

.0019

.9668

4.882

$-.0001$

Bi

5.044

$-.0127$

.1594

$-.0120$

$-.0136$

$-.0185$

$-1.186$

$: 2066$

5.139

$-.0157$

$-1.042$

$-1.266$

$-.6345$

$-1.821$

$-.1127$

$-.0822$

-.0023

-.0390

5.054

$-.0100$

$\mathrm{Cu}$

5. 086

$-.0005$

.0181

$-.0102$

.4547
4. 923

$-.0009$

i1 63

243.7

243.2

.0007

47.98

49.56

4. 933

.0043

36.16

36.83

52.54

51.85

.0220

$-.0070$

245.7

245.0

4.915

.0043

Ca

4. 960

.0022

.2230

250.3

251.5

-.0009

.2224

.1641

5.017

.0036

.1627

.1058

.1770

.1732

18.49

18.42

251.9

250.9

4.977

.0017

Eu

$-.0015$

.0003

.0011

-.0378
-.0390
5.216

$-.0014$

.2348

.0002

.0171

.0147

.3681

.5830

5.245

.0163

.4463

.8012

.2161

$-.0246$

.0212

.0218

.0249

.0124

5.240

.0025

$\mathrm{Cd}$

$\mathrm{Ge}$

$5.021 \quad 4.952$

.0001

Q. 0128

.0020

.9434

.0002

.0097

.0602

5.028

.0005

.0478

.0099

.0218

$-.0084$

.0012

.0001

.0041

.9452

5.006

.0009

Fe

4.672

$-.0007$

.0950

94.49

94.39
B

Ba

5.086

.0060

.1058

-.0004

$-.0019$

.0040

.0614

51227

.0020

.2256

.2053

.1641

.0612

1.678

1. 708

$-.0032$

.0032

5.136

.0060

$\mathrm{Ce}$

.0087

.2134

.0136

.0185

.0043

.3048

.2075

4.982

.0154

.2083

.2092

.1906

.4343

.0140

.0227

.0192

0168

4.959

.0028

K

\subsection{2}

4. 942

.4749

.0018

$-.0320$

5.025

.0002

.1018

.0002

.4818

.0001

.0130

. 0148

.

.0001

.0150

.0109

.0189

.0256

.0254

.0003

.4830

5.020

.0000

5. 106

$-.0001$

.0109

0002

.4806

$-.0002$

-.0017

.0093

$-.0002$

$-.0016$

.0038

.0093

$-.0017$

.0001

-.0005

0003

.4882

5.155 
HAF-SDWAA-DP-245, REV.QNAt-14, 97

Averages

Wed 04-30-97 11:17:34 AM

page 3

Analys $f$ seport

\# Sample Name

6 PREPBLKTJA

7 S97T000518

8 597T000518_D

$9 \mathrm{CCV}$

$10 \mathrm{CCB}$

il $597 \mathrm{TO00523}$

12 S97T000523 D

135979000524

14 S97T000524_D

15 S97T000647

$16597 \mathrm{~T} 000647 \mathrm{D}$

17 ICSA

18 ICSAB

$19 \mathrm{CCV} 1$

$20 \mathrm{CCB}_{-}-1$
$\mathrm{Cu} \quad \mathrm{Eu}$

$\begin{array}{ll}.0003 & .0006 \\ .0218 & .0053 \\ .0481 & -.0010 \\ 5.116 & -.0016 \\ .0003 & .0005 \\ .0131 & .0122 \\ .0394 & .0080 \\ .0300 & .0015 \\ .0148 & .0123 \\ .0092 & .0148 \\ .0096 & .0150 \\ .0097 & -.0403 \\ .4633 & -.0375 \\ 5.117 & -.0019 \\ .0002 & .0003\end{array}$

$\mathrm{Mg}$

1. ICV

2 ICB

3 ILS

4 ICSA

5 ICSAB

6 PREPBLKTJA

7 S97T000518

8 S97T000518_D

$9 \mathrm{CCV}$

$10 \mathrm{CCB}$

i. $\$ 97 \mathrm{~T} 000523$

12 S97T000523_D

13 S97T000524-

$14597 \mathrm{~T}^{15000524}$

16 \$97T000647_D

17 ICSA

18 IGSAB

$19 \operatorname{CCV} 1$

If Sample Name

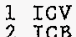

3 LXS

4 ICSA

5 ICSAB

6 PREPBLKTJA

7 S97T000518

8 \$97T000518_D

$9 \mathrm{CCV}$

$10 \mathrm{CCB}$

11 S97T000523

$5.039 \quad 4.926$

$-.0019$

.2215

254.6

254.3

$-.0022$

.0352

.0710

5.043

.0093

$-.3861$

.0379

.0504

1820

3.904

3.886

257.1

256.3

5.024

.0013

$P$

4.92

.0002

$-.0057$

.4479

.0023

.2099

.2244

4.923

.0003

. 3319

.3231

.1752

.0895

.0890

$-.0058$

.4466

4.896

.0004

$\mathrm{Pb}$

4. 904

5. 204

$\therefore 0066$

.4122

.0032

.0019

$-.0047$

3. 755

2.985

5.192

$-.0005$

.0020

.2281

.0491

.9934

$-.0058$

- .3252

.5190

4.890

$-.0056$

.2051
$\mathrm{Fe}$

K

La

Li

$-.0012$

4649 Q297.6

$4953 \quad 11420$.

4.699

.0004

.5563

1.315

4177
5682

1831

1807

95.09

94.72

4.675

$-.0007$

Ko

5.185

.0008

.1077

$-.0059$

$-.0127$

.0001

.1513

.2325

5. 194

.0010

.1752

.1833

.1918

.2154

.0056

.0001

$-.0112$

. .0053

$\mathrm{Na}$

161

.0012

$\mathrm{s}$

Q.1013

$\mathrm{Sb}$
5.054

.0117

.2165

$-.0228$

$-.0391$

.0039

9.474

9.943

5. 053

7.0031
12030 .

4.876

1. 1038

11170 .

$11720^{\circ}$.

12700 .

.7344

.9012

$-.0106$

.0983

4.974

$-.1401$

5.150

.0056

.2121

197.2

195.8

427.5

427.3

5.151

.0039

398.7

404.2

408.2

410.9

4.308

4.290

198.9

198.0

5.137

.0082

\section{.0003}

.0261

.0329

5.068

.0020

.0046

.0192

.0638

.0013

$-.0035$

$\therefore .0040$

5.050

.0007

$\mathrm{Nd}$

5. 019

.0015

.2055

.0016

.0060

.0009

.0834

.0538

5.062

.0037

.1484

. 1986

. 0558

.1362

.0041

0091

.0026

.0036

5.019

.0023

Se

4. 902

.0069

.1156

.0023

.0094

.0074

.3357

$-.0268$

4. 922

.0062

.3733
$\mathrm{Ni}$

Si

.0007

.0556

.0456

4.974

.0011

$-.0049$

.0052
0204

.0456

.0049

.0059

.0031

1. 063

.0006

4. 972

.0056

.0437

-.0064

9180

Q. 2675

2.106

2.143

4.995

$-.0011$

2.289

2. 559

1. 529

1. 634

.0046

.0011

-.0106

.9238

4.941

-.0015 
Analysis Report

Averages

-HNF-SA-AMADP-245,REV OMt

Analysis th

$\mathrm{P}$

$\mathrm{Pb}$

wed 04-30-97 $11: 17: 34$ AM page 4

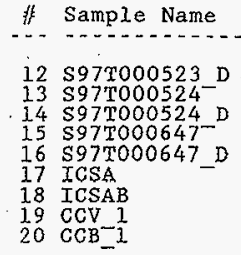

\# Sample Name

$\begin{array}{ll}2.927 & -.0842 \\ 4.332 & 1.926 \\ 4.202 & -.2930 \\ -.0043 & -.0397 \\ -.0337 & -.0315 \\ .0075 & .0206 \\ .0097 & -9848 \\ 5.169 & 4.867 \\ -.0161 & .0059\end{array}$

$s$

$\mathrm{Sb}$

Se

Si

7.458
10.18
10.63
6.310
6.306
-.0067
-.0141
5.049
-.0049

.0153
.2679
.3496
.0025
.0086
.0049
.0071
4.899
.0066

1.020

1.053

.9687

.0772

.0310

$-.0230$

$-.0075$

4.805

.0296

$1 \mathrm{ICV}$

$\frac{1}{3}$ ICB

4 ICSA

5 ICSAB

6 PREPBLKTJA

7 597T000518

8 S97T000518_D

$9 \mathrm{CCV}$

$10 \mathrm{GCB}$

11 S97T000523

12 597T000523 D

13 S97T000524-

$14597 T 000524 \mathrm{D}$

15 \$97T000647

16 S97T000647 D

17 ICSA.

18 ICSAB

$3.9 \mathrm{CCV} 1$

$\mathrm{Sm}$

$\mathrm{Sr}$

Th

TI

4.928

.0017

4: 986

.

.0103

.0171

.0061

.0195

4. 0021

.0084

.0390

.1508

1
-.1269

.2262

.0183

.0301

.0043

.0037

4.958

.0068

.0002

.0023

.0022

.0003

.0135

.0113

.0001

.0135

.0113

.0113

.0135

.0135

.0881

.0022

$-.0096$

$-.0108$

.0047

$-.0175$

$-.0197$

.0003

.2026

.4089

$-.0077$

.0005

.2566

.2123

.4141

.2939

$-.0026$

-.0025

.013

5.005

.0034

5.015

.0003

.0214

.0010

.0015

.0003

$-.0085$

5.0077

TI

.0000

.0131

.0031

.0243

.0010

.0015

.0012

50010

.0005

.9150
.6284
.7400
5.350
5.347
.0107
.0016
4.940
.0103

\# Sample Name $\mathrm{V}$ $\mathrm{Y}$

$Z_{\mathrm{n}}$

$2 x$

U

4.816

$-.0316$

.4013

$-.0310$

.0183

$-.0166$

$-.3746$

-.7713
4.803

$-.0167$

$-1.290$

$-1.113$

-.0511

$-1.309$

$-.0344$

$-.0930$

.0056

.0134

4.755

$-.0228$

9.879

.0178

.5588

.0569

.0710

$-.0614$

$-2.588$

$-2.760$

9.962

.0350

$-2.786$

$-2.518$

$-3.391$

$-2.992$

.1730

.1974

.0369

9.908

.0202

$1 \mathrm{ICV}$

2 ICB

3 ILS

4 ICSA

5 ICSAB

6 PREPBLKTJA

7 S97T000518

8 S97T0005I8D

$9 \mathrm{CCV}$

$1.0 \mathrm{GCB}$

i1 $597 \mathrm{~T} 000523$

$12597 T 000523 \mathrm{D}$

13 597T000524-

$14597 T 000524$ D

15 S97T000647

16 \$97T000647 D

17 ICSA

5.182
.0008
.1088
.0022
.4812
.0019
.0038
5.0130
5.203
.0013
.0205
.0611
.0118
.0206
.0031
.0030
.0018

.0054

.0002

.0009

.0068

0073

.0003

.0075

.0044

.0052

.0005

.0108

.0107

.0014

.0173

.0012

.0016

.0067
4.936

$-.0000$

.0220

.0058

.9493

$-.0020$

$-.0142$

.0208

4.927

.0005

$-.0046$

.0122

$-.0015$

. 1042

.1014
...

5.075

.0001

$-.0008$

.0008

.0008

$-.0290$

$-.0283$

5.099

.0011

$-.0013$

.0175

$-.0439$

$-.0041$

.0019

.0026

$-.0018$ 


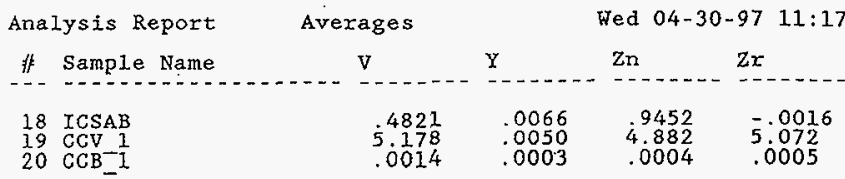

$\frac{1}{2}$
0
0
0
0
0
0
0
0
0
0
0
0 


\section{LABCORE Data Entry Template for Worklist\# 19295}

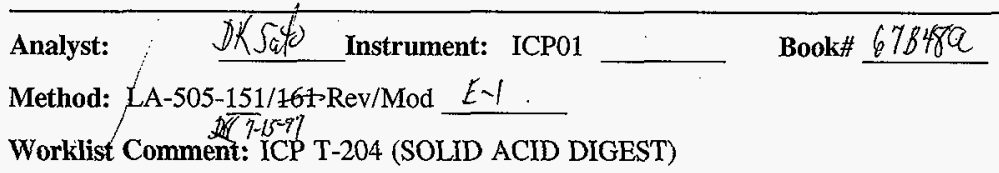

\begin{tabular}{|c|c|c|c|c|c|c|c|c|c|}
\hline $\bar{s}$ & Type & Sample\# & RA & Test & Matrix & Group\# & Projec & & \\
\hline 1 & $\mathrm{ICV}$ & & & $@ I C P-Q C$ & $Q C$ & & & & \\
\hline 2 & $I C B$ & & & DICD-QC & $\mathrm{QC}$ & & & & \\
\hline 3 & IISS & & & @ICD-QC & $Q C$ & & & & \\
\hline 4 & $\operatorname{ICSA}$ & & & @ICP-QC & $Q \mathrm{C}$ & & & & \\
\hline 5 & $I C S A B$ & & & $@ I C P-Q C$ & $\mathrm{QC}$ & & & & \\
\hline 6 & PREPSTDARI & & & QICP-A0I & SOLID & & & & \\
\hline 7 & PREPBIKARL & & & QICP-AOI & SOLID & & & & \\
\hline 8 & SERDIL & S97T001199 & $0 \mathrm{~A}$ & @ICP-AOI & SOLID & & & & \\
\hline \multirow[t]{8}{*}{9} & SAMPEE & S97T001199 & $O \mathrm{~A}$ & @ICP-A.OI & SOLID & 970002 & $261 T-204$ & & \\
\hline & & Analytes Reque & ested & $1: A G-A-01$ & , $A L-A-01$ & $A S-A-01$ & , B-A-OI & , $\mathrm{BA}-\mathrm{A}-01$ & . , \\
\hline & & & & $\mathrm{BE}-\mathrm{A}-01$ & , BI-A-01 & $C A-A-0 I$ & $C D-A-01$ & , CE-A-OI & - , $\mathrm{CO}-\mathrm{A}-01$ \\
\hline & & & & $C R-A-01$ & , $\mathrm{CU}-\mathrm{A}-01$ & $F E-A-01$ & $K-A-01$ & , LA-A-01 & , LI-A-01 \\
\hline & & & & $M G-A-01$ & MN-A-01 & $\mathrm{MO}-\mathrm{A}-01$ & $N A-A-01$ & $\mathrm{ND}-\mathrm{A}-01$ & $\therefore$ NI-A-01 \\
\hline & & & & $\mathrm{P}-\mathrm{A}-\mathrm{OI}$ & , $\mathrm{PB}-\mathrm{A}-01$ & $S-A-01$ & , SB-A-0I & , SI-A-01 & $\therefore S M-A-01$ \\
\hline & & & & SR-A-O1 & TI-A-01 & TL-A-01 & $\mathrm{U}-\mathrm{A}-\mathrm{OI}$ &,$V-A-0 I$ & , $\mathrm{zN}-\mathrm{A}-01$ \\
\hline & & & & $\mathrm{ZR}-\mathrm{A}-01$ & & & & & \\
\hline
\end{tabular}

10 DUP

S97T001199 O A QICP-A01 SOLID

11 SPK-PREDIG S97T001199 O A BICP-A01 SOLID

12 ICSA

13 ICSAB

$14 \mathrm{CCV}$

$15 \mathrm{CCB}$
(a)P-QC QC

QICP-QC QC

@ICP-QC QC

@ICP-QC QC

Data Entry Comments:

$\bar{S}=$ Worklist Slot Number, $R=$ Replicate Number, $A=$ Aliquot Code . 


\section{LABCORE Data Entry Template for Worklist\# 19295}

S Type

Sample\#

R A Test

Matrix

Group\# Project

Final page for worklist \# 19295

Dinais $x \cdot 20100 \%-15-97$

Analyst Signature

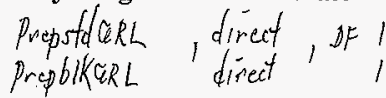

$5977001199-2,4.4-28 \quad 10$

$5977001199 \quad 4-4$

$597 T 001199,20 \quad 4-4$

S97T001199-5 4-4

$5977001199-x \quad 4-4-1-9 \quad 20$

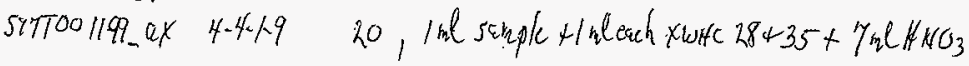

Post spike:

$$
\begin{aligned}
& A g=\frac{\left(\frac{167.772}{20}\right)-0}{10} \times 100=83.9 \% \\
& \beta_{i}=\frac{\left(\frac{579.454}{20}\right)-\left(\frac{370.554}{20}\right)}{10} \times 100=104.4 \% \\
& F e=\frac{\left(\frac{230.882}{20}\right)-\left(\frac{28.434}{20}\right)}{10} \times 100=101.2 \% \\
& k=\underbrace{\left(\frac{237.583}{20}\right)-\left(\frac{48.237}{20}\right)}_{10} \times 100=94.7 \% \\
& L_{a}=\frac{\left(\frac{286.589}{20}\right)-\left(\frac{80.735}{20}\right)}{10} \times 100=102.9 \% \\
& M_{n}=\frac{\left(\frac{308.370}{20}\right)-\left(\frac{104.562}{20}\right)}{10} \times 100= \\
& \left.\mu_{a}=\frac{\left(\frac{431.156}{20}\right)-\left(\frac{232 \cdot 149}{20}\right)}{10}\right)_{x / 0}=
\end{aligned}
$$

Data Entry Comments:

$S=$ Worklist Slot Number, $R=$ Replicate Number, $A=$ Aliquot Code . 
$07 / 15 / 97 \quad 10: 14 \quad 8509372$
$F_{i} / e^{2}: 9707 / 5 G .76 T$

$$
H_{1}: 970715 \mathrm{a} .50 .5
$$

Identity 1: ICV Identity 2: Quality control Task name : OPIIMA Sample Weight : 1.0000 Solution volune :

$\begin{array}{lll}\text { Sample Weight : } \quad 1.0000 & \text { Solution Volune : } & 1.00 \\ \text { on-Peak Integrations }: 3 & \text { off-Peak Integrations : } & 1\end{array}$

\section{HNF-SD-WM-DP-255, REV. 0}

9:57 AN July 15, 1997

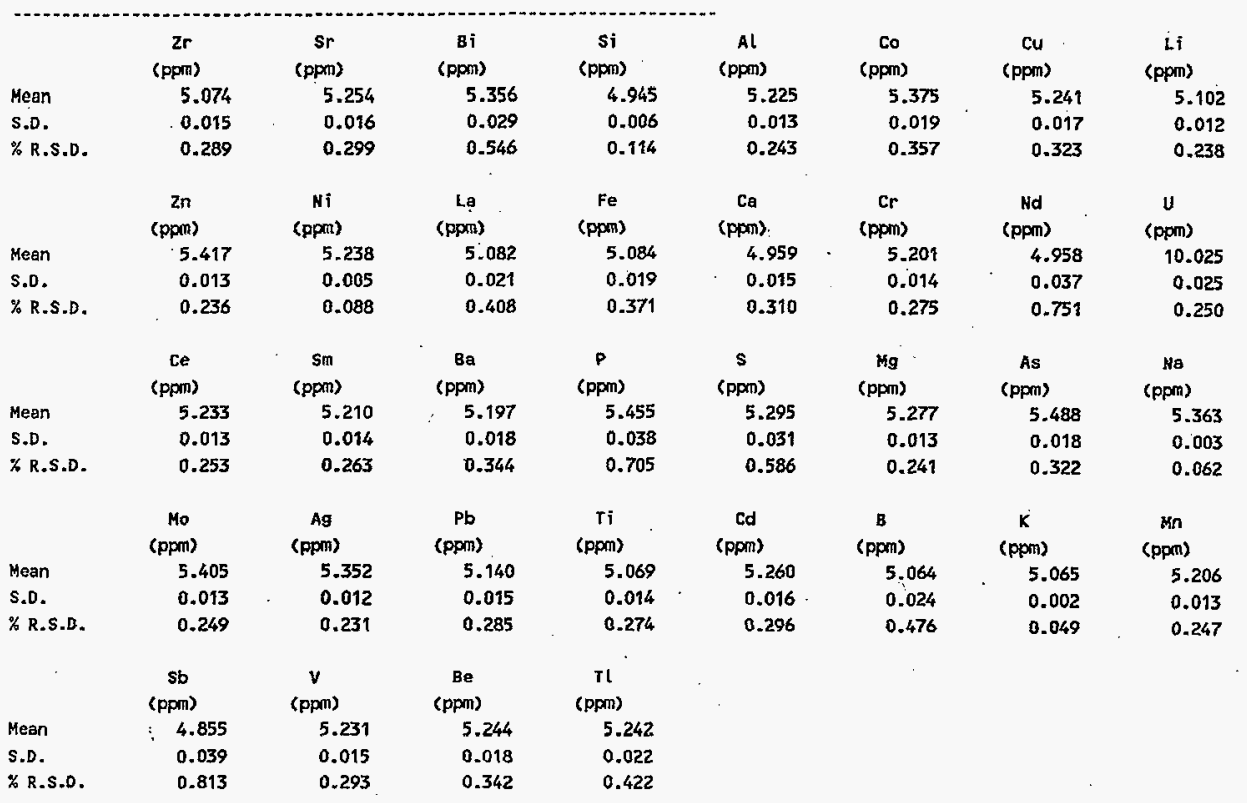

$$
\begin{aligned}
& \text { DK \&4O } \\
& 07-15-97 \\
& T=204
\end{aligned}
$$




\section{HNF-SD-WM-DP-255, REV. 0}

Identity 1: ICB Task name : OPTIKA Sample Weight : Identity 2: Quality control 10:03 AM July 15, 1997 on-Peak Integrations : 3 off-Peak integrations : 1

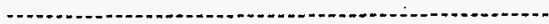

\begin{tabular}{lrrrrr} 
& Zr & \multicolumn{1}{c}{ Sr } & \multicolumn{1}{c}{ Bi } & \multicolumn{1}{c}{ si } & \multicolumn{1}{c}{ Al } \\
& (ppm) & (ppm) & (ppm) & \multicolumn{1}{c}{ (ppm) } & (ppm) \\
Mean & -0.001 & 0.000 & 0.008 & -0.001 & -0.007 \\
S.D. & 0.001 & 0.000 & 0.003 & 0.001 & 0.001 \\
X R.S.D. & 38.618 & 173.205 & 35.122 & 94.474 & 9.075
\end{tabular}

Mean

S.D.

\% R.S.D.

Mean

S.D.

\% R.S.D.

$$
\text { zn }
$$$$
0.000
$$

$$
0.000
$$

4024.826

Hean

S.D.

\% R.S.D.

ce
$(\mathrm{pp}))$
-0.013
0.003
24.803

$\mathrm{Ni}$
$(\mathrm{ppm})$
-0.005
0.002
38.750

Le
(ppm)
-0.000
0.004

Sit $\quad B a$

(PPFin)
-0.027
0.003

$$
9.723
$$

Mo
(ppm)
-0.001
0.000

(ppm)

0.000

0.001

49.486

110.157

(ppom)

Mean

S.D.

\% R.S.D.

$V$
$(p p m)$
-0.001
0.001
70.874

(ppin)

0.000
0.000
624.500

\begin{tabular}{cc} 
P & \multicolumn{1}{c}{$s$} \\
(ppm) & (ppm) \\
0.004 & -0.010 \\
0.007 & 0.005 \\
163.123 & 47.985
\end{tabular}

$\mathrm{Pb}$
(ppm)
0.003
0.008
248.333

$T i$
(ppm)
-0.000
0.000
16.225
cd
(ppm)
$-0.000$
0.000
228.616

$\begin{array}{cc}\text { Be } & T l \\ \text { (ppm) } & \text { (ppm) } \\ -0.000 & 0.005 \\ 0.000 & 0.005 \\ 56.796 & 111.813\end{array}$

56.796
738.611

Fe

(ppm)

$-0.000$

0.001

227.899

Ca
(pprn)
0.001
0.000
6.186

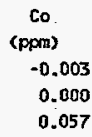

Cr

(ppm)

$$
0.001
$$

0.002

259.655

Hg
(ppm)
-0.000
.0 .000
86.665

$$
B
$$

$$
\begin{aligned}
& \text { (ppm) } \\
& .-0.000 \\
& 0.000 \\
& 101.228
\end{aligned}
$$

Cu
(ppm)
-0.001
0.000
22.776

Li

(ppm)

$$
0.000
$$

0.000

915816.136

\section{Nd}

(PPm)

$-0.000$

0.004

1473.167

u

(ppm)

$-0.023$

0.004

19.356

As No

(ppm)

$-0.001$

0.003

472.843

(ppom)

0.001

0.001

86.633

$K \quad$ Hn

(ppm) (ppm)

$-0.056 \quad-0.001$

$0.007 \quad 0.000$

$11.845 \quad 34.759$ 


\section{HNF-SD-WWM-DP-255, REV. 0}

Identity 1: LLS Identity 2: Quatity Control 10:06 AM July 15, 1997

Task name : OPTIMA

Sample Height : $\quad 1.0000$ solution Volume : $\quad \mathbf{1 . 0 0}$

On-Peak Integrations : 3 off-Peak integrations : 1

\begin{tabular}{|c|c|c|c|c|c|c|c|c|}
\hline & $\begin{array}{c}2 r \\
(p p m)\end{array}$ & $\begin{array}{c}\mathrm{Sr} \\
(\mathrm{ppm})\end{array}$ & $\underset{\text { (pprn) }}{\text { Bi }}$ & $\begin{array}{c}\text { si } \\
(\mathrm{ppm})\end{array}$ & $\begin{array}{c}\text { Al } \\
\text { (ppm) }\end{array}$ & $\begin{array}{c}\text { Co } \\
\text { (ppm) }\end{array}$ & $\begin{array}{c}\mathrm{cu} \\
(\mathrm{ppm})\end{array}$ & $\begin{array}{c}\text { Li } \\
\text { (ppm) }\end{array}$ \\
\hline Mean & 0.019 & 0.021 & 0.216 & 0.108 & 0.111 & 0.043 & 0.021 & 0.022 \\
\hline S.D. & 0.001 & 0.000 & 0.005 & 0.001 & 0.004 & $0: 005$ & 0.000 & 0.000 \\
\hline \multirow[t]{2}{*}{ * R.S.D. } & 5.737 & 0.191 & 2.101 & 0.532 & 3.978 & 10,504 & 1.212 & 0.498 \\
\hline & $\begin{array}{c}\mathrm{Zn} \\
(\mathrm{ppm})\end{array}$ & $\underset{\text { (ppm) }}{\mathrm{Ni}}$ & $\begin{array}{c}\text { La } \\
\text { (ppm) }\end{array}$ & $\begin{array}{c}\text { Fe } \\
\text { (ppm) }\end{array}$ & $\begin{array}{c}\mathrm{Ca} \\
\text { (ppm) }\end{array}$ & $\begin{array}{c}\mathrm{Cr} \\
\text { (ppom) }\end{array}$ & $\begin{array}{c}\text { Nd } \\
\text { (ppm) }\end{array}$ & $\underset{(p p m)}{U}$ \\
\hline Mean & 0.022 & 0.041 & 0.100 & 0.106 & 0.209 & 0.024 & 0.219 & 0.366 \\
\hline S.D. & 0.000 & 0.001 & 0.005 & 0.005 & 0.000 & 0.001 & 0.010 & 0.038 \\
\hline \multirow[t]{2}{*}{ \& R.S.D. } & 0.029 & 2.335 & 4.515 & 4.617 & $: \quad 0.082$ & 2.969 & 4.418 & 10.377 \\
\hline & $\begin{array}{c}\mathrm{Ce} \\
(\mathrm{ppm})\end{array}$ & $\begin{array}{r}\mathrm{Sm} \\
(\mathrm{ppm})\end{array}$ & $\begin{array}{c}\text { Ba } \\
\text { (ppon) }\end{array}$ & $\begin{array}{c}P \\
(p p m)\end{array}$ & $\begin{array}{c}s \\
\text { (ppm) }\end{array}$ & $\begin{array}{c}\mathrm{Mg} \\
\text { (ppon) }\end{array}$ & $\begin{array}{c}\text { As } \\
\text { (ppm) }\end{array}$ & $\begin{array}{c}\mathrm{Ma} \\
\text { (ppm) }\end{array}$ \\
\hline Hean & 0.222 & 0.185 & 0.106 & $0.438^{\circ}$ & 0.206 & 0.227 & 0.217 & 0.215 \\
\hline S.D. & 0.008 & 0.007 & 0.000 & 0.006 & 0.003 & 0.000 & 0.002 & 0.001 \\
\hline \multirow[t]{2}{*}{ \% R.S.D. } & 3.439 & 3.901 & 0.156 & 1.455 & 1.559 & 0.148 & 0.817 & 0.585 \\
\hline & $\begin{array}{c}\text { Ho } \\
\text { (ppm) }\end{array}$ & $\begin{array}{c}\text { Ag } \\
\text { (PPM) }\end{array}$ & $\begin{array}{c}\mathrm{Pb} \\
\text { (Ppm) }\end{array}$ & $\begin{array}{c}\text { Ti } \\
\text { (ppm) }\end{array}$ & $\begin{array}{c}\text { cd } \\
\text { (ppm) }\end{array}$ & (ppon) & $\begin{array}{c}\mathrm{K} \\
\text { (ppm) }\end{array}$ & $\begin{array}{c}\text { Mn } \\
\text { (ppn) }\end{array}$ \\
\hline Mean & 0.106 & 0.026 & 0.239 & 0.019 & 0.011 & 0.107 & 0.590 & 0.021 \\
\hline S.D. & 0.001 & 0.001 & 0.001 & 0.000 & 0.001 & 0.001 & 0.009 & 0.000 \\
\hline \% R.S.D. & 1.410 & 2.022 & 0.548 & 1.174 & 9.115 & 0.636 & 1.488 & 1.781 \\
\hline
\end{tabular}

\begin{tabular}{|c|c|c|c|c|}
\hline & $\begin{array}{c}\text { sb } \\
\text { (ppm) }\end{array}$ & $\begin{array}{c}v \\
\text { (ppm) }\end{array}$ & $\begin{array}{c}\mathrm{Be} \\
\text { (ppm) }\end{array}$ & $\begin{array}{c}\mathrm{Tl} \\
\text { (pom) }\end{array}$ \\
\hline Mean & 0.127 & 0.108 & 0.012 & 0.424 \\
\hline S.D. & 0.017 & 0.001 & 0.000 & 0.022 \\
\hline \% R.S.D. & 13.444 & 0.669 & 3.500 & 5.135 \\
\hline
\end{tabular}




\section{HNF-SD-WM-DP-255, REV. 0}

Identity is ICSA Task name : OPTIMA Sample Weight : Identity 2: Quality Control $10: 09$ AM July 15, 1997

On-Peak Integrations : 3 off-Peak Integrations : 1

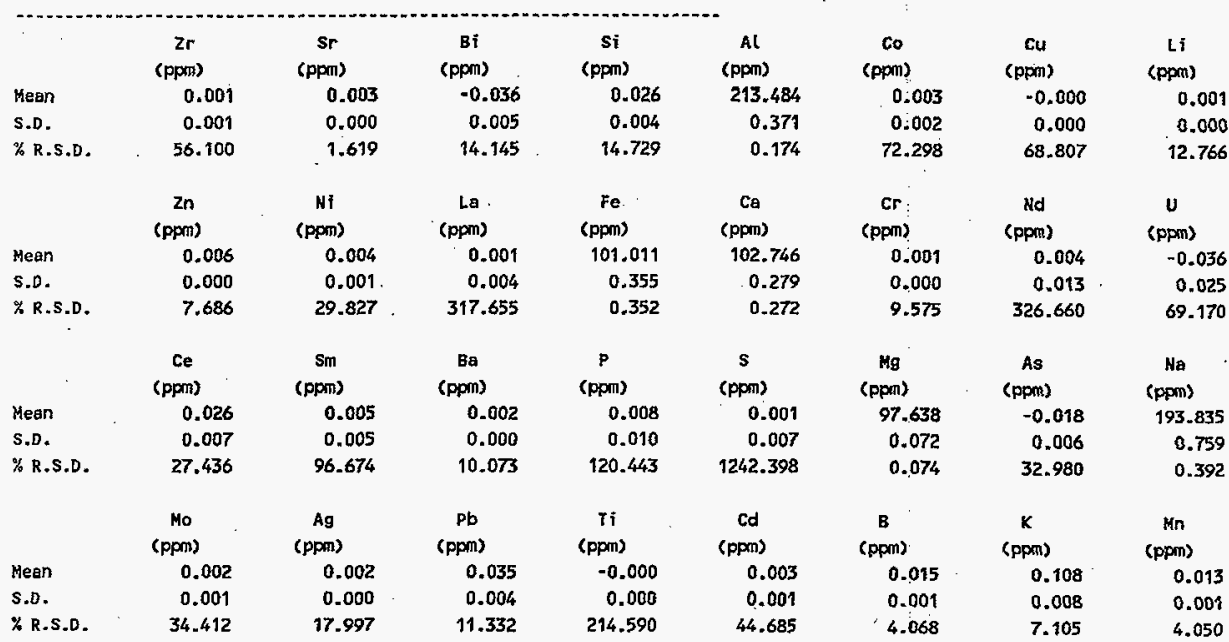

\begin{tabular}{lrrrr} 
& Sb & $v$ & Be & \multicolumn{1}{c}{$r$ r } \\
& (ppm) & \multicolumn{1}{c}{ (ppm) } & \multicolumn{1}{c}{ (ppm) } & \multicolumn{1}{c}{ (ppm) } \\
Mean & 0.017 & 0.001 & 0.003 & 0.004 \\
S.D. & 0.012 & 0.001 & 0.000 & 0.001 \\
\% R.S.D. & 69.905 & 106.268 & 14.070 & 24.561
\end{tabular}




\section{HNF-SD-WM-DP-255, REV. 0}

Identity 1: ICSAB Identity 2: Qual ity Control

10:14 AM July 15, 1997

Task name : OPTIMA

$\begin{array}{lll}\text { Sample Height : } \quad 1.0000 & \text { Solution Volcmie : } & 1.00 \\ \text { On-Peak Integrations }: 3 \text { off-Peak Integrations : } & 1\end{array}$

\begin{tabular}{|c|c|c|c|c|c|c|c|c|}
\hline & $\begin{array}{c}\mathrm{Zr} \\
(\mathrm{PPR})\end{array}$ & $\begin{array}{c}\mathrm{sr} \\
\text { (ppm) }\end{array}$ & $\begin{array}{c}\text { Bi } \\
\text { (ppm) }\end{array}$ & $\begin{array}{c}\text { si } \\
\text { (pppn) }\end{array}$ & $\begin{array}{c}\text { Al } \\
\text { (ppm) }\end{array}$ & $\begin{array}{c}\text { ca: } \\
\text { (ppom) }\end{array}$ & $\begin{array}{c}\mathrm{Cu} \\
\text { (ppm) }\end{array}$ & $\begin{array}{c}L i \\
(p p m)\end{array}$ \\
\hline Mean & -0.002 & 0.002 & -0.030 & 0.021 & 212.264 & 0.520 & 0.507 & 0.959 \\
\hline S.D. & 0.001 & 0.000 & 0.006 & 0.003 & 0.525 & 0.004 & 0.001 & 0.002 \\
\hline \multirow[t]{2}{*}{ \% R.S.D. } & 71.900 & 3.125 & 18.664 & 16.758 & 0.248 & 0.751 & 0.290 & 0.217 \\
\hline & $\underset{(p p m)}{2 n}$ & $\begin{array}{c}\mathrm{Ni} \\
\text { (ppm) }\end{array}$ & $\begin{array}{c}\text { La } \\
\text { (ppm) }\end{array}$ & $\begin{array}{c}\text { Fe } \\
\text { (ppm) }\end{array}$ & $\begin{array}{c}c a \\
\text { (ppm) }\end{array}$ & $\begin{array}{r}\text { Cr: } \\
\text { (ppril) }\end{array}$ & $\begin{array}{c}\text { Nd } \\
\text { (ppm) }\end{array}$ & $\begin{array}{c}U \\
(\mathrm{ppm})\end{array}$ \\
\hline Mean & 1.039 & 1.039 & 0.003 & 100.399 & 102.420 & 0.532 & -0.001 & 0.042 \\
\hline S.D. & 0.002 & 0.003 & 0.001 & 0.166 & 0.112 & 0.007 & 0.004 & 0.050 \\
\hline \multirow[t]{2}{*}{ \% R.S.O. } & 0.171 & 0.278 & 25.784 & 0.165 & 0.109 & 1.317 & 352.914 & 118.457 \\
\hline & $\begin{array}{c}\text { Ce } \\
(\mathrm{ppm})\end{array}$ & $\begin{array}{c}\mathrm{Sin} \\
\text { (ppm) }\end{array}$ & $\begin{array}{c}\text { Ba } \\
\text { (ppm) }\end{array}$ & $\stackrel{p}{(p p m)}$ & $\stackrel{s}{(p p m)}$ & $\begin{array}{c}\mathrm{Mg} \\
\text { (ppm) }\end{array}$ & $\begin{array}{c}\text { As } \\
\text { (ppm) }\end{array}$ & $\begin{array}{c}\mathrm{Na} \\
\text { (pprn) }\end{array}$ \\
\hline Mean & 0.016 & 0.027 & 0.529 & 0.014 & -0.013 & 94.333 & -0.023 & 192.604 \\
\hline S.D. & .0 .012 & 0.008 & 0.000 & 0.008 & 0.007 & 0.188 & 0.007 & 0.346 \\
\hline \multirow[t]{2}{*}{ \% R.S.D. } & 75.326 & 29.483 & 0.070 & 53.207 & 54.654 & 0.199 & 32.647 & 0.180 \\
\hline & $\begin{array}{c}\text { Mo } \\
\text { (p;m) }\end{array}$ & $\begin{array}{c}\mathrm{Ag} \\
(\mathrm{ppm})\end{array}$ & $\begin{array}{c}\mathrm{Pb} \\
\text { (ppma) }\end{array}$ & $\begin{array}{c}T i \\
\text { (ppm) }\end{array}$ & $\begin{array}{c}\text { Cd } \\
\text { (ppm) }\end{array}$ & $\begin{array}{c}\text { B } \\
\text { (ppm) }\end{array}$ & $\underset{\text { (ppm) }}{K}$ & $\begin{array}{c}\mathrm{Mn} \\
\text { (ppm) }\end{array}$ \\
\hline Meen & -0.001 & 1.109 & 1.079 & 0.001 & 1.045 & 0.014 & 0.003 & 0.534 \\
\hline S.D. & 0.001 & 0.003 & 0.029 & 0.001 & 0.001 & 0.000 & 0.016 & 0.000 \\
\hline \%R.S.D. & 134.238 & 0.263 & 2.665 & 108.896 & 0.109 & 3.159 & 549.239 & 0.049 \\
\hline
\end{tabular}

\begin{tabular}{lrrrr} 
& \multicolumn{1}{c}{ Sb } & $V$ & \multicolumn{1}{c}{ Be } & \multicolumn{1}{c}{$T$} \\
& (ppm) & (ppm) & \multicolumn{1}{c}{ (ppm) } & \multicolumn{1}{c}{ (ppm) } \\
Mean & 0.018 & 0.510 & 0.518 & 0.017 \\
S.0. & 0.009 & 0.000 & 0.001 & 0.025 \\
\% R.S.D. & 48.218 & 0.070 & 0.188 & 149.188
\end{tabular}


Identity 1: PREPSTDARL Identity 2: Direct

10:18 AM suty 15, 1997

Task name : OPTIMA

Sample Weight : 1.0000 Solution Volume : 1.00

on-Peak Integrations : 3 off-Peak. Integrations : 1 .

\begin{tabular}{|c|c|c|c|c|c|c|c|c|}
\hline & $\begin{array}{c}z r \\
\text { (ppm) }\end{array}$ & $\begin{array}{c}\mathrm{Sr} \\
(\mathrm{Fpm})\end{array}$ & $\begin{array}{c}\mathrm{Bi} \\
\text { (ppm) }\end{array}$ & $\begin{array}{c}s i \\
(p p m)\end{array}$ & $\begin{array}{c}\text { Al } \\
\text { (ppm) }\end{array}$ & $\begin{array}{r}\text { Co } \vdots \\
\text { (ppm) }\end{array}$ & $\begin{array}{c}\mathrm{Cu} \\
(\mathrm{ppm})\end{array}$ & $\underset{(p p m)}{L i}$ \\
\hline Hean & 4.933 & 5.084 & 4.964 & 8.020 & 5.140 & $5: 082$ & 4.767 & 4.957 \\
\hline s.o. & 0.004 & 0.002 & 0.023 & 0.013 & 0.017 & 0.008 & 0.006 & 0.016 \\
\hline \multirow[t]{2}{*}{ \% R.S.D. } & 0.090 & 0.030 & 0.471 & 0.158 & 0.321 & $\begin{array}{c}0: 159 \\
\vdots\end{array}$ & 0.135 & 0.326 \\
\hline & $\begin{array}{c}\mathrm{Zn} \\
\text { (ppm) }\end{array}$ & $\begin{array}{c}M i \\
(p p m)\end{array}$ & Lo & $\begin{array}{c}\text { Fe } \\
\text { (ppm) }\end{array}$ & $\begin{array}{c}\text { Ca } \\
\text { (ppm) }\end{array}$ & $\begin{array}{c}\mathrm{Cr} \\
\text { (ppm) }\end{array}$ & $\begin{array}{c}\text { Nd } \\
\text { (ppm) }\end{array}$ & $\underset{\text { (ppm) }}{u}$ \\
\hline Mean & 4.871 & 5.099 & 5.110 & 4.898 & 4.982 & 4.912 & 5.076 & 9.692 \\
\hline \$.0. & 0.009 & 0.013 & 0.008 & 0.032 & 0.007 & $0: 012$ & 0.007 & 0.021 \\
\hline \multirow[t]{2}{*}{ \% R.S.D. } & 0.176 & 0.257 & 0.151 & 0.649 & 0.134 & $\begin{array}{c}0.240 \\
\vdots\end{array}$ & 0.131 & $\begin{array}{c}0.213 \\
.\end{array}$ \\
\hline & $\begin{array}{c}\text { Ce } \\
\text { (ppm) }\end{array}$ & $\underset{(p m m)}{S m}$ & $\begin{array}{c}\text { Ba } \\
\text { (pppm) }\end{array}$ & $\underset{\text { (ppon) }}{P}$ & (ppm) & $\begin{array}{c}\mathrm{Mg} \\
(\mathrm{ppm})\end{array}$ & $\begin{array}{c}\text { As } \\
\text { (pprn) }\end{array}$ & $\begin{array}{c}\mathrm{Ha} \\
(\mathrm{ppm})\end{array}$ \\
\hline Mean & 5.234 & 5.132 & 5.064 & 5.033 & 5.883 & 5.046 & 5.250 & 5.144 \\
\hline S.D. & 0.012 & 0.003 & 0.004 & 0.012 & 0.020 & 0.013 & 0.015 & 0.015 \\
\hline \multirow[t]{2}{*}{ \% R.S.D. } & 0.231 & 0.056 & 0.080 & 0.243 & 0.333 & 0.263 & 0.288 & 0.283 \\
\hline & $\begin{array}{c}\text { Mo } \\
\text { (ppon) }\end{array}$ & $\begin{array}{c}\text { Ag } \\
\text { (ppm) }\end{array}$ & $\begin{array}{c}\mathrm{Pb} \\
\text { (ppra) }\end{array}$ & $\begin{array}{c}\text { Ti } \\
\text { (ppm) }\end{array}$ & $\begin{array}{c}\mathrm{Cd} \\
\text { (ppmit) }\end{array}$ & $\begin{array}{c}\text { B } \vdots \\
\text { (ppm) }\end{array}$ & $\underset{\text { (pprn) }}{\mathrm{x}}$ & $\begin{array}{c}\text { Mn } \\
\text { (ppm) }\end{array}$ \\
\hline Mean & 4.982 & 1.200 & 4.801 & 4.474 & 4.960 & 4.959 & 4.624 & 4.966 \\
\hline S.D. & 0.002 & 0.003 & 0.023 & 0.004 & 0.017 & 0.014 & 0.028 & 0.013 \\
\hline \% R.S.D. & 0.037 & 0.228 & 0.481 & 0.085 & 0.342 & 0.279 & 0.602 & 0.263 \\
\hline
\end{tabular}

\begin{tabular}{|c|c|c|c|c|}
\hline & $\begin{array}{c}\mathrm{sb} \\
(\mathrm{ppm})\end{array}$ & $\begin{array}{c}v \\
\text { (ppm) }\end{array}$ & $\begin{array}{c}\text { Be } \\
\text { (ppm) }\end{array}$ & $\begin{array}{c}\mathrm{Tl} \\
\text { (ppm) }\end{array}$ \\
\hline Mean & 4.820 & 4.898 & 5.347 & 4.888 \\
\hline S.D. & 0.017 & 0.009 & 0.005 & 0.028 \\
\hline \% R,S,D. & 0.361 & 0.190 & 0.101 & 0.569 \\
\hline
\end{tabular}


HNF-SD-WM-DP-255, REV. 0

Identity 1: PREPBLK Identity 2: Quality control Task name : OPT IMA

Sample weight :

1.0000 solution volume : On-Peak Integrations : 3 off-Peak Integrations : 1

$10: 22$ AM July 15, 1997

\begin{tabular}{|c|c|c|c|c|c|c|c|c|}
\hline & $\begin{array}{c}2 r \\
\text { (ppm) }\end{array}$ & $\begin{array}{c}S r \\
\text { (ppon) }\end{array}$ & $\underset{(\mathrm{ppm})}{\mathrm{Bi}}$ & $\begin{array}{c}\mathbf{s i} \\
\text { (pprn) }\end{array}$ & $\underset{\text { (pp:3) }}{A l}$ & $\begin{array}{c}\text { Co: } \\
\text { (ppm) }\end{array}$ & $\underset{\text { (ppm) }}{\mathrm{Cu}}$ & $\underset{\text { (ppom) }}{\mathrm{Li}}$ \\
\hline Mean & 0.001 & 0.002 & -0.001 & 2.447 & 0.169 & $0: 006$ & 0.017 & 0.001 \\
\hline S.D. & 0.001 & 0.000 & 0.005 & 0.016 & 0.006 & $0: 002$ & 0.000 & 0.000 \\
\hline \multirow[t]{2}{*}{ X R.S.D. } & 40.966 & 4.348 & 586.673 & 0.673 & 3.705 & 34610 & 1.168 & 5.249 \\
\hline & $\begin{array}{c}2 n \\
\text { (PPR) }\end{array}$ & $\begin{array}{c}\mathrm{Hi} \\
(\mathrm{ppm})\end{array}$ & $\begin{array}{c}\text { La } \\
\text { (ppm) }\end{array}$ & $\begin{array}{c}\text { Fe } \\
\text { (ppom) }\end{array}$ & $\underset{\text { (ppm) }}{\mathrm{Ca}}$ & $\begin{array}{r}\mathrm{cr} \\
\text { (ppm) }\end{array}$ & $\begin{array}{c}\text { Nd } \\
\text { (ppm) }\end{array}$ & $\begin{array}{c}u \\
(\mathrm{ppm})\end{array}$ \\
\hline Mean & 0.037 & 0.001 & 0.001 & 0.037 & 0.208 & -0.022 & 0.020 & -0.146 \\
\hline s.o. & 0.000 & 0.003 & 0.000 & 0.600. & 0.001 & 0.002 & 0.003 & 0.015 \\
\hline \multirow[t]{2}{*}{ \% R.S.D. } & 1.268 & 396.672 & 0.521 & 0.985 & 0.307 & $7: 406$ & 17.118 & 10.005 \\
\hline & $\begin{array}{c}\mathrm{Ce} \\
\text { (ppm) }\end{array}$ & $\underset{(\mathrm{ppm})}{\mathrm{sm}}$ & $\begin{array}{c}\mathbf{B a} \\
(\mathrm{ppm})\end{array}$ & $\stackrel{P}{P}$ & $\begin{array}{c}s \\
\text { (ppm) }\end{array}$ & $\underset{(\mathrm{Ppm})}{\mathrm{Mg}}$ & $\begin{array}{c}\text { As } \\
\text { (ppm) }\end{array}$ & $\begin{array}{c}\mathrm{Na} \\
\text { (ppm) }\end{array}$ \\
\hline Mean & 0.031 & -0.022 & 0.002 & 0.041 & 1.299 & 0.040 & 0.000 & 0.336 \\
\hline s.p. & 0.004 & 0.005 & 0.000 & 0.010 & 0.010. & 0.001 & 0.002 & 0.002 \\
\hline \multirow[t]{2}{*}{ \% R.S.D. } & 12.379 & 24.413 & 2.619 & 24.149 & 0.735 & 1.925 & 563.387 & 0.649 \\
\hline & $\begin{array}{c}\text { Mo } \\
\text { (ppm) }\end{array}$ & $\underset{(p p * 1)}{\mathbf{A g}}$ & $\begin{array}{c}\mathrm{Pb} \\
\text { (ppm) }\end{array}$ & $\begin{array}{c}\mathbf{T i} \\
\text { (ppm) }\end{array}$ & $\underset{\text { (pppm) }}{c d}$ & $\begin{array}{c}\text { B } \\
\text { (ppm) }\end{array}$ & $\underset{\text { (pprii) }}{\mathbf{X}}$ & $\underset{\text { (ppm) }}{M n}$ \\
\hline Mean & 0.000 & 0.002 & 0.049 & 0.001 & 0.001 & 0.119 & 0.150 & 0.001 \\
\hline s.o. & 0.001 & 0.000 & 0.010 & 0.000 & 0.800 & 0.002 & 0.089 & 0.000 \\
\hline K R.S.D. & 2440.827 & 23.829 & 19.786 & 38.512 & 94.061 & 1.587 & 6.312 & 41.805 \\
\hline
\end{tabular}


Identity 1: $5977001199 \_\mathrm{I}$ Identity 2: acid dig,4-4-2-8 ml Task name : OPTIMA Sample Height: $\quad 1.0000$ solution Volume : 10.00 On-Peak Integrations : 3 off-Pekk Integrations : 1

\begin{tabular}{|c|c|c|c|c|}
\hline - & $\mathbf{z r}$ & sr & Bi & si \\
\hline & (ppxin) & (ppm) & $(\mathrm{ppm})$ & (ppm) \\
\hline Mean & 0.008 & 3.465 & 368.878 & 10.949 \\
\hline S.D. & 0.006 & 0.009 & 0.608 & 0.018 \\
\hline \multirow[t]{3}{*}{ \% R.S.B. } & 73.465 & 0.252 & 0.165 & 0.162 \\
\hline & $2 n$ & $\mathrm{Ni}$ & La & $\mathrm{Fe}$ \\
\hline & (ppin) & (ppm) & (ppm) & (ppm) \\
\hline Hean & 0.407 & 1.732 & 80.566 & 28.215 \\
\hline S.D. & 0.006 & 0.015 & 0.037 & 0.222 \\
\hline \multirow[t]{3}{*}{ \% R.S.D. } & 1.441 & 0.872 & 0.046 & 0.786 \\
\hline & Ce & Sm & Ba & $\mathbf{p}$ \\
\hline & (ppni) & (ppm) & (ppm) & (Ppril) \\
\hline Mean & 0.380 & -0.249 & -0.076 & 19.000 \\
\hline S.D. & 0.127 & 0.064 & 0.001 & 0.334 \\
\hline \multirow[t]{3}{*}{ \% R.S.D. } & 33.455 & 25.862 & 1.639 & 1.756 \\
\hline & Mo & Ag & $\mathrm{Pb}$ & . $\mathbf{T i}$ \\
\hline & (ppm) & (ppm) & (ppm) & (ppn) \\
\hline Hean & -0.014 & -0.014 & 2.510 & 0.020 \\
\hline s.o. & 0.011 & 0.005 & 0.084 & 0.001 \\
\hline \multirow[t]{3}{*}{ X R.S.D. } & 72.640 & 34.076 & 3.339 & 2.893 \\
\hline & sb & v & $\mathrm{Be}$ & TI \\
\hline & (ppm) & (ppm) & (ppm) & (ppm) \\
\hline Mean & 0.114 & 0.035 & 0.002 & 2.652 \\
\hline S.D. & 0.078 & 0.006 & 0.000 & 0.242 \\
\hline \% R.S.D. & 68.235 & 16.693 & 3.127 & 9.141 \\
\hline
\end{tabular}

10:27 AM July 15, 1997:

At
(ppm)
0.343
0.017
4.844

Co:
(ppon)
$0: 070$
$0: 022$
$31: 817$

cu
(ppm)
0.045
0.001
2.277

Li

(ppm)

Ca

cr:

(ppm)

1.526

0.002

33.041

0.093

0.128

0.283

s

(ppm)

$-0.005$

0.031

616.481

(ppr)

cd

(ppin)

\subsection{6}

0.009

150.510

(Ppr)

0.276

0.001

0.464

B

(ppm)

0.524

0.008

1.517
4

(ppm)

$-1.891$

0.332

17.560

Na

(ppm)

230.439

1.688

0.733

\begin{tabular}{cc}
\multicolumn{1}{c}{$K$} & \multicolumn{1}{c}{ Nn } \\
(ppm) & (ppm) \\
46.536 & 104.141 \\
0.296 & 0.092 \\
0.637 & 0.089
\end{tabular}


1dentity 1: 5971001199 Identity 2: acid dfg,4-4 ml

10:31 AH July 15, 1997 Task name : OPTIMA

Sample Hejght :

1.0000 solution Volume :

2.00

On-Peak Integrations : 3 off-Peak Integrations : 1

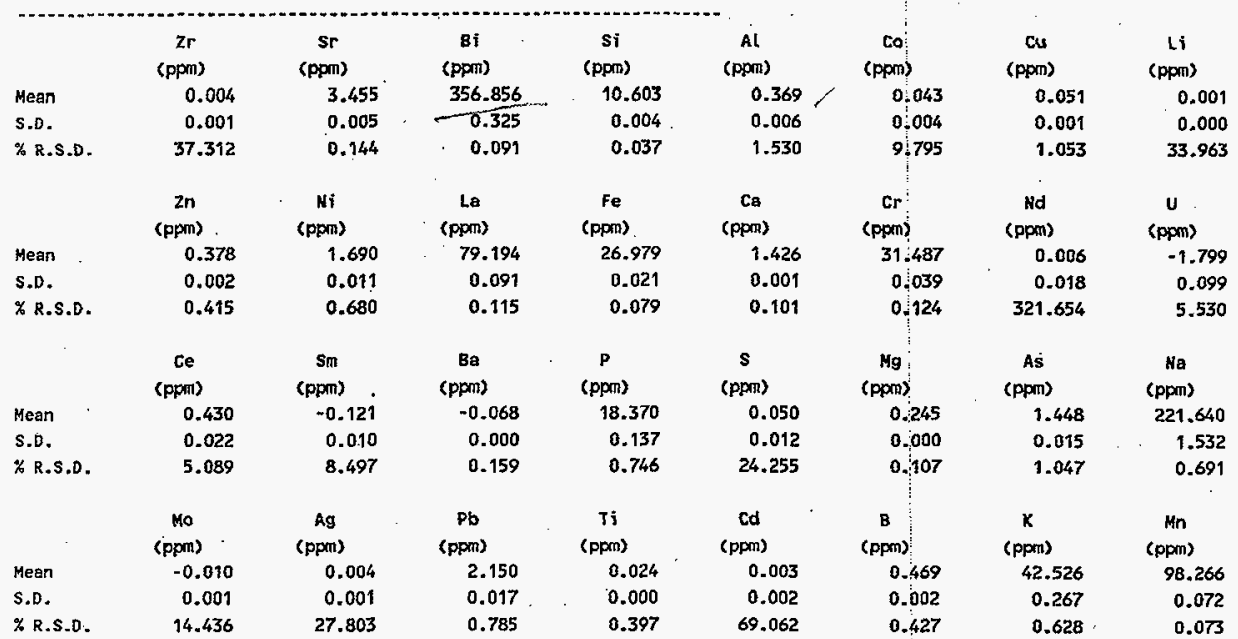

\begin{tabular}{lrrrr} 
& $s b$ & $v$ & \multicolumn{1}{c}{ Be } & \multicolumn{1}{c}{ Tl } \\
& (ppm) & \multicolumn{1}{c}{$\begin{array}{c}\text { (ppm) } \\
\text { (ppm) }\end{array}$} \\
Mean & 0.213 & 0.029 & 0.001 & 2.025 \\
S.D. & 0.034 & 0.000 & 0.000 & 0.033 \\
\%R.S.D. & 15.927 & 1.241 & 64.485 & 1.633
\end{tabular}




\section{HNF-SD-WM-DP-255, REV. 0}

Identity 1: $5971001199 \_$Identity 2: acid dig,4-4 ml $\quad$ 10:34 AM July 15, 1997 Tosk name : OPTIMA

Sample Weight : 1.0000 Solution volume : 2.00

On-Peak Integrations : 3 off-Peak Integrations : 1

\begin{tabular}{|c|c|c|c|c|c|c|c|c|}
\hline & $\begin{array}{c}\mathrm{Zr} \\
\text { (PPn) }\end{array}$ & $\begin{array}{c}\mathrm{sr} \\
\text { (ppm) }\end{array}$ & $\begin{array}{c}\mathrm{Bi} \\
(\mathrm{ppm})\end{array}$ & $\underset{\text { (ppm) }}{\mathbf{s i}}$ & $\begin{array}{c}\text { Al } \\
\text { Sppom })\end{array}$ & $\begin{array}{r}\text { co! } \\
\text { (ppon) }\end{array}$ & $\begin{array}{c}\mathrm{Cu} \\
\text { (ppon) }\end{array}$ & $\underset{(p+\infty)}{L i}$ \\
\hline Mean & 0.006 & 3.574 & 369.493 & 10.477 & 0.387 & 0.049 & 0.046 & 0.002 \\
\hline S.D. & 0.001 & 0.004 & 0.301 & 0.011 & 0.015 & 0,009 & 0.001 & 0.000 \\
\hline \% R.S.D. & 21.296 & 0.119 & 0.081 & 0.101 & 3.820 & $17: 729$ & 1.387 & 19.365 \\
\hline
\end{tabular}

\begin{tabular}{|c|c|c|c|c|c|c|c|c|}
\hline & $\begin{array}{c}\mathrm{Zn} \\
\text { (ppm) }\end{array}$ & $\begin{array}{c}\text { Ni } \\
\text { (ppm) }\end{array}$ & $\begin{array}{c}\text { La } \\
\text { (ppm) }\end{array}$ & $\begin{array}{c}\text { Fe } \\
\text { (ppm) }\end{array}$ & $\begin{array}{c}\text { Ca } \\
\text { (ppr) }\end{array}$ & $\underset{(\mathrm{ppm})}{\mathbf{C r}}$ & $\begin{array}{c}\text { Nd } \\
\text { (ppm) }\end{array}$ & $\begin{array}{c}\mathbf{U} \\
\text { (ppm) }\end{array}$ \\
\hline Mean & 0.508 & 1.720 & 83.266 & 29.967 & 1.479 & 31.849 & 0.020 & -1.834 \\
\hline S.o. & 0.002 & 0.015 & 0.120 & 0.035 & 0.001 & 0.084 & 0.006 & 0.038 \\
\hline \% R.S.D. & 0.473 & 0.851 & 0.144 & 0.116 & 0.067 & 0.264 & 29.281 & 2.086 \\
\hline
\end{tabular}

Mean

S.D.

\% R.S.D.

Ce
(ppm)
0.458
0.011
2.464

Hean

S.D.

\% R.S.D.

No

$-0.013$

0.002

18.412

Sm
(ppm)
-0.152
0.006
4.145

Ba

(ppn)

$-0.052$

0.000

0.830

P
(ppm)
18.947
0.172
0.909

s

(ppm)

$-0.167$

0.013

7.638

\begin{tabular}{cc} 
Ag & \multicolumn{1}{c}{$\mathrm{Pb}$} \\
(ppm) & (ppm) \\
0.008 & 2.221 \\
0.001 & 0.005 \\
15.609 & 0.240
\end{tabular}

Ti
(ppm)
0.025
0.001
5.522

cd

(ppon)

0.004
0.001
17.841

$\mathrm{Mg}$

(ppm)

0.243

0.001

0.262

B

(ppr)

0.875

0.004

0.479

Tt

(ppon)

2.191

0.022

1.016

\begin{tabular}{cr}
\multicolumn{1}{c}{ As } & \multicolumn{1}{c}{$\mathrm{Na}$} \\
(ppm) & \multicolumn{1}{c}{ (ppm) } \\
1.486 & 226.929 \\
0.024 & 1.387 \\
1.640 & 0.611
\end{tabular}

$K$

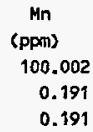


Identity 1: S97r001199_s Itentity 2; acid dig,4-4 ml

$10: 37$ AM July 15, 1997 Task name : OPTIMA

Sample Weight : $\quad 1.0000$ Solution Volune : $\quad 2.00$

on-Peak Integrations : 3 off-Peak Integrations : 1

\begin{tabular}{lrrrrr} 
& $2 r$ & Sr & Bi & \multicolumn{1}{c}{ si } & \multicolumn{1}{c}{ Al } \\
& (ppm) & (pprn) & (ppm) & \multicolumn{1}{c}{ (ppm) } & (ppm) \\
Mean & 5.002 & 8.441 & 365.921 & 16.002 & 5.516 \\
S.D. & 0.013 & 0.012 & 0.263 & 0.045 & 0.021 \\
\% R.S.D. & 0.253 & 0.138 & 0.072 & 0.279 & 0.384
\end{tabular}

Mean

$$
\text { (ppon) }
$$

5.384

S.D.

\% R.S.D.

0.029

0.530

Ni
(ppm)
6.958
0.021
0.296

ce
(ppni)

5.779

Mean

S.D.

\% R.S.D.

0.054

0.927

Sm
(ppm)
4.931
0.016
0.316

$$
\begin{aligned}
& \text { La } \\
& \text { (ppm) } \\
& 87.075 \\
& 0.126 \\
& 0.145
\end{aligned}
$$

Fe
(ppm)
93.926
0.167
0.178

Ca

(ppm)

6.353

0.009

0.141

Ba
(ppm)
5.058
0.006
0.116

$p$

(ppm)

24.076

0.129

0.537

$s$

(ppm)

4.862

0.031

0.641

$\begin{array}{cc}\text { Mo } & \text { Ag } \\ \text { (ppm) } & \text { (ppm) } \\ 5.068 & 1.268 \\ 0.011 & 0.005 \\ 0.220 & 0.361 .\end{array}$

$\mathrm{Pb}$
(ppm)
7.166
0.042
0.584

$\mathrm{Ti}$
(ppm)
4.556
0.012
0.267

cd

(ppm)

5.078

0.007

0.143

TL

(ppm)

\begin{tabular}{cc} 
Be & \multicolumn{1}{c}{$T \mathrm{~L}$} \\
(ppp) & (ppm) \\
5.168 & 7.307 \\
0.013 & 0.063 \\
0.242 & 0.869
\end{tabular}

$\checkmark$

5.043

0.013

0.260

0.869

\% R.S.D.

1.377

$0.260 \quad 0.242$

(1)

$\mathrm{Cu}$

(ppm)

4.815

0.009

0.179

Hd

(ppm)

5.047

0.024

0.472

As
(ppm)

6.840

0.023

0.338

K
(ppm)
49.467
0.188
0.380

(ppm)

(ppm)

5.785

0.009

0.150

.150

106.052

0.315

0.297 
Identity 1: s971001199_X Identity 2: acid dig,4-4-1-9 ml Tesk name : OPTIMA

Sample Weight : $\quad 1.0000$ solution Volure : $\quad 20.00$

On-Peak Integrations: 3 off-Peak Integrations : 1

\begin{tabular}{lrrrrr} 
& Zr & Sr & \multicolumn{1}{c}{ Bi } & \multicolumn{1}{c}{ si } & \multicolumn{1}{c}{ Al } \\
Hean & (ppm) & (ppm) & (ppm) & \multicolumn{1}{c}{ (ppm) } & \multicolumn{1}{c}{ (ppm) } \\
S.D. & 0.024 & 3.477 & 370.594 & 11.256 & 0.228 \\
\% R.S.D. & 0.010 & 0.013 & 1.987 & 0.031 & 0.072 \\
& 41.922 & 0.375 & 0.536 & 0.275 & 31.624
\end{tabular}

\begin{tabular}{|c|c|c|c|c|c|}
\hline & $\begin{array}{c}\text { Zn } \\
\text { (ppm) }\end{array}$ & $\begin{array}{c}\text { Hi } \\
\text { (ppm) }\end{array}$ & $\begin{array}{c}\text { La } \\
\text { (popn). }\end{array}$ & $\underset{\text { (ppon) }}{\mathrm{Fe}}$ & $\begin{array}{c}\mathrm{Ca} \\
\text { (pom) }\end{array}$ \\
\hline Mean & 0.397 & 1.716 & 80.735 & 28.434 & 1.6 \\
\hline Sid. & 0.006 & 0.028 & 0.393 & 0.300 & $0: 0$ \\
\hline \% R.S.D. & 1.419 & 1.613 & 0.486 & 1.054 & 0.3 \\
\hline
\end{tabular}

\begin{tabular}{lrrrrr} 
& \multicolumn{1}{c}{ Ce } & \multicolumn{1}{c}{ Sm } & \multicolumn{1}{c}{ Ba } & \multicolumn{1}{c}{$p$} & \multicolumn{1}{c}{$s$} \\
(ppm) & \multicolumn{1}{c}{$\begin{array}{c}\text { (ppm) } \\
\text { (ppm) }\end{array}$} & \multicolumn{1}{c}{ (ppm) } & \multicolumn{1}{c}{ (ppm) } \\
Mean & 0.159 & 0.123 & -0.083 & 19.485 & -0.022 \\
S.D. & 0.109 & 0.069 & 0.003 & 0.160 & 0.040 \\
\%R.S.D. & 68.819 & 56.377 & 3.907 & 0.822 & 180.586
\end{tabular}

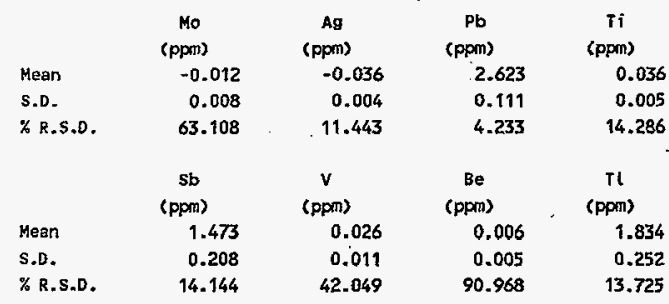

HNF-SD-WMA-DP-255, REV. 0

10:43 AM July 15, 1997: 


\section{Task name : OPTIMA}

Identity 1: 5971001199_AX Identity 2: acid dig,4-4-1-9 ml

Sample Weight : 1.0000 Solution Volume : 20.00

on-Peak Integrations : 3 . off-Peak Integrations : 1

\begin{tabular}{lrrrrr} 
& \multicolumn{1}{c}{ Zr } & \multicolumn{1}{c}{ Sr } & \multicolumn{1}{c}{ Bi } & \multicolumn{1}{c}{ si } & \multicolumn{1}{c}{ Al } \\
(ppm) & (ppm) & \multicolumn{1}{c}{ (ppm) } & \multicolumn{1}{c}{ (ppm) } & \multicolumn{1}{c}{ (ppm) } \\
Hean & 205.379 & 214.478 & 579.454 & 217.858 & 210.426 \\
S.D. & 0.540 & 0.716 & 1.302 & 0.260 & 0.660 \\
\& R.S.D. & 0.263 & 0.334 & 0.225 & 0.120 & 0.314
\end{tabular}

\begin{tabular}{lrrrrr} 
& \multicolumn{1}{c}{ Zn } & \multicolumn{1}{c}{ Ni } & \multicolumn{1}{c}{ La } & \multicolumn{1}{c}{ Fe } & \multicolumn{1}{c}{ Ca } \\
& (ppm) & \multicolumn{1}{c}{ (ppm) } & \multicolumn{1}{c}{ (ppm) } & \multicolumn{1}{c}{ (ppm) } & \multicolumn{1}{c}{ (ppm) } \\
Mean & 207.752 & 212.173 & 286.569 & 230.882 & 206.357 \\
S.0. & 0.782 & 0.497 & 0.323 & 0.646 & 0.283 \\
\% R.S.D. & 0.376 & 0.234 & 0.113 & 0.280 & 0.137
\end{tabular}

\begin{tabular}{|c|c|c|c|c|c|}
\hline & Ce & SiII & $\mathbf{B a}$ & P & $\mathbf{s}$ \\
\hline & (ppm) & (ppm) & (ppm) & (pprn) & (ppm) \\
\hline Kean & 215.547 & 207.511 & 208.073 & 233.577 & 207.082 \\
\hline S.D. & 1.549 & 0.404 & 0.254 & 2.045 & 2.159 \\
\hline \% R.S.D. & 0.718 & 0.195 & 0.122 & 0.876 & 1.04 \\
\hline
\end{tabular}

\begin{tabular}{|c|c|c|c|c|}
\hline & $\begin{array}{c}\text { No } \\
\text { (ppm) }\end{array}$ & $\begin{array}{c}\text { Ag } \\
\text { (ppm) }\end{array}$ & $\begin{array}{c}\mathrm{Pb} \\
\text { (ppm) }\end{array}$ & $\begin{array}{c}\mathrm{Ti} \\
\text { (ppm) }\end{array}$ \\
\hline Mean & 212.281 & 167.772 & 210.911 & 206.058 \\
\hline S.D. & $\uparrow .027$ & 0.716 & 0.919 & 0.693 \\
\hline \% R.s.D. & 0.484 & 0.427 & 0.436 & 0.336 \\
\hline & $\begin{array}{c}\text { sb } \\
\text { (ppm) }\end{array}$ & $\underset{(\mathrm{ppm})}{v}$ & $\begin{array}{c}\text { Be } \\
\text { (ppm) }\end{array}$ & $\begin{array}{c}\mathrm{Tl} \\
\text { (ppm) }\end{array}$ \\
\hline Mean & 202.356 & 206.512 & 205.185 & 218.233 \\
\hline S.D. & 0.753 & 0.512 & 0.340 & 1.103 \\
\hline X R.S.D. & 0.372 & 0.248 & 0.166 & 0.505 \\
\hline
\end{tabular}


Identity 1: ICSA Task name : CPT IMA Sample Wefight : on-Peak Integrat
Identity 2: Quality Control

1.0000 Solution Volune :

off-Peak Integrations

\begin{tabular}{|c|c|c|c|c|c|}
\hline & $\begin{array}{c}\mathrm{Zr} \\
(\mathrm{ppm})\end{array}$ & $\begin{array}{c}\mathrm{sr} \\
(\mathrm{ppom})\end{array}$ & $\begin{array}{c}\text { Bi } \\
(\mathrm{ppm})\end{array}$ & $\begin{array}{c}\mathrm{si} \\
\text { (ppm) }\end{array}$ & $\begin{array}{c}A 6 \\
\text { (ppm) }\end{array}$ \\
\hline Mean & 0.003 & 0.002 & -0.020 & 0.036 & 216.312 \\
\hline S.D. & $0.001^{\circ}$ & 0.000 & 0.009 & 0.005 & 0.172 \\
\hline \% R.S.D. & 18.733 & 4.537 & 46.882 & 12.784 & 0.079 \\
\hline . & $\underset{\text { (ppm) }}{\mathrm{Zn}}$ & $\begin{array}{c}\mathrm{Ni} \\
\text { (ppn) }\end{array}$ & $\begin{array}{c}\text { La } \\
\text { (ppm) }\end{array}$ & $\begin{array}{c}\mathrm{Fe} \\
\text { (ppm) }\end{array}$ & $\begin{array}{c}\mathrm{Ca} \\
(\mathrm{ppm})\end{array}$ \\
\hline Mean & 0.006 & 0.002 & 0.003 & 101.534 & 103.497 \\
\hline S.D. & 0.000 & 0.002 & 0.001 & 0.061 & 0.161 \\
\hline \multirow[t]{2}{*}{ \% R.S.D. } & 7.077 & 83.239 & 26.359 & 0.060 & 0.155 \\
\hline & $\begin{array}{c}\text { Ce } \\
\text { (ppm) }\end{array}$ & $\begin{array}{c}\mathrm{Sm} \\
(\mathrm{ppm})\end{array}$ & (ppm) & $\underset{(p p m)}{P}$ & $\begin{array}{c}\$ \\
\text { (ppm) }\end{array}$ \\
\hline Mean & 0.021 & 0.016 & 0.002 & 0.006 & 0.005 \\
\hline S.D. & 0.011 & 0.008 & 0.000 & 0.006 & 0.005 \\
\hline \multirow[t]{2}{*}{ \% R.S.D. } & 53.273 & 51.053 & 16.013 & 97.415 & 92.908 \\
\hline & $\begin{array}{c}\text { Mo } \\
\text { (ppom) }\end{array}$ & $\begin{array}{c}\text { Ag } \\
\text { (ppm) }\end{array}$ & $\begin{array}{c}\mathrm{Pb} \\
(\mathrm{ppm})\end{array}$ & $\begin{array}{c}T i \\
(p p m)\end{array}$ & $\cdot \frac{C d}{(p p m)}$ \\
\hline Mean & 0.000 & 0.028 & 0.000 & 0.001 & 0.003 \\
\hline S.D. & 0.001 & 0.002 & 0.008 & 0.000 & 0.002 \\
\hline \multirow[t]{2}{*}{ \% R.S.D. } & 300.002 & 8.881 & 1591.243 & 24.427 & 73.055 \\
\hline & $\begin{array}{c}\text { sb } \\
\text { (ppm) }\end{array}$ & $\underset{(p p m)}{v}$ & $\begin{array}{c}\text { Be } \\
\text { (ppon) }\end{array}$ & $\begin{array}{c}T l \\
\text { (pprs) }\end{array}$ & \\
\hline Mean & 0.069 & 0.001 & 0.003 & 0.005 & . \\
\hline S.D. & 0.039 & 0.001 & 0.000 & 0.028 & \\
\hline * R.S.D. & 56.381 & 72.346 & 4.696 & 604.621 & \\
\hline
\end{tabular}

11:01 AM July 15, 1997 
Identity 1: ICSAB Identity 2: Quality Control

11:04 AM July 15, 1997
Sample Weight : $\quad 1.0000$ Solution Volume : $\quad 1.00$

on-Peik Integrations : 3 off-Peak Integrations : 1

\begin{tabular}{|c|c|c|c|c|c|c|c|c|}
\hline & $\begin{array}{c}\mathrm{Zr} \\
\text { (ppm) }\end{array}$ & $\begin{array}{c}S r \\
\text { (ppm) }\end{array}$ & $\begin{array}{c}\mathbf{8} \mathbf{i} \\
\text { (ppm) }\end{array}$ & $\begin{array}{c}\text { si } \\
\text { (ppm) }\end{array}$ & $\begin{array}{c}\text { Al } \\
\text { (ppm) }\end{array}$ & $\begin{array}{c}C 0 \\
\text { (ppm) }\end{array}$ & $\begin{array}{c}\mathrm{cu} \\
\text { (ppom) }\end{array}$ & $\underset{\text { (pp:1) }}{\mathbf{L i}}$ \\
\hline Mean & -0.003 & 0.003 & -0.031 & 0.021 & 215.202 & 0.529 & 0.507 & 0.944 \\
\hline S.D. & 0.001 & 0.000 & 0.006 & 0.004 & 0.269 & 0.004 & 0.002 & 0.007 \\
\hline \multirow[t]{2}{*}{ KR.S.D. } & 31.368 & 0.080 & 19.298 & 20.629 & 0.125 & $0^{i} 756$ & 0.315 & 0.722 \\
\hline & $\frac{2 n}{(p p m)}$ & $\underset{(p p m)}{N i}$ & $\begin{array}{c}\text { La } \\
\text { (ppm) }\end{array}$ & $\begin{array}{c}\text { Fe } \\
\text { (ppm) }\end{array}$ & $\begin{array}{c}\text { Ca } \\
\text { (ppm) }\end{array}$ & $\begin{array}{r}\mathrm{Cr} ! \\
(\mathrm{ppm})\end{array}$ & $\begin{array}{c}\text { Nd } \\
\text { (Ppm) }\end{array}$ & $\underset{\text { (ppom) }}{U}$ \\
\hline Mean & 1.043 & 1.047 & -0.000 & 99.877 & 102.165 & 0 & 0.022 & 0.033 \\
\hline S.D. & 0.003 & 0.001 & 0.001 & 0.267 & 0.096 & 0002 & 0.013 & 0.021 \\
\hline \multirow[t]{2}{*}{ \% R.S.D. } & 0.288 & 0.113 & 584.656 & 0.267 & 0.094 & $0: 450$ & 58.428 & 63.896 \\
\hline & $\underset{(p p m)}{C e}$ & $\begin{array}{c}\mathrm{Sm} \\
\text { (ppm) }\end{array}$ & $\begin{array}{c}8 \mathbf{8} \\
(\mathrm{ppm})\end{array}$ & $\frac{P}{\text { (ppm) }}$ & $\begin{array}{c}\mathrm{s} \\
\text { (ppm) }\end{array}$ & $\begin{array}{r}\mathrm{Hg} \\
\text { (ppom) }\end{array}$ & $\begin{array}{c}\text { As } \\
\text { (ppm) }\end{array}$ & $\begin{array}{c}\mathrm{Na} \\
\text { (ppm) }\end{array}$ \\
\hline Mean & 0.025 & 0.016 & 0.534 & 0.021 & 0.027 & 95.790 & -0.005 & 191.031 \\
\hline S.D. & 0.007 & 0.009 & 0.002 & 0.0 .06 & 0.004 & 0.245 & 0.008 & 1.281 \\
\hline \multirow[t]{2}{*}{ \% R.S.D. } & 29.086 & 54.245 & 0.301 & 28.033 & 14.982 & 0.256 & 137.389 & 0.670 \\
\hline & $\begin{array}{c}\text { Mo } \\
\text { (ppm) }\end{array}$ & $\begin{array}{c}\mathrm{Ag} \\
(\mathrm{ppm})\end{array}$ & $\begin{array}{c}\mathrm{Pb} \\
\text { (ppm) }\end{array}$ & $\begin{array}{c}T i \\
\text { (ppm) }\end{array}$ & $\begin{array}{c}\text { Cd } \\
\text { (ppmi) }\end{array}$ & $\begin{array}{c}\text { B } \\
(p p m)\end{array}$ & $\begin{array}{c}K \\
\text { (ppm) }\end{array}$ & $\begin{array}{c}\text { Mn } \\
\text { (ppm) }\end{array}$ \\
\hline Mean & -0.003 & 1.111 & 1.055 & -0.000 & 1.052 & 0.012 & 0.035 & 0.532 \\
\hline S.D. & 0.001 & 0.003 & 0.025 & 0.000 & 0.004 & 0.000 & 0.011 & 0.002 \\
\hline \% R.S.D. & 48.087 & 0.242 & 2.359 & 1067.639 & 0.333 & 2.343 & 31.787 & 0.292 \\
\hline
\end{tabular}




\begin{tabular}{|c|c|c|c|c|c|}
\hline & $2 r$ & $\mathbf{s r}$ & Bi & si & Al \\
\hline & (ppm) & (ppm) & (ppm) & (ppm) & (ppm) \\
\hline Mean & 5.105 & 5.221 & 5.371 & 4.959 & 5.248 \\
\hline S.D. & 0.003 & 0.005 & 0.046 & 0.012 & 0.015 \\
\hline \multirow[t]{2}{*}{ \% R.S.D. } & 0.056 & 0.100 & 0.853 & 0.243 & 0.285 \\
\hline & $\begin{array}{c}2 n \\
\text { (ppm) }\end{array}$ & $\begin{array}{c}\mathrm{Ni} \\
(\mathrm{ppm})\end{array}$ & $\begin{array}{c}\text { La } \\
\text { (ppm) }\end{array}$ & $\begin{array}{c}\mathrm{Fe} \\
\text { (ppm) }\end{array}$ & $\begin{array}{c}\mathrm{Ca} \\
(\mathrm{Ppm})\end{array}$ \\
\hline Mean & 5.524 & 5.325 & 5.163 & 5.226 & 4.966 \\
\hline s.D. & 0.011 & 0.006 & 0.008 & 0.019 & 0.005 \\
\hline \multirow[t]{2}{*}{ \% R.S.D. } & 0.196 & 0.122 & 0.150 & 0.360 & 0.093 \\
\hline & $\begin{array}{c}\text { Ce } \\
\text { (ppm) }\end{array}$ & $\begin{array}{c}\mathrm{Sm} \\
\text { (ppm) }\end{array}$ & $\begin{array}{c}\mathrm{BB} \\
(\mathrm{ppm})\end{array}$ & $\underset{\text { (ppm) }}{P}$ & $\stackrel{s}{(p p m)}$ \\
\hline Mean & 5.188 & 5.118 & 5.133 & 5.478 & 5.289 \\
\hline S.D. & 0.028 & 0.008 & 0.002 & 0.026 & 0.008 \\
\hline \multirow[t]{2}{*}{ \% R.S.D. } & 0.544 & 0.151 & 0.038 & 0.470 & 0.159 \\
\hline & $\begin{array}{c}\text { Ho } \\
\text { (ppor) }\end{array}$ & $\begin{array}{c}A g \\
(\mathrm{ppm})\end{array}$ & $\begin{array}{c}\mathrm{Pb} \\
\text { (ppm) }\end{array}$ & $\begin{array}{c}\mathrm{Ti} \\
\text { (ppm) }\end{array}$ & $\begin{array}{c}\text { cd } \\
\text { (ppm) }\end{array}$ \\
\hline Mean & 5.445 & 5.447 & 5.240 & 5.069 & 5.358 \\
\hline S.D. & 0.020 & 0.012 & 0.014 & 0.009 & 0.010 \\
\hline \multirow[t]{2}{*}{ \% R.S.D. } & 0.376 & 0.221 & 0.276 & 0.170 & 0.191 \\
\hline & $\begin{array}{c}\mathrm{sb} \\
\text { (ppm) }\end{array}$ & $\begin{array}{c}v \\
\text { (ppm) }\end{array}$ & $\begin{array}{c}\mathrm{Be} \\
(\mathrm{ppm})\end{array}$ & $\begin{array}{c}T l \\
(p p m)\end{array}$ & \\
\hline Mean & 4.853 & 5.316 & 5.291 & 5.293 & \\
\hline 5.0 & 0.023 & 0.009 & 0.005 & 0.018 & \\
\hline X R.S.B. & 0.478 & 0.166 & 0.093 & 0.336 & \\
\hline
\end{tabular}


Identity 1: СCB Task name : OPTIMA Sample Height : Identity 2: Quality Control 11:18 AN July 15, 1997 on-Peak Integrat

1.0000 solution Volume :

1.00

: 3 off-Peak Integrations : 1

\begin{tabular}{|c|c|c|c|c|c|}
\hline & $\begin{array}{c}2 r \\
(p p m)\end{array}$ & $\begin{array}{c}\text { sr } \\
(p p m)\end{array}$ & $\underset{\text { (ppm) }}{\mathbf{B i}}$ & $\begin{array}{c}\text { si } \\
\text { (ppm) }\end{array}$ & $\begin{array}{c}\text { Al } \\
\text { (ppm) }\end{array}$ \\
\hline Mean & -0.000 & -0.000 & -0.008 & 0.006 & -0.006 \\
\hline s.o. & 0.000 & 0.000 & 0.002 & 0.000 & 0.005 \\
\hline \multirow[t]{2}{*}{ \% R.S.D. } & 530.505 & 21.651 & 23.715 & 6.736 & 84.625 \\
\hline & $\begin{array}{c}2 n \\
(p p m)\end{array}$ & $\underset{\text { (ppm) }}{n i}$ & $\begin{array}{c}2 a \\
\text { (ppm) }\end{array}$ & - $\underset{(\mathrm{ppm})}{\mathrm{Fe}}$ & $\begin{array}{c}\mathrm{Ca} \\
(\mathrm{ppm})\end{array}$ \\
\hline Mean & -0.000 & -0.000 & 0.002 & 0.603 & 0.001 \\
\hline S.D. & 0.000 & 0.001 & 0.002 & 0.003 & 0.000 \\
\hline \multirow[t]{2}{*}{ \% R.S.D. } & 150.464 & 302.636 & 113.380 & 82.568 & 10.000 \\
\hline & $\begin{array}{c}\mathrm{Ce} \\
\text { (ppm) }\end{array}$ & $\underset{(\mathrm{ppm})}{\mathrm{Sm}}$ & $\begin{array}{c}\mathrm{Ba} \\
(\mathrm{ppm})\end{array}$ & $\underset{(p p m)}{p}$ & (ppm) \\
\hline Mean & -0.029 & 0.003 & -0.000 & $=-0.006$ & -0.008 \\
\hline S.D. & 0.005 & 0.003 & 0.000 & 0.004 & 0.001 \\
\hline \multirow[t]{2}{*}{ \% R.S.D. } & 16.225 & 85.631 & 12.385 & 70.830 & 8.448 \\
\hline & $\begin{array}{c}\text { Mo } \\
\text { (ppm) }\end{array}$ & $\begin{array}{c}\text { Ag } \\
\text { (ppm) }\end{array}$ & $\begin{array}{c}\mathrm{Pb} \\
(\mathrm{ppm})\end{array}$ & $\underset{\text { (ppm) }}{\mathbf{T i}}$ & $\begin{array}{c}c d \\
\text { (ppm) }\end{array}$ \\
\hline Mean & 0.001 & -0.000 & 0.031 & 0.000 & -0.000 \\
\hline S.D. & 0.001 & 0.000 & 0.007 & 0.000 & 0.000 \\
\hline \multirow[t]{2}{*}{ \% R.S.D. } & 77.273 & 54.330 & 23.777 & 41.089 & 100.077 \\
\hline & $\begin{array}{c}\text { sb } \\
(\mathrm{ppm})\end{array}$ & $\begin{array}{c}v \\
\text { (ppm) }\end{array}$ & $\begin{array}{c}\mathrm{Be} \\
\text { (ppm) }\end{array}$ & $\begin{array}{c}T l \\
\text { (ppon) }\end{array}$ & \\
\hline Mean & 0.021 & -0.001 & 0.000 & -0.016 & \\
\hline s.o. & 0.020 & 0.000 & 0.000 & 0.007 & \\
\hline \# R.S.B. & 96.806 & 34.177 & 1886.097 & 41.493 & . \\
\hline 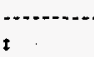 & & & & & . \\
\hline
\end{tabular}

HNF-SD-WHA-DP-255, REV. 0 


\section{LABCORE Completed Worklist Report for Worklist\# 18752}

Analyst: krm

Instrument: CARB2

Book\# $\frac{25 N I 2 A}{260012 A}$

Method: $\angle A-342-100$ Rev/Mod $E-O$

Worklist Comment: T-204 @TICTOC1 *RERUN*

HNF-SD-WM-DP-255, REV. 0

\begin{tabular}{llllll}
\hline Seq Type & Sample\# R A & Test & Matrix Actual Found DL or Yield Unit
\end{tabular}

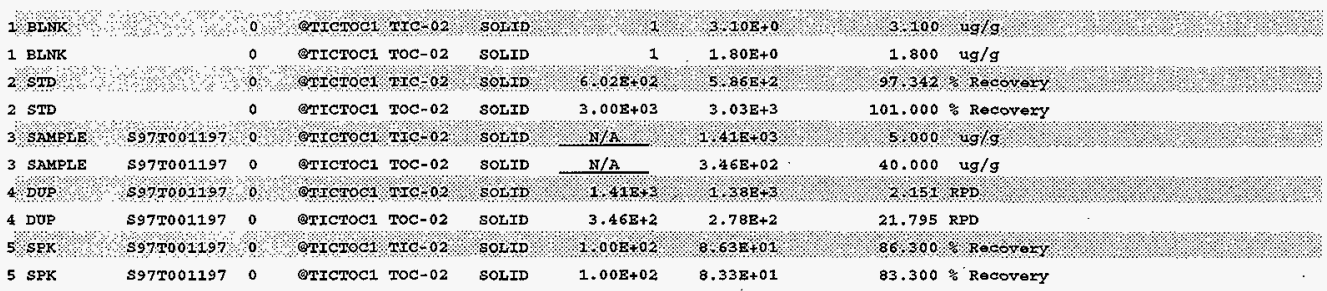

\section{Final page for worklist\# 18752}

Analyst Signature

Date

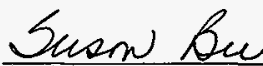

Analyst Signature

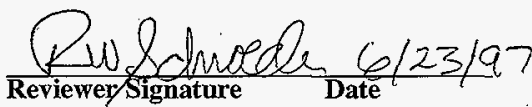




\section{LABCORE Data Entry Template for Worklist\# 18752}

Analyst: $\quad K / P M$ Instrument: CARB2 $\ldots$ Book\# $25 \mathrm{~N} / 2 \mathrm{NA}$

Method: LA-342-100 Rev/Mod

HNF-SD-WM-DP-255, REV. 0

Worklist Comment: T-204 @TICTOC1 *RERUN*

\begin{tabular}{llllll}
\hline S Type & Sanplef & Test Matrix & Group F Project
\end{tabular}

1. BLNK

ETICTOC1. SOLID

2 STD

ETICTOC1 SOLID

3 SAMPLE

S97T0011970

erICTOC1 SOLID

$97000261 \mathrm{~T}-204$

Analytes Requested: TIC-02, TOC-02
4 DUP
S97T0011970
ETICTOC1 SOLID
$5 \mathrm{SPK}$
S97T001197 0
ETICTOCI SOLID

\section{Final page for worklist \# 18752}

$$
6-3 /-9>
$$




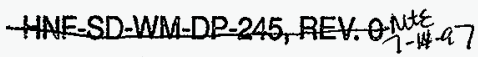

WORKBOOK PAGE: BLANK1

TIC/TOC : LA-342-100 (E-0) SOLIDS

\begin{tabular}{|c|c|c|c|c|}
\hline TIC/TOC : LA & 2-100 (E-0) & & TIC & TOC \\
\hline$\sqrt{\text { Type }}$ & Sample Size in $\mathrm{g}$ & (SS) & 0.2262 & 0.2262 \\
\hline BLNK & Dilution Factor & (DF) & 1. & 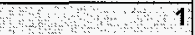 \\
\hline Work List & $\mu \mathrm{g}$ of Carbon in Sample & (C1) & 9.4 & 29.6 \\
\hline 18752 & $\mu \mathrm{g}$ of Carbon from Baseline & (C2) & 6.3 & 31.4 \\
\hline
\end{tabular}

YWest Codo @TICTOC1

$\frac{\text { Matrix }}{\text { SOLID }}$

HNF-SD-WM-DP-255, REV. 0

Batch Nümber 97002841

Rerun $\mu \mathrm{g}$ of Carbon $=\left|\mathrm{C}_{1}-\mathrm{C}_{2}\right|$

0 $\frac{\text { Sample Prep }}{\text { N/A }}$

Sample \# BLANK

Instrument Code CARB2

PreparedBy SKB

Chemist RWS

Analyst

Date Complete 06/23/97

Analysis Date 06/21/97

Analy sis Time 05:00 AM T-204

\begin{tabular}{|l|l||l|}
\hline Method Detection Limit in ug/g & TIC & TOC \\
\hline$\mu g$ of Carbon & 5 & \\
\hline
\end{tabular}


WORKBOOK PAGE: STD2

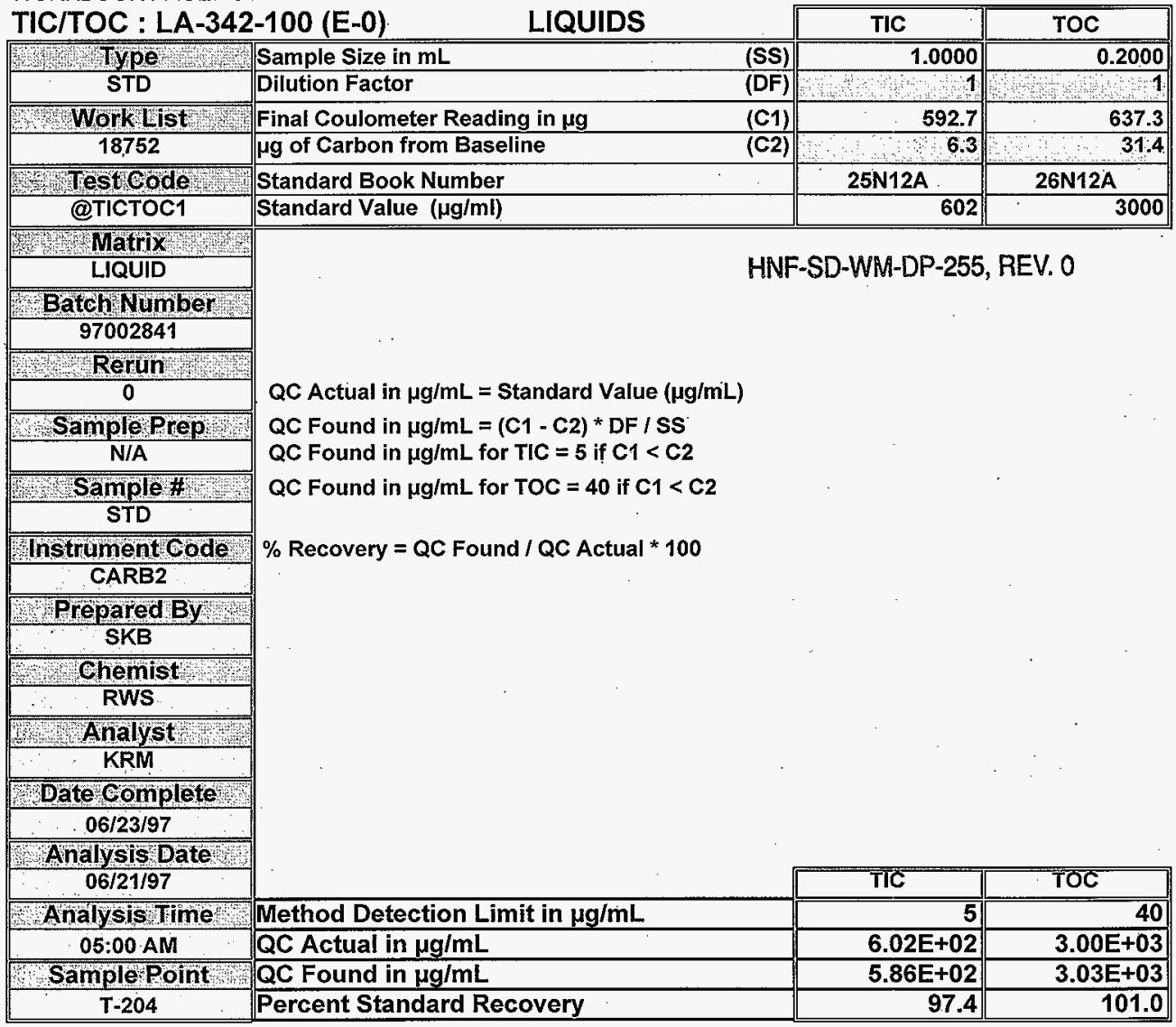

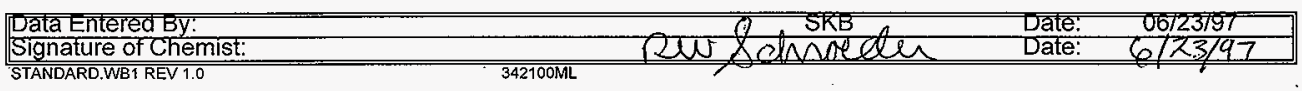


HNF-SQWWA-DR-245, REV Onute

WORKBOOK PAGE: SAM3

\begin{tabular}{|c|c|c|c|c|}
\hline TIC/TOC : LA-342 & SOLIDS & & TIC & TOC \\
\hline S Type & Sample Size in $\mathrm{g}$ & (SS) & 0.2262 & 0.2262 \\
\hline SAMPLE & Dilution Factor & (DF) & 1 & के \\
\hline Work Wist & ug of Carbon in Sample & (C1) & 324.4 & 109.6 \\
\hline 18752 & $\mu \mathrm{g}$ of Carbon from Baseline & (C2) & 6.3 & 31.4 \\
\hline TestCode & & & & \\
\hline @TICTOC1 & & & & \\
\hline Matrix & & & & \\
\hline SOLID & & HNF-S & WAADP 255 & DFY \\
\hline Batch Namber & & IINA-O & 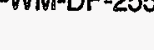 & , nev. \\
\hline 97002841 & & & & \\
\hline Rerun & $\mu \mathrm{g}$ of Carbon $/ \mathrm{g}=(\mathrm{C} 1-\mathrm{C} 2) * \mathrm{DF} / \mathrm{SS}$ & & & \\
\hline $\mathbf{0}$ & $\mu \mathrm{g}$ of Carbon $/ \mathrm{g}$ for $\mathrm{TIC}=5$ if $\mathrm{C} 1<\mathrm{C} 2$ & & & \\
\hline SamplePrep & $\mu \mathrm{g}$ of Carbon/g for TOC $=40$ if $\mathrm{C} 1<\mathrm{C} 2$ & & & \\
\hline N/A & & & & \\
\hline Sample\# & & & & \\
\hline S97T001197 & & & & \\
\hline Instrument Code & . & & & \\
\hline CARB2 & & & & \\
\hline Prepared By & & & & \\
\hline SKB & & & & \\
\hline Chemist thes & & & & \\
\hline RWS & & & & \\
\hline Analyst & , & & & \\
\hline KRM & & & & \\
\hline Date Complete & & & & \\
\hline $06 / 23 / 97$ & & & & \\
\hline Analysis Date & & & & \\
\hline$\therefore \quad 06 / 21 / 97$ & & & TTC & TOC \\
\hline Analysis Time, & Method Detection Limit in ug/g & & 5 & 40 \\
\hline 05:00 AM & & & & \\
\hline Sample Point & $\mu \mathrm{g}$ of Carbon $/ \mathrm{g}$ & & 1.41E+03 & $3.46 \mathrm{E}+02$ \\
\hline
\end{tabular}

\begin{tabular}{|c|c|c|c|}
\hline Data Entered By: & SKB & Date: & $06 / 23 / 97$ \\
\hline Signature of Chemist: & edle & Date: & $6 / 23 / 97$ \\
\hline
\end{tabular}




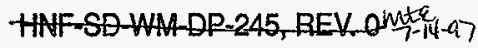

WORKBOOK PAGE: DUP4

TIC/TOC : LA-342-100 (E-0) SOLIDS

\begin{tabular}{|c|c|c|c|c|}
\hline Type & Sample Size in $\mathrm{g}$ & (SS) & 0.3003 & 0.3003 \\
\hline DUP & Dilution Factor & (DF) & 1 & 1) \\
\hline Work Eist & ug of Carbon in Sample & (C1) & 421.7 & 114.8 \\
\hline 18752 & ug of Carbon from Baseline & (C2) & 6.3 & 31.4 \\
\hline Test Code & Known $\mu \mathrm{g}$ of C from Original Sample & & $141 E+3$ & $3.46 E+2$ \\
\hline @TICTOC1 & & & & \\
\hline
\end{tabular}

\begin{tabular}{|l} 
Matrix \\
\hline SOLID
\end{tabular}

HNF-SD-WM-DP-255, REV. 0

Batch Number 97002841

$\frac{1}{2 \text { Rerun }}$

$\frac{\text { Sample Prep }}{\text { N/A }}$

Sample H

Instrument Code CARB2

Prepared By SKB

Chemist RWS

Analyst

Date complete 06/23/97

Analysis Date 06/21/97

Analysis Time 05:00 AM

Sample Point T-204 $\mu \mathrm{g}$ of Carbon/g $=(\mathrm{C} 1-\mathrm{C} 2)^{*} \mathrm{DF} / \mathrm{SS}$

$\mu \mathrm{g}$ of Carbon/g for TIC $=5$ if $\mathrm{C}_{1}<\mathrm{C}_{2}$

$\mu \mathrm{g}$ of Carbon/g for TOC $=40$ if $\mathrm{C} 1<\mathrm{C} 2$

\begin{tabular}{|l|r|r|}
\hline \multicolumn{1}{|c|}{} & \multicolumn{1}{|c|}{ TIC } & TOC \\
\hline Method Detection Limit in $\mathrm{ug} / \mathrm{g}$ & 5 & \\
\hline & & \\
\hline $\mathrm{gg}$ of Carbon/g & $1.38 \mathrm{E}+03$ & $2.78 \mathrm{E}+02$ \\
\hline
\end{tabular}

\begin{tabular}{|c|c|c|c|}
\hline Data Entered By: & SKB & Date: & $06 / 23 / 97$ \\
\hline Signature of Chemist: & Ca & Date: & $6 / 25 / 97$ \\
\hline
\end{tabular}


WORKBOOK PAGE: SPIKE5

TIC/TOC : LA-342-100 (E-0)

HWF-SD-WM-EP-245, REV.0inte 7497

\begin{tabular}{|c|c|c|c|}
\hline Type & Sample Vial Data & TIC & TOC \\
\hline SPK & Sample Size in $\mathrm{g}$ & 0.2262 & 0.2262 \\
\hline Work List & Final Coulometer Reading in $\mu \mathrm{g}$ & 324.4 & 109.6 \\
\hline 18752 & Spiked Vial Data & & \\
\hline Testcode & Sample Size in $\mathrm{g}$ & (0.3147 & 0.3147 \\
\hline @TICTOC1 & Amount of Spike Std. in $\mathrm{mL}$ & 0.500 & 0.100 \\
\hline Matrix & Final Coulometer Reading in $\mu \mathrm{g}$ & 711 & 402.5 \\
\hline SOLID & Spike Book Number & 25N12A & 26N12A \\
\hline Batch Number & Spike Standard Value in $\mu \mathrm{g} / \mathrm{ml}$ & 602 & 3000 \\
\hline 97002841 & \multirow{20}{*}{$\begin{array}{l}\text { QC Actual in } \mu \mathrm{g} / \mathrm{mL}=\text { Spike Val } \\
\text { QC Found in } \mu \mathrm{g} / \mathrm{mL}=(\text { Percent S }\end{array}$} & & \\
\hline Rerun & & & \\
\hline $\mathbf{0}$ & & & \\
\hline SamplèPrep & & & \\
\hline N/A & & & \\
\hline Sample\# & & $((\mathrm{SPK}$ CONC$) *$ & SPK VOL) ${ }^{* 1}$ \\
\hline S97T001197 & & & \\
\hline Instrument $C o d e$ & & & \\
\hline CARB2 & & Actual) / 100 & \\
\hline Prepared/By & & & \\
\hline SKB & & & \\
\hline Chemist & & & \\
\hline RWS & & & \\
\hline Analyst & & & \\
\hline KRM & & & \\
\hline Bate Complete & & & \\
\hline $06 / 23 / 97$ & & & \\
\hline Analysis Date & & & \\
\hline $06 / 21 / 97$ & & & \\
\hline Analysis Time & & TIC & TOC \\
\hline 05:00 AM & QC Actual in $\mu \mathrm{g} / \mathrm{mL}$ & $6.02 \mathrm{E}+02$ & 3.00E+03 \\
\hline Sample Point & QC Found in $\mu \mathrm{g} / \mathrm{mL}$ & $5.19 \mathrm{E}+02$ & $2.50 \mathrm{E}+03$ \\
\hline T-204 & Percent Spike Recovery & 86.3 & 83.3 \\
\hline
\end{tabular}

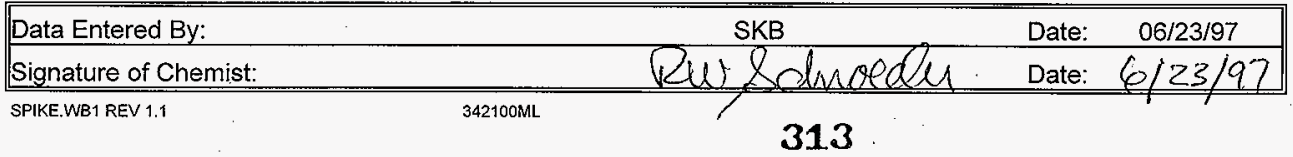


HAF-SO-WAA-DP-245, REV.0 0 intr, 74

TIC- TOTAL INORGANIC CARBON ANALYSIS REPORT

TICTOC REV 2.0

$\ll$ BLANK ANALYSIS $\gg$ HNF-SD-WM-DP-255, REV. 0

Sample: BASEl

Sample Size $=1 \mathrm{uL}$

Dil Factor $=1$

Blank ID \# = BASE 1

Blank Value $=\mathrm{N} / \mathrm{A}$
Date: $06 / 20 / 97$

Time: $21: 33: 21$

$$
\begin{array}{ll}
\text { Analyst : } & \text { KR MONTEITH } \\
\text { Min Readings }= & 22 \\
\text { Max Readings }= & 22 \\
\% \text { Difference }= & 10
\end{array}
$$

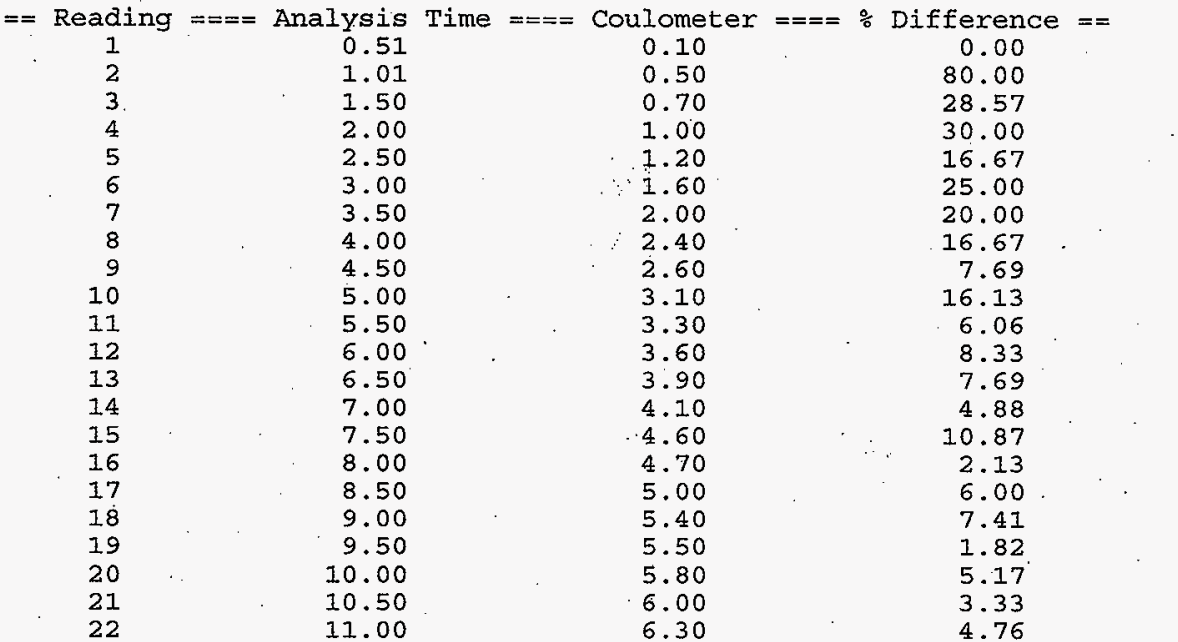

BLANK VAIUE $=6.3 \mathrm{micrograms}$ carbon

BLANK FACTOR $=6.3 / 10.99768=$

$+5.7 E-0 I \quad u g / m i n$ Carbon

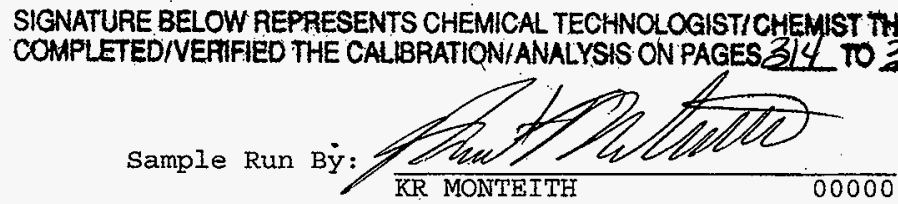


TOC- TOTAL OREAANIC CARBON ANALYSIS REPORT

\section{$\ll$ BLANK ANALYSIS $\gg$ HNF-SD-WM-DP-255, REV. 0}

Sample: BASE1

$$
\begin{aligned}
& \text { Sample Size }=1 \mathrm{uL} \\
& \text { Dil Factor }=1 \\
& \text { Blank ID \# }=\text { BASE1 } \\
& \text { Blank Value }=\mathrm{N} / \mathrm{A} .
\end{aligned}
$$

Date: $06 / 20 / 97$

$==$ Reading $====$ Analysis Til

$\begin{array}{rr}1 & 0.51 \\ 2 & 1.01 \\ 3 & 1.51 \\ 4 & 2.01 \\ 5 & 2.51 \\ 6 & 3.00 \\ 7 & 3.50 \\ 8 & 4.00 \\ 9 & 4.50 \\ 10 & 5.00 \\ 11 & 5.50 \\ 12 & 5.00 \\ 13 & 6.50 \\ 14 & 7.00 \\ 15 & 7.50 \\ 16 & 8.00 \\ 17 & 8.50 \\ 18 & 9.00 \\ 19 & 9.50 \\ 20 & 10.00 \\ 21 & 10.50 \\ 22 & 11.00\end{array}$

Time: $21: 46: 33$

$$
\begin{aligned}
& \text { Analyst : } \quad \text { KR MONTEITH } \\
& \text { Min Readings }=22 \\
& \text { Max Readings }=22 \\
& \% \text { Difference }=10
\end{aligned}
$$

Time ==== Coulometer $====\%$ Difference $==$

$\begin{array}{rr}: 0.40 & 0.00 \\ 0.80 & 50.00 \\ 1.20 & 33.33 \\ 5.00 & 76.00 \\ 12.00 & 58.33 \\ 18.00 & 33.33 \\ 22.00 & 18.18 \\ 24.40 & 9.84 \\ 25.70 & 5.06 \\ 26.60 & 3.38 \\ 27.20 & 2.21 \\ 27.70 & 1.81 \\ 28.20 & 1.77 \\ 28.70 & 1.74 \\ 29.10 & 1.37 \\ 29.40 & 1.02 \\ 29.80 & 1.34 \\ 30.10 & 1.00 \\ 30.50 & 1.31 \\ 30.80 & 0.97 \\ 31.10 & 0.96 \\ 31.40 & 0.96\end{array}$

BLANKK VALUE $=31.4$ micrograms carbon

BLANK FACTOR $=3 i: 4 / 10.99878=+2.85 \mathrm{E}+00$ ug $/ \mathrm{min}$ Carbon $\ll$ WARNING - BLANK VALUE EXCEEDS $1.5 \mathrm{ug} / \mathrm{min}$ Carbon!!!!! $\gg>$ 
HAF-SD-WMAPP-245, REV.QMTE

TIC- TOTAL INORGANIC CARBON ANALYSIS REPORT

TICTOC REV 2.0

HNF-SD-WM-DP-255, REV. 0

Sample: STD1

Date: $06 / 20 / 97$

Time: $22: 17: 21$

Sample Size $=1000$ uL

Dil Factor $=1$

Analyst : $\quad \mathrm{KR}$ MONTEITH

Blank ID \# =

Blank Value $=.57 \mathrm{ug} / \mathrm{minute} \mathrm{C}$ Min Readings $=22$

Max Readings $=22$

$\div$ Difference $=10$

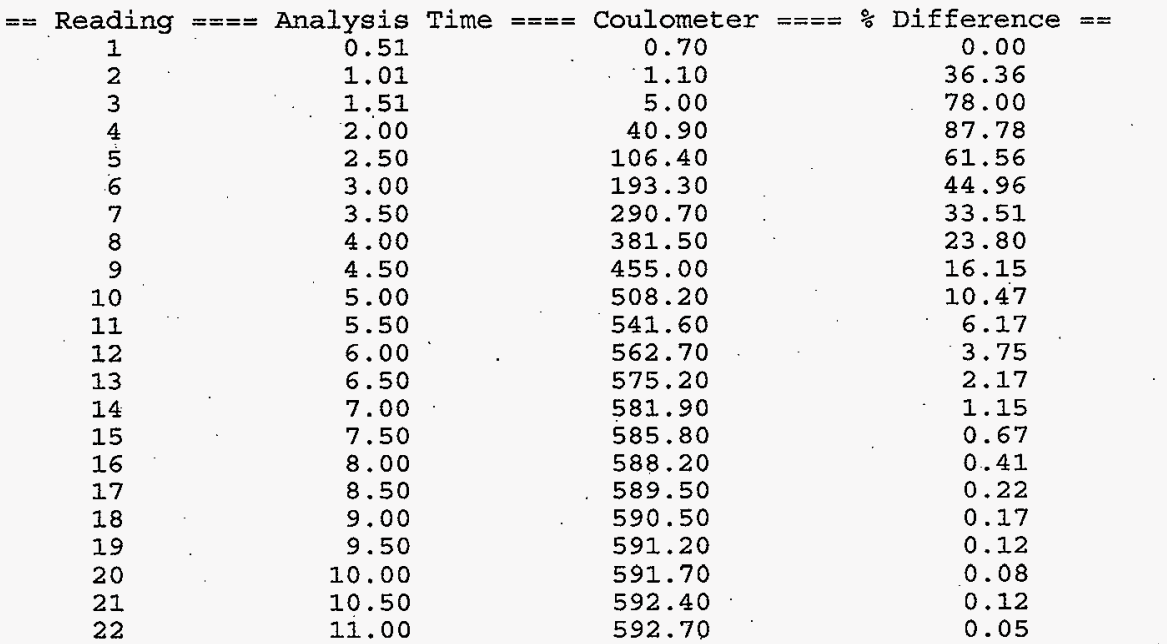

USER INPUT BLANK VALUE

BLANK VALUE $=6.269304$ micrograms carbon

BLANK FACTOR $=6.269304 / 10.99878=+5.7 \mathrm{E}-01 \mathrm{ug} / \mathrm{min}$ Caxbon

SAMPLE RESULTS :

$(592.7-6.268817)(1) /(1000)=$

$(592.7-6.268817)(1) /(1000)(12)=$

$+5.864 \mathrm{E}-01$

$+4.887 \mathrm{E}-02$
g/L Carbon

Molar Carbon

Sample Run Bÿ: 


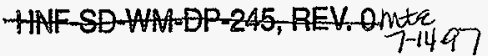

TOC- TOTAL ORGANIC CARBON ANALYSIS REPORT

TICTOC REV 2.0

HNF-SD-WM-DP-255, REV. 0
Sample: STD1

Sample Size $=200 \mathrm{uI}$

Dil Factor $=1$

Blank ID \# =

Blank Value $=2.85 \mathrm{ug} / \mathrm{minute} \mathrm{C}$
Time: $22: 30: 30$

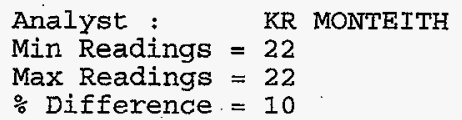

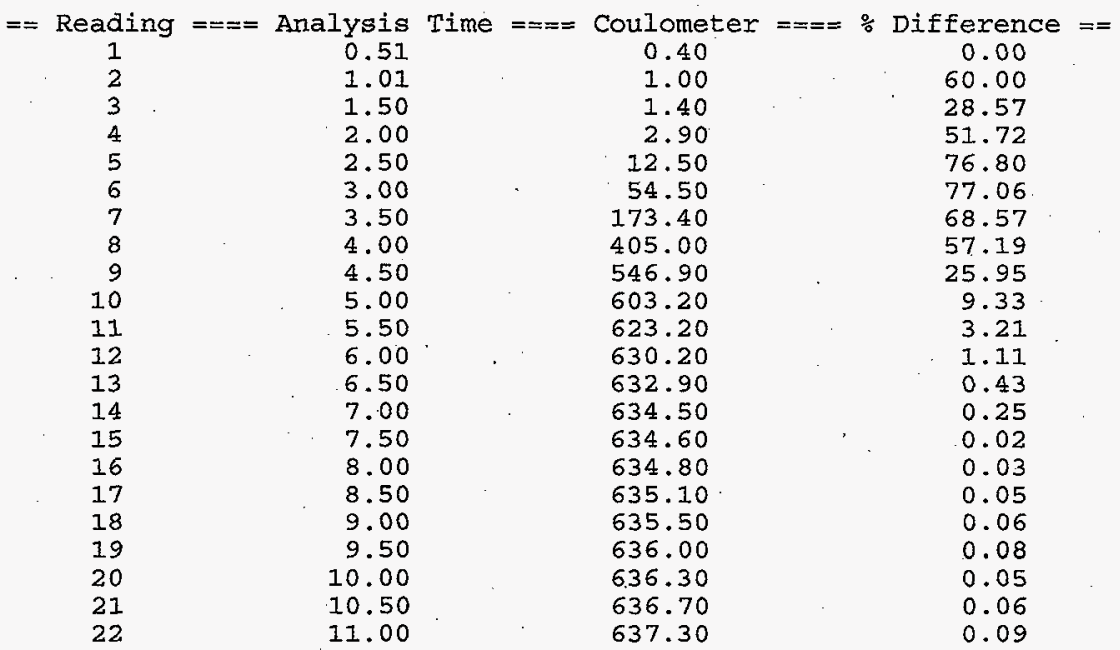

USER INPUT BLANK VALUE

BLANK VALUE $=31.34652$ micrograms carbon

BLANK FACTOR $=31.34652 / 10.99878=+2.8 \mathrm{E}+00 \quad \mathrm{ug} / \mathrm{min}$ Carbon

SAMPLE RESULTS :

$\left\{\begin{array}{l}637.3-31.34408)(1) /(200) \\ 637.3-31.34408)(1) /(200)(12)=\end{array}\right.$

$\ll<$ WARNING - BLANK VALUE EXCEEDS $1.5 \mathrm{ug} / \mathrm{min}$ Carbon!!!!! $\gg>>$

$+3.030 \mathrm{E}+00 \mathrm{~g} / \mathrm{L}$ Carbon

$+2.525 \mathrm{E}-01$ Molar.Carbon

Sample Run By: 


\section{HAE-SD-WM-DP-245, REV.0Mt $7-44-97$ \\ TIC- TOTAL INORGANIC CARBON ANALYSIS REPORT \\ TICTOC REV 2.0 \\ HNF-SD-WM-DP-255, REV. 0}

Sample: BLK1

Sample Size $=1 \mathrm{uL}$
Dil Factor $=1$
Blank ID $=$
Blank Value $=.57 \mathrm{ug} /$ minute $\mathrm{C}$

$==$ Reading $===$ Analysis Time $====$ Coulometer $====\%$ Difference $==$

$\begin{array}{rrrr}1 & 0.51 & 0.80 & 0.00 \\ 2 & 1.01 & 1.40 & 42.86 \\ 3 & 1.51 & 1.90 & 26.32 \\ 4 & 2.00 & 2.40 & 20.83 \\ 5 & 2.50 & 2.90 & 17.24 \\ 6 & 3.00 & 3.50 & 17.14 \\ 7 & 3.50 & 4.00 & 12.50 \\ 8 & 4.00 & 4.40 & 9.09 \\ 9 & 4.50 & 4.80 & 8.33 \\ 10 & 5.00 & 5.20 & 7.69 \\ 11 & 5.50 & 5.60 & 7.14 \\ 12 & 6.00 & 6.00 & 6.67 \\ 13 & 6.50 & 6.40 & 6.25 \\ 14 & 7.00 & 6.70 & 4.48 \\ 15 & 7.50 & 7.20 & 6.94 \\ 16 & 8.00 & 7.40 & 2.70 \\ 17 & 8.50 & 7.80 & 5.13 \\ 18 & 9.00 & 8.20 & 4.88 \\ 19 & 9.50 & 8.40 & 2.38 \\ 20 & 10.00 & 8.80 & 4.55 \\ 21 & 10.50 & 9.10 & 3.30 \\ 22 & 11.00 & 9.40 & 3.19\end{array}$

USER INPUT BIANK VALUE

BIANK VALUE $=6.269304$ micrograms carbon

BLANK FACTOR $=6.269304 / 10.99878=+5.7 \mathrm{E}-01 . \mathrm{ug} / \mathrm{min}$ Carbon

SAMPLE RESULTS:

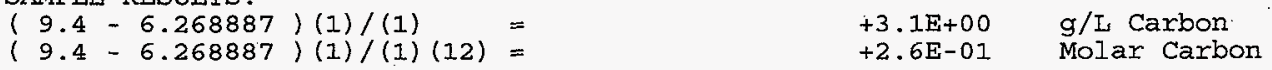

Sample Run Bì:

$\overline{K R}$ MONTEITH 00000


TOC- TOTAL ORGGANIC CARBON ANALYSIS REPORT

Sample: BLK1

Sample Size $=1$ uL

Dil Factor $=1$

Blank ID \# =

Blank Value $=2.85 \mathrm{ug} / \mathrm{minute} \mathrm{C}$

Time: $22: 56: 37$

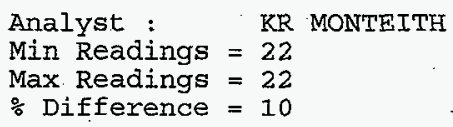

$==$ Reading $====$ Analysis Time $====$ Coulometer $====\%$ Difference $==$

\begin{tabular}{|c|c|c|c|}
\hline$I$ & 0.51 & 0.40 & 0.00 \\
\hline 2 & 1.01 & 0.80 & 50.00 \\
\hline 3 & 1.51 & 1.70 & 52.94 \\
\hline 4 & 2.00 & 5.80 & 70.69 \\
\hline 5 & 2.50 & 10.40 & 44.23 \\
\hline 6 & 3.00 & 15.10 & 31.13 \\
\hline 7 & 3.50 & 18.60 & 18.82 \\
\hline 8 & 4.00 & 21.20 & 12.26 \\
\hline 9 & 4.50 & 22.90 & 7.42 \\
\hline 10 & 5.00 & 24.00 & 4.58 \\
\hline 11 & 5.50 & 24.90 & 3.61 \\
\hline 12 & 6.00 & 25.50 & 2.35 \\
\hline 13 & 6.50 & 26.10 & 2.30 \\
\hline 14 & 7.00 & 26.50 & 1.51 \\
\hline 15 & 7.50 & 27.00 & 1.85 \\
\hline 16 & 8.00 & 27.40 & 1.46 \\
\hline 17 & 8.50 & 27.80 & 1.44 \\
\hline 18 & 9.00 & 28.10 & 1.07 \\
\hline 19 & 9.50 & 28.50 & 1.40 \\
\hline 20 & 10.00 & 28.80 & 1.04 \\
\hline 21 & 10.50 & 29.20 & 1.37 \\
\hline 22 & 11.00 & 29.60 & 1.35 \\
\hline
\end{tabular}

USER INPUT BLANK VALUE

BLANK VALUE $=31.34652$ micrograms carbon

BLANK FACTOR $=31.34652 / 10.99878=+2.8 \mathrm{E}+00 \mathrm{ug} / \mathrm{min}$ Carbon

SAMPLE RESULTS :

$(29.6-31.34652)(1) /(1)=<5.00 \mathrm{E}-3 \mathrm{~g} / \mathrm{I}$ Carbon

$(29.6-31.34652)(1) /(1)(12)=\quad<4.17$ E-4 Molar Carbon

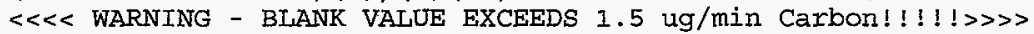

Sample Run By:

KR MONTEITH 00000


Sample: S97T1197-1

Sample Size = 1 uI

Dil Factor $=1$

Blank ID \# =

Blank. Value $=.57 \mathrm{ug} / \mathrm{minute} \mathrm{C}$
Time: $23: 22: 10$

$$
\begin{array}{ll}
\text { Analyst : } & \text { KR MONTEITH } \\
\text { Min Readings }= & 22 \\
\text { Max Readings }= & 22 \\
\% \text { Difference }= & 10
\end{array}
$$

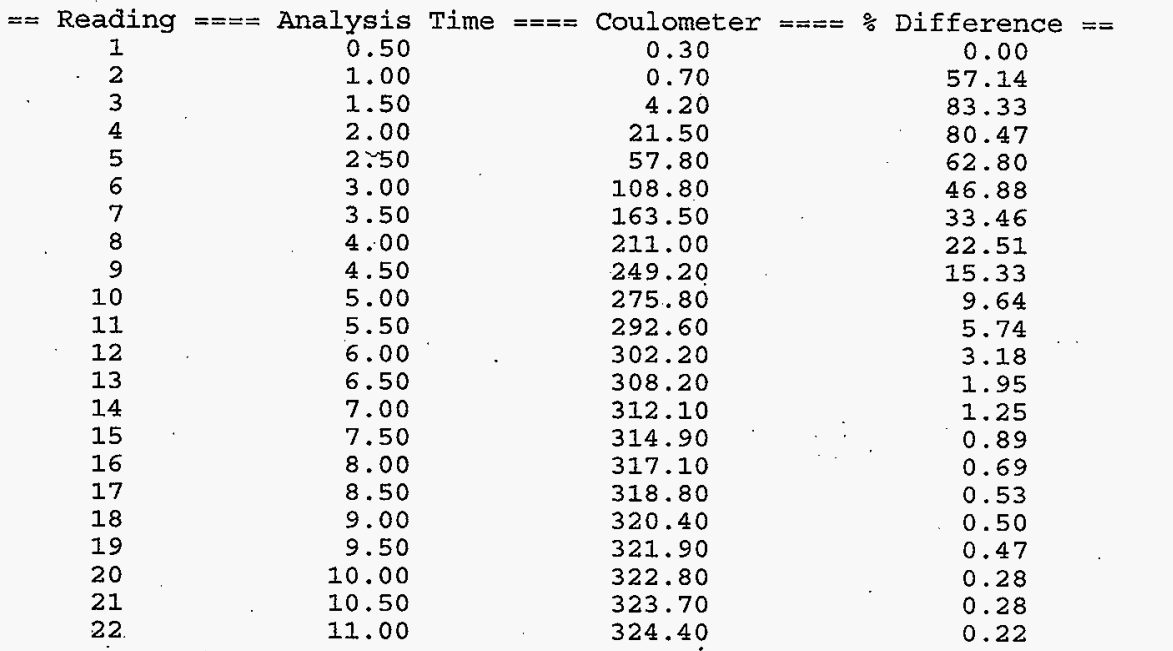

USER INPUT BLANK VALUE

BLANK VALUE $=6.269304$ micrograms carbon

BLANK FACTOR $=6.269304 / 10.99878=$

$+5.7 E-01 \quad u g / m i n$ Carbon

SAMPLE RESULTS:

$\left(\begin{array}{l}324.4-6.26833)(1) /(1) \\ (324.4-6.26833)(1) /(1)(12)=\end{array}\right.$

$+3.181 \mathrm{E}+02 \mathrm{~g} / \mathrm{I}$ Carbon

$+2.651 \mathrm{E}+01$ Molar Carbon

Sample Run Bỳ:

KR MONTEITH

00000 


\section{HNF-SA WAMDP-245, REV.OMTt 7497}

TOC- TOTAL ORGANIC CARBON ANALYSIS REPORT

TICTOC REV 2.0

HNF-SD-WM-DP-255, REV, 0

Sample: S97T1197-1

Date: $06 / 20 / 97$

Time: $23: 34: 17$

Sample Size $=1$ uI

Dil Factor $=1$

Blank ID \# =

Blank Value $=2.85 \mathrm{ug} /$ minute $\mathrm{C}$

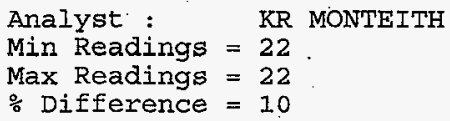

Analyst :

KR MONTEITH

Min Readings $=22$.

Max Readings $=22$

$\%$ Difference $=10$

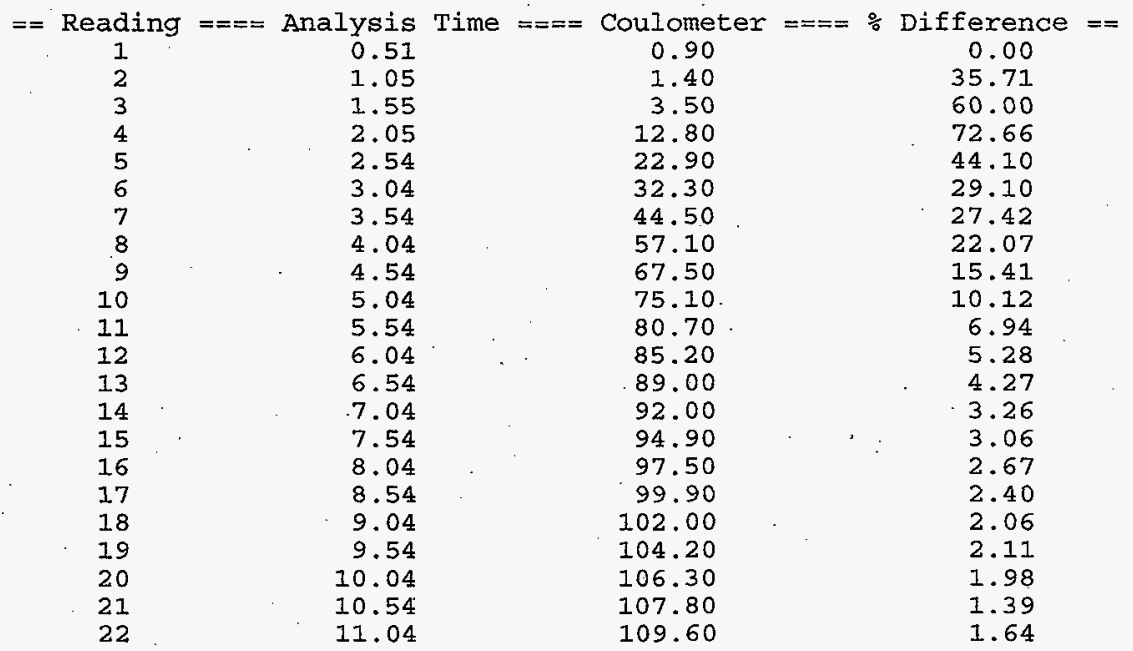

USER INPUT - BT.ANK VALUE

BLANK. VALUE $=31.34652$ micrograms carbon

BLANK FACTOR $=31.34552 / 10.99878=+2.8 \mathrm{E}+00 \quad \mathrm{ug} / \mathrm{min}$ Carbon

SAMPLE RESULTS:

$\left(\begin{array}{l}109.6-31.45854)(1) /(1) \\ 109.6-31.45854)(1) /(1)(12)=\end{array}\right.$

$+7.814 \mathrm{E}+01$

$+6.512 \mathrm{E}+00$

$\ll<$ WARNING - BLANK VAIUE EXCEEDS $1.5 \mathrm{ug} / \mathrm{min}$ Carbon!!!!! $\gg>>$
g/I Carbon

Molar Carbon

Sample Run By:

$\overline{\text { KR MONTEITH }} 000000$




\section{HAF-SD WAA-DP-245, REV ONAE, 7 - 97}

\section{TIC- TOTAL INÖRGANIC CARBON ANALYSIS REPORT}

TICTOC REV 2.0

HNF-SD-WM-DP-255, REV. 0

Sample: S97T1197DUP-1 Date: 06/21/97

Sample Size $=1 \mathrm{uL}$

DiI Factor $=1$

Blank ID \# =

Blank Value $=.57 \mathrm{ug} / \mathrm{minute} \mathrm{C}$
Time: $01: 33: 33$

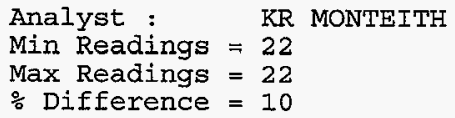

$\begin{array}{crcc}\text { Reading }===\text { Analysis } & \text { Time }===\text { Coulometer }===\% & \text { Difference }== \\ 1 & 0.51 & 0.30 & 0.00 \\ 2 & 1.01 & 0.80 & 62.50 \\ 3 & 1.50 & 2.60 & 69.23 \\ 4 & 2.00 & 13.60 & 80.88 \\ 5 & 2.50 & 41.70 & 67.39 \\ 6 & 3.00 & 83.10 & 49.82 \\ 7 & 3.50 & 132.50 & 37.28 \\ 8 & 4.00 & 183.80 & 27.91 \\ 9 & 4.50 & 232.40 & 20.91 \\ 10 & 5.00 & 273.60 & 15.06 \\ 11 & 5.50 & 305.50 & 10.44 \\ 12 & 6.00 & 331.30 & 7.79 \\ 13 & 6.50 & 352.00 & 5.88 \\ 14 & 7.00 & 369.00 & 4.61 \\ 15 & 7.50 & 382.20 & 3.45 \\ 16 & 8.00 & 392.70 & 2.67 \\ 17 & 8.50 & 400.70 & 2.00 \\ 18 & 9.00 & 407.20 & 1.60 \\ 19 & 9.50 & 411.80 & 1.12 \\ 20 & 10.00 & 415.70 & 0.94 \\ 21 & 10.50 & 419.10 & 0.81 \\ 22 & 11.00 & 421.70 & 0.62\end{array}$

USER INPUT BLANK VAIUE

BI_ANK VALUE $=6.269304$ micrograms carbon

BLANK FACTOR $=6.269304 / 10.99878=$

+5.7E-01 ug/min Carbon

SAMPLE RESULTS:

$(421.7-6.268856)(1) /(1)=$

$(421.7-6.268856)(1) /(1)(12)=$

+4.154E+02 $\mathrm{g} / \mathrm{L}$ Carbon

$+3.462 \mathrm{E}+01$ Molar Carbon

Sample Run By:

$\overline{\text { KR MONTEITH }} 00000$




\section{HAFSOWAAR-245, REV OMt 7 - $-14-97$}

TOC- TOTAL ORGANIC CARBON ANALYSIS REPORT

TICTOC REV 2.0

HNF-SD-WM-DP-255, REV. 0

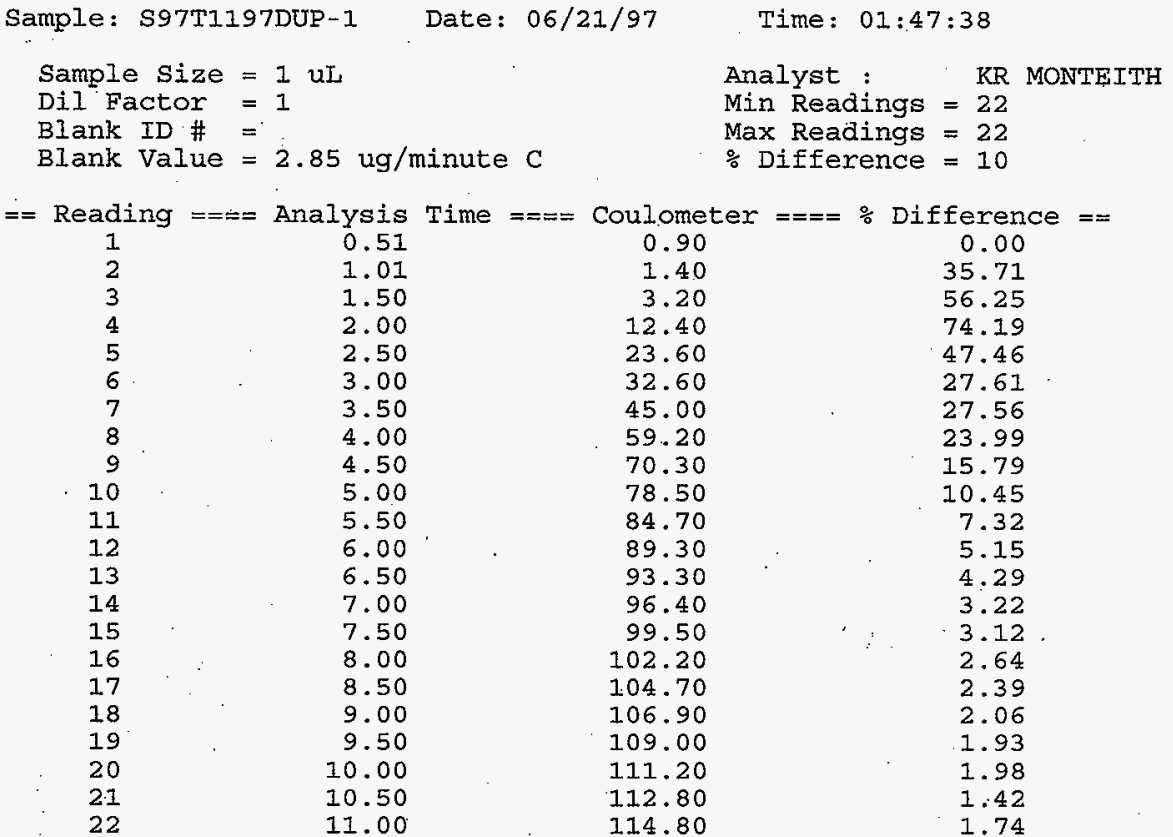

USER INPUT BLANK VAIUEE

BLANK VALUE $=31.34652$ micrograms carbon

BLANK FACTOR $=31.34652 / 10.99878=+2.8 \mathrm{E}+00 \mathrm{ug} / \mathrm{min}$ Carbon

SAMPLE RESULTS :

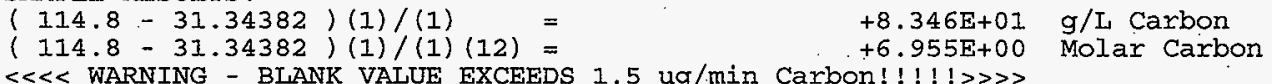

Sample Run By:

KR MONTEITH

00000 


\section{HNF-SD-WAPP-245,REV.Onte -97 \\ TIC- TOTAL INORGANIC CARBON ANALYSIS REPORT \\ TICTOC REV 2.0 \\ HNF-SD-WM-DP-255, REV. 0}

Sample: S97T1197SPK-1 Date: 06/21/97

Time: $02: 41: 21$

Sample Size $=1 \mathrm{uL}$

DiI Factor $=1$

Blank ID \# =

Blank Value $=.57 \mathrm{ug} / \mathrm{minute} \mathrm{C}$

Analyst : . KR MONTEITH

Min Readings $=22$

Max Readings $=22$

Difference $=10$

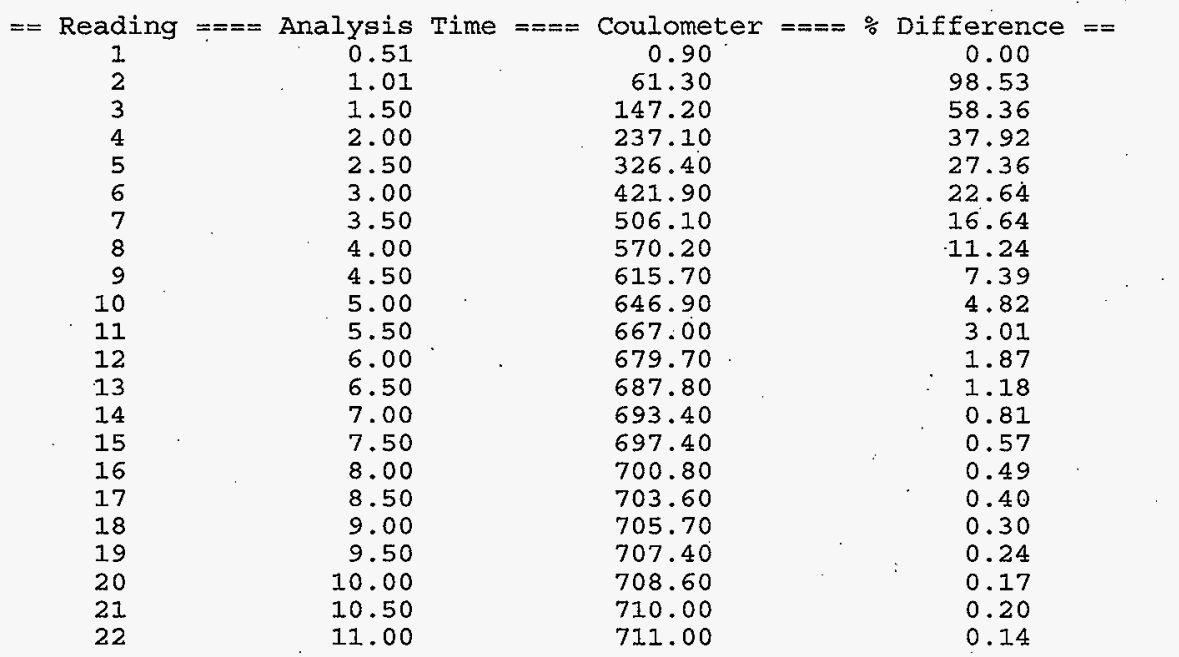

USER INPUT BLANK VALUE

BLLANK VALUE $=6.269304$ micrograms carbon

BLANK FACTOR $=6.269304 / 10.99878=+5.7 \mathrm{E}-01 \mathrm{ug} / \mathrm{min}$ Carbon

SAMPLE RESULTS:

$(711-6.268278)(1) /(1)=$

$+7.047 \mathrm{E}+02 \mathrm{~g} / \mathrm{I}$ Carbon

$(711-6.268278)(1) /(1)(12)=$

$+5.873 \mathrm{E}+01$ Molar Carbon

Sample Run Bÿ:

KR MONTEITH
00000 


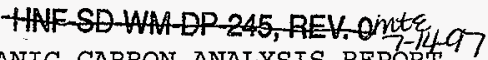 \\ TOC- TOTAL ORGANIC CARBON ANALYSIS REPORT \\ TICTOC REV 2.0 \\ HNF-SD-WM-DP-255, REV. 0}

Sample: S97T1197SPK-1 Date: 06/21/97

Time: $02: 53: 21$

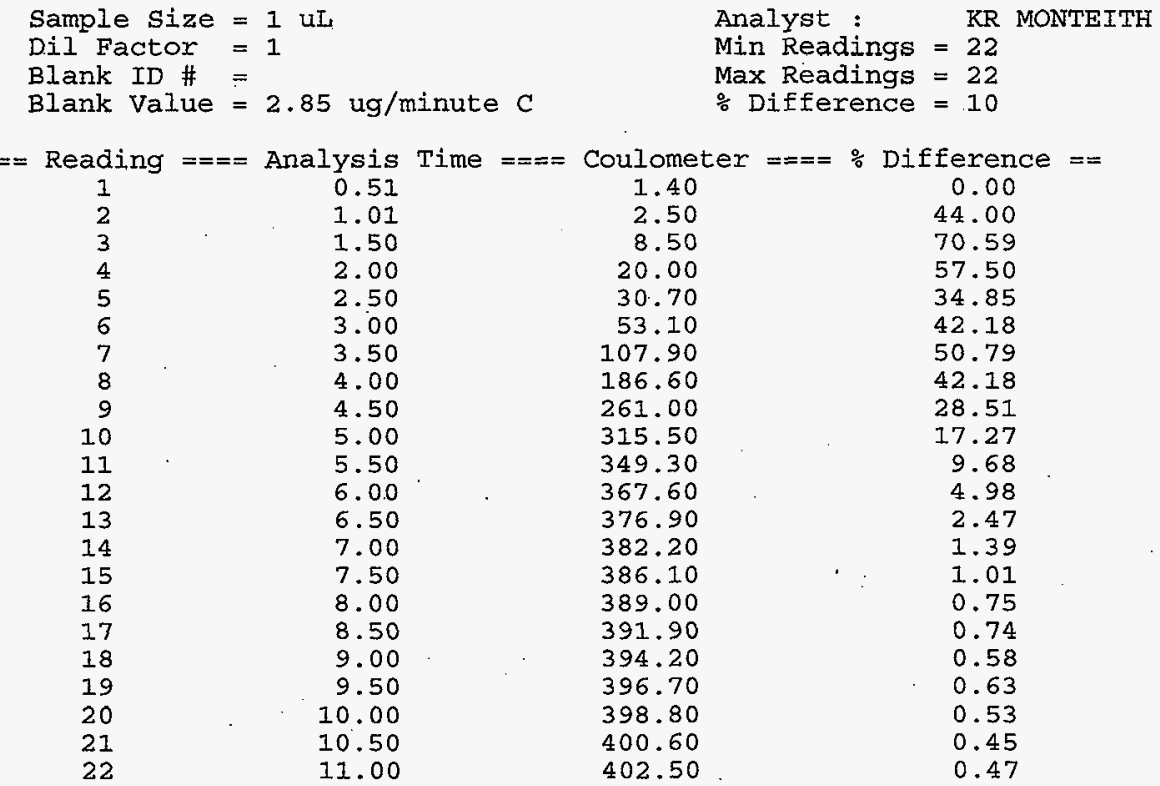

USER INPUT BI.ANK VALUE

BL_ANK VALUE $=31.34652$ micrograms carbon

BLANK FACTOR $=31.34652 / 10.99878=+2.8 \mathrm{E}+00 \quad \mathrm{ug} / \mathrm{min}$ Carbon

SAMPLE RESULTS:
$(402.5-31.34665)(1) /(1)=$
$+3.712 \mathrm{E}+02$
g/L Carbon
$+3.093 E+01$
Molar Carbon

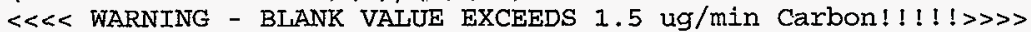

Sample Run By:

KR MONTEITH 00000


HNF-SD-WM-DP-255, REV. O

RADIOCHEMICAL ANALYSES 
HNF-SD-WM-DP-255, REV. 0

THIS PAGE INTENTIONALLY LEFT BLANK 


\section{LABCORE Completed RadChem Report for Worklist\#: 17815}

Analyst: scl

Instrument: $\mathrm{AB} 15$

Book\#

Method:

Rev/Mod

HNF-SD-WM-DP-255, REV. 0

Worklist Comment: T204, @ALPHA01, Deter s.s. by Ludlum. Std: 1.0mL skm

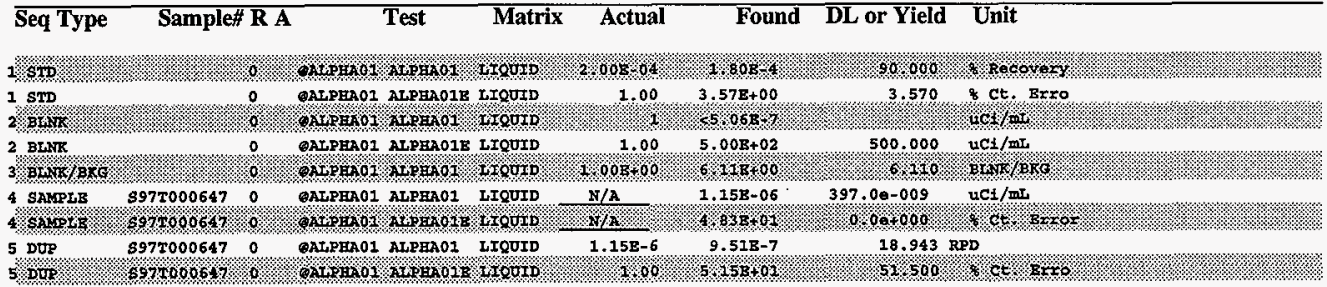

\section{Final page for worklist\# 17815}

Analyst Signature

\section{Date}

Date

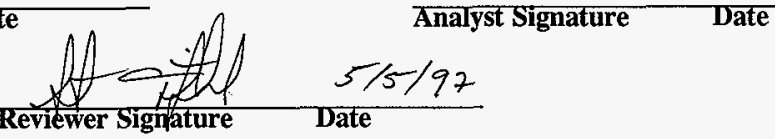

$$
\begin{aligned}
& \text { Blok unlue is rear detection limit, Value } \\
& \text { is aceptable. }
\end{aligned}
$$

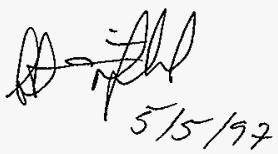

Units shown for $Q C(B L K / B K G)$ may not reflect the actual units. 


\section{LABCORE Data Entry Template for Worklist\# 17815}

Analyst:

S. L Instrument: $\mathrm{AB} 00$

15

Book\# 29B 56

Method: LA-508-101 Rev/Mod_G-0

HNF-SD-WM-DP-255, REV. 0

Worklist Comment: T204, @ALPHA01, Deter s.s. by Ludlum. Std: 1.0mL skm

\begin{tabular}{llll}
\hline Type Sample\# & A Test , Matrix Group\# Project
\end{tabular}

1 STD

@ALPHAO1 LIQUID

2 BLNK

QALPHA01 LIQUID

3 BLNK/BKG

@ALPHAO1 LIQUID

4 SAMPLE

S97T000647 0

@ALPHA01 LIQUID

$97000261 \mathrm{~T}-204$

Analytes Requested: ALPHAO1, ALPHAO1E

5 DUP

$\$ 97 T 000647 \quad 0$

@AI,PHA01 LIQUID

\section{Final page for worklist \# 17815}

$\frac{\mathscr{L}_{x}}{\text { Snalyst Signature }} \quad 5-2-97$

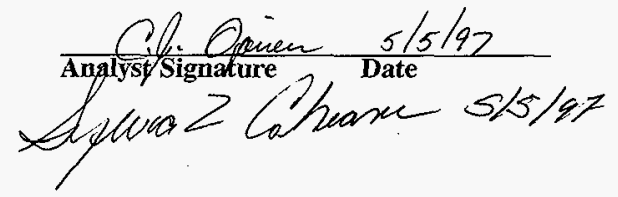

Data Entry Comments:

Boil dowa samplo 
WORKBOOK PAGE: STD1

AT : LA-508-101 (E-1)

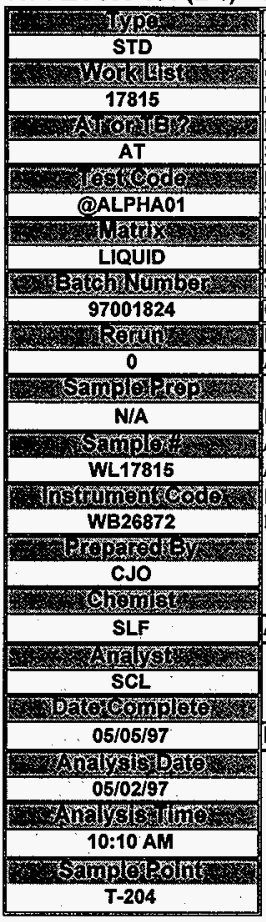

LA-508-113 (B-0) STANDARD

DETECTOR NUMBER

DISH SIZE $\quad(1,2$, or 5$)$

GROSS COUNTS

COUNT TIME in MINUTES

BACKGROUND in cpm

SAMPLE SIZE in $\mathrm{mL}$

DILUTION FACTOR

STANDARD BOOK NUMBER

EFFICIENCY FACTOR

Lc, Rmax, or Rs,(SAMPLE RATE) as APPROPRIATE

Standard Value in $\mu \mathrm{Ci} / \mathrm{mL}$

Concentration in $\mu \mathrm{Ci} / L$

Replicate Concentration in $\mu \mathrm{Ci} / \mathrm{L}$

AVERAGE CONCENTRATION in $\mu \mathrm{Ci} / L$

Rs (Sample Count Rate) $=(\mathrm{TC} / \mathrm{CT})-\mathrm{BKG}$

ALPHA TOTAL $\mu \mathrm{Ci} / \mathrm{L}=\mathrm{Rs} * 1000 \mathrm{~mL} /{ }^{*} \mathrm{DF} /(\mathrm{EFF} * \mathrm{SS} * 2220000 \mathrm{dpm} / \mu \mathrm{Ci}$ )

ALPHA TOTAL $\mu \mathrm{Ci} / \mathrm{mL}=$ ALPHA TOTAL $\mu \mathrm{Ci} / \mathrm{L} / 1000 \mathrm{~mL} /$

Relative Counting Error $=[\mid(T$ The Square Root of TC + BKG * CT) $/($ TC - BKG * CT) $] * 1.96$ * 100

Detection Levels and Less Than Values are determined from Procedure LA-508-002.

\begin{tabular}{|c|c|c|c|}
\hline \multirow[t]{3}{*}{ ALPHA TOTAL CONCENTRATION in $\mu \mathrm{Ci} / \mathrm{mL}$} & $=$ & $1.80 \mathrm{E}-04$ & \multirow{2}{*}{$\begin{array}{c}\text { DETECTION } \\
\text { LEVEL }\end{array}$} \\
\hline & & & \\
\hline & & & 3.97E-07 \\
\hline RELATIVE COUNTING ERROR & $\equiv$ & $3.6 \%$ & $\mu \mathrm{Ci} / \mathrm{mL}$ \\
\hline
\end{tabular}

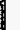

Analyst:

Signature of Chemist:

STANDARD.WB1 Rev. 1.0

\section{장}


HNF-SD-WM-DP-255, REV. 0

WORKBOOK PAGE: BLANK2

HNF-SOWA-DP-245,REV, ON 7 - $14-97$

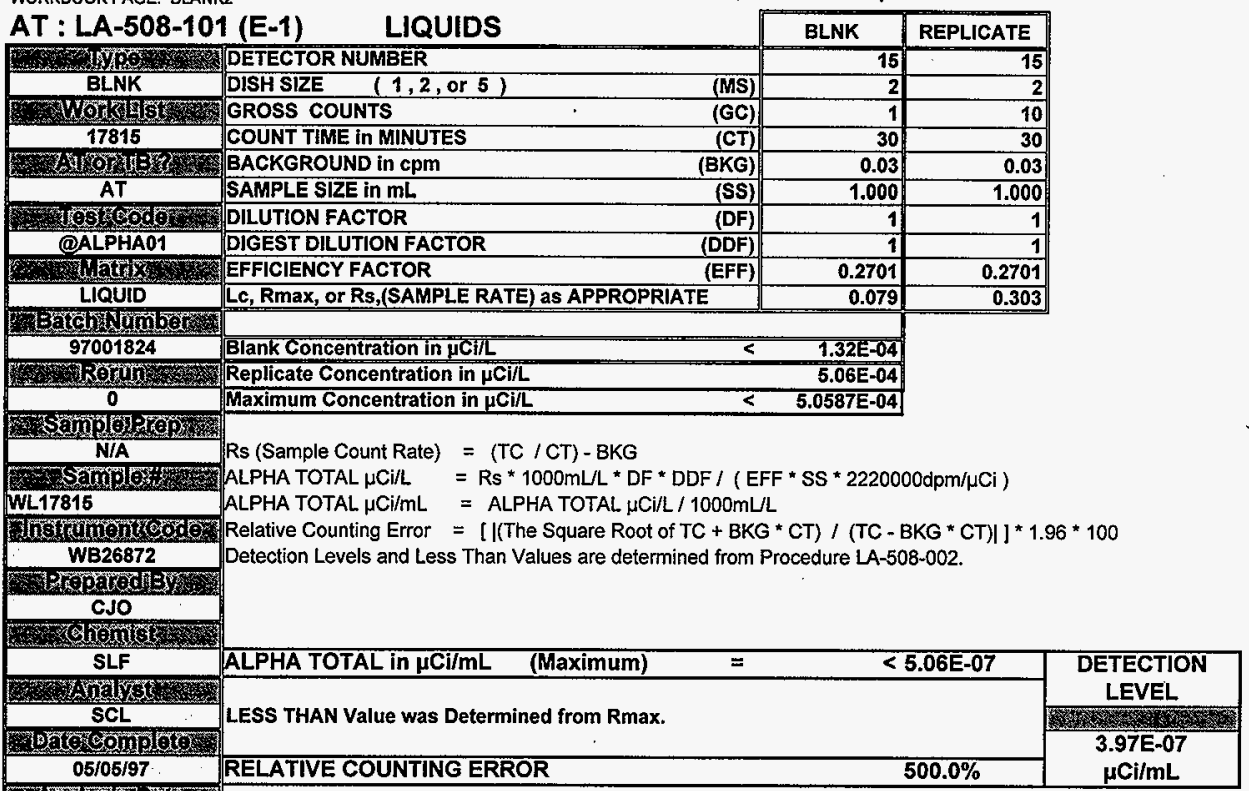

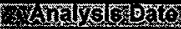
$05 / 02 / 97$

and 1 s 3 uno 10:10 AM

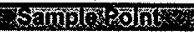
T-204

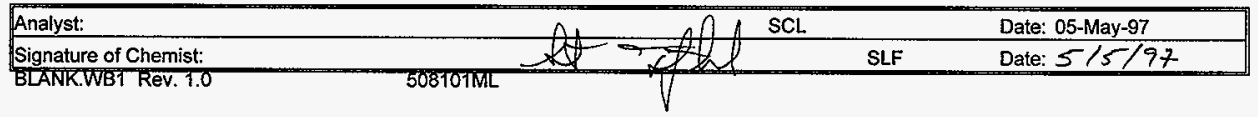


HNF-SD-WM-DP-255, REV. 0

HNF-SD-WAA-DP-245,REV-OntE $7-14-a 7$

WORKBOOK PAGE: SAM4

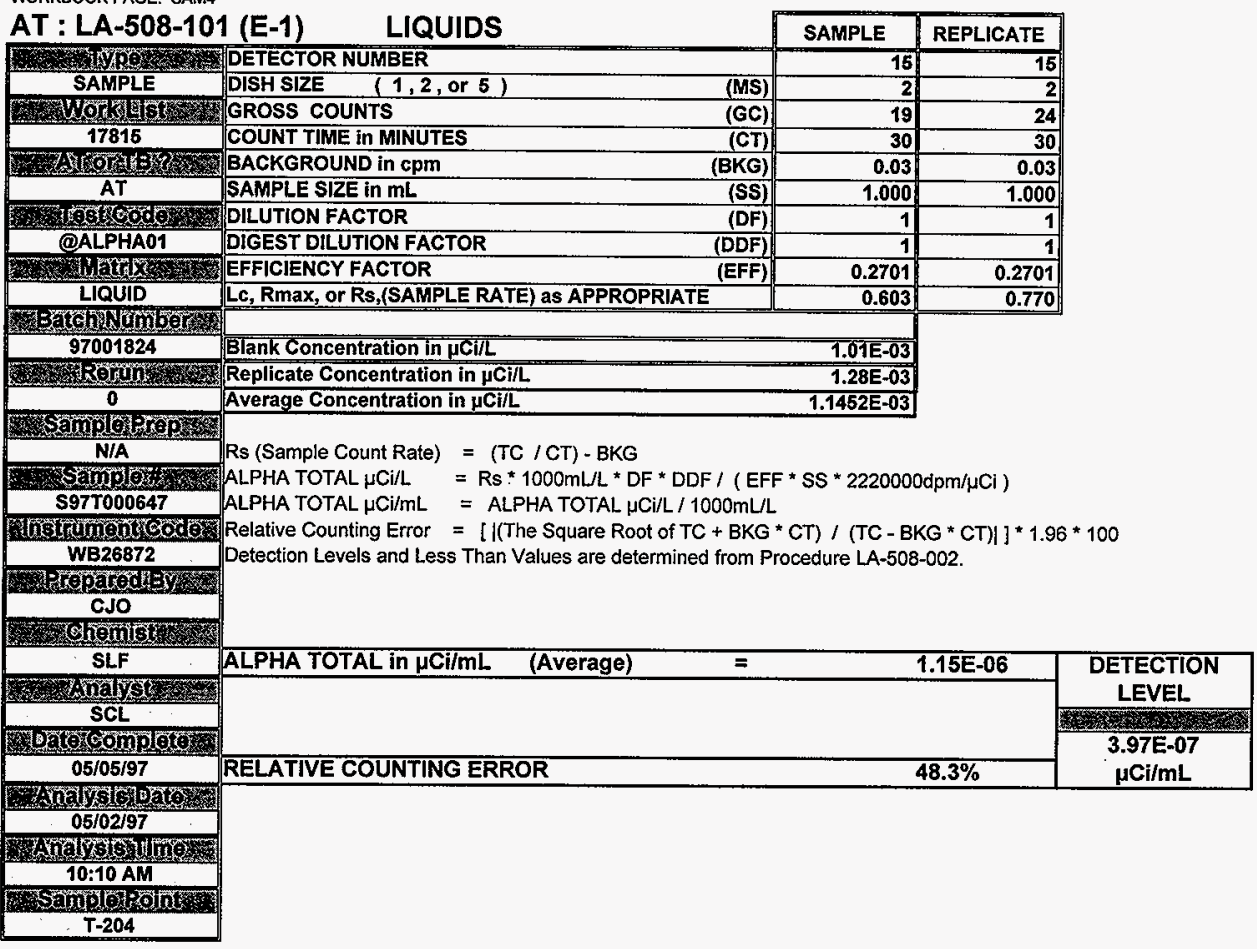

\begin{tabular}{llll} 
Analyst: & Signature of Chemist: \\
\hline SAMPLE.WB1 Rev. 1.0 & 508101ML
\end{tabular}


HNF-SD-WM-DP-255, REV. 0

HIF-SD-WAT-OP-245,REV-OMUE,

WORKBOOKPAGE: DUP5

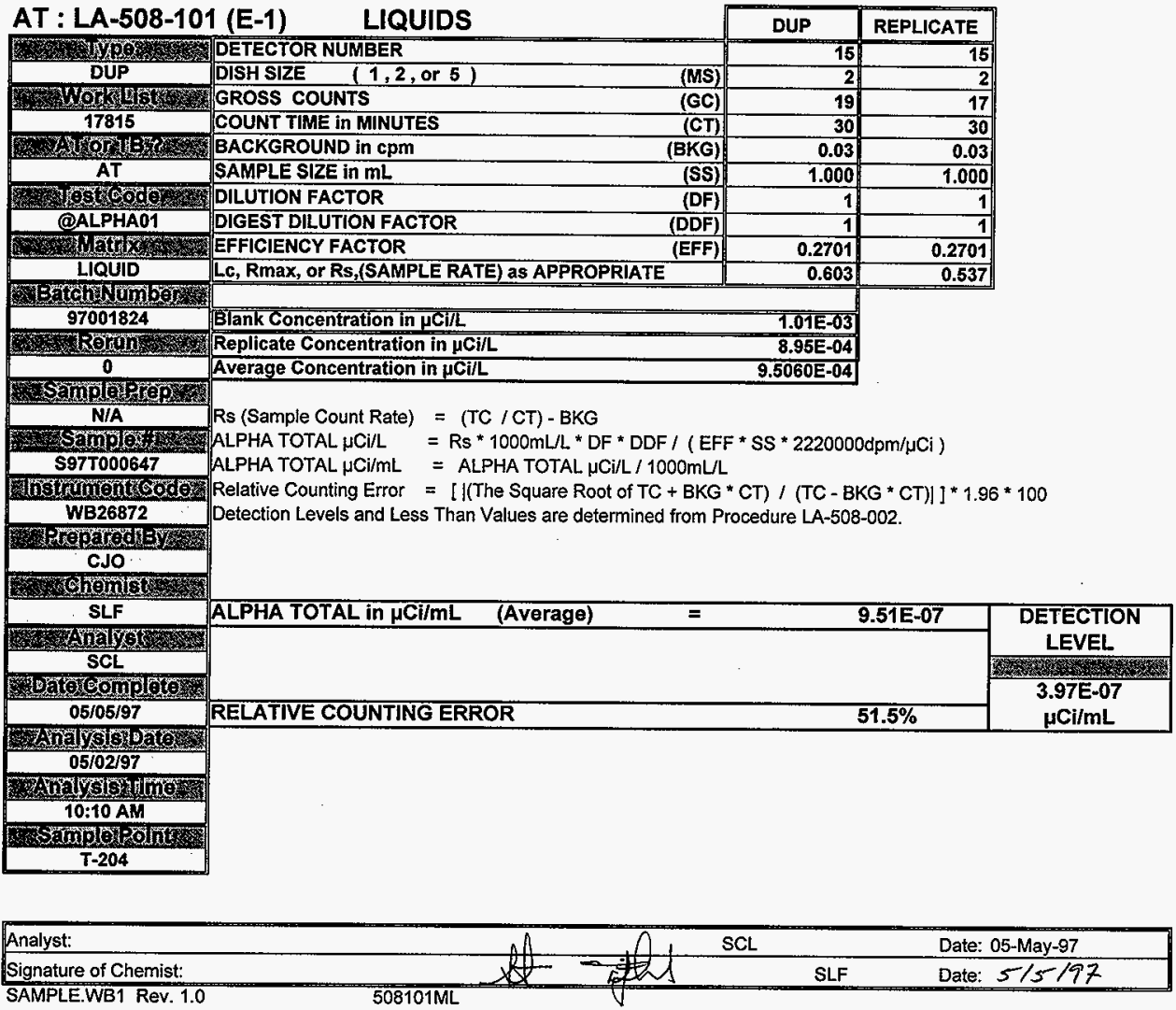




\section{LABCORE Completed RadChem Report for Worklist\#: 17817}

Analyst: adp

Method:
Instrument: $\mathrm{AB} 14$

Rev/Mod
Book\#

HNF-SD-WM-DP-255, REV. 0

Worklist Comment: T204, @ALPHA01, Deter s.s. by Ludlum. Std: $1.0 \mathrm{~mL}$ skm.

\begin{tabular}{lllll}
\hline Seq Type & Sample\# R A & Test & Matrix Actual Found DL or Yield Unit
\end{tabular}

\begin{tabular}{|c|c|c|c|c|c|}
\hline 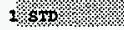 & & $\alpha$ & $\alpha+2,100$ & 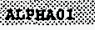 & solst \\
\hline 1 STD & & 0 & QAYPFAO1 & O1B & SOLI \\
\hline 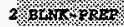 & & 0 & $01 \%$ & or & oh \\
\hline 2 BIMR-PREP & & 0 & OAEPFAO1 & ALPE & sour \\
\hline 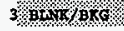 & & \% & 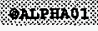 & AIPAAO & 801 \\
\hline 4 SAMLE & 8975000590 & $O F$ & AAPHAO1 & ALFEAO1 & SOLT \\
\hline $48 x_{4}$ & $27005=0$ & $0 \%$ & $4 p_{10}$ & $4+2=11$ & som \\
\hline DUP & $\$ 977000590$ & $0 F$ & ALPHAO1 & AIIPE & SOIT \\
\hline 5\%OP & 597000590 & $0 \%$ & 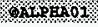 & 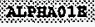 & $801-1$ \\
\hline SPK & s977000590 & Q. $:$ & QALPFAO2 & $A 2 P E$ PO2 & so: \\
\hline 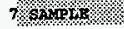 & 39700052 & 0. & 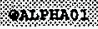 & $\times 12=401$ & 808 \\
\hline SAYPLE & s97T000621 & 0.7 & OAIPAAO1 & AUPHAO 18 & soly \\
\hline 820 & 394500621 & 0 & 08 & ALP & sor \\
\hline 8 DUP & $597 T 000621$ & $0 F$ & PFAO1 & ALPHA 012 & SoLI \\
\hline 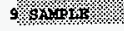 & 8978000622 & $0 \%$ & fol & 41 & sol \\
\hline SAMpLB & 5971000622 & 0.7 & OALPAAO1 & ALPHAO18 & sotI \\
\hline ou & & $0 \%$ & $\triangle A-1=A \gamma$ & $4128+1$ & 04 \\
\hline & & & & 01 & th \\
\hline
\end{tabular}

\begin{tabular}{|c|c|}
\hline 400804 & $1 / 18$ - \\
\hline 1.00 & $3,65 \mathrm{~g}+00$ \\
\hline & $2 / 518 \times$ \\
\hline 1.00 & $1,43 \mathrm{~B}+02$ \\
\hline $1 / 005000$ & $5 / 71=00$ \\
\hline $\mathbb{N} / \mathrm{A}$ & $1,38 \mathrm{~B}-01$ \\
\hline M & $868 \mathrm{~B}+00$ \\
\hline $1.38 \mathrm{~B}-1$ & $1.36 \mathrm{E}-1$ \\
\hline 100 & $8.94 \mathrm{~B}+00$ \\
\hline $2.633-02$ & $2.048-02$ \\
\hline s/a & 1427801 \\
\hline $\mathbb{N} / \mathrm{A}$ & $9.10 \mathrm{~B}+00$ \\
\hline $1278-2$ & 1651 \\
\hline 1.00 & $7.92 \mathrm{~B}+00$ \\
\hline Nto & 424001 \\
\hline $\mathrm{N} / \mathrm{A}$ & $9.06 \mathrm{~B}+00$ \\
\hline $1 / 2921$ & 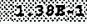 \\
\hline 1.00 & $8.36 \mathrm{z}+00$ \\
\hline
\end{tabular}

\begin{tabular}{|c|c|}
\hline $80<500$ & 2. p. $010 x y$ \\
\hline 3.650 & \& Ct. Exro \\
\hline 25100,004 & act $\%$ \\
\hline 143.000 & uCi/g \\
\hline 8710 & $8 \mathrm{H} / \mathrm{Bro}$ \\
\hline $240.00-005$ & $\mathrm{uCi} / \mathrm{g}$ \\
\hline $60 \% 000$ & 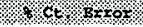 \\
\hline 1.460 & $R P D$ \\
\hline 840 & SctKrys \\
\hline 77.567 & 8 Recovery \\
\hline 24800005 & $4 \times 1 \%$ \\
\hline $0.00+000$ & \& Ct. Error \\
\hline 6027 & Rp \\
\hline 7.920 & \& Ct. Brfo \\
\hline $250 \times 0005$ & and $/ \%$ \\
\hline $0.00+000$ & $8 \mathrm{ct} \cdot \mathrm{zrror}$ \\
\hline $6 \% 2$ & 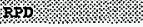 \\
\hline
\end{tabular}

\section{Final page for worklist\# 17817}

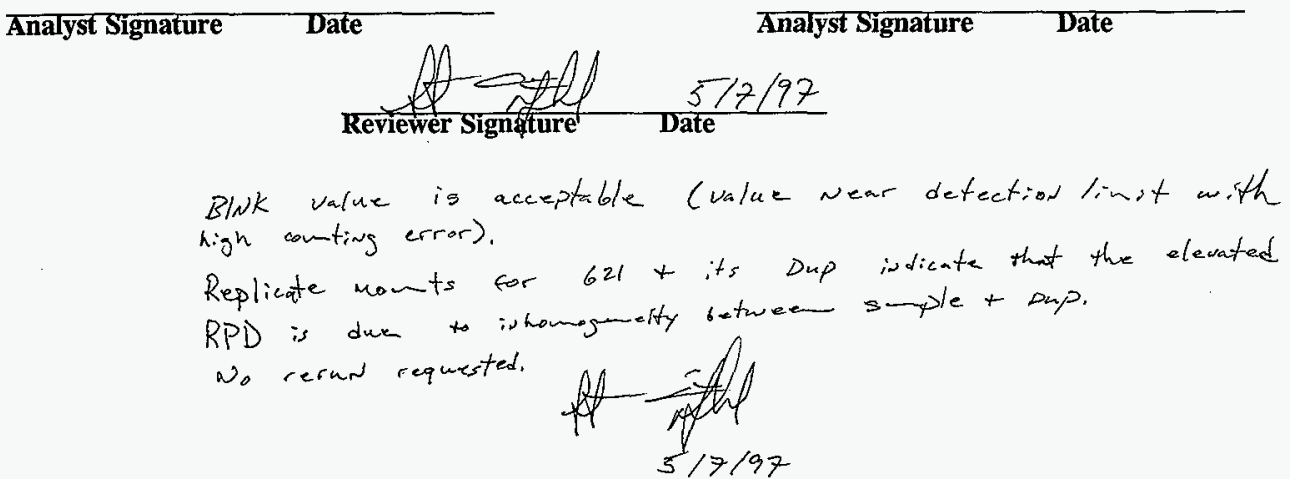

Units shown for $Q \overline{Q C}(B L K / B \bar{K} G)$ may not reflect the actual units. 


\section{LABCORE Data Entry Template for Worklist\# 17817}

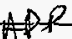

Analyst:

S.L
Instrument: $\mathrm{ABOO}$ 14

Method: LA-508-101 Rev/Mod 6-C

Book\# 79 B 56

HNF-SD-WM-DP-255, REV. 0

Worklist Comment: T204, @ALPHA01, Deter s.s. by Ludlum. Std: 1.0mL skm

S Type Sample\# R A Test Matrix Group\# Project

1 STD

$@$ ALPHAO2 SOLID

2 BLNK-PREP

@ALPHA01 SOLID

$3 B L N \dot{K} / B K G$

@ALPHA01 SOLID

4 SAMPLE

S97T000590 O F OALPHA01 SOLID

Analytes Requested: ALPHAO1, ALPHAO1E

97000261 T-204

5 DUP

S97T000590 O F @ALPHA01 SOLID

$6 \mathrm{SPK}$

S97T000590 O F @ALPHA01 SOLID

7 SAMPLE

S97T000621 O F @ALPHAO1 SOLID

$97000261 \quad T-204$

Analytes Requested: ALPHA01 , ALPHAO1E

8 DUP

S97T000621 $0 F$ @ALPHA01 SOLID

9 SAMPLE

S97T000522 O F @ALPHA01 SOLID

Analytes Requested: ALPHAO1, ALPHAOIE

\section{Final page for worklist \# 17817}
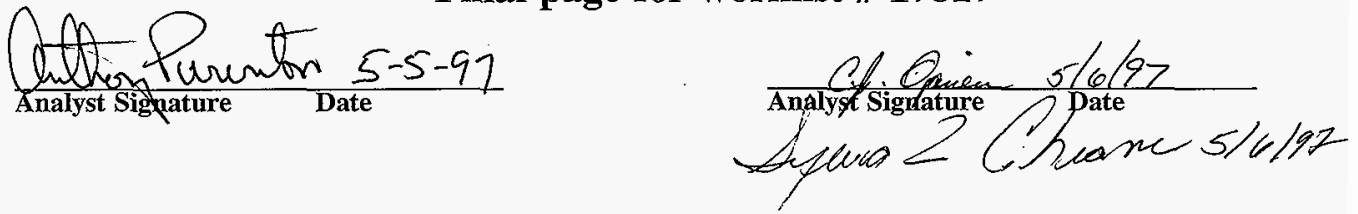

Data Entry Comments:

toil down sanples

spike\# 131843 
HNF-SD-WM-DP-255, REV. 0

HAF-SD-WM-OP-245, REV. $0 M \frac{9 t \varepsilon}{7-14-97}$

WORKBOOK PAGE: STD

AT : LA-508-101 (E-1)

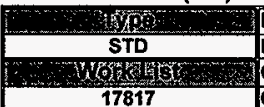

7atrotid

AT

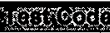

@ALPHA01

irtarts.

LIQUID

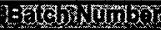
97001826

3

0

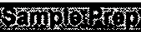

N/A

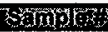

WL17817

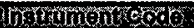
WB27807

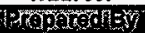
CJO

(6)

SLF

ANTW 8

ADP

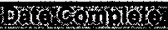
05/06/97

Whar $05 / 05 / 97$

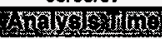
03:30 AM

38. Whe T-204

\begin{tabular}{|c|c|c|}
\hline LA-508-113 (B-0) STANDARD & STANDARD & REPLICATE \\
\hline DETECTOR NUMBER & 14 & 14 \\
\hline$(1,2$, or 5$)$ & 2 & 2 \\
\hline GROSS COUNTS & 2885 & 2904 \\
\hline COUNT TIME in MINUTES & 30 & 30 \\
\hline BACKGROUND in cpm & 0.07 & 0.07 \\
\hline SAMPLE SIZE in $\mathrm{mL}$. & 1.000 & 1.000 \\
\hline DILUTION FACTOR & 1. & 1 \\
\hline STANDARD BOOK NUMBER & 79856 & $79 B 56$ \\
\hline EFFICIENCY FACTOR & 0.2692 & 0.2692 \\
\hline LG, Rmax, or Rs,(SAMPLE RATE) as APPROPRIATE & 96.097 & 96.730 \\
\hline Standard Value in $\mu \mathrm{C} / \mathrm{mL}$ & $2.00 \mathrm{E}-04$ & \\
\hline Concentration in $\mu \mathrm{Ci} / \mathrm{L}$ & 1.61E-01 & \\
\hline Replicate Concentration in $\mu \mathrm{C} i / \mathrm{L}$ & 1.62E-01 & \\
\hline AVERAGE CONCENTRATION in & $1.6133 E-01$ & \\
\hline
\end{tabular}

Rs (Sample Count Rate) $=(\mathrm{TC} / \mathrm{CT})-\mathrm{BKG}$

ALPHA TOTAL $\mu \mathrm{Ci} / \mathrm{L}=\mathrm{Rs} * 1000 \mathrm{~mL} / \mathrm{LF} /($ EFF * $\mathrm{SS} * 2220000 \mathrm{dpm} / \mu \mathrm{Ci})$

ALPHA TOTAL $\mu \mathrm{Ci} / \mathrm{mL}=$ ALPHA TOTAL $\mu \mathrm{CI} / \mathrm{L} / 1000 \mathrm{~mL} / \mathrm{L}$

Relative Counting Error = [|(The Square Root of TC + BKG * CT) / (TC - BKG * CT) $]$ ] $1.96 * 100$

Detection Levels and Less Than Values are determined from Procedure LA-508-002.

\begin{tabular}{|lll|c|}
\hline ALPHA TOTAL CONCENTRATION in $\mu \mathrm{Ci} / \mathrm{mL}$ & $=$ & $1.61 \mathrm{E}-04$ & $\begin{array}{c}\text { DETECTION } \\
\text { LEVEL }\end{array}$ \\
& & & \\
\hline RELATIVE COUNTING ERROR & $=$ & $3.7 \%$ & $\begin{array}{c}5.29 E-07 \\
\mu \mathrm{Ci} / \mathrm{mL}\end{array}$ \\
\hline
\end{tabular}

\begin{tabular}{lllll}
\hline Analyst: & & CJO & Date: 06-May-97 \\
\hline Signature of Chemist: & & SLF & Date: $5 / 2 / 97$ \\
\hline STANDARD.WB1 Rev. 1.0 & $508101 \mathrm{ML}$ & & \\
\hline
\end{tabular}

\section{6}


HNF-SD-WM-DP-255, REV. 0

WORKBOOK PAGE: BLANK2

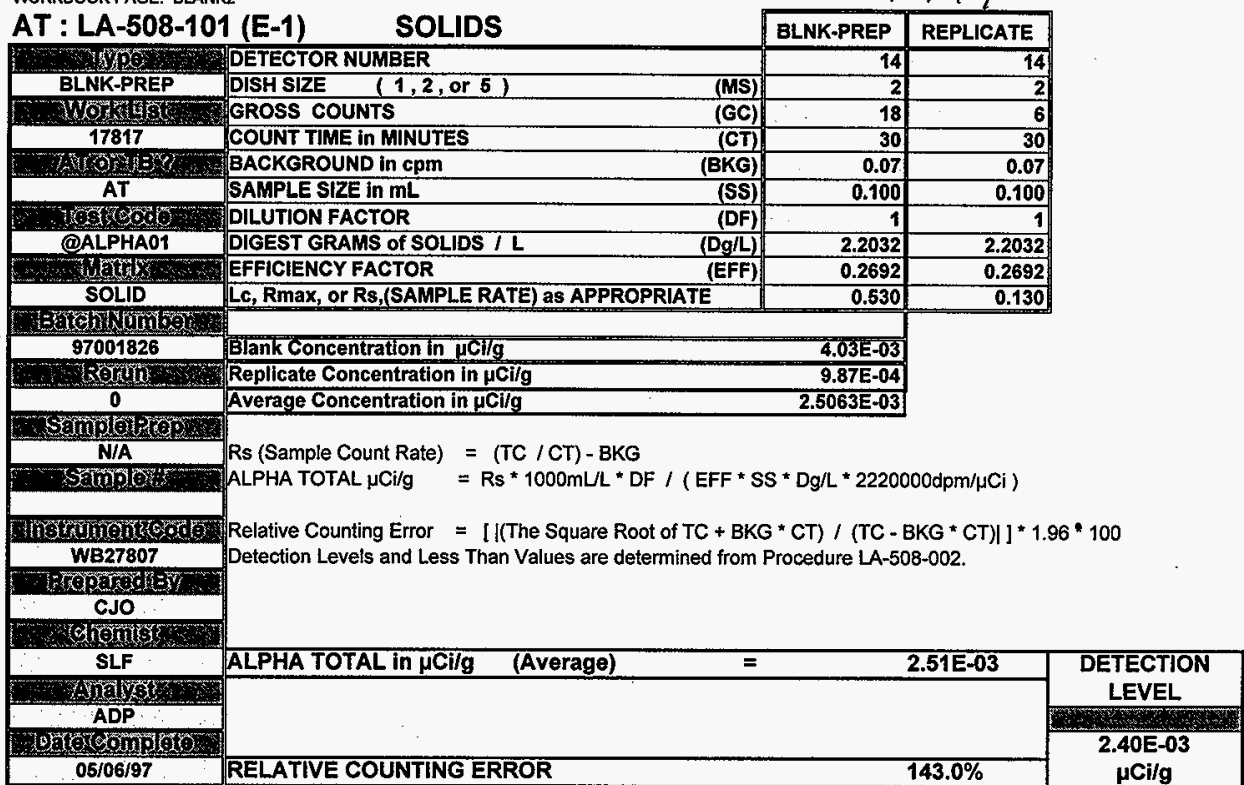

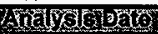
$06 / 05 / 97$.

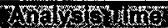
03:30 AM

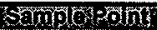
T-204

Analyst:
Signature of Chemist:
BLANK.WBT Rev. T.0


HNF-SD-WM-DP-255, REV. 0

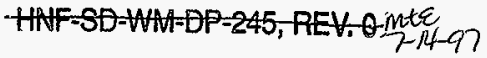

WORKBOOK PAGE: SAM4

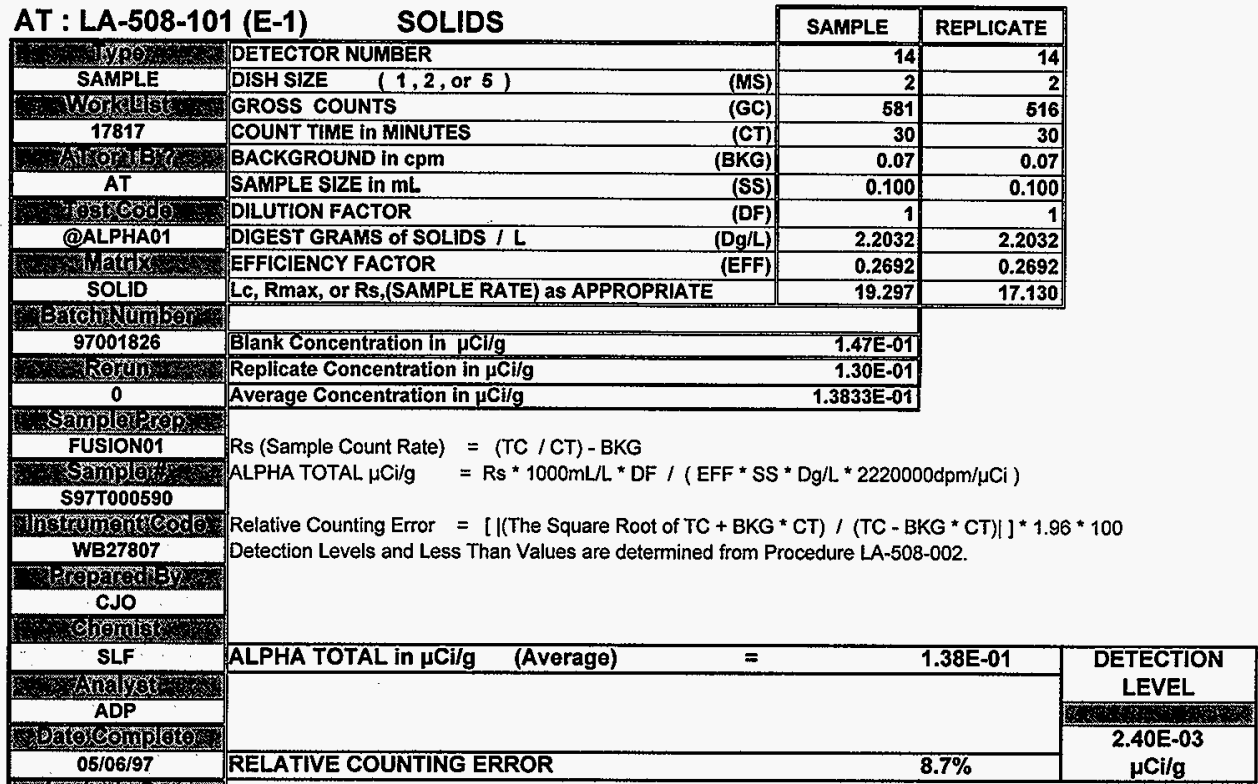

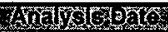
$05 / 05 / 97$

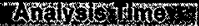
03:30 AM T-204

Analyst:

Signature of Chemist:

SAMPLE.WB1 Rev. 1.0
DETECTOR NUMBER GROSS COUNTS COUNT TLME in MINUTES EFFICIENCY FACTOR

Lc, Rmax, or Rs,(SAMPLE RATE) as APPROPRIATE

Blank Concentration in $\mu \mathrm{Cl} / \mathrm{g}$

1.47E-01

Replicate Concentration in $\boldsymbol{\mu C i / g}$

Rs (Sample Count Rate) $=(T C / C T)-B K G$

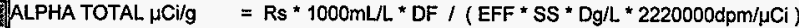

Relative Counting Error $=[$ [(The Square Root of TC + BKG * CT) / (TC - BKG * CT) $]]^{\star} 1.96 * 100$

Detection Levels and Less Than Values are determined from Procedure LA-508-002.

RELATIVE COUNTING ERROR

$\mathbf{8 . 7 \%}$ 
HNF-SD-WM-DP-255, REV. 0

HAF-SD-WAPP-245, REV. Qint-14-97

WORKBOOK PAGE: DUP5

\begin{tabular}{|c|c|c|c|c|}
\hline AT : LA-50 & SOLIDS & & & \\
\hline & DETECTOR NUMBER & Dur & REPLICAIE & \\
\hline DUP & \begin{tabular}{|l|} 
DETECTOR NUMBER \\
DISH SIZE $(1,2$, or 5$)$
\end{tabular} & 14 & 14 & \\
\hline 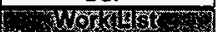 & GROS SIE COUNTS $(1,2$, or 5$)$ & 2 & & \\
\hline 17817 & 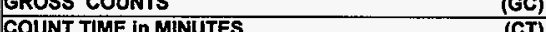 & 487 & 582 & \\
\hline Dox & BACKGROUND in cpm & 0.07 & 0.07 & \\
\hline AT & SAMPLE SIZE in $\mathrm{mL}$ & 0.100 & 0.100 & \\
\hline & DILUTION FACTOR & 1 & 1 & \\
\hline @ALPHA01 & DIGEST GRAMS of SOLIDS / L & 2.1864 & 2.1864 & \\
\hline Whater & EFFICIENCY FACTOR & 0.2692 & 0.2692 & \\
\hline SOLID & LC, Rmax, or RS,(SAMPLE RATE) as APPROPRIATE & 16.163 & 19.330 & \\
\hline 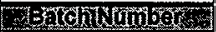 & & & & \\
\hline 97001826 & Blank Concentration in $\mu$ Cig & 1.24E-01 & & \\
\hline Roron & Replicate Concentration in $\mu \mathrm{Ci} / \mathrm{g}$ & $1.48 \mathrm{E}-01$ & & \\
\hline 0 & Average Concentration in $\mu \mathrm{Ci} / \mathrm{g}$ & $1.3582 \mathrm{E}-01$ & & \\
\hline 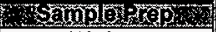 & & & & \\
\hline FUSIONO1 & Rs (Sample Count Rate) & & & \\
\hline Sampirats & $=R s * 1000 \mathrm{~mL} / \mathrm{L} * \mathrm{DF} /(\mathrm{EFF} * \mathrm{Ss}$ & $\mathrm{Dg} / \mathrm{L} * 222000$ & $\mathrm{Odpm} / \mathrm{\mu Ci})$ & \\
\hline $\mathbf{5 9 7 T 0 0 0 5 9 0}$ & & & & \\
\hline astanowenters & |(The Square Root of TC + BK & $\mathrm{CT}) /(\mathrm{TC}-\mathrm{B}$ & $\mathrm{KG} * \mathrm{CT}) \mid \mathrm{J}^{*} 1.96$ & \\
\hline WB27807 & Detection Levels and Less Than Values are determined from $F$ & cedure LA-508 & & \\
\hline 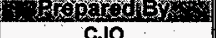 & & & & \\
\hline CJo & & & & \\
\hline SLF & ALPHA TOTAL in $\mu$ Ci/g (Average) & & $1.36 E-01$ & \\
\hline Anas & & & & LEVEL \\
\hline 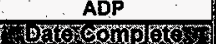 & & & & \\
\hline $05 / 06 / 97$ & RELATIVE COUNTING ERROR & & $8.9 \%$ & $\begin{array}{l}2.42 \mathrm{E}-\mathrm{U} \\
\mu \mathrm{Ci} / \mathrm{g}\end{array}$ \\
\hline
\end{tabular}

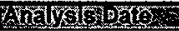
$05 / 05 / 97$.

Whass 03:30 AM

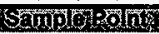
T-204

Analyst:
Signature of Chemist:
SAMPLE.WB1 Rev. 1.0


HNF-SD-WAM-DP-255, REV. 0

WORKBOOKPAGE: SPK6

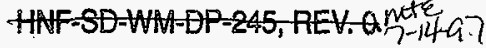

\begin{tabular}{|c|c|c|c|}
\hline \multirow{2}{*}{\multicolumn{2}{|c|}{ AT : LA-508-101 (E-1) LA-508-113 (B-0) SPIKED SAMPLE }} & \multirow[b]{2}{*}{ SPIKE } & \multirow[b]{2}{*}{ REPLICATE } \\
\hline & & & \\
\hline 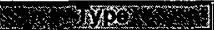 & DETECTOR NUMEER & 14 & 14 \\
\hline SPK & DISH SIZE & 2 & 2 \\
\hline yon & TOTAL COUNTS & 36956 & 37144 \\
\hline 17817 & COUNT TIME in MINUTES & 30 & 30 \\
\hline stortsole & BACKGROUND in cpm & 0.07 & 0.07 \\
\hline AT & SAMPLE VOLUME in $\mathrm{mL} \quad$ (Spiked Vial) & 0.100 & 0.100 \\
\hline Worost & SAMPLE DILUTION FACTOR (Spiked Vial) & 1 & 1 \\
\hline @ALPHA01 & DIGEST GRAMS Of SOLIDS /L & 2.2032 & 2.2032 \\
\hline ula & SPIKE VOLUME in $\mathrm{mL}$ & 0.100 & 0.100 \\
\hline SOLID & SPIKE DILUTION FACTOR & 1 & 1 \\
\hline $\operatorname{ros} 000$ & SPIKE BOOK NUMBER & $131 \mathrm{~B} 43$ & 131843 \\
\hline 97001826 & SPIKE VALUE in $\mu \mathrm{CB} / \mathrm{mL}$ & 2.6295E-02 & 2.6295E-02 \\
\hline (3) & INSTRUMENT EFFICIENCY FACTOR & 0.2692 & 0.2692 \\
\hline 0 & SAMPLE + SPIKE $\mu \mathrm{CI} / \mathrm{g}$ & $9.36 E+00$ & $9.40 E+00$ \\
\hline 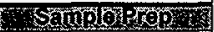 & AVERAGE or MAXIMUM $\mu$ Ci/g in SAMPLE & $1.3833 E-01$ & \\
\hline FUSIONO1 & & & \\
\hline somplom & Rs (Sample Count Rate) & & \\
\hline 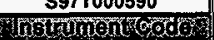 & SAMPLE + SPIKE $\mu \mathrm{Ci} / \mathrm{g}=R \mathrm{~s} * 1000 \mathrm{mLL} * \mathrm{DF} /(\mathrm{EFF} * \mathrm{~S}$ & $* \mathrm{Dg} / \mathrm{L} * 222000$ & $\mathrm{OOdpm} / \mu \mathrm{Ci})$ \\
\hline WB27807 & QC ACTUAL = SVal & & \\
\hline Horowl & QC FOUND $=\left(\left((S+S \mu C i / g-S A M P L E \mu C i / g) *\left(\left(S D F /\left(S V_{0}\right)^{*}\right.\right.\right.\right.$ & $000)) /(D F / S S / D$ & $\lg (L))))$ \\
\hline CiJO & PERCENT SPIKE RECOVERY = (QC FOUND / QC ACTUAL) & 100 & \\
\hline 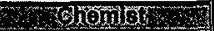 & & & \\
\hline$\because \mathbf{S L F}$ & & & \\
\hline AAnarg & & & \\
\hline ADP & & & \\
\hline 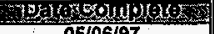 & & & \\
\hline $05 / 06 / 97$ & & & \\
\hline Wors 8 & & & \\
\hline $05 / 05 / 97$ & QC ACTUAL & $2.63 E-02$ & \\
\hline 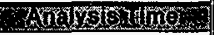 & QCFOUND & 2.04E-02 & \\
\hline $03: 30 \mathrm{AM}$ & AVG. PERCENT SPIKE RECOVERY & $77.4 \%$ & \\
\hline 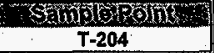 & & & \\
\hline
\end{tabular}

\begin{tabular}{l} 
Analyst: \\
Signature of Chemist: \\
\hline SPIKE.WB1 Rev. 1.0
\end{tabular}


HNF-SD-WM-DP-255, REV. 0

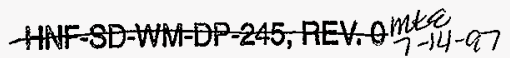

WORKBOOK PAGE: SAM7

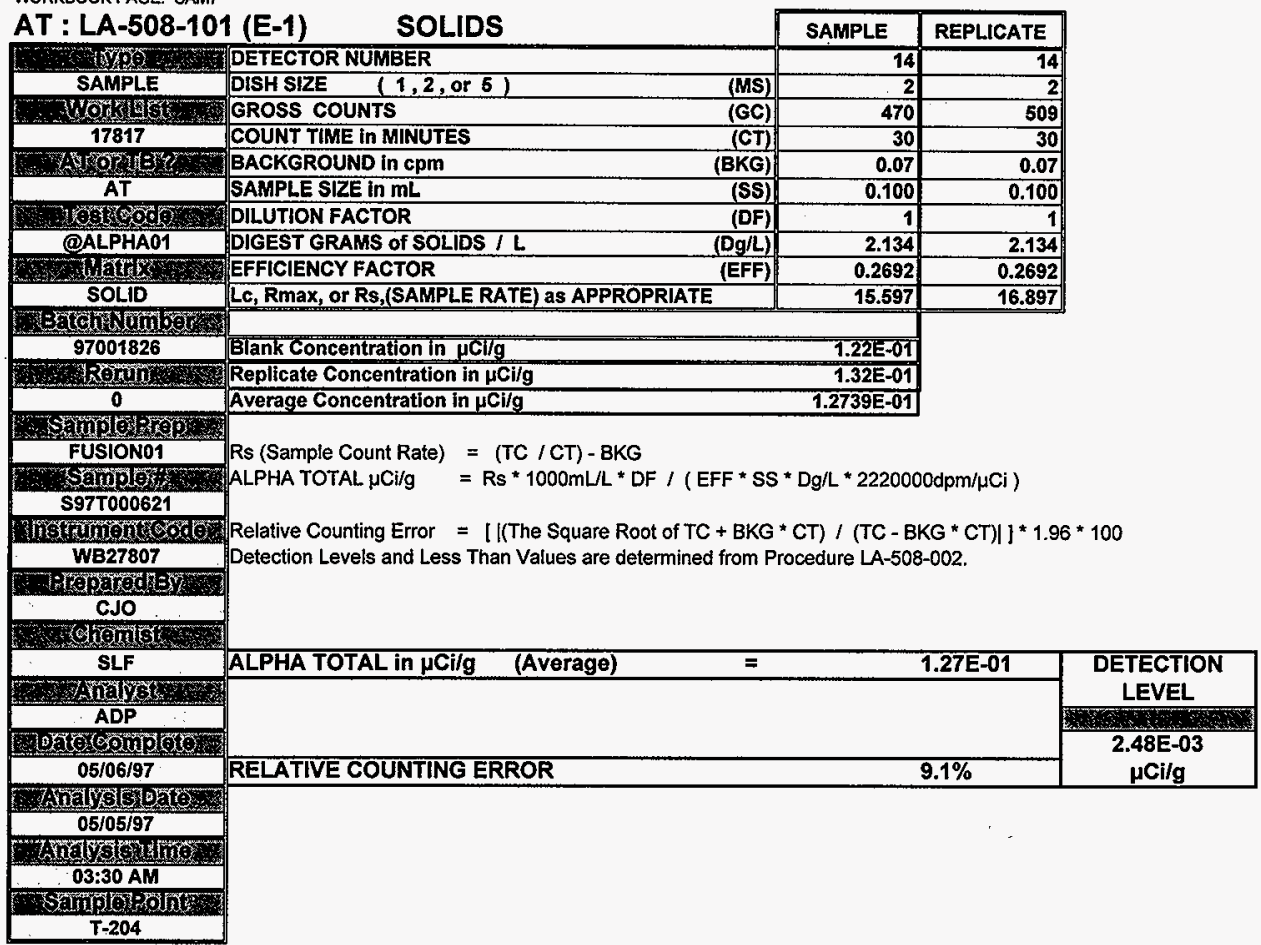

\begin{tabular}{lll}
\hline Analyst: & Signature of Chemist: \\
\hline SAMPLE.WB1 Rev. 1.0 & 508101ML
\end{tabular}


WORKBOOKPAGE: DUPB

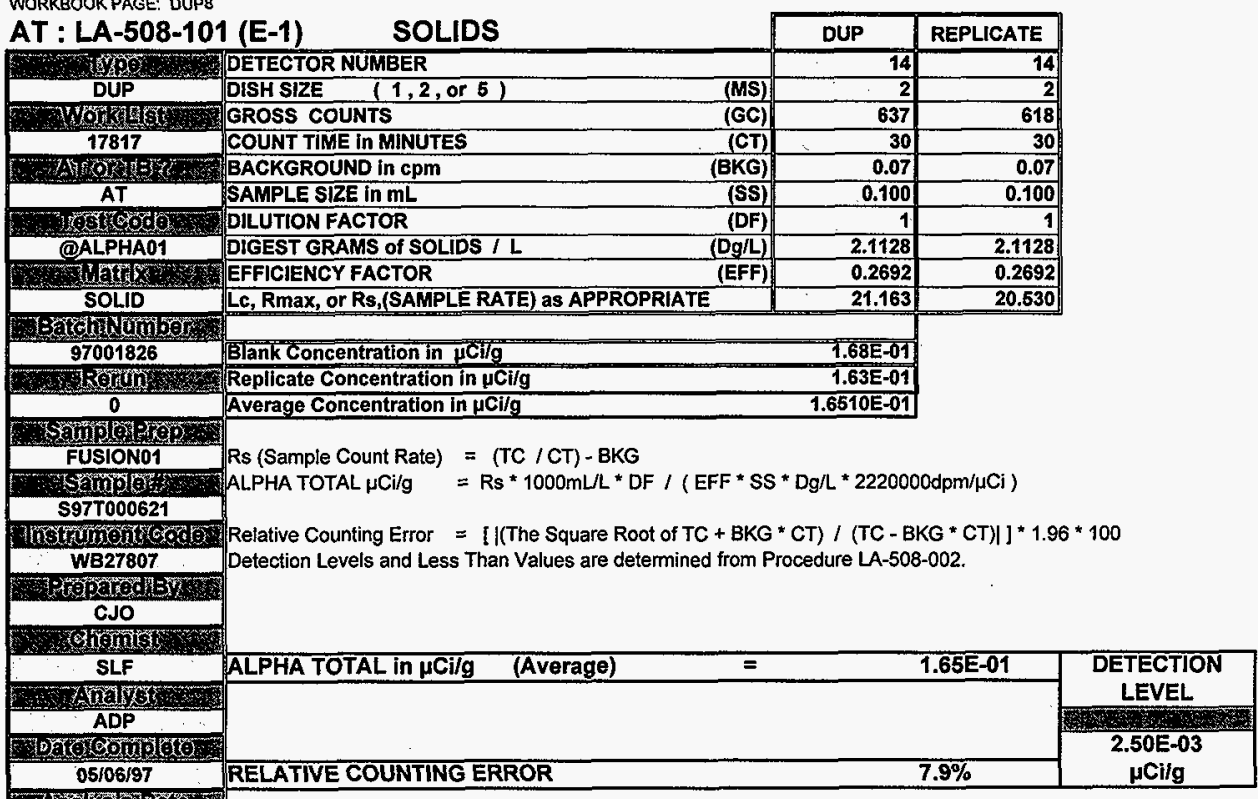

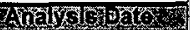
$05 / 05 / 97$

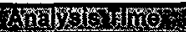
03:30 AM

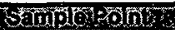
T-204

\begin{tabular}{ll}
\hline Analyst: & Signature of Chemist: \\
\hline SAMPLE.WB1 Rev. 1.0 & $508101 \mathrm{ML}$
\end{tabular}


WORKBOOK PAGE: SAM9

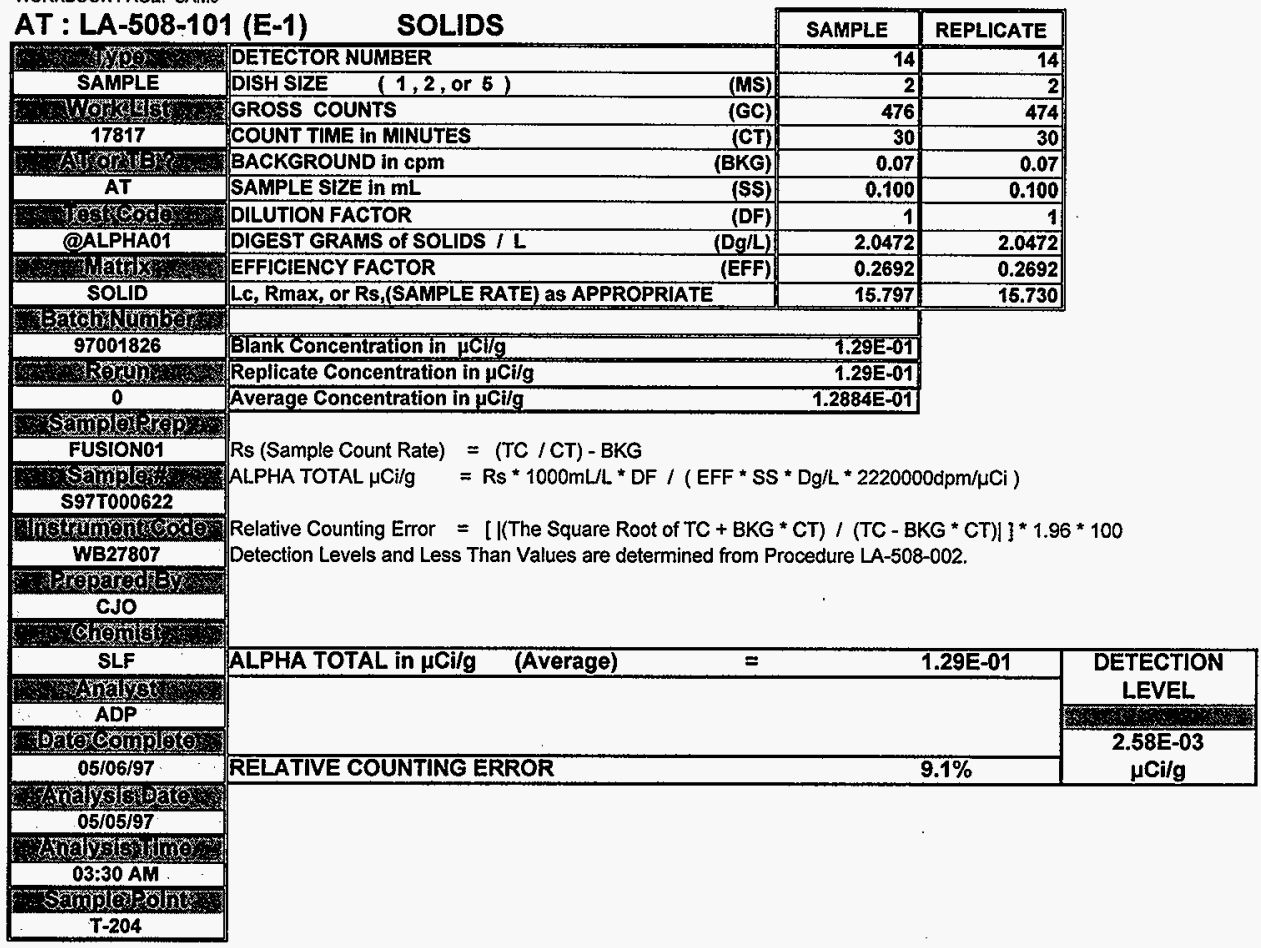

Analyst:
Signature of Chemist:
SAMPLE.WB1 Rev. 1.0


WORKBOOK PAGE: DUP10

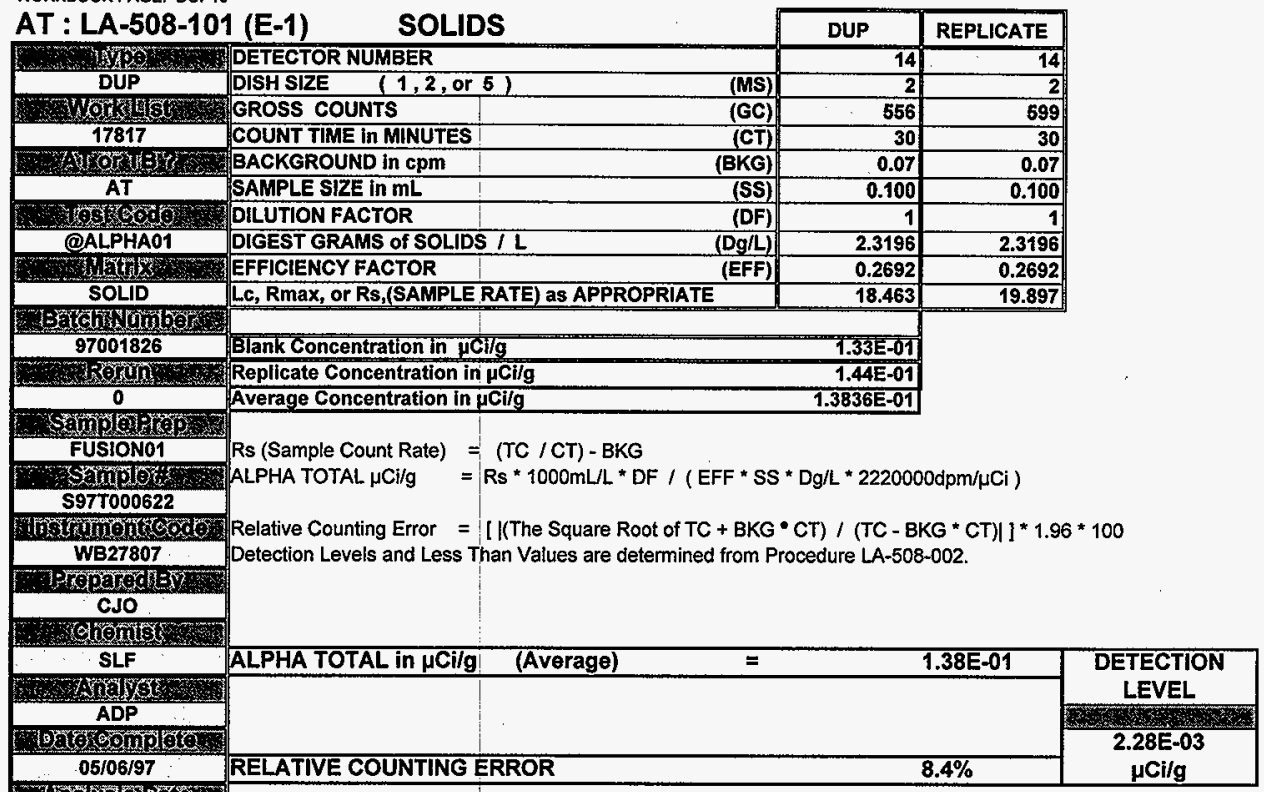

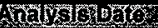
$05 / 05 / 97$

3 and 9 s 03:30 AM

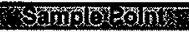
T-204

Analyst:

Signature of Chemist:

SAMPLE.WB1 1 Rev. 1.0 


\section{LABCORE Completed RadChem Report for Worklist\#: 17901}

Analyst: scl

Method: Rev/Mod
Book\#

Worklist Comment: T204, @ALPHA01 Deter s.s. by Ludlum. Std: 1.0mL skm

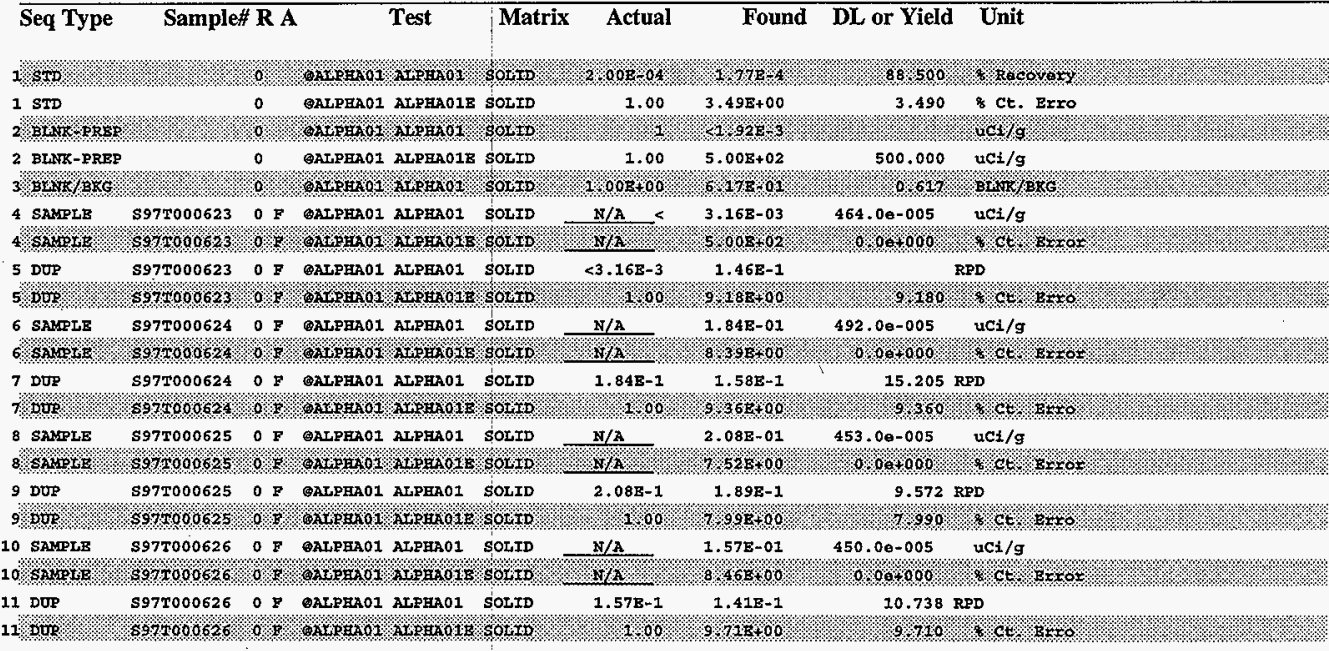

\section{Final page for worklist\# 17901}

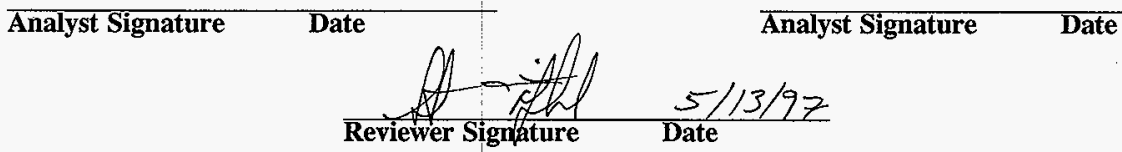

Units shown for $Q C(B L K / B K G)$ may not reflect the actual units. 


\section{LABCORE Data Entry Template for Worklist\# 17901}

Analyst: $\quad$ S.L Instrument: $A B 00 \quad 15 \quad$ Book\# 79856

Method: LA-508-101 Rev/Mod G

Worklist Comment: T204, @ALPHA01 Deter s.s. by Ludlum. Std: $1.0 \mathrm{~mL}$ skm

\begin{tabular}{|c|c|c|c|c|c|c|}
\hline S Type & Sample\# & R A & Test & Matrix & Group\# & Project \\
\hline 1 STD & & & $@ \mathrm{ALPF}$ & SOLID & & \\
\hline 2 BLNK-PREP & & & $@ A L P F$ & SOLID & & \\
\hline 3 BLNK/BKG & & & $@ A L P Y$ & SOLID & & \\
\hline
\end{tabular}

4 SAMPLE S97T000623 O F @ALPHAOI SOLID

97000261 T-204

Analytes Requested: ALPHAO1 , ALPHAO1E

5 DUP S97T000623 O F @ALPHAOI SOLID

6 SAMPLE S97T000624 O F @ALPHA0I SOLID

Analytes Requested: ALPHAO1 , ALPHA01E

97000261 T-204

7 DUP S97TO00624 OF @ALPHAOI SOLID

8 SAMPLE S97T000625 O F @ALPHAO1 SOLID Analytes Requested: ALPHAO1, ALPHAOIE

$97000261 \quad T-204$

9 DUP S97T000625 O F @ALPHA01 SOLID

10 SAMPJE

S97T000626 O F @ALPHAO1 SOLID

Analytes Requested: ALPHAO1, ALPHA01E

97000261 T-204

11 DUP

S97T000626 O F @ALPHA01 SOLID

Final page for worklist \# 17901

$\frac{\mathscr{L}_{2}}{\text { Analyst Signature }} \frac{5-7-97}{\text { Date }}$

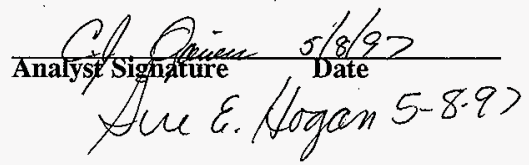

Data Entry Comments:

$\bar{S}=$ Worklist Slot Number, $R=$ Replicate Number, $A=$ Aliquot Code. 


\section{5 \\ HNF-SD-WM-DP-247, REV. 0}

WORKBOOK PAGE: STD1

AT : LA-508-101 (E-1)

LA-508-113 (B-0) STANDARD

DETECTOR NUMBER

STD

Worth

17901

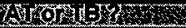

AT

DISH SIZE $(1,2$, or 5$)$

GROSS COUNTS

COUNT TIME in MINUTES

BACKGROUND in cpm

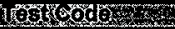

SAMPLE SIZE in $\mathrm{mL}$

@ALPHA01

intry

DILUTION FACTOR

STANDARD BOOK NUMBER

LIQUID

EFFICIENCY FACTOR

LC, Rmax, or Rs,(SAMPLE RATE) as APPROPRIATE

Barch Noribot

97001917

Standard Value in $\mu \mathrm{Ci} / \mathrm{mL}$

Concentration in $\mu \mathrm{CI} / \mathrm{L}$

STANDARD REPLICATE

0

Replicate Concentration in $\mu \mathrm{Ci} / \mathrm{L}$

AVERAGE CONCENTRATION in $\mu \mathrm{Ci} / L$

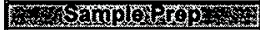

N/A

Rs (Sample Count Rate) $=($ TC /CT) - BKG

3amion

WL17901

ALPHA TOTAL $\mu \mathrm{Ci} / \mathrm{L}=\mathrm{Rs}$ * $1000 \mathrm{~mL} /{ }^{*} \mathrm{DF} /(\mathrm{EFF} * \mathrm{SS} * 2220000 \mathrm{dpm} / \mu \mathrm{Ci})$

ALPHA TOTAL $\mu \mathrm{Ci} / \mathrm{mL}=$ ALPHA TOTAL $\mu \mathrm{CI} / \mathrm{L} / 1000 \mathrm{~mL} / \mathrm{L}$

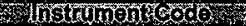
WB26872

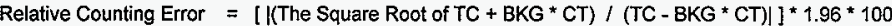

Detection Levels and Less Than Values are determined from Procedure LA-508-002.

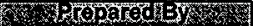

cjo

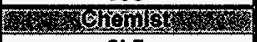

SLF

ALPHA TOTAL CONCENTRATION in $\mu \mathrm{Ci} / \mathrm{mL}=$

$=$

$1.77 \mathrm{E}-04$

DETECTION

LEVEL.

SCL

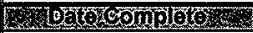

05/08/97

RELATIVE COUNTING ERROR

$8.90 \mathrm{E}-07$

$\mu \mathrm{Ci} / \mathrm{mL}$

\begin{tabular}{|c|c|}
\hline \multirow{2}{*}{\multicolumn{2}{|c|}{$\frac{2 \text { And }}{05 / 07 / 97}$}} \\
\hline & \\
\hline 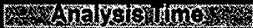 & \\
\hline $01: 30 \mathrm{PM}$ & \\
\hline inploso & \\
\hline 20 & \\
\hline
\end{tabular}

Analyst:
Signature of Chemist:
STANDARD.WB1 Rev. 1.0




\section{5 \\ HNF-SD-WM-DP-247, REV. 0}

WORKBOOK PAGE: BLANK2

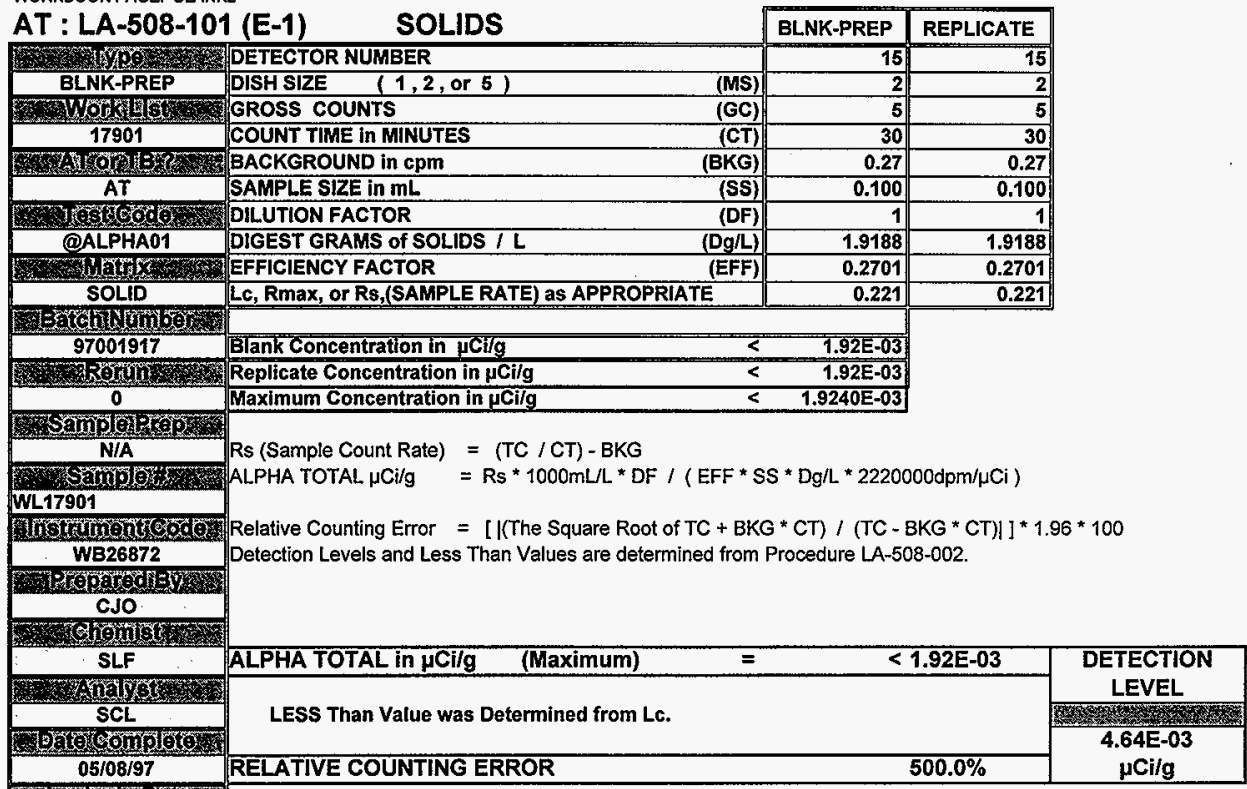

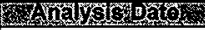
$05 / 07 / 97$

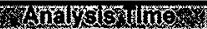
01:30 PM

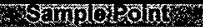
T-204
Analyst:

Signature of Chemist:

BLANK.WB1 Rev. 1.0

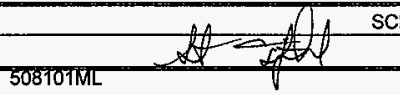

$\mathrm{SCL}$ Date: 08-May-97 SLF Date: $5 / 13 / 97$ 
HNF-SD-WM-DP-247, REV. 0

WORKBOOK PAGE: SAM4

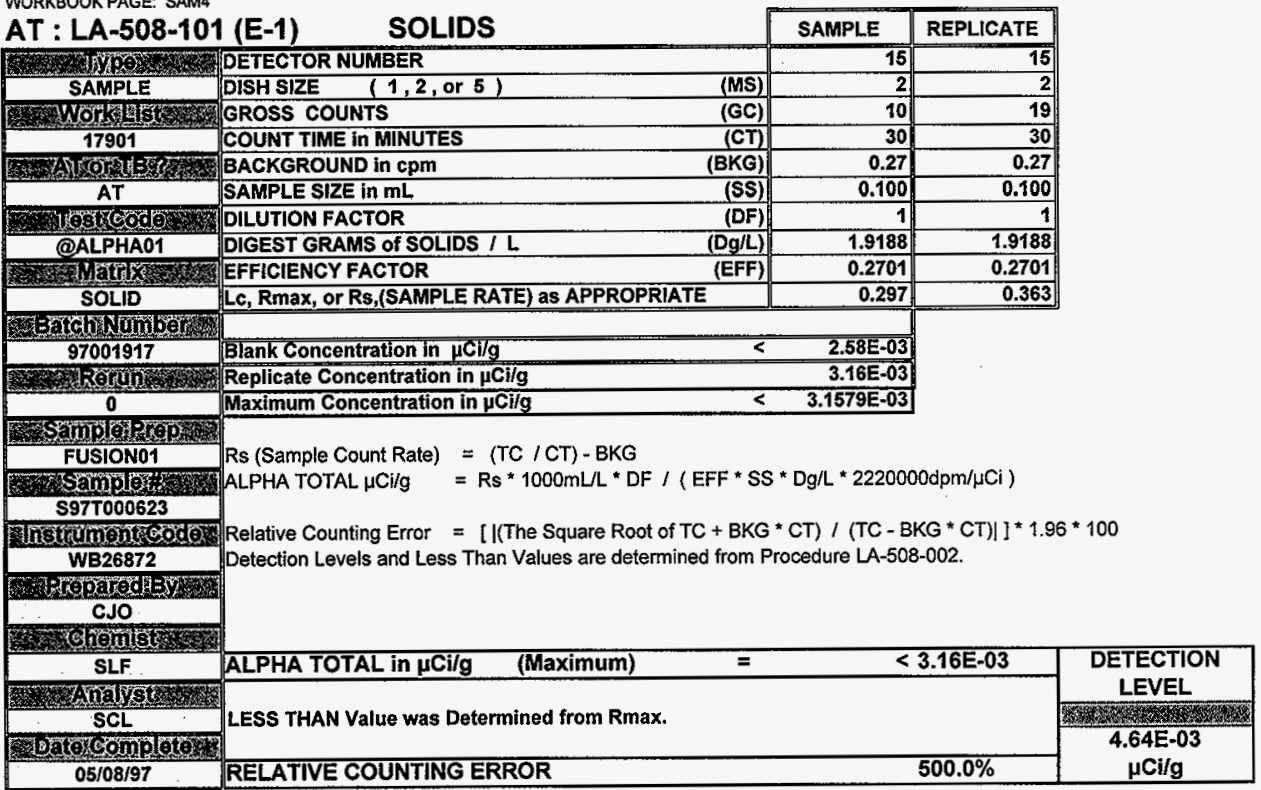

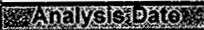
$05 / 07 / 97$

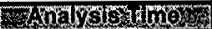
01:30 PM

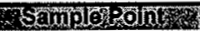
$T-204$

\begin{tabular}{l} 
Analyst: \\
\hline Signature of Chemist: \\
SAMPLE.WB1 Rev. 1.0 \\
$508101 \mathrm{ML}$
\end{tabular}


WORKBOOKPAGE: DUP5

\begin{tabular}{|c|c|c|c|c|}
\hline : LA-508-10 & SOLIDS & DUP & REPLICATE & \\
\hline 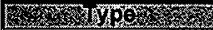 & DETECTOR NUMBER & 15 & 15 & \\
\hline DUP & DISH SIZE $\quad(1,2$, or 5$)$ & 2 & 2 & \\
\hline 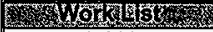 & GROSS COUNTS & 480 & 546 & \\
\hline 17901 & COUNT TIME in MINUTES & 30 & 30 & \\
\hline 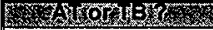 & BACKGROUND in cpm & 0.27 & 0.27 & \\
\hline AT & SAMPLE SIZE in $\mathrm{mL}$ & 0.100 & 0.100 & \\
\hline a 20000 & DILUTION FACTOR & 1 & 1 & \\
\hline @ALPHA01 & DIGEST GRAMS of SOLIDS / L & 1.9192 & 1.9192 & \\
\hline - & EFFICIENCY FACTOR & 0.2701 & 0.2701 & \\
\hline SOLID & Lc, Rmax, or Rs,(SAMPLE RATE) as APPROPRIATE & 15.730 & 17.930 & \\
\hline sonoxmbe & & & & \\
\hline 97001917 & Blank Concentration in $\mu \mathrm{Ci} / \mathrm{g}$ & T.37E-01 & & \\
\hline 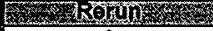 & Replicate Concentration in $\mathrm{\mu Ci} / \mathrm{g}$ & $1.56 \mathrm{E}-01$ & & \\
\hline 0 & Average Concentration in $\mathrm{\mu Ci} / \mathrm{g}$ & 1.4625E-01 & & \\
\hline 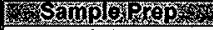 & \multirow{3}{*}{\multicolumn{4}{|c|}{$\begin{array}{l}\text { Rs }(\text { Sample Count Rate })=(\text { TC } / \mathrm{CT})-\mathrm{BKG} \\
\text { ALPHA TOTAL } \mu \mathrm{Ci} / g \quad=\mathrm{Rs} * 1000 \mathrm{~mL} / \mathrm{L} * \mathrm{DF} /(\mathrm{EFF} * \mathrm{SS} * \mathrm{Dg} / \mathrm{L} * 2220000 \mathrm{dpm} / \mu \mathrm{Ci})\end{array}$}} \\
\hline FUSIONO1 & & & & \\
\hline $\begin{array}{l}\text { S5mplow } \\
\text { S97T000623 }\end{array}$ & & & & \\
\hline 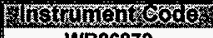 & \multirow{4}{*}{\multicolumn{4}{|c|}{$\begin{array}{l}\text { Relative Counting Error }=[\mid(\text { The Square Root of TC }+ \text { BKG * CT }) /(\text { TC }- \text { BKG * CT) }]]^{*} 1.96 * 100 \\
\text { Detection Levels and Less Than Values are determined from Procedure LA-508-002. }\end{array}$}} \\
\hline WB26872 & & & & \\
\hline 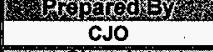 & & & & \\
\hline Anowing & & & & \\
\hline SLF & ALPHA TOTAL in $\mu \mathrm{Ci} / \mathrm{g}$ & & 1.46E-01 & \multirow{2}{*}{$\begin{array}{l}\text { DETECTION } \\
\text { LEVEL }\end{array}$} \\
\hline 2. & & & & \\
\hline 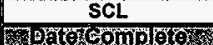 & & & & $\frac{4}{4.64 E-03}$ \\
\hline $05 / 08 / 97$ & RELATIVE COUNTING ERROR & & $9.2 \%$ & $\mu \mathrm{Ci} / \mathrm{g}$ \\
\hline
\end{tabular}

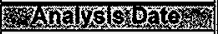
$05 / 07 / 97$

wanarsis 01:30 PM

F⿻ T-204

\begin{tabular}{l}
\hline Analyst: \\
\hline Signature of Chemist: \\
SAMPLE.WB1 Rev. 1.0 \\
$508101 \mathrm{ML}$
\end{tabular}




\section{5 \\ HNF-SD-WM-DP-247, REV. 0}

WORKBOOK PAGE: SAM6

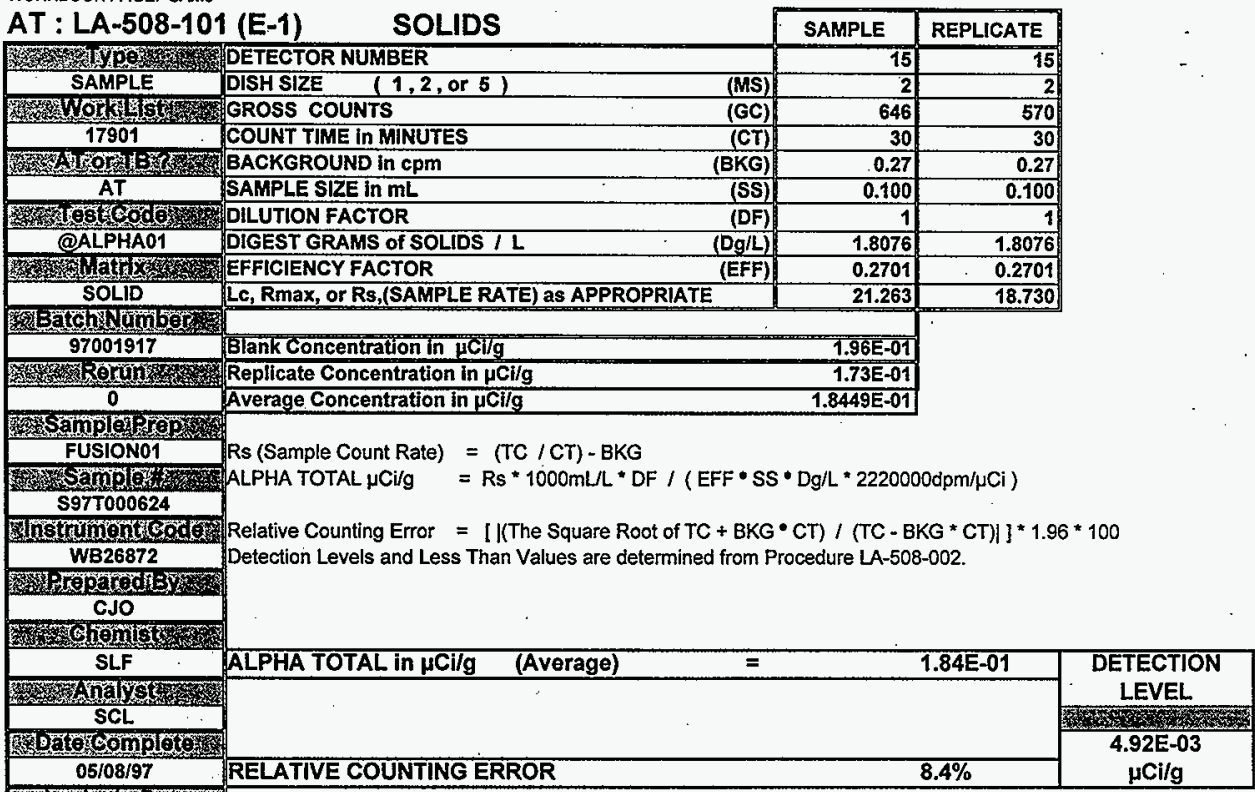

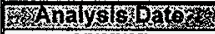
$05 / 07 / 97$

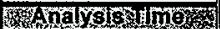
01:30 PM

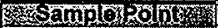
T-204

\begin{tabular}{|c|c|c|}
\hline Analyst: & SCL & Date: 08-May-97 \\
\hline Signature of Chemist: & & Date: $5 / / 3 / 97$ \\
\hline
\end{tabular}




\section{HNF-SD-WM-DP-247,REV. 0}

WORKBOOK PAGE: DUP7

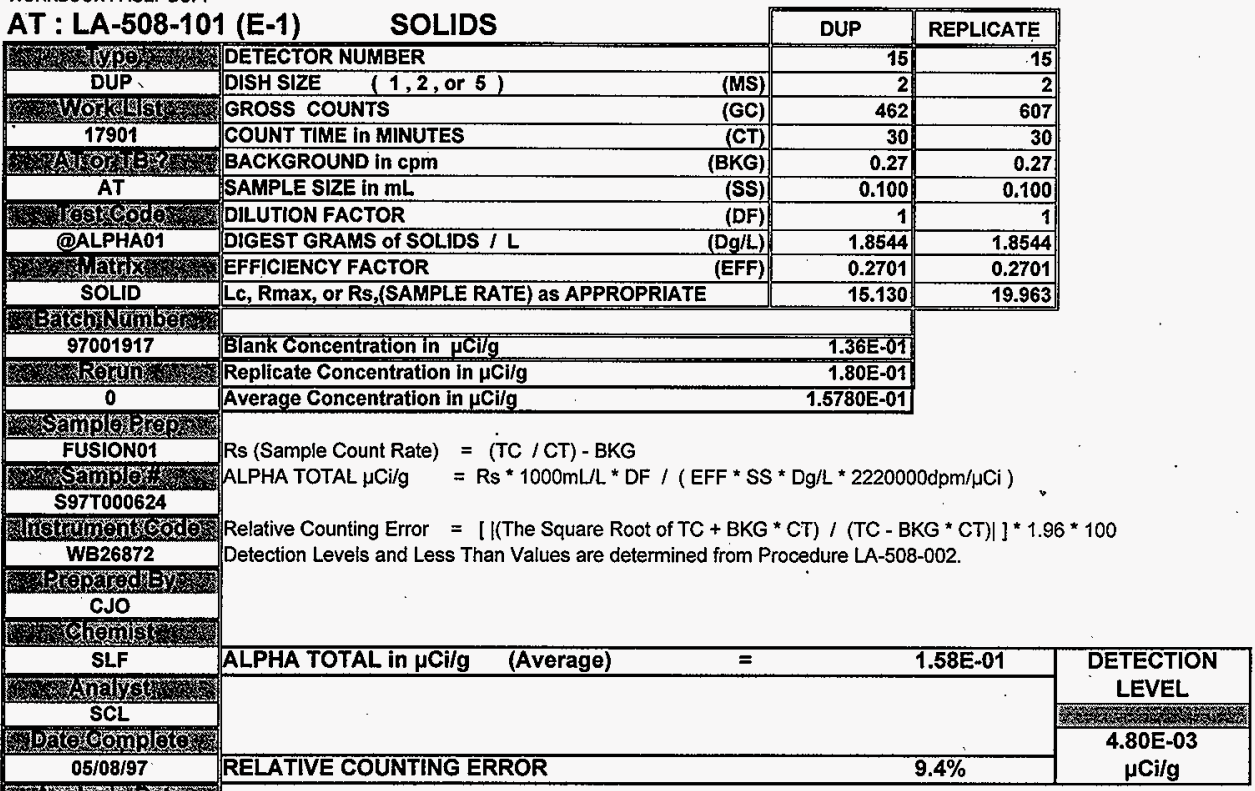

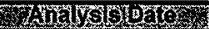
05/07/97

1017s 01:30 PM

W s $\mathrm{T}-204$

\begin{tabular}{l}
\hline Analyst: \\
\hline Signature of Chemist: \\
\hline SAMPLE.WB1 Rev. 1.0
\end{tabular}




\section{HNF-SD-WM-DP-247, REV. 0}

WORKBOOK PAGE: SAMB

\begin{tabular}{|c|c|c|c|c|}
\hline \multirow{2}{*}{\multicolumn{2}{|c|}{ AT : LA-508-101 (E-1) SOLIDS }} & \multirow{2}{*}{\multicolumn{2}{|c|}{ SAMPLE REPLICATE }} & \\
\hline & & & & \\
\hline 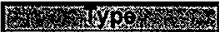 & DETECTOR NUMBER & 15 & 15 & \\
\hline SAMPLE & DISH SIZE $\quad(1,2$, or 5$)$ & 2 & 2 & \\
\hline 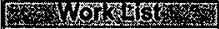 & GROSS COUNTS & 778 & 704 & . \\
\hline 17901 & COUNT TIME in MINUTES & 30 & 30 & \\
\hline 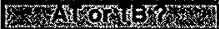 & BACKGROUND in cpm & 0.27 & 0.27 & \\
\hline AT & SAMPLE SIZE in mL & 0.100 & 0.100 & \\
\hline Trand & DILUTION FACTOR & 1. & 1 & \\
\hline @ALPHA01 & DIGEST GRAMS of SOLIDS / L & 1.962 & 1.962 & \\
\hline 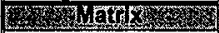 & EFFICIENCY FACTOR & 0.2701 & 0.2701 & \\
\hline SOLID & Lc, Rmax, or RS,(SAMPLE RATE) as APPROPRIATE & 25.663 & 23.197 & \\
\hline Batch $10 \mathrm{mb}$ & & & & \\
\hline 97001917 & Blank Concentration in $\mu$ Cilg & 2.18E-0T & & \\
\hline 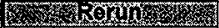 & Replicate Concentration in $\mu \mathrm{Ci} / \mathrm{g}$ & 1.97E-01 & & \\
\hline 0 & Average Concentration in $\mu \mathrm{Ci} / \mathrm{g}$ & $2.0766 \mathrm{E}-01$ & & \\
\hline WSampletoo & & & & \\
\hline FUSIONO1 & Rs (Sample Count Rate) $=(\mathrm{TC} / \mathrm{CT})-\mathrm{BKG}$ & & & \\
\hline Fanolor & $=R^{*}{ }^{*} 1000 \mathrm{~mL} /{ }^{*} \mathrm{DF} /\left(\mathrm{EFF}{ }^{*} \mathrm{SS}\right.$ & $\mathrm{Dg} / \mathrm{L}$ * 222000 & Odpm/ $\mu \mathrm{Ci})$ & \\
\hline S97T000625 & & & & \\
\hline 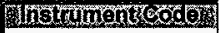 & Relative Counting Error $=$ [I(The Square Root of $\mathrm{TC}+\mathrm{BKG}$ & $\mathrm{CT}) /(\mathrm{TC}-\mathrm{Bl}$ & $\langle G * C T) \|]^{*} 1.96$ & * 100 \\
\hline WB26872 & Detection Levels and Less Than Values are determined from $P$ & cedure LA-508 & -002 . & \\
\hline 20 & & & & \\
\hline CJO & & & & \\
\hline 5owis & & & & \\
\hline SLF & ALPHA TOTAL in $\mu \mathrm{Ci} / \mathrm{g}$ & & $2.08 \mathrm{E}-01$ & DETECTION. \\
\hline 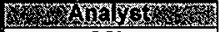 & & & & LEVEL \\
\hline 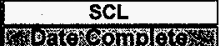 & & & & $\frac{1}{4.53 E-03}$ \\
\hline $05 / 08 / 97$ & RELATIVE COUNTING ERROR & & $7.5 \%$ & $\mu \mathrm{Ci} / \mathrm{g}$ \\
\hline
\end{tabular}

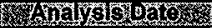
05/07/97

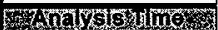
04:30 PM

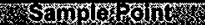
$T-204$

\begin{tabular}{|l} 
Analyst: \\
Signature of Chemist: \\
SAMPLE.WB1 Rev. 1.0
\end{tabular}




\section{HNF-SD-WM-DP-247,REV. 0}

WORKBOOK PAGE: DUP9

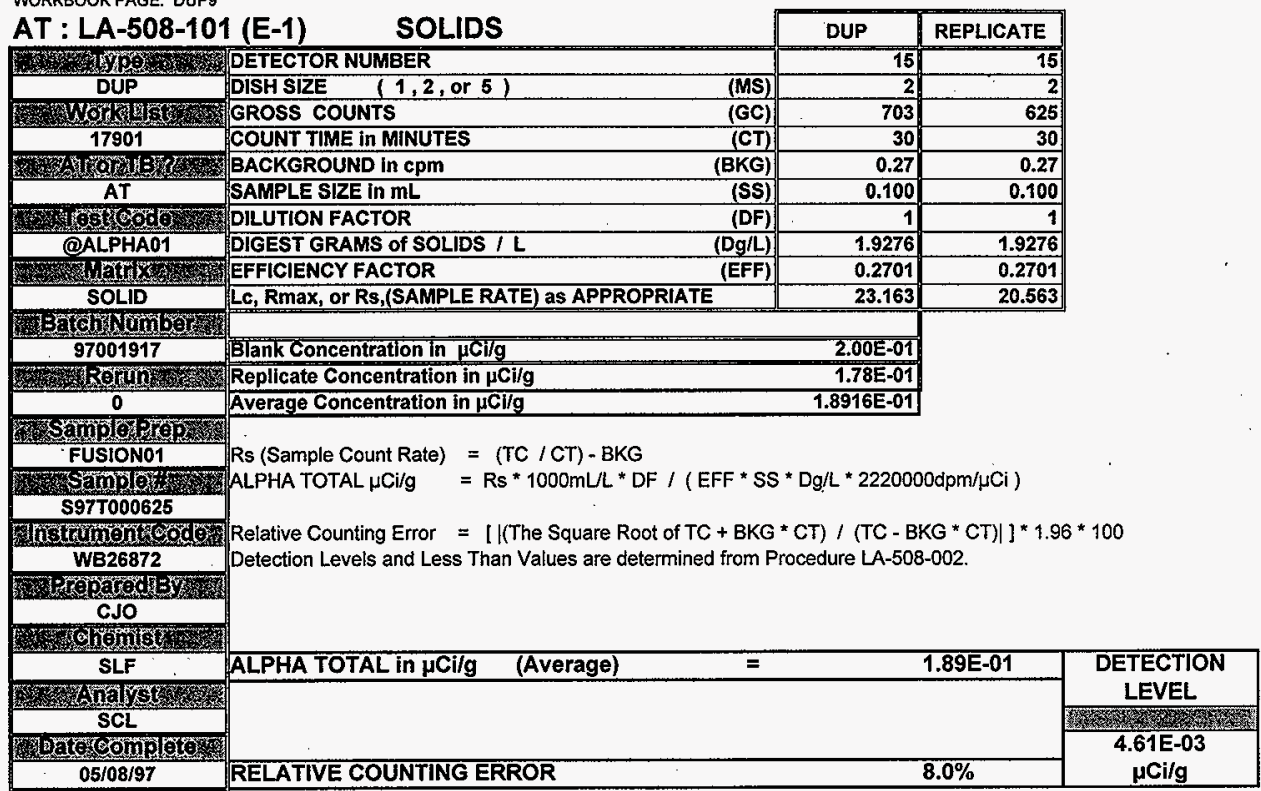

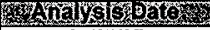
05/07/97

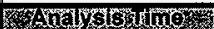
01:30 PM

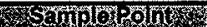
T-204

\begin{tabular}{|l|lll}
\hline Analyst: & Signature of Chemist: & Date: 08-May-97 \\
\hline SAMPLE.WB1 Rev. 1.0 & $508101 \mathrm{ML}$ & SLF
\end{tabular}


WORKBOOKPAGE: SAM10

AT : LA-508-101 (E-1)

HNF-SD-WM-DP-247, REV. 0

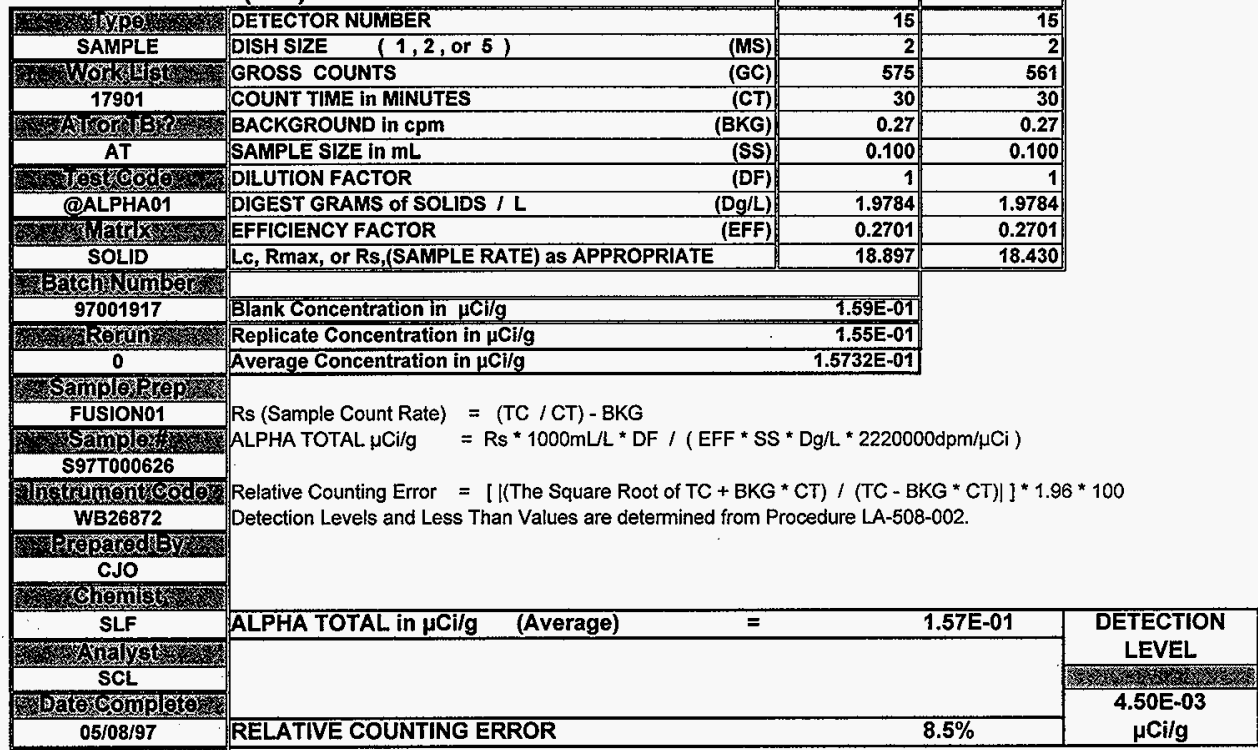

- D.

$05 / 07 / 97$

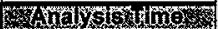

01:30 PM

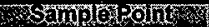

T-204

Analyst:

Signature of Chemist:

SAMPLE.WB1 Rev. 1.0

$508101 \mathrm{ML}$ 
HNF-SD-WM-DP24, REV. 0

WORKBOOK PAGE: DUP11

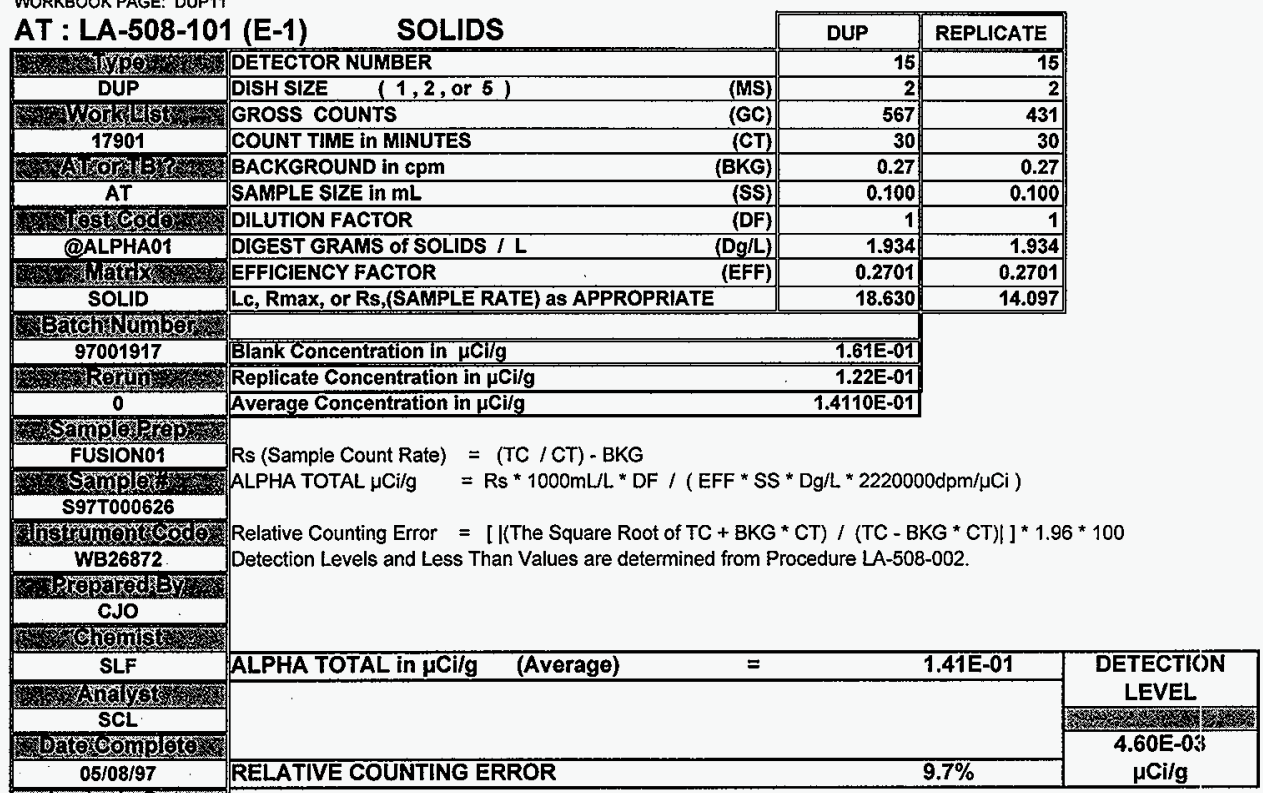

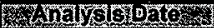
$05 / 07 / 97$

\% 4 TaI 01:30 PM

Formolo T-204

\begin{tabular}{|l}
\hline Analyst: \\
Signature of Chemist: \\
\hline SAMPLE.WB1 Rev. 1.0
\end{tabular}




\section{LABCORE Completed RadChem Report for Worklist\#: 17950}

Analyst: scl

Instrument: $\mathrm{AB} 15$

Method:

Rev/Mod
Book\#

HNF-SD-WM-DP-255, REV. 0

Worklist Comment: Use $.1 \mathrm{~mL}$ sample size. Rerun \#1. SLF

\begin{tabular}{|c|c|c|c|c|c|c|c|c|}
\hline$\overline{\text { Seq Type }}$ & Sample & $\mathbf{R A}$ & & Test & Matrix & Actual & Found & DL or Yield Unit \\
\hline 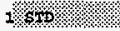 & & 0 & \% 1,3501 & \% & solvt & $2 \% 00804$ & $1228=4$ & \% $18=0 \% 0$ \\
\hline 1 STP & & 0 & Gargha01 & Axpmo1s & SOLID & 1.00 & $3.52 \mathrm{E}+00$ & $8 \mathrm{ct}$. Brro \\
\hline $2 \% 14 \times 4 \%$ & & $\%$ & $\% \alpha_{4}=1 \times 1$ & 4 & som I & 1 & $2 \times 478 \%$ & ichlo \\
\hline 2 BLNK-PREP & & 0 & OALPHA 1 & ALpmo1E & SOLID & 1.00 & $5.008+02$ & 500.000 \\
\hline $3: 10=4 \%$ & & 0 & 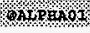 & Arpapor & $501+p$ & 1000500 & $2 \times 3340 \%$ & 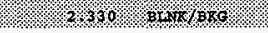 \\
\hline 4 SAYPLB & S97T000587 & $0 F$ & OATPHAO1 & AIPHA01 & SOLID & $\mathbf{n / 2}$ & $1.76 \mathrm{E}-01$ & $296.08-005$ \\
\hline $4540+1$ & $s 020005 \%$ & (0) & 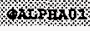 & $x_{2}+x_{01}$ & Sol-1) & $4 x$ h & $8028+\%$ & 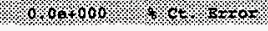 \\
\hline $5 \mathrm{DNP}$ & 5975000587 & $0 F$ & OALPAAOI & AIPRAOI & SOIID & $1.76 \mathrm{~F}-1$ & $1.51 \mathrm{E}-1$ & $15.291 \mathrm{RPD}$ \\
\hline $5 \% 0$ & $59700058 \%$ & $0 \%$ & \% & $x_{4}=1019$ & sol 40 & 100 & $408+00$ & 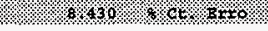 \\
\hline 6 SAMPLF & s97T000588 & $0 F$ & OALPAPO01 & ALPEA01 & SOLID & $N / 2$ & $1.38 \mathrm{~B}-01$ & $315,00-005$ \\
\hline $6.9 \times 1 \times+10$ & 5912000580 & $8 \%$ & $47=7+201$ & $4=1 \times 01$ & sol-10 & $2 / 2$ & 217840 & 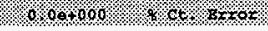 \\
\hline 7 DUP & \$97T000588 & $0 F$ & OATPHAO1 & ALPEAOH & SOLID & $1.38 \mathrm{~g}-1$ & $1.49 \mathrm{E}-1$ & $7.656 \mathrm{RPD}$ \\
\hline $7 \times 109$ & 497200058 & $0 \%$ & $64 x+101$ & Hop 014 & Sod $=0$ & 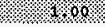 & 606000 & 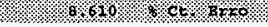 \\
\hline $8 \mathrm{SPK}$ & 5975000588 & $0 F$ & OALPHAO01 & AxpHa01 & SOLID & $2.635-02$ & $1.98 \mathrm{~B}-02$ & \&ecovery \\
\hline 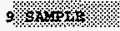 & 897000589 & 60 & 6 A & $2 \times 1+1401$ & sotith & 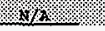 & 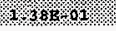 & 212000005 \\
\hline 9 SAMPLP & 5977000589 & $0 F$ & OALPHAO1 & ALPFAOIB & SOLID & $\mathrm{N} / \mathrm{A}$ & $9.18 \mathrm{E}+00$ & $\because \mathrm{ct} . \mathrm{Error}$ \\
\hline $10 \%$ on & 897000589 & $80 \%$ & 6418401 & AI $1=101$ & solin & 10827 & $1468 \times$ & $17328 \times 0 p$ \\
\hline 0 DUP & s97T000589 & $0=$ & GALPEAON & ALPEA01B & SOLID & 1.00 & $1.08 \mathrm{E}+01$ & \& Ct. Exro \\
\hline
\end{tabular}

Final page for worklist\# 17950

Analyst Signature Date

Units shown for $Q C(B L K / B K G)$ may not reflect the actual units. 


\section{LABCORE Data Entry Template for Worklist\# 17950}

Analyst: $\quad$ S.L Instrument: AB00 15 Book\# 79656

Method: LA-508-101 Rev/Mod G-O

Worklist Comment: Use $.1 \mathrm{~mL}$ sample size. Rerun \#1. SLF

HNF-SD-WM-DP-255, REV, 0

S Type Sample\# R A Test Matrix Group\# Project

1 STD

@ALPHAOI SOLID

2 BLNK-PREP

@ALPHAO1 SOLID

3 BLNK/BKG

@ALPHAO1 SOLID

4 SAMPLE

S97T000587 0 F @ALPHA01 SOLID

Analytes Requested: ALPHAO1, ALPHA01E

97000261 T-204

5 DUP

S97T000587 O F OALPHAO1 SOLID

6 SAMPLE

S97T000588 0 F @ALPHAO1 SOLID

$97000261 \mathrm{~T}-204$

Analytes Requested: ALPHA01, ALPHA01E

7 DUP S97TO00588 O F @ALPHAOI SOLID

8 SPK $\quad$ S97TO00588 0 F @ALPHA01 SOLID

9 SAMPLE

S97T000589 0 F @ALPHA01 SOLID

Analytes Requested: AIPHAO1, ALPHA01E

$97000261 \mathrm{~T}-204$

\section{Final page for worklist \# 17950}

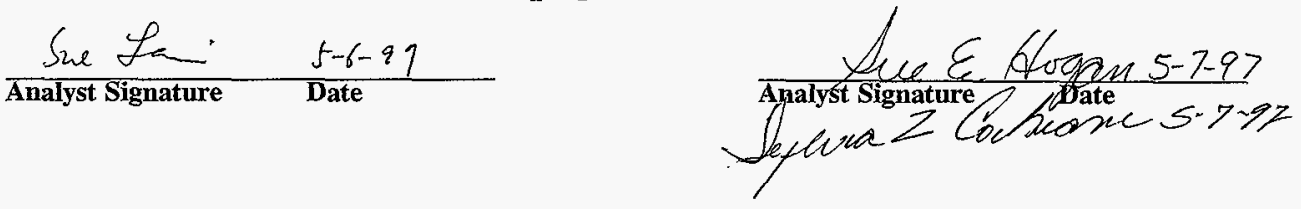

Data Entry Comments:

boil down samples 


\section{HNF-SD-WM-DP-255, REV. 0}

WORKBOOK PAGE: STD1

AT : LA-508-101 (E-1) LA-508-113 (B-0) STANDARD

STANDARD REPLICATE

A.

STD

DETECTOR NUMBER

7. Wofk

17950

DISH SIZE $(1,2$, or 5$)$

ROSS COUNTS

COUNT TIME in MINUTES

S 2 -

AT

BACKGROUND in cpm

SAMPLE SIZE in $\mathrm{mL}$

7\% 7rom

@ALPHA01 DILUTION FACTOR

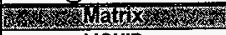

STANDARD BOOK NUMBER

LIQUID

Lc, Rmax, or Rs, (SAMPLE RATE) as APPROPRIATE

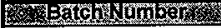
97001966

W. 3 KerM

Standard Value in $\mu \mathrm{Cl} / \mathrm{mL}$

(BKC)

(DF)

\begin{tabular}{|r|r|}
\hline 15 & 15 \\
\hline 2 & 2 \\
\hline 3263 & 2947 \\
\hline 30
\end{tabular}

T)

Replicate Concentration in $\mu \mathrm{Cl} / \mathrm{L} \quad 1.64 \mathrm{E}-0.1$

0

AVERAGE CONCENTRATION in $\mu \mathrm{CI} / L$

$=$

$1.7244 E-01$

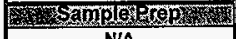

N/A

Rs (Sample Count Rate) $=(\mathrm{TC} / \mathrm{CT})-\mathrm{BKG}$

W

WL17950

ALPHA TOTAL $\mu \mathrm{Cl} / \mathrm{L}=\mathrm{RS} * 1000 \mathrm{~mL} / \mathrm{L} * \mathrm{DF} /(\mathrm{EFF} * \mathrm{SS} * 2220000 \mathrm{dpm} / \mu \mathrm{Ci})$

ALPHA TOTAL $\mu \mathrm{Ci} / \mathrm{mL}=$ ALPHA TOTAL $\mu \mathrm{Ci} / \mathrm{L} / 1000 \mathrm{~mL} / \mathrm{L}$

7xastrumenterocers WB26872

Relative Counting Error $=[$ [(The Square Root of TC + BKG * CT) $/(T C-B K G * C T)]$ * $1.96 * 100$

Detection Levels and Less Than Values are determined from Procedure LA-508-002.

wrandepared

SEH

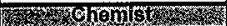

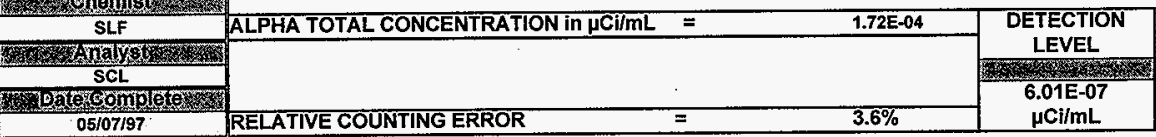

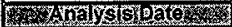
$05 / 06 / 97$

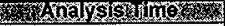

03:10 PM

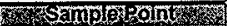

T-204

\begin{tabular}{|l|lll}
\hline Analyst: & Signature of Chemist: & SLF & Date: $5 / 8 / 92$ \\
\hline STANDARD.WB1 Rev. 1.0 & $508101 \mathrm{ML}$ &
\end{tabular}




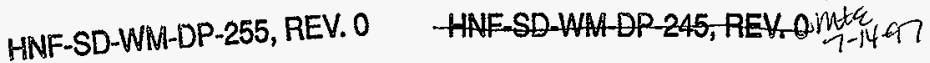

WORKBOOKPAGE: BLANK2

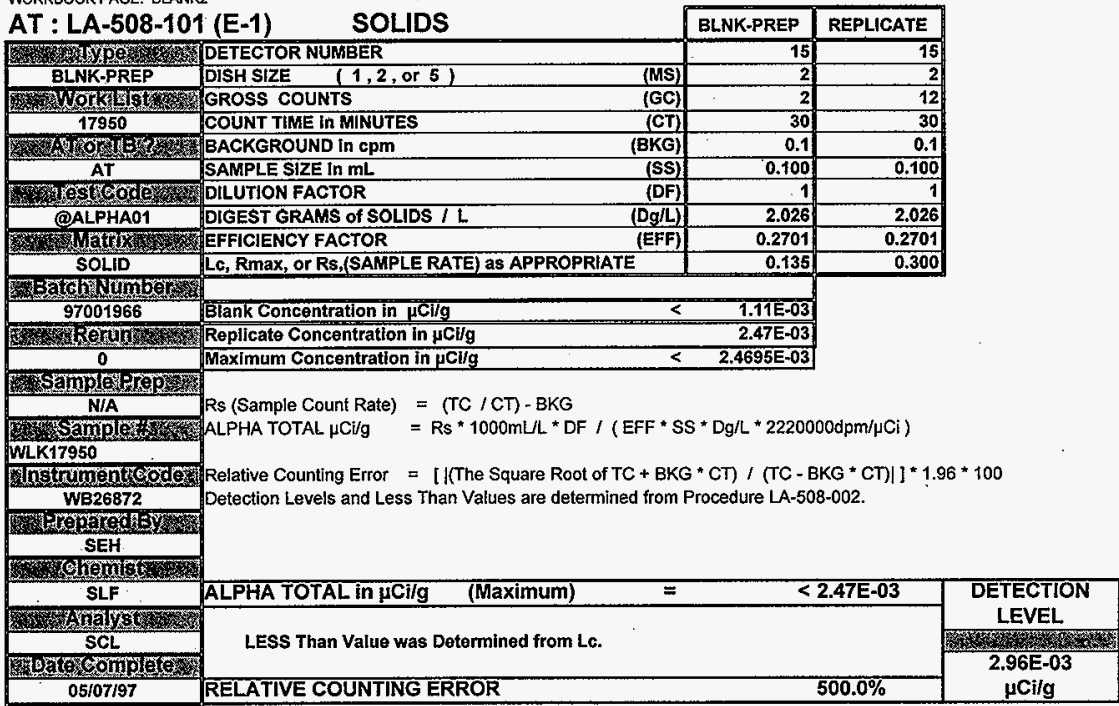

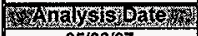
$05 / 06 / 97$

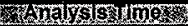
03:10 PM

S Sampley T-204

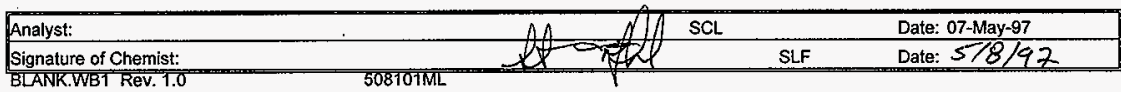




\section{HNF-SD-WM-DP-255, REV. 0}

HNF-SD-WM-BP-245, REV.0, MtE -97

WORKBOOKPAGE: SAM4

AT : LA-508-101 (E-1)

SOLIDS

\begin{tabular}{|c|c|}
\hline SAMPLE & REPLICATE \\
\hline 15 & 15 \\
\hline 2 & 2 \\
\hline 680 & 606 \\
\hline 30 & 30 \\
\hline 0.1 & 0.1 \\
\hline 0.100 & 0.100 \\
\hline 1 & 1 \\
\hline 2.026 & 2.026 \\
\hline 0.2701 & 0.2701 \\
\hline 22.567 & 20.100 \\
\hline
\end{tabular}

\begin{tabular}{|c|c|c|}
\hline 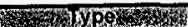 & DETECTOR NUMBER & \\
\hline SAMPLE & DISH SIZE $\quad(1,2$, or 5$)$ & (MS) \\
\hline 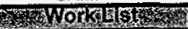 & GROSS COUNTS & (GC) \\
\hline 17950 & COUNT TIME in MINUTES & (CT) \\
\hline $6 \times 1001410$ & BACKGROUND in cpm & (BKG) \\
\hline AT & SAMPLE SIZE in $\mathrm{mL}$ & (SS) \\
\hline K3: & DILUTION FACTOR & (DF) \\
\hline (9ALPHA01 & DIGEST GRAMS of SOLIDS $/ \mathrm{L}$ & $(\mathrm{Dg} / \mathrm{l})$ \\
\hline 76 L L Trus & EFFICIENCY FACTOR & (EFF) \\
\hline SOLID & LC, Rmax, or RS, (SAMPLE RATE & \\
\hline
\end{tabular}

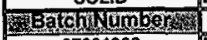

97001966

Blank Concentration in $\mu \mathrm{Cllg}$

1.86E-01

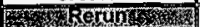

1

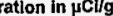

$7561 \mathrm{E}-01$

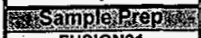

FUSIONO1

Average Concentration in $\mu \mathrm{Cl} / \mathrm{g}$

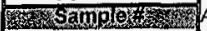

Rs (Sample Count Rate) $=(\mathrm{TC} / \mathrm{CT})-\mathrm{BKG}$

$\$ 97 \mathrm{~T} 000587$

mostumenterader

WB26872

ALPHA TOTAL $\mu \mathrm{Cl} / \mathrm{g}=\mathrm{Rs} * 1000 \mathrm{~mL} / \mathrm{L} * \mathrm{DF} /(\mathrm{EFF} * \mathrm{SS} * \mathrm{Dg} / \mathrm{L} * 2220000 \mathrm{dpm} / \mu \mathrm{Ci})$

$\underset{\text { SEH }}{\text { WB26872 }}$

Relative Counting Error $=\left[\right.$ [(The Square Root of TC + BKG * CT) $/\left(T C-B K G\right.$ * CT) I ${ }^{*} 1.96$ * 100

Detection Levels and Less Than Values are determined from Procedure LA-508-002.

meristis

\begin{tabular}{|c|}
\hline SLF \\
\hline W \\
\hline SCL \\
\hline Sorterompras \\
\hline $05 / 07 / 97$ \\
\hline 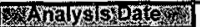 \\
\hline $05 / 06 / 97$ \\
\hline 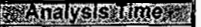 \\
\hline 03:10 PM \\
\hline ampeson \\
\hline$T-204$ \\
\hline
\end{tabular}

\begin{tabular}{|c|c|c|}
\hline ALPHA TOTAL in $\mu \mathrm{Ci} / \mathrm{g}$ & 1.76E-01 & \multirow{2}{*}{$\begin{array}{l}\text { DETECTION } \\
\text { LEVEL }\end{array}$} \\
\hline & & \\
\hline & & 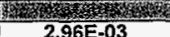 \\
\hline RELATIVE COUNTING ERROR & $8.0 \%$ & $\mu \mathrm{Ci} / \mathrm{g}$ \\
\hline
\end{tabular}

\section{(1)} $\mu \mathrm{Ci} / \mathrm{g}$

\begin{tabular}{lll}
\hline Analyst: & Signature of Chemist: & Date: $07-M a y-97$ \\
SAMPLE.WB1 Rev.1.0 & $508101 \mathrm{ML}$ & Date: $5 / 8 / 92$ \\
\hline
\end{tabular}


HNF-SD-WM-DP-255, REV. 0

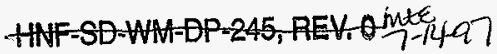

WORKBOOK PAGE: DUP5

\begin{tabular}{|c|c|c|c|c|}
\hline \multirow{2}{*}{\multicolumn{2}{|c|}{ AT : LA-508-101 (E-1) $\quad$ SOLIDS }} & \multirow{2}{*}{\multicolumn{2}{|c|}{\begin{tabular}{|l|l|} 
DUP & REPLICATE \\
\end{tabular}}} & \\
\hline & & & & \\
\hline Wy & DETECTOR NUMBER & 15 & 15 & \\
\hline DUP & $(1,2$, or 5$)$ & 2 & 2 & \\
\hline 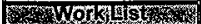 & GROSS COUNTS & 549 & 578 & \\
\hline 17950 & COUNT TIME In MINUTES & 30 & 30 & \\
\hline 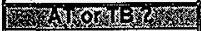 & BACKGROUND in cpm & 0.1 & 0.1 & \\
\hline AT & SAMPLE SIZE in $\mathrm{mL}$ & 0.100 & 0.100 & \\
\hline Whos & DILUTION FACTOR & 1 & 1 & \\
\hline @ALPHA01 & DIGEST GRAMS of SOLIDS / $\mathrm{L}$ & 2.0688 & 2.0688 & \\
\hline 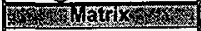 & EFFICIENCY FACTOR & 0.2701 & 0.2701 & \\
\hline SOLID & Lc, Rmax, or RS, (SAMPLE RATE) as APPROPRIATE & 18.200 & 19.167 & \\
\hline \multicolumn{3}{|c|}{ Sarob } & & \\
\hline 97001966 & Blank Concentration in $\mu$ Ci/g & 1.47E-01 & & \\
\hline W & Replicate Concentration in $\mu \mathrm{Ci} / \mathrm{g}$ & 1.55E-01 & & \\
\hline 1 & Average Concentration in $\mu \mathrm{C} / \mathrm{g}$ & 1.5061E-01 & & \\
\hline \multirow{2}{*}{ 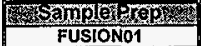 } & \multirow{9}{*}{\multicolumn{4}{|c|}{$\begin{array}{l}\text { Rs (Sample Count Rate) }=(\text { TC } / \mathrm{CT})-\mathrm{BKG} \\
\text { ALPHA TOTAL } \mu \mathrm{Ci} / \mathrm{g}=\mathrm{Rs} * 1000 \mathrm{~mL} / \mathrm{L}^{*} \mathrm{DF} /\left(\mathrm{EFF} * \mathrm{SS}{ }^{*} \mathrm{Dg} / \mathrm{L} * 2220000 \mathrm{dpm} / \mu \mathrm{Ci}\right) \\
\text { Relative Counting Error }=\left[\mid\left(T \text { The Square Root of TC }+\mathrm{BKG}{ }^{*} \mathrm{CT}\right) /\left(\mathrm{TC}-\mathrm{BKG}{ }^{*} \mathrm{CT}\right) \mid\right] * 1.96 * 100 \\
\text { Detection Levels and Less Than Values are determined from Procedure LA-508-002. }\end{array}$}} \\
\hline & & & & \\
\hline \multirow{2}{*}{ 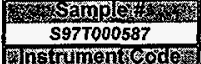 } & & & & \\
\hline & & & & \\
\hline 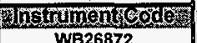 & & & & \\
\hline \multirow{3}{*}{ 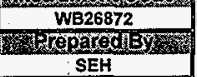 } & & & & \\
\hline & & & & \\
\hline & & & & \\
\hline 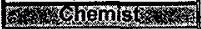 & & & & \\
\hline SLF & ALPHA TOTAL in $\mu$ Cilg & & 1.51E-01 & \multirow{2}{*}{$\begin{array}{l}\text { DETECTION } \\
\text { LEVEL }\end{array}$} \\
\hline 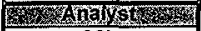 & & & & \\
\hline SCL & & & & \multirow{3}{*}{$\begin{array}{c}2.90 \mathrm{E}-03 \\
\mu \mathrm{Ci} / \mathrm{g}\end{array}$} \\
\hline Porexomperen & & & & \\
\hline $05 / 07 / 97$ & RELATIVE COUNTING ERROR & & $8.4 \%$ & \\
\hline
\end{tabular}

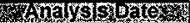

$05 / 06 / 97$

nolys

03:10 PM

\%

$T-204$

\begin{tabular}{lll|l|l|}
\hline Analyst: & Date: $07-\mathrm{May}-97$ \\
\hline Signature of Chemist: & Date: $5 / 8 / 97$ \\
\hline SAMFLE.WB1 Rev. 1.0
\end{tabular}




\section{HNF-SD-WM-DP-255, REV. 0}

\section{HNF-SD-WM-DP-245,REV, 0 inte $74-97$}

WORKBOOK PAGE: SAMG

\begin{tabular}{|c|c|c|c|c|}
\hline AT : LA-508-101 & SOLIDS & SAMPLE & REPLICATE & \\
\hline 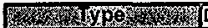 & DETECTOR NUMBER & 15 & 15 & \\
\hline SAMPLE & DISH SIZE $\quad(1,2$, or 5$)$ & 2 & 2 & \\
\hline 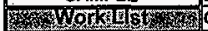 & GROSS COUNTS & 488 & 466 & \\
\hline 17950 & COUNT THME In MINUTES & 30 & 30 & \\
\hline 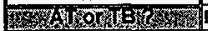 & BACKGROUND in $\mathrm{cpm}$ & 0.1 & 0.1 & \\
\hline AT & SAMPLE SIZE in $\mathrm{mL}$ & 0.100 & 0.100 & \\
\hline 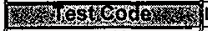 & DILUTION FACTOR & 1 & 1. & \\
\hline @ALPHA01 & DIGEST GRAMS Of SOLIDS / L & 1.9084 & 1.9084 & $\therefore$ \\
\hline 17\% & EFFICIENCY FACTOR & 0.2701 & 0.2701 & \\
\hline SOLID & LC, Rmax, or Rs, (SAMPLE RATE) as APPROPRIATE & 16.167 & 15.433 & \\
\hline sach & & & & \\
\hline 97001966 & Blank Concentration in $\mu \mathrm{Cl} / \mathrm{g}$ & 1.4TE-01 & & \\
\hline Xrorar & Replicate Concentration in $\mu \mathrm{Ci} / \mathrm{g}$ & $1.36 \mathrm{E}-01$ & & \\
\hline 1 & Average Concentration in $\mu \mathrm{Cl} / \mathrm{g}$ & $1.3807 \mathrm{E}-01$ & & \\
\hline 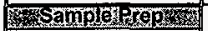 & & & . & \\
\hline FUSIONO1 & Rs (Sample Count Rate) $=(\mathrm{TC} / \mathrm{CT})-\mathrm{BKG}$ & & & \\
\hline 9. & $=R \mathrm{~s} * 1000 \mathrm{~mL} / \mathrm{L} * \mathrm{DF} /(\mathrm{EFF} * \mathrm{SS}$ & $\mathrm{Dg} / \mathrm{L} * 222000$ & Odpm/ $\mu \mathrm{Ci})$ & \\
\hline \$97T000588 & Relative Counting Error $=[1($ The Square Root of TC + BKG & $\mathrm{CT}) /(\mathrm{TC}-\mathrm{BK}$ & $\left.\left\langle G{ }^{*} \mathrm{CT}\right) \mid\right]^{*} 1.96$ & \\
\hline WB26872 & Detection Levels and Less Than Values are determined from $P$ & cedure LA-508 & 002. & \\
\hline Xringred $3 y$ & & & & \\
\hline SEH & & & & \\
\hline mestomist & & & & \\
\hline SLF & ALPHA TOTAL in $\mu$ Cigg (Average) & & 1.38E-01 & DETECTION \\
\hline Wharas & & & & LEVEL \\
\hline $\mathrm{SCL}$ & & & & 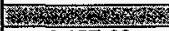 \\
\hline 20.jexomprom & & & & 3.15E-03 \\
\hline $05 / 07 / 97$ & RELATIVE COUNTING ERROR & & $9.2 \%$ & $\mu \mathrm{Ci} / \mathrm{g}$ \\
\hline 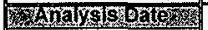 & & & & \\
\hline $05 / 06 / 97$ & & & & \\
\hline 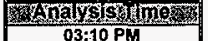 & & & & \\
\hline Samplojoms & . & & & \\
\hline$T-204$ & & & & \\
\hline
\end{tabular}

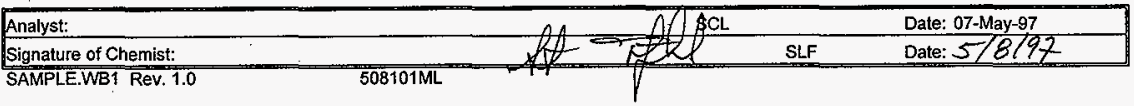




\section{HNF-SD-WM-DP-255, REV. 0 HAF-SDWAR-OP-245, REV. 9 ME}

WORKBOOK PAGE: DUP7

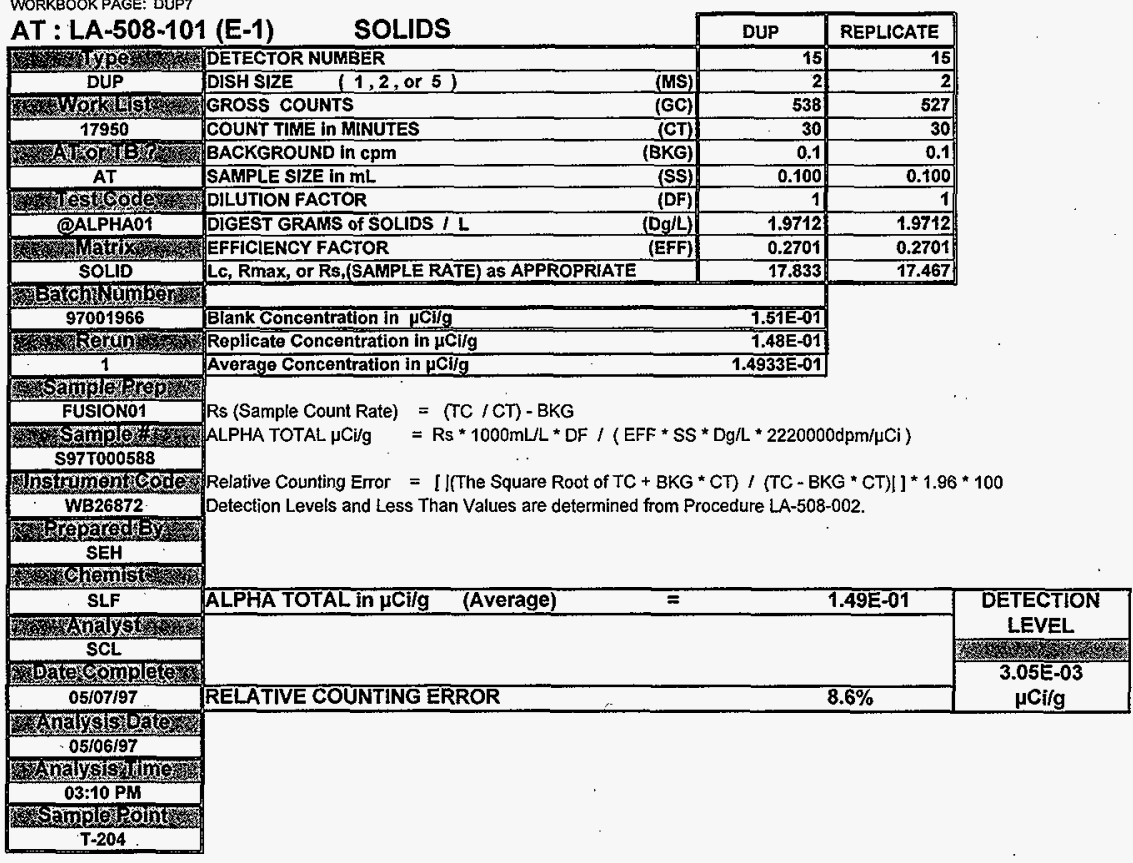

Analyst:
Signature of Chemist:
SAMPLE.WB1 Rev. 1.0




\section{HNF-SD-WM-DP-255, REV. 0}

WORKBOOK PAGE: SPK8

HNF-SD-WA-DP-245,REV.Onte

AT : LA-508-101 (E-1) LA-508-113 (B-0) SPIKED SAMPLE

A

SPK

17950

DISH SIZE

1,2 or 5

COUNT TIME in MINUTES

W\%

AT

BACKGROUND in cpm

SAMPLE VOLUME in $\mathrm{mL}$

(Spiked Vial)

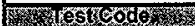

@ALPHAO 1

Kind

SOLID

DIGEST GRAMS of SOLIDS $/ L$

SPIKE VOLUME in $\mathrm{mL}$

SPIKE DILUTION FACTOR

Farch

97001966

SPIKE BOOK NUMBER

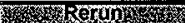

SPIKE VALUE in $\mu \mathrm{Cl} / \mathrm{mL}$

TRUMENT EFFICIENCY FACTOR

SAMPLE + SPIKE $\mu \mathrm{CI} / \mathrm{g}$

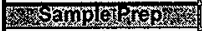

AVERAGE Or MAXIMUM $\mu$ CI/g in SAMPLE

FUSION01

Frample

S97T000588

Rs (Sample Count Rate) $=$ (TC / CT) $-B K G$

Finstrimentodo

WB26872

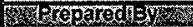

SEH

SAMPLE + SPIKE $\mu \mathrm{Ci} / 9$

$=R S * 1000 \mathrm{~mL} / \mathrm{L} * \mathrm{DF} /(\mathrm{EFF} * \mathrm{SS} * \mathrm{Dg} / \mathrm{L} * 2220000 \mathrm{dpm} / \mu \mathrm{Ci})$

QC ACTUAL $=S V a l$

QC FOUND $=\left(\left((S+S \mu \mathrm{Ci} / \mathrm{g}-\mathrm{SAMPLE} \mu \mathrm{Ci} / \mathrm{g}){ }^{*}\left(\left(\mathrm{SDF} /\left(\mathrm{SVO} \mathbf{l}^{*} 1000\right)\right) /(\mathrm{DF} / \mathrm{SS} / \mathrm{Dg} / \mathrm{L})\right)\right)\right)$

PERCENT SPIKE RECOVERY $=(Q C$ FOUND $/$ QC ACTUAL $) * 100$

WFims

SLF

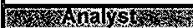

SCL

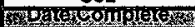

$05 / 07 / 97$

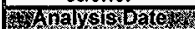

$05 / 06 / 97$

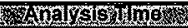

03:10 PM

QC ACTUAL

$=\quad 2.63 \mathrm{E}-02$

QC FOUND

$=1.98 \mathrm{E}-02$

AVG. PERCENT SPIKE RECOVERY

$=75.3 \%$

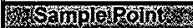

$T-204$

\begin{tabular}{llll}
\hline Analyst: & Date: 07-May-97 \\
Signature of Chemist: & Date: $5 / 8 / 97$ \\
\hline SPIKE.WB1 Rev. 1.0 & 508101ML
\end{tabular}




\section{HNF-SD-WM-DP-255, REV. 0}

WORKBOOK PAGE: SAM9

\section{AT : LA-508-101 (E-1) SOLIDS}

C6.

SAMPLE

DISH SIZE $(1,2$, or 5

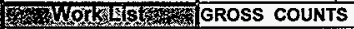

17950

H.

AT

COUNT TIME In MINUTES

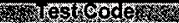

(aALHA01

BACKGROUND in cpm

SAMPLE SIZE in $\mathrm{mL}$

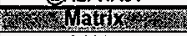

UTION FACTOR

DIGEST GRAMS Of SOLIDS / L

SOLID

EFFICIENCY FACTOR

Lc, Rmax, or Rs, (SAMPLE RATE) as APPROPRIATE

HNF-SD-WAA-DP-245,-REV.0-2,

$\frac{\text { Batchojumberm }}{97001966}$

Blank Concentration in $\mu \mathrm{Cl} / \mathrm{g}$

SAMPLE REPLICATE

(MS)

(GS)

(CT)

(BKG)

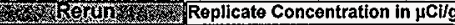

Average Concentration in $\mu \mathrm{C} / / \mathrm{g}$

(SS)

(DF)

$(\mathrm{Dg} / \mathrm{L})$

(EFF)

\begin{tabular}{|c|c|c|}
\hline (A)) & 1.9248 & 1.9248 \\
\hline & 0.2701 & 0.2701 \\
\hline & 15.400 & 16.367 \\
\hline
\end{tabular}

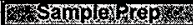

FUSIONO1

Rs (Sample Count Rate) $=(\mathrm{TC} / \mathrm{CT})-\mathrm{BKG}$

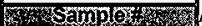

\$97T000589

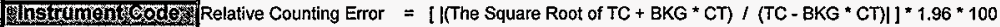

WB26872

Detection Levels and Less Than Values are determined from Procedure LA-508-002.

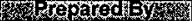

SEH

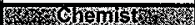

\begin{tabular}{|c|c|c|c|}
\hline SLF & ALPHA TOTAL in $\mu \mathrm{Ci} / \mathrm{g}$ & $1.38 \mathrm{E}-01$ & \multirow{2}{*}{$\begin{array}{l}\text { DETECTION } \\
\text { LEVEL }\end{array}$} \\
\hline 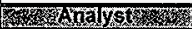 & & & \\
\hline $\mathrm{SCL}$ & & & 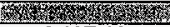 \\
\hline 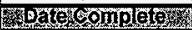 & & & 3.12E-03 \\
\hline $05 / 07 / 97$ & RELATIVE COUNTING ERROR & $9.2 \%$ & $\mu \mathrm{Ci} / \mathrm{g}$ \\
\hline
\end{tabular}

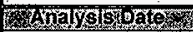
$05 / 06 / 97$

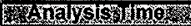

03:10 PM

Sranp T-204

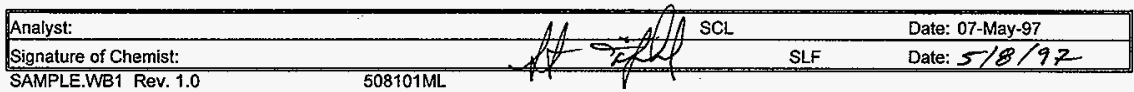


HNF-SD-WM-DP-255, REV. 0

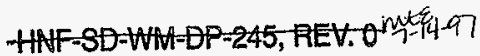

WORKBOOK PAGE: DUP10

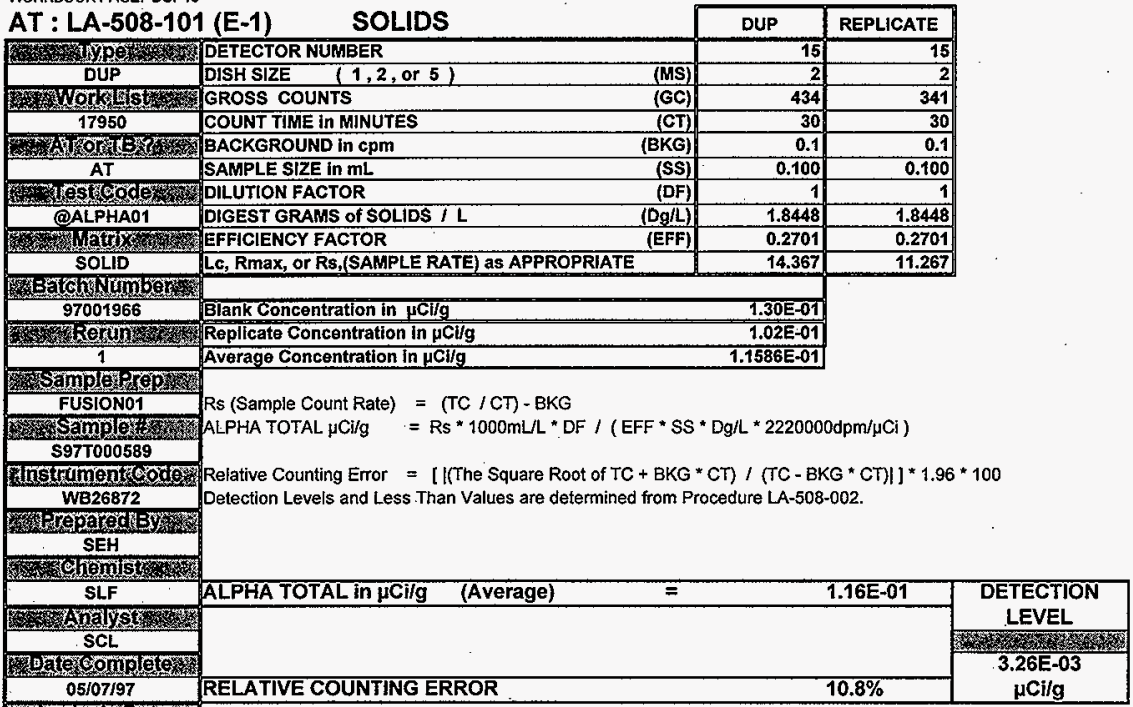

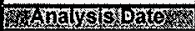
$05 / 06 / 97$

Fanarsisutmorman 03:10 PM

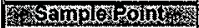
T-204

\begin{tabular}{llll}
\hline Analyst: & SCL & Date: 07-May-97 \\
\hline Signature of Chemist: & $508101 \mathrm{ML}$ & SLF & Date: $5 / 8 / 97$ \\
\hline SAMPLE.WB1 Rev. 1.0 & & &
\end{tabular}




\section{LABCORE Completed RadChem Report for Worklist\#: 18873}

Analyst: rdm

Method:

Method: $\longrightarrow$ Rev/Mod

\author{
Book\# \\ HNF-SD-WM-DP-255, REV。
}

Worklist Comment: T204, GEA. Use $5.0 \mathrm{~mL}$ sample size. $1 \mathrm{~mL}$ std. RERUN! llf

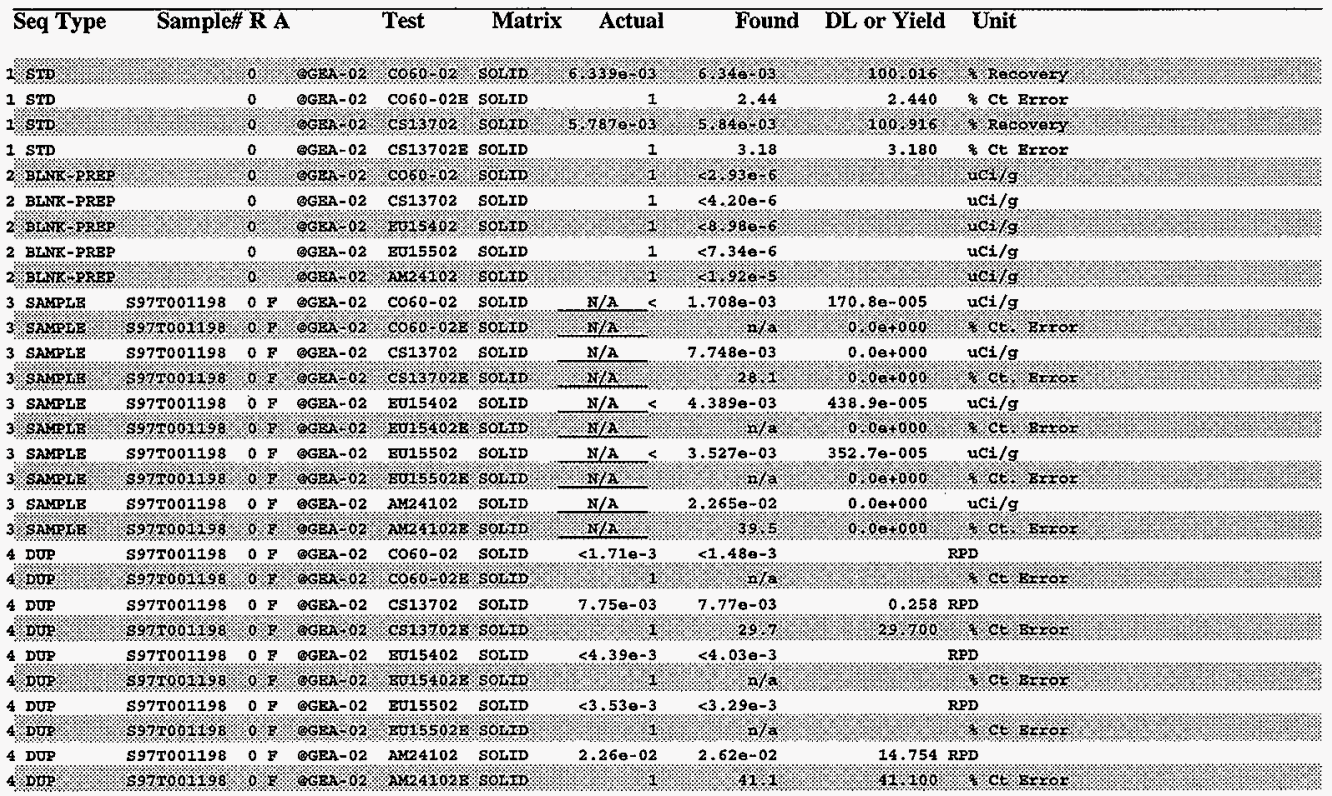

\section{Comments Section:}

Comments for sample\# S97T001198 and test @GEA-02 .

$\mathrm{DL}=0=>\mathrm{n} / \mathrm{a}$.

$\mathrm{DL}=0=>\mathrm{n} / \mathrm{a}$.

Units shown for $Q C(B L K / B K G)$ may not reflect the actual units. 
worklistrad Version 1.0 05/09/96

06/30/97 13:56

HNF-SD WAA-DR-245, REV O-

\section{LABCORE Completed RadChem Report for Worklist\#: 18873}

Seq Type Sample\# RA

Found DL or Yeld Unit

Final page for worklist\# 18873

Analyst Sïgnature

Date

Analyst Signature

Date

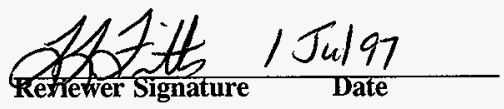




\section{LABCORE Data Entry Template for Worklist\# 18873}

Analyst: $\quad$ GDh Instrument: GEA00 Z Book\# 75856

Method: LA-548-121 Rev/Mod

HNF-SD-WM-DP-255, REV. 0

Worklist Comment: T204, GEA. Use $5.0 \mathrm{~mL}$ sample size. $1 \mathrm{~mL}$ std. RERUN! llf

S Type Sample\# R A Test Matrix Group\# Project

1 STD @GEA-02 SOIMD

2 BLNK-PREP @GEA-02 SOLID

3 SAMPLE S97T001198 O F @GEA-02 SOLID 97000261 T-204

Analytes Requested: AM24102, AM24102E, CO60-02, CO60-02E, CS13702,

CS13702E, EU15402, EU15402E, EU15502, EU15502E

4 DUP S97T001198 O F @GEA-02 SOLID

\section{Final page for worklist \# 18873}

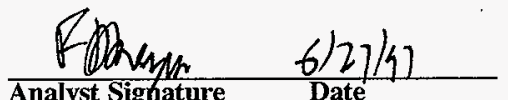

Analyst Sigaature
Date

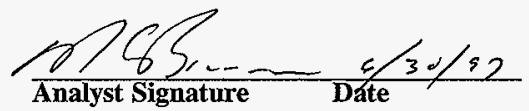

Data Entry Comments:

$S=$ Worklist Slot Number, $R=$ Replicate Number, $A=$ Aliquot Code. 


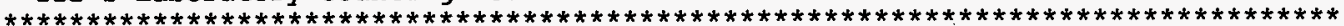

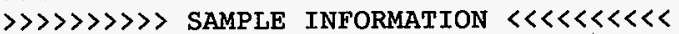

Worklist \#:

Sample ID:

Sample Size:

Dilution Factor:

$\gg \gg>>>>>>$ COUNT INFORMATION $<<<<<<<<<~$

Detector ID:

File Number:

Geometry:

Count Time:

Real Time:

Dead Time:
18873

WL18873-STD

$1.00000 \mathrm{E}-03 \mathrm{~L}$

$1.00000 \mathrm{E}+00$

GEA3

dka300: [ spec.GEA3] 3g3357. cnf

42

0 00:50:00.00 sec

$000: 50: 02.62 \mathrm{sec}$

$0.1 \%$

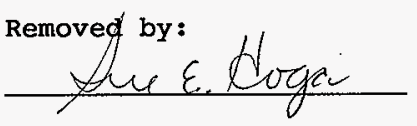

Verified by:

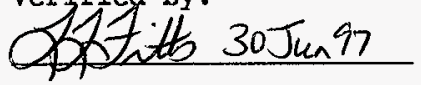

fet $27-14-9$

HAF-SOWATPR245, REV.O

HNF-SD-WM-DP-255, REV. 0
$>>>>>>>>$ ANALYSIS INFORMATION $<<<<<<<<<~$ Sample Count Time: Decayed to:

Standard Deviations :

Analysis Library:

Analyst:

Background Subtract:
26 -JUN- $1997 \quad 09: 55: 45.27$

26-JUN-1997 09:55:45.27

2

ENVGEA

CJO

DKA 300 : [ SPEC. GEA3] 3GBACK

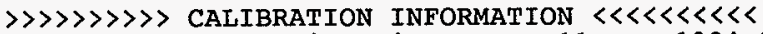

Date of last energy calibration: 11-MAR-1994 $11: 47: 01.11$

Date of last efficiency calibration: 15-MAR-1994 10:28:40.20

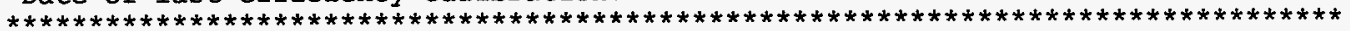

Post-NID Peak Search Report

It Energy

$0661.91 *$

$0 \quad 1172.93$

$0 \quad 1332.02$
Area

FWHM Channel

Left PW :Err

Fit

Nuclides

Activity $\mathrm{uCi} / \mathrm{L}$

5169
4105
3557

$1.57 \quad 1324.00$

1317

15

1.862346 .13

2338

$2.08 \quad 2664.42 \quad 2656$

3.2

3.5

$17 \quad 3.4$

CS -137

5.84

$\mathrm{CO}-60$

6.47

$\mathrm{CO}-60$

6.23 
Summary of Nuclide Activity Sample ID : WL18873-STD

Total number of lines in spectrum Number of unidentified lines Number of lines tentatively identified by NID 3

Nuclide Type :

$\begin{array}{lrrr}\text { Nuclide } & \text { Hlife } & \text { Decay } & \begin{array}{c}\text { Uncurrected } \\ \text { uCi/L }\end{array} \\ \text { CO-60 } & 5.27 Y & 1.00 & 6.344 \mathrm{E}+00 \\ \text { CS-137 } & 30.00 Y & 1.00 & 5.842 \mathrm{E}+00 \\ & \text { Total Activity }: & 1.219 \mathrm{E}+01\end{array}$

Grand Total Activity : $1.219 \mathrm{E}+01$

Flags: " $K "$ = Keyline not found
"E" = Manually edited mee -149

HNF-SO-WAMPR-245,REVO Page : 2

Acquisition date : 26-JUN-1997 09:55:45

HNF-SD-WM-DP-255, REV. 0 $100.00 \%$
Wtd Mean

Decay Corr Decay Corr 2-Sigma uCi/L

$6.344 \mathrm{E}+00$

$5.842 \mathrm{E}+00$

$-\cdots-1-\cdots$

2-Sigma Error $\%$ Error Flags

$0.155 \mathrm{E}+00 \quad 2.44$

$0.186 \mathrm{E}+00 \quad 3.18$
$1.219 \mathrm{E}+01$

"M" = Manually accepted

"A" = Nuclide specific abn. limit 
Mininum Detectable Activity Report Sample ID : WL18873-STD

Nuclide

Bckgnd

$\mathrm{BE}-7$

$\mathrm{NA}-22$

NA-24

$\mathrm{K}-40$

CR-51

$\mathrm{MN}-54$

$\mathrm{CO}-56$

$\mathrm{CO}-57$

$\mathrm{CO}-58$

$\mathrm{FE}-59$

$\mathrm{SE}-75$

$\mathrm{SR}-85$

$\mathrm{Y}-88$

NB-94

ZRNB-95

$\mathrm{RU}-103$

RURH-106

AG-108m

CD-109

AG-110M

$\mathrm{SN}-113$

$T E-123 \mathrm{~m}$

$\mathrm{SB}-124$

SB-125

TE-125m

I -131

CS-134

$\mathrm{BA}-140$

LA -140

CEPR-144

EU-152

EU-154

$\mathrm{EU}-155$

HG-203

TL-208

BI -212

PB-212

BI -214

$\mathrm{PB}-214$

$\mathrm{RA}-224$

$\mathrm{RA}-226$

AC- 228

$\mathrm{TH}-228$

$\mathrm{TH}-229$

$\mathrm{PA}-233$

UTH-233

$\mathrm{PA}-234 \mathrm{M}$

$\mathrm{TH}-234$

$\mathrm{U}-235$

NP -237

NP-239

$\begin{array}{rr}311 . & 477.59 \\ 33 . & 1274.53 \\ 13 . & 1368.55 \\ 127 . & 1460.75 \\ 312 . & 320.08 \\ 174 . & 834.83 \\ 186 . & 846.76 \\ 372 . & 122.06 \\ 188 . & 810.78 \\ 213 . & 1099.25 \\ 400 . & 264.66 \\ 273 . & 514.01 \\ 2 . & 1836.06 \\ 216 . & 871.09 \\ 157 . & 724.18 \\ 220 . & 497.08 \\ 173 . & 621.93 \\ 154 . & 722.94 \\ 327 . & 88.03 \\ 240 . & 657.76 \\ 336 . & 391.69 \\ 370 . & 159.00 \\ 167 . & 602.73 \\ 306 . & 427.89 \\ 330 . & 109.27 \\ 302 . & 364.48 \\ 176 . & 604.70 \\ 182 . & 537.31 \\ 8 . & 1596.21 \\ 374 . & 133.51 \\ 10 . & 1408.01 \\ 33 . & 1274.51 \\ 329 . & 105.31 \\ 384 . & 279.20 \\ 386 . & 277.36 \\ 160 . & 727.18 \\ 469 . & 238.63 \\ 208 . & 609.31 \\ 333 . & 351.92 \\ 463 . & 240.99 \\ 401 . & 186.10 \\ 240 . & 911.21 \\ 341 . & 84.37 \\ 319 . & 312.17 \\ 334 . & 1001.03 \\ 428 . & 63.03 \\ 215 . & 86.48 \\ 281 . & \\ 403 . & \\ 346 . & \\ 341 . & \end{array}$

\section{Energy}

(kev)

477.59

1274.53

1460.75

320.08

836.76

122.06

264.66

836.06

871.09

724.18

497.08

722.94

391.69

159.00

602.73

427.89

364.48

604.70

596.21

133.51

1408.01

274.51
105.31

279.20

277.36

727.18

238.63

609.31

240.99

911.21

84.37

88.47
312.17

245.34

1001.03

85.71

106.12
HNF-SD-WATDP-245, REV.O

$M+\varepsilon 7-14-97$ Page :

Acquisition date : 26-JUN-1997 09:55:45

\section{MDA}

(uCi/L) HNF-SD-WM-DP-255, REV 0

6.0051E-01

$4.9898 \mathrm{E}-02$

$3.5239 \mathrm{E}-02$

9.6946E-01

4.5209E-01

$7.5269 \mathrm{E}-02$

$7.8793 \mathrm{E}-02$

$3.4407 \mathrm{E}-02$

$7.6581 \mathrm{E}-02$

$1.8619 \mathrm{E}-01$

7. 6485E-02

$6.4475 \mathrm{E}-02$

$2.0280 \mathrm{E}-02$

8. $6662 \mathrm{E}-02$

$1.4539 \mathrm{E}-01$

$5.9582 \mathrm{E}-02$

$1.1765 \mathrm{E}+00$

$6.9235 E-02$

$1.0692 \mathrm{E}+00$

$7.5784 \mathrm{E}-02$

8.5922E-02

$3.5277 \mathrm{E}-02$

$5.7161 E-02$

$1.9193 \mathrm{E}-01$

$1.0566 \mathrm{E}+01$

6.1009E-02

$5.8844 \mathrm{E}-02$

$2.1648 \mathrm{E}-01$

3. $3055 \mathrm{E}-02$

$5.2112 \mathrm{E}-01$

$1.5063 \mathrm{E}-01$

$1.4489 \mathrm{E}-01$

$1.4085 \mathrm{E}-01$

5.5908E-02

7. 1997E-01

$9.6707 \mathrm{E}-01$

$1.0270 \mathrm{E}-01$

1. $3974 \mathrm{E}-01$

$2.5160 \mathrm{E}-01$

$1.1336 \mathrm{E}+00$

9.6471E-01

3. $5622 \mathrm{E}-01$

$3.3627 \mathrm{E}+00$

$1.5309 \mathrm{E}-01$

$1.1982 \mathrm{E}-01$

$4.0907 \mathrm{E}+01$

$1.6521 \mathrm{E}-01$

$2.0635 \mathrm{E}+00$

$5.8782 \mathrm{E}-02$

$3.3027 \mathrm{E}-01$

$1.3613 \mathrm{E}-01$ 
Minimu Detectable Activity Report (continued)

Sample ID : WL18873-STD

Acquisition date : 26-JUN-1997 09:55:45

Nuclide

PU-239

$\mathrm{AM}-241$

$\mathrm{AM}-243$

\section{Bckgnd}

Sum

373.

323.

321.
Energy

(kev)

$$
\begin{array}{r}
129.30 \\
59.54 \\
74.67
\end{array}
$$

MDA ( $\mathrm{UCi} / \mathrm{L}$ )

HNF-SD-WM-DP-255, REV. 0

$$
\begin{aligned}
& 4.5304 E+02 \\
& 3.8917 E-01 \\
& 9.1243 E-02
\end{aligned}
$$

HAE?-14-97 


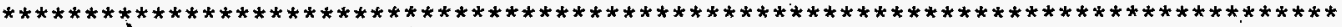

* 222-S 'Laboratory Counting Room 26-JUN-1997 13:22:34.96

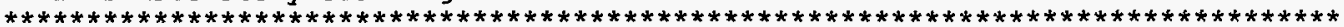
$>>>>>>>>>$ SAMPLE INFORMATION $<<<<<<<<<~$

Worklist \#:

Sample ID:

Sample Size:

Dilution Factor:

$\gg \gg \gg \gg \gg \gg \gg>$ COUNT INFORMATION $<<<<<<<<<~$

Detector ID:

File Number:

Geometry:

Count Time:

Real Time:

Dead Time:
18873

WL18873-BLK

$5.00000 \mathrm{E}-03 \mathrm{I}$

$1.00000 \mathrm{E}+00$

GEA3

dka300: [ spec.GEA3] 3g3358.cnf

42

$002: 30: 00.00 \mathrm{sec}$

0 02:30:01.21 sec

$0.0 \%$

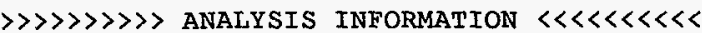

Sample Count Time: 26-JUN-1997 10:51:38.49

Decayed to:

Standard Deviations:

Analysis Library:

Analyst: 26-JUN-1997 10:51:38.49

2

ENVGEA

SEH

Background Subtract: DKA300:[SPEC.GEA3]3GBACK

Remoyed by:

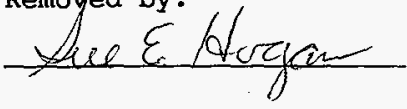

Verified by:

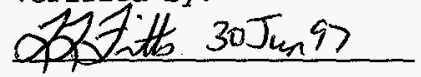

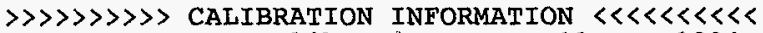

Date of last energy calibration: 11-MAR-1994 11:47:01.11

Date of last efficiency calibration: 15-MAR-1994 10:28:40.20

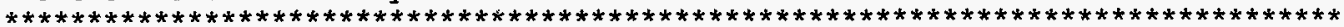

Post-NID Peak Search Report

It Energy

$0 \quad 84.90$

$661.89 *$

$1119.61 *$

1312.97

1441.41
Area FWHM Channel

Left $\mathrm{PW}$ \% $\mathrm{Err}$

167

1318

2232

2620

2877
885.4

1398.8

1457.3

12116.6

9126.3
Fit Nuclides Activity $\mathrm{uCi} / \mathrm{L}$

TH-228 0.160

CS-137 4.199E-03 
Minimum Detectable Activity Report Sample ID : WL18873-BLK

Nuclide

Bckgnd

Sum

Energy (keV)

$\mathrm{BE}-7$

NA -22

NA-24

$\mathrm{K}-40$

$\mathrm{CR}-51$

$\mathrm{MN}-54$

$\mathrm{CO}-56$

$\mathrm{CO}-57$

$\mathrm{CO}-58$

$\mathrm{FE}-59$

$\mathrm{CO}-60$

$\mathrm{SE}-75$

$\mathrm{SR}-85$

$\mathrm{Y}-88$

NB-94

ZRNB-95

RU-103

RURH-106

AG- $108 \mathrm{~m}$

CD-109

AG- $110 \mathrm{M}$

SN-113

TE-123m

SB-124

SB- 125

$\mathrm{TE}-125 \mathrm{~m}$

I-131

CS-134

$\mathrm{BA}-140$

LA- 140

CEPR-144

EU-152

EU-154

EU-155

HG-203

TL-208

BI -212

PB-212

BI-214

PB-214

$\mathrm{RA}-224$

RA -226

$\mathrm{AC}-228$

TH-229

$\mathrm{PA}-233$

UTH-233

$P A-234 \mathrm{M}$

$\mathrm{TH}-234$

$\mathrm{U}-235$

NP -237

NP-239

$\begin{array}{rr}90 . & 477.59 \\ 28 . & 1274.53 \\ 18 . & 1368.55 \\ 374 . & 1460.75 \\ 136 . & 320.08 \\ 35 . & 834.83 \\ 42 . & 846.76 \\ 226 . & 122.06 \\ 59 . & 810.78 \\ 35 . & 1099.25 \\ 23 . & 1332.50 \\ 164 . & 264.66 \\ 156 . & 514.01 \\ 11 . & 1836.06 \\ 47 . & 871.09 \\ 53 . & 724.18 \\ 90 . & 497.08 \\ 62 . & 621.93 \\ 55 . & 722.94 \\ 163 . & 88.03 \\ 67 . & 657.76 \\ 119 . & 391.69 \\ 231 . & 159.00 \\ 74 . & 602.73 \\ 116 . & 427.89 \\ 208 . & 109.27 \\ 120 . & 364.48 \\ 75 . & 604.70 \\ 91 . & 537.31 \\ 14 . & 1596.21 \\ 253 . & 133.51 \\ 23 . & 1408.01 \\ 28 . & 1274.51 \\ 198 . & 105.31 \\ 197 . & 279.20 \\ 165 . & 277.36 \\ 57 . & 727.18 \\ 265 . & 238.63 \\ 142 . & 609.31 \\ 169 . & 351.92 \\ 259 . & 240.99 \\ 228 . & 186.10 \\ 87 . & 911.21 \\ 161 . & 88.47 \\ 186 . & 312.17 \\ 197 . & 1001.03 \\ 46 . & 63.29 \\ 161 . & 86.48 \\ 240 . & 106.12 \\ 215 . & \\ 197 . & \end{array}$

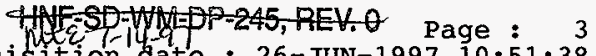

Acquisition date : 26-JUN-1997 10:51:38

\author{
$\mathrm{MDA}$
$\mathrm{uCi} / \mathrm{L})$ HNF-SD-WM-DP-255, REV. 0
}

$2.2077 \mathrm{E}-02$

$3.0922 \mathrm{E}-03$

$2.8608 \mathrm{E}-03$

$1.0865 \mathrm{E}-01$

$2.0220 \mathrm{E}-02$

$2.3824 \mathrm{E}-03$

$2.6171 \mathrm{E}-03$

$1.8016 \mathrm{E}-03$

$2.9611 \mathrm{E}-03$

$5.3328 \mathrm{E}-03$

$2.9307 \mathrm{E}-03$

$3.3185 \mathrm{E}-03$

$3.2871 \mathrm{E}-03$

$2.7087 \mathrm{E}-03$

$2.8100 \mathrm{E}-03$

$5.8075 \mathrm{E}-03$

$2.6027 \mathrm{E}-03$

4. $8465 \mathrm{E}-02$

$2.8289 E-03$

$5.1014 \mathrm{E}-02$

$2.7447 \mathrm{E}-03$

$3.4793 \mathrm{E}-03$

$1.8730 \mathrm{E}-03$

$2.5882 \mathrm{E}-03$

8.0482E-03

$5.6412 \mathrm{E}-01$

$2.6264 \mathrm{E}-03$

$2.6201 \mathrm{E}-03$

$1.0410 \mathrm{E}-02$

$2.8963 \mathrm{E}-03$

$2.8774 \mathrm{E}-02$

$1.4484 \mathrm{E}-02$

8.9837E-03

$7.3433 \mathrm{E}-03$

$2.6974 \mathrm{E}-03$

3. $1863 \mathrm{E}-02$

$3.9494 \mathrm{E}-02$

5.1953E-03

$7.7574 \mathrm{E}-03$

2. $6897 \mathrm{E}-02$

$5.7407 \mathrm{E}-02$

$4.8968 \mathrm{E}-02$

$1.4610 \mathrm{E}-02$

$7.3411 \mathrm{E}-03$

$6.0270 \mathrm{E}-03$

$1.8729 \mathrm{E}+00$

$5.3365 \mathrm{E}-03$

$1.0509 \mathrm{E}-01$

$3.0468 \mathrm{E}-03$

$1.7485 \mathrm{E}-02$

$7.0463 \mathrm{E}-03$ 
Minimum Detectable Activity Report (continued)

Sample' ID : WL18873-BLK

Acquisition date : 26-JUN-1997 10:51:38

Nuclide

PU-239

$\mathrm{AM}-241$

$A M-243$

\section{Bckgnd}

sum

208.

173.

196.
Energy (kev)

129.30

59.54

74.67
MDA

( $\mathrm{uCi} / \mathrm{L}$ ) HNF-SD-WM-DP-255, REV. 0

$2.2775 \mathrm{E}+01$

$1.9209 \mathrm{E}-02$

$4.7987 \mathrm{E}-03$

WHEZ-149 


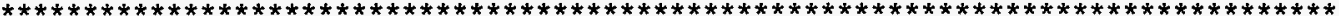

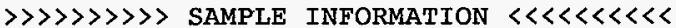

Worklist \#:

Sample ID:

Sample Size:

Dilution Factor:
18873

S97T001198-SAM

$5.00000 \mathrm{E}-03 \mathrm{~L}$

$1.00000 \mathrm{E}+00$
Removed by:

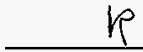

Detector ID:

COUNT INFORMATION $<\ll<く<く<く<~$

File Number:

GEA3

Geometry:

dka 300 : [ spec. GEA3] 3g3359.cnf

42

Count Time:

0 02:30:00.00 sec

$0 \quad 02: 30: 01.24$ sec

Real Time:

Dead Time:

$0.0 \%$

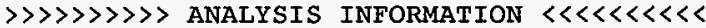

Sample Count Time:

26-JUN-1997 14:11:02.96

Decayed to:

26-JUN-1997 14:11:02.96

Standard Deviations: 2

Analysis Library:

Analyst:

ENVGEA

SEH

Background Subtract: DKA300: [SPEC.GEA3] 3GBACK

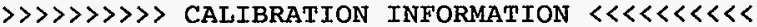

Date of last energy calibration: 11-MAR-1994 11:47:01.11

Date of last efficiency calibration: 15-MAR-1994 10:28:40.20

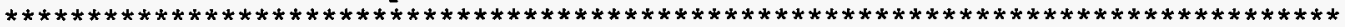

Post-NID Peak Search Report

It Energy

Area FWHM Channel Left Pw \%Err

Fit

Nuclides

Activity uCi/L

$\begin{array}{lrrrrrrr}0 & 59.41 & 165 & 1.11 & 119.36 & 115 & 9 & 39.5 \\ 0 & 661.87 * & 225 & 1.46 & 1323.92 & 1316 & 13 & 28.1 \\ 0 & 911.05 * & 30 & 1.02 & 1822.27 & 1816 & 12 & 96.7\end{array}$

AM-241

CS -137

$4.970 \mathrm{E}-02$

$1.698 \mathrm{E}-02$ 
Summary of Nuclide Activity

Total number of lines in spectrum Number of unidentified lines

Number of lines tentatively identified by NID Sample ID : S97T001198-SAM

Adquisition date : 26-JUN-1997 14:11:02

\section{Nuclide Type :}

$\begin{array}{lrrr}\text { Nuclide } & \text { Hlife } & \text { Decay } & \begin{array}{c}\text { Uncorrected } \\ \text { uCi } / \mathrm{L}\end{array} \\ \text { CS-137 } & 30.00 \mathrm{Y} & 1.00 & 1.698 \mathrm{E}-02 \\ \text { AM-241 } & 432.20 \mathrm{Y} & 1.00 & 4.970 \mathrm{E}-02 \\ & \text { Total Activity }: & 6.668 \mathrm{E}-02\end{array}$

Grand Total Activity : $6.668 \mathrm{E}-02$

$6.668 \mathrm{E}-02$

Flags: "K" = Keyline not found

"E" = Manually edited
3

0

$3 \quad 100.00 \%$
HNF-SD-WM-DP-255, REV. 0
"M" = Manually accepted

"A" = Nuclide specific abn. limit 
Minimum Detectable Activity Report Sample ID : S97T001198-SAM

Nuclide

$\mathrm{BE}-7$

NA -22

NA-24

$\mathrm{K}-40$

CR-5I

$\mathrm{MN}-54$

$\mathrm{CO}-56$

$\mathrm{CO}-57$

$\mathrm{CO}-58$

$\mathrm{FE}-59$

$\mathrm{CO}-60$

$\mathrm{SE}-75$

$\mathrm{SR}-85$

$\mathrm{Y}-88$

NB-94

ZRNB-95

$\mathrm{RU}-103$

RURH-106

AG $-108 \mathrm{~m}$

CD-109

AG-110M

$\mathrm{SN}-113$

TE-123m

SB-124

SB-125

TE-125m

I -131

CS-134

$\mathrm{BA}-140$

$\mathrm{LA}-140$

CEPR-144

EU-152

$\mathrm{EU}-154$

EU-155

HG-203

TL-208

BI -212

PB-212

BI-214

$\mathrm{PB}-214$

RA-224

RA-226

$\mathrm{AC}-228$

$\mathrm{TH}-228$

TH-229

$P A-233$

UTH-233

PA-234M

TH-234

$\mathrm{U}-235$

NP -237

Bckgnd
Sum

$114 . \quad 477.59$

33. $\quad 1274.53$

25. 1368.55

363. 1460.75

150. $\quad 320.08$

52. 834.83

56. 846.76

222 .

46 .

22 .

40 .

209 .

137 .

10 .

41 .

52 .

92 .

69.

50 .

189.

64 .

126.

223.

64 .

102 .

235 .

137 .

72 .

89.

17 .

249 .

28.

33.

220 .

196.

190.

69.

267.

146 .

177.

249 .

230 .

91.

210.

183.

149.

203.

34 .

181.

221.

200 .

122.06

810.78

1099.25

1332.50

264.66

514.01

1836.06

871.09

724.18

497.08

621.93

722.94

88.03

657.76

391.69

159.00

602.73

427.89

109.27

364.48

604.70

537.31

1596.21

133.51

1408.01

1274.51

105.31

279.20

277.36

727.18

238.63

609.31

351.92

240.99

186.10

911.21

84.37

88.47

312.17

245.34

1001.03

63.29

185.71

86.48 $20+\varepsilon-14-9$

HAF-SD-WHAP-245,REV O Page : Acquisition date : 26-JUN-1997 14:11:02
MDA

( $\mathrm{UCi} / \mathrm{L})$

HNF-SD-WM-DP-255, REV. 0
$2.4739 \mathrm{E}-02$

$3.2899 \mathrm{E}-03$

$1.0715 \mathrm{E}-01$

$2.1233 \mathrm{E}-02$

$2.8413 \mathrm{E}-03$

$2.9667 \mathrm{E}-03$

$1.7848 \mathrm{E}-03$

$2.6430 \mathrm{E}-03$

$4.2912 \mathrm{E}-03$

$3.7464 \mathrm{E}-03$

$3.7230 \mathrm{E}-03$

$3.0881 \mathrm{E}-03$

$2.6191 \mathrm{E}-03$

$2.6450 \mathrm{E}-03$

$5.7494 \mathrm{E}-03$

$2.6247 \mathrm{E}-03$

5.0702E-02

$2.7218 \mathrm{E}-03$

$5.4691 \mathrm{E}-02$

$2.6982 \mathrm{E}-03$

$3.5780 \mathrm{E}-03$

$1.8399 \mathrm{E}-03$

$2.4293 \mathrm{E}-03$

$7.5701 \mathrm{E}-03$

$5.9755 \mathrm{E}-01$

$2.7908 \mathrm{E}-03$

$2.5691 \mathrm{E}-03$

$1.0274 \mathrm{E}-02$

$3.1226 \mathrm{E}-03$

$2.8551 \mathrm{E}-02$

$1.6029 \mathrm{E}-02$

$9.6295 \mathrm{E}-03$

$7.7376 \mathrm{E}-03$

$2.6927 \mathrm{E}-03$

$3.4060 \mathrm{E}-02$

$4.3167 \mathrm{E}-02$

$5.2070 E-03$

$7.8660 \mathrm{E}-03$

2.7489E-02

$5.6362 \mathrm{E}-02$

$4.9200 \mathrm{E}-02$

$1.4927 \mathrm{E}-02$

$1.7732 \mathrm{E}-01$

$7.8109 \mathrm{E}-03$

$5.4270 \mathrm{E}-03$

$1.9027 \mathrm{E}+00$

$4.6096 \mathrm{E}-03$

$1.1137 \mathrm{E}-01$

$2.9284 \mathrm{E}-03$

$1.6905 E-02$
$3.3164 \mathrm{E}-03$ 
Minimum Detectable Activity Report (continued)

Page : 4

Sample ID : S97T0011.98-SAM

Acquisition date : 26-JUN-1997 14:11:02

Nuclide

NP -239

$\mathrm{PU}-239$

$\mathrm{AM}-243$

\section{Bckgnd}

Sum

219.

245 .

222 .
Energy

( $\mathrm{keV})$

106.12

129.30

74.67

\section{MDA \\ (uCi/L)}

$7.4054 \mathrm{E}-03$ HNF-SD-WM-DP-255, REV. 0

$2.4662 \mathrm{E}+01$

$5.0866 \mathrm{E}-03$ iter $2-14 \Omega$

HAF-SD-WHA-DP-245, REV.O 


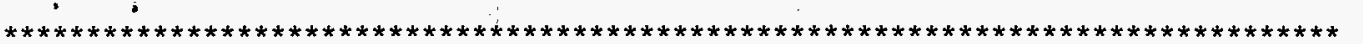

* 222-S Laboratory Counting Room 26-JUN-1997 19:31:52.51

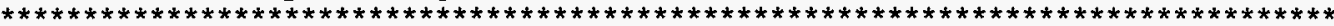

Worklist \#:

Sample ID:

Sample Size:

Dilution Factor:

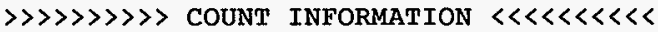

Detector ID:

File Number:

Geometry:

Count Time:

Real Time:

Dead Time:

18873

S97T1198-DUP

$5.00000 \mathrm{E}-03 \mathrm{~L}$

$1.00000 \mathrm{E}+00$

GEA3

42

0 02:30:00.00 sec

$002: 30: 01.24 \mathrm{sec}$

$0.0 \%$ dka300: [ spec.GEA3] 3g3361.cnf

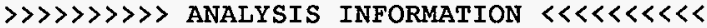

Sample Count Time:

Decayed to:

Standard Deviations:

Analys is Library:

Analyst:

Background Subtract:
26-JUN-1997 $17: 01: 13.73$

$26-J U N-1997 \quad 17: 01: 13.73$

2

ENVGEA

VR

DKA300: [ SPEC. GEA3] 3GBACK
Removed by:

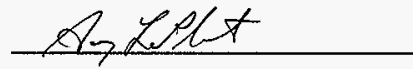

Verified by:

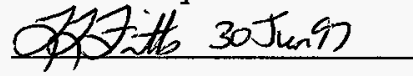

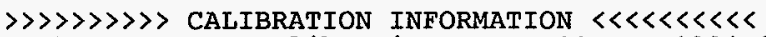

Date of last energy calibration: 11-MAR-1994 11:47:01.11

Date of last efficiency calibration: 15-MAR-1994 10:28:40.20

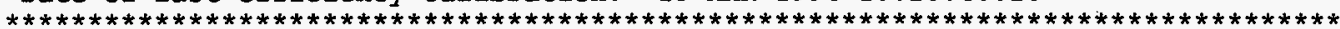

Post-NID Peak Search Report

It Energy

Area FWHM Channel Left PW $\%$ Err

Fit Nuclides

Activity uCi/L

$0 \quad 59.61$

$\begin{array}{lll}190 & 1.18 & 119.77\end{array}$

115

1241.1

$224 \quad 1.67 \quad 1324.12$

$\begin{array}{lll}1318 & 15 & 29.7\end{array}$

AM-241

CS-137

$5.703 \mathrm{E}-02$

1. $689 \mathrm{E}-02$ 
Summary of Nuclide Activity Sample ID : S97T1198-DUP

Total number of lines in spectrum Number of unidentified lines

Number of lines tentatively identified by NID
HNF-SD-WIN-DP-245, REV. Qnat $\varepsilon-14-97$

Page :

Acquisition date : 26-JUN-1997 17:01:13
Nuclide Type :

\begin{tabular}{|c|c|c|c|}
\hline $\begin{array}{l}\text { Nuclide } \\
\text { CS-137 } \\
\text { AM-241 }\end{array}$ & $\begin{array}{r}\text { H1ife } \\
30.00 Y \\
432.20 Y\end{array}$ & $\begin{array}{r}\text { Decay } \\
1.00 \\
1.00\end{array}$ & $\begin{array}{c}\text { Uncorrectec } \\
\text { uCi/L } \\
1.689 \mathrm{E}-02 \\
5.703 \mathrm{E}-02\end{array}$ \\
\hline & Total & : & 7.3921 \\
\hline
\end{tabular}

Grand Total Activity : $7.392 \mathrm{E}-02$

Flags: " $\mathrm{K}$ " = Keyline not found

"E" = Manually edited
2 HNF-SD-WM-DP-255, REV. 0

$100.00 \%$
Wtd Mean

Decay Corr Decay Corr 2-Sigma

uCi/L 2-Sigma Error \%Error Flags

$1.689 \mathrm{E}-02$

$5.703 E-02$

$0.502 \mathrm{E}-02$

29.73

$2.347 \mathrm{E}-02 \quad 41.15$

$7.392 \mathrm{E}-02$

$7.392 E-02$

"M" = Manually accepted

"A" = Nuclide specific abn. limit 
Minimum Detectable Activity Report Sample ID : S97T1198-DUP

\begin{tabular}{|c|c|c|}
\hline Nuclide & $\begin{array}{l}\text { Bckgnd } \\
\text { Sum }\end{array}$ & $\begin{array}{l}\text { Energy } \\
(\mathrm{keV})\end{array}$ \\
\hline $\begin{array}{l}\text { BE-7 } \\
\text { NA-22 } \\
\text { NA-24 } \\
\mathrm{K}-40 \\
C R-51 \\
M N-54 \\
\mathrm{CO}-56 \\
\mathrm{CO}-57 \\
\mathrm{CO}-58 \\
\mathrm{FE}-59 \\
\mathrm{CO}-60 \\
\mathrm{SE}-75 \\
\mathrm{SR}-85 \\
\mathrm{Y}-88 \\
\mathrm{NB}-94 \\
\mathrm{ZRNB}-95 \\
\mathrm{RU}-103 \\
\mathrm{RURH} \\
\mathrm{AG}-108 \mathrm{~m} \\
\mathrm{CD}-109 \\
\mathrm{AG}-110 \mathrm{~m} \\
\mathrm{SN}-113 \\
\mathrm{TE}-123 \mathrm{~m} \\
\mathrm{SB}-124 \\
\mathrm{SB}-125 \\
\mathrm{TE}-125 \mathrm{~m} \\
\mathrm{I}-131 \\
\mathrm{CS}-134 \\
\mathrm{BA}-140 \\
\mathrm{LA}-140 \\
\mathrm{CEPR}-144 \\
\mathrm{EU}-152 \\
\mathrm{EU}-154 \\
\mathrm{EU}-155 \\
\mathrm{HG}-203 \\
\mathrm{TL}-208 \\
\mathrm{BI}-212 \\
\mathrm{~PB}-212 \\
\mathrm{BI}-214 \\
\mathrm{~PB}-214 \\
\mathrm{RA}-224 \\
\mathrm{RA}-226 \\
\mathrm{AC}-228 \\
\mathrm{TH}-228 \\
\mathrm{TH}-229 \\
\mathrm{PA}-233 \\
\mathrm{UTH}-233 \\
\mathrm{PA}-234 \mathrm{M} \\
\mathrm{TH}-234 \\
\mathrm{U}-235 \\
\mathrm{NP}-237 \\
\end{array}$ & 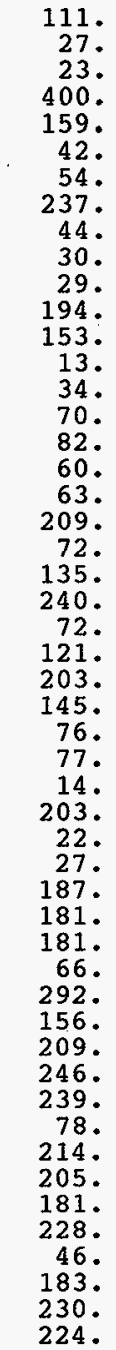 & $\begin{array}{r}477.59 \\
1274.53 \\
1368.55 \\
1460.75 \\
320.08 \\
834.83 \\
846.76 \\
122.06 \\
810.78 \\
1099.25 \\
1332.50 \\
264.66 \\
514.01 \\
1836.06 \\
871.09 \\
724.18 \\
497.08 \\
621.93 \\
722.94 \\
88.03 \\
657.76 \\
391.69 \\
159.00 \\
602.73 \\
427.89 \\
109.27 \\
364.48 \\
604.70 \\
537.31 \\
1596.21 \\
133.51 \\
1408.01 \\
1274.51 \\
105.31 \\
279.20 \\
277.36 \\
727.18 \\
238.63 \\
609.31 \\
351.92 \\
240.99 \\
186.10 \\
911.21 \\
84.37 \\
88.47 \\
312.17 \\
245.34 \\
1001.03 \\
63.29 \\
185.71 \\
86.48\end{array}$ \\
\hline
\end{tabular}

HAF-SD-WMIDP-245, REV.O-

put? $]$ - Page : 3

Accuisition date : 26-JUN-1997 17:01:13

$\mathrm{MDA}$
$(\mathrm{uC} i / L) \quad$ HNF-SD-WM-DP-255, REV. 0

$2.4392 \mathrm{E}-02$

$3.0205 \mathrm{E}-03$

$3.1315 \mathrm{E}-03$

1. $1228 \mathrm{E}-01$

$2.1814 \mathrm{E}-02$

$2.5696 \mathrm{E}-03$

$2.9306 \mathrm{E}-03$

$1.8425 \mathrm{E}-03$

$2.5798 \mathrm{E}-03$

$4.9424 \mathrm{E}-03$

$3.2267 \mathrm{E}-03$

$3.5891 \mathrm{E}-03$

$3.2503 \mathrm{E}-03$

$2.9123 \mathrm{E}-03$

$2.4199 \mathrm{E}-03$

$6.6262 \mathrm{E}-03$

2. $4909 \mathrm{E}-03$

4. $7431 \mathrm{E}-02$

$3.0328 \mathrm{E}-03$

5. $7449 \mathrm{E}-02$

2. $8449 \mathrm{E}-03$

3. $6954 \mathrm{E}-03$

$1.9067 \mathrm{E}-03$

2. 5595E-03

8. 2070E-03

$5.5675 \mathrm{E}-01$

2.8691E-03

$2.6436 \mathrm{E}-03$

$9.6267 \mathrm{E}-03$

$2.8573 \mathrm{E}-03$

2. $5873 \mathrm{E}-02$

$1.4196 \mathrm{E}-02$

$8.7637 \mathrm{E}-03$

$7.1451 \mathrm{E}-03$

$2.5960 \mathrm{E}-03$

3. $3263 \mathrm{E}-02$

4. 2422E-02

5. $4385 \mathrm{E}-03$

8.1176E-03

$2.9783 \mathrm{E}-02$

5. $6013 \mathrm{E}-02$

$5.0066 \mathrm{E}-02$

I. $3880 \mathrm{E}-02$

1. $7895 \mathrm{E}-01$

$8.2405 \mathrm{E}-03$

$5.9407 \mathrm{E}-03$

$2.0116 \mathrm{E}+00$

5. 3143E-03

$1.1187 \mathrm{E}-01$

$2.9861 \mathrm{E}-03$

$1.7822 \mathrm{E}-02$ 
Minimum Detectable Activity Report (continued)

Sample ID : S97T1198-DUP

Acquisition date : 26-JUN-1997 17:01:13

Nuclide

NP-239

$\mathrm{PU}-239$

AM-243
Bckgnd

Sum

191.
232.
207.
Energy (kev)

106.12

129.30

74.67
MDA

( $\mathrm{UC} i / L)$

$6.9359 \mathrm{E}-03$

$2.4033 \mathrm{E}+01$ HNF-SD-WM-DP-255, REV. 0 4.9151E-03
Page 4 


\section{LABCORE Completed Worklist Report for Worklist\# 18756}

Analyst: $\mathrm{krq}$

Instrument: $\mathrm{AB} 12$

Book\#

Method:

$\operatorname{Rev} / \mathrm{Mod}$

HNF-SD-WM-DP-255, REV. 0

Worklist Comment: T204, @SR90-01 S.S. by Ludlum. Std: 1.0mL skm

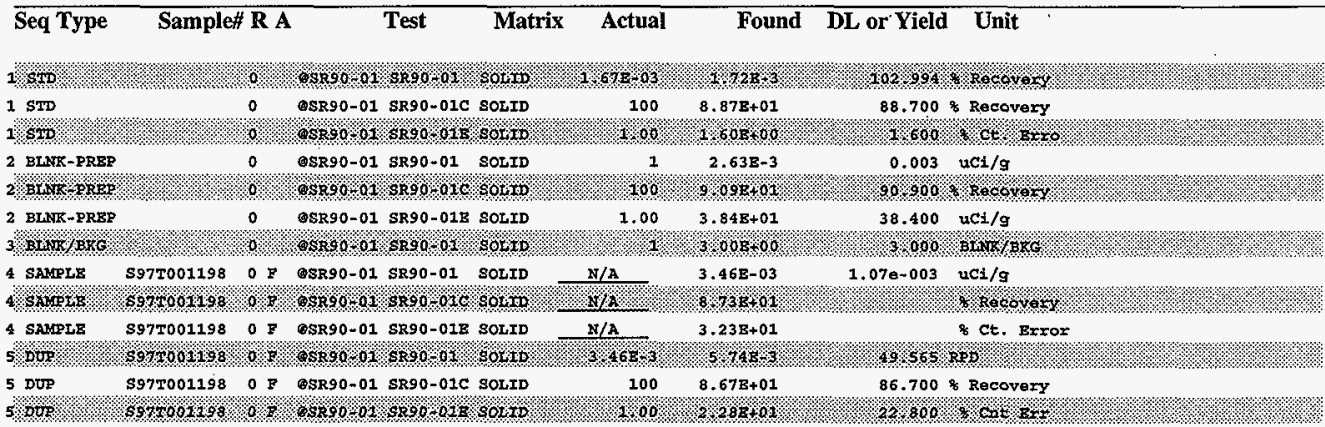

\section{Final page for worklist\# 18756}

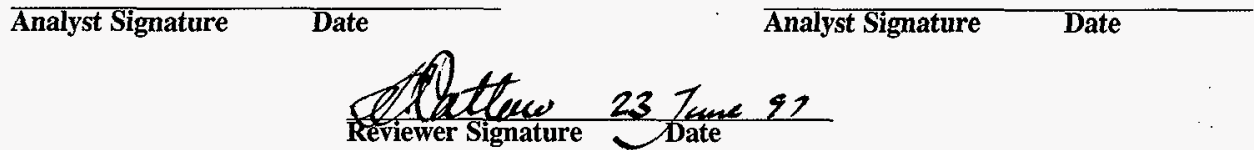

Sample/Dup RPD is high, however, count rates for both are very low with high counting uncertainties. Resalts will be accepted. sae. 


\section{LABCORE Data Entry Template for Worklist\# 18756}

\section{Analyst:}

Instrument: AB00

12

Method: LA-220-101 Rev/Mod

Book\# 751556

HNF-SD-WM-DP-255, REV. 0

Worklist Comment: T204, @SR90-01 S.S. by Ludlum. Std: 1.0mL skm

\begin{tabular}{|c|c|c|c|c|c|c|c|}
\hline$s$ & Type & Sample\# & R A & Test & Matrix & Group\# & Project \\
\hline 1. & STD & & & @SR90-01 & SOLID & & \\
\hline 2 & BLNK-PREP & & & @SR90-01 & SOLID & & \\
\hline 3 & BLNK/BKG & & & @SR90-01 & SOLID & & \\
\hline 4 & SAMPLE & $\begin{array}{l}\text { S97T001198 } \\
\text { Analytes Reque }\end{array}$ & $\begin{array}{l}0 \mathrm{~F} \\
\text { ested }\end{array}$ & $\begin{array}{r}\text { @SR90-01 } \\
\text { d. } \quad \text { SR90-01 }\end{array}$ & $\begin{array}{l}\text { SOLID } \\
\text {, SR90-01C, }\end{array}$ & $\begin{array}{l}97000261 \\
\text { SR90-01E }\end{array}$ & $\mathrm{T}-204$ \\
\hline & DUP & S97T001198 & $0 F$ & @SR90-01 & SOLID & & \\
\hline
\end{tabular}

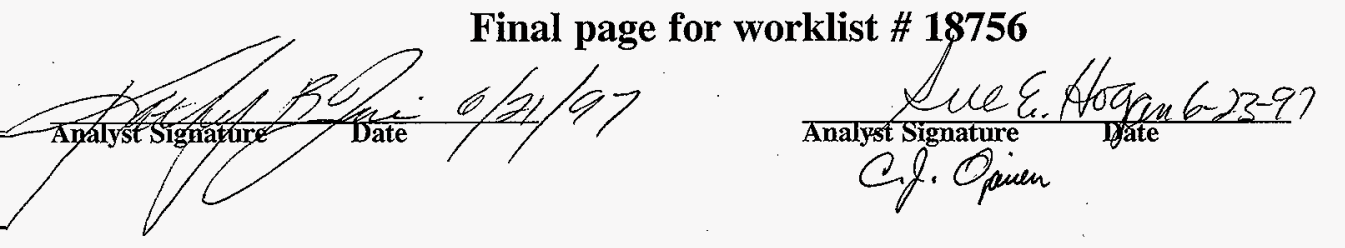

Data Entry Comments:

$S=$ Worklist Slot Number,$R=$ Replicate Number, $A=$ Aliquot Code 
HNF-SD-WMA-DP-255, REV. 0

\section{WORKBOOK PAGE: STD1}

Sr-89/90 : LA-220-101 (D-1), 102 (E-3), 104 (D-1) LIQUIDS

\begin{tabular}{|c|c|c|c|c|}
\hline Non & DETECTOR NUMBER & 12 & CARRIER ADDED in mL & 1.000 \\
\hline STD & TOTAL COUNTS & 15048 & GROSS WEIGHT & 7.1613 \\
\hline Now & COUNT TIME In MINUTES & 10 & TARE WEIGHT & 7.0726 \\
\hline 18766 & BACKGROUND in $\mathrm{cpm}$ & 2.6 & NET WEIGHT & 0.0887 \\
\hline Nathorde & SAMPLE VOLUME in $\mathrm{mL}$ & 1.000 & DELTA TIME (HOURS) & 5.17 \\
\hline$@ S R 90.01$ & DILUTION FACTOR & & & \\
\hline WWatix & DIGEST DILUTION FACTOR & & & \\
\hline LIQUID & SAMPLE COUNT RATE & 1502.20 & SR-90 EFFICIENCY FACTO & 0.4180 \\
\hline Bat 1 WTIn & CRITICAL LEVEL & 0.97 & Y.90 EFFICIENCY FACTOR & 0.4660 \\
\hline 97002839 & TIME OF SEPARATION & $02: 35$ & $\operatorname{Rmax}$ & N/A \\
\hline Wont & DATE OF SEPARATION & $06 / 21 / 97$ & DETECTION LIMIT & 2.03 \\
\hline 0 & TIME OF COUNT & $07: 45$ & Sr-89/90 CONC. in $\mu \mathrm{Ci} / \mathrm{L}$ & $1.7210 \mathrm{E}+00$ \\
\hline STinplerix & DATE OF COUNT & $06 / 21 / 97$ & & \\
\hline N/A & STANDARD BOOK & $75 \mathrm{B56}$ & & \\
\hline S & STANDARD VALUE in $\mu \mathrm{Ci} / \mathrm{mL}$ & $1.6688 \mathrm{E}-03$ & & \\
\hline WL18756 & \\
\hline 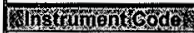 & & & & \\
\hline WB27811 & \multicolumn{4}{|c|}{ Sample Count Rate (Rs) $=($ Total Counts (TC) / Count Time (CT)) - Background in cpm (BKG) } \\
\hline Ropara & \multirow{3}{*}{\multicolumn{4}{|c|}{ 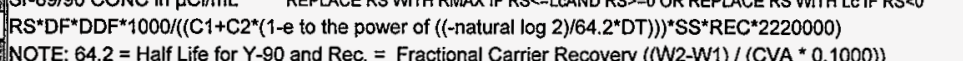 }} \\
\hline SEH & & & & \\
\hline 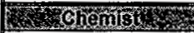 & & & & $.1000))$ \\
\hline SAC & \multicolumn{4}{|c|}{ Relative Counting Error $=$ The Square Root of $\left(\left(\mathrm{TC}+\mathrm{BKG}{ }^{*} \mathrm{CT}\right) /\left(\mathrm{TC}-\mathrm{BKG}^{*} \mathrm{CT}\right)^{* 1.96)}\right.$} \\
\hline 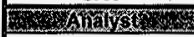 & \multirow{2}{*}{\multicolumn{4}{|c|}{ Percent Carrier Recovery $=($ Net Weight $/$ Expected weight $) * 100$}} \\
\hline KRQ & & & & \\
\hline 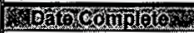 & \multicolumn{4}{|c|}{ Detection Levels and Less Than Values are determined from Procedure LA-508-002. } \\
\hline $06 / 23 / 97$ & \multirow{2}{*}{\multicolumn{3}{|c|}{ Delta Time (hours) $=((\mathrm{DOC}-\mathrm{SO}) * 24)+(\mathrm{TOC}-\mathrm{ST}) / 100$}} & \multirow{4}{*}{$\begin{array}{l}\text { DETECTION } \\
\text { LEVEL }\end{array}$} \\
\hline 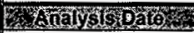 & & & & \\
\hline 06/21/97 & \multicolumn{2}{|l|}{ Sr-89/90 CONCENTRATION } & $1.72 \mathrm{E}-03$ & \\
\hline 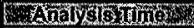 & & \\
\hline $05: 30 \mathrm{AM}$ & \multicolumn{2}{|l|}{ RELATIVE COUNTING ERROR } & $1.6 \%$ & \multirow{3}{*}{$\begin{array}{c}2.33 \mathrm{E}-06 \\
\mu \mathrm{Ci} / \mathrm{L}\end{array}$} \\
\hline SSAm & \multirow{2}{*}{\multicolumn{3}{|c|}{ PERCENT CARRIER RECOVERY }} & \\
\hline$T-204$ & & & & \\
\hline
\end{tabular}

\begin{tabular}{|c|c|c|c|}
\hline Analyst: & KRQ & Date: & 23-Jun-97 \\
\hline Signature of Chemist: & SAC & Date: & Tun 7 \\
\hline
\end{tabular}


HNF-SD-WM-DP-255, REV. 0 teq $-14-97$

WORKBOOK PAGE: BLANK2

LA-220-101 I D-1 Sr-89/90 : LA-220-101 (D-1), 102 (E-3), 104 (D-1)

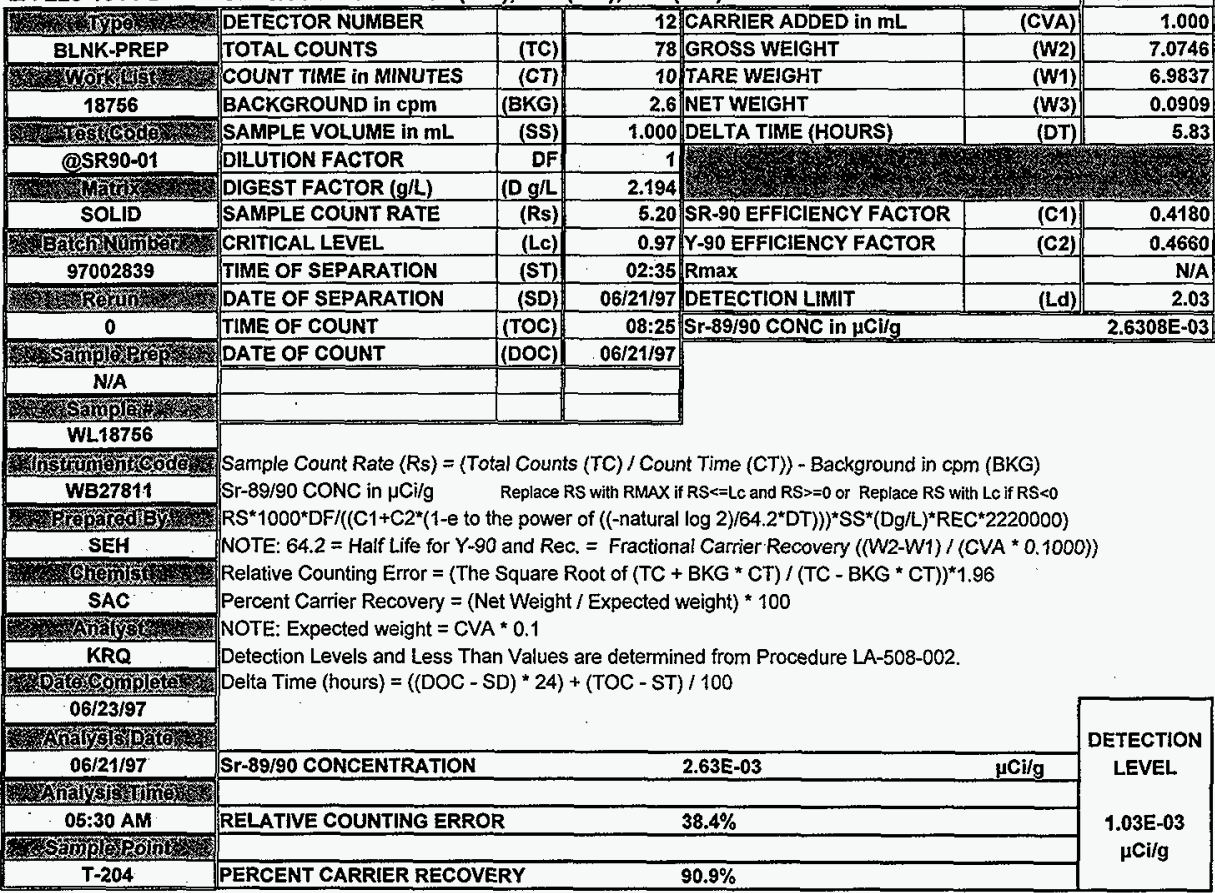

\begin{tabular}{|c|c|c|c|}
\hline Analyst: & KRQ & Date: & 23-Jun-97 \\
\hline Signature of Chemist: & SAC & Date: & 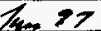 \\
\hline
\end{tabular}




\section{HNF-SD-WM-DP-255, REV. 0}

WORKBOOK PAGE: SAM4

Sr-89/90 : LA-220-101 (D-1), 102 (E-3), 104 (D-1)

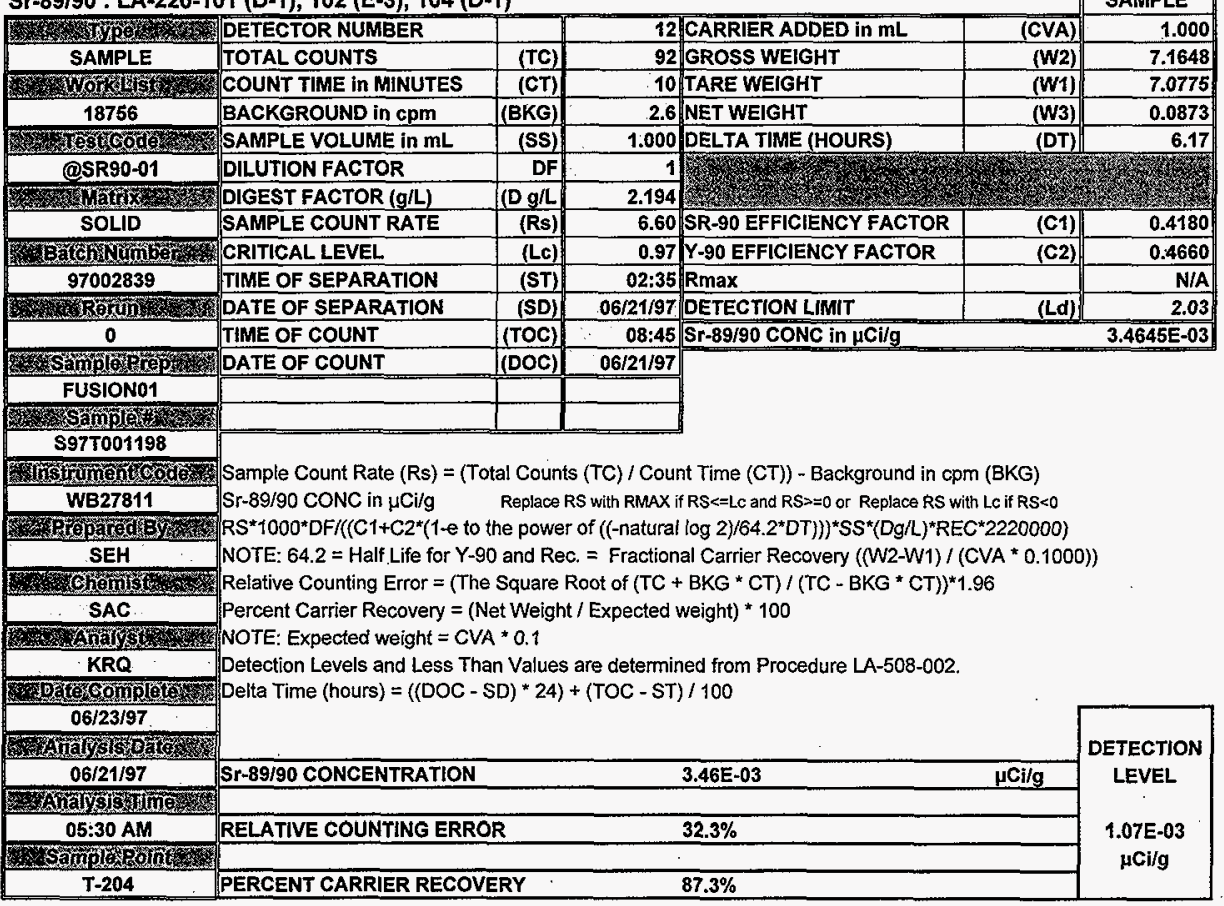

\begin{tabular}{|c|c|c|c|}
\hline Analyst: & KRQ & Date: & 23-Jun-97 \\
\hline Signature of Chemist: & SAC & Date: & $7 a+7$ \\
\hline
\end{tabular}


WORKBOOK PAGE: DUP5

Sr-89/90 : LA-220-101 (D-1), 102 (E-3), 104 (D-1)

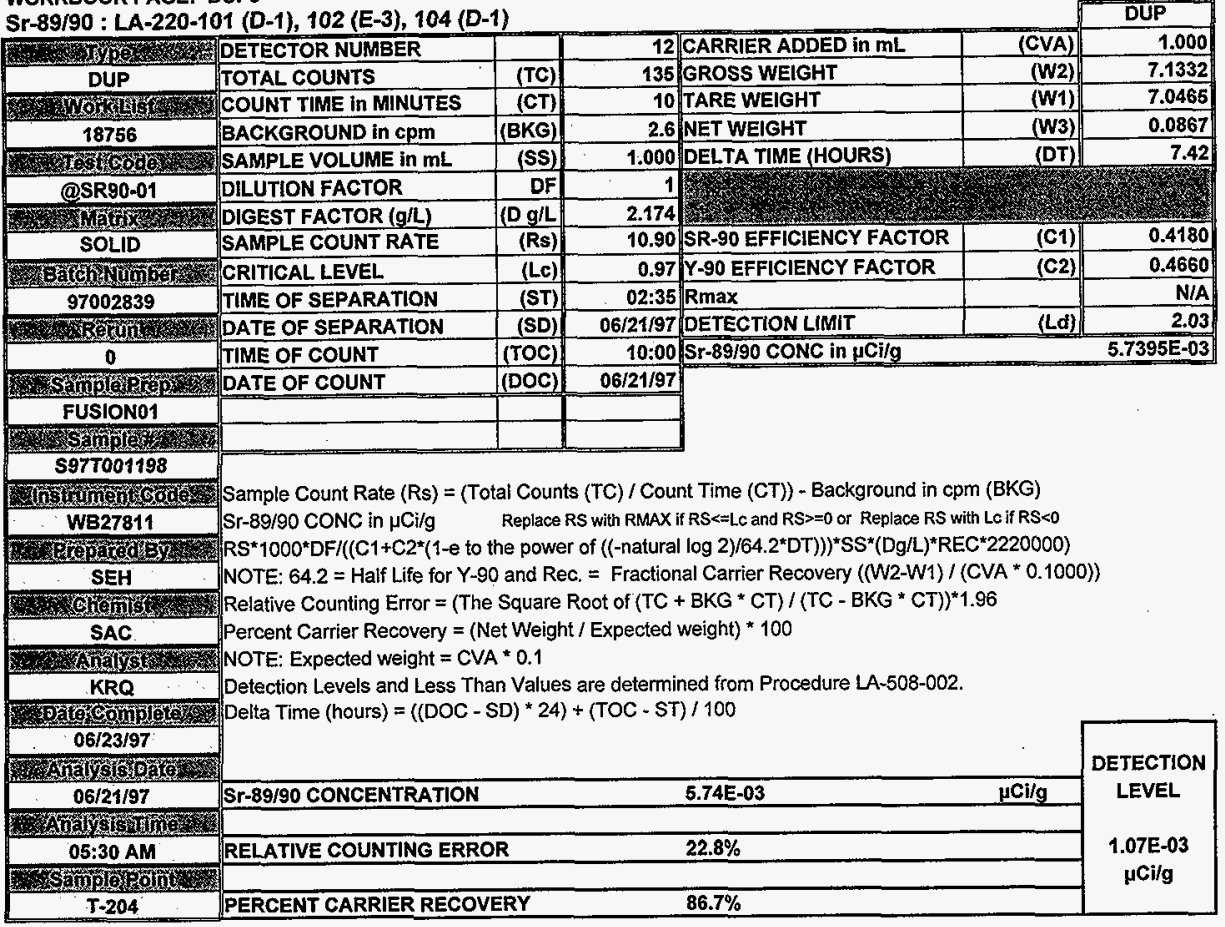

\begin{tabular}{|c|c|c|c|}
\hline Analyst: & KRQ & Date: & 23-Jun-97 \\
\hline Signature of Chemist: & SAC & Date: & $7 \tan 2$ \\
\hline
\end{tabular}

\section{2}




\section{DISTRIBUTION SHEET}

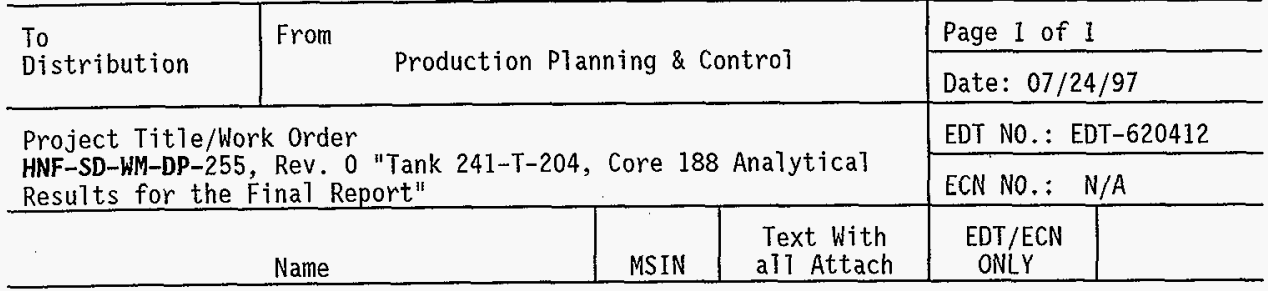

DE\&S Hanford, Inc.

J. E. Meacham S7-14 $\quad X$

U.S. Department of Energy, RL

C. A. Babel

S7-54 $\quad x$

Lockheed Martin Hanford Corp.

J. N. Appe ?

G3-21

K. G. Carothers

R2-11

T. J. Kelley

S7-21

M. J. Kupfer

H5-49

J. B. Schaffer

R2-12

L. A. Stauffer

R2-11

J. A. Voogd

H5- 03

W. D. Winkelman

$\mathrm{R} 2-12$

A. E. Young

R1-10

$X$
$X$

$X$
$X$
$X$
$X$
$X$
$X$
$X$

Lockheed Martin Services, Inc.

Central Files

A3-88

1

Los Alamos Technical Associates

M. .T. Ellsworth

T6-06

$X^{*}$

Pacific Northwest National Laboratory

S. J. Harris

K7-22

$\mathrm{K} 9-08$

$x$

K. L. Silvers

$X$

Waste Management of Hanford, Inc.

D. C. Hetzer

56-31

J. L, Nuzum

K. L. Powell

C. M. Seidel

T6-06

T6-04

T6-04

LTIC

T6-03

$\begin{array}{ll}x & x \\ x & x\end{array}$

SGN Eurisys Services Corp.

B. A. Higley

H5-27

$x$

Defense Nuclear Federal Safety Board

Rich Tontodonato

$x$

625 Indiana Ave. N. W.

Washington D.C. 20004

* Needs only releasing paperwork, not a copy of the released document. 